FHWA/IN/JTRP-2000/20

Final Report

REAL-TIME DETERMINATION OF SOIL TYPE, WATER CONTENT, AND DENSITY USING ELECTROMAGNETICS

Vincent P. Drnevich

Chih-Ping Lin

Quanghee Yi

Xiong Yu

Janet Lovell

August 17, 2001 
Final Report

FHWA/IN/JTRP-2000/20

\title{
REAL-TIME DETERMINATION OF SOIL TYPE, WATER CONTENT, AND DENSITY USING ELECTROMAGNETICS
}

\author{
Vincent P. Drnevich $\square$ \\ Chih-Ping Lin 2 \\ Quanghee $\mathrm{Yi}^{\square}$ \\ Xiong $\mathrm{Yu}^{3}$ \\ Janet Lovel 4 \\ School of Civil Engineering \\ Purdue University \\ Joint Transportation Program \\ Project No. C-35-5T \\ File No. 6-6-20 \\ SPR-2201 \\ Prepared in Cooperation with the \\ Indiana Department of Transportation and \\ The U.S. Department of Transportation \\ Federal Highway Administration
}

The contents of this report reflect the views of the authors who are responsible fo the facts and the accuracy of the data presented herein. The contents do not necessarily reflect the official views or policies of the Federal Highway Administration and the Indiana Department of Transportation. This report does not constitute a standard, specification or regulation.

\author{
Purdue University \\ West Lafayette, Indiana \\ August 17, 2001
}

\footnotetext{
${ }^{1}$ Principal Investigator and Professor of Civil Engineering

${ }^{2}$ Former Graduate Assistant, now Assistant Professor of Civil Engineering, National Chiao Tung Univ., Hinghu, Taiwan

${ }^{3}$ Graduate Assistant

${ }^{4}$ Laboratory Manager, Geotechnical and Materials Laboratories
} 


\section{ACKNOWLEDGEMENTS}

This project was blessed with creative ideas, encouragement, and excellent participation from the appointed Study Advisory Committee led by Tommy E. Nantung (Project Administrator). Peter Capon (Rieth-Riley Construction), Don Johnson (FHWA, Indianapolis), Tom Kuhn (Kuhn Construction), Sam Mansukani (FHWA, Chicago), Wes Shaw (INDOT, Crawfordsville District), and Nyyar Zia (INDOT, Division of Materials and Test) all were very helpful and supportive. The authors wish to acknowledge the comments and suggestions of past graduate students Dr. Wei Feng and Dr. Shafiq Siddiqui, the work of Mr. Jie Zhang who performed the tests upon which temperature effects were based, and undergraduate student Vincent Sovis who helped with some of the programming. Dr. Jody Tishmack (Purdue's Ash Management Coordinator) provided significant understanding, help, and ideas to the authors, who are most grateful. The authors are most appreciative of the leadership provided by Dr. Kumares Sinha (Director of the JTRP), Donald Lucas (recently retired Chief Engineer, INDOT), and Firooz Zandi (Acting Chief Engineer). Project personnel enjoyed exceptional service from Karen S. Hatke (Program

Manager, JTRP). Finally, the authors are most appreciative of FHWA/INDOT/JTRP for the support of this project. 
TECHNICAL REPORT STANDARD TITLE PAGE

\begin{tabular}{|c|c|c|}
\hline $\begin{array}{l}\text { 1. Report No. } \\
\text { FHWA/IN/JTRP-2000/20 }\end{array}$ & 2. Government Accession No. & 3. Recipient's Catalog No. \\
\hline \multirow{2}{*}{\multicolumn{2}{|c|}{$\begin{array}{l}\text { 4. Title and Subtitle } \\
\text { Real-Time Determination Of Soil Type, Water Content, and Density Using } \\
\text { Electromagnetics }\end{array}$}} & $\begin{array}{l}\text { 5. Report Date } \\
\text { August } 17,2001\end{array}$ \\
\hline & & 6. Performing Organization Code \\
\hline \multicolumn{2}{|c|}{$\begin{array}{l}\text { 7. Author(s) } \\
\text { Vincent Drnevich, Chih-Ping Lin, Quanghee Yi, Janet Lovell }\end{array}$} & $\begin{array}{l}\text { 8. Performing Organization Report No. } \\
\text { FHWA/IN/JTRP-2000/20 }\end{array}$ \\
\hline \multirow{2}{*}{\multicolumn{2}{|c|}{$\begin{array}{l}\text { 9. Performing Organization Name and Address } \\
\text { Joint Transportation Research Program } \\
\text { 1284 Civil Engineering Building } \\
\text { Purdue University } \\
\text { West Lafayette, Indiana } 47907-1284\end{array}$}} & 10. Work Unit No. \\
\hline & & $\begin{array}{l}\text { 11. Contract or Grant No. } \\
\text { SPR-2201 }\end{array}$ \\
\hline \multirow{2}{*}{\multicolumn{2}{|c|}{$\begin{array}{l}\text { 12. Sponsoring Agency Name and Address } \\
\text { Indiana Department of Transportation } \\
\text { State Office Building } \\
100 \text { North Senate Avenue } \\
\text { Indianapolis. IN } 46204\end{array}$}} & $\begin{array}{l}\text { 13. Type of Report and Period Covered } \\
\text { Final Report }\end{array}$ \\
\hline & & 14. Sponsoring Agency Code \\
\hline
\end{tabular}

15. Supplementary Notes

Prepared in cooperation with the Indiana Department of Transportation and Federal Highway Administration.

\section{Abstract}

The primary objective of this study was to explore the potential use of electromagnetic characteristics of soils using the Time Domain Reflectometry (TDR) technique to identify physical properties of soils. Three fundamental studies in this exploration are the frequencydependent electromagnetic properties of soils, the wave propagation in a TDR system, and the inverse analyses of TDR waveforms. The three-phase model for soils was extended to a four-phase model so as to account for the interface effect and hence the soil fineness. The physical parameters of the four-phase model are related to the frequency-dependent dielectric permitivity of the soil through a semiempirical volumetric mixing model. A spectral analysis method was developed to simulate wave propagation in the TDR system in a realistic way. Inverse analysis based on the material model and the wave propagation model was formulated using Bayesian statistics to calibrate the TDR system and to infer material properties from the measured TDR waveform. A TDR probe system was used to measure the dielectric properties of soils in a compaction mold and in the field. An experimental program was carried out to make TDR measurements of 5 different soils with different water contents and densities. The result of the inverse analysis matched the TDR waveform very well and determined the dielectric spectrum of soils. With the ability to measure the dielectric spectrum of soils, the apparent dielectric constant at a particular frequency can be calculated. Results showed that the apparent dielectric constant at $1 \mathrm{GHz}$ gave much better correlation with soil water content and density than the apparent dielectric constant obtained by measuring the time between reflections from the soil surface and the one from the end of the probe. Additional work was done to assess the effects of temperature of the soil on measured dielectric constant and it showed that temperature effects were relatively small and could be accounted for in a systematic way. The use of the method for soils having large particle sizes was examined and it was found that the method was applicable for particle sizes passing the $19 \mathrm{~mm}(3 / 4-i n$.) sieve as long as the percentage of particles in the larger size range was limited. Finally, the method was examined for use with stabilizing additives for soil. While test results were obtained modified soils, the measured apparent dielectric constant changed with time after compaction whereas the water content by oven drying did not change appreciably. It is conjectured that changes in apparent dielectric constant are sensitive to the hydration caused by these additives. Thus, the use of the TDR method can be a tool to monitor changes in soils containing additives. Detailed study of this phenomenon is continuing.

\section{Key Words}

Time Domain Reflectometry, electromagnetic wave propagation, soils, gravels, sands, silts, clays, water content, compaction, density, dry density, apparent dielectric constant, permitivity, conductivity.

\begin{tabular}{|c|c|c|l|}
\hline $\begin{array}{c}\text { 19. Security Classif. (of this report) } \\
\text { Unclassified }\end{array}$ & $\begin{array}{c}\text { 20. Security Classif. (of this page) } \\
\text { Unclassified }\end{array}$ & $\begin{array}{c}\text { 21. No. of Pages } \\
320\end{array}$ & 22. Price \\
\hline
\end{tabular}




\section{TECHNICAL Summary}

INDOT Research

Technology Transfer and Project Implementation Information

TRB Subject Code: 62-6 Soil Compaction and Stabilization

August 2001

Publication No.: FHWA/IN/JTRP-2000/20, SPR-2201

Final Report

\section{Real-Time Determination of Soil Type, Water Content, and Density Using Electromagnetics}

\section{Introduction}

This study explored the electromagnetic characteristics of soils using the Time Domain Reflectometry (TDR) technique to identify their physical properties. It is the third in a series of projects supported by the Joint Transportation Research Program at Purdue University. The first was to examine feasibility of TDR for measurement of soil water content in conjunction with field density testing for construction control. A major breakthrough occurred in this project; a technique was developed that made it possible to measure both water content and density. The procedure was written up, published, and is the source of three U.S. patents (Patent No. 5,801,537, September 1, 1998, U.S. Patent No. 5,933,015, August 3, 1999, and one about to be issued).

The second project focused on developing an automated procedure for this test and for creating a draft ASTM Standard for the method. The automated, computerbased procedure is called $\mathrm{TDR}^{++}$and a draft standard was developed. The second project also developed procedures to remove the effects of the apparatus and cables.

\section{Findings}

Three fundamental studies in this exploration are the frequency-dependent electromagnetic properties of soils, the wave propagation in a TDR system, and the inverse analyses of TDR waveforms. The three-phase model for soils was extended to a four-phase model so
This project focused on obtaining a rational mathematical model for the propagation of an electromagnetic wave in the soil specimen so that the model could be used to better understand the testing process and provide more information about the soil being tested. An expansion to this project focused on examining the effects of temperature and establishing the validity of the method for soils with large particle sizes and for soils with additives like fly ash, lime, etc.

The TDR method developed by this research is totally new and a radical departure from the current procedures used in geotechnical practice for measuring water content and density for soil. The techniques developed as part of these research projects is becoming known as the Purdue TDR Method. It is generating widespread interest. Project personnel produced thirteen technical publications and project reports and three dissertations as part of these research projects. 
spectral analysis method was developed to simulate wave propagation in the TDR system in a realistic way. Inverse analysis based on the material model and the wave propagation model was formulated using Bayesian statistics to calibrate the TDR system and to infer material properties from the measured TDR waveform.

A TDR probe system was designed to measure the dielectric properties of soils in a compaction mold and in the field. An experimental program was carried out to make TDR measurements of 5 different soils with different water contents and densities. The result of the inverse analysis matched the TDR waveform very well and determined the dielectric spectrum of soils. With the ability to measure the dielectric spectrum of soils, the apparent dielectric constant at a particular frequency can be calculated. Results showed that the apparent dielectric constant at $1 \mathrm{GHz}$ gave much better correlation with soil water content and density than the apparent dielectric constant obtained by traditional method.

Temperature of the soil at the time of test has an effect on the measured apparent dielectric constant, and hence, measured water content. The effect is quite small for cohesionless soils and generally can be ignored for test temperatures of $20^{\circ} \mathrm{C} \pm 5^{\circ} \mathrm{C}$. A recommended Temperature Correction Function for use with all cohesionless soils will provide sufficient accuracy for test temperatures between $4^{\circ} \mathrm{C}$ and $40^{\circ} \mathrm{C}$.

This research also confirmed a recently identified "anomaly" in the behavior with temperature for cohesive soils where the apparent dielectric constant increases with temperature rather than decreasing as it does for pure water and cohesionless soils containing water. The source of this behavior is the subject of current research in the TDR community.

Similar to that for cohesionless soils, a Temperature Correction Function also was generated for cohesive soils. This function corrects the values of apparent dielectric constant at the temperature of the soil at the time of test to values at a temperature of $20^{\circ} \mathrm{C}$, assuming that the temperature at the time of test is between $4^{\circ} \mathrm{C}$ and $40^{\circ} \mathrm{C}$. Since the apparent dielectric constant for ice is significantly different from water, the TDR method is not applicable for testing frozen soils.

For the TDR method to be practical, it must be accurate for wide range of soil types and for soils with larger particle sizes. It was found that the method was applicable for soils having a maximum particle size of 19 $\mathrm{mm}$ (3/4-in.) as long as the percentage of soil particles in the size range from $9.5 \mathrm{~mm} \mathrm{(3/8-}$ in.) to $19 \mathrm{~mm}(3 / 4-i n$.$) is less than twenty$ percent and the percentage with sizes greater than the No. 4 sieve is less than fifty percent. These are the same particle size restrictions associated with the Standard Compaction Test.

Use of the TDR method for soils with additives appears promising, but is not as simple to address as was originally expected. Introduction of additives like cement, fly ash, and lime generally cause hydration to occur. The hydration process removes some of the free water and it is exothermic. The dielectric properties of hydrated water appear to be different from free water. Because the TDR method is relatively fast and non destructive, it has the ability to monitor changes in the modified soil with time after addition of the additives and compacting the mixture.

\section{Implementation}

A follow-on project, SPR 2489, started in September 2000 that will involve twelve agencies/firms/universities around the country in a joint effort to Beta Test the Purdue TDR Method for a variety of soils.
The list includes the U.S. Army Corps of Engineers, the U.S. Bureau of Reclamation, several state DOT's, three universities, and six engineering consulting firms. 
A draft ASTM Standard on the method, developed as part of the previous and present projects, is in the balloting process and has been submitted to the appropriate AASHTO Standards Committee. A relationship has been established with a major international supplier of TDR equipment to provide custom TDR electronics and software for this method. The Beta Testing project will make use of this equipment.

\section{Contact}

For more information:

Prof. Vincent Drnevich

Principal Investigator

School of Civil Engineering

Purdue University

West Lafayette, IN 47907

Phone: (765) 494-5029

Fax: (765) 496-1364
There is an international symposium TDR2001 that will be held in September 5-7, 2001 at Northwestern University in Evanston, Illinois. The Purdue TDR Group will have four papers in that symposium and will be putting on a half-day workshop on the Purdue TDR Method for Measuring Water Content and Density of Soil.
Indiana Department of Transportation

Division of Research

1205 Montgomery Street

P.O. Box 2279

West Lafayette, IN 47906

Phone: (765) 463-1521

Fax: (765) 497-1665

\section{Purdue University}

Joint Transportation Research Program

School of Civil Engineering

West Lafayette, IN 47907-1284

Phone: (765) 494-9310

Fax: (765) 496-1105 


\section{TABLE OF CONTENTS}

LIST OF TABLES

\section{CHAPTER 1 - INTRODUCTION}

1.1 Overview of TDR Research at Purdue Supported by the JTRP ................................... 1

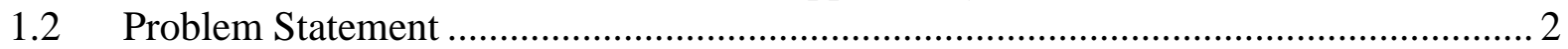

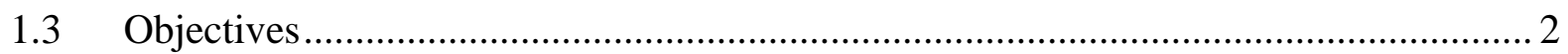

1.4 Scope of Work

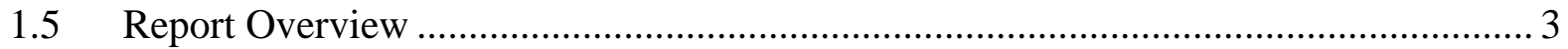

\section{CHAPTER 2 - ELECTROMAGNETIC PROPERTIES OF SOILS}

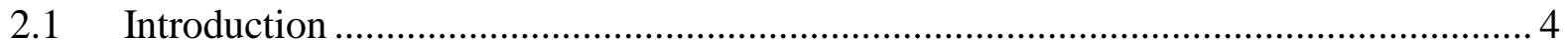

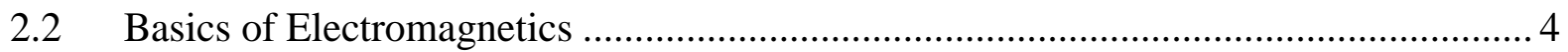

2.2.1 Governing Equations of Electromagnetics....................................................... 4

2.2.2 Constitutive Relations of Electromagnetics ..................................................... 6

2.2.3 Spectral Analysis of Electromagnetics ......................................................... 8

2.3 Dielectric Properties of Homogeneous Materials ....................................................... 9

2.3.1 Characteristics of Dielectrics in a Static Field ............................................... 10

2.3.2 Polarization Mechanisms ……………………………................................... 16

2.3.3 Characteristics of Dielectrics in a Time-Varying Field..................................... 18

2.3.4 Conductivity …………………………………................................... 24

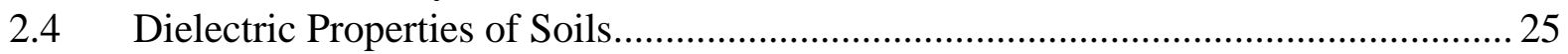

2.4.1 Dielectric Properties of Earth Materials …………………………………... 25

2.4.1.1 Dielectric Properties of Pure Water................................................... 26

2.4.1.2 Dielectric Properties of Saline Water................................................... 27

2.4.2 Interfacial Polarization Mechanisms …………………………………….... 28

2.4.3 Dielectric Spectrum of Soils ......................................................................... 30

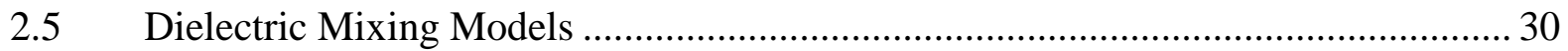

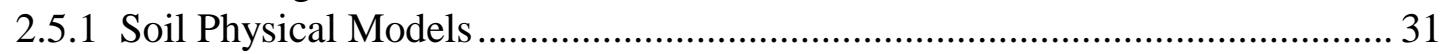

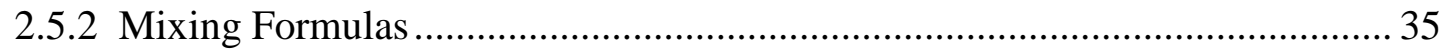

2.5.2.1 Theoretical Polarization Models ……………..................................... 35

2.5.2.2 Semi-empirical Volumetric Mixing Models .......................................... 37

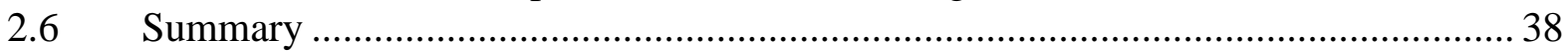

\section{CHAPTER 3 - ANALYSES OF TDR SYSTEMS}

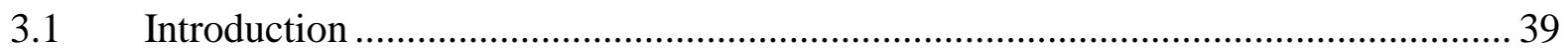

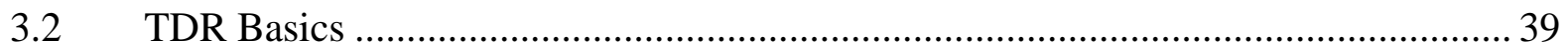

3.2.1 System Analysis of TDR Waveform ............................................................. 41 
3.2.2 Simplified Analysis.

3.2.2.1 DC Analysis

3.2.2.2 Travel Time Analysis ....... 43

3.2.2.3 Amplitude Attenuation Analysis.....

.

3.3 Advanced Analysis of Wave Propagation in the TDR System .................................. 47

3.4 Transmission Line Equations ................................................................................. 50

3.4.1 Basic Assumptions …………………………………………………..... 51

3.4.1.1 Transverse ElectroMagnetic (TEM) Mode ………........................... 51

3.4.1.2 Uniform Transmission Line ............................................................. 51

3.4.1.3 Perfect Conductor or Conductors of Small Losses ............................ 52

3.4.2 Plane Wave Equation ...............................................................................52

3.4.3 Transmission Line Equations of a Two-Conductor Line ………………….....56

3.4.4 Transmission Line Equations of a Multi-Conductor Line................................. 63

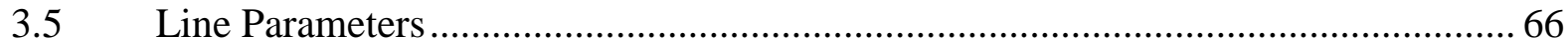

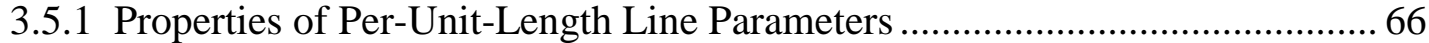

3.5.2 Calculation and Calibration of Line Parameters …………………………........6 68

3.6 Spectral Analysis of Wave Equation of a Transmission Line................................... 70

3.6.1 Spectral Analysis of the Wave Equation ........................................................ 70

3.6.1.1 General Solution of the Wave Equation............................................. 70

3.6.1.2 Terminal Condition and Particular Solutions....................................... 73

3.6.1.3 Input Impedance Method..................................................................... 74

3.6.1.4 ABCD Matrix Method ...................................................................... 76

3.6.2 Spectral Analysis of Non-Uniform Transmission Line.................................... 78

3.6.2.1 Input Impedance Method.................................................................. 80

3.6.2.2 ABCD Matrix Method ....................................................................... 80

3.6.2.3 Interpretation of Wave Propagation ................................................. 81

3.7 Simulation of the TDR Waveform ....................................................................... 85

3.7.1 Response of a Linear System to Aperiodic Signals.......................................... 86

3.7.2 Practical Issues of the Numerical Implementation .......................................... 89

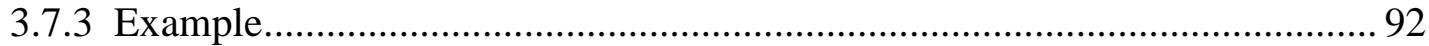

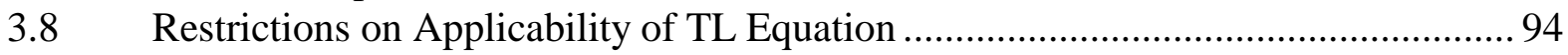

3.8.1 Higher Order Mode .................................................................................. 94

3.8.2 Discontinuity Effect ……………………………….................................95

3.8.3 Imperfect Conductor.................................................................................. 96

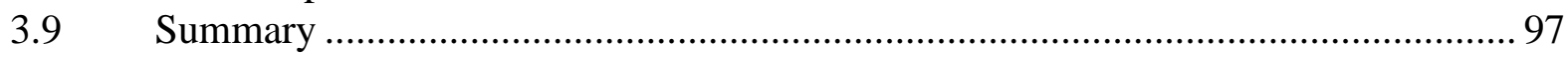

\section{CHAPTER 4 - INVERSE ANALYSIS OF THE TDR SYSTEM}

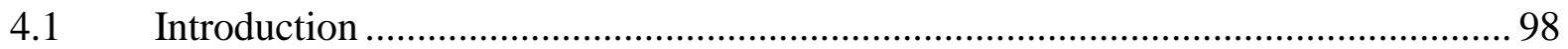

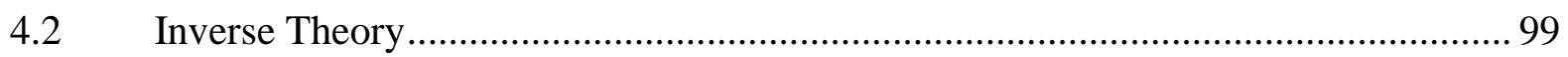

4.2.1 Definition of the Inverse Problem ............................................................... 99

4.2.2 Methods of Solution .................................................................................. 99

4.2.2.1 Direct Inversion ......................................................................... 99

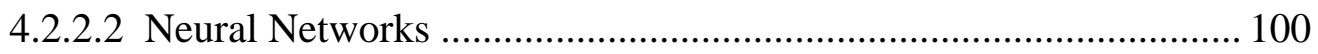


Page

4.2.2.3 Inversion Based on a Forward Model ............................................ 102

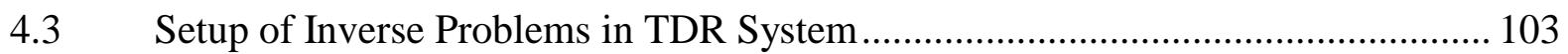

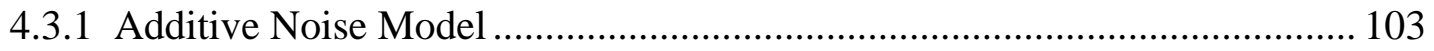

4.3.2 Interpretation of the Stochastic System......................................................... 104

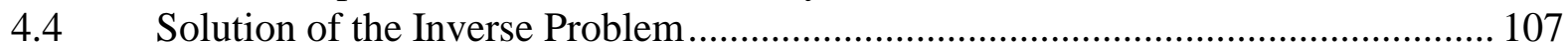

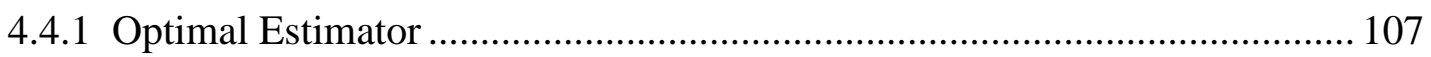

4.4.2 Uncertainty Analysis ............................................................................ 110

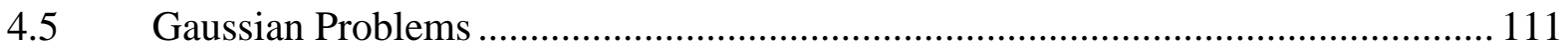

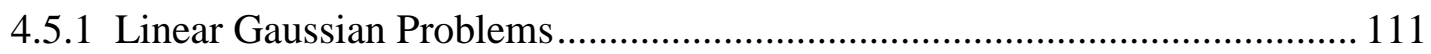

4.5.1.1 Optimal Estimator ............................................................................ 111

4.5.1.2 Uncertainty Analysis (Model Covariance) ........................................ 113

4.5.1.3 Model Resolution Operator ............................................................... 116

4.5.1.4 Data Covariance and Resolution ................................................... 117

4.5.2 Nonlinear Gaussian Problem................................................................... 119

4.5.2.1 Optimal Estimator ......................................................................... 119

4.5.2.2 Uncertainty and Resolution Analysis .............................................. 119

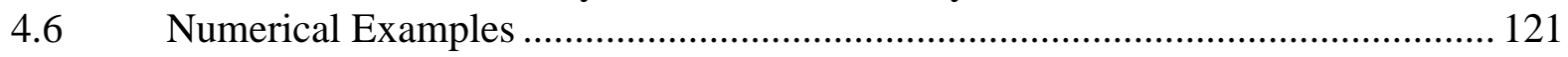

4.6.1 Inversion of an Impedance Profile ............................................................ 122

4.6.2 Inversion of Mixing Model Parameters........................................................ 122

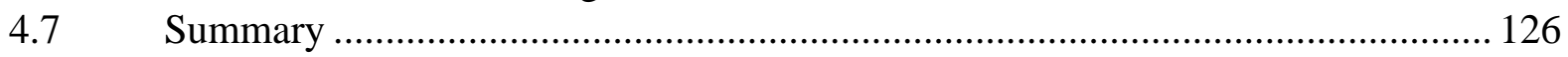

\section{CHAPTER 5 - MEASUREMENT OF DIELECTRIC PROPERTIES}

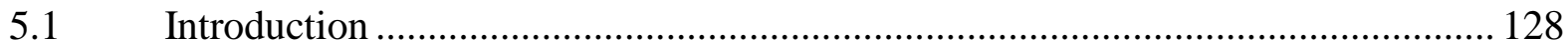

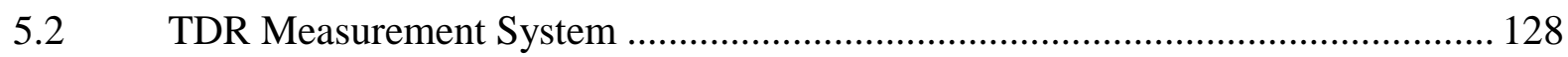

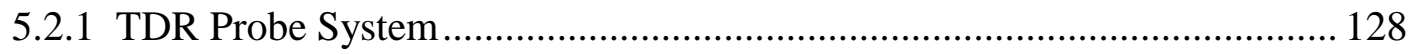

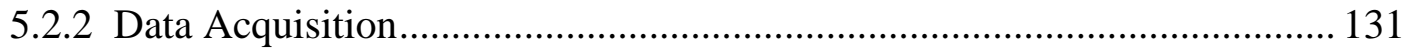

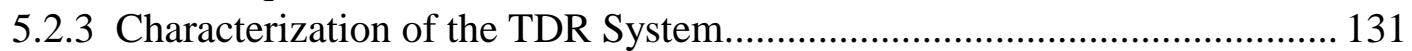

5.2.4 Data Error and Bandwidth of the TDR System......................................... 133

5.3 Calibration of the TDR System Parameters ......................................................... 139

5.3.1 Front Panel Aberration ............................................................................ 140

5.3.2 Line Parameters of the Coaxial Cable .......................................................... 142

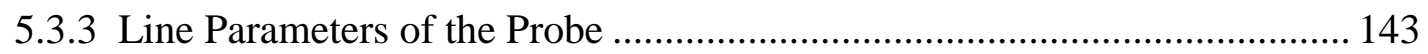

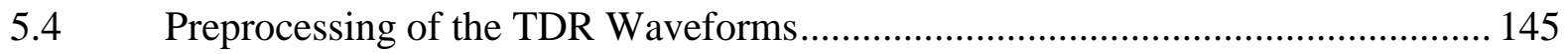

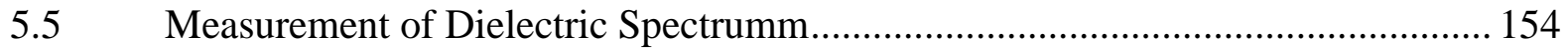

5.5.1 Measurement by Solving the Scattering Function......................................... 154

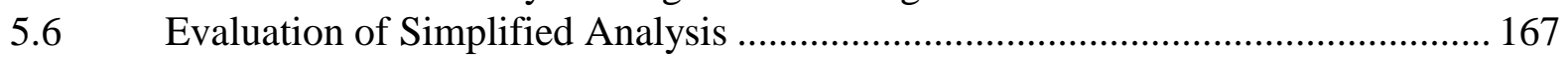

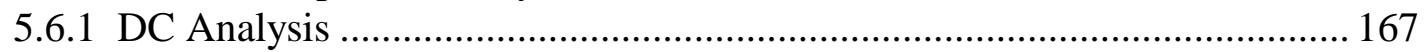

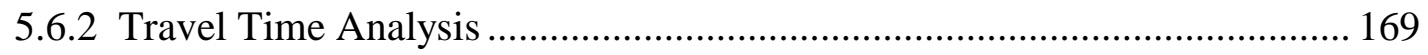

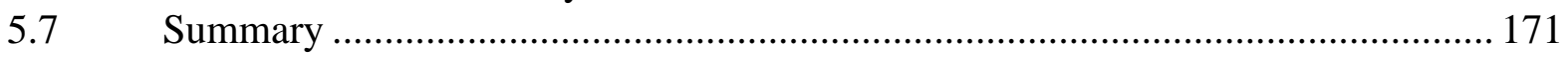




\section{CHAPTER 6 - EXPERIMENTAL STUDY OF SOIL PROPERTIES}

$6.1 \quad$ Introduction $\quad$ Page

6.2 Measurements of TDR Waveforms on Soils................................................... 175

6.2.1 Materials and Methods ........................................................................... 175

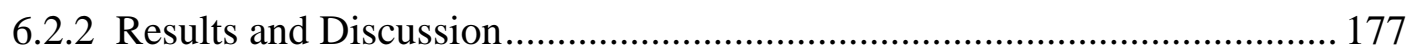

6.3 Soil Properties by Simplified Analysis ....................................................... 180

6.3.1 Apparent Dielectric Constant ............................................................... 180

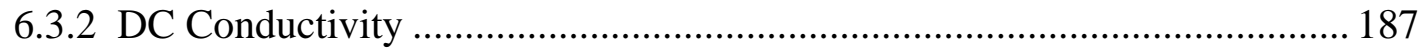

6.4 Soil Properties by the Full Waveform Analysis .............................................. 192

6.4.1 Sensitivity Analysis of the Volumetric Mixing Model ............................. 193

6.4.2 Dielectric Spectrum by Dielectric Mixing Model ..................................... 199

6.4.3 Apparent Dielectric Constant Revisited .................................................... 206

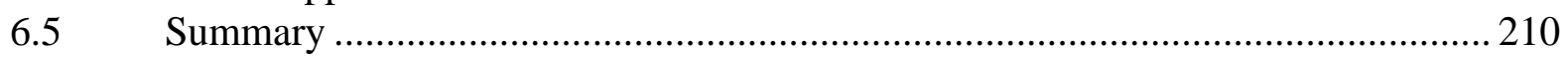

\section{CHAPTER 7 - TEMPERATURE EFFECTS}

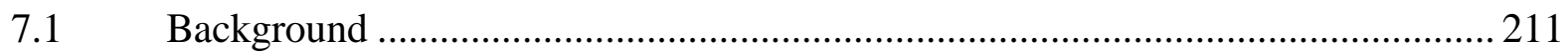

Test Procedures and Test Results ............................................................... 212

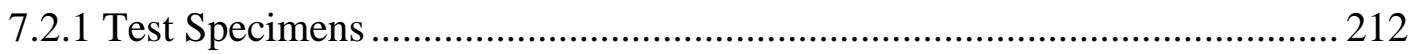

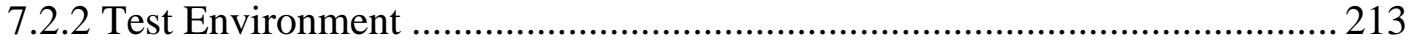

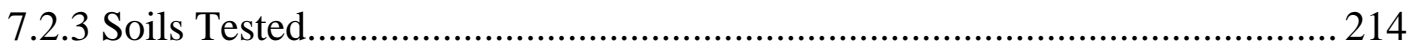

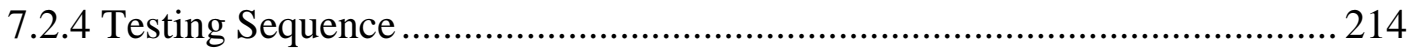

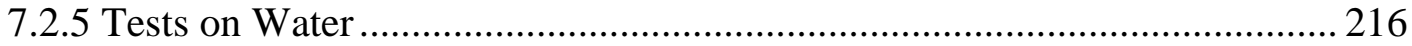

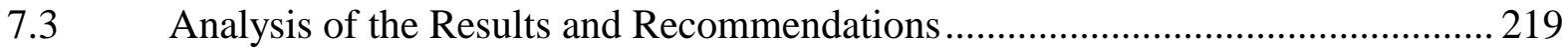

7.3.1 Normalized Apparent Dielectric Constant ............................................ 219

7.3.2 Effects of Water Content on Behavior of Cohesive Soils ........................ 220

7.3.3 Temperature Adjustments to Measured Values of Apparent Dielectric

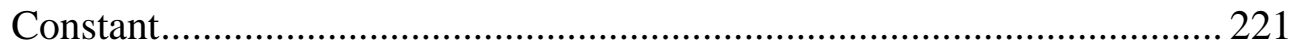

Summary and Conclusions ...................................................................... 222

\section{CHAPTER 8 - PARTICLE SIZE LIMITATIONS}

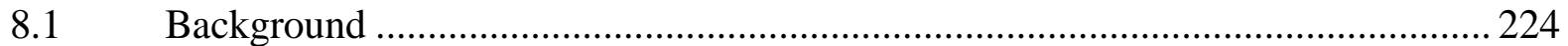

8.2 Materials and Methods ................................................................................ 224

8.2.1 Water Content by Oven Drying ............................................................. 224

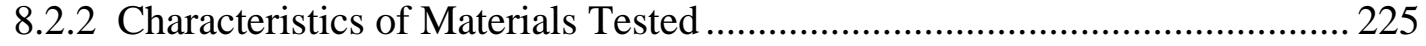

8.2.3 Determination of Soil-Specific Constants, $a$ and $b$.............................. 225

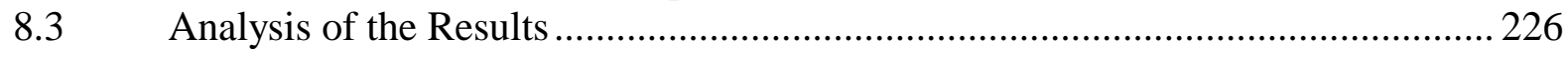

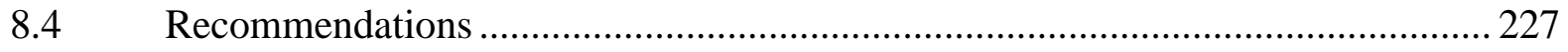

\section{CHAPTER 9 - SOIL ADDITIVES}

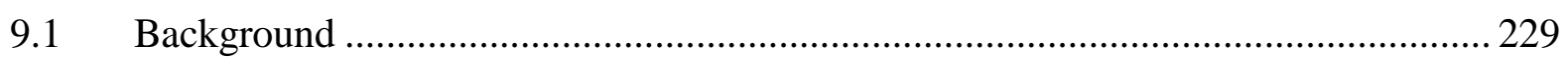

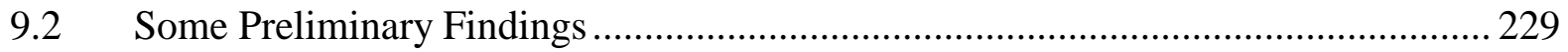


9.3 Page

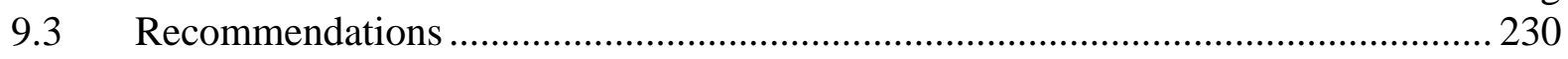

\section{CHAPTER 10 - SUMMARY, CONCLUSIONS, AND RECOMMENDATIONS}

10.1 Summary and Conclusions ................................................................................. 232

10.1.1 Fundamental Studies of Electromagnetic Wave Propagation ...................... 232

10.1.2 Effects of Temperature, Particle Size and Soil Additives ........................... 234

10.2 Recommendations for Further Research ........................................................ 235

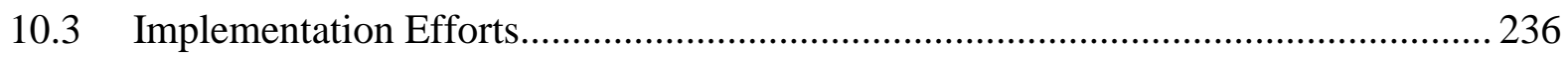

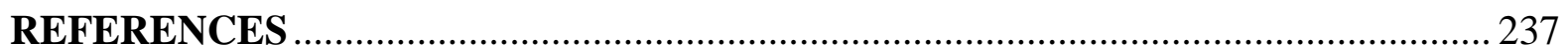

\section{APPENDICES}

A1. List of Presentations Resulting from this Research.............................................. 245 


\section{LIST OF TABLES}

Table

Page

2.1 Notation of electromagnetic field quantities ................................................. 5

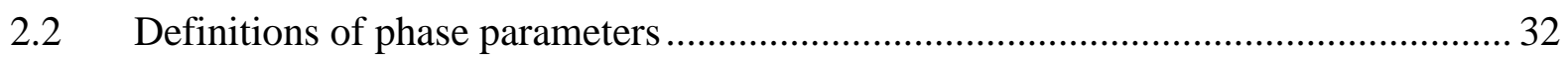

2.3 Three-Phase Relation Described by $\rho_{\mathrm{s}}$, water content and density of soil $\left(\mathrm{V}_{\mathrm{f}}=1\right) \ldots \ldots .32$

2.4 Four-Phase Relation Described y $A_{c}, \rho_{s}$, water content and density of soil $\left(V_{f}=1\right) \ldots .35$

4.1 Relationship between Linear Gaussian problem and Linear Algebraic solutions..... 114

4.2 Covariance of the Linear Algebraic Solutions ................................................ 115

4.3 Summary of the random vectors in the linear Gaussian inverse problem ............... 118

4.4 The inverse solution of the impedance profile ............................................... 122

4.5 Assumed values of dielectric parameters of soil components............................. 126

4.6 The inverse solution of the mixing model parameters ........................................ 126

5.1 The prior and estimated values of the line parameters of the coaxial cable............ 143

5.2 The prior and estimated values of the line parameters for the coaxial probe........... 144

5.3 Comparison of the measured and predicted TDR waveforms measured in liquids .. 145

5.4 Estimation of the dielectric parameters for the aqueous samples ......................... 164

5.5 The assumed values of volumetric mixing parameters ..................................... 165

5.6 Estimation of Debye parameters and conductivity from the fabricated TDR waveform generated by volumetric mixing model............................................ 165

5.7 The Simplified dc analysis of the aqueous samples .......................................... 169

6.1 Percentage of three components of mixed soils .............................................. 176

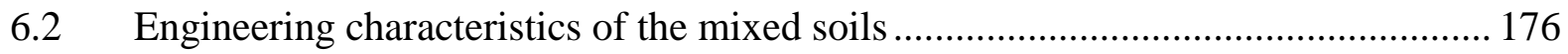

6.3 Results of regression analysis for the constants in Eq. (6.9) ............................... 190 
6.4 Results of regression analysis for the mixed soils using Eq. (6.10) ....................... 192

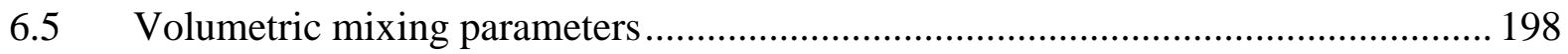

6.6 The prior and estimated values of the volumetric mixing parameters .................... 205

6.7 Matrix of coefficient of correlation for the volumetric mixing model.................... 205

6.8 Resolution matrix for the volumetric mixing model ........................................... 205

6.9 Results of parameter calibration for mixed soil M1 ........................................ 207

6.10 Results of parameter calibration for mixed soil M2 ...................................... 207

6.11 Results of parameter calibration for mixed soil M3 ........................................ 208

6.12 Results of parameter calibration for mixed soil M4 .......................................... 208

6.13 Results of parameter calibration for mixed soil M1 ............................................ 209

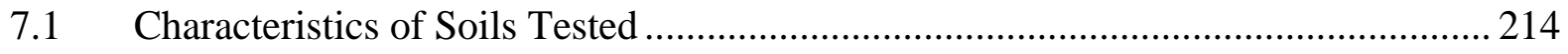

7.2 Testing Program and Results of Tests on Soils .............................................. 218

8.1 Compositions in Percent by Weight and Comparison of $a, b$, and $R^{2} \ldots \ldots \ldots \ldots \ldots \ldots . . . . .225$ 


\section{LIST OF FIGURES}

Figure $\quad$ Page

2.1 Electrical field and potential of a dipole of charge (modified from Krauss, 1984)..... 11

2.2 Uniform polarization of a dielectric material ..................................................... 12

2.3 A dielectric with a non-uniform polarization .................................................... 13

2.4 Polarization of the atoms of a dielectric by a pair of equal positive charges (modified from Ramo et al., 1994) ........................................................................ 15

2.5 (a) A symmetrical molecule/atom has no permanent polarization.

(b) An external electric field induces a polarization in the molecule/atom

2.6 (a) The water molecule, $\mathrm{H}_{2} \mathrm{O}$, has a permanent polarization resulting from its bent geometry, (b) Randomly oriented water dipole, (c) Dipole alignment with the electric field

2.7 Equivalent mechanical model of the electronic polarization (Krauss, 1984)............. 20

2.8 Frequency response of permittivity and loss factor for a hypothetical dielectric showing various contributing phenomena (Ramo, et al., 1994).............................. 22

2.9 Polarization mechanisms occurring in soils (Santamarina and Fam, 1997).............. 29

2.10 Qualitative representation of dielectric properties of wet soils as a function of frequency (modified from Hilhorst and Dirkson, 1994) ........................................ 30

2.11 Soil phase diagram (three phases) where $V$ is volume, $M$ is mass and $\rho$ is density for each phase.

2.12 Soil phase diagram (four phases) where $V$ is volume, $M$ is mass and $\rho$ is density for each phase.

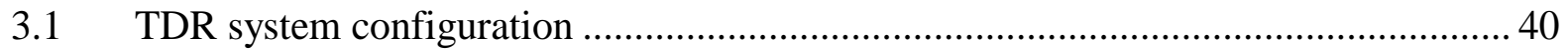

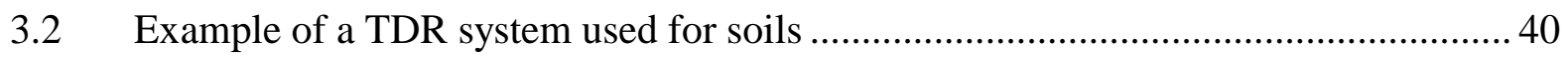

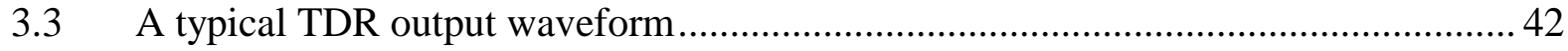

3.4 Direct interpretation of the TDR waveform to estimate for dielectric permittivity $\varepsilon_{\mathrm{s}}$ and dc activity $\sigma_{\mathrm{dc}}$ 
Figure

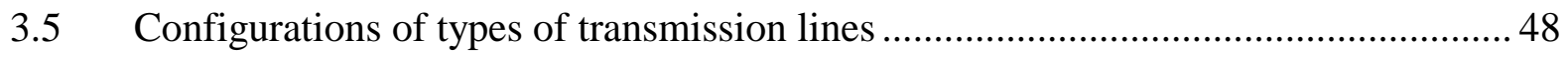

3.6 Components of a transmission line in the TDR system ...................................... 49

3.7 Illustration of the TEM mode of propagation ...................................................... 53

3.8 Contours and surfaces for the derivation of transmission-line equation for two-conductor transmission lines: (a) longitudinal plane, (b) transverse plane (modified from Paul, 1994)

3.9 The per-unit length equivalent circuit model, where $1, \mathrm{~g}$, and $\mathrm{c}$ are the per-unit-length inductance, conductance, and capacitance, respectively

3.10 Contours and surfaces for the derivation of transmission-line equation for multi-conductor transmission lines: (a) longitudinal plane, (b) transverse plane (modified from Paul, 1994)

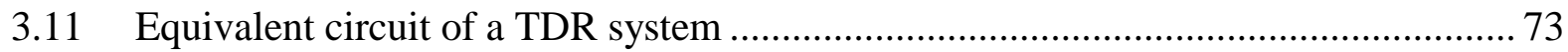

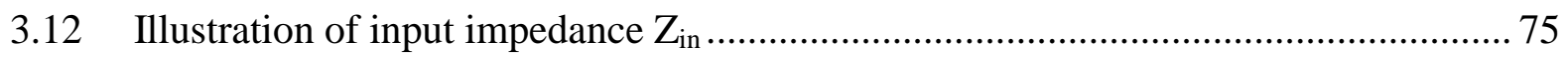

3.13 Representation of a non-uniform line as a cascade of uniform sections where $\mathrm{z}_{\mathrm{i}}{ }^{\prime}$ is the local coordinate of each section and $\mathrm{z}$ is the global coordinate

3.14 A semi-infinite transmission line

3.15 The reflected wave $\left(\mathrm{V}_{\mathrm{r}}\right)$ and transmitted wave $\left(\mathrm{V}_{\mathrm{t}}\right)$ at the interface of two lines with impedance $\mathrm{Z}_{1}$ and $\mathrm{Z}_{2}$.....

3.16 The sampling voltage in terms an incident wave and a reflected wave from the transmission line

3.17 The flow chart of the spectral algorithm

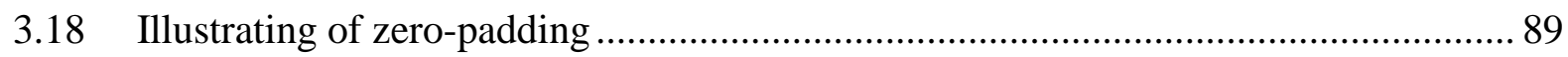

3.19 Duality relation between time domain and frequency domain ................................ 90

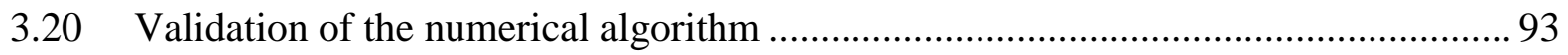

3.21 The per-unit-length equivalent circuit accounting for imperfect conductors (modified from Ramo et al., 1994). 
Figure

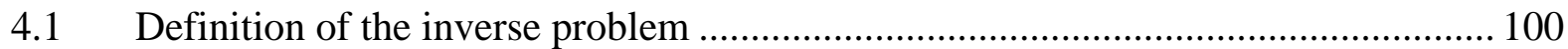

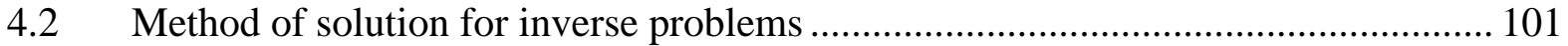

4.3 Typical neural network architecture ................................................................. 101

4.4 Stochastic system of the observed data (Classical Interpretation) ............................ 104

4.5 Stochastic system of the observed data (Bayesian Interpretation) .......................... 105

4.6 Cost functions: (a) square error, (b) absolute error, (c) uniform cost..................... 108

4.7 Example of inversion of impedance profile .................................................. 123

4.8 The comparison between simulated spectrum and predicted spectrum ................... 125

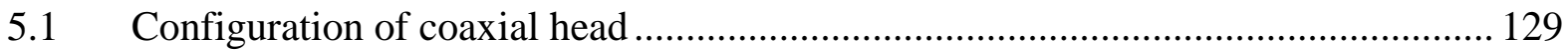

5.2 Configuration of CC and MRP transmission lines ............................................ 130

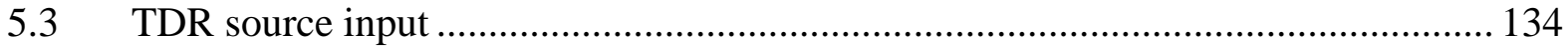

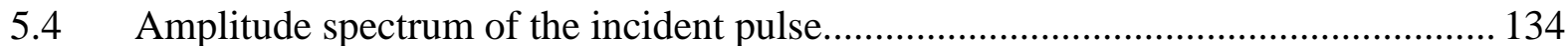

5.5 Error of amplitude spectrum $\left.\left(\mathrm{A}_{\text {input }}-\mathrm{A}_{\text {modified input }}\right) / \mathrm{A}_{\text {modified input }}\right)$ due to aberration, where A represent the amplitude spectrum ......................................................... 135

5.6 Standard deviation in ten measurements of TDR waveform in air ......................... 136

5.7 Standard deviation in ten measurements of system function in air ....................... 136

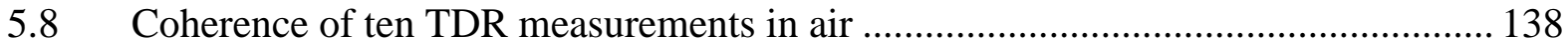

5.9 Noise-to-signal ratio of ten TDR measurements in air.......................................... 138

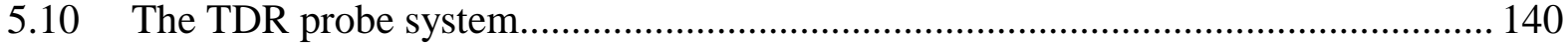

5.11 The impedance profile of the front panel aberration ............................................ 141

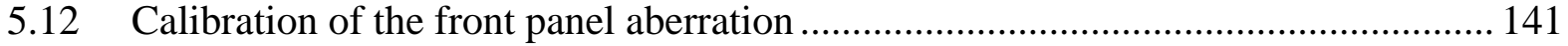

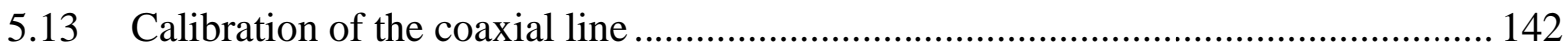




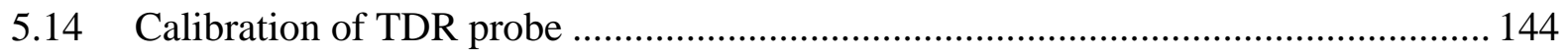

5.15 Comparison of the measured and predicted TDR waveforms measured in liquids .. 145

5.16 Preprocessing of the TDR waveforms........................................................... 146

5.17 Real part of scattering function after layer-peeling processing ........................... 148

5.18 Time-domain waveform after layer-peeling processing ................................... 148

5.19 Effect of conductive loss on layer-peeling processing $(\sigma=0.5 \mathrm{~S} / \mathrm{m}) \ldots \ldots \ldots \ldots \ldots \ldots \ldots . . . \ldots . . . . .149$

5.20 The scattering function and TDR waveform after preprocessing for

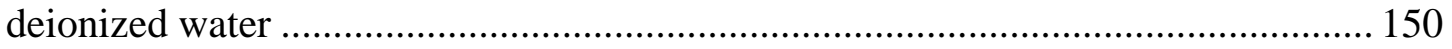

5.21 The scattering function and TDR waveform after preprocessing for tap water ....... 151

5.22 The scattering function and TDR waveform after preprocessing for ethynol alcohol

5.23 The scattering function and TDR waveform after preprocessing for butanol alcohol

5.24 Dielectric spectrum of butanol alcohol with zero conductivity measured by fabricated data

5.25 Dielectric spectrum of butanol alcohol with conductivity equal to $0.5 \mathrm{~S} / \mathrm{m}$ measured by fabricated data

5.26 Estimated dielectric spectrum of deionized water.

5.27 Estimated dielectric spectrum of tap water ................................................... 157

5.28 Estimated dielectric spectrum of ethanol alcohol.......................................... 158

5.29 Estimated dielectric spectrum of butanol alcohol ......................................... 158

5.30 Sensitivity of the TDR waveform on Debye's parameters evaluated at $\left(\varepsilon_{\mathrm{s}}, \varepsilon_{\infty}, \mathrm{f}_{\mathrm{rel}}, \sigma\right)=(25,15,30 \mathrm{MHz}, 0.02 \mathrm{~S} / \mathrm{m})$

5.31 Sensitivity of the TDR waveform on Debye's parameters evaluated at $\left(\varepsilon_{\mathrm{s}}, \varepsilon_{\infty}, \mathrm{f}_{\mathrm{rel}}, \sigma\right)=(25,15,3 \mathrm{GHz}, 0.02 \mathrm{~S} / \mathrm{m})$ 160 
Figure

5.32 Measured dielectric spectrum of butanol alcohol from the estimation of Debye's parameters

5.33 Measured dielectric spectrum of ethanol alcohol from the estimation of Debye's parameters

5.34 Measured dielectric spectrum of deionized water from the estimation of Debye's parameters

5.35 Measured dielectric spectrum of tap water from the estimation of Debye's parameters

5.36 Comparison of the dielectric spectra based on volumetric mixing equation and Debye equation.

5.37 Comparison of the time-domain waveforms based on volumetric mixing equation

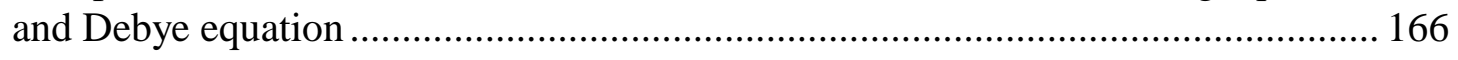

5.38 Simplified dc analysis for a low-loss material ............................................. 167

5.39 Simplified de analysis for a high-loss material ............................................. 168

5.40 The loss factor and phase velocity of a typical soil dielectric ............................ 171

5.41 Comparison of apparent dielectric constant with the apparent dielectric spectrum and real part of dielectric

6.1 Example of an abnormal waveform in M3-1 .................................................. 177

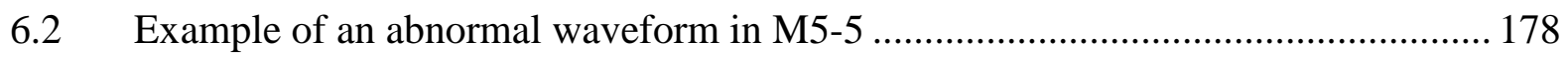

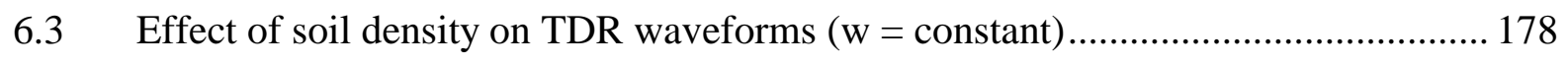

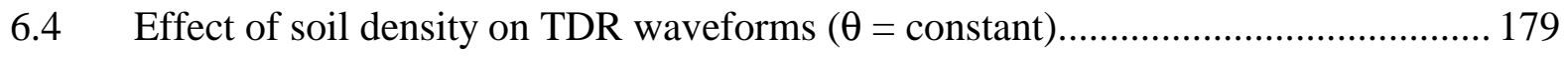

6.5 Effect of soil type on TDR waveforms................................................... 180

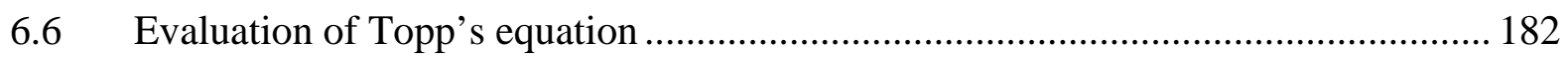

$6.7 \sqrt{K_{a}}$ vs. volumetric water content $(\theta)$ relationship ...................................... 183

6.8 Error in volumetric water content measured by Topp's equation versus dry density 
Figure

$6.9 \quad\left(\sqrt{K_{a}}-\mathrm{c}\right) \rho_{\mathrm{w}} / \rho_{\mathrm{a}}$ vs. gravimetric water content (w) relationship

$6.10\left(\sqrt{K_{a}}\right) \rho_{\mathrm{w}} / \rho_{\mathrm{a}}$ vs. gravimetric water content $(\mathrm{w})$ relationship

6.11 Soil type effect on the relationship between the apparent dielectric constant and water content

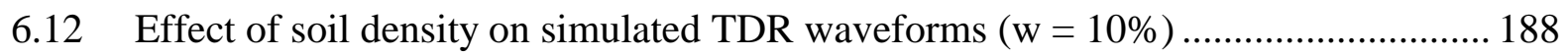

6.13 Effect of soil type on simulated TDR waveforms .......................................... 189

6.14 Conductivities calculated from simulated TDR waveforms ............................... 190

$6.1 \quad\left(\sqrt{ } \sigma_{d c}\right.$-c $) \rho_{w} / \rho_{d}$ vs. w relationship for the fictitious soils based on volumetric mixing model

$6.16\left(\sqrt{ } \sigma_{d c}-\mathrm{c}\right) \rho_{w} / \rho_{d}$ vs. w relationship for the mixed soils

6.17 Parametric study of volumetric mixing parameters in TDR

6.18 Parametric study of volumetric mixing parameters in $\operatorname{Re}[\varepsilon(f)]$

6.19 Parametric study of volumetric mixing parameters in $-\operatorname{Im}[\varepsilon(f)]$

6.20 Normalized sensitivity of the TDR waveform to the volumetric mixing parameters 201

6.21 Normalized sensitivity of $\operatorname{Re}[\varepsilon(f)]$ to the volumetric mixing parameters .202

6.22 Normalized sensitivity of $-\operatorname{Im}[\varepsilon(f)]$ to the volumetric mixing parameters 203

6.23 Waveform matching by the volumetric mixing model .204

6.24 Estimated dielectric spectrum by the volumetric mixing model. 204

$6.25 \sqrt{ } K_{a}$-c) $\rho_{w} / \rho_{d}$ vs. $w$ relationship using the apparent dielectric constant at $1 \mathrm{GHz}\left(\varepsilon_{a, 1 \mathrm{GHz}}\right)$ for each soil type.

7.1 Center Rod Being Driven into Specimen Through Guide Template 212 
Figure

7.2 Mold with Center Rod and Adapter Ring Ready for Multiple Rod Probe.....

7.3 Multiple Rod Probe Head Ready for Making TDR Measurements

7.4 Tests on Crosby Till at a Target Water Content of $21 \%$ with Tests at All

Temperatures Done with Separate Soil

7.5 Tests on Crosby Till at a Target Water Content of $41 \%$ with Tests at All

Temperatures Done on the Same Soil

7.6 Apparent Dielectric Constant Variation with Temperature for Water 217

7.7 Apparent Dielectric Constant Variation with Temperature for Water

7.8 Change of Slope of Normalized Temperature Effects Lines with Water Content for Crosby Till.

7.9 Slope of Normalized Apparent Dielectric Constant with Water Content Normalized by the Plasticity Limit 220

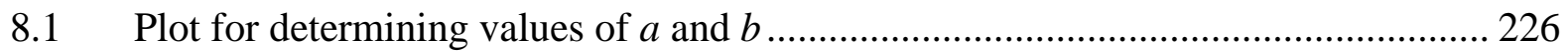

8.2 Comparison of TDR measured water content with oven dry water content for soils with large particle sizes

9.1 Water Content Comparisons of Fly Ash Stabilized Crosby Till with Oven Drying 230 


\section{CHAPTER 1 - INTRODUCTION}

\subsection{Overview of TDR Research at Purdue Supported by the JTRP}

This project is the third in a series supported by the Joint Transportation Research Program at Purdue University. The first was to examine its feasibility for measurement of soil water content in conjunction with field density testing for construction control (Siddiqui and Drnevich, 1995). A major breakthrough occurred in this project; a technique was developed that made it possible to measure both water content and density. The procedure was written up, published, and is the source of three U.S. patents (Patent No. 5,801,537, September 1, 1998, U.S. Patent No. 5,933,015, August 3, 1999, and one about to be issued).

The second project focused on developing an automated procedure for this test and for creating a draft ASTM Standard for the method (Feng, et al., 1998). The automated, computerbased procedure is called TDR ${ }^{++}$and a draft standard was developed. The second project also developed procedures to remove the effects of the apparatus and cables.

This project focused on obtaining a rational mathematical model for the propagation of an electromagnetic wave in the soil specimen so that the model could be used to better understand the testing process and provide more information about the soil being tested. An extension to the third project focused on examining the effects of temperature and establishing the validity of the method for soils with large particle sizes and for soils with additives like fly ash, lime, etc.

The TDR method developed by this research is totally new and a radical departure from the current procedures used in geotechnical practice for measuring water content and density for soil. The techniques developed as part of these research projects is becoming known as the Purdue TDR Method. It is generating widespread interest. Thirteen technical publications and project reports and three dissertations were produced as part of these research projects by project personnel. They are included in the list references at the end of this report. Additionally, there have been thirteen presentations on various aspects of this research. Those presentations also are listed in Appendix A1. 


\subsection{Problem Statement}

Observations made in the previous research indicate that the complex dielectric constant of soil is frequency dependent. Understanding of the complex dielectric permittivity as a function of basic material properties is very important to interpreting the test results and for increasing the accuracy of the method. Ultimately, it will allow for simplifying the method by reducing the method to a single step method rather than the two-step method generated from previous research.

\section{$1.3 \quad$ Objectives}

The project objectives were to understand the fundamental nature of one-dimensional electromagnetic wave propagation in soil and to relate the wave propagation to soil properties that are of interest for geotechnical engineering. An understanding of electromagnetic wave propagation in the apparatus, cables, and probe head also must be known and accounted before basic soil properties can be measured with the TDR method. With this understanding, the TDR signals can be generated and compared with those measured on soil and other types of materials.

\subsection{Scope of Work}

Study the variation of dielectric constant with frequency for different soils using advanced signal processing techniques. This material dielectric property is as important as constitutive modeling, e.g. describing stress-strain behavior, in soil mechanics.

System modeling and numerical analysis of the TDR wave propagation are necessary to perform a thorough simulation of electro-magnetic wave propagation in the TDR system. The work developed a complete analytical waveform generation and inverse analysis and compared these with experimental results.

The research examined neural networks and other modern methods for enhancing the inverse problem. The objective was to be able to take a TDR signature and from an analysis of that signature, identify some basic soil properties that include water content, density and others to identify the soil.

In the summer of 1999, the Study Advisory Committee recommended expansion of the project to cover effects of temperature, particle size, and additives mixed in with the soil. 


\subsection{Report Overview}

This report highlights the important findings of this project, including the work completed as part of the expansion of the project. Chapters 2 through 6 feature the work completed by Dr. Chih-Ping Lin and are excerpted from his dissertation (Lin, C-P., 1999). This work is fundamental in nature and opened the door for a much better understanding of the TDR process. It serves as a powerful base for future research and development of the TDR method. For this reason, the work is included in this report in its entirety for the sake of completeness and to acknowledge that it was developed with the support of the FHWA/INDOT/JTRP.

Chapters 7 through 9 focus on the work done in the expansion, namely temperature effects, large particle size effects, and effects of soil additives. Chapter 7 describes the work on temperature effects that pushes the state of the art and for the first time provides a simple method for taking temperatures into consideration in measuring soil water content with TDR.

Chapter 8 provides test results and analyses show that the Purdue TDR Method works well for soils with larger particle sizes, an important consideration if the method is to be practical for field use.

Chapter 9 gives some preliminary results when soils are modified with additives such as fly ash, lime, or cement. The additives cause water in the soil to hydrate. Conventional ovendrying drives some of the hydrated water off. The TDR method appears to monitor the hydration process with time. This is an important finding because it will allow the TDR method to monitor the efficacy of additives with time while conventional oven-drying is totally oblivious to this effect.

The report concludes with a summary, conclusions, and recommendations for future research, Chapter 10. An exhaustive set of references is included to substantiate work done in the project and assist with future research. 


\section{CHAPTER 2 - ELECTROMAGNETIC PROPERTIES OF SOILS}

\subsection{Introduction}

Soil response under loading or changes in the environment depends strongly on the volumetric fraction of the soil components, soil type, and chemical characteristics of the soil water. The determination of the intrinsic physical properties of the soil is essential for many areas including geotechnical, geo-environmental, and agricultural applications. It is not surprising that the electrical response of soils to an external electromagnetic field is affected by the same soil physical properties that control the engineering behavior of the soil. The electromagnetic properties of soils have been studied and used to correlate with soil physical properties for many years. Early studies of electromagnetic properties provided only a qualitative interpretation or an empirical correlation with soil properties, but not general quantitative results. A fundamental understanding of the dielectric properties of soils will facilitate the development of a sound relationship between the electromagnetic permittivity and the soil physical properties.

\subsection{Basics of Electromagnetics}

Similar to the field of mechanics, electromagnetics consists of a set of governing equations and constitutive relations from which solutions for applications are derived. The basic governing equations and constitutive relations will be discussed below which will serve as the basis for the study of dielectric polarizability in the following sections and transmission line equations in the next chapter. The spectral version of electromagnetics will also be presented which transform the problem from the time domain to the frequency domain. Spectral analysis in the frequency domain can account for frequency-dependent material properties and dispersion phenomena in electromagnetic wave propagation.

\subsubsection{Governing Equations of Electromagnetics}

The macroscopic phenomena (with dimensions large compared to atomic dimensions) of the electric and magnetic fields are governed by Maxwell's equations. Theses include Faraday's law, Ampere's Law, and Gauss' Law as: 
(Differential form)

(Integral form)

$$
\begin{array}{ll}
\nabla \cdot \boldsymbol{D}=\rho & \oint_{s} \boldsymbol{D} \cdot d \boldsymbol{s}=\int_{v} \rho d v \quad(\text { Gauss's Law for } \mathbf{E}) \\
\nabla \cdot \boldsymbol{B}=0 & \oint_{s} \boldsymbol{B} \cdot d \boldsymbol{s}=0 \quad(\text { Gauss's Law for B) } \\
\nabla \times \boldsymbol{E}=-\frac{\partial \boldsymbol{B}}{\partial t} & \oint_{c} \boldsymbol{E} \cdot \mathrm{d} \boldsymbol{l}=-\frac{d}{d t} \int_{s} \boldsymbol{B} \cdot d \boldsymbol{s} \quad \text { (Faraday's Law) } \\
\nabla \times \boldsymbol{H}=\boldsymbol{J}+\frac{\partial \boldsymbol{D}}{\partial t} & \oint_{c} \boldsymbol{H} \cdot d \boldsymbol{l}=\int_{s} \boldsymbol{J} \cdot d \boldsymbol{s}+\frac{d}{d t} \int_{s} \boldsymbol{D} \cdot d \boldsymbol{s} \quad \text { (Ampere's Law) }
\end{array}
$$

Table 2.1 summarizes the variables and units in Eq. (2.1). Note that the bold face is used to represent a vector quantity. The differential form and integral form are related by the Divergence theorem and Stokes' theorem in vector analysis. These equations are applicable when the dimensions of interest are large compared to atomic dimensions.

Table 2.1 Notation of electromagnetic field quantities.

\begin{tabular}{c|ll}
\hline Field Notation & Quantity & SI Units \\
\hline $\boldsymbol{E}(x, y, z, t)$ & Electric field density & Volt $/ \mathrm{m}$ \\
$\boldsymbol{D}(x, y, z, t)$ & Electric flux density & Coulomb $/ \mathrm{m}^{2}$ \\
$\boldsymbol{H}(x, y, z, t)$ & Magnetic field density & Ampere $/ \mathrm{m}$ \\
$\boldsymbol{B}(x, y, z, t)$ & Magnetic flux density & Weber $/ \mathrm{m}^{2}$ \\
$\boldsymbol{J}(x, y, z, t)$ & Current density & Ampere $/ \mathrm{m}^{2}$ \\
$\rho(x, y, z, t)$ & Volume charge density & Coulomb $/ \mathrm{m}^{3}$ \\
\hline
\end{tabular}

The equation of continuity or conservation of charge is implicit in Maxwell's equation and can be written explicitly as: 


$$
\begin{array}{lc}
\text { (Differential form) } & \text { (Integral form) } \\
\nabla \cdot \boldsymbol{J}=-\frac{\partial \rho}{\partial t} & \oint_{s} \boldsymbol{J} \cdot d \boldsymbol{s}=-\int_{v} \frac{\partial \rho}{t} d v=-\frac{\partial}{\partial t} Q
\end{array}
$$

where $Q$ is the charge in the volume $V$, enclosed by a surface $S$. It should be noted that the four Maxwell's equations in (2.1) are not all independent. If we take the divergence of (2.1c) and (2.1d) and note that $\nabla \cdot(\nabla \times \mathbf{A})=0$ from vector analysis, we find that

$$
\begin{aligned}
& \frac{\partial}{\partial t}(\nabla \cdot \boldsymbol{B})=0 \\
& \nabla \cdot \boldsymbol{J}=-\frac{\partial}{\partial t}(\nabla \cdot \boldsymbol{D})
\end{aligned}
$$

According to the continuity equation in Eq. (2.2), equation (2.3b) implies Eq. (2.1a). In the absence of proof of the existence of any isolated magnetic sources, equation (2.3a) is equivalent to Eq. (2.1b). Therefore, equations (2.1c) and (2.1d) can be considered the only two independent governing equations in electromagnetics.

\subsubsection{Constitutive Relations of Electromagnetics}

Maxwell's equations in (2.1) contain 12 unknowns: $E_{x}, E_{y}, E_{z}, H_{x}, H_{y}, H_{z}, D_{x}, D_{y}, D_{z}, B_{x}$, $B_{y}, B_{z}$. Since only the curl equations (2.1c) and (2.1d) are independent, we have six equations (each vector equation contains three scalar equations in terms of vector components). Thus we need six additional equations relating these components. The constitutive relations of the medium provide these additional equations.

$$
\begin{aligned}
& \boldsymbol{D}=f_{D}(\boldsymbol{E}) \\
& \boldsymbol{B}=f_{B}(\boldsymbol{H})
\end{aligned}
$$


Each equation relates three field vector components, giving a total of six equations. In addition, if we treat $\boldsymbol{J}$ in (2.1d) as unknown, we introduce three additional unknowns, $J_{x}, J_{y}$, and $J_{z}$. Thus, we need three additional equations given by

$$
\boldsymbol{J}=f_{J}(\boldsymbol{E})
$$

where $f_{D}, f_{B}$, and $f_{J}$ represent the properties of dielectric permittivity, magnetic permeability, and electric conductivity, respectively. The type of medium will determine these specific functional relationships between the various field vectors. Materials react to applied electromagnetic fields in a variety of ways including displacement of both free and bound electrons by electric fields and the orientation of atomic moments by magnetic fields. In many cases, these responses can be treated as linear (proportional to the applied fields) over the useful ranges of field magnitudes. If the response is independent of the direction of the applied field, the material is called isotropic. For a homogeneous, linear, and isotropic medium, Eq. (2.4) can be characterized by the scalar parameters of $\varepsilon, \mu, \sigma$.

$$
\begin{aligned}
& \boldsymbol{D}=\varepsilon \boldsymbol{E} \\
& \boldsymbol{B}=\mu \boldsymbol{H} \\
& \boldsymbol{J}=\sigma \boldsymbol{E}
\end{aligned}
$$

where $\varepsilon$ is the dielectric permittivity, $\mu$ is the magnetic permeability, and $\sigma$ is the electric conductivity. These scalars often depend on the frequency $f$ of the fields, so we will write $\varepsilon(f)$, $\mu(f)$ and $\sigma(f)$. In this case, it would be improper to write Eq. (2.5) as shown, since the field vectors are time-domain quantities: i.e., $\boldsymbol{D}(x, y, z, t)$, etc. In later sections, we will concentrate on the spectral analysis of electromagnetic waves. In that case, (2.5) will relate these field vectors in their spectral representations, and it would be proper to use frequency-dependent parameters. 


\subsubsection{Spectral Analysis of Electromagnetics}

Electromagnetic field quantities are functions of space and time. It has long been known that an arbitrary time signal can be thought of as a superposition of many sinusoidal components, i.e., it has a distribution or spectrum of components. Working in terms of a spectrum is called spectral analysis (Sneddon, 1951). If the time variation of the field quantities is of interest at a particular point in space, then a Fourier Series can describe the spectral representation.

$$
U(x, y, z, t)=\sum_{k=-\infty}^{+\infty} \hat{U}_{k}\left(x, y, z, f_{k}\right) e^{j 2 \pi f_{k} t}
$$

where

$$
\hat{U}_{k}\left(x, y, z, f_{k}\right)=\frac{1}{T_{p}} \int_{T_{p}} U(x, y, z, t) e^{-j 2 \pi f_{k} t} d t
$$

in which $U$ represent any electromagnetic field quantity listed in Table 2.1, $\hat{U}_{k}$ are the spatially dependent Fourier coefficients, $f_{k}=k / T_{p}$ is the frequency of the $k^{\text {th }}$ frequency coefficient, and $T_{p}$ is the fundamental period of the field quantity.

In spectral analysis, the governing equations can then be simplified to be only a function of space, and the frequency dependency of the constitutive relations are taken into account naturally. Notice that the Fourier coefficients are functions of frequency and thus there is no reduction in the total number of independent variables. However, using a spectral representation, the differentiation and integration with respect to time in the partial differential equations become simple algebraic operations. Using Eq. (2.6), Eq. (2.1) is then simplified to 
(Differential form) (Integral form)

$$
\begin{array}{ll}
\nabla \cdot \hat{\boldsymbol{D}}=\hat{\rho} & \oint_{S} \hat{\boldsymbol{D}} \cdot d \boldsymbol{s}=\int_{v} \hat{\rho} d v \\
\nabla \cdot \hat{\boldsymbol{B}}=0 & \oint_{s} \hat{\boldsymbol{B}} \cdot d \boldsymbol{s}=0 \\
\nabla \times \hat{\boldsymbol{E}}=-j 2 \pi f \hat{\boldsymbol{B}} & \oint_{c} \hat{\boldsymbol{E}} \cdot \mathrm{d} \boldsymbol{l}=-j 2 \pi f \int_{s} \hat{\boldsymbol{B}} \cdot d \boldsymbol{s} \\
\nabla \times \hat{\boldsymbol{H}}=\hat{\boldsymbol{J}}+j 2 \pi \hat{\boldsymbol{D}} & \oint_{c} \hat{\boldsymbol{H}} \cdot d \boldsymbol{l}=\int_{s} \hat{\boldsymbol{J}} \cdot d \boldsymbol{s}+j 2 \pi f \int_{s} \hat{\boldsymbol{D}} \cdot d \boldsymbol{s}
\end{array}
$$

Notice that the subscript $k$ is dropped, but it will be understood that the above equations must be solved at each frequency. The frequency-dependent constitutive relations can be written as

$$
\begin{aligned}
\hat{\boldsymbol{D}} & =\varepsilon(f) \hat{\boldsymbol{E}} \\
\hat{\boldsymbol{B}} & =\mu(f) \hat{\boldsymbol{H}} \\
\hat{\boldsymbol{J}} & =\sigma(f) \hat{\boldsymbol{E}}
\end{aligned}
$$

All materials have some response to magnetic fields but, except for ferromagnetic and ferrimagnetic materials, this is usually small, and differs from free space $\left(\mu_{0}=1\right)$ by a negligible fraction (Ramo et al., 1994). For materials with moderate-to-low conductivities (say, $\sigma \leq 1$ Seimen/m), then the conductivity is not frequency dependent $\left(\sigma \cong \sigma_{d c}\right)$ within microwave frequencies, where $\sigma_{d c}$ is the conductivity at zero frequency. In the following sections, the dielectric permittivity, $\varepsilon(f)$, of materials will be discussed in greater detail.

\subsection{Dielectric Properties of Homogeneous Materials}

Soil is a heterogeneous material, which consists of three types of materials. In order to understand the dielectric behavior of soil, it is necessary to study the dielectric properties of 
individual materials first. The mechanisms of dielectric polarization and their frequencydependent properties are discussed in the following sections.

\subsubsection{Characteristics of Dielectrics in a Static Field}

In this section, we consider linear, isotropic dielectrics in a static field to explain the connection between microscopic effects of polarization and their representation by a permittivity. In a static field, there is no interaction between the electric field and magnetic field. Gauss's law in Eq. (2.1a) and the constitutive relation in Eq. (2.5a) describe the governing equation of electric field in free space as

$$
\nabla \cdot \varepsilon_{0} \boldsymbol{E}=\rho_{f}
$$

where $\boldsymbol{E}=$ electric field, $\rho_{f}=$ charge density, and $\varepsilon_{0}=$ permittivity of free space (vacuum). Instead of having air or a vacuum as the medium, we shall consider material media, specifically dielectrics. If a source charge is placed in a dielectric medium, the resulting electric field will be different from that in a free space. This can be explained by the phenomenon of polarization. As opposed to conductors, dielectrics have few free (mobile) charges. The charges are mostly bound to the parent atoms. Even though a dielectric is electrically neutral, an externally applied electric field may cause microscopic separations of the centers of positive and negative charges, which thus behave like dipoles of charges. The charge separation distances are on the order of the atomic dimensions, but the vast numbers of dipoles may result in a significant effect. This phenomenon is referred to as the polarization of the dielectric.

The separation of centers of positive and negative charges due to polarization phenomena can be modeled as an equivalent electric dipole composed of equal and opposite point charges $q_{b}$ separated by a distance $\boldsymbol{l}$, as shown in Fig. 2.1. The charge $q_{b}$ is referred to as a bound charge since it is not normally available for conduction except under the application of large electric fields. The product $q_{b} \boldsymbol{l}$ is defined as the dipole moment $\boldsymbol{p}$. That is 


$$
\boldsymbol{p}=q_{b} \boldsymbol{l}
$$

where $\boldsymbol{l}$ is the vector pointing from $-q_{b}$ to $+q_{b}$ as shown in Fig. 2.1. The field due to a charge dipole is a typical example of an electric field and has been studied in almost every textbook on electromagnetics. The electrical potential $\left(V_{A}\right)$ and field $\left(\boldsymbol{E}_{\boldsymbol{A}}\right)$ at point, $A$, for the electrical dipole can be written as (Ramo et al., 1994)

$$
\begin{aligned}
& V_{A}=\frac{\boldsymbol{p} \cdot \boldsymbol{r}}{4 \pi \varepsilon_{0} r^{2}} \\
& \boldsymbol{E}_{A}=-\nabla V_{A}
\end{aligned}
$$

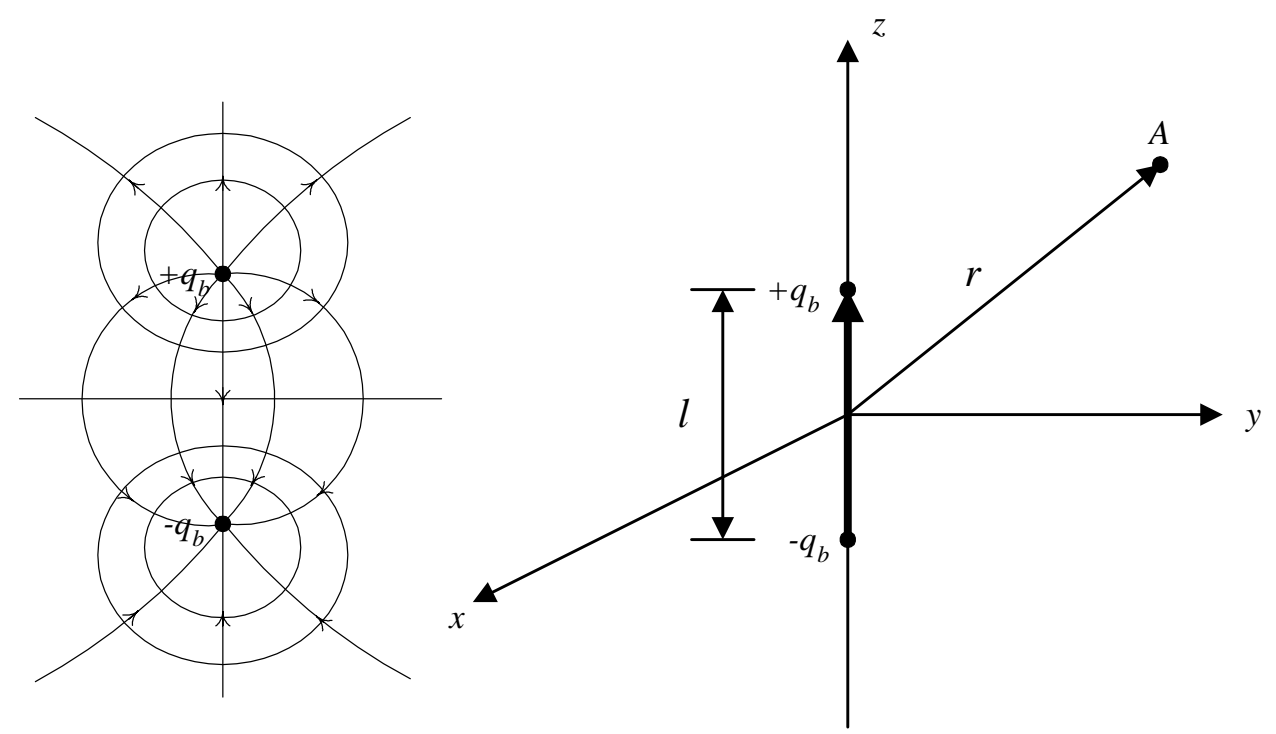

Fig. 2.1 Electrical field and potential of a dipole of charge (modified from Krauss, 1984)

The polarization results in the production of charge dipoles in dielectrics. We may view the result as a large number of dipoles in free space, each composed of charges $-q_{b}$ and $+q_{b}$. 
Assuming now a block of a continuous dielectric material, a polarization vector $\boldsymbol{P}$ is defined as the dipole moment per unit volume

$$
\boldsymbol{P}=\lim _{\Delta v \rightarrow 0} \frac{\sum_{i} \boldsymbol{p}_{\boldsymbol{i}}}{\Delta v}=\frac{d \boldsymbol{p}}{d v}
$$

in which we sum all of the individual dipole moments vectorially in the volume $\Delta v$. For the block of dielectric material immersed in an electric field shown in Fig. 2.2, the tendency of the dipoles to align with the field causes charges to appear at the surfaces of the material. Thus, it appears that an overall separation of charge has been achieved between the surfaces of the material.

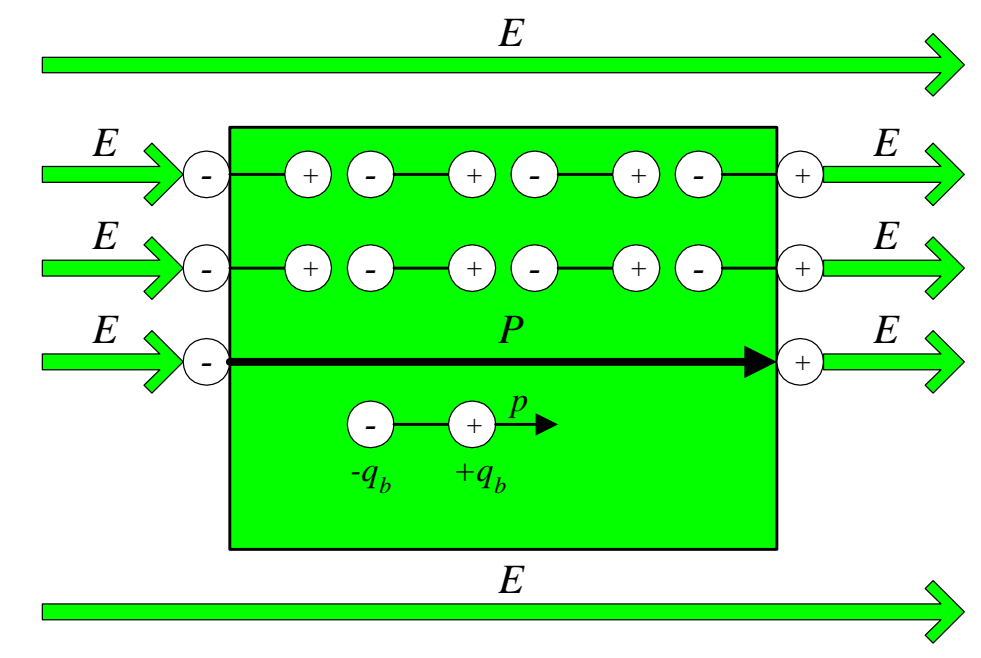

Fig. 2.2 Uniform polarization of a dielectric material

Figure 2.1 illustrates the electric potential and field of a single dipole, and Fig. 2.2 illustrates the effect of a dielectric with uniform polarization. However, in general, we need to consider the effects of the presence of non-uniformly polarized dielectrics, as shown in Fig. 2.3. 


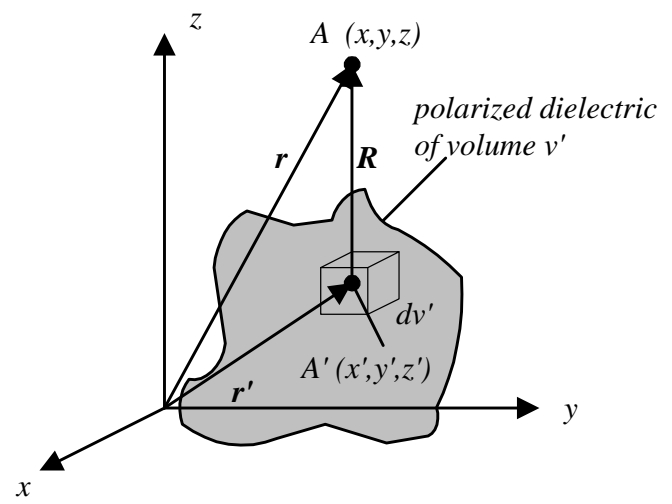

Fig. 2.3 A dielectric with a non-uniform polarization

Using Eq. (2.11), the electric potential at point $A(x, y, z)$ due to a small volume of the polarized dielectric, $d v^{\prime}$, at $A^{\prime}\left(x^{\prime}, y^{\prime}, z^{\prime}\right)$ is

$$
d V_{A}=\frac{d \boldsymbol{p} \cdot \tilde{\boldsymbol{R}}}{4 \pi \varepsilon_{0} R^{2}}=\frac{\boldsymbol{P} \cdot \tilde{\boldsymbol{R}} d v^{\prime}}{4 \pi \varepsilon_{0} R^{2}}
$$

where $\widetilde{\boldsymbol{R}}$ is a unit vector of $\boldsymbol{R}$ pointing in the direction from $A^{\prime}$ to $A, v^{\prime}$ is the volume of the polarized dielectric. It can shown that using $\nabla(1 / \mathrm{R})=\widetilde{\boldsymbol{R}} / R^{2}$ and the vector identity $\nabla \cdot(\psi \Phi)=$ $\psi \nabla \cdot \Phi+\Phi \cdot \nabla \psi$, Eq. (2.14) becomes

$$
d V_{A}=\frac{1}{4 \pi \varepsilon_{0}}\left[\nabla \cdot\left(\frac{\boldsymbol{P}}{R}\right)-\frac{1}{R} \nabla \cdot \boldsymbol{P}\right] d v^{\prime}
$$

and

$$
V_{A}=\frac{1}{4 \pi \varepsilon_{0}} \int_{v}\left[\nabla \cdot\left(\frac{\boldsymbol{P}}{R}\right)-\frac{1}{R} \nabla \cdot \boldsymbol{P}\right] d v^{\prime}
$$


The first term on the right-hand side of (2.16) may be transformed into a surface integral by the divergence theorem. So the electrical potential at point $A$ due to the polarized dielectric of volume $v^{\prime}$ is

$$
V_{A}=\oint_{s^{\prime}} \frac{\boldsymbol{P} \cdot d \boldsymbol{s}^{\prime}}{4 \pi \varepsilon_{0} R}-\int_{v^{\prime}} \frac{\nabla \cdot \boldsymbol{P}}{4 \pi \varepsilon_{0} R} d v^{\prime}
$$

where $d \boldsymbol{s}^{\prime}$ is the small surface area vector pointing out of the closed surface of the polarized dielectric. We can also obtain the potential at point $A$ from the equivalent bound surface charge and volume charge, $\rho_{s b}$ and $\rho_{v b}$, as

$$
V_{A}=\oint_{s^{\prime}} \frac{\rho_{s b} d s^{\prime}}{4 \pi \varepsilon_{0} R}-\int_{v^{\prime}} \frac{\rho_{v b} d v^{\prime}}{4 \pi \varepsilon_{0} R}
$$

Comparing Eq. (2.17) to Eq. (2.18), we have

$$
\begin{aligned}
& \rho_{s b}=P_{n} \\
& \rho_{v b}=-\nabla \cdot \boldsymbol{P}
\end{aligned}
$$

where $P_{n}$ is the component of $\boldsymbol{P}$ normal to the surface, $d \boldsymbol{s}^{\prime}$. This shows that a polarized dielectric can be replaced by an equivalent surface and volume charge distribution for the purpose of determining the resulting electric field and electric potentials. Therefore, conceptually, we can remove the dielectric and replace it with the dipoles in free space. Consider a region of space containing a dielectric material subjected to electric field due to some free charges as shown in Fig. 2.4. If we replace the dielectric with the bound charges of dipoles in free space, the total charge that influences the resultant electric field is the sum of free charges and bound charges, $\rho_{f}$ 
$+\rho_{b}$. Consequently, using Gauss's law in differential form for this free-space region, we may write

$$
\begin{aligned}
& \nabla \cdot\left(\varepsilon_{0} \boldsymbol{E}\right)=\rho_{f}+\rho_{b}=\rho_{f}-\nabla \cdot \boldsymbol{P} \\
& \nabla \cdot\left(\varepsilon_{0} \boldsymbol{E}+\boldsymbol{P}\right)=\rho_{f}
\end{aligned}
$$

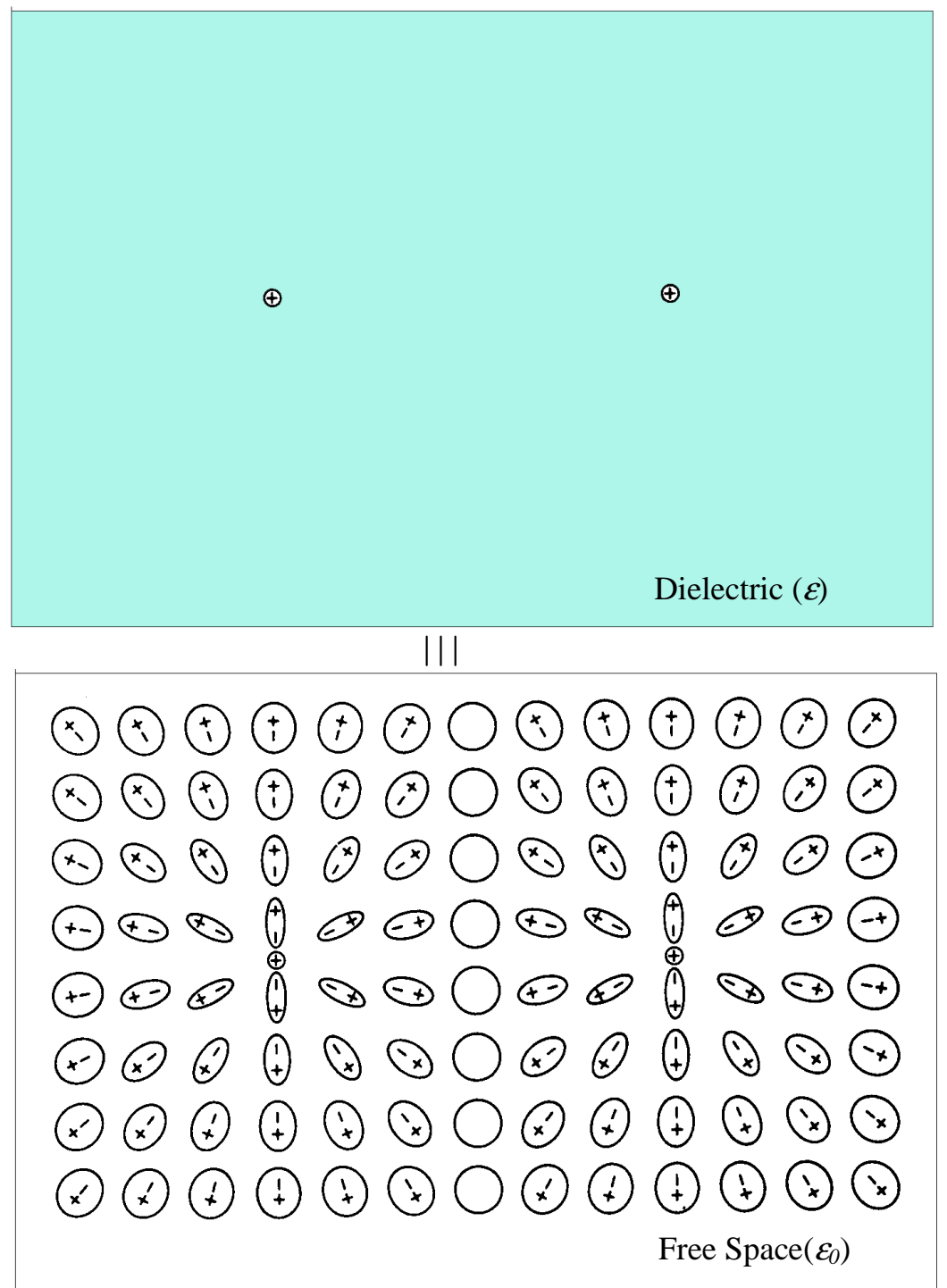

Fig. 2.4 Polarization of the atoms of a dielectric by a pair of equal positive charges (modified from Ramo et al., 1994) 
Because of the polarization, it is convenient in handling electric field problems to introduce another vector more directly related to free charges than is electric field vector $\boldsymbol{E}$. The electric flux density vector $\boldsymbol{D}$ is defined as

$$
\boldsymbol{D}=\varepsilon_{0} \boldsymbol{E}+\boldsymbol{P}
$$

For an isotropic, linear material the polarization is proportional to the field intensity and we can write the linear relation (Ramo et al., 1994)

$$
\boldsymbol{P} \approx \chi \varepsilon_{0} \boldsymbol{E}
$$

where $\chi$ is called the electric susceptibility. Substituting this into Eq. (2.23) we obtain

$$
\boldsymbol{D}=\varepsilon_{0}(1+\chi) \boldsymbol{E}=\boldsymbol{\varepsilon} \boldsymbol{E}=\varepsilon_{\mathrm{r}} \varepsilon_{0} \boldsymbol{E}
$$

where $\varepsilon$ is known as the permittivity of the dielectric. Notice that for free space, $\chi$ must be zero since $\boldsymbol{P}=0$ (no bound charge and hence no polarization vector); thus, $\varepsilon=\varepsilon_{0}$. It is common to classify materials according to a relative permittivity or dielectric constant $\mathcal{E}_{r}=\mathcal{E} / \mathcal{E}_{0}$, which gives a measure of the polarizability of a material relative to free space. As an example, air has a relative permittivity of $\mathcal{E}_{r}=1$. Distilled water has $\mathcal{E}_{r}=80$, and dry soil typically has $\mathcal{E}_{r}=3$.

\subsubsection{Polarization Mechanisms}

The polarization of a dielectric material due to some externally applied electric field may occur as a result of three effects: (1) electronic polarization; (2) ionic polarization, and (3) orientational polarization. Electronic polarization occurs when the externally applied electric field 
causes a shift in the atom's positive and negative charges. Equilibrium is attained when the internal Coulomb attractive force produced by the charge separation balances the applied force. When a charge separation occurs, we essentially have a microscopic electric dipole.

Ionic polarization occurs in molecules composed of positively and negatively charged ions (cations and anions). An externally applied electric field again results in a microscopic separation of charge centers thus resembling a dipole of charge. The electronic and ionic polarizations are called induced polarization, which is illustrated in Fig. 2.5.
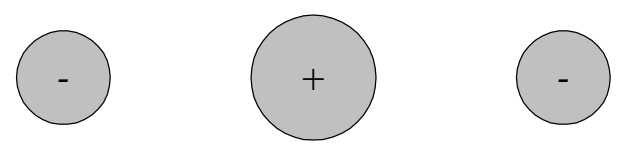

(a)

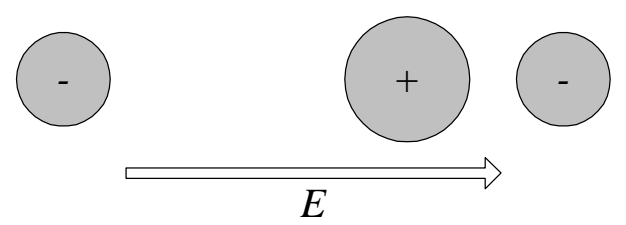

(b)

Fig. 2.5 (a) A symmetrical molecule/atom has no permanent polarization. (b) An external electric filed induces a polarization in the molecule/atom

Orientational polarization, on the other hand, occurs in materials that possess permanent, microscopic separations of charge centers. For example, consider the geometry of a water molecule (Fig. 2.6a). The molecule is arranged so that the negative oxygen atom is bonded to the positively charged hydrogen atoms with a $105^{\circ}$ angle between two bonds. The center of positive charge is at the oxygen atom, and the center of negative charge lies at a point midway along the line joining the hydrogen atoms. In the absence of an applied electric field, these permanent dipoles are randomly oriented (Fig. 2.6b). In the presence of an applied electric field, these permanent dipoles tend to rotate to align with the applied field (Fig. 2.6c). 


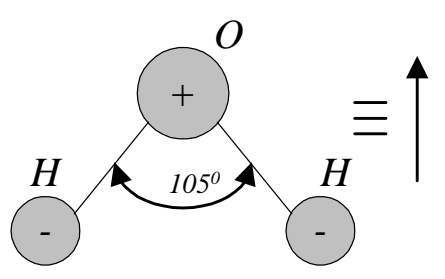

(a)

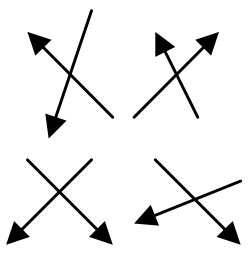

(b)

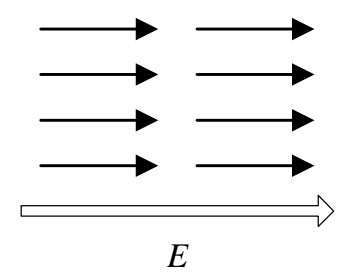

(c)

Fig. 2.6 (a) The water molecule, $\mathrm{H}_{2} \mathrm{O}$, has a permanent polarization resulting from its bent geometry (b) Randomly oriented water dipole (c) Dipole alignment with the electric field

If there are $N$ molecules of the same type, the polarization may be written as (Ramo et al., 1994).

$$
\boldsymbol{P}=\varepsilon_{0} \chi_{e} \boldsymbol{E}=N \alpha_{T} \boldsymbol{E}_{l o c}=N \alpha_{T} g \boldsymbol{E}
$$

where $\alpha_{T}$ is the molecular polarizability, and $g$ is the ratio between local field $\boldsymbol{E}_{l o c}$ acting on the molecule and the applied field $\boldsymbol{E}$. The local field differs from the applied field because of the effect of the surrounding molecules. Comparing Eq. (2.24) and (2.26), we may write the relative permittivity as

$$
\varepsilon_{r}=1+\chi_{e}=\frac{N \alpha_{T} g}{\varepsilon_{0}}
$$

If the surrounding molecules act in a spherically symmetric fashion on the molecules for which $\boldsymbol{E}_{l o c}$ is being calculated, it can be shown that $g=\left(2+\varepsilon_{r}\right) / 3$ (Von Hippel, 1954), so (2.27) can be written as

$$
\frac{\varepsilon_{r}-1}{\varepsilon_{r}+2}=\frac{N \alpha_{T}}{3 \varepsilon_{0}}
$$


which is known as Clausius-Mossotti relation. As discussed above, the molecular polarizability has contributions from several different atomic or molecular effects. The three effects together constitute the total molecular polarizability,

$$
\alpha_{T}=\alpha_{e}+\alpha_{i}+\alpha_{d}
$$

where $\alpha_{e}, \alpha_{i}$, and $\alpha_{d}$ are electronic, ionic, and permanent dipole contributions, respectively.

\subsubsection{Characteristics of Dielectrics in a Time-Varying Field}

The permittivity of dielectrics may also depend on frequency. If the electric field is a result of a sinusoidal source the dipoles in the dielectric also tend to align with each resulting change in direction of the field produced by the alternating source. However, atomic and molecular restoring forces prevent an instantaneous alignment. As the frequency is increased, the tendency of the dipole alignment to lag behind the directional change of the field becomes more pronounced.

The mechanism of the electronic polarization may be modeled by the classical mechanical system, as shown in Fig. 2.7. Any displacement of the charge cloud from its central ion produces a restoring force and its interaction with the inertia of the moving charge cloud produces a resonance as in a mechanical spring-mass system. Similarly, the displacement of one ion from another produces resonance in the ionic polarizability but it occurs at lower frequencies than the electronic contribution because of larger masses in motion. There are also losses or damping in each of the resonances, envisioned as arising from radiation or interaction with other charges. The Lorentz model of an atom in which damping is proportional to the velocity of oscillating charge is expressed by equation of motion (Krauss et al., 1984) 


$$
\frac{d^{2} l}{d t^{2}}+\Gamma \frac{d l}{d t}+\left(2 \pi f_{0}\right)^{2} l=\frac{q}{m} E_{l o c}
$$
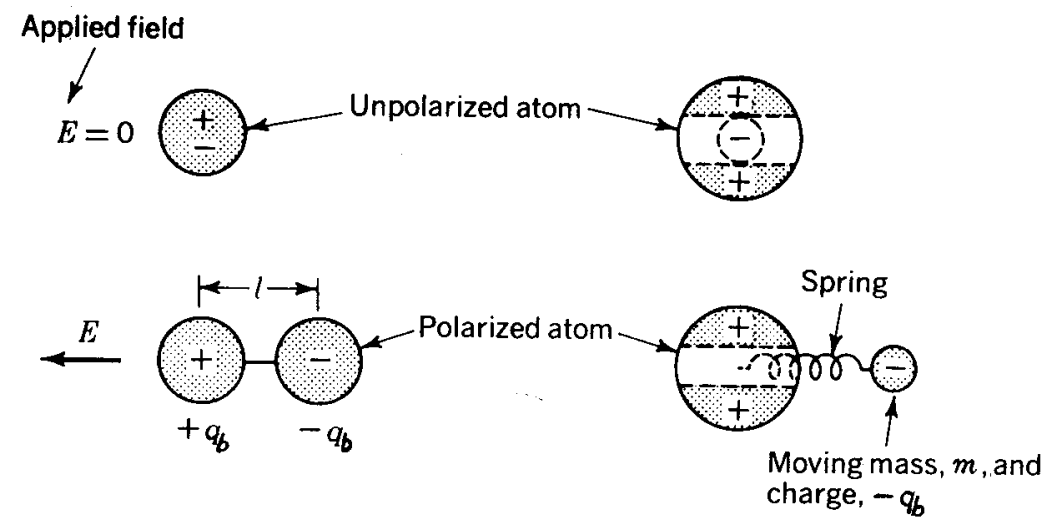

$$
E=0
$$

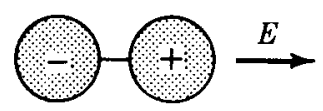

Atomic dipole

(a)
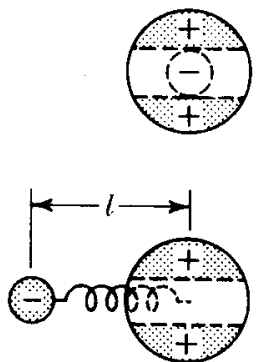

Equivalent mechanical system

(b)

Fig. 2.7 Equivalent mechanical model of the electronic polarization (Krauss, 1984)

where $\Gamma$ is a damping constant and natural frequency $f_{0}$ is related to the restoring force. Eq. (2.30) is most easily solved by spectral analysis. Using spectral representation for the displacement and field, Eq. (2.30) becomes an algebraic equation and one easily finds

$$
\hat{l}=\frac{(q / m) \hat{E}_{l o c}}{\left(\left(2 \pi f_{0}\right)^{2}-(2 \pi f)^{2}\right)+j 2 \pi f \Gamma}
$$

where $j=\sqrt{ }-1$. The electronic polarizability, $\alpha_{e}$, is the ratio of the dipole moment $p=q l$ to the local field where 


$$
\alpha_{e}=\frac{\hat{p}}{\hat{E}_{l o c}}=\frac{q \hat{l}}{\hat{E}_{l o c}}=\frac{\left(q^{2} / m\right)}{\left(\left(2 \pi f_{0}\right)^{2}-(2 \pi f)^{2}\right)+j 2 \pi f \Gamma}
$$

Equation (2.32) can be generalized to represent both electronic and ionic resonant responses:

$$
\alpha_{k}=\frac{F_{k}}{\left(\left(2 \pi f_{0}\right)^{2}-(2 \pi f)^{2}\right)+j 2 \pi f \Gamma_{k}}
$$

where $F_{k}$ measures the strength of the kth resonance. Notice that $\alpha_{k}$ in Eq. (2.33) is in general complex and a function of the frequency of the electric field. The expression for relative permittivity due to electronic and ionic polarization can be obtained by substituting (2.33) into (2.28). The resulting expression for relative permittivity is also complex and a function of frequency. The real and imaginary parts of (2.33) contribute to $\varepsilon_{r}{ }^{\prime}$ (real part of $\varepsilon_{r}$ ) and $\varepsilon_{r}{ }^{\prime \prime}$ (imaginary part of $\varepsilon_{r}$ ), respectively, in a manner shown by the electronic and ionic resonance pictured for hypothetical dielectric in Fig. 2.8. Near resonance, $\varepsilon_{r}{ }^{\prime \prime}$ goes through a peak. The contribution to $\varepsilon_{r}^{\prime}$ from a given resonance has peaks of opposite sign on either side of resonance.

The complex number, $\varepsilon_{r}$, is a result of the spectral representation of the polarization (i.e. in the frequency domain). The real part of the permittivity is often called the dielectric constant. It is a measure of how much energy from an external electric field is stored in a material. Notice that the imaginary part represents the dielectric loss (or damping). If $\Gamma_{k} \approx 0$ in equation (2.33), then the frequency-dependent dielectric permittivity $\varepsilon_{r}$ has only a real part.

The dynamic response of the permanent dipole contribution to permittivity is different from electronic and ionic polarization in that the force opposing complete alignment of the dipole in the direction of applied field is related to thermal effects. It acts as a viscous force and the dynamic response is 'over-damped'. The frequency response of such an over-damped system is of the form (Von Hippel, 1954) 


$$
\alpha_{d}=\frac{p^{2}}{3 K_{B} T(1+j 2 \pi f \tau)}
$$

in which $T$ is the temperature, $K_{B}$ the Boltzmann's constant, $p$ the permanent dipole moment, and $\tau$ the relaxation time for the effect (i.e. the time for polarization to fall to $\mathrm{e}^{-1}$ of the original value if the orienting fields are removed). The dipole contribution to the permittivity is also illustrated for a hypothetical dielectric in Fig. 2.8. It produces a smoother decrease in $\varepsilon_{r}$ as one goes through the range $2 \pi f \tau \approx 1$, along with a peak of absorption.

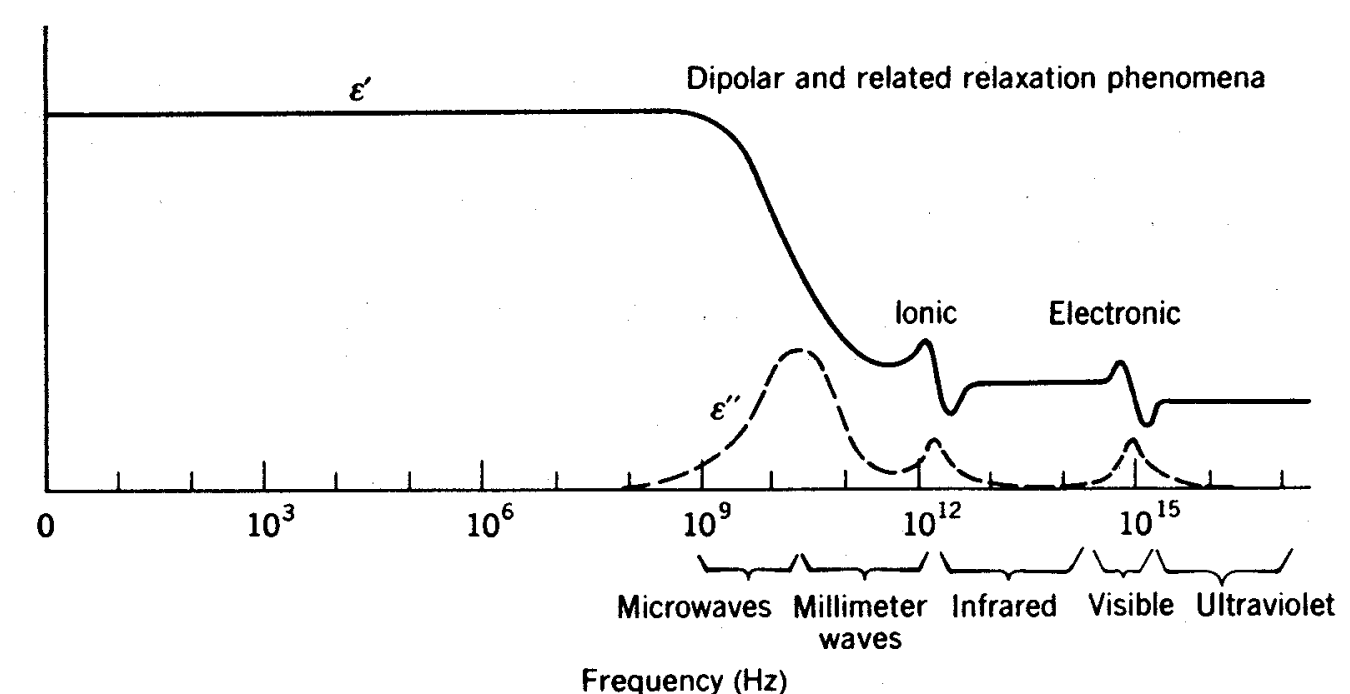

\section{Fig. 2.8 Frequency response of permittivity and loss factor for a hypothetical dielectric showing various contributing phenomena (Ramo, et al., 1994)}

Now let's consider the frequency dependency of the Clausius-Mossotti relation explicitly by substituting (2.33) and (2.34) into (2.28). As will be explained in Chapter 5, the frequency range of interest in this study is $\leq 2 \mathrm{GHz}$ due to the bandwidth of the measurement device. We can consider the frequency dependency due to permanent dipole polarization only because $\alpha_{e}$ and $\alpha_{i}$ remain constant for frequencies less than $10^{12} \mathrm{~Hz}$ as shown in Fig. 2.8. Let $\alpha_{e}+\alpha_{i}=\alpha_{0} \approx$ constant, then 


$$
\frac{\varepsilon_{r}-1}{\varepsilon_{r}+2}=\frac{N \alpha_{T}}{3 \varepsilon_{0}}=\frac{N}{3 \varepsilon_{0}}\left(\alpha_{0}+\alpha_{d}\right)=\frac{N}{3 \varepsilon_{0}}\left(\alpha_{0}+\frac{p^{2}}{3 K_{B} T(1+j 2 \pi f \tau)}\right)
$$

Let $\varepsilon_{s}$ represents the relative permittivity at $f$ goes to 0 , then Eq. (2.35) becomes

$$
\frac{\varepsilon_{s}-1}{\varepsilon_{s}+2}=\frac{N}{3 \varepsilon_{0}}\left(\alpha_{0}+\frac{p^{2}}{3 K_{B} T}\right)
$$

Similarly, let $\varepsilon_{\infty}$ be the relative permittivity at $f$ goes to the infinity, then Eq. (2.35) becomes

$$
\frac{\varepsilon_{\infty}-1}{\varepsilon_{\infty}+2}=\frac{N \alpha_{0}}{3 \varepsilon_{0}}
$$

Using the definitions for $\varepsilon_{s}$ and $\varepsilon_{\infty}, \varepsilon_{r}$ can be rewritten as

$$
\varepsilon_{r}=\varepsilon_{\infty}+\frac{\left(\varepsilon_{s}-\varepsilon_{\infty}\right)}{1+j \omega\left(\frac{\varepsilon_{s}+2}{\varepsilon_{\infty}+2} \tau\right)}
$$

It is convenient to define a new relaxation time parameter, $\tau_{r}$, as

$$
\tau_{r}=\frac{\varepsilon_{s}+2}{\varepsilon_{\infty}+2} \tau
$$

and Eq. (2.38) becomes 


$$
\varepsilon_{r}=\varepsilon_{\infty}+\frac{\left(\varepsilon_{s}-\varepsilon_{\infty}\right)}{1+j 2 \pi f \tau_{r}}
$$

or

$$
\varepsilon_{r}=\varepsilon_{\infty}+\frac{\left(\varepsilon_{s}-\varepsilon_{\infty}\right)}{1+j\left(f / f_{r}\right)}
$$

Equation (2.40) is the well-known Debye's equation, in which $f_{r}$ is the relaxation frequency where $f_{r}=1 /\left(2 \pi \tau_{r}\right)$ (analogous to a resonant frequency).

\subsubsection{Conductivity}

The real part and imaginary part of Eq. (2.40) can be written explicitly as

$$
\varepsilon_{r}=\varepsilon_{r}{ }^{\prime}-j \varepsilon_{r}{ }^{\prime \prime}
$$

where

$$
\varepsilon_{r}^{\prime}=\varepsilon_{\infty}+\frac{\varepsilon_{s}-\varepsilon_{\infty}}{1+\left(f / f_{r}\right)^{2}}
$$

and

$$
\varepsilon^{\prime \prime}{ }_{r}=\frac{\left(\varepsilon_{s}-\varepsilon_{\infty}\right) f / f_{r}}{1+\left(f / f_{r}\right)^{2}}
$$

The real part is a measure of how much energy from an external electric field is stored in the material. The imaginary part is a measure of how dissipative or lossy a material is to an external electric field. The DC conductivity, $\sigma_{d c}$, of the dielectric material adds to the loss of external electric fields. As will be discussed in the next chapter, it is convenient in electric field analysis to combine the dielectric loss and conductive loss terms. The resulting equivalent complex permittivity becomes 


$$
\varepsilon_{r}{ }^{*}=\varepsilon_{r}{ }^{\prime}-j \varepsilon_{r}{ }^{i i}=\varepsilon_{r}{ }^{\prime}-j\left(\varepsilon_{r}{ }^{\prime}+\frac{\sigma_{d c}}{2 \pi f \varepsilon_{0}}\right)
$$

in which $\varepsilon_{r}^{i i}=\varepsilon_{r}{ }^{\prime \prime}+\sigma_{d c} d\left(2 \pi f \varepsilon_{0}\right)$ is the equivalent imaginary part of the permittivity. Sometimes the imaginary part of Eq. (2.42a) is expressed in terms of the equivalent conductivity, $\sigma_{e q}=$ $\sigma_{d c}+2 \pi f \varepsilon_{0} \varepsilon_{r}{ }^{\prime \prime}$. Equation (2.42a) then becomes

$$
\varepsilon_{r}^{*}=\varepsilon_{r}{ }^{\prime}-j \frac{\sigma_{e q}}{2 \pi f \varepsilon_{0}}=\varepsilon_{r}{ }^{\prime}-j \frac{\left(\sigma_{d c}+2 \pi f \varepsilon_{0} \varepsilon_{r}{ }^{\prime \prime}\right)}{2 \pi f \varepsilon_{0}}
$$

\subsection{Dielectric Properties of Soils}

The dielectric properties of soil components can be described using the results above however, the heterogeneity of soils adds to the complexity of the dielectric properties of soils due to interface effects. The interfacial polarization mechanisms and their effects on dielectric properties of soils are discussed below in Section 2.4.2 below.

\subsubsection{Dielectric Properties of Earth Materials}

Soil is a three-phase system consisting of air, solid particles, and water. The dielectric permittivity of air is approximately equal to 1.0 (i.e. no polarization in a free space). The conductivity of air is equal to 0 . Solid particles in a soil are non-polar materials. Their dielectric polarization is only due to electronic and ionic polarization mechanisms, which have relaxation frequencies above $1 \mathrm{THz}\left(10^{12} \mathrm{~Hz}\right)$. Therefore, they have a low value of dielectric permittivity $(\varepsilon \approx 5)$, and are nearly lossless, independent of frequency and temperature at frequencies less than 1 THz. The conductivity of soil particles is also equal to 0. For polar materials, such as water, the 
dipolar polarization adds to the electronic and ionic polarization, resulting in a much higher value of dielectric permittivity.

\subsubsection{Dielectric Properties of Pure Water}

The frequency dependence of the dielectric permittivity of pure water, $\varepsilon_{w}$, is given by Debye's equation in Eq. (2.40) with parameters $\mathcal{E}_{w 0}, \mathcal{E}_{w \infty}$, and $\tau_{w r}$ as the static dielectric constant, high-frequency limit, and relaxation time of $\varepsilon_{w}$, respectively. In addition to the dependence on frequency, the dielectric permittivity of water is also temperature dependent because the dielectric loss of the orientational polarization results from thermal effects (see Eq. (2.34)).

Stogryn (1971) determined the high-frequency dielectric constant for pure water to be $\mathcal{E}_{w \infty}$ $=4.9$. At high frequency, the contribution of the dielectric constant is from the electronic and ionic polarization, which are a mechanical effect rather than a thermal effect. The dependence of $\varepsilon_{w \infty}$ on temperature is so weak that for computational purpose $\varepsilon_{w \infty}$ may be considered a constant, thus

$$
\varepsilon_{w \infty}=4.9
$$

Stogryn (1971) obtained an expression for $\tau_{w r}$ by fitting a polynomial to the data reported by Grant et al. (1957), where

$$
2 \pi \tau_{w r}(T)=1.1109 \times 10^{-10}-3.824 \times 10^{-12} T+6.938 \times 10^{-14} T^{2}-5.096 \times 10^{-16} T^{3}
$$

where $T$ is in ${ }^{\circ} \mathrm{C}$. The relaxation frequency of pure water, $f_{w r}=1 /\left(2 \pi \tau_{w r}\right)$, lies in the microwave region where $f_{w r}\left(0^{\circ} \mathrm{C}\right) \approx 9 \mathrm{GHz}$ and $f_{w r}\left(20^{\circ} \mathrm{C}\right) \approx 17 \mathrm{GHz}$. Klein and Swift (1977) generated a regression fit for $\varepsilon_{w 0}(T)$ from dielectric measurements conducted at $1.43 \mathrm{GHz}$ and $2.65 \mathrm{GHz}$ (with $\mathcal{E}_{w \infty}$ and $\tau_{w r}$ given by Eqs. (2.43) and (2.44)) this resulted in 


$$
\varepsilon_{w 0}(T)=88.045-0.4147 T+6.295 \times 10^{-4} T^{2}+1.075 \times 10^{-5} T^{3}
$$

\subsubsection{Dielectric Properties of Saline Water}

Saline water is water containing dissolved salts. The salinity, $S$, of a solution is defined as the total mass of solid salt in grams dissolved in one kilogram of solution. Thus, $S$ is expressed in parts per thousand (ppt) on a weight basis. The frequency dependency of the dielectric permittivity of pure water, $\varepsilon_{s w}$, is given by Debye's equation, Eq. (2.40), with parameters $\varepsilon_{s w 0}, \varepsilon_{s w \infty}$, and $\tau_{s w r}$ as the static dielectric constant, high-frequency limit, and relaxation time of $\varepsilon_{s w}$, respectively.

Stogryn (1971) pointed out that there is no evidence to indicate that $\mathcal{E}_{w \infty}$ depends on salinity. Hence, $\varepsilon_{s w \infty}=\varepsilon_{w \infty}=4.9$. Klein and Swift (1977) obtain an expression for $\varepsilon_{s w 0}$ in terms of salinity and temperature in the range of $4 \mathrm{ppt}<\mathrm{S}<35 \mathrm{ppt}$ as

$$
\varepsilon_{s w 0}(T, S)=\varepsilon_{s w 0}(T, 0) \cdot a(T, S)
$$

where

$$
\varepsilon_{s w 0}(T, 0)=87.134-1.949 \times 10^{-1} T-1.276 \times 10^{-2} T^{2}+2.491 \times 10^{-4} T^{3}
$$

and

$$
a(T, S)=1.0+1.613 \times 10^{-5} T S-3.656 \times 10^{-3} S+3.210 \times 10^{-5} S^{2}-4.232 \times 10^{-7} S^{3}
$$

The form used in Eq. (2.44) can also be used to define the relaxation time. Klein and Swift (1977) modified the expression for $\tau_{s w r}$ to include the effect of salinity in the range of $0<\mathrm{S}<157 \mathrm{ppt}$,

$$
\tau_{s w r}(T, S)=\tau_{s w r}(T, 0) \cdot b(T, S)
$$

where 


$$
2 \pi \tau_{\text {swr }}(T, 0)=1.1109 \times 10^{-10}-3.824 \times 10^{-12} T+6.938 \times 10^{-14} T^{2}-5.096 \times 10^{-16} T^{3}
$$

and

$$
b(T, S)=1.0+2.282 \times 10^{-5} T S-7.638 \times 10^{-4} S-7.760 \times 10^{-6} S^{2}+1.105 \times 10^{-8} S^{3}(2.47 \mathrm{c})
$$

Similarly, the DC conductivity of sea water was derived for $0<\mathrm{S}<40$ ppt by Stogryn (1971) as

$$
\sigma(T, S)=\sigma(25, S) e^{-\phi}
$$

where

$$
\sigma(25, S)=S\left(0.18252-1.4619 \times 10^{-3} S+2.093 \times 10^{-5} S^{2}-1.282 \times 10^{-7} S^{3}\right)
$$

and

$$
\begin{aligned}
\phi=\Delta & {\left[2.033 \times 10^{-2}+1.266 \times 10^{-4} \Delta+2.464 \times 10^{-6} \Delta^{2}\right.} \\
& \left.-S\left(1.849 \times 10^{-5}-2.551 \times 10^{-7} \Delta+2.551 \times 10^{-8} \Delta^{2}\right)\right]
\end{aligned}
$$

\subsubsection{Interfacial Polarization Mechanisms}

The dielectric property of each soil phase can be described by the dielectric mechanisms mentioned above. At frequencies below the microwave region, the dielectric permittivity of the earth materials is almost independent of frequency. The heterogeneity of soil, however, adds to the complexity of its dielectric properties. There are three major effects due to this heterogeneity: bound water polarization (Fig. 2.9e), the Maxwell-Wagner effect (Fig. 2.9d), and double layer polarization (Fig. 2.9f).

The bound water polarization results from the fact that water can be bound to the soil matrix. The degree of binding varies from unbound or free water at a great distance from the matrix surface, to heavily bound or absorbed water. If water becomes bound to the soil matrix, it 
is not capable of doing as much work and hence has lost energy. The relaxation frequency of bound water is less than that of free water (Hilhorst, 1998).

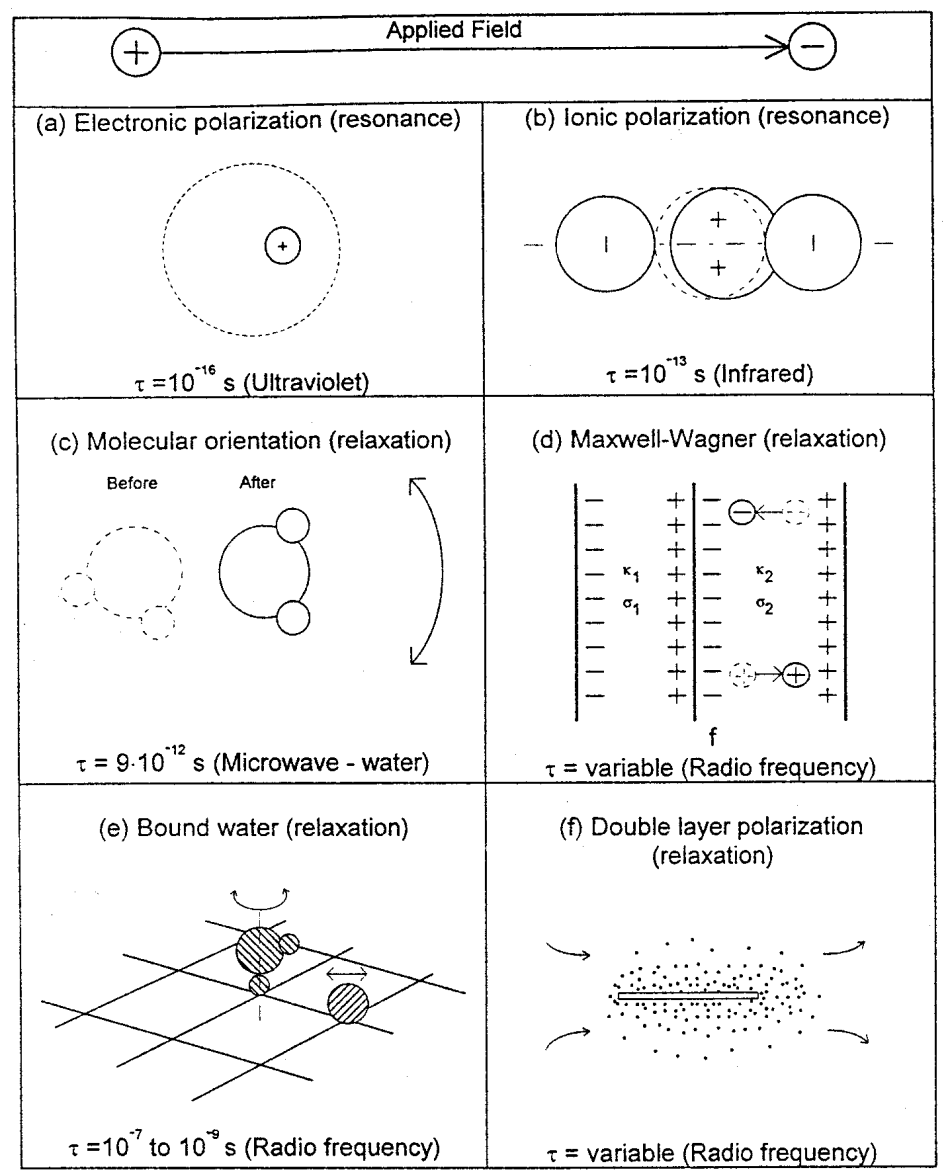

Fig. 2.9 Polarization mechanisms occurring in soils (Santamarina and Fam, 1997)

The double layer polarization is due to separation of cations and anions in an electric double layer around a clay plate. It is a surface phenomenon that is dominant at frequencies $<100$ $\mathrm{kHz}$ (Chew, 1982). Since this study focuses on the dielectric properties of soil in the TDR frequency between $1 \mathrm{MHz}$ and $1 \mathrm{GHz}$ as shown in Chapter 5, double layer polarization will be neglected.

The Maxwell-Wagner effect is the most important phenomenon that affects the lowfrequency end of the dielectric spectrum of soils. The Maxwell-Wagner effect is a macroscopic phenomenon that depends on the differences in dielectric properties of the soil constituents. It is a result of the distribution of conducting and non-conducting areas in the soil matrix. This 
interfacial effect is dominant at frequencies less than $150 \mathrm{MHz}$, below the frequencies where bound water relaxation plays a dominant role (Hilhorst, 1998).

\subsubsection{Dielectric Spectrum of Soils}

A qualitative representation of the dielectric properties of wet soils is presented in Fig. 2.10 (Hilhorst and Dirkson, 1994). The dielectric spectrum can be roughly divided into two parts with the dividing frequency at about $150 \mathrm{MHz}$. The higher frequencies are dominated by the bound water relaxation and the lower frequencies are dominated by the Maxwell-Wagner effect. The TDR frequencies lie from the higher end of the Maxwell-Wagner effect to the lower end of free water relaxation. Dielectric spectra of soils will be described in terms of soil physical properties in the next section. The results may be used to determine the soil type, water content and density.

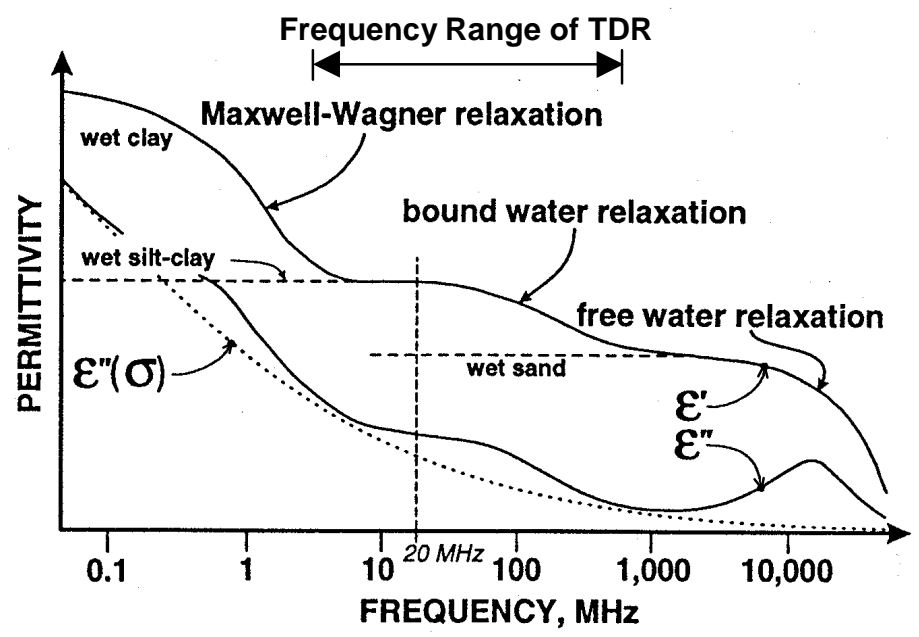

Fig. 2.10 Qualitative representation of dielectric properties of wet soils as a function of frequency (modified from Hilhorst and Dirkson, 1994)

\subsection{Dielectric Mixing Models}

Soil is a porous medium consisting of various types and concentrations of pore fluids, air and soil particles of different mineralogy, size, shape and orientation. The average dielectric permittivity and conductivity is related to (1) the volumetric fractions of the components (i.e. 
water content and density of the matrix); (2) characteristics of each component and their interaction; (3) microstructure of the soil matrix (i.e. the shape, orientation, and arrangement of the particles and pores). These factors are grouped in such a way that they represent different levels of characterization. They also represent the factors that affect different parts of the dielectric spectrum from high frequencies to low frequencies (Fig. 2.10). In the frequency of interest (the TDR frequency range), the average dielectric permittivity of a soil can be expressed as a function of volumetric fractions of the components and the characteristics of each component and their interactions. Functions that determine the dielectric permittivity in terms of the soil physical properties are called dielectric mixing models.

\subsubsection{Soil Physical Models}

It is conventional to schematically represent the three phases of a soil in a phase diagram in which each of the three phases is shown separately as in Fig. 2.11. In engineering practice, we usually measure the total volume $V_{t}$, the mass of water $M_{w}$, and the mass of dry solids $M_{s}$. Then we calculate the rest of the values and the mass-volume relationships that we need. If we look at the phase diagram carefully we can see that, for a unit volume of soil, only three quantities, $M_{s}$, $M_{w}$, and $\rho_{s}$, are required to completely define the phase relations. In geotechnical engineering, several physical parameters are defined for the sake of convenience in particular problems. These basic definitions are summarized in Table 2.2. Notice that these physical parameters are not independent. Depending on the problem, some parameters are more relevant than the others.
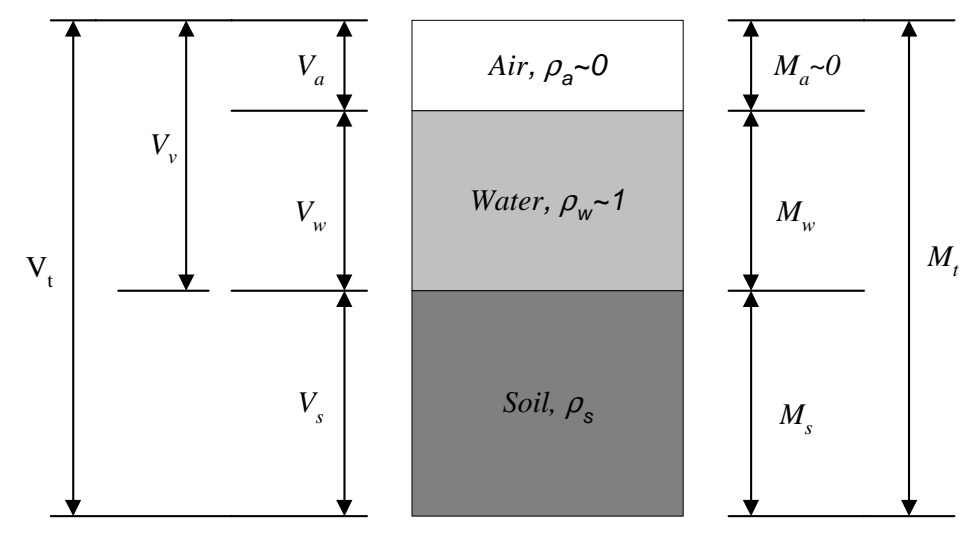

Fig. 2.11 Soil phase diagram (three phases) where $V$ is volume, $M$ is mass and $\rho$ is density for each phase 
Density of solid $\rho_{s}$, water content $\theta$ or $w$, density $\rho_{d}$ or $\rho_{t}$ form three independent parameters which completely describe the phase relations. The results are summarized in Table 2.3.

Table 2.2 Definitions of phase parameters.

\begin{tabular}{c|lcc}
\hline Description of Phase & \multicolumn{1}{|c}{ Terminology } & Definition & Unit \\
& & & \\
\hline \multirow{5}{*}{ Volumetric Ratio } & Void Ratio, $e$ & $V_{v} / V_{s}$ & Dimensionless \\
& Porosity, $n$ & $V_{v} / V_{t}$ & Dimensionless \\
& Degree of Saturation, $S$ & $V_{w} / V_{v}$ & $\%$ \\
& Volumetric Water & $V_{w} / V_{t}$ & Dimensionless \\
& Content, $\theta$ & & \\
\hline \multirow{2}{*}{ Mass Ratio } & Gravimetric Water & $M_{w} / M_{s}$ & $\%$ \\
& Content, $w$ & & \\
\hline \multirow{5}{*}{ Density } & Density of Solid, $\rho_{s}$ & $M_{s} / V_{s}$ & $\mathrm{Mg} / \mathrm{m}^{3}$ \\
& Dry Density, $\rho_{d}$ & $M_{s} / V_{t}$ & $\mathrm{Mg} / \mathrm{m}^{3}$ \\
& Total (Wet) Density, $\rho_{t}$ & $M_{t} / V_{t}$ & $\mathrm{Mg} / \mathrm{m}^{3}$ \\
& Saturated Density, $\rho_{s a t}$ & $\left(M_{s}+M_{w}\right) / V_{t}$, & $\mathrm{Mg} / \mathrm{m}^{3}$ \\
& & $S=100 \%$ & \\
& Submerged density, $\rho$ & $\rho_{s a t}-\rho_{w}$ & $\mathrm{Mg} / \mathrm{m}^{3}$ \\
\hline
\end{tabular}

Table 2.3 Three-Phase Relation Described by $\rho_{s}$, water content and density of soil $\left(V_{t}=1\right)$

\begin{tabular}{l|c|c|c|c|}
\hline Independent & \multicolumn{2}{|c|}{ LHS of Phase Diagram } & \multicolumn{2}{c}{ RHS of Phase Diagram } \\
\cline { 2 - 5 } Parameters & $V_{s}$ & $V_{w}$ & $M_{s}$ & $M_{w}$ \\
\hline$\rho_{s}, \theta, \rho_{d}$ & $\rho_{d} / \rho_{s}$ & $\theta$ & $\rho_{d}$ & $\theta \rho_{w}$ \\
$\rho_{s}, w, \rho_{d}$ & $\rho_{d} / \rho_{s}$ & $w \rho_{d} / \rho_{w}$ & $\rho_{d}$ & $w \rho_{d}$ \\
$\rho_{s}, \theta, \rho_{t}$ & $\left(\rho_{t^{-}} \theta \rho_{w}\right) / \rho_{s}$ & $\theta$ & $\rho_{t^{-}} \theta \rho_{w}$ & $\theta \rho_{w}$ \\
$\rho_{s}, w, \rho_{t}$ & $\left.\rho_{t} /(1+w) \rho_{s}\right]$ & $w \rho_{t} /\left[(1+w) \rho_{w}\right]$ & $\rho_{t} /(1+w)$ & $w \rho_{t} /(1+w)$ \\
\hline
\end{tabular}

Interatomic and intermolecular bonding forces hold matter together. Unbalanced forces exist at phase boundaries. All liquids and solids terminate at a surface, or phase boundary, on the other side of which is matter of a different composition or state. In solids, atoms are bonded into a three-dimensional structure, and the termination of this structure at a surface, or phase boundary, produces unbalanced force fields. Each unsatisfied bond force is significant relative to the weight of atoms and molecules, but is infinitesimal compared to the weight of a piece of gravel or a grain 
of sand. However, when particle size decreases, specific surface area increases. Experience shows that for many materials when particle size is reduced to 1 or $2 \mu \mathrm{m}$ or less, then the surface forces begin to exert a distinct influence on the soil behavior (Mitchell, 1993). The mechanical properties of soils depend directly on interactions of these phases with each other and with applied potentials (e.g., stress, hydraulic head, electrical potential, and temperature difference). Because of these interactions, we cannot understand soil behavior in terms of each component alone.

The air-water and air-soil interactions are not important on the behavior of soil systems. The major phase interaction is between soil particles and water. Water is a dipolar molecule. Even though water is electrically neutral, it has two separate centers of charge, one positive and one negative. Thus the water molecule is electrostatically attracted to the surface of the clay crystals. Water can also be held to the clay crystal by hydrogen bonding (hydrogen of the water is attracted to the oxygens or hydroxyls on the surface of the clay). The negatively charged clay surface also attracts cations present in the water. Since all cations are hydrated to some extent, depending on the ion, cations also contribute to the attraction of water to the clay surface (Holtz and Kovacs, 1981).

The water contained in the soil can be divided into two fractions: (a) bound water and (b) free water as shown in Fig. 2.12. Bound water refers to water molecules that are contained in the first few molecular layers surrounding the soil particles and therefore are tightly held by the soil particles due to the influence of matric forces.
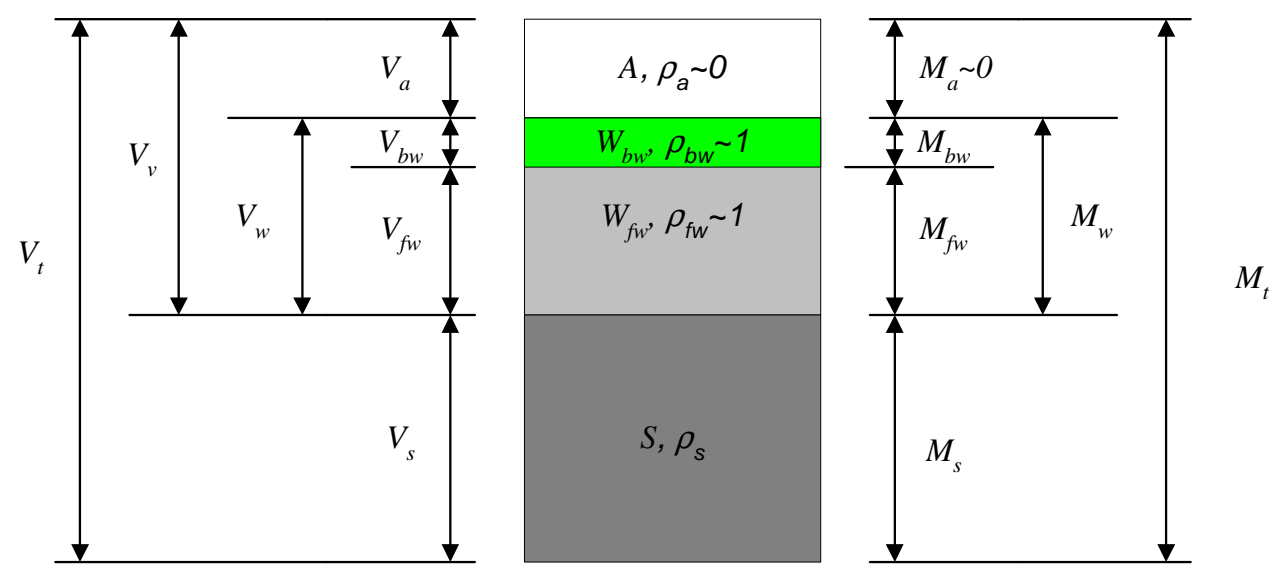

Fig. 2.12 Soil phase diagram (four phases) where $V$ is volume, $M$ is mass and $\rho$ is density for each phase 
Because the matric forces acting on a water molecule decrease rapidly with increasing distance from the soil particles, water molecules that are located several molecular layers away from soil particles are able to move within the soil medium with relative ease, and hence referred to as "free water".

Dividing the water into bound and free water fractions, we get a four-phase soil model to account for the soil-water interaction. This is only an approximate description of the actual distribution of water molecules in the soil medium and is based on a somewhat arbitrary criterion for establishing the transition point between bound and free water layers. Using the four-phase soil model and assuming that a bound water layer of uniform thickness exists on all soil particles, the volumetric faction of bound water can be calculated as

$$
\theta_{b}=\delta \rho_{d} A_{s}
$$

in which $A_{s}$ is the specific surface which is the ratio of the surface area of a material to its mass and $\delta$ is the uniform thickness of the bound water. The variable, $\delta$, is a function of type of mineral of soil particle and electrolyte while $A_{s}$ is a function of particle size. Depending on the mineralogy of the soil particle and electrolytes in the water, the value of $\delta$ may vary. We define the effective specific surface, $A_{e s}$, as the equivalent specific surface such that Eq. (2.49) gives the volumetric bound water content for the four-phase soil model assuming $\delta=\delta_{m}=3 \times 10^{-10} \mathrm{~m}$, in which $\delta_{m}$ is the thickness of a molecular water layer (Dobson et al., 1985). Therefore, Eq. (2.49) becomes

$$
\theta_{b}=\delta_{m} \rho_{d} A_{e s}
$$

By introducing the fourth phase and the definition of effective specific surface, Table 2.3 can be modified to Table 2.4. Effective specific surface directly reflects the volumetric bound water content through the dry density. It also reflects the combined effect of fineness of the soil 
particles, soil mineralogy and water salinity. Therefore, it is closely related to both the mechanical and electromagnetic behavior of soils.

Table 2.4 Four-Phase Relation Described by $A_{\mathrm{es}}, \rho_{\mathrm{s}}$, water content and density of soil $\left(V_{t}=1\right)$

\begin{tabular}{l|c|c|c|c|c}
\hline Independent & \multicolumn{3}{|c|}{ LHS of Phase Diagram } & \multicolumn{2}{c}{ RHS of Phase Diagram } \\
\cline { 2 - 6 } Parameters & $V_{s}$ & $V_{w}$ & $V_{b w}$ & $M_{s}$ & $M_{w}$ \\
\hline$A_{e s}, \rho_{s}, \theta, \rho_{d}$ & $\rho_{d} / \rho_{s}$ & $\theta$ & $\delta_{m} \rho_{d} A_{e s}$ & $\rho_{d}$ & $\theta \rho_{w}$ \\
$A_{e s}, \rho_{s}, w, \rho_{d}$ & $\rho_{d} / \rho_{s}$ & $w \rho_{d} / \rho_{w}$ & $\delta_{m} \rho_{d} A_{e s}$ & $\rho_{d}$ & $w \rho_{d}$ \\
$A_{e s}, \rho_{s}, \theta, \rho_{t}$ & $\left(\rho_{t}-\theta \rho_{w}\right) / \rho_{s}$ & $\theta$ & $\delta_{m} \rho_{t} A_{e s} /(1+$ & $\rho_{t^{-}} \theta \rho_{w}$ & $\theta \rho_{w}$ \\
& & & $w)$ & & \\
$A_{e s}, \rho_{s}, w, \rho_{t}$ & $\rho_{t} /[(1+w)$ & $w \rho_{t} /[(1+w) \rho$ & $\delta_{m} \rho_{d} A_{e s} /(1+$ & $\rho_{t} /(1+w)$ & $w \rho_{t} /(1+w)$ \\
& $\left.\rho_{s}\right]$ & $w$ & $w)$ & & \\
\hline
\end{tabular}

\subsubsection{Mixing Formulas}

Given a mixture made up of different components, each with its own dielectric and shape characteristics, can the dielectric behavior of the mixture as a whole be predicted? To answer this question, numerous dielectric mixing models for heterogeneous material have been developed in physics and engineering. These models can be grouped into two categories: theoretical polarization models and semi-empirical volumetric mixing models.

\subsubsection{Theoretical Polarization Models}

Dielectric permittivity of a mixture depends primarily on the volume fractions of the components and their 'pure' permittivities and secondarily on the shape of the components, their orientation to the applied field, and the effect of the interaction of the component permittivities on the local field (Sihvola and Lindell, 1992). In a theoretical model, usually the substance with the highest volume fraction is regarded as the host material, or continuous medium, and the other substances are regarded as inclusions. To relate the average dielectric permittivity of a mixture to factors mentioned above, it is necessary to relate the average electric field within the mixture as a whole to the field within the inclusions. If the inclusions are randomly dispersed within the host material, it is not possible to derive an exact solution for the fields within the inclusions because the mutual interactions of the inclusions (through their polarization fields) are dependent upon their positions relative to each other. Tinga et al. (1973) provided a concise review of the various 
approximations that have been proposed in the literature for solving the interaction problem. These approximations range from the ones ignoring short-range interactions (between inclusions) altogether (by restricting the validity of the dielectric mixing model to only those mixtures characterized by a low concentration of inclusions), to those assigning an effective dielectric constant to the immediate surroundings of an included particle in an attempt to account for shortrange interactions (De Loor, 1968), and finally to those accounting for first-order inclusion interactions by solving Maxwell's equations with appropriate boundary conditions (Tinga et al., 1973).

Most of the available theoretical models have been developed for media containing either ellipsoidal particles (or special types such as spheres, needles, and disc) or confocal ellipsoidal shells. Also, it is assumed in all cases that dimensions of the inclusions are much smaller than the wavelength of the radiation propagating in the mixture medium. Tinga (1992) gives a list of the major classical mixture theories from which most of the others can be derived.

De Loor's formula (De Loor, 1968) was adopted and modified for soils by Dobson et al. (1985). The dielectric permittivity of the soil mixture, $\varepsilon_{m}$ is given as

$$
\varepsilon_{m}=\frac{3 \varepsilon_{s}+2 v_{f w}\left(\varepsilon_{f w}-\varepsilon_{s}\right)+2 v_{b w}\left(\varepsilon_{b w}-\varepsilon_{s}\right)+2 v_{a}\left(\varepsilon_{a}-\varepsilon_{s}\right)}{3+v_{f w}\left(\frac{\varepsilon_{s}}{\varepsilon_{f w}}-1\right)+v_{b w}\left(\frac{\varepsilon_{s}}{\varepsilon_{b w}}-1\right)+v_{a}\left(\frac{\varepsilon_{s}}{\varepsilon_{a}}-1\right)}
$$

where $v$ is the volumetric fraction of the soil component, the subscripts $b w, f w, a$ and $s$ refer to bound water, free water, air, and soil solid, respectively. This formula is capable of describing the complex dielectric permittivity of soils measured at high frequencies $(>1.45 \mathrm{GHz})$. However, it is not capable of describing the complex dielectric permittivities measured at the lower frequencies. This is because De Loor's model was developed for the high frequency range (> 500 $\mathrm{MHz}$ ), where interface effects do not play a role (Heimovaara et al. 1994). The equation can be modified for the lower frequency region, however, by considering an equivalent circuit for the Maxwell-Wagner dispersion (Hilhorst, 1998). 


\subsubsection{Semi-empirical Volumetric Mixing Models}

A different group of published mixing formulas is based on Birchak's exponential model (Birchak et al., 1974):

$$
\left(\varepsilon_{m}\right)^{\alpha}=\sum_{i=1}^{n} v_{i}\left(\varepsilon_{i}\right)^{\alpha}
$$

where $v_{i}$ and $\varepsilon_{i}$ are the volumetric fraction and permittivity of each component. The exponent $\alpha$ is an empirical constant that summarizes the geometry of the medium with respect to the applied electric field. For $\alpha=1$, Eq. (2.52) reduces to the sum of the volume fractions of the permittivities of each component. In this case the material can be modeled as a parallel connection of capacitors. For $\alpha=-1$ this model is a series connection of capacitors. The $\alpha$ values of 1 and -1 are considered the extremes. All other constituent orientations should fall in between. For $\alpha=0.5$, Eq. (2.52) becomes the refractive index mixing formula. Eq. (2.52) was applied to soils by Dobson and Ulaby (1985) and Heimovaara et al. (1994) to predict the frequencydependent complex permittivity. It can be rewritten in terms of soil physical parameters as

$$
\varepsilon_{m}^{\alpha}=\left(\frac{\rho_{d}}{\rho_{s}}\right) \varepsilon_{s}^{\alpha}+\left(\theta-\theta_{b w}\right) \varepsilon_{f w}^{\alpha}+\theta_{b w} \varepsilon_{b w}^{\alpha}+\left(1-\frac{\rho_{d}}{\rho_{s}}-\theta\right) \varepsilon_{a}^{\alpha}
$$

where $\theta_{b w}$ is the volumetric bound water content. If the inhomogeneous mixture consists of a homogeneous matrix of one material in which particles of a second material are imbedded (the particle size being small compared to the wavelength of the electromagnetic wave), then $\alpha$ becomes 0.5 (Birchak et al., 1974). Lediu et al. (1986) and Siddiqui and Drnevich (1994) showed that the water content of the soil was a linear function of velocity of electromagnetic wave in the soil. This also suggests $\alpha=0.5$ for homogeneous and isotropic soils. 


\subsection{Summary}

The fundamental concepts of polarization and dielectric permittivity have been highlighted from a macroscopic point of view, and their implication with regard to soil dielectric properties examined. The heterogeneity of soils adds to the complexity of their dielectric properties due to interface effects. The three-phase model for soils has been extended to a four-phase model to account for the interface effects and hence the soil fineness. The physical parameters of the fourphase model have been shown to relate to the soil dielectric permittivity through a mixing model. The dielectric mixing models will be examined and compared using data measured by the TDR technique in the following chapters. A satisfactory mixing formula can then be used for nondestructive testing of soil physical properties. 


\section{CHAPTER 3 - ANALYSES OF TDR SYSTEMS}

\subsection{Introduction}

Permittivity generally is measured by placing the substance between two plates of a capacitor (at low frequencies) or into a coaxial line and measuring the complex impedance. A number of measurements over a wide frequency range are required for a complete characterization, which is time consuming and demands a considerable investment in instrumentation, particularly in the microwave region. Fellner-Feldegg (1969) showed that one could obtain the same information over a wide frequency range (1 $\mathrm{MHz}$ to several $\mathrm{GHz}$ ) by making measurements not in the frequency domain but in the time domain using a time domain reflectometer (TDR). Since then, TDR has been used extensively to measure the complex dielectric permittivity of polar and non-polar liquids (Giese and Tiemann, 1975, Clarkson et al., 1977). It has also been applied to measure dielectric properties of soils.

In the past 20 years, dramatic development has been made in the area of time domain reflectometry. It has become a valuable tool for the measurement of soil water content and bulk soil electrical conductivity. The two major features of TDR waveforms used for water content and conductivity calculations are the travel time of the TDR waveform in the probe and the voltage amplitude of the waveform at long times. However, much more information on the dielectric properties of the soil is contained in the waveforms measured with TDR. To extract this extra information requires a more fundamental understanding of the response of TDR probes inserted into the soil. A technique to realistically model the TDR waveform is introduced in this chapter.

\subsection{TDR Basics}

The instruments used for time domain reflectometry (TDR) are basically composed of a pulse generator and a sampling oscilloscope. These instruments are sometimes called cable radar. The pulse generator sends an electrical pulse along a coaxial cable and the oscilloscope is used to observe the echoes returning back to the input. Such instruments have been used since 1930's for cable testing prior to Fellner-Feldegg (1969) using them for measuring the permittivity of liquids. Fig. 3.1 shows the configuration of the TDR system. Fig. 3.2 shows an example of such a TDR 
system used for measuring dielectric properties of soils. The measurement probe is an extension of the coaxial probe that allows electromagnetic wave to propagate into the material being tested.

\section{TDR Device}

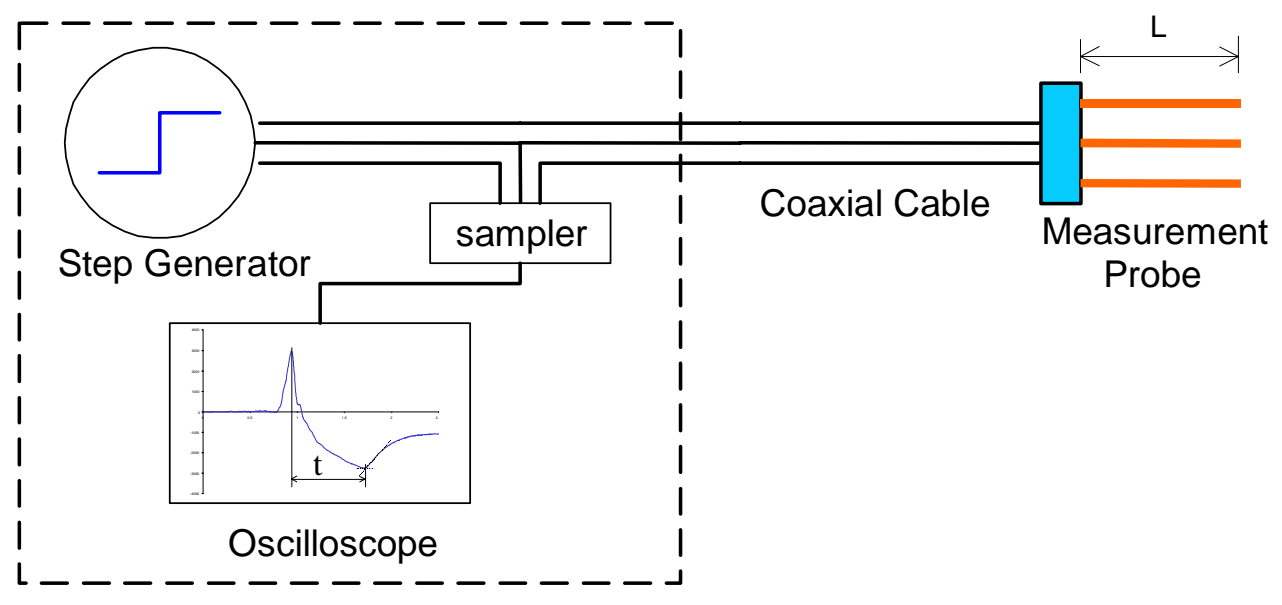

Fig. 3.1 TDR system configuration

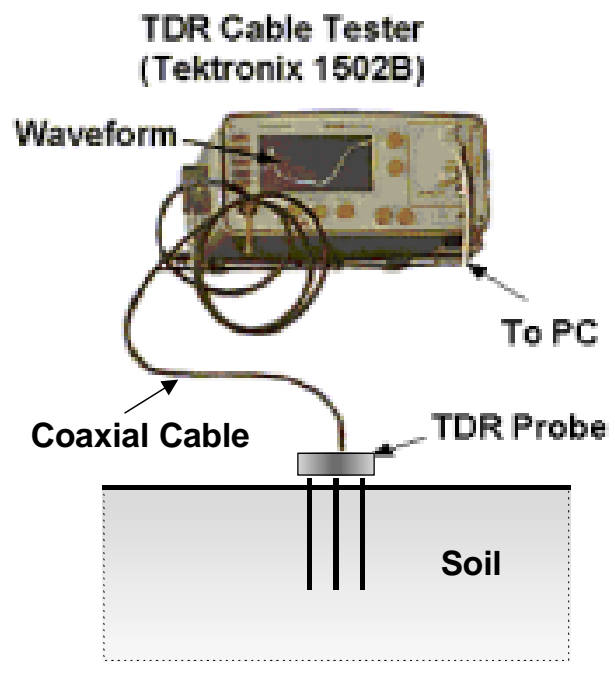

Fig. 3.2 Example of a TDR system used for soils 


\subsubsection{System Analysis of TDR Waveform}

The propagation of an electromagnetic field in a transmission line is governed by the wave equation derived from Maxwell's equations. Because of the special field structure within the transmission line, electromagnetic waves can be described by the propagation of a line voltage or a current. As will be discussed later in this chapter, two important components of the solution of the wave equation are the characteristic impedance $Z$ and the propagation constant $\gamma$. The characteristic impedance is the ratio of voltage to current propagating along the line. It is a function of the geometry of the transmission line and the dielectric permittivity of the insulating material. For a coaxial transmission line, the characteristic impedance is derived as

$$
Z=\frac{\ln \left(\frac{b}{a}\right)}{2 \pi} \sqrt{\frac{\mu_{0}}{\varepsilon_{0}}} \frac{1}{\sqrt{\varepsilon_{r}{ }^{*}}}=\frac{Z_{p}}{\sqrt{\varepsilon_{r}{ }^{*}}}
$$

where $b$ is the inner diameter of the outer conductor, $a$ is the outer diameter of the inner conductor, $\varepsilon_{0}$ is the vacuum permittivity $\left(8.854 \times 10^{-12} \mathrm{~F} / \mathrm{m}\right), \mu_{0}$ is the vacuum permeability $\left(4 \pi \times 10^{-7} \mathrm{H} / \mathrm{m}\right), \varepsilon_{r}^{*}$ is the equivalent dielectric permittivity, and $Z_{p}$ is defined as the impedance of the same line filled with air as the medium (Krauss, 1984). The characteristic impedance is an intrinsic property of the transmission line. For a line with sections having different impedances, reflection and transmission of waves can occur at the section interfaces.

The propagation constant is the other intrinsic property of a transmission line, which controls the speed and decay of a wave traveling along the line. It is only a function of the dielectric permittivity of the insulating material. For a coaxial transmission line, it is derived as

$$
\gamma=\frac{j 2 \pi f}{c} \sqrt{\varepsilon_{r}^{*}}=\alpha+j \beta
$$


in which $c$ is the velocity of electromagnetic waves in free space, and $\alpha$ and $\beta$ are the real and imaginary parts of the propagation constant, respectively. The real part represents the attenuation of the wave. The imaginary part is the spatial frequency, which gives the velocity of wave propagation when divided by temporal frequency $(2 \pi f)$. Since the dielectric permittivity of the insulating material depends on frequency, the propagation velocity is also a function of frequency. The TDR waveform recorded by the sampling oscilloscope is a result of multiple reflections and dispersion. A typical TDR output waveform is shown in Fig. 3.3.

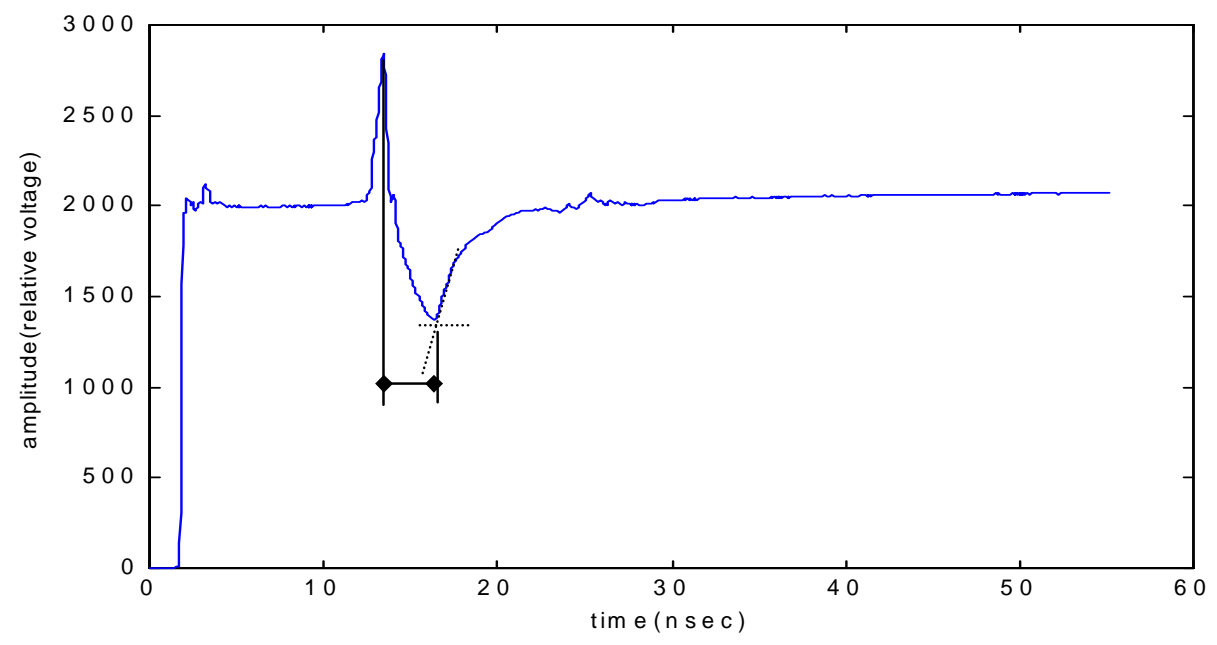

Fig. 3.3 A typical TDR output waveform

The experimental time-domain information may be treated by various procedures in order to obtain the dielectric permittivity. In the time-domain, the permittivity may be obtained by integral and convolution methods while in the frequency domain the permittivity can be obtained via numerical Fourier transformation of the time-domain signals. Clarkson et al. (1977) made a comparison between the real-time analysis and the frequency-domain analysis. Unless some special requirement exists, processing the data in frequency domain is preferred. Taking the ratio of the Fourier transform of a reflected transient from a sample to that of the input signal corresponds to deconvolving the sample response from the input signal, and the resulting quantity will usually (depending on the experimental arrangement) be related to the complex permittivity via a system function (or transfer function). 


$$
S\left(\varepsilon_{r}{ }^{*}, f\right)=\frac{Y(f)}{X(f)}
$$

in which $S(f)$ is the theoretical system function; $Y(f)$ is the Fourier transform of the output waveform; $X(f)$ is the Fourier transform of input step pulse. The system function is a function of impedance, propagation constant, and boundary conditions and it has different forms depending on the nature of the TDR experiments. It may be derived by considering the total reflection coefficient as being obtained from the sum of all the signals reaching the sampler from various successive reflections and transmissions at the line interfaces. For TDR systems that use coaxial probes and have only one mismatch (between the cable and the probe), analytical solutions of the system function have been derived (Giese and Tiemann, 1975). The permittivity at each frequency can then be evaluated numerically, e.g., by use of the Newton-Raphson iteration procedure.

A recent application of this type of system to measure dielectric permittivities of soils can be found in Heimovaara (1994) and Heimovaara et al. (1996). They designed a small 7-rod probe to emulate a coaxial probe and used a delicate probe head to avoid additional impedance mismatches between the cable and the probe so that the single-layer system function derived by Giese and Tiemann (1975) could be used. A probe that uses fewer rods and a mismatched transition unit between the cable and the probe are necessary for practical field measurements. The focus of this chapter is to develop a numerical wave propagation model that can deal with a non-coaxial probe and multiple- impedance mismatches so that robust probes with fewer rods and a mismatched transition unit can be used for the field measurements.

\subsubsection{Simplified Analysis}

\subsubsection{DC Analysis}

Limiting cases of time domain solutions provide some useful direct interpretation of the TDR waveforms (Giese and Tiemann, 1975). These can be used to estimate the DC permittivity and conductivity. If the length $L$ of the sample is chosen so that the thin-sample condition 


$$
|\chi|<<1
$$

is satisfied within the frequency range of the measurement, the dc conductivity $\sigma_{d c}$ and dc dielectric constant $\varepsilon_{s}$ can be estimated as

$$
\begin{aligned}
& \sigma_{d c}=\frac{\varepsilon_{0} c}{\left(\frac{Z_{1}}{Z_{p}}\right) L}\left(2 \frac{V_{0}}{V_{\infty}}-1\right) \\
& \varepsilon_{s}=\frac{A c}{\left(\frac{Z_{1}}{Z_{p}}\right) L V_{\infty}}+1
\end{aligned}
$$

where $Z_{1}$ is the impedance of the cable, $S_{e p}$ is the impedance of the probe filled with air, $V_{0}$ is the amplitude of the signal coming from the TDR system, $V_{\infty}$ is the asymptotic value of the reflected signal, and $A$ is the area between a reference waveform obtained for a fictitious dielectric of the assumed permittivity $\mathcal{E}_{r}^{*}=1-j \sigma_{d c} / \omega \varepsilon_{0}$ and the measured waveform for the dielectric under test with the permittivity $\varepsilon_{r}{ }^{*}=\varepsilon_{r}-j \sigma_{d c} / 2 \pi f \varepsilon_{0}$ as shown in Fig. 3.4, where $\varepsilon_{r}$ and $\sigma_{d c}$ are the dielectric permittivity and dc conductivity of the material being tested. The rational behind these formulations is that zero frequency in the frequency domain corresponds with infinite times in the time domain. 

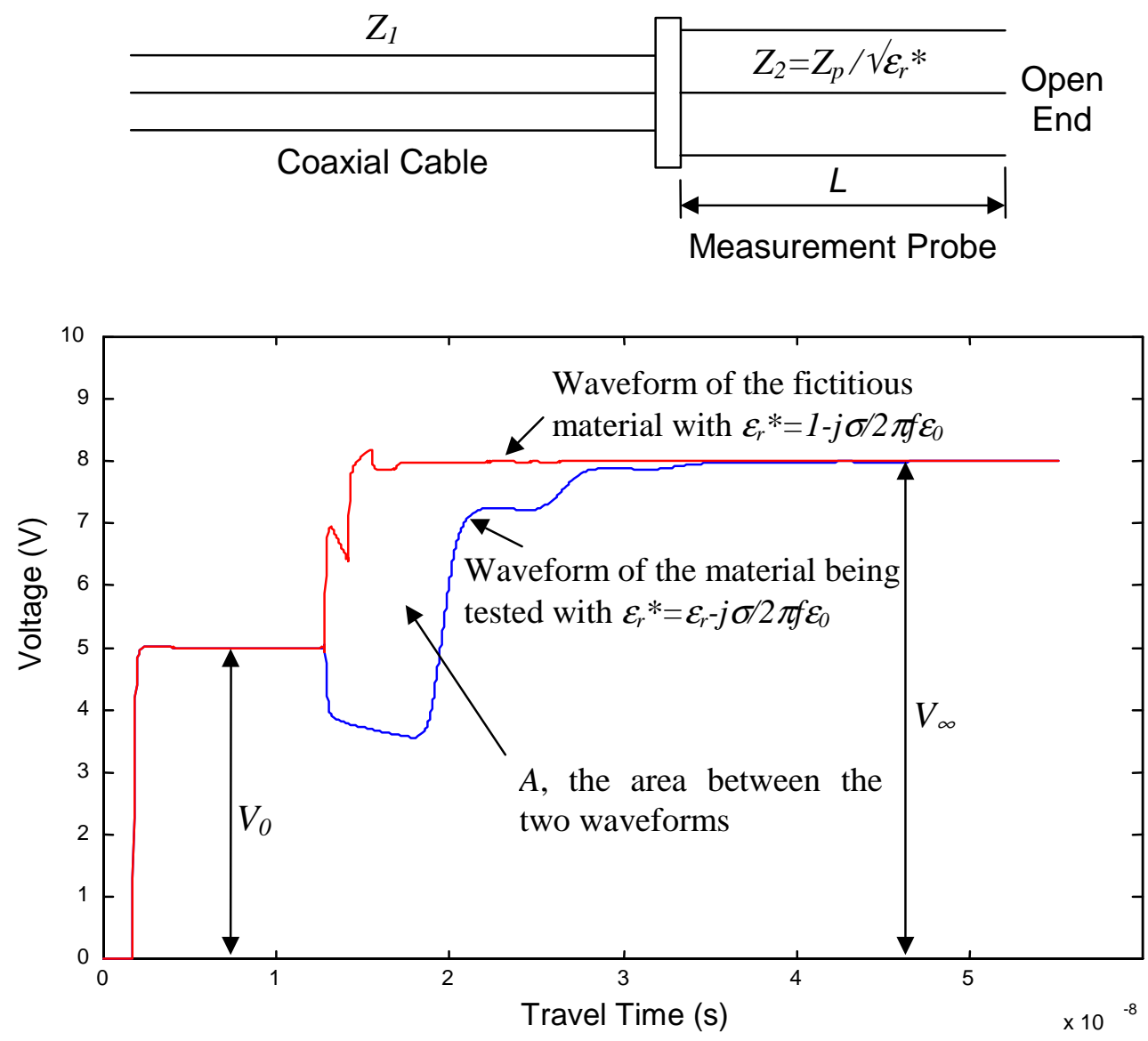

\section{Fig. 3.4 Direct interpretation of the TDR waveform to estimate dc dielectric permittivity $\varepsilon_{s}$ and de conductivity $\sigma_{d c}$}

\subsubsection{Travel Time Analysis}

In general, permittivity is a complex number and is a function of the applied frequency of the electric field in the frequency domain. The frequency-dependent propagation velocity of the electromagnetic wave traveling in a material with equivalent dielectric permittivity $\varepsilon_{r}^{*}$ (Eq. 2.42a) is determined as

$$
v(f)=\frac{2 \pi f}{\beta}=\frac{c}{\sqrt{\frac{\varepsilon_{r}{ }^{\prime}(f)}{2}\left(1+\sqrt{1+\left(\frac{\varepsilon_{r}{ }^{i i}(f)}{\varepsilon_{r}{ }^{\prime}(f)}\right)^{2}}\right)}}
$$


Davis and Annan (1977) showed that the real part of the permittivity of soils is not strongly frequency dependent over the frequency range of $1 \mathrm{MHz}$ to $1 \mathrm{GHz}$. They also showed that the imaginary part was considerably less than the real part in this frequency range. Based on these conclusions, Topp et al. (1980) defined the apparent dielectric constant, $K_{a}$, as the quantity determined from the measured velocity of the electromagnetic wave traveling through a transmission line. The apparent propagation velocity, $v$, of an electromagnetic wave in a transmission line is related to the apparent dielectric constant, $K_{a}$, as

$$
v=\frac{c}{\sqrt{K_{a}}}
$$

The apparent velocity of the electromagnetic wave traveling through a transmission line is obtained by travel time analysis using a tangent line approximation to find the inflection points (Fig. 3.3). The TDR device sends a step pulse down the cable that is reflected from both the beginning and end of the probe due to impedance mismatches. The two reflections cause two discontinuities in the resulting signal. The time difference between these two discontinuities is the time $(t)$ required by the signal to travel twice the length $(L)$ of the probe in soil. So the wave propagation velocity in soil is

$$
v=\frac{2 L}{t}
$$

and the apparent dielectric constant of soil then becomes (using Eq. 3.8 and 3.9)

$$
K_{a}=\left(\frac{c t}{2 L}\right)^{2}
$$


TDR has become a useful tool for the measurement of soil water content since Topp et al. (1980) published their empirical correlation equation between $K_{a}$ and volumetric water content.

\subsubsection{Amplitude Attenuation Analysis}

Similar to travel time analysis, simple amplitude attenuation analysis was introduced by Dalton et al. (1984) and Topp et al. (1988) to measure DC conductivity. The dielectric was assumed to be non-dispersive in the attenuation analysis, which violates the fact that a dielectric with non-zero conductivity is dispersive. In addition, the interpretation of amplitude attenuation is difficult because of the dispersion phenomenon. Therefore, amplitude attenuation analysis is not a useful tool for estimating DC conductivity. The DC analysis (Eq. 3.6) given in Section 3.2.2.1 should be a better way to determine DC conductivity both theoretically and practically.

\subsection{Advanced Analysis of Wave Propagation in the TDR System}

Currently, the system analysis of TDR system is limited to a single-section system. The time-domain analysis is limited to the simplified analysis. However, the TDR waveform contains much more information. In order to extract this extra information, a more fundamental understanding of the response of probes inserted in the material being tested is required. The main objective in this chapter is to develop a wave propagation model that can analyze the system function of a multi-section system and simulate the full time-domain waveform. The model will facilitate parametric studies to gain insight into the dielectric behavior of soils.

Transmission lines (TL) for TDR measurements are primarily of two types: coaxial type and $n+1$ wire type. The coaxial type of probe is composed of a cylindrical cylinder (CC) acting as the outer conductor and a rod along the centerline of the cylinder acting as a central conductor. The coaxial probe is called coaxial line $(\mathrm{CL})$. The $\mathrm{n}+1$ wire type of probe is composed of $\mathrm{n}$ wires acting as the outer conductors and a center wire as the inner conductor. The $n+1$ wire probe is also called a multiple rod probe (MRP) when rods are used rather than wires. The transmission line can be submerged in the media or floating on top of the media. Fig. 3.5 illustrates the useful types and configurations of transmission lines in a TDR system. The coaxial type of probe is adopted for laboratory measurements such as in the compaction mold or in a Shelby tube, using 
the cylindrical cylinder as the outer conductor with the inner conductor being a rod inserted along the centerline of the soil in the mold. The MRP probes can be used for in-place measurements. Several researchers (Topp et al., 1980, Zegelin et al., 1989, Heimovaara, 1993, Siddiqui and Drnevich, 1995) use these two types of transmission lines. Selker and et al., 1993 showed that a non-invasive MRP can be adopted for truly non-destructive field probes.
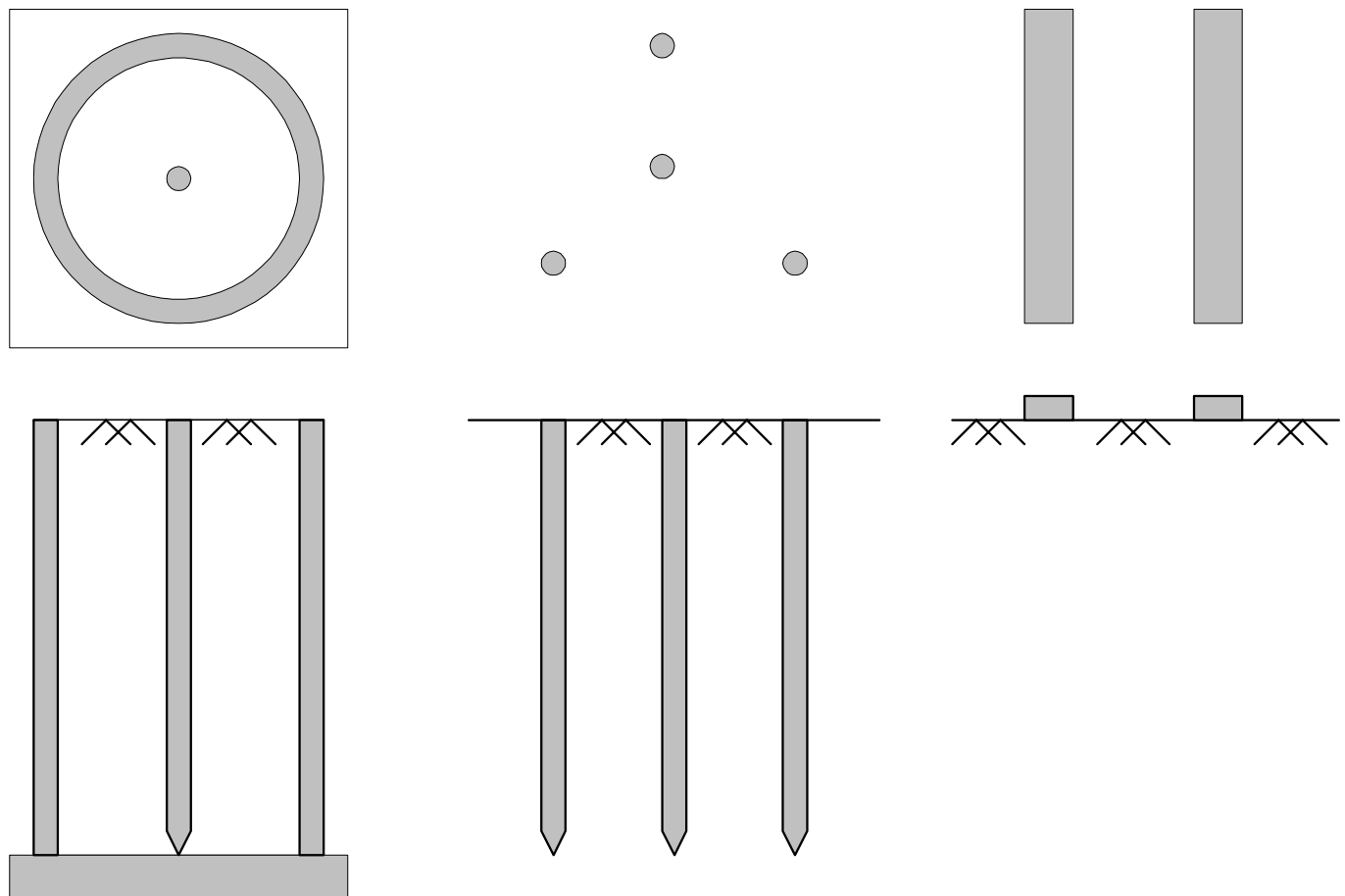
(a) Coaxial Line
(b) MRP
(c) Non-invasive MRP

Fig. 3.5 Configurations of types of transmission lines

Coaxial lines and 2-rod multiple-rod probes are two-conductor transmission lines. The analysis of such transmission lines consisting of two parallel conductors of uniform cross section is a fundamental and well-understood subject in electrical engineering. A multiple-rod probe with more than 2 rods is a multi-conductor transmission line (MTL). The analysis of such lines consisting of more than two conductors is not as well understood. 
In a TDR system, there can be more than one type of transmission line. A $50-\Omega$ cable connecting the cable tester and the TDR probes is a coaxial transmission line. The measurement probe could be either a coaxial line or MRP. We also need a transitional device (or probe head) to connect the cable and measurement probe. Therefore, most likely, the TDR system consists of cable tester (e.g., Tektronix 1502B) and non-uniform transmission line as shown in Fig. 3.6. A Transmission line with a uniform cross section is called a uniform transmission line, otherwise it is called a non-uniform transmission line. The wave propagating in a uniform transmission line can be analyzed easily by solving the wave equation for a uniform transmission line. The wave propagation in a non-uniform transmission line, however, is more difficult to analyze because each section of the non-uniform line has a different governing wave equation.

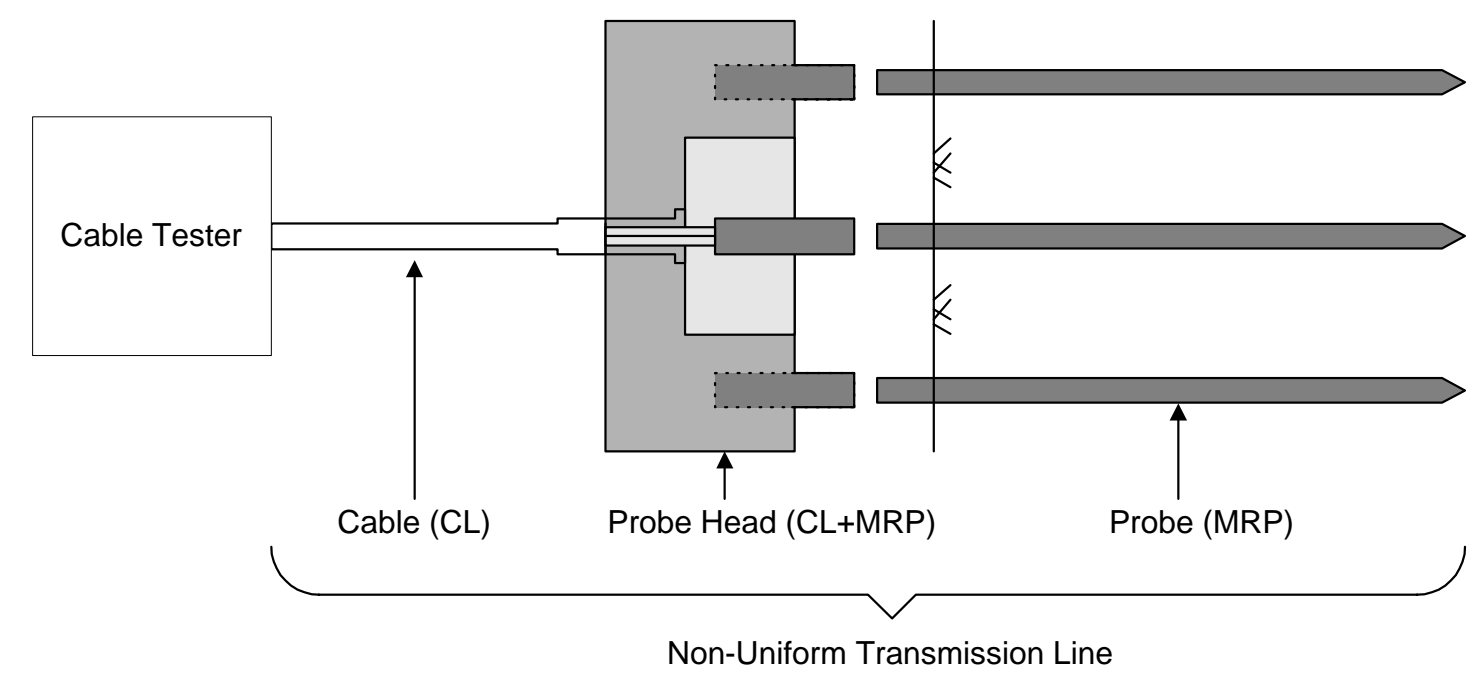

Fig. 3.6 Components of a transmission line in the TDR system

In developing solutions for the wave propagation in transmission lines, we find similarity with formulations in pile dynamics and structural dynamics. The same mathematical framework can be used to develop the solutions. The framework to analyze the wave propagation in a transmission line is as follows: 
1. Derive the governing differential equations using an infinitesimal physical element. The governing equation will typically involve per-unit-length parameters of the problem (Section 3.4).

2. Determine the per-unit-length parameters for a given geometry and material (Section 3.5).

3. Obtain the general solution by solving the resulting governing equations using either analytical or numerical approaches (Section 3.6).

4. Incorporate the boundary conditions to determine the unknown coefficients in the general form of the solution (Section 3.6).

This four-step process is exactly how we can obtain the solution for the line voltage and currents in the frequency domain in a TDR system. Inverse Fourier transformation is carried out numerically to obtain the time-domain waveform (Section 3.7)

\subsection{Transmission Line Equations}

The interaction between electric and magnetic energy gives rise to the propagation of electromagnetic waves. More specifically, the magnetic fields that change with time induce electric fields as explained by Faraday's law, and the time-varying electric fields induce magnetic fields as explained by the generalized Ampere's law. These interrelationships also occur along conducting or dielectric boundaries, and can give rise to waves that are guided by such boundaries. Due to the special field structure inside the transmission line, electromagnetic waves can be described by the propagation of line voltage or current. In this section, the governing equations of a uniform transmission line will be derived. Both 2-conductor and multi-conductor transmission line will be discussed.

The transmission-line equation can be derived using one of the following methods: (1) from the integral form of Maxwell's equations; (2) from the differential form of Maxwell's equations; and (3) from the per-unit-length equivalent circuit. The first two derivations are rigorous and can illustrate many important concepts and restrictions of the formulation. However, 
the transmission-line equations are usually derived from a representation of the line as lumped circuit elements distributed along the line. We will first derive the transmission line equation using the integral form of Maxwell's equations because it is rigorous and not only gives us the resulting governing equation, but also gives the meaning and the properties of the per-unit-length parameters. The derivation using a per-unit-length equivalent circuit will also be discussed because it is simple and useful. Basic assumptions of the derivation are introduced first.

\subsubsection{Basic Assumptions}

\subsubsection{Transverse ElectroMagnetic (TEM) Mode}

The fundamental assumption for all transmission-line formulas and analyses, whether for a two-conductor or a multi-conductor line, is that the field surrounding the conductors is a Transverse Electro-Magnetic (TEM) field. A TEM field is one in which the electric and magnetic fields in the space surrounding the line conductors are transverse or perpendicular to the line axis. Transmission lines having electrically large cross-sectional dimensions can have, in addition to the TEM mode of propagation, other higher-order modes of propagation (Leviaton and Adams, 1982). An analysis of the fields using the transmission-line formulation would then only predict the TEM mode component and not represent a complete analysis. Other aspects, such as imperfect line conductors, also may invalidate the TEM mode transmission-line equation description.

Justification of the assumption of only TEM propagation will be discussed in a later section, so the effect of non-TEM fields will not be considered in this study. In general, for transmission lines where the cross-sectional dimensions are much less than a wavelength, the solution of the TEM transmission line equations gives the primary contribution to the field. This is referred to as the quasi-TEM approximation and is an implicit assumption throughout this study (Lindell, 1981).

\subsubsection{Uniform Transmission Line}

If the cross-sectional dimensions of the line vary along the line axis $(z)$, then the per-unitlength parameters will be functions of the position variable, $z$. This makes the resulting transmission-line equations very difficult to solve. Such transmission lines are said to be non- 
uniform lines. If the cross-sectional dimensions of both the line conductors and the surrounding medium are constant along the line axis, the line is said to be a uniform line whose resulting differential equations are relatively easy to solve. The TDR system is an example of a nonuniform line as shown in Fig. 3.6 because the conductor cross sections and dielectric media are different in the coaxial cable, probe head, and soil specimen. Therefore, the per-unit-length parameters will be functions of $z$. A common way of handling this type of problem is to divide the line into several uniform sections, analyze each separately and combine the results using the boundary conditions at the interfaces.

\subsubsection{Perfect Conductor or Conductors of Small Losses}

Unlike losses in the surrounding medium, lossy conductors implicitly invalidate the TEM field structure assumption. The line current flowing through the imperfect line conductor generates a nonzero electric field along the conductor surface, which is directed along the line axis violating the basic assumption of the TEM field structure in the surrounding medium. However, if the conductor losses are small, the resulting field structure is approximately TEM. This is referred to as the quasi-TEM assumption. Although the transmission-line equations are no longer strictly valid, they are assumed to be representative because the small losses can be accounted for by including the per-unit-length resistance parameter, $r$.

An inhomogeneous surrounding medium (in the cross section) also invalidates the basic assumption of a TEM field structure because a TEM field structure must have one and only one velocity of propagation of the waves in the medium. This cannot be the case for an inhomogeneous medium. An approximate way of characterizing this situation of an inhomogeneous medium is to obtain an effective dielectric constant (Lindell, 1981). Baker and Lascano (1989), Knight (1992), and Siddiqui and Drnevich (1995) have studied the spatial sensitivity of the effective dielectric permittivity.

\subsubsection{Plane Wave Equation}

The fundamental field on a transmission line is the transverse electromagnetic (TEM) field. Therefore, it is appropriate to examine the general properties of this TEM mode of propagation. A TEM field is one in which the electric and magnetic field vectors at each point in space lie in a plane transverse or orthogonal to the direction of propagation. 
Consider a rectangular coordinate system shown in Fig. 3.7 illustrating a propagating TEM wave in which the field vectors are assumed to lie in a plane (the $x-y$ plane) that is transverse to the direction of propagation (the $z$-axis). These field vectors are denoted with a subscript $t$ to denote the transverse direction. Combining Eq. (2.7) and (2.8), Maxwell's equations at a source free location $(\rho=0)$ become

$$
\begin{aligned}
& \nabla \cdot \hat{\boldsymbol{E}}_{\boldsymbol{t}}=0 \\
& \nabla \cdot \hat{\boldsymbol{H}}_{\boldsymbol{t}}=0 \\
& \nabla \times \hat{\boldsymbol{E}}_{\boldsymbol{t}}=-j 2 \pi f \mu \hat{\boldsymbol{H}}_{\boldsymbol{t}} \\
& \nabla \times \hat{\boldsymbol{H}}_{\boldsymbol{t}}=\sigma \hat{\boldsymbol{E}}_{\boldsymbol{t}}+j 2 \pi f \varepsilon \hat{\boldsymbol{E}}_{\boldsymbol{t}}
\end{aligned}
$$

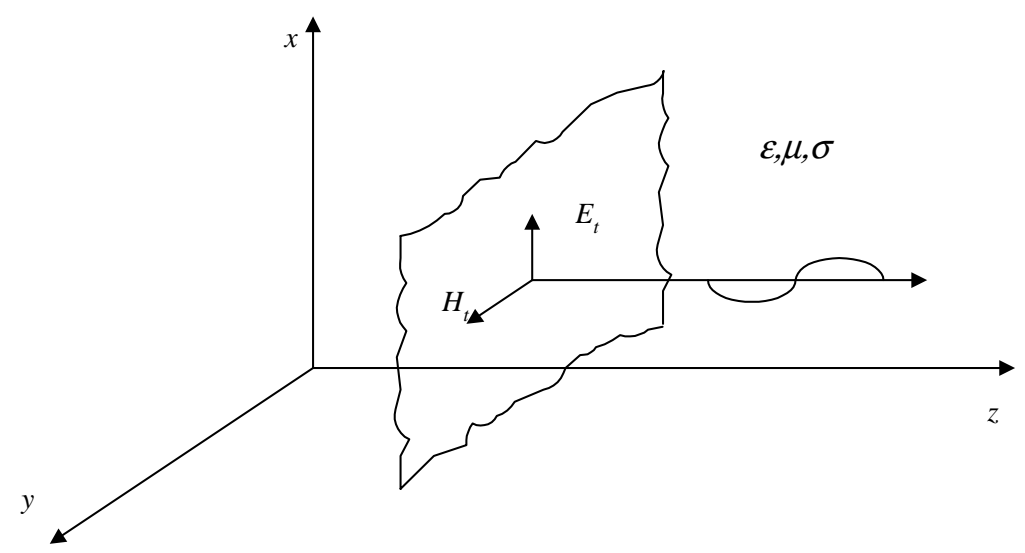

Fig. 3.7 Illustration of the TEM mode of propagation

The "del" operator, $\nabla$, can be broken into two components, one component in the $z$ direction and one component in transverse plane as

$$
\nabla=\nabla_{t}+\nabla_{z}
$$


where $\nabla_{t}=\tilde{\boldsymbol{x}} \frac{\partial}{\partial x}+\tilde{\boldsymbol{y}} \frac{\partial}{\partial y}, \nabla_{z}=\tilde{z} \frac{\partial}{\partial z}$, and $\tilde{\boldsymbol{x}}, \tilde{\boldsymbol{y}}$, and $\tilde{\boldsymbol{z}}$ are unit vectors in the direction of $\mathrm{x}, \mathrm{y}$, and z. Using Eq. (3.12) to separate Eq. (3.11) by equating those components in the $z$ direction and in the transverse plane gives

$$
\begin{aligned}
& \nabla_{t} \cdot \hat{\boldsymbol{E}}_{\boldsymbol{t}}=0 \\
& \nabla_{t} \cdot \hat{\boldsymbol{H}}_{t}=0 \\
& \nabla_{t} \times \hat{\boldsymbol{E}}_{\boldsymbol{t}}=0 \\
& \nabla_{t} \times \hat{\boldsymbol{H}}_{\boldsymbol{t}}=0 \\
& \nabla_{z} \cdot \hat{\boldsymbol{E}}_{t}=0 \\
& \nabla_{z} \cdot \hat{\boldsymbol{H}}_{\boldsymbol{t}}=0 \\
& \nabla_{z} \times \hat{\boldsymbol{E}}_{\boldsymbol{t}}=\tilde{z} \times \frac{\partial \hat{\boldsymbol{E}}_{t}}{\partial z}=-j 2 \pi f \mu \hat{\boldsymbol{H}}_{t} \\
& \nabla_{z} \times \hat{\boldsymbol{H}}_{\boldsymbol{t}}=\tilde{z} \times \frac{\partial \hat{\boldsymbol{H}}_{t}}{\partial z}=\sigma \hat{\boldsymbol{E}}_{\boldsymbol{t}}+j 2 \pi \varepsilon \hat{\boldsymbol{E}}_{\boldsymbol{t}}
\end{aligned}
$$

In the transverse plane, Eq. (3.13) is identical to the equation for a static field. This permits a unique definition of voltage and current for a non-static variation of the field vectors in a fashion similar to the static case (Ramo et al., 1994). The voltage between two points at distance $z$ in a transverse plane can be defined uniquely as the line integral of the transverse electric field between those two points. Similarly, the current in the $z$ direction can be uniquely defined as the line integral of the transverse magnetic field around any closed contour lying solely in the transverse plane at distance $z$. Thus, 


$$
\begin{aligned}
& \hat{V}(z)=-\int_{P_{1}}^{P_{2}} \hat{\boldsymbol{E}}_{\boldsymbol{t}} \cdot d \boldsymbol{l} \\
& \hat{I}(z)=-\oint_{c_{t}} \hat{\boldsymbol{H}}_{\boldsymbol{t}} \cdot d \boldsymbol{l}
\end{aligned}
$$

In the $z$ direction, Eq. (3.14a) and (3.14b) are automatically satisfied by aTEM field (i.e., $E_{z}$ and $H_{z}$ equal to zero). Equations (3.14c) and (3.14d) are coupled governing equations for the interaction between the $\hat{\boldsymbol{E}}$ field and the $\hat{\boldsymbol{H}}$ field. In order to solve the equations it is better to transfer them to two de-coupled equations. We take the cross product of the $z$-direction unit vector with (3.14c) and (3.14d), which gives

$$
\begin{aligned}
& -\frac{\partial \hat{\boldsymbol{E}}_{t}}{\partial z}=-j 2 \pi f \mu\left(\tilde{z} \times \hat{\boldsymbol{H}}_{t}\right) \\
& -\frac{\partial \hat{\boldsymbol{H}}_{t}}{\partial z}=\sigma\left(\tilde{z} \times \hat{\boldsymbol{E}}_{t}\right)+j 2 \pi f \varepsilon\left(\tilde{z} \times \hat{\boldsymbol{E}}_{t}\right)
\end{aligned}
$$

Taking the partial derivative of both sides of Eq. (3.16) with respect to $z$ and substituting into Eq. (3.14) gives

$$
\begin{aligned}
& \frac{\partial^{2} \hat{\boldsymbol{E}}_{t}}{\partial z^{2}}=j 2 \pi f \mu(\sigma+j 2 \pi f \varepsilon) \hat{\boldsymbol{E}}_{\boldsymbol{t}}=(j 2 \pi f)^{2} \mu \varepsilon^{*} \hat{\boldsymbol{E}}_{\boldsymbol{t}} \\
& \frac{\partial^{2} \hat{\boldsymbol{H}}_{\boldsymbol{t}}}{\partial z^{2}}=j 2 \pi f \mu(\sigma+j 2 \pi f \varepsilon) \hat{\boldsymbol{H}}_{\boldsymbol{t}}=(j 2 \pi f)^{2} \mu \varepsilon^{*} \hat{\boldsymbol{H}}_{\boldsymbol{t}}
\end{aligned}
$$


where it is convenient to define the equivalent dielectric permittivity as $\varepsilon^{*}=\varepsilon+\sigma /(j 2 \pi f)$ to represent the total effect of the dielectric permittivity and conductivity. The Eq. (3.17) is the wave equation for a plane wave in a source free region. Since the fields in a transmission line are assumed to be TEM, wave propagation in a transmission line satisfies Eq. (3.17). However, we are looking for the transmission line equation in terms of voltage and current which are quantities that can be readily measured. Note that we can derive Eq. (3.17) using integral form. Since there are no boundaries in a general free space, the differential form is used to illustrate the TEM properties. In the next section, the integral form is used to illustrate the current and voltage and per-unit-length parameters when deriving the TL equations.

\subsubsection{Transmission Line Equations of a Two-Conductor Line}

Consider the two-conductor transmission line shown in Fig. 3.8 and assume all the assumptions made in previous section are valid. Starting with Faraday's law of Maxwell's equations in integral form we get

$$
\oint_{c} \hat{\boldsymbol{E}} \cdot d \boldsymbol{l}=-j 2 \pi f \mu \int_{s} \hat{\boldsymbol{H}} \cdot d \mathrm{~s} \quad \text { (Faraday's Law) }
$$

Since we assume only TEM fields about the conductors in any cross-sectional plane is valid, as indicated in Fig. 3.8b, there are no $z$ components in the $\hat{\boldsymbol{E}}$ and $\hat{\boldsymbol{H}}$ fields (i.e. $E_{z}=H_{z}=0$ ). Therefore, we may uniquely define voltage and current between conductors, independent of path, so long as we take the path to lie in a transverse plane. Eq. (3.15) becomes

$$
\begin{aligned}
& \hat{V}(z)=-\int_{0}^{1} \hat{\boldsymbol{E}}_{\boldsymbol{t}} \cdot d \boldsymbol{l}_{\boldsymbol{0} \boldsymbol{1}} \\
& \hat{I}(z)=\oint_{\mathrm{c}_{\mathrm{xy}}} \hat{\boldsymbol{H}}_{\boldsymbol{t}} \cdot d \boldsymbol{l}_{\boldsymbol{x} \boldsymbol{y}}
\end{aligned}
$$


where 0 and 1 in the integral are any points along conductors 0 and 1 (see Fig. 3.8), and $c_{x y}$ is a closed contour lying in the transverse plane as shown in Fig. 3.8b.

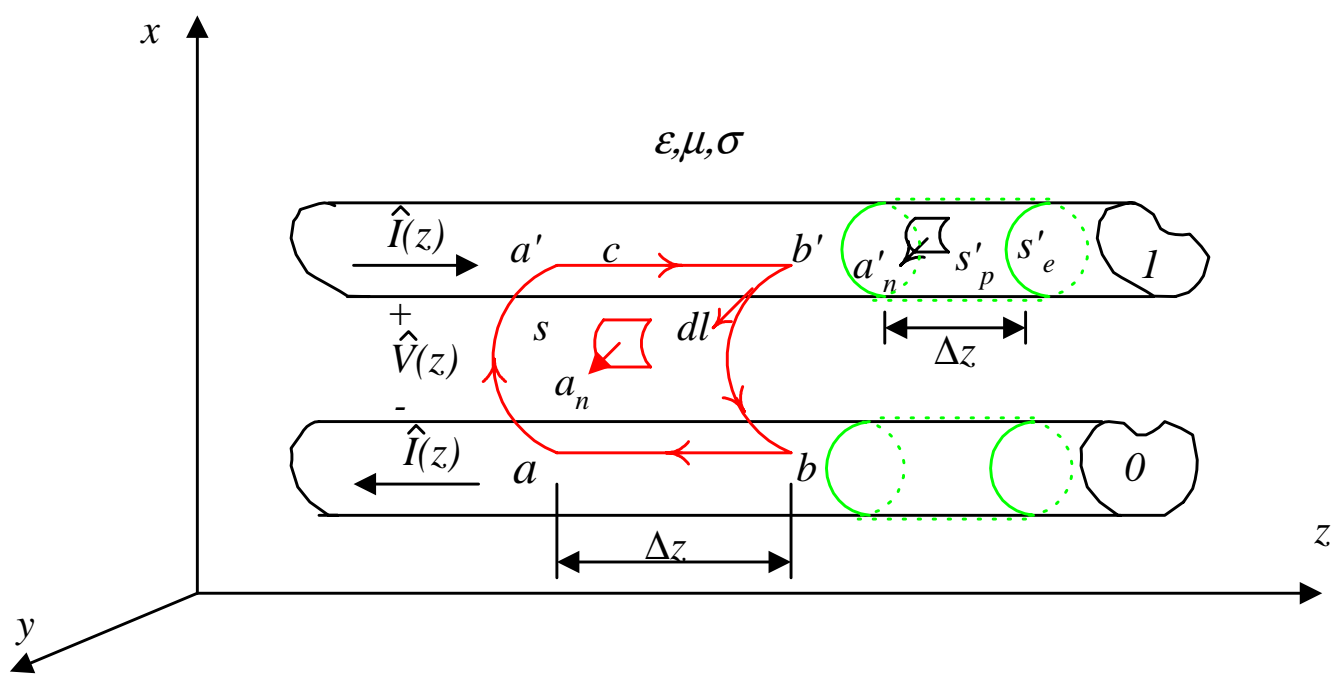

(a)

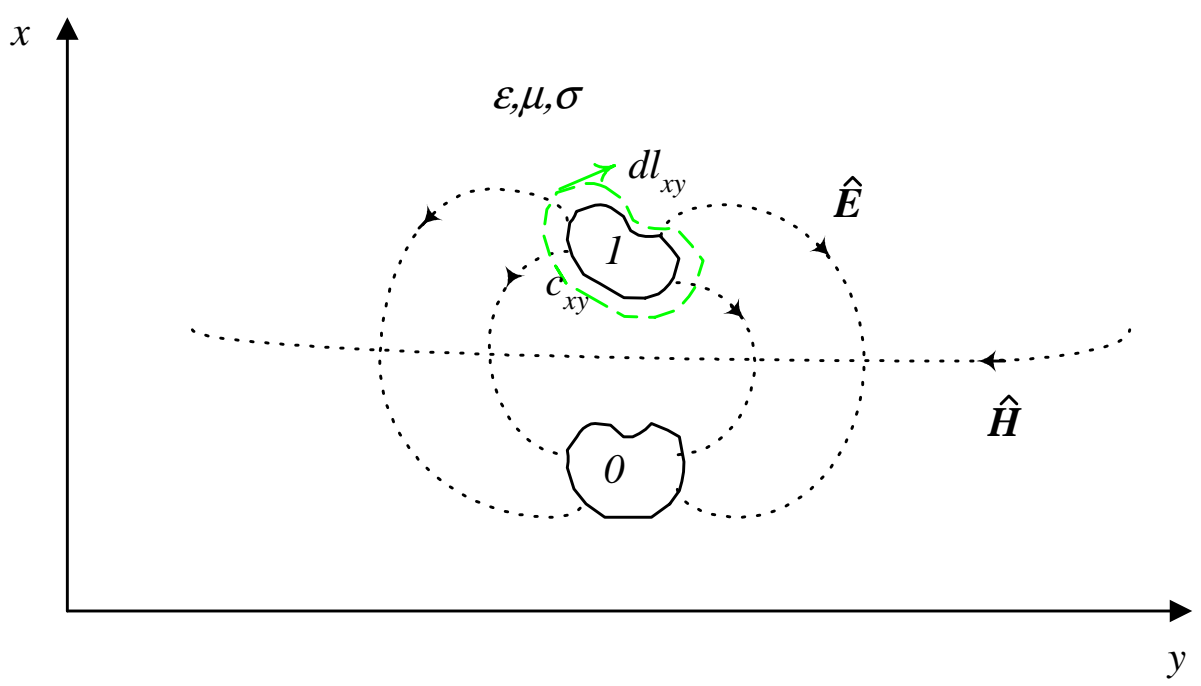

(b)

Fig. 3.8 Contours and surfaces for the derivation of transmission-line equation for twoconductor transmission lines: (a) longitudinal plane, (b) transverse plane (modified from Paul, 1994) 
We now turn to the derivation of the transmission-line equations in terms of the voltage and current as defined above. In Fig. 3.8a, choose an open surface $s$ where the unit normal of $s$ lies in the $x-y$ (transverse) plane. Integrating Faraday's law around this open surface

$$
\int_{a}^{a^{\prime}} \hat{\boldsymbol{E}}_{\boldsymbol{t}} \cdot d \boldsymbol{l}+\int_{a^{\prime}}^{b^{\prime}} \hat{\boldsymbol{E}}_{z} \cdot d \boldsymbol{l}+\int_{b^{\prime}}^{b} \hat{\boldsymbol{E}}_{\boldsymbol{t}} \cdot d \boldsymbol{l}+\int_{b}^{a} \hat{\boldsymbol{E}}_{z} \cdot d \boldsymbol{l}=j 2 \pi f \mu \int_{s} \hat{\boldsymbol{H}}_{\boldsymbol{t}} \cdot \boldsymbol{a}_{\boldsymbol{n}} d s
$$

Observe that the second and fourth integrals on the left-hand side are zero since these are along the surfaces of the perfect conductors; and that the negative sign vanishes because of the direction chosen for the line integral (or $\boldsymbol{a}_{\boldsymbol{n}}$ ) and the right-hand rule. When the voltages between the two conductors are defined as in Eq. (3.19a), Eq. (3.20) becomes

$$
-\hat{V}(z)+\hat{V}(z+\Delta z)=j 2 \pi f \mu \int_{s} \hat{\boldsymbol{H}}_{\boldsymbol{t}} \cdot \boldsymbol{a}_{\boldsymbol{n}} d s
$$

Dividing both sides by $\Delta \mathrm{z}$ and taking the limit $\Delta \mathrm{z} \rightarrow 0$ gives

$$
\frac{d \hat{V}(z)}{d z}=j 2 \pi f \mu \lim _{\Delta z \rightarrow 0} \frac{1}{\Delta z} \int_{s} \hat{\boldsymbol{H}}_{\boldsymbol{t}} \cdot \boldsymbol{a}_{\boldsymbol{n}} d s
$$

The integral on the right hand side of (3.22) is the magnetic flux penetrating the surface, s. This magnetic flux can be interpreted as an inductance of the loop formed between the two conductors. Considering the current defined as (3.19b), the inductance for a section of length $\Delta z$ is

$$
L=\frac{\Psi}{\hat{I}}=\frac{-\mu \int_{s} \hat{\boldsymbol{H}}_{\boldsymbol{t}} \cdot \boldsymbol{a}_{\boldsymbol{n}} d s}{\oint_{c_{x y}} \hat{\boldsymbol{H}}_{\boldsymbol{t}} \cdot d \boldsymbol{l}_{\boldsymbol{x} \boldsymbol{y}}}
$$


Then the per-unit-length inductance, $l$, can be defined for any cross section (since the line is uniform) as

$$
l=\lim _{\Delta z \rightarrow 0} \frac{L}{\Delta z}=\frac{-\mu}{\hat{I}} \lim _{\Delta z \rightarrow 0} \frac{1}{\Delta z} \int_{s} \boldsymbol{H}_{\boldsymbol{t}} \cdot \boldsymbol{a}_{\boldsymbol{n}} d s=-\mu \frac{\int_{a}^{a^{\prime}} \hat{\boldsymbol{H}}_{\boldsymbol{t}} \cdot \boldsymbol{a}_{\boldsymbol{n}} d l}{\oint_{\mathrm{c}_{\mathrm{xy}}} \hat{\boldsymbol{H}}_{\boldsymbol{t}} \cdot d \boldsymbol{l}_{\boldsymbol{x y}}}
$$

Combining Eq. (3.24) with (3.22) gives the first transmission-line equation,

$$
\frac{d \hat{V}(z)}{d z}=-j 2 \pi f l \hat{I}(z)
$$

To derive the second transmission-line equation, recall the continuity equation which states that the net outflow of current from a closed surface equals the time rate of decrease of the charge enclosed by that surface. By enclosing each conductor with a closed surface, $s^{\prime}$, of length $\Delta \mathrm{z}$ just off the surface of the conductor as shown in Fig. 3.8a, the continuity equation over this closed surface gives

$$
\oint_{s_{e}} \hat{\boldsymbol{J}} \cdot d \boldsymbol{s}^{\prime}+\oint_{s_{p}^{\prime}} \hat{\boldsymbol{J}} \cdot d \boldsymbol{s}^{\prime}=-j 2 \pi f Q_{\text {enc }}
$$

The portion of this closed surface at the ends is denoted by $s_{e}^{\prime}$ whereas the portion of the surface around the perimeter is denoted by $s_{p}^{\prime}$. The LHS terms in Eq. (3.26) are

$$
\oint_{s_{e}} \hat{\boldsymbol{J}} \cdot d \boldsymbol{s}^{\prime}=\hat{I}(z+\Delta z)-\hat{I}(z)
$$




$$
\oint_{s_{p}^{\prime}} \hat{\boldsymbol{J}} \cdot d \boldsymbol{s}^{\prime}=\sigma \oint_{s_{p}^{\prime}} \hat{\boldsymbol{E}}_{\boldsymbol{t}} \cdot d \boldsymbol{s}^{\prime}
$$

The right-hand side of Eq. (3.26) can be defined in terms of per-unit-length capacitance. The total charge enclosed by the surface is, according to Gauss's law,

$$
Q_{e n c}=\varepsilon \int_{s^{\prime}} \hat{\boldsymbol{E}}_{\boldsymbol{t}} \cdot d \boldsymbol{s}^{\prime}=\varepsilon \int_{s_{p}} \hat{\boldsymbol{E}}_{\boldsymbol{t}} \cdot d \boldsymbol{s}^{\prime}
$$

The capacitance, $C$, between the conductors for a length of $\Delta z$ of the line is

$$
C=\frac{Q_{e n c}}{V}
$$

and the per-unit-length capacitance is

$$
c=\lim _{\Delta z \rightarrow 0} \frac{C}{\Delta z}=\frac{\varepsilon}{V} \lim _{\Delta z \rightarrow 0} \frac{1}{\Delta z} \int_{s^{\prime}} \hat{\boldsymbol{E}}_{\boldsymbol{t}} \cdot d \boldsymbol{s}^{\prime}=\varepsilon \frac{\oint_{c_{x y}} \hat{\boldsymbol{E}}_{\boldsymbol{t}} \cdot \boldsymbol{a}_{\boldsymbol{n}} d \boldsymbol{l}}{-\int_{0}^{1} \hat{\boldsymbol{E}}_{\boldsymbol{t}} \cdot d \boldsymbol{l}_{\boldsymbol{0 1}}}
$$

Similarly, a conductance between the two conductors for a length of $\Delta \mathrm{z}$ may be defined as

$$
G=\frac{\int_{s_{p}^{\prime}} \hat{\boldsymbol{J}} \cdot d \boldsymbol{s}^{\prime}}{\hat{V}(z)}
$$

This leads to the definition of a per-unit-length conductance from (3.27b) as 


$$
g=\lim _{\Delta z \rightarrow 0} \frac{G}{\Delta z}=\frac{\sigma}{V} \lim _{\Delta z \rightarrow 0} \frac{1}{\Delta z} \int_{s_{p}^{\prime}} \hat{\boldsymbol{E}}_{\boldsymbol{t}} \cdot d \boldsymbol{s}^{\prime}=\sigma \frac{\oint_{c_{x y}} \hat{\boldsymbol{E}}_{\boldsymbol{t}} \cdot \boldsymbol{a}_{\boldsymbol{n}} d \boldsymbol{l}}{-\int_{0}^{1} \hat{\boldsymbol{E}}_{\boldsymbol{t}} \cdot d \boldsymbol{l}_{\boldsymbol{0 1}}}
$$

Substituting (3.27a), (3.30) and (3.32) into (3.26), dividing both sides by $\Delta \mathrm{z}$, and taking the limit as $\Delta \mathrm{z} \rightarrow 0$ gives the second transmission-line equation,

$$
\frac{d \hat{I}(z)}{d z}=-(g+j 2 \pi f c) \hat{V}(z)=-j 2 \pi f c^{*} \hat{V}(z)
$$

where it is convenient to define the equivalent capacitance parameter as $c^{*}=c+g /(j 2 \pi f)$ to represent the total effect of capacitance and conductance. Equations (3.24) and (3.33) are referred to as the transmission-line equations and represent a coupled set of first-order, partial differential equations describing the line voltage, $\hat{V}(z, t)$, and line current, $\hat{I}(z, t)$.

The derivation of transmission line equations from the integral form of Maxwell's equations was rigorous and illustrated many important concepts. The same results can be derived from an equivalent distributed parameter, lumped circuit. The concept stems from the fact that lumped-circuit concepts are only valid for structures whose largest dimension is electrically small, i.e., much less than a wavelength, at the frequency of excitation. If a structural dimension is electrically large, we may break it into the union of electrically small substructures and can then represent each substructure with a lumped circuit model (Ramo et al., 1994). Therefore, we may characterize a section of the line of length $\Delta z$ with a lumped capacitance, a lumped inductance, and a lumped conductance as shown in Fig. 3.9a. Applying Kirchhoff's law,

$$
\hat{V}(z+\Delta z)-\hat{V}(z)=-j 2 \pi f l \Delta z \hat{I}(z)
$$




$$
\hat{I}(z+\Delta z)-\hat{I}(z)=-g \Delta z \hat{V}(z+\Delta z)-j 2 \pi f c \Delta z \hat{V}(z+\Delta z)
$$

where $l, g$, and $c$ are the per-unit-length inductance, conductance, and capacitance, respectively.

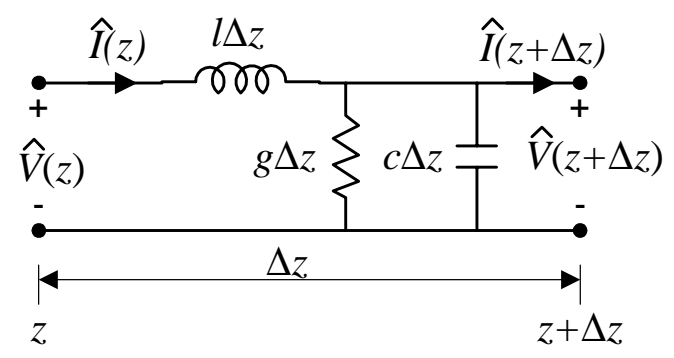

Fig. 3.9 The per-unit-length equivalent circuit model, where $l, g$, and $c$ are the per-unitlength inductance, conductance, and capacitance, respectively

Dividing (3.34) by $\Delta z$ and taking the limit $\Delta z \rightarrow 0$ gives

$$
\begin{aligned}
& \frac{d \hat{V}(z)}{d z}=-j 2 \pi f l \hat{I}(z) \\
& \frac{d \hat{I}(z)}{d z}=-(g+j 2 \pi f c) \hat{V}(z)=-j 2 \pi f c^{*} \hat{V}(z)
\end{aligned}
$$

The uncoupled version of Eq. (3.35) may be obtained by differentiating both equations with respect to $z$ and substituting. The governing wave equation of the 2-conductor transmission line becomes

$$
\frac{d^{2} \hat{V}(z)}{d z^{2}}=j 2 \pi f l(g+j 2 \pi f c) \hat{V}(z)=(j 2 \pi f)^{2} l c^{*} \hat{V}(z)
$$




$$
\frac{d^{2} \hat{I}(z)}{d z^{2}}=j 2 \pi f l(g+j 2 \pi f c) \hat{I}(z)=(j 2 \pi f)^{2} l c * \hat{I}(z)
$$

\subsubsection{Transmission Line Equations of a Multi-conductor Line}

The previous section discussed the development and derivation of transmission equation for two-conductor transmission lines. Under the same assumptions made in Section 3.3.1, the development and derivation of the MTL equations parallels the developments for two-conductor lines. In fact, the developed MTL equations have, using matrix notation, a form identical to those equations. Two-conductor lines and multi-conductor lines are analogous to single-degree-offreedom systems and multiple-degree-of-freedom systems in structural dynamics.

Consider a general multi-conductor line in Fig. 3.10. It consists of $n$ conductors and a reference conductor (denoted as the zero ${ }^{\text {th }}$ conductor) to which the $\mathrm{n}$ line voltages will be referenced. Making all of the assumptions of Section 3.1, which leads to a TEM field structure, voltage between the $i^{\text {th }}$ conductor and the reference conductor (positive on the $i^{\text {th }}$ conductor) can be uniquely defined as

$$
\begin{aligned}
& \hat{V}_{i}(z)=-\int_{0}^{i} \hat{\boldsymbol{E}}_{\boldsymbol{t}} \cdot d \boldsymbol{l}_{\boldsymbol{0} \boldsymbol{i}} \\
& \hat{I}_{i}(z)=\oint_{C_{i x y}} \hat{\boldsymbol{H}}_{\boldsymbol{t}} \cdot d \boldsymbol{l}_{i x y}
\end{aligned}
$$

For each conductor $(i)$ and the reference conductor (0), the transmission-line equation can be derived in a similar fashion to the case of two-conductor line. Applying Farady's law around the contour $c_{0 i}$, which encloses surface $s_{i}$ between the reference conductor and the $i^{\text {th }}$ conductor, gives the first transmission-line equation. Applying the continuity equation to the closed surface $\hat{s}_{i}$, of length $\Delta z$ just off the surface of the $i^{\text {th }}$ conductor, gives the second transmission-line equation. Using matrix notation, the transmission-line equations of a multi-conductor transmission line can be written as (Paul, 1994) 


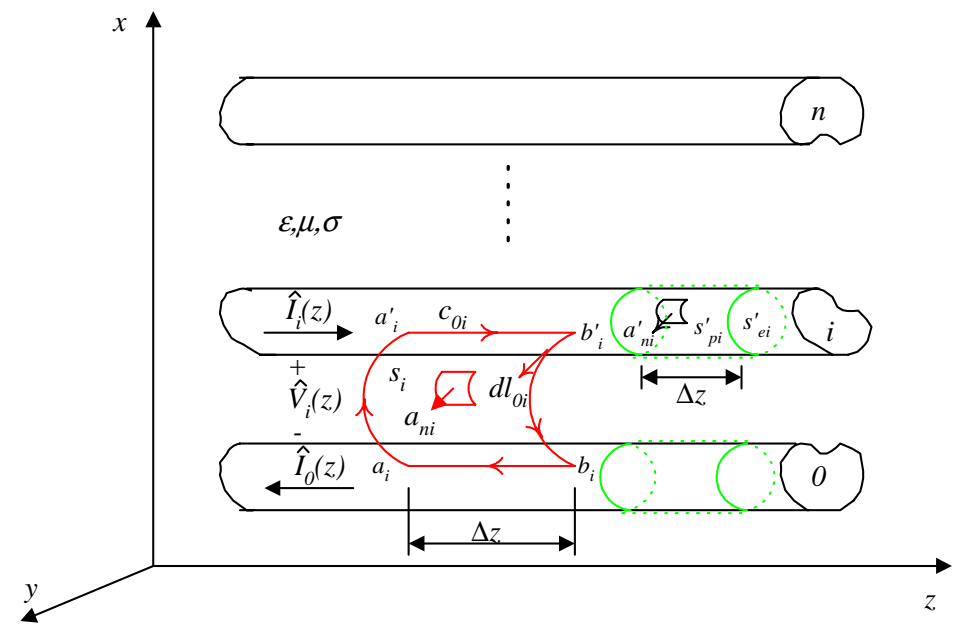

(a)

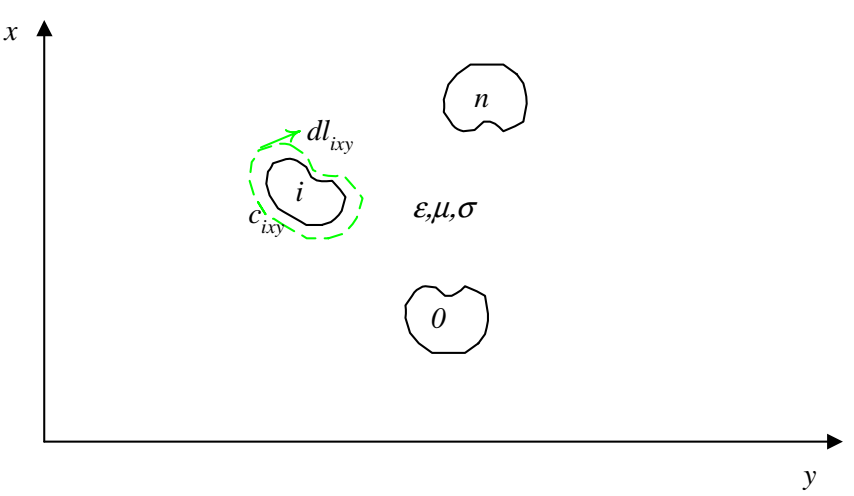

(b)

Fig. 3.10 Contours and surfaces for the derivation of transmission-line equation for multiconductor transmission lines: (a) longitudinal plane, (b) transverse plane (modified from Paul, 1994)

$$
\begin{aligned}
& \frac{d}{d z} \hat{\boldsymbol{V}}(z)=-j 2 \pi f \boldsymbol{L} \hat{\boldsymbol{I}}(z) \\
& \frac{d}{d z} \hat{\boldsymbol{I}}(z)=-(\boldsymbol{G}+j 2 \pi f \boldsymbol{C}) \hat{\boldsymbol{V}}(z)
\end{aligned}
$$

where 


$$
\begin{aligned}
& \hat{\boldsymbol{V}}(z)=\left[\begin{array}{c}
\hat{V}_{1}(z) \\
\hat{V}_{2}(z) \\
\vdots \\
\hat{V}_{n}(z)
\end{array}\right] \\
& \hat{\boldsymbol{I}}(z)=\left[\begin{array}{c}
\hat{I}_{1}(z) \\
\hat{I}_{2}(z) \\
\vdots \\
\hat{I}_{n}(z)
\end{array}\right] \\
& \boldsymbol{L}=\left[\begin{array}{cccc}
l_{11} & l_{12} & \cdots & l_{1 n} \\
l_{21} & l_{22} & \cdots & l_{2 n} \\
\vdots & \vdots & \ddots & \vdots \\
l_{n 1} & l_{n 2} & \cdots & l_{n n}
\end{array}\right] \\
& \boldsymbol{G}=\left[\begin{array}{cccc}
\sum_{k=1}^{n} g_{1 k} & -g_{12} & \cdots & g_{1 n} \\
-g_{21} & \sum_{k=1}^{n} g_{2 k} & \cdots & -g_{2 n} \\
\vdots & \vdots & \ddots & \vdots \\
-g_{n 1} & -g_{n 2} & \cdots & \sum_{k=1}^{n} g_{n k}
\end{array}\right] \\
& \boldsymbol{C}=\left[\begin{array}{cccc}
\sum_{k=1}^{n} c_{1 k} & -c_{12} & \cdots & c_{1 n} \\
-c_{21} & \sum_{k=1}^{n} c_{2 k} & \cdots & -c_{2 n} \\
\vdots & \vdots & \ddots & \vdots \\
-c_{n 1} & -c_{n 2} & \cdots & \sum_{k=1}^{n} c_{n k}
\end{array}\right] \\
& l_{i j}=\left.\frac{\hat{\psi}_{i}}{\hat{I}_{j}}\right|_{\hat{I}_{1}=\cdots=\hat{I}_{i-1}=\hat{I}_{i+1}=\cdots=\hat{I}_{n}=0}=\frac{-\mu \int_{0}^{i} \hat{\boldsymbol{H}}_{\boldsymbol{t}} \cdot \boldsymbol{a}_{\boldsymbol{n} \boldsymbol{i}} d l_{0 i}}{\oint_{c_{j x y}} \hat{\boldsymbol{H}}_{\boldsymbol{t}} \cdot d \boldsymbol{l}_{\boldsymbol{j} \boldsymbol{x y}}} \\
& g_{i j}=\left.\frac{I_{i}}{V_{j}}\right|_{V_{1}=\cdots=V_{i-1}=V_{i+1}=\cdots=V_{n}=0}=\frac{\oint_{c_{i x y}} \mathrm{H}_{t} \cdot d \mathrm{l}_{i x y}}{-\int_{0}^{j} \mathrm{E}_{t} \cdot d \mathrm{l}_{0 \mathrm{j}}}
\end{aligned}
$$




$$
c_{i j}=\left.\frac{\hat{q}_{i}}{\hat{V}_{j}}\right|_{\hat{V}_{1}=\cdots=\hat{V}_{i-1}=\hat{V}_{i+1}=\cdots=\hat{V}_{n}=0}=\frac{\varepsilon \oint_{c_{i x y}} \hat{\boldsymbol{E}}_{\boldsymbol{t}} \cdot \boldsymbol{a}_{\boldsymbol{n}} d l_{i x y}}{-\int_{0}^{j} \hat{\boldsymbol{E}}_{t} \cdot d \boldsymbol{l}_{\boldsymbol{0 j}}}
$$

in which, $\hat{\psi}_{i}$, is the total magnetic flux penetrating the $i^{\text {th }}$ circuit (i.e. surface $s_{i}$ per unit length), $\hat{q}_{i}$ is the total charge residing on the $i^{\text {th }}$ conductor surface (i.e. surface $s_{i}^{\prime}$ per unit length), and $\boldsymbol{L}, \boldsymbol{G}$, and $\boldsymbol{C}$ are the per-unit-length inductance, conductance, and capacitance matrix, respectively. Multi-conductor transmission-line equation (3.38) is much more difficult to solve than the twoconductor transmission-line equation (3.35). The MRP line used for measurement can be and should be designed to be axially symmetric. Hence, (3.38) may be reduced to an equivalent twoconductor transmission line. The difference between this equivalent wave equation and (3.35) is just the per-unit-length parameters in the equations.

\subsection{Line Parameters}

All cross-sectional information about a particular line that distinguishes it from other lines is contained in the per-unit-length parameters. The MTL equations are identical in form for all lines except that the per-unit-length parameters are different. Without a determination of the perunit-length parameters for the specific line, one cannot solve the resulting MTL equations because the coefficients in those equations will be unknown.

\subsubsection{Properties of Per-Unit-Length Line Parameters}

Since the field structure is assumed to be TEM in the transmission line, there should be a relationship between the transmission line equations and the general TEM plane wave equations. Performing the line integral between two points, $a$ and $a$ ' in Fig. 3.8, on the conductors in a transverse plane on both sides of plane wave equation (3.17a) and recalling the definition of voltage given in $(3.19 \mathrm{a})$ yields

$$
\frac{d^{2} \hat{V}(z)}{d z^{2}}=j 2 \pi f \mu(\sigma+j 2 \pi f \varepsilon) \hat{V}(z)=(j 2 \pi f)^{2} \mu \varepsilon^{*} \hat{V}(z)
$$


Similarly, performing the contour integral around the top conductor in a transverse plane, $c_{x y}$, on both sides of plane wave equation (3.17b) and recalling the definition of current from (3.19b) yields

$$
\frac{d^{2} \hat{I}(z)}{d z^{2}}=j 2 \pi f \mu(\sigma+j 2 \pi f \varepsilon) \hat{I}(z)=(j 2 \pi f)^{2} \mu \varepsilon^{*} \hat{I}(z)
$$

Comparing (3.39) to (3.36), we identify the three important properties of the per-unitlength parameters. These are

$$
\begin{aligned}
& l c=\mu \varepsilon \\
& g l=\sigma \mu \\
& l c^{*}=\mu \varepsilon^{*}
\end{aligned}
$$

Similarly, for the case of a multi-conductor transmission line, the following identity may be obtained.

$$
\begin{aligned}
& \boldsymbol{L C}=\boldsymbol{C L}=\mu \boldsymbol{I}_{\boldsymbol{n}} \\
& \boldsymbol{L} \boldsymbol{G}=\boldsymbol{G} \boldsymbol{L}=\mu \sigma \boldsymbol{I}_{\boldsymbol{n}} \\
& \boldsymbol{L} \boldsymbol{C}^{*}=\boldsymbol{C}^{*} \boldsymbol{L}=\mu \varepsilon^{*} \boldsymbol{I}_{\boldsymbol{n}}
\end{aligned}
$$


The identities in Eq. (3.40) or Eq. (3.41) are valid only for lines with perfect conductors and homogeneous surrounding media, as is the assumption of a TEM field structure and the resulting TL equations. We can extend the TL equation representation, in an approximate manner, to include inhomogeneous media as well as imperfect conductors under the quasi-TEM assumption. Because of the identities in Eq. (3.41), we need to determine only one of the perunit-length parameter matrices since Eq. (3.41) can be written as

$$
\begin{aligned}
& \boldsymbol{L}=\mu \varepsilon \boldsymbol{C}^{-1} \\
& \boldsymbol{G}=\frac{\sigma}{\varepsilon} \boldsymbol{C} \\
& \boldsymbol{C}=\mu \varepsilon \boldsymbol{L}^{-1}
\end{aligned}
$$

\subsubsection{Calculation and Calibration of Line Parameters}

The line parameters can be calculated using their definitions defined in the derivation of the TL equations. However, there exist very few transmission-line structures for which the perunit-length parameters can be determined analytically. The two-wire probe and coaxial line are two examples and the per-unit-length capacitance and inductance are derived in many electromagnetics textbooks. For two-wire probes, the line parameters are derived as (Ramo, 1994)

$$
\begin{aligned}
& c=\frac{\pi \varepsilon}{\cosh ^{-1}\left(\frac{s}{d}\right)} \\
& l=\mu \varepsilon c^{-1}=\frac{\mu}{\pi} \cosh ^{-1}\left(\frac{s}{d}\right) \\
& g=\frac{\sigma}{\varepsilon} c=\frac{\pi \sigma}{\cosh ^{-1}\left(\frac{s}{d}\right)}
\end{aligned}
$$


where $\mathrm{d}$ is the diameter of the two wires and $\mathrm{s}$ is the spacing between the two wires. For coaxial cables, the line parameters become

$$
\begin{aligned}
& c=\frac{2 \pi \varepsilon}{\ln \left(\frac{b}{a}\right)} \\
& l=\mu \varepsilon c^{-1}=\frac{\mu_{0}}{2 \pi} \ln \left(\frac{b}{a}\right) \\
& g=\frac{\sigma}{\varepsilon} c=\frac{2 \pi \sigma}{\ln \left(\frac{b}{a}\right)}
\end{aligned}
$$

where $a$ is the outer diameter of the inner conductor and $b$ is the inner diameter of the outer conductor. The three line parameters are functions of the cross-sectional geometry of the transmission line and the electromagnetic properties of the insulating medium. But they are not independent. It is interesting to note that only one of the three line parameters and the electromagnetic properties of the surrounding medium are needed to determine the three line parameters. Hence, instead of trying to calculate the values of the line parameters, they can be experimentally determined using material of known electromagnetic properties.

It will become obvious in the next section that the solution of the wave equation can be characterized by defining two new parameters, the characteristic impedance and the propagation constant, as functions of the line parameters. The propagation constant reflects the effect of the electromagnetic properties of the insulating medium, while the characteristic impedance reflects the effect of the cross-sectional geometry. Therefore, it is convenient to experimentally determine or calibrate the line impedance instead of the line parameters. This is an inverse problem and will be discussed in the next chapter. 


\subsection{Spectral Analysis of Wave Equation of a Transmission Line}

In the previous two sections, the TL equations and per-unit-length parameters were derived. The solution for the EM wave propagation in a TL requires two additional steps: 1) the general solution to the TL equations; and 2) applying the boundary condition to get particular solutions. The general solution for a uniform transmission line will be solved first. It will then be used as a basis for the solution of a non-uniform transmission line. It is important to note that the transmission line equation and its solution are here derived in the frequency domain (i.e. spectral analysis). It is necessary to perform an inverse transformation to obtain the time-domain waveform. The numerical technique to implement the spectral analysis will be discussed in next section.

\subsubsection{Spectral Analysis of the Wave Equation}

\subsubsection{General Solution of the wave equation}

The wave equation of a two-conductor transmission line was derived as Eq. (3.36). Also, it was pointed out that the MRP could be reduced to an equivalent two-conductor line if it is axially symmetric. The only difference is the values of the line parameters. The wave equation is often rewritten as

$$
\begin{aligned}
& \frac{d^{2} \hat{V}(z)}{d z^{2}}=\gamma^{2} \hat{V}(z) \\
& \frac{d^{2} \hat{I}(z)}{d z^{2}}=\gamma^{2} \hat{I}(z)
\end{aligned}
$$

where the propagation constant (wave number), $\gamma$, is

$$
\gamma=\sqrt{j 2 \pi f l(g+j 2 \pi f c)}=j 2 \pi f \sqrt{l c^{*}}
$$


Using the properties of the line parameters in Eq. (3.40) and the definition of the equivalent dielectric permittivity in Eq. (2.42), Eq. (3.46) can be reduced to

$$
\gamma=j 2 \pi f \sqrt{\mu_{0} \varepsilon_{0}} \sqrt{\varepsilon_{r}^{*}}=\frac{j 2 \pi f}{c} \sqrt{\varepsilon_{r}^{*}}
$$

It is interesting to note that the propagation constant is independent of line parameters (i.e. type and geometry of the transmission line). It only depends on the electromagnetic properties of the insulating material. The general solution of the ordinary differential equation (3.45) can be easily obtained as

$$
\begin{aligned}
& \hat{V}(z)=\hat{V}^{+} e^{-\gamma}+\hat{V}^{-} e^{\gamma} \\
& \hat{I}(z)=\hat{I}^{+} e^{-\gamma}+\hat{I}^{-} e^{\gamma}
\end{aligned}
$$

where $\hat{V}^{+}, \hat{V}^{-}, \hat{I}^{+}$, and $\hat{I}^{-}$are the unknown constants in the general solution. Eq. (3.48) can be interpreted as the sum of forward- and backward-traveling waves with 2 unknown coefficients. Note that $\hat{V}$ and $\hat{I}$ are not independent according to the transmission line equation (3.35). Substituting (3.48a) into (3.35a), we obtain

$$
\frac{d \hat{V}}{d z}=-\gamma\left(\hat{V}^{+} e^{-\gamma}-\hat{V}^{-} e^{\gamma z}\right)=-j 2 \pi f l \hat{I}
$$

Therefore, $\hat{I}$ can be written in terms of $\hat{V}$ as 


$$
\hat{I}(z)=\frac{\gamma}{j 2 \pi f l}\left(\hat{V}^{+} e^{-\gamma}-\hat{V}^{-} e^{\gamma}\right)=\frac{\hat{V}^{+}}{Z_{c}} e^{-\gamma}+\frac{\hat{V}^{-}}{Z_{c}} e^{\gamma}
$$

where $Z_{c}$ is defined as the characteristic impedance

$$
Z_{c}=\frac{j 2 \pi f l}{\gamma}=\sqrt{\frac{j 2 \pi f l}{g+j 2 \pi f c}}=\sqrt{\frac{l}{c^{*}}}
$$

Using the properties of the line parameters in Eq. (3.40) and the definition of the equivalent dielectric permittivity in Eq. (2.42), Eq. (3.51) can be reduced to

$$
Z_{c}=\frac{l}{\sqrt{\mu_{0} \varepsilon_{0}}} \frac{1}{\sqrt{\varepsilon_{r}^{*}}}=\frac{Z_{p}}{\sqrt{\varepsilon_{r}^{*}}}
$$

where the reference impedance $Z_{p}=l / \sqrt{\mu_{0} \varepsilon_{0}}$ is the impedance of the transmission line filled with air, which is only a function of the cross-sectional geometry of the transmission line. Therefore, the general solution to a uniform transmission line is

$$
\begin{aligned}
& \hat{V}(z)=\hat{V}^{+} e^{-\gamma z}+\hat{V}^{-} e^{\gamma} \\
& \hat{I}(z)=\frac{\hat{V}^{+}}{Z_{c}} e^{-\gamma}+\frac{\hat{V}^{-}}{Z_{c}} e^{\gamma}
\end{aligned}
$$


Note that the two parameters in the general solutions, $\gamma$ and $Z_{c}$ can be determined from $\varepsilon_{r}{ }^{*}$ and $Z_{p}$ instead of the three line parameters. They represent the dielectric property of the insulating material and geometry of the transmission line, respectively.

\subsubsection{Terminal Conditions and Particular Solutions}

A transmission line will have terminations at the left and right ends that consist of independent voltage and/or current sources and lumped elements such as resistors, capacitors, inductors, diodes, transistors, etc. These terminal constraints provide the additional two equations (one for the left termination and one for the right termination) that can be used to explicitly determine the two undetermined coefficients in the general solution.

Consider a TDR system shown in Fig. 3.1. In the case of a uniform transmission line (i.e. only the cable without the Measurement Probe connected to the TDR device), it can be represented by an equivalent circuit shown in Fig. 3.11. The line is terminated at the load end, $z=$

$l$, with a load impedance, $\hat{Z}_{L}$. At the source end, $z=0$, an independent voltage source $V_{S}$ and a source impedance, $Z_{S}$, terminate the line. Thus, the boundary conditions (terminal constraints) are

$$
\begin{aligned}
& \hat{V}(0)=\hat{V}_{S}-Z_{S} \hat{I}(0) \\
& \hat{V}(l)=Z_{L} \hat{I}(l)
\end{aligned}
$$

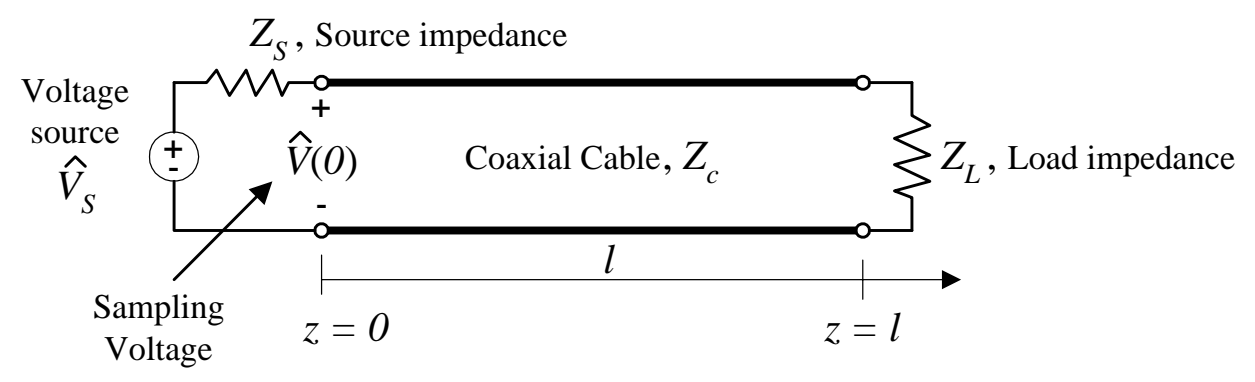

Fig. 3.11 Equivalent circuit of a TDR system 
At this point, we could apply the boundary conditions to solve for unknown coefficients $\hat{V}^{+}$and $\hat{V}^{-}$(two unknowns, two equations). Substituting the solution of $\hat{V}^{+}$and $\hat{V}^{-}$back to Eq. (3.53) we can then solve for the voltage or current along the transmission line. The solution of special interest is the voltage at $z=0$, which is the sampling voltage display on the oscilloscope. However, in order to extend the usefulness of the solution, more systematic approaches are taken. Three methods are discussed below. These methods may not seem straightforward as directly applying boundary conditions to the general solution in the case of a uniform transmission line, but they will become useful when solving the wave equation for a non-uniform transmission line.

\subsubsection{Input Impedance Method}

The first method uses the concept of input impedance in circuit analysis. The concept is similar to the equivalent stiffness in mechanical dynamics in which force and displacement are analogous to the voltage and current. As shown in Fig. 3.12, the input impedance $Z_{\text {in }}(z)$ is the equivalent impedance when looking into the circuit (i.e. the uniform transmission line) at position $z$ (Magnusson et al., 1992). It is defined as

$$
Z_{\text {in }}(z)=\frac{\hat{V}(z)}{\hat{I}(z)}=Z_{c} \frac{\hat{V}^{+} e^{-\gamma}+\hat{V}^{-} e^{\gamma}}{\hat{V}^{+} e^{-\gamma}-\hat{V}^{-} e^{\gamma z}}=Z_{c} \frac{1+\hat{V}^{-} / \hat{V}^{+} e^{2 \gamma}}{1-\hat{V}^{-} / \hat{V}^{+} e^{2 \gamma}}=Z_{c} \frac{1+\rho_{L} e^{2 \gamma(z-l)}}{1-\rho_{L} e^{2 \gamma(z-l)}}
$$

in which the reflection coefficient at the load end, $\rho_{L}$ is defined as

$$
\rho_{L}=\left.\frac{\hat{V}^{-} e^{-\gamma}}{\hat{V}^{+} e^{\gamma}}\right|_{z=l}=\frac{\hat{V}^{-}}{\hat{V}^{+}} e^{-2 \varkappa}
$$

If the input impedance at the source end (i.e. the impedance at the source end looking into the transmission line) can be determined, the sampling voltage $\hat{V}(0)$ can be calculated by the boundary condition at the source end (Eq. (3.54a)). In order to find the input impedance at the 
source end $(z=0)$, we first examine the input impedance at the load end $(z=l)$. From the definition of the input impedance in Eq. (3.55)

$$
Z_{\text {in }}(l)=Z_{c} \frac{1+\rho_{L}}{1-\rho_{L}}
$$

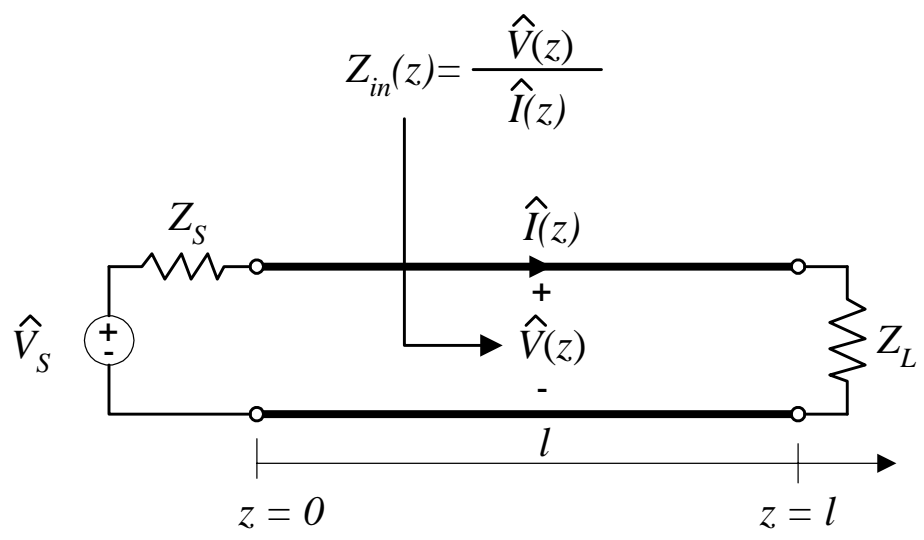

Fig. 3.12 Illustration of input impedance $Z_{\text {in }}$

The boundary condition at the load end (Eq. (3.54b)) can be written as

$$
Z_{\text {in }}(l)=\frac{\hat{V}(l)}{\hat{I}(l)}=Z_{L}
$$

The reflection coefficient $\rho_{L}$ is determined from (3.57) and (3.58) as

$$
\rho_{L}=\frac{Z_{L}-Z_{c}}{Z_{L}+Z_{c}}=\frac{Z_{\text {in }}(l)-Z_{c}}{Z_{\text {in }}(l)+Z_{c}}
$$


Substituting Eq. (3.59) into (3.55), the impedance at $z=0$ can be written as a function of the input impedance at $\mathrm{z}=l$ as

$$
Z_{\text {in }}(0)=Z_{c} \frac{Z_{\text {in }}(l)+Z_{c} \tanh (\mathcal{\prime})}{Z_{c}+Z_{\text {in }}(l) \tanh (\mathcal{\prime})}
$$

where

$$
\tanh (x)=\frac{e^{\varkappa}-e^{-x}}{e^{x}+e^{\varkappa}}=\frac{1-e^{-2 \chi}}{1+e^{-2 \chi}}
$$

Using the terminal condition at $z=0$ and $\hat{I}(0)=\hat{V}(0) / Z_{\text {in }}(0), \hat{V}(0)$ is determined as

$$
\hat{V}(0)=\frac{Z_{\text {in }}(0)}{Z_{\text {in }}(0)+Z_{S}} \hat{V}_{S}
$$

\subsubsection{ABCD Matrix Method}

Several matrices have been introduced for scattering problems (i.e. wave propagation in a non-uniform media in a transmission line) in geophysics and electromagnetics, such as the scattering matrix, the transmission matrix, and the ABCD matrix (Proponotarios and Wing, 1967, Claerbout, 1976, Frolik and Yagle, 1997). They are useful when dealing with non-uniform transmission lines. These matrices are correlated. In this section, the ABCD matrix is used since its derivation is simpler.

Recall the general solution to the uniform transmission line (3.53) and the terminal constraint (3.54). There are four unknown quantities in the terminal conditions (voltage and current at both ends). However, they are related by Eq. (3.53). Evaluation of the voltage and current at the two ends $(z=0, z=l)$ can be obtained as 


$$
\begin{aligned}
& {\left[\begin{array}{l}
\hat{V}(0) \\
\hat{I}(0)
\end{array}\right]=\left[\begin{array}{cc}
1 & 1 \\
\frac{1}{Z_{c}} & -\frac{1}{Z_{c}}
\end{array}\right]\left[\begin{array}{l}
\hat{V}^{+} \\
\hat{V}^{-}
\end{array}\right]} \\
& {\left[\begin{array}{l}
\hat{V}(l) \\
\hat{I}(l)
\end{array}\right]=\left[\begin{array}{cc}
e^{-x} & e^{\varkappa} \\
\frac{e^{-x}}{Z_{c}} & -\frac{e^{\varkappa}}{Z_{c}}
\end{array}\right]\left[\begin{array}{c}
\hat{V}^{+} \\
\hat{V}^{-}
\end{array}\right]}
\end{aligned}
$$

The relationship between the line voltage/current at $z=0$ and the line voltage/current at $z=l \mathrm{can}$ be obtained by equating $\hat{V}^{+}$and $\hat{V}^{-}$in Eq. (3.63) and (3.64) as

$$
\left[\begin{array}{l}
\hat{V}(l) \\
\hat{I}(l)
\end{array}\right]=\left[\begin{array}{cc}
\cosh (\chi) & -Z_{c} \sinh (\chi) \\
-\frac{1}{Z_{c}} \sinh (\chi) & \cosh (\chi)
\end{array}\right]\left[\begin{array}{c}
\hat{V}(0) \\
\hat{I}(0)
\end{array}\right]
$$

where

$$
\begin{aligned}
& \cosh (x)=\frac{e^{x}+e^{-x}}{2} \\
& \sinh (x)=\frac{e^{x}-e^{-x}}{2}
\end{aligned}
$$

Therefore, $\hat{V}^{+}$and $\hat{V}^{-}$are eliminated by putting the solution (3.53) in the form of (3.65). The $2 \times 2$ matrix relating voltage and current at two ends is called the ABCD matrix $(\Phi)$.

$$
\Phi=\left[\begin{array}{ll}
A & B \\
C & D
\end{array}\right]=\left[\begin{array}{cc}
\cosh (\chi) & -Z_{c} \sinh (\chi) \\
-\frac{1}{Z_{c}} \sinh (\chi) & \cosh (\chi)
\end{array}\right]
$$


This representation relates the line voltage and current at one end of line, $z=0$, to the line voltage and current at the other end of the line, $z=l$. In fact, the chain parameter matrix can be used to relate the voltage and current at any point on the line by replacing the $l$ with $z$. Now that the general solution in terms of the ABCD matrix has been obtained, we incorporate the terminal constraints in order to solve for terminal voltages and currents (four equations and four unknowns in Eqs. (3.54) and (3.65)). The solution of particular interest is $\hat{V}(0)$

$$
\hat{V}(0)=\frac{Z_{L} D-B}{\left(Z_{L} D-B\right)-Z_{s}\left(Z_{L} C-A\right)} \hat{V}_{s}=\frac{\frac{Z_{L} D-B}{A-Z_{L} C}}{\frac{Z_{L} D-B}{A-Z_{L} C}+Z_{s}} \hat{V}_{s}
$$

where $Z_{L}$ is the load impedance, $Z_{S}$ is the source impedance, $\hat{V}_{S}$ is the source voltage, and $A, B, C$, and $D$ are from Eq. (3.67). Equation (3.62) makes use of input impedance to relate current and voltage at a point, while Eq. (3.68) makes use of the ABCD (chain)-matrix to relate voltage and current at two ends. Comparing Eq. (3.68) and Eq. (3.62), input impedance at $z=0$ is related to the ABCD matrix by

$$
\hat{Z}_{\text {in }}(0)=\frac{Z_{L} D-B}{A-Z_{L} C}
$$

\subsubsection{Spectral Analysis of Non-Uniform Transmission Line}

A transmission line can be non-uniform in either geometrical shape or material. The whole transmission line in a TDR system is typically not uniform. It consists of a section of $50 \Omega$ cable, a transition head, and a soil probe as shown in Fig. 3.6. The probe head and the soil probe both can be non-uniform transmission lines. A non-uniform line can be represented as a line with piecewise-constant parameters as shown in Fig. 3.13. 


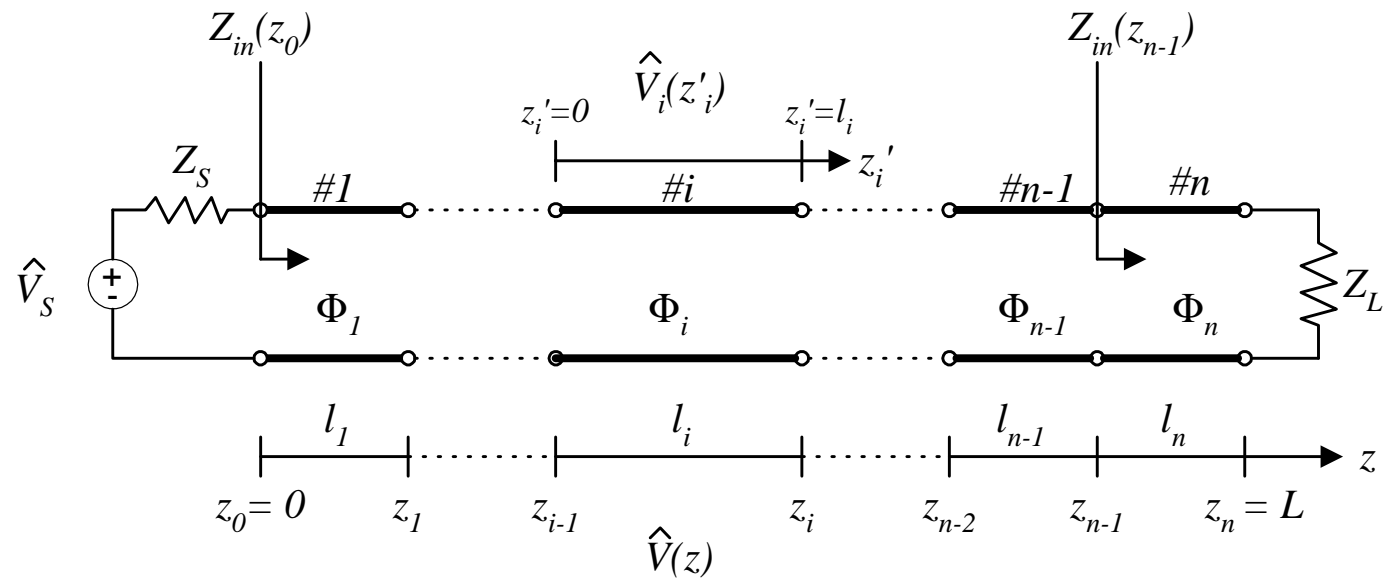

\section{Fig. 3.13 Representation of a non-uniform line as a cascade of uniform sections where $z_{i}{ }^{\prime}$ is the local coordinate of each section and $z$ is the global coordinate}

In a general non-uniform transmission line, the per-unit-length parameter will be functions of $\mathrm{z}$. In this case the transmission line differential equations become non-constant-coefficient differential equations. Although the differential equations remain linear (if the surrounding medium is linear), they are difficult to solve. However, if we can approximate the non-uniform line as a discretely uniform line as shown in Fig. 3.13, Eq. (3.53) still represents the general solution for each uniform section.

For each of the $n$ uniform sections, the general solution consists of the sum of forward and backward travelling waves with two unknown coefficients. Therefore, there are a total of $2 n$ unknown coefficients. The terminal conditions are the same as in Eq. (3.54). This leaves $2 n$ unknown coefficients with only two boundary conditions. The continuity constraints at the discontinuities between the terminations provide $2(n-1)$ more equations as

$$
\begin{aligned}
& \hat{V}_{i}\left(z_{i}^{\prime}=l_{i}\right)=\hat{V}_{i+1}\left(z_{i+1}^{\prime}=0\right) \\
& \hat{I}_{i}\left(z_{i}^{\prime}=l_{i}\right)=\hat{I}_{i+1}\left(z_{i+1}^{\prime}=0\right)
\end{aligned}
$$

in which $z_{i}^{\prime}$ denotes the local coordinate for each section as shown in Fig. 3.13. 
Again, at this point we could apply the $2 n$ boundary conditions to solve for $2 n$ unknown coefficients $\left(\hat{V}_{i}^{+}, \hat{V}_{i}^{-}\right)$. This is done more conveniently by numerical solution. However, the effort made in the previous section enables a more systematic explicit procedure.

\subsubsection{Input Impedance Method}

Instead of solving the simultaneous equation, the simplified procedure involves calculating the input impedance from the end termination to the source termination. The analysis starts with the point farthest from the signal source, transforming the impedance back successively to the next discontinuity until the input is reached. This is done using Eq. (3.60) in a bottom-up fashion, which relates the impedance at the two ends of the uniform section of transmission line.

$$
\begin{aligned}
& Z_{i n}\left(z_{n}\right)=Z_{L} \\
& Z_{i n}\left(z_{n-1}\right)=Z_{c, n} \frac{Z_{L}+Z_{c, n} \tanh \left(\gamma_{n} l_{n}\right)}{Z_{c, n}+Z_{L} \tanh \left(\gamma_{n} l_{n}\right)} \\
& Z_{i n}\left(z_{n-2}\right)=Z_{c, n-1} \frac{Z_{i n}\left(z_{n-1}\right)+Z_{c, n-1} \tanh \left(\gamma_{n-1} l_{n-1}\right)}{Z_{c, n-1}+Z_{i n}\left(z_{n-1}\right) \tanh \left(\gamma_{n-1} l_{n-1}\right)} \\
& \quad \vdots \\
& Z_{\text {in }}(0)=Z_{c, 1} \frac{Z_{i n}\left(z_{1}\right)+Z_{c, 1} \tanh \left(\gamma_{1} l_{1}\right)}{Z_{c, 1}+Z_{i n}\left(-z_{1}\right) \tanh \left(\gamma_{1} l_{1}\right)}
\end{aligned}
$$

where $Z_{c, i}, \gamma_{i}$, and $l_{i}$, are the characteristic impedance, propagation constant, and length of each section. Once the input impedance looking into the entire line is obtained by use of Eq. (3.71), the sampling voltage $\hat{V}(0)$ can then be solved using Eq. (3.62).

\subsubsection{ABCD Matrix Method}

As shown in Eq. (3.65), the ABCD matrix $\left(\Phi_{\mathrm{i}}\right)$ relates the line voltages and currents of the two ends of the $i$ th uniform section of a non-uniform transmission line.

$$
\left[\begin{array}{l}
\hat{V}\left(z_{i}\right) \\
\hat{I}\left(z_{i}\right)
\end{array}\right]=\Phi_{i}\left[\begin{array}{l}
\hat{V}\left(z_{i-1}\right) \\
\hat{I}\left(z_{i-1}\right)
\end{array}\right]
$$


The overall $\mathrm{ABCD}$ matrix $(\Phi)$ of the entire line is the matrix that relates the voltages and currents at the two boundaries.

$$
\left[\begin{array}{c}
\hat{V}(l) \\
\hat{I}(l)
\end{array}\right]=\Phi\left[\begin{array}{c}
\hat{V}(0) \\
\hat{I}(0)
\end{array}\right]
$$

The $\Phi$ can be obtained as the product (in the appropriate order) of the ABCD matrices of the individual uniform sections as

$$
\Phi=\Phi_{n} \Phi_{n-1} \cdots \Phi_{1}
$$

Once the overall ABCD (chain) matrix of the entire line is obtained by use of Eq. (3.74), the sampling voltage can be obtained by Eq. (3.68). The major advantage of ABCD matrix method over input impedance method is that the overall ABCD matrix can be obtained without the recursion in a bottom-up fashion.

\subsubsection{Interpretation of Wave Propagation}

As discussed previously, the solution of the wave equation can be interpreted as waves traveling along the transmission line. Consider the transmission line in Fig. 3.14 with $l=\infty$. Substituting $l=\infty$ into Eq. (3.60), the input impedance at source end becomes $Z_{\text {in }}(0)=Z_{c}$. From Eq. (3.62), the sampling voltage becomes

$$
\hat{V}(0)=\frac{Z_{c}}{Z_{c}+Z_{S}} \hat{V}_{S}
$$




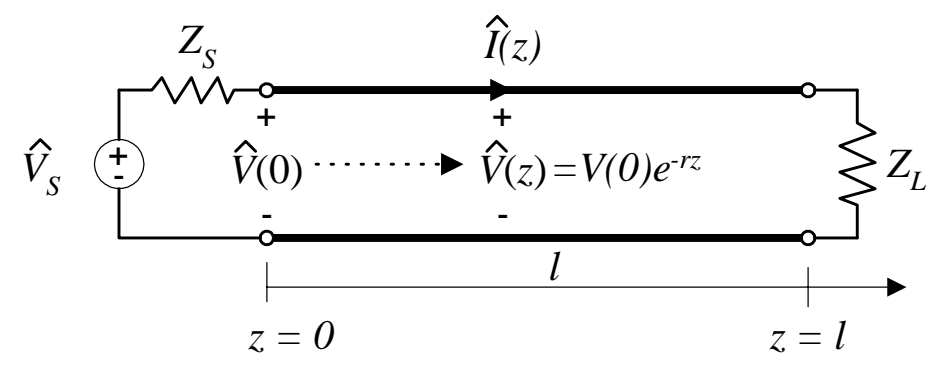

Fig. 3.14 A semi-infinite transmission line

Comparing Eq. (3.75) with Eq. (3.53a), we obtain $\hat{V}^{-}=0$ and $\hat{V}^{+}=\hat{V}(0)$. Therefore, the voltage at any point $z$ becomes

$$
\hat{V}(z)=\hat{V}(0) e^{-\gamma}=\hat{V}(0) T(z)
$$

Equation (3.76) represents a wave traveling in the $z$ direction (i.e. from source down to the transmission line). $T(z)=e^{-\gamma z}$ is defined as the traveling wave function. As $\hat{V}(0)$ propagates down to the transmission line, the attenuation and phase shift are changed by the traveling wave function. There is no wave component traveling in the negative $z$ direction because this is a semiinfinite line. When there is a line termination or impedance mismatch, the wave will be reflected and transmitted as shown in Fig. 3.15.

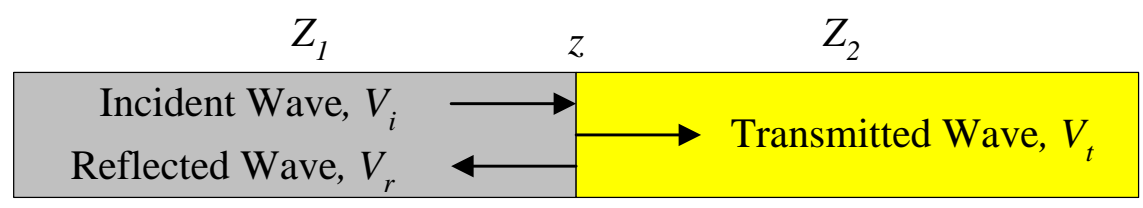

Fig. 3.15 The reflected wave $\left(V_{r}\right)$ and transmitted wave $\left(V_{t}\right)$ at the interface of two lines with impedance $Z_{1}$ and $Z_{2}$.

The voltage and current at point $\mathrm{z}$ becomes 


$$
\begin{aligned}
& \hat{V}(z)=\hat{V}_{i}+\hat{V}_{r}=\hat{V}_{t} \\
& \hat{I}(z)=\frac{\hat{V}_{i}}{Z_{1}}-\frac{\hat{V}_{r}}{Z_{1}}=\frac{\hat{V}_{t}}{Z_{2}}
\end{aligned}
$$

where $V_{i}=\hat{V}(-l) e^{\mathcal{L}}$ represents the wave traveling in a infinite transmission line, $V_{r}$ is the reflected wave, $V_{t}$ is the transmitted wave, $Z_{l}$ is the characteristic impedance for the section of the transmission line in which the reflected wave propagates; and $Z_{2}$ the characteristic impedance for the section of the transmission line in which the transmitted wave propagates. Equaton (3.77) represents the interface condition at $z$. The reflected wave and transmitted wave can be obtained by solving two unknowns in Eq. (3.77) as

$$
\begin{aligned}
& \hat{V}_{r}=\rho \hat{V}_{i} \\
& \hat{V}_{t}=\tau \hat{V}_{i}
\end{aligned}
$$

where reflection coefficient and transmission coefficient are defined as

$$
\begin{aligned}
& \rho=\frac{V_{r}}{V_{i}}=\frac{Z_{2}-Z_{1}}{Z_{2}+Z_{1}} \\
& \tau=\frac{V_{t}}{V_{i}}=\frac{2 Z_{2}}{Z_{2}+Z_{1}}=1+\rho
\end{aligned}
$$

The solution of the sampling voltage in Eq. (3.62) can be rewritten in terms an incident wave and a reflected wave from the transmission line as 


$$
\hat{V}(0)=\frac{Z_{\text {in }}(0)}{Z_{\text {in }}(0)+Z_{S}} \hat{V}_{S}=\frac{\hat{V}_{S}}{2}+\left(\frac{Z_{\text {in }}(0)-Z_{S}}{Z_{\text {in }}(0)+Z_{S}}\right) \frac{\hat{V}_{S}}{2}=\hat{V}_{\text {in }}+S_{11} \hat{V}_{\text {in }}
$$

where the incident wave $\hat{V}_{\text {in }}$ and the scattering function $S_{11}$ are defined as

$$
\begin{aligned}
& \hat{V}_{\text {in }}=\frac{\hat{V}_{S}}{2} \\
& S_{11}=\frac{Z_{\text {in }}(0)-Z_{S}}{Z_{\text {in }}(0)+Z_{S}}
\end{aligned}
$$

The scattering function can be seen as the reflection coefficient of the whole transmission line as shown in Fig. 3.16. The ratio of the sampling voltage $(\hat{V}(0))$ to the source voltage $\left(\hat{V}_{S}\right)$ is called the system function $H$ while the ratio of the reflected wave $\left(S_{11} \hat{V}_{i n}\right)$ to the incident wave $\left(\hat{V}_{\text {in }}\right)$ of a whole transmission line is called the scattering function. From Eq. (3.62), they have the following relationship

$$
H=\frac{Z_{\text {in }}(0)}{Z_{\text {in }}(0)+Z_{S}}=\frac{1+S_{11}}{2}
$$

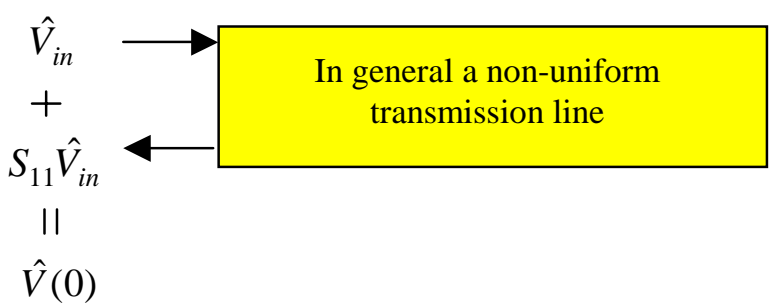

Fig. 3.16 The sampling voltage in terms an incident wave and a reflected wave from the transmission line 


\subsection{Simulation of the TDR Waveform}

The solution of the sampling voltage of a TDR system with a uniform transmission line is derived in Eq. (3.62) using input impedance or in Eq. (3.68) using the ABCD matrix. For nonuniform transmission lines, these two equations are still valid if the overall input impedance or $\mathrm{ABCD}$ matrix is calculated by Eq. (3.71) or Eq. (3.74). Denoting the voltage source $V_{S}(t)$ by a input signal $X(t)$ and sampling voltage $V(-l, t)$ by a output signal $Y(t)$, Eq. (3.62) or Eq. (3.68) can be rewritten as a

$$
\hat{Y}_{k}\left(f_{k}\right)=H_{k}\left(f_{k}\right) \cdot \hat{X}_{k}\left(f_{k}\right)=\frac{1+S_{11, k}\left(f_{k}\right)}{2} \hat{X}_{k}\left(f_{k}\right)
$$

where the subscript $k$ represent the $k$ th component of the Fourier coefficients, which was omitted in the derivation for convenience.

Note that the solution is the Fourier coefficients of the spectral representation of the sampling voltage. In order to obtain the TDR waveform in the time domain, recall the spectral representation of a signal using Fourier Series (assuming the signal is periodic) as

$$
U(t)=\sum_{k=-\infty}^{+\infty} \hat{U}_{k}\left(f_{k}\right) e^{j 2 \pi f_{k} t}
$$

where

$$
\hat{U}_{k}\left(f_{k}\right)=\frac{1}{T_{p}} \int_{T_{p}} U(t) e^{-j 2 \pi f_{k} t} d t
$$

in which $U$ represent any continuous-time signal, $\hat{U}_{k}$ are the Fourier coefficients, $f_{k}=k / T_{p}$ is the frequency at $k^{\text {th }}$ frequency coefficient, and $T_{p}$ is the fundamental period of the signal. Hence, the TDR waveform can be calculated as 


$$
Y(t)=\sum_{k=-\infty}^{+\infty}\left(\hat{X}_{k} \hat{H}_{k}\right) e^{j 2 \pi f_{k} t}
$$

The basic algorithm to obtain the TDR waveform is shown as Fig. 3.17. Briefly, the input $X(t)$ is transformed to its frequency spectrum $\hat{X}_{k}$ by Eq. $(3.85 \mathrm{~b})$. The solution in the frequency

domain is then obtained by evaluating the product $\hat{H}_{k} \hat{X}_{k}$ at each frequency. The solution in the time domain is obtained by use of Eq. (3.85a). The signal reconstruction can be viewed as a superposition of infinitely many signals with different frequencies. The summation of infinite number of terms in (3.85a) is not a problem if the signal is band-limited, which is the case for most of the practical signals. If the signal is not band-limited, then the reconstruction has to be approximated by summation of finite number of terms.

In the actual numerical implementation, Eq. (3.85) is not used. Instead, a discrete Fourier transform (DFT) is used, in which the continuous-time signal is discretized, because there exists a very efficient fast algorithm, the Fast Fourier Transform (FFT), for computing the DFT (Prokis and Manolakis, 1992). Some practical issues of implementing the FFT are discussed in a later section.

\subsubsection{Response of a Linear System to Aperiodic Signals}

If the input to a linear system is a periodic signal with fundamental period $T_{p}$, the spectral representation of such signals uses Fourier series (an infinite number of linear combinations of complex exponentials). Since such a signal exists from $-\infty$ to $+\infty$, the total response of the system calculated at any time instant as shown in Fig. 3.17, is simply equal to the steady-state response. On the other hand, if the signal is applied at some finite instant in time, say at $t=0$, whether it is aperiodic or periodic after $t=0$, the response of the system consists of two terms, the transient response and steady-state response. In such cases, the spectral representation of the signal should use a Fourier integral (or Fourier transformation) rather than Fourier series, in which the spectrum becomes continuous, and Eq. (3.85) becomes 


$$
\begin{aligned}
& U(t)=\int_{-\infty}^{+\infty} \hat{U}(f) e^{j 2 \pi f t} d f \\
& \hat{U}(f)=\int_{-\infty}^{+\infty} U(t) e^{-j 2 \pi f t} d f
\end{aligned}
$$

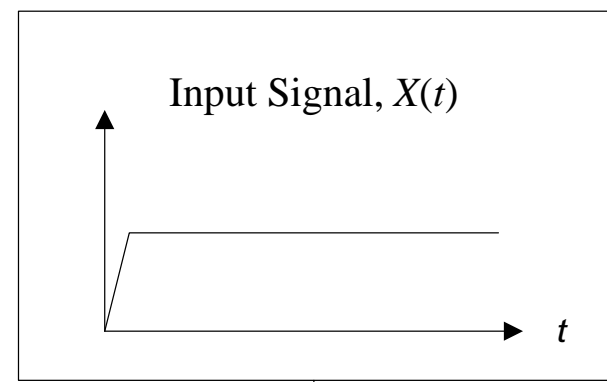

Eq. (2.6b)

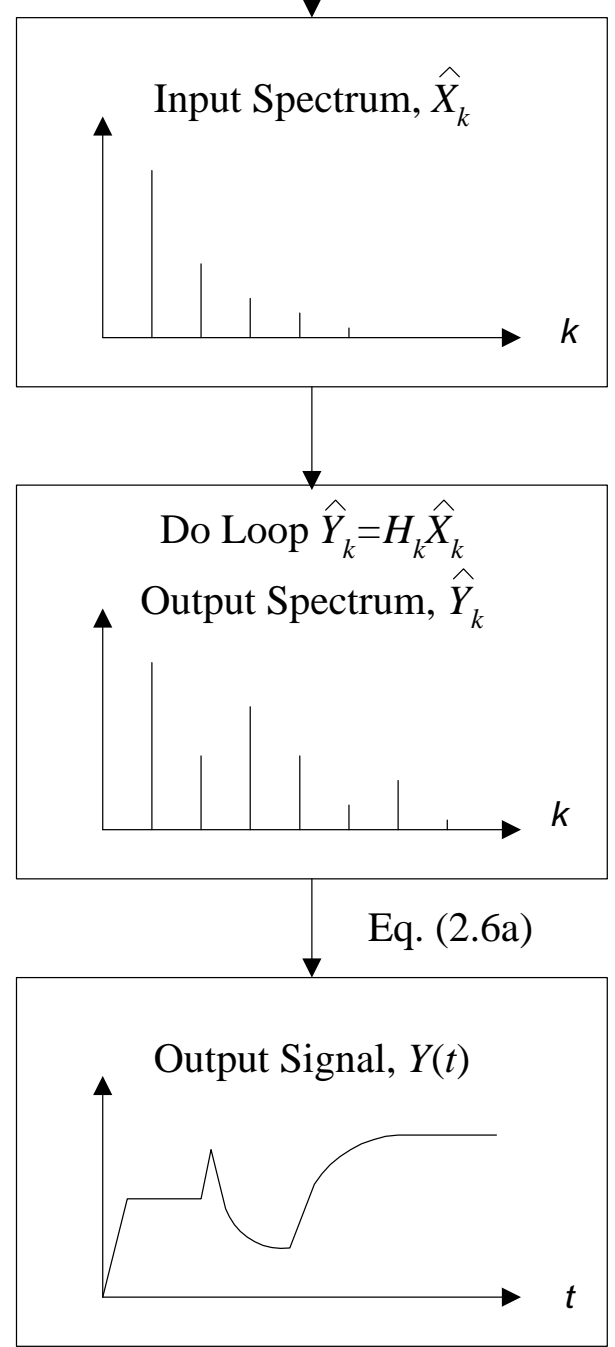

Fig. 3.17 The flow chart of the spectral algorithm 
If the system function is evaluated at continuous frequencies, it can be shown that the Fourier transform of the output of a linear system is the product of the Fourier transform of the input and the system function of that system (Prokis and Manolakis, 1992).

$$
\hat{Y}(f)=H(f) \cdot \hat{X}(f)
$$

The TDR waveform with an aperiodic input can then be determined in the same way as shown in Fig. 3.17, in which Eq. (3.87) is used instead of Eq. (3.85). However, the inverse transform (i.e. Eq. (3.87b)) is very difficult to do in an exact analytical manner.

It is desirable to determine the TDR waveform with an aperiodic input using Fourier series because the problem of the inverse transform can be avoided and the fast algorithm can be used. However, if the aperiodic signal is truncated at $t=T_{r}$ and repeated with fundamental period of $T_{r}$, the output signal obtained by the procedure depicted in Fig. 3.17 contains only the steady state periodic response. In order to obtain the total solution including the transient part, we can choose the input waveform over a period of time $\left(0 \leq t \leq T_{r}\right)$ to be that of the desired output waveform, then pad zeros and choose a repetition frequency low enough such that the output response reaches the zero steady state before the onset of the next pulse. This is shown in Fig. 3.18. For the TDR system, the repetition frequency can be chosen as $2 T_{r}$ such that the output signal will reach steady state at $t \leq T_{r}$. The input waveform is padded with zeros after $t>T_{r}$ so that the output signal will reach the zero steady state at $t \leq 2 T_{r}$. The time $T_{r}$ required for the output signal to reach steady state depends on the length of the probe and the dielectric permittivity of the material under test. The TDR input signal has a zero header (a beginning portion of zeros before the step pulse). The output waveform should also have a zero header if $T_{r}$ is sufficiently long. Insufficiency of $T_{r}$ is detected when the output waveform has a non-zero header (i.e. wrap around). 


\subsubsection{Practical Issues of the Numerical Implementation}

As discussed above, the simulation of a TDR waveform is actually implemented by discrete Fourier transform (DFT) because there exists a fast algorithm, the FFT, for the evaluation. In the

DFT, the continuous time $X(t)$ is discretized and becomes $X(n)$ with time spacing $\Delta t$ such that $\mathrm{X}(t=n \Delta t)=\mathrm{X}(n)$. The spectral representation of a discrete signal becomes (Prokis and Manolakis, 1992)

$$
\begin{aligned}
& X(n)=\sum_{k=0}^{N-1} \hat{X}_{k} e^{j(2 \pi / N) k n} \\
& \hat{X}_{k}=\frac{1}{N} \sum_{n=0}^{N-1} X(n) e^{-j(2 \pi / N) k n}
\end{aligned}
$$
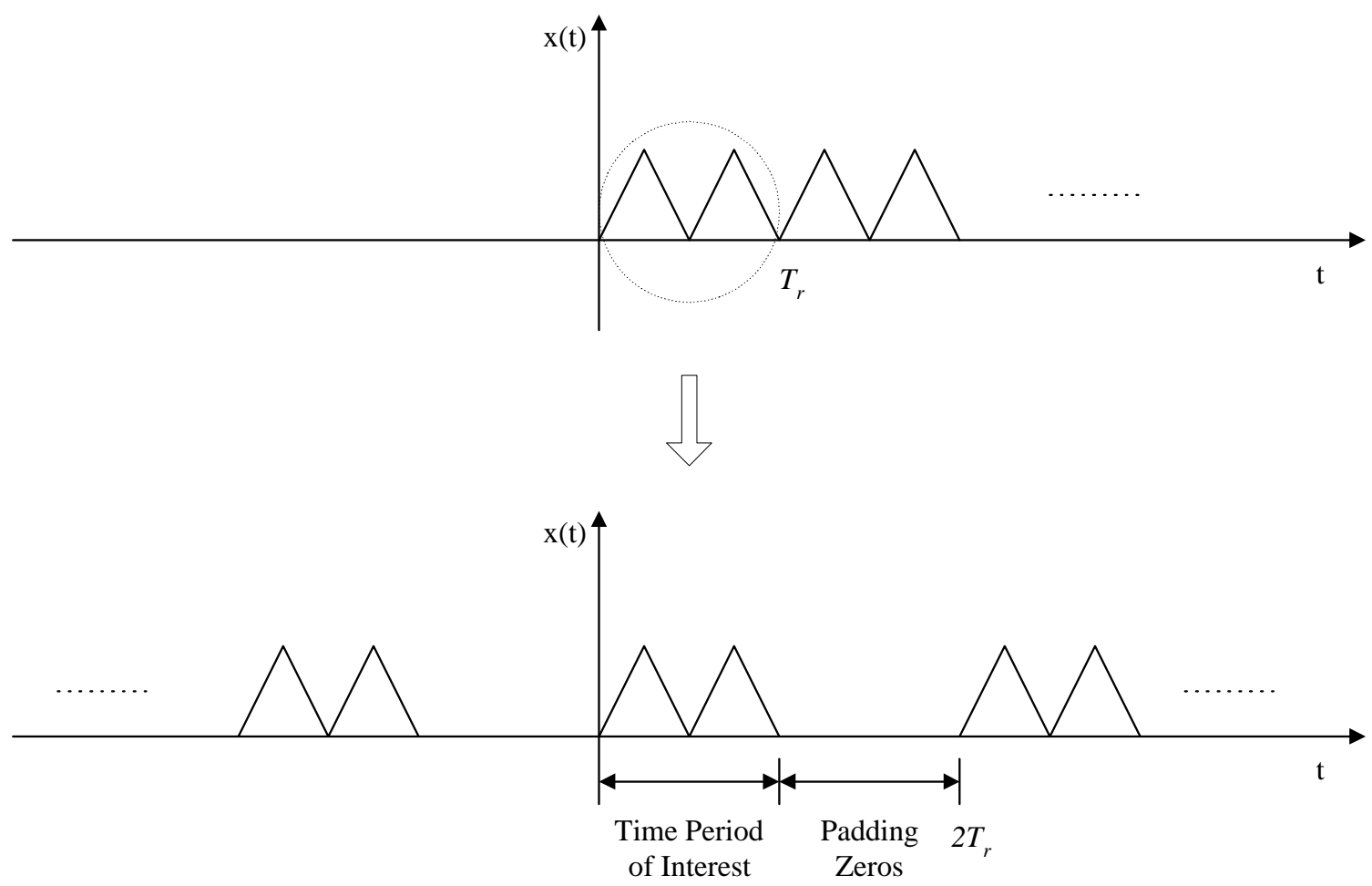

Fig. 3.18 Illustrating of zero-padding 
For a periodic signal with fundamental period equal to $T_{p}$, the spectrum is discrete (i.e. the Fourier coefficient of Fourier Series) with frequency spacing of $1 / T_{p}$. On the other hand, if the signal is discretized with time spacing of $\Delta t$, the spectrum is periodic with fundamental period equal to $1 / \Delta t$. One of the most important properties of the discrete Fourier transform is the duality relation between the time domain and the frequency domain, where periodicity with period $\alpha$ in one domain automatically implies discretization with spacing of $1 / \alpha$ in the other domain, and vice versa as shown in Fig. 3.19. The amount of information contained in one domain is limited by the length of the window of that domain (i.e. the period $\alpha$ ). Therefore, the sampling process reduces the information contained in a continuous-time signal. If the spectrum of the analog signal can be recovered from the spectrum of the discrete-time signal, there is no loss of information. The criterion of selecting a sampling rate is dictated by the sampling theorem (Prokis and Manolakis, 1992).
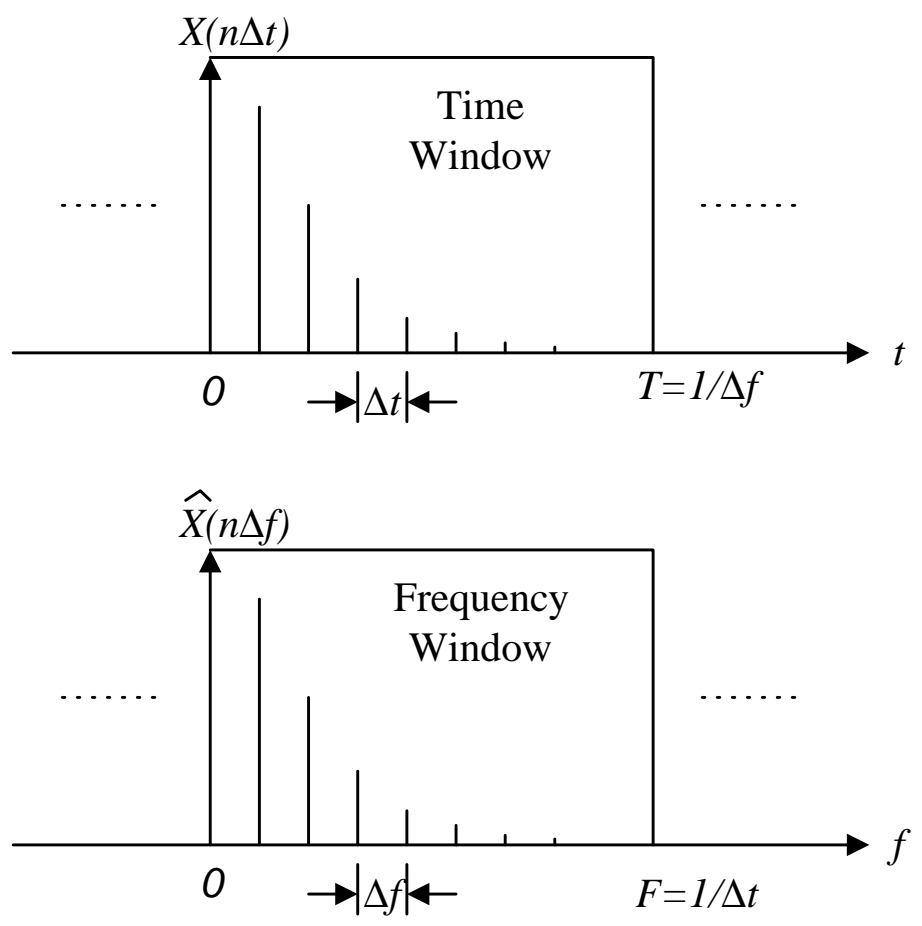

Fig. 3.19 Duality relation between time domain and frequency domain 
Therefore, the most important issue of the DFT implementation is how to choose the sampling rate $(\Delta t$ or $\Delta f$ ) and the length of window (i.e. fundamental period $T$ or $F$ ) both in time domain and frequency domain. In order to approach the real spectral representation of a broadband spectrum of an input signal, the size of window in frequency domain has to be large enough. According to the sampling theorem

$$
F \geq 2 f_{\max }=\frac{1}{\Delta t}
$$

where $f_{\max }$ is the bandwidth (i.e. the highest frequency) of a band-limited, continuous-time signal. This requirement will determine the resolution in time domain and the size of window in frequency domain. On the other hand, to allow the wave to propagate in the time domain and reach steady state, the length of the time window has to be large enough.

$$
T \geq 2 t_{\max }=\frac{1}{\Delta f}
$$

where $t_{\max }$ is the time that is required for the output signal to reach steady state. This requirement will determine the resolution in frequency domain and the size of window in time domain.

In the process, it is necessary to realize that $H_{k} \hat{X}_{k}$ is evaluated only up to the Nyquist frequency (i.e. $F / 2$ ) and the remainder is obtained from the complex conjugate of the initial part. This ensures that the reconstructed time history is real only. An additional feature of note is that the dc (zero frequency) component is undetermined since it does not propagate. For example, the equivalent dielectric permittivity in the system function is undetermined at zero frequency as shown in Eq. (2.42a). It is advantageous to remove its arbitrariness by imposing that the first value of the reconstruction be zero, that is, 


$$
\hat{Y}_{0}=-\sum_{1}^{N-1} \hat{Y}_{k}
$$

This is consistent with the idea that the system is quiescent before the arrival of the wave. Furthermore, it is good practice to give all signals a zero header (a beginning portion of zeros before the actual signal) to emphasize this point.

\subsubsection{Example}

Let the voltage source of the TDR be denoted by $X$, the sampling voltage be denoted by $Y$, and the FFT algorithm by function $F F T($ ). The simulation of a TDR waveform takes the following steps:

1. Determine $F$ (and hence $\Delta t$ ) and $T$ (and hence $\Delta f$ ) using Eq. (3.90) and (3.91).

2. $\hat{X}_{k}=F F T(X)$ (Equivalent to Eq. (3.89b)).

3. Determine $H_{k}$ from Eqs. (3.62) and (3.71) using input impedance method or Eqs. (3.68) and (3.74) using ABCD matrix method. In order to calculate the $H_{k}$, we need to know the length $l_{i}$, the reference impedance $Z_{p i}$, and the equivalent dielectric permittivity $\varepsilon_{r i}{ }^{*}$ of each uniform section of the non-uniform transmission line and the terminal impedances, $Z_{S}$ and $Z_{L}$.

4. Calculate $\hat{Y}_{k}$ using Eqs. (3.84) and (3.92)

5. $Y=\operatorname{IFFT}\left(\hat{Y}_{k}\right)$ (Equivalent to Eq. (3.89a)).

A very simple example, which can be solved analytically in the time domain (Magnusson et al., 1992), is used to validate the above procedure. The problem and result are shown in Fig. 3.20. It is obvious that the numerical procedure produces the same result as the analytical solution. The calibration of the reference impedances in a TDR system and the validation of above procedure using TDR waveforms of materials with known dielectric permittivity will be shown in subsequent chapters. 


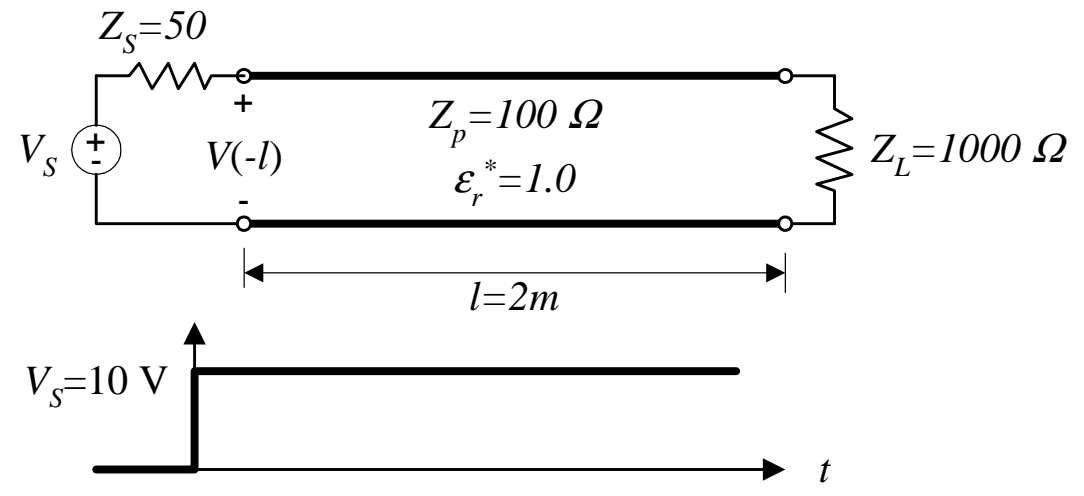

(a)

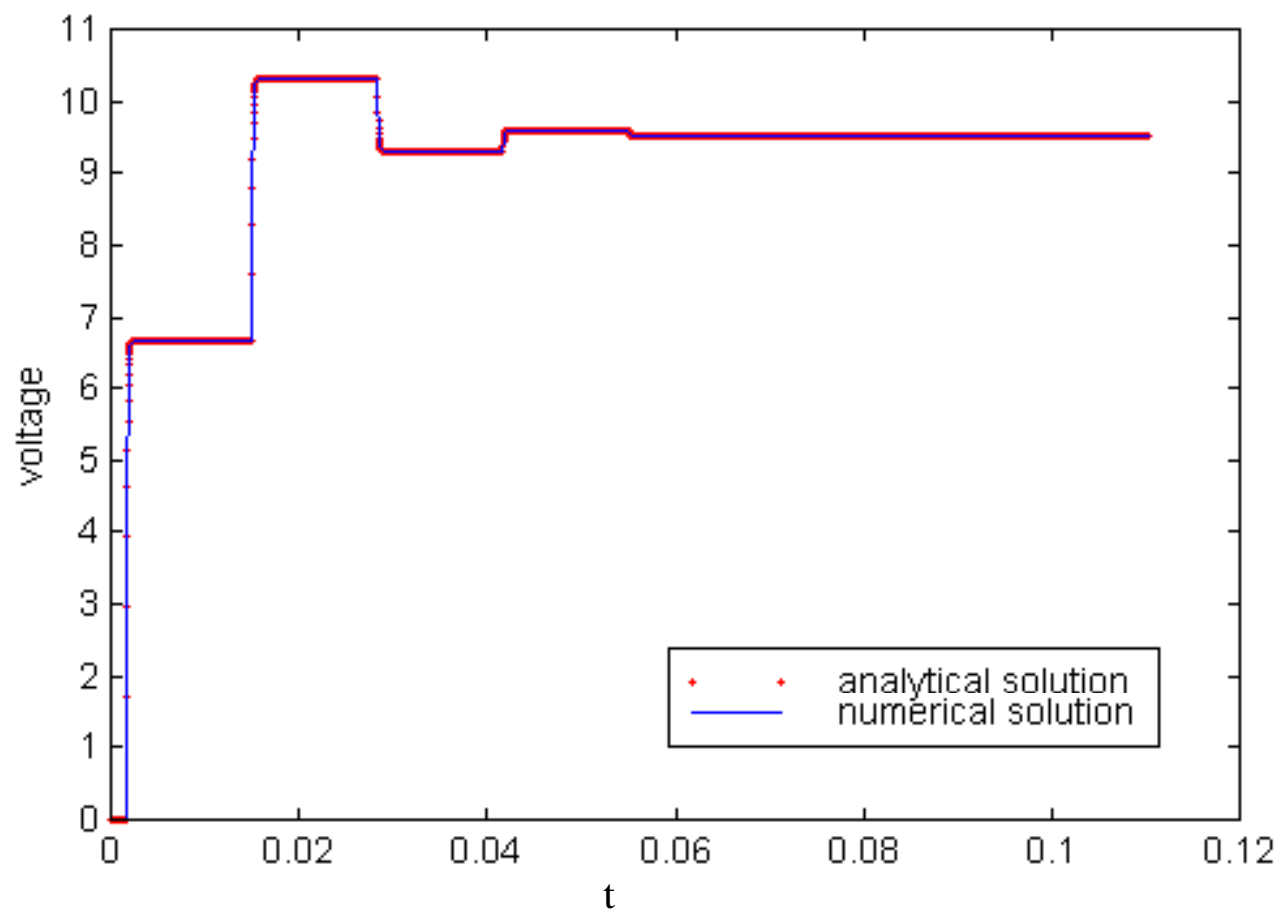

(b)

Fig. 3.20 Validation of the numerical algorithm 


\subsection{Restrictions on Applicability of TL Equation}

The basic assumptions of the derivation of TL equations have been discussed in section 3.3.1. Here we would like to look at some of the quantitative restriction on these assumptions.

\subsubsection{Higher Order Mode}

Transmission-line structures having electrically large cross-sectional dimensions have, in addition to the TEM mode of propagation, other higher-order modes of propagation (Leviaton and Adams, 1982). The higher-order modes include transverse magnetic (TM) modes and transverse electric (TE) modes. The TEM formulation is complete up to some cutoff frequency where the conductor separations are relatively smaller than the cutoff wavelength above which higher-order modes begin to propagate. The cutoff frequency of a propagation mode is the frequency below which the wave can not propagate in that propagation mode. The cutoff frequency of the TEM mode is dc while the cutoff frequencies of TM and TE are much higher.

It is a formidable task to obtain the analytical solution of Maxwell's equations in order to consider the total effect of all modes. For a closed system transmission line such as the coaxial transmission line, the general solution to Maxwell's equations for the fields and modes between the inner wire and outer shield was solved in Ramo et al. (1994). The cutoff wavelength for the lowest-order TE mode can be written as

$$
\lambda_{c}=\pi(a+b)
$$

Similarly, for the lowest-order TM mode, the cutoff wavelength is

$$
\lambda_{c} \approx 2(b-a)
$$

where $a$ is the outer diameter of the inner conductor and $b$ is the inner diameter of the outer conductor. The relationship between the wavelength and frequency is. 


$$
f_{c}=\frac{v}{\lambda_{c}} \approx \frac{c}{\lambda_{c} \sqrt{K_{a}}}
$$

where $c$ is the velocity of light, and $K_{a}$ is the apparent dielectric constant. The frequency bandwidth of the TDR system with 200 ps rise time (i.e. Tektronix 1502b) is about $1 \mathrm{GHz}$. For typical coaxial cables, $\lambda_{c} \approx 0.01 m$ and $K a \approx 2$. Hence, The cutoff frequency for the coaxial cable is about $40 \mathrm{GHz}$, which is well below the frequency range of the TDR system. So the assumption of TEM is justified for coaxial cables. However, the dimensions of the soil probe may be much larger. Considering the size of a standard compaction mold and the apparent dielectric constant of soils, $\lambda_{c} \approx 0.1 m$ and $K_{a} \approx 20$. In such a case, the cutoff frequency is about $0.7 \mathrm{GHz}$, which is slightly lower than the bandwidth of the TDR system. There will be some small contributions from the high-order modes within the TDR bandwidth. Therefore, the quasi-TEM is assumed and the probe impedance should be calibrated experimentally.

\subsubsection{Discontinuity Effect}

A discontinuity occurs whenever our assumption that the line can be modeled as a uniform line is violated. A discontinuity effect (or fringing effect) may occur in transmission line at the plane of sudden change of geometry and cause reflections, apart from reflections caused by any sudden change of the characteristic impedance of the line (Somlo, 1967). It is very difficult to model, especially at the interface of two different types of transmission lines. The discontinuity

effect is neglected in the formulation. But when the line parameters are calibrated, they may include the discontinuity effect. So the line parameters obtained from calibration are equivalent line parameters rather than actual ones.

When a line is terminated at the end of probe, a fringing effect may also occur. In fact, this terminal effect has been used to measure dielectric permittivity of materials (Athey et al., 1982, El-Rayes and Ulaby, 1987). The terminal fringing effect is a function of the cross-sectional geometry at the end of the line (i.e. $b / a$ ratio). However, $b / a=\infty$ for the soil probe because the rods are coneshaped at their ends to facilitate installation. Therefore, the terminal fringing effect may be neglected in such a case. 


\subsubsection{Imperfect Conductor}

The previous derivations assumed that the two conductors are perfect conductors. Lossy conductors invalidate the TEM field structure assumption. However, under the assumption of quasi-TEM mode, small conductor losses can be handled by including the per-unit-length resistance, $r$, in series with the inductance element in the equivalent circuit in an approximate manner as shown in Fig. 3.21. The transmission line equations become

$$
\begin{aligned}
& \frac{d \hat{V}(z)}{d z}=-(r+j 2 \pi f l) \hat{I}(z) \\
& \frac{d \hat{I}(z)}{d z}=-(g+j 2 \pi f c) \hat{V}(z)
\end{aligned}
$$

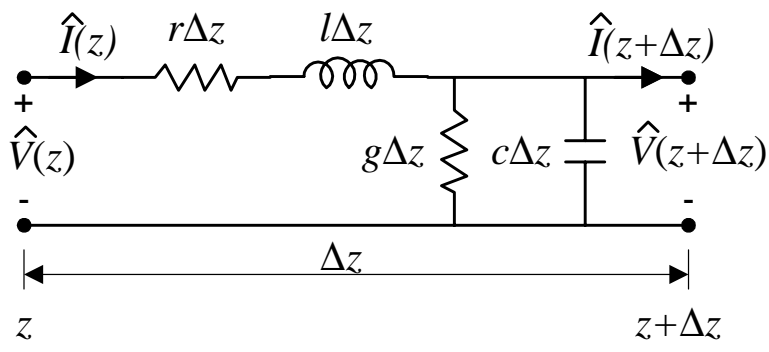

Fig. 3.21 The per-unit-length equivalent circuit accounting for imperfect conductors (modified from Ramo et al., 1994)

The per-unit-length resistance, $r$, is a function of cross-sectional geometry of the line and the skineffect surface resistivity of conductor. For 2-wire probes, it is derived as (Ramo, 1994)

$$
r=\frac{2 R_{s}}{\pi d}\left(\frac{s / d}{\sqrt{(s / d)^{2}-1}}\right)
$$


where $R_{s}$ is the skin effect surface resistivity of conductor. For a coaxial cable, the per-unitlength resistance becomes

$$
r=\frac{R_{s}}{2 \pi}\left(\frac{1}{a}+\frac{1}{b}\right)
$$

The line resistance is neglected in this study because of the low surface resistivity of the conductors and short transmission line.

\subsection{Summary}

The TDR basics have been reviewed and the insufficiency of current analysis methods were highlighted from the field measurement perspective. The governing wave equations and their limitations were reexamined in order to obtain a fundamental understanding of transmission lines. It was shown that a multiple-conductor transmission line could be reduced to a twoconductor transmission line. It was further shown that the line parameters are not independent and they may be determined experimentally. A spectral analysis method was then developed to simulate wave propagation in a non-uniform and dispersive transmission line. The fast FFT algorithm can be used to implement the method very efficiently. The numerical wave propagation model is a powerful tool for probe design, parametric studies, data interpretation, and inverse analyses. 


\section{CHAPTER 4 INVERSE ANALYSIS OF THE TDR SYSTEM}

\subsection{Introduction}

Given the dielectric properties of the material as described in Chapter 2 and the characteristics of a transmission line, the TDR waveform can be predicted using the methods developed in Chapter 3. However, the applications of the TDR technique are typically inverse problems. We are interested in the dielectric properties of the material or the characteristics of the transmission line from the measured TDR waveform.

In recent years, TDR has found applications in soil science and civil engineering. The application of TDR in civil engineering has been of two types. The first type of application uses a material of known dielectric properties. The goal of the waveform interpretation is to detect the anomalies along the line caused by the environment change surrounding the line. Examples of this type of application are monitoring of rock deformation, localized failure planes in soil, and structural deformation. The second type of application uses the material that requires measurement of the physical properties as the media inside the transmission line and the line is kept as uniform as possible. The goal of waveform interpretation is to determine the electromagnetic parameters of the material. The electromagnetic parameters are in turn related to other physical properties of interest. In both cases, the measurement system needs to be calibrated before making any measurements. The two types of applications and the system calibration are all inverse problems.

A probabilistic framework of the inverse solution is developed based on a forward model (i.e. material models described in Chapter 2 and/or wave propagation model developed in Chapter 3). The use of probability naturally incorporates the data uncertainties and facilitates the evaluation of the uncertainty of the estimation of the model parameters. 


\subsection{Inverse Theory}

\subsubsection{Definition of the Inverse Problem}

In this study, the data and model parameters are discrete or discretized. They are conveniently represented by vectors. Denote the data by vector $\boldsymbol{d}$ and model parameter by vector $m$.

$$
\begin{aligned}
& \boldsymbol{d}=\left[d_{1}, d_{2}, d_{3}, \ldots \ldots . ., d_{N D}\right]^{\mathrm{T}} \\
& \boldsymbol{m}=\left[m_{1}, m_{2}, m_{3}, \ldots . ., m_{N M}\right]^{\mathrm{T}}
\end{aligned}
$$

in which subscript $N D$ represents the size of the data and $N M$ represents the number of model parameters. The model parameters and the data are in some way related and this relationship is called the model. Usually the model may take the form of analytical formulas or a computer simulation code that the data and model parameters are expected to follow. Symbolically, the data and model parameters are related as

$$
\boldsymbol{d}=g(\boldsymbol{m})
$$

in which $g(\boldsymbol{m})$ represents the prediction of the data by the model and the model parameters. The inverse problem is defined as the process of inferring the model parameters from the data, i.e., starting with data (and a general principle or model), it determines estimates of the model parameters. As opposed to the forward problem, the inverse problem is illustrated in Fig. 4.1 for TDR applications.

\subsubsection{Methods of Solution}

\subsubsection{Direct Inversion}

Some methods of inversion are known as "direct" or "exact". They are based on the physics of the forward problem and derived by discovering a mathematical operator that is applied to the observed data (often recursively) to derive a model. The inversion operator can be derived 
easily only for simple problems. For models that are more complicated or presented in a computer code, the inverse operator is very difficult if not impossible to find. One of the few examples is the layer-stripping method for the inverse scattering problem in geophysics and electromagnetics (Aki and Richards, 1980, Yagle and Levy, 1983, 1985, Jeffrey and Yagle, 1997). These methods cannot deal with data uncertainty and data redundancy in a natural manner. Any noise present in the data will cause errors that grow rapidly and accumulate because of the recursion. They are of interest for solving mathematical inverse problems, but not always for data interpretation. Methods to obtain solutions for inverse problems are summarized in Fig. 4.2.

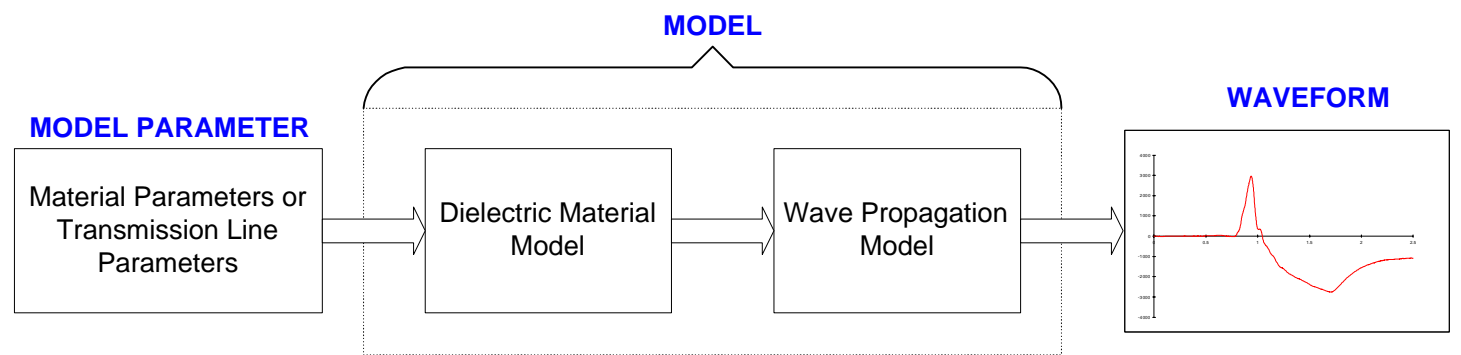

(a) Forward Problem

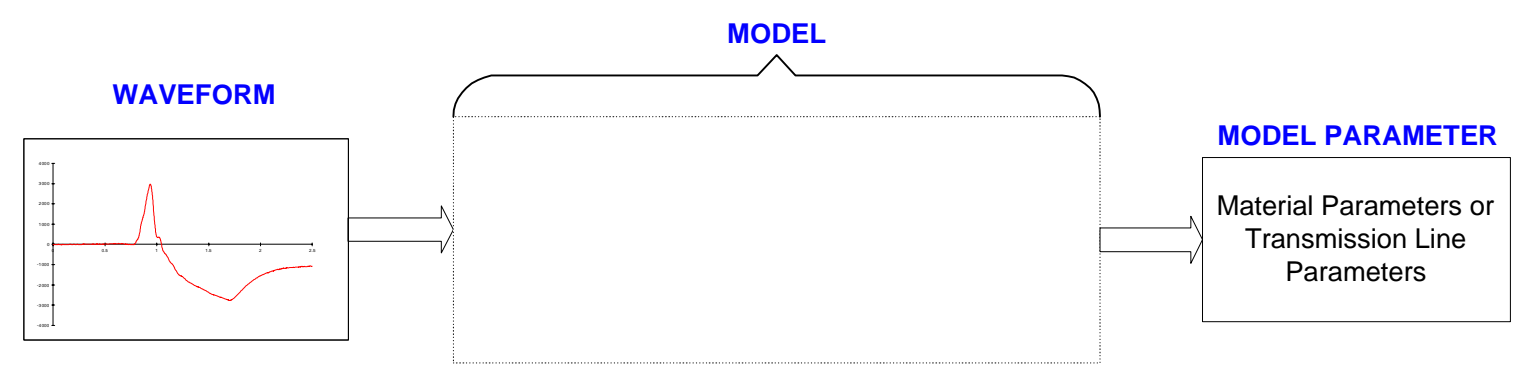

(b) Inverse Problem

Fig. 4.1 Definition of the inverse problem

\subsubsection{Neural Networks}

Artificial neural networks (ANN) are systems that are inspired by modeling networks of real (biological) neurons in the brain. A neural network consists of a number of interconnected processing units as shown in Fig. 4.3. These units are commonly referred to as neurons. Each neuron receives an input signal from neurons to which it is connected. Each of these connections has numerical weights associated with it. These weights determine the nature and strength of the 
influence between the interconnected neurons. The signals from each input are then processed through a weighted sum of the inputs, and the processed output is then transmitted to other neurons via a transfer or activation function. Fig. 4.3 shows the architecture of a typical neural network consisting of three layers of interconnected neurons. Each neuron is connected to all the neurons in the next layer. There is an input layer where data are presented to the neural network, and an output layer that holds the response of the network to input. It is the intermediate layers, also known as hidden layers that enable these networks to distributively represent and compute complicated associations between patterns. The neural network learns by updating the weights of the connections (Hagan et al., 1996).

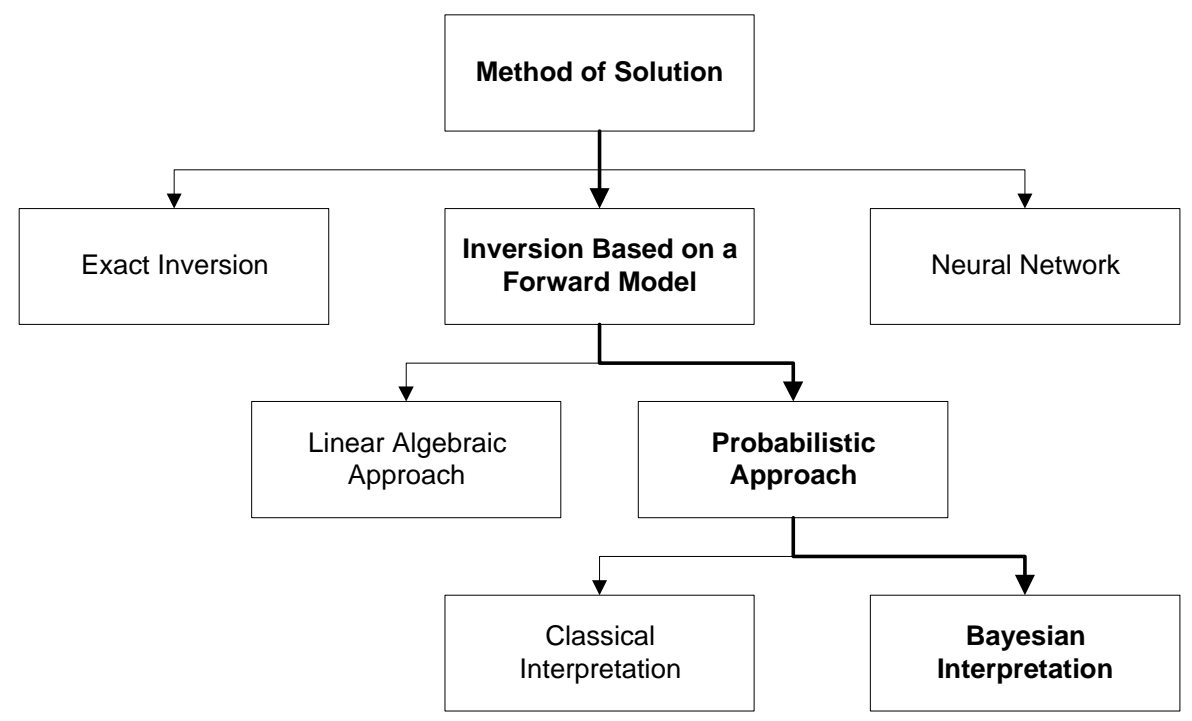

Fig. 4.2 Method of solution for inverse problems

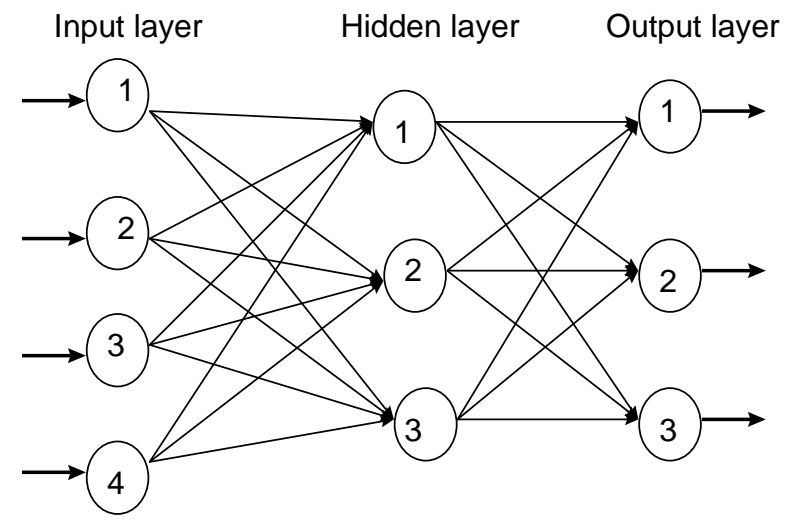

Fig. 4.3 Typical neural-network architecture 
Back-propagation networks are multiplayer, feed-forward networks with the backpropagation learning algorithm. They are probably the most used neural networks. The basic mathematical concepts of the back-propagation algorithm are found in the literature (Rumelhart et al., 1986). Training of the neural network is essentially carried out through the presentation of a series of example patterns of associated input and target output values. The neural network learns by modifying the weights of the neurons in response to the errors between the actual output values and the target output values. It has been shown that a multiplayer, back-propagation network, with as few as one hidden layer, using arbitrary squashing activation functions and linear or polynomial integration functions, can approximate virtually any (Borel measurable) function of interest to any desired degree of accuracy, providing a sufficient number of hidden units are available (Hornick et al., 1989). The theorem establishes multiplayer, back-propagation networks as a class of universal approximators. It assures the representation capability of the structures of the back-propagation networks if there are enough hidden units.

Because of the distributed representation, there is no need to specify a mathematical relationship between the input and output variables. Neural networks can be effective for establishing patterns and relationships not previously known. Inverse mappings can be developed as easily as the direct mappings, and there is considerable flexibility in defining the contents of the input and output layers. Both quantitative and qualitative information can be considered with neural networks. Unlike direct inversion, neural networks are trained to deal with inherent noisy and imprecise data. However, use of a neural network requires that the relationship being mapped from input to output should be unique, and that a large amount of training data is available. The main criticism of the neural network methodology is its inability to trace and explain the step-bystep logic used to arrive at the outputs from the given inputs.

\subsubsection{Inversion Based on a Forward Model}

Inverse theory based on a forward model and observation is a set of mathematical techniques for obtaining useful information about the physical world on the basis of inferences drawn from observations. Geophysical applications of inverse theory are given by Menke (1989) and Tarantola (1987). As defined above, the data and model parameters are in some way related and can be denoted by Eq. (4.2). No claims are made either that the equation $g(\boldsymbol{m})=\boldsymbol{d}$ contains enough information to specify the model parameters uniquely or that they are even consistent. 
One of the purposes of inverse theory is to answer these kinds of questions and to provide means of dealing with the problems that they imply.

Linear algebraic methods are classical approaches that have been used to study the inversion based on a forward model. These methods assume a linear relationship between the data and the model or they linearize a nonlinear relationship. Several useful concepts have been developed including concepts of non-uniqueness and data (or model) resolution. Menke (1989) and Santamarina and Fratta (1997) provide detailed descriptions of these methods. These methods do not account for data errors explicitly and can not analyze the inverse problem in a truly nonlinear manner. Another approach used to study inverse problems is to use a probabilistic framework. The probabilistic framework can be further categorized into two groups: classical interpretation and Bayesian interpretation. Tarantola (1987) formulated the inverse problem using a strong theoretical information theory in the Bayesian paradigm and showed how different states of information can be combined to describe an answer to the inverse problem. This chapter illustrates how to use Bayesian interpretation to set up inverse problems and use the theory of random variables to derive and interpret solutions. The approach for choosing the general framework for inverse problems in geotechnical engineering is also depicted in Fig. 4.2. Linear algebraic solutions will be shown to be special cases in the probabilistic framework.

\subsection{Setup of Inverse Problems in TDR System}

\subsubsection{Additive Noise Model}

Equation (4.2) represents a perfect relationship between data and model parameters. However, all the measured data are subject to noise that leads to uncertainty. One way to deal with the uncertainty is to use the additive noise model (Tarantola, 1987). In the forward model some assumptions have been made in order to make the solution possible. We might correct the model (it would probably be more accurate to call it "erroring the observations") by attaching modeling errors, $\Delta_{m}$, to the predicted data values.

$$
D_{\text {corrected }}=g(\boldsymbol{m})+\Delta_{m}
$$


Note that capital letters are used to denote data and noise in Eq. (4.3). To be consistent with the notation of probability theory, a capital letter is used to denote a random variable and small letter is used to denote the deterministic variable or values taken by random variables. We can also add measurement errors, $\Delta_{d}$, to Eq. (4.3) to account for noise in the data.

$$
D_{\text {obs }}=\boldsymbol{D}_{\text {corrected }}+\Delta_{d}
$$

Substituting Eq. (4.3) into (4.4) leads to

$$
\boldsymbol{D}_{\text {obs }}=g(\boldsymbol{m})+\Delta_{m}+\Delta_{d}=g(\boldsymbol{m})+\Delta
$$

in which $\Delta$ is the total noise, the sum of modeling errors $\Delta_{m}$ and data errors $\Delta_{d}$. Therefore, the observed data is the output of a stochastic system described in Fig. 4.4.

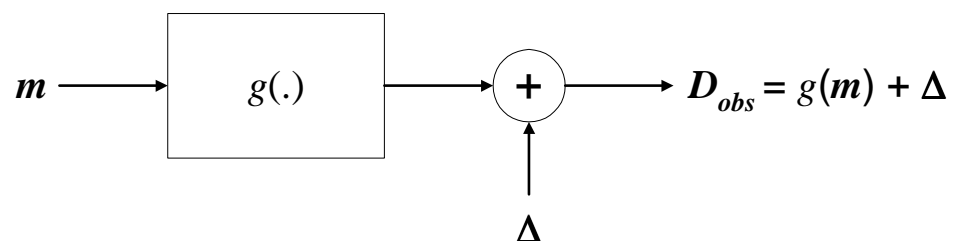

Fig. 4.4 Stochastic system of the observed data (Classical Interpretation)

\subsubsection{Interpretation of the Stochastic System}

The two possible interpretations of an additive noise model are classical interpretation and Bayesian interpretation. In the classical interpretation, model parameters are deterministic but unknown. There are several estimators that estimate the model parameters from the observations under the classical statistical model, such as the method of moments, maximum likelihood, and uniform minimum variance unbiased estimators (UMVUE). Casella and Berger (1990) provide 
detailed descriptions of these methods. These approaches have tended to deal only with estimates of model parameters rather than with probability distributions. Bayesian interpretation takes the viewpoint that the most general way of describing a state of information of a parameter set is by defining a probability density over the corresponding parameter space. In this sense, model parameters are considered as random variables, like noise $\Delta$ and data $\boldsymbol{D}_{\boldsymbol{o b s}}$. So Fig. 4.4 has to be modified by replacing $\boldsymbol{m}$ with $\boldsymbol{M}$ as a random variable as shown in Fig. 4.5. Therefore, Eq. (4.5) becomes

$$
D_{o b s}=g(M)+\Delta
$$

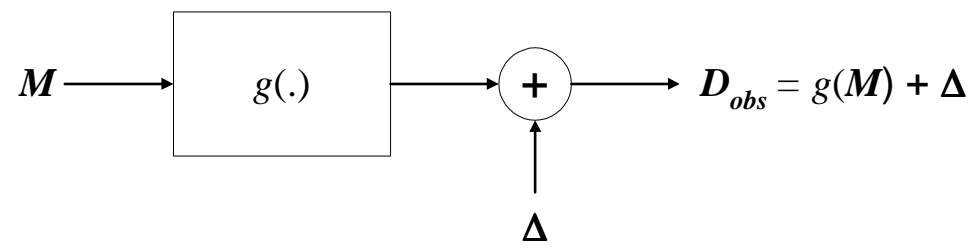

Fig. 4.5 Stochastic system of the observed data (Bayesian Interpretation)

Using the Bayesian interpretation, $\boldsymbol{M}$ and $\boldsymbol{D}_{\boldsymbol{o b s}}$ become jointly distributed random variables having joint density $p\left(\boldsymbol{d}_{\boldsymbol{o b s}}, \boldsymbol{m}\right)$. Before the experiment or the observation of $\boldsymbol{D}_{\boldsymbol{o b s}}$ is made, the model parameters are unknown or poorly known. Regardless of the ignorance of the model parameters, we have some idea (prior information) about $\boldsymbol{M}$ (i.e. the range and distribution of $\boldsymbol{M}$ ). After conducting the experiments or making the observations, the ignorance (very weak prior information) can be improved and model parameters can be better estimated. Hence, when using the Bayesian concept to solve the inverse problem, the appropriate question to ask is: given a priori information on some model parameters, $p(\boldsymbol{m})$, and given a stochastic system (uncertain physical law, $\mathrm{g}(\boldsymbol{M})+\boldsymbol{\Delta})$ that relates some observable parameters to the model parameters, how should the a priori information be modified to improve the knowledge about the model parameters based upon some observations? Following the property of conditional probability and the total 
probability law, Bayes' theorem states that the posterior probability (probability distribution of model parameters conditioned on a certain observation) is (Papoulis, 1991)

$$
p\left(\boldsymbol{m} \mid \boldsymbol{d}_{\text {obs }}\right)=\frac{p\left(\boldsymbol{d}_{\text {obs }} \mid \boldsymbol{m}\right) p(\boldsymbol{m})}{p\left(\boldsymbol{d}_{\text {obs }}\right)}=\frac{p\left(\boldsymbol{d}_{\text {obs }} \mid \boldsymbol{m}\right) p(\boldsymbol{m})}{\int_{\Lambda} p\left(\boldsymbol{d}_{\text {obs }} \mid \boldsymbol{m}\right) p(\boldsymbol{m}) d \boldsymbol{m}}
$$

in which $\Lambda$ represents the model space such that $m \in \Lambda\left(\Lambda_{1} \times \Lambda_{2} \times \ldots \times \Lambda_{M}\right)$.

Accordingly, to make inferences about physical systems from data one must answer two fundamental questions.

1. What is known about the model parameters, $\boldsymbol{M}$, independent of the data? (i.e. what is the prior information $\mathrm{p}(\boldsymbol{m})$ ?)

2. What is the accuracy of the data and the modeling, $\Delta=\Delta_{d}+\Delta_{m}$ ? (i.e. what is $\mathrm{p}\left(\boldsymbol{d}_{\boldsymbol{o b s}} \mid \boldsymbol{m}\right)$, which can be calculated from Eq. (4.6) and probability distribution of $\Delta$, to account for the measurement and modeling errors?)

If these two questions can be reasonably answered, the probability distribution of the model parameters after considering the data observations can be calculated by Eq. (4.7). Equation (4.7) leads very naturally to the analysis of error and to tests of the significance of answers even for highly nonlinear problems. This is why Tarantola [1987] strongly takes the viewpoint that "the most general formulation of inverse problems is obtained when using the language of probability calculus, and when using a Bayesian interpretation of probability." The work of Savage (1972) and De Finetti (1974, 1975), and their successors have proven the inadequacy of classical statistics and shown theoretically that no logically consistent inference maker can behave in a nonBayesian way. Although the state of practice for data analysis is still classical statistics, Lindley (1975) has predicted Bayesian statistics to be the statistics of the twenty first century. 


\subsection{Solution of the Inverse Problem}

Using the Bayesian statistical model, the solution of inverse problems and the analysis of errors can be performed in a fully nonlinear way (but perhaps with a prohibitively large amount of computing time). In fact, Eq. (4.7) is the solution to the inverse problem. The most general way of studying the information obtained regarding the parameter values is by a direct study of the a posterior probability density $p\left(\boldsymbol{m} \mid \boldsymbol{d}_{\boldsymbol{o b s}}\right)$. But there is no way to display it in a multidimensional space if the number of model parameters is greater than two. One of the most comprehensive understandings is obtained by calculating the marginal probability distribution of a particular model parameter. The marginal distribution can be calculated as

$$
\begin{aligned}
p\left(m_{i} \mid \boldsymbol{d}_{\boldsymbol{o b s}}\right) & =\int_{\boldsymbol{\Lambda}_{1}} d m_{1} \int_{\boldsymbol{\Lambda}_{2}} d m_{2} \cdots \int_{\boldsymbol{\Lambda}_{i-1}} d m_{i-1} \int_{\boldsymbol{\Lambda}_{i+1}} d m_{i+1} \cdots \int_{\boldsymbol{\Lambda}_{M}} d m_{M} p\left(\boldsymbol{m} \mid \boldsymbol{d}_{\boldsymbol{o b s}}\right) \\
& =\frac{\int_{\boldsymbol{\Lambda}_{1}} d m_{1} \int_{\boldsymbol{\Lambda}_{2}} d m_{2} \cdots \int_{\boldsymbol{\Lambda}_{i-1}} d m_{i-1} \int_{\boldsymbol{\Lambda}_{i+1}} d m_{i+1} \cdots \int_{\boldsymbol{\Lambda}_{M}} d m_{M} p\left(\boldsymbol{d}_{\boldsymbol{o b s}} \mid \boldsymbol{m}\right) p(\boldsymbol{m})}{\int_{\boldsymbol{\Lambda}} p\left(\boldsymbol{d}_{\boldsymbol{o b s}} \mid \boldsymbol{m}\right) p(\boldsymbol{m}) d \boldsymbol{m}}
\end{aligned}
$$

In general, Eq. (4.8) involves multidimensional numerical integration. Since $\mathrm{p}\left(\boldsymbol{d}_{o b s} \mid \boldsymbol{m}\right)$ is a function of $\boldsymbol{m}$ through the forward model, $\mathrm{g}(\boldsymbol{m})$, numerical integration is prohibitively expensive if the time to evaluate $\mathrm{g}(\boldsymbol{m})$ is computational intensive. Efficient methods to evaluate the multidimensional integral such as Monte-Carlo integration and importance sampling techniques are discussed in detail by Sen and Stoffa $(1995,1996)$. The meaning of "solving" an inverse problem depends on the type of practical application. Usually we are interested in obtaining the "best" estimate in some sense.

\subsubsection{Optimal Estimator}

The sense of optimality is determined by combining information obtained from the inverse problem solution and economic considerations when the solution of the inverse problem is used to make decisions in design or construction. Denote the observation space by $\Gamma$ and model space by $\Lambda$, so that $\boldsymbol{d}_{o b s} \in \Gamma$ and $\boldsymbol{m} \in \Lambda$. The goal of the optimal parameter estimation is to find a function $\hat{\boldsymbol{m}}: \Gamma \rightarrow \Lambda$ such that $\hat{\boldsymbol{m}}\left(\boldsymbol{d}_{\text {obs }}\right)$ is in a sense the best estimator of the true value of the model 
parameters ( $\boldsymbol{m}_{\text {true }}$ ) given $\boldsymbol{d}_{\text {obs }}$. The sense of optimality is defined as the minimization of a certain error cost. Consider a one-parameter inverse problem and suppose a function $C: R \times R \rightarrow R$ is called a cost function, for example, $C(\hat{m}, m)$ represents the cost of estimating a true value of $m b y$ $\hat{m}$. Some typical cost functions are shown in Fig. 4.6. They corresponds to the following cases

$$
\begin{aligned}
& C(\hat{m}, m)=(\hat{m}-m)^{2} \quad \text { (Squared Error) } \\
& C(\hat{m}, m)=|\hat{m}-m| \text { (Absolute Error) } \\
& C(\hat{m}, m)=\left\{\begin{array}{ll}
0 & \text { if }|\hat{m}-m| \leq \varepsilon \\
1 & \text { if }|\hat{m}-m|>\varepsilon
\end{array}\right. \text { (Uniform cost) }
\end{aligned}
$$

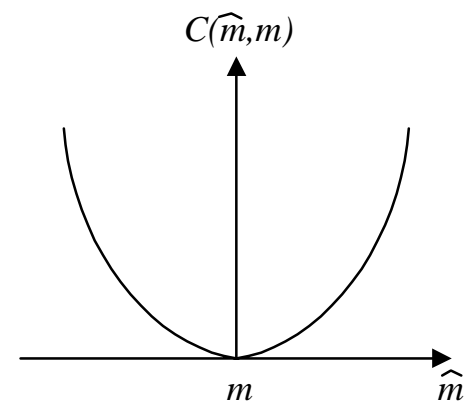

(a)

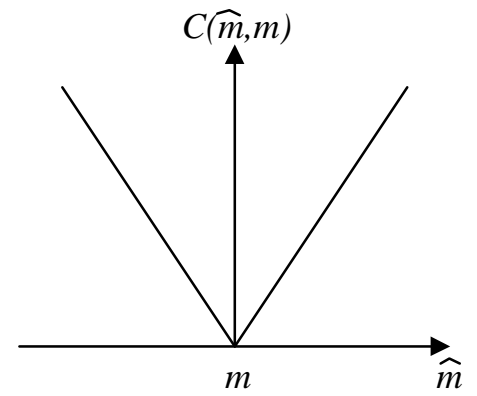

(b)

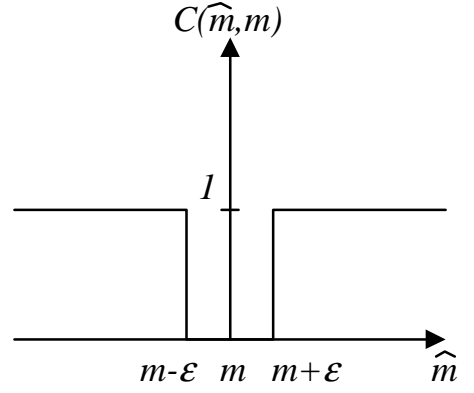

(c)

Fig. 4.6 Cost functions: (a) square error; (b) absolute error; (c) uniform cost

Given a cost function $C$, we can define conditional risk (risk given some certain true model parameter), $R_{M}(\hat{m})$ as

$$
R_{M}(\hat{m})=E\left\{C\left(\hat{m}\left(\boldsymbol{D}_{\text {obs }}\right), M\right) \mid M=m\right\}
$$


where $E\{Y \mid X=x\}$ is the conditional expectation of $Y$ given $X=x$. Now since the parameter $m$ is a realization of a random variable $M$, the average risk can be defined as

$$
\begin{aligned}
r(\hat{m}) & =E\left\{R_{M}(\hat{m})\right\}=E\{E\{C(\hat{m}, M) \mid M=m\}\} \\
& =E\{C(\hat{m}, M)\}=E\left\{E\left\{C(\hat{m}, M) \mid \boldsymbol{D}_{\text {obs }}=\boldsymbol{d}_{\boldsymbol{o b s}}\right\}\right\}
\end{aligned}
$$

The goal of the estimation is to pick $\hat{m}$ that minimizes $r(\hat{m})$. This may be found by minimizing $E\left\{C(\hat{m}, M) \mid \boldsymbol{D}_{\text {obs }}=\boldsymbol{d}_{\text {obs }}\right\}$ in Eq. (4.11). Therefore, the optimal estimator is

$$
\hat{m}\left(\boldsymbol{d}_{\boldsymbol{o b s}}\right)=\underset{\hat{m}}{\arg \min } \int_{\boldsymbol{\Lambda}} C\left(\hat{m}\left(\boldsymbol{d}_{\boldsymbol{o b s}}\right), m\right) p\left(m \mid \boldsymbol{d}_{\boldsymbol{o b s}}\right) d m
$$

where $\arg \min Y(x)$ represents the argument $x$ that minimizes the function $Y(x)$. It has been shown, for example by Poor (1988), that by using the different cost functions in Eq. (4.9), the solution to Eq. (4.12) becomes the mean, medium, and maximum of the posterior probability distribution, respectively. The cost functions and derivation are extended and the same results are obtained for a multi-dimensional model (i.e. $\boldsymbol{m}$ is a vector) in Poor (1988) such that

$$
\begin{aligned}
& \hat{\boldsymbol{m}}_{M M S E}=\int_{\Lambda} \boldsymbol{m} p\left(\boldsymbol{m} \mid \boldsymbol{d}_{\boldsymbol{o b s}}\right) d \boldsymbol{m} \\
& \hat{\boldsymbol{m}}_{M M A E}=\hat{\boldsymbol{m}} \quad \text { such that } \hat{m}_{i}=\operatorname{median}\left[p\left(m_{i} \mid \boldsymbol{d}_{\boldsymbol{o b s}}\right)\right] \\
& \hat{\boldsymbol{m}}_{M A P}=\underset{\boldsymbol{m}}{\arg \min } p\left(\boldsymbol{m} \mid \boldsymbol{d}_{\text {obs }}\right)
\end{aligned}
$$

These estimators are called Minimum Mean Square Error (MMSE) estimator, Minimum Mean Absolute Error (MMAE) estimator and Maximum A Posterior (MAP) estimator, 
respectively. In general, the solution of the posterior mean (MMSE) and the posterior median (MMAE) requires numerical integration. The posterior maximum (MAP) can be found using optimization algorithms. Among these optimal estimators the MAP estimator is the most popular because efficient optimization schemes can be used to solve for the MAP point. Since the posterior density function is a function of the model $g($.$) , it can be arbitrarily complex depending$ on the model $g($.$) . If the posterior density function is multi-modal, the optimization may converge$ in the local minimum. Global optimization techniques such as Simulated Annealing and Generic Algorithms are used to find the global minimum in Sen and Stoffa $(1995,1996)$.

\subsubsection{Uncertainty Analysis}

When the solution of the inverse problem is given as an optimal estimator (such as mean, median and maximum posterior), it is necessary to discuss the uncertainty. The most common way to represent the uncertainty is to calculate the covariance of the posterior distribution.

$$
C_{M \mid D_{o b s}}=\int_{\Lambda}\left(\boldsymbol{m}-\hat{\boldsymbol{m}}_{M M S E}\right)\left(\boldsymbol{m}-\hat{\boldsymbol{m}}_{M M S E}\right)^{T} p\left(\boldsymbol{m} \mid \boldsymbol{d}_{o b s}\right) d \boldsymbol{m}
$$

However, if the posterior density function is multi-modal, the covariance may not be very meaningful. The most comprehensive understanding of the uncertainty is obtained by directly discussing the probability that the value of the model parameters lies in a given range around the optimal estimator. The probability that the model parameters lies in a given range around the optimal estimator, denoted by $\Lambda^{\prime}$, is

$$
P\left(\boldsymbol{m} \in \Lambda^{\prime}\right)=\int_{\Lambda^{\prime}} p\left(\boldsymbol{m} \mid \boldsymbol{d}_{o b s}\right) d \boldsymbol{m}=\frac{\int_{\boldsymbol{\Lambda}^{\prime}} p\left(\boldsymbol{d}_{o b s} \mid \boldsymbol{m}\right) p(\boldsymbol{m}) d \boldsymbol{m}}{\int_{\Lambda} p\left(\boldsymbol{d}_{o b s} \mid \boldsymbol{m}\right) p(\boldsymbol{m}) d \boldsymbol{m}}
$$




\subsection{Gaussian Problems}

Note that marginal posterior (Eq. (4.8)), mean (Eq. (4.13a)), median (Eq. (4.13b)), covariance (Eq. (4.14)), and Eq. (4.15) all require intensive numerical integration. However, analytical solutions can be obtained for a large number of problems when prior information and data error can be described by Gaussian distributions. A multi-variable Gaussian distribution is defined as

$$
p(x)=\frac{1}{\sqrt{(2 \pi)^{N}\left|C_{x}\right|}} \exp \left\{-\frac{1}{2}\left(x-\mu_{x}\right)^{T} C_{x}^{-1}\left(x-\mu_{x}\right)\right\}
$$

and denoted as $\mathrm{N}\left(\mu_{x}, C_{x}\right)$, in which $\mu_{x}$ is the mean vector and $C_{x}$ is the covariance matrix. Assuming a Gaussian distribution for the prior model $\boldsymbol{M}$ and the noise $\Delta$ (i.e. $\boldsymbol{M} \sim \mathrm{N}\left(\boldsymbol{m}_{\boldsymbol{p}}, C_{M}\right)$, and $\left.\Delta \sim \mathrm{N}\left(0, C_{\Delta}\right)\right)$, leads to $p(\boldsymbol{m}) \sim \mathrm{N}\left(\boldsymbol{m}_{\boldsymbol{p}}, C_{M}\right)$ and $p\left(\boldsymbol{d}_{\boldsymbol{o b s}} \mid \boldsymbol{m}\right) \sim \mathrm{N}\left(\mathrm{g}(\boldsymbol{m}), C_{\Delta}\right)$, and the posterior probability density, Eq. (4.7), becomes

$$
\begin{aligned}
& p\left(\boldsymbol{m} \mid \boldsymbol{d}_{\boldsymbol{o b s}}\right)=\text { const } \cdot \frac{1}{p\left(\boldsymbol{d}_{\boldsymbol{o b s}}\right)(2 \pi)^{N / 2} \sqrt{\left|C_{\boldsymbol{\Delta}} \| C_{M}\right|}} \cdot \\
& \quad \exp \left\{-\frac{1}{2}\left[\left(\boldsymbol{d}_{\boldsymbol{o b s}}-g(\boldsymbol{m})\right)^{T} C_{\boldsymbol{\Delta}}{ }^{-1}\left(\boldsymbol{d}_{\boldsymbol{o b s}}-g(\boldsymbol{m})\right)+\left(\boldsymbol{m}-\boldsymbol{m}_{\boldsymbol{p}}\right)^{T} C_{M}{ }^{-1}\left(\boldsymbol{m}-\boldsymbol{m}_{\boldsymbol{p}}\right)\right]\right\}
\end{aligned}
$$

The linear case where $g(\boldsymbol{m})=A \boldsymbol{m}$ will be discussed first in detail to gain insight into inverse solutions. The solutions are derived using the results of a multivariate Gaussian random variable. It will be shown that the linear algebraic solutions are special cases of Linear Gaussian problems. The linear Gaussian problem will be extended to nonlinear cases by linearization.

\subsubsection{Linear Gaussian Problems}

\subsubsection{Optimal Estimator}

If $\mathrm{g}(\boldsymbol{m})=A \boldsymbol{m}$, the stochastic system of the linear observation model becomes 


$$
D_{o b s}=A M+\Delta
$$

in which $A$ is a deterministic matrix, and $\boldsymbol{M}$ and $\Delta$ are independent Gaussians. From a Lemma of multivariate Gaussian distribution (see Appendix A), it can be shown that $\boldsymbol{D}_{\boldsymbol{o b s}}$ and $\boldsymbol{M}$ are jointly Gaussian

$$
\left[\begin{array}{c}
\boldsymbol{M} \\
\boldsymbol{D}_{\text {obs }}
\end{array}\right] \sim \mathrm{N}\left(\left[\begin{array}{c}
\boldsymbol{m}_{p} \\
A \boldsymbol{m}_{p}
\end{array}\right],\left[\begin{array}{cc}
C_{M} & C_{M} A^{T} \\
A C_{M} & A C_{M} A^{T}+C_{\Delta}
\end{array}\right]\right)
$$

Let the estimate of the model parameters $\boldsymbol{M}_{e s t}=\boldsymbol{M} \mid \boldsymbol{D}_{o b s}$ (random vector of $\boldsymbol{M}$ conditioned on $\boldsymbol{D}_{o b s}$ ) be the random vector having the posterior probability distribution. By another Lemma of multivariate Gaussian distribution (also see Appendix A), it can be shown that the conditional distribution of $\boldsymbol{M}$ conditioned on $\boldsymbol{D}_{o b s}$ (posterior probability) is also a Gaussian with

$$
\begin{aligned}
& E\left\{\boldsymbol{M}_{\text {est }}\right\}=\hat{\boldsymbol{m}}=\boldsymbol{m}_{p}+C_{M} A^{T}\left(A C_{M} A^{T}+C_{\boldsymbol{\Delta}}\right)^{-1}\left(\boldsymbol{d}_{\text {obs }}-A \boldsymbol{m}_{p}\right) \\
& \operatorname{Cov}\left\{\boldsymbol{M}_{\text {est }}\right\}=C_{M_{\text {est }}}=C_{M}-C_{M} A^{T}\left(A C_{M} A^{T}+C_{\boldsymbol{\Delta}}\right)^{-1} A C_{M}
\end{aligned}
$$

Therefore, $\boldsymbol{M}_{\text {est }} \sim \mathrm{N}\left(\hat{\boldsymbol{m}}, C_{\text {Mest }}\right)$. Note that in this case $\hat{\boldsymbol{m}}$ is the MMSE, MMAE and MAP estimator. Define a stochastic inversion operator $A_{s t}{ }^{-1}$ as the linear inverse operator

$$
A_{s t}^{-1}=C_{M} A^{T}\left(A C_{M} A^{T}+C_{\Delta}\right)^{-1}
$$

Equation (4.20) can be simplified to 


$$
\begin{aligned}
& \hat{\boldsymbol{m}}=\boldsymbol{m}_{p}+A_{s t}^{-1}\left(\boldsymbol{d}_{\text {obs }}-A \boldsymbol{m}_{p}\right) \\
& C_{M_{\text {est }}}=C_{M}-A_{s t}^{-1} A C_{M}
\end{aligned}
$$

It can be shown that the following equality holds (See Appendix B)

$$
\begin{aligned}
A_{s t}{ }^{-1} & =C_{M} A^{T}\left(A C_{M} A^{T}+C_{\Delta}\right)^{-1} \\
& =\left(A^{T} C_{\Delta}{ }^{-1} A+C_{M}{ }^{-1}\right)^{-1} A^{T} C_{\Delta}{ }^{-1}
\end{aligned}
$$

Using Eqs. (4.22a) and (4.23), the relationship between the Linear Gaussian case and the Linear Algebraic solutions can be seen. Following the notation of Menke (1989), Table 4.1 shows that all the linear algebraic estimations can be obtained by choosing appropriate Gaussian parameters for the prior information and the error term. For example, the least square solution assumes no prior information at all (i.e. $\boldsymbol{M} \sim \mathrm{N}(\mathbf{0},[\infty])$ ) and the data errors are identically distributed, independent Gaussians (i.e. $\Delta \sim \mathrm{N}(\mathbf{0}, c \mathrm{I})$, where $\mathrm{I}$ is the unit matrix and $c$ is the squared data error).

\subsubsection{Uncertainty Analysis (Model Covariance)}

Using the equality in Eq. (4.23), the covariance matrix can be rewritten as

$$
\begin{aligned}
C_{M_{\text {est }}} & =C_{M}-C_{M} A^{T}\left(A C_{M} A^{T}+C_{\Delta}\right)^{-1} A C_{M} \\
& =C_{M}-\left(A^{T} C_{\Delta}^{-1} A+C_{M}{ }^{-1}\right)^{-1} A^{T} C_{\Delta}^{-1} A C_{M} \\
& =\left(A^{T} C_{\Delta}^{-1} A+C_{M}^{-1}\right)^{-1}
\end{aligned}
$$

Therefore, using the Bayesian inversion framework, the covariance of the linear algebraic estimator can be easily obtained by Eq. (4.24) as shown in Table 4.2. The most trivial use of the posterior covariance $C_{\text {Mest }}$ is to interpret the square roots of the diagonal elements (variance) as 
"error bars" that describes the one-standard deviation uncertainties of the posterior values of the model parameters. A direct examination of the off-diagonal elements of $C_{\text {Mest }}$ is not easy. It is better to calculate the coefficients of correlation between model parameters as

$$
\rho_{i j}=\frac{C_{M_{e s t}, i j}}{\sqrt{C_{M_{e s t}, i i} C_{M_{e s t}, j j}}}
$$

which has the well-known property that $-1 \leq \rho_{i j} \leq 1 . \rho_{i j}=0$ means the posterior model parameters are not correlated, $\rho_{i j}=1$ means highly correlated, and $\rho_{i j}=-1$ means highly anti-correlated. A strong correlation between two model parameters means that they have not been independently resolved by the data set and that only some linear combinations of the model parameters are resolved.

\section{Table 4.1 Relationship between Linear Gaussian problem and Linear Algebraic solutions}

Problem Definition: $\boldsymbol{D}_{\text {obs }}=A \boldsymbol{M}+\boldsymbol{\Delta}$, where $\boldsymbol{M} \sim \mathrm{N}\left(\boldsymbol{m}_{p}, C_{M}\right), \Delta \sim \mathrm{N}\left(\boldsymbol{0}, \varepsilon^{2} C_{\Delta}\right)$

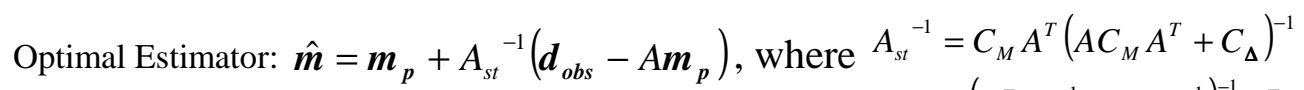

$$
=\left(A^{T} C_{\Delta}^{-1} A+C_{M}^{-1}\right)^{-1} A^{T} C_{\Delta}^{-1}
$$

\begin{tabular}{|c|c|c|}
\hline $\begin{array}{l}\text { Type of Linear } \\
\text { Algebraic Solution }\end{array}$ & Linear Algebraic Inverse Estimate & $\begin{array}{c}\text { Equivalent Bayesian Problem } \\
\text { (prior and data error) }\end{array}$ \\
\hline Least Squares & $\hat{\boldsymbol{m}}=\left[A^{T} A\right]^{-1} A^{T} \boldsymbol{d}_{\boldsymbol{o b s}}$ & $\boldsymbol{M} \sim \mathrm{N}(\mathbf{0},[\infty]), \Delta \sim \mathrm{N}(\mathbf{0}, c \mathrm{I})$ \\
\hline Minimum Norm & $\hat{\boldsymbol{m}}=A^{T}\left[A A^{T}\right]^{-1} \boldsymbol{d}_{\boldsymbol{o b s}}$ & $\boldsymbol{M} \sim \mathrm{N}(\mathbf{0}, c \mathrm{I}), \Delta \sim \mathrm{N}(\mathbf{0},[0])$ \\
\hline $\begin{array}{l}\text { Damped Least } \\
\text { Squares }\end{array}$ & $\begin{array}{l}\hat{\boldsymbol{m}}=\left[A^{T} A+\varepsilon^{2} I\right]^{-1} A^{T} \boldsymbol{d}_{\boldsymbol{o b s}} \\
=A^{T}\left[A A^{T}+\mathcal{E}^{2} I\right]^{-1} \boldsymbol{d}_{\boldsymbol{o b s}}\end{array}$ & $\begin{array}{c}\boldsymbol{M} \sim \mathrm{N}\left(\mathbf{0}, c \varepsilon^{-2} \mathrm{I}\right), \Delta \sim \mathrm{N}(\mathbf{0}, c \mathrm{I}), \text { or } \\
\boldsymbol{M} \sim \mathrm{N}(\mathbf{0}, c \mathrm{I}), \Delta \sim \mathrm{N}\left(\mathbf{0}, c \varepsilon^{2} \mathrm{I}\right)\end{array}$ \\
\hline $\begin{array}{l}\text { Weighted Least } \\
\text { Squares }\end{array}$ & $\hat{\boldsymbol{m}}=\left[A^{T} W_{e} A\right]^{-1} A^{T} W_{e} \boldsymbol{d}_{\boldsymbol{o b s}}$ & $\boldsymbol{M} \sim \mathrm{N}(\mathbf{0},[\infty]), \Delta \sim \mathrm{N}\left(\mathbf{0}, c W_{e}^{-1}\right)$ \\
\hline $\begin{array}{l}\text { Weighted Minimum } \\
\text { Norm }\end{array}$ & $\hat{\boldsymbol{m}}=\boldsymbol{m}_{p}+W_{m}^{-1} A^{T}\left[A W_{m}^{-1} A^{T}\right]^{-1}\left[\boldsymbol{d}_{\boldsymbol{o b s}}-A \boldsymbol{m}_{p}\right]$ & $\boldsymbol{M} \sim \mathrm{N}\left(\boldsymbol{m}_{p}, c W_{m}^{-1}\right), \Delta \sim \mathrm{N}(\mathbf{0},[0])$ \\
\hline Weighted Damped & $\hat{\boldsymbol{m}}=\boldsymbol{m}_{p}+\left[A^{T} W_{e} A+\mathcal{E}^{2} W_{m}\right]^{-1} A^{T} W_{e}\left[\boldsymbol{d}_{\boldsymbol{o b s}}-A \boldsymbol{m}_{p}\right]$ & $\begin{array}{c}\boldsymbol{M} \sim \mathrm{N}\left(\boldsymbol{m}_{p}, c \varepsilon^{-2} W_{m}^{-1}\right), \Delta \sim \mathrm{N}\left(\mathbf{0}, \mathrm{c} W_{e}^{-1}\right) \\
\text { or }\end{array}$ \\
\hline Least Squares & $=\boldsymbol{m}_{p}+W_{m}^{-1} A^{T}\left[A W_{m}^{-1} A^{T}+\varepsilon W_{e}^{-1}\right]^{-1}\left[\boldsymbol{d}_{o b s}-A \boldsymbol{m}_{p}\right]$ & $\boldsymbol{M} \sim \mathrm{N}\left(\boldsymbol{m}_{p}, c W_{m}^{-1}\right), \boldsymbol{\Delta \sim N}\left(\mathbf{0}, c \varepsilon^{2} W_{e}^{-1}\right)$ \\
\hline
\end{tabular}


Note: 1 . The Linear Algebraic Solutions can be obtained by minimizing $E+\varepsilon^{2} L$, where $E=\left(\boldsymbol{d}_{\boldsymbol{o b s}}-A \boldsymbol{m}\right)^{T} W_{e}\left(\boldsymbol{d}_{\boldsymbol{o b s}}-A \boldsymbol{m}\right)$, and $L=\left(\boldsymbol{m}-\boldsymbol{m}_{p}\right)^{T} W_{m}\left(\boldsymbol{m}-\boldsymbol{m}_{p}\right)$. (See Menke, 1989)

$W_{e}$ : error weighting matrix $\quad W_{m}$ : model weighting matrix $\quad \varepsilon$. damping constant

2. $c$ : arbitrary constant $=$ variance of the uncorrelated data error or variance of the uncorrelated prior

[0]: matrix containing all zeros; $\quad[\infty]$ : matrix containing all $\infty$; $\quad$ I: unit matrix

\section{Table 4.2 Covariance of the Linear Algebraic Solutions}

Problem Definition $\quad: \boldsymbol{D}_{\boldsymbol{o b s}}=A \boldsymbol{M}+\boldsymbol{\Delta}$, where $\boldsymbol{M} \sim \mathrm{N}\left(\boldsymbol{m}_{p}, C_{M}\right), \Delta \sim \mathrm{N}\left(\mathbf{0}, \varepsilon^{2} C_{\Delta}\right)$

Covariance of Estimate: $C_{M_{\text {est }}}=C_{M}-C_{M} A^{T}\left(A C_{M} A^{T}+C_{\Delta}\right)^{-1} A C_{M}=C_{M}-\left(A^{T} C_{\Delta}^{-1} A+C_{M}^{-1}\right)^{-1} A^{T} C_{\Delta}^{-1} A C_{M}$ $=\left(A^{T} C_{\Delta}^{-1} A+C_{M}^{-1}\right)^{-1}$

\begin{tabular}{|c|c|c|}
\hline $\begin{array}{c}\text { Type of Linear } \\
\text { Algebraic Solution }\end{array}$ & $\begin{array}{c}\text { Equivalent Bayesian Problem } \\
\text { (prior and data error) }\end{array}$ & Covariance Matrix \\
\hline Least Squares & $\boldsymbol{M} \sim \mathrm{N}(\mathbf{0},[\infty]), \Delta \sim \mathrm{N}(\mathbf{0}, c \mathrm{I})$ & $c\left(A^{T} A\right)^{-1}$ \\
\hline Minimum Norm & $\boldsymbol{M} \sim \mathrm{N}(\mathbf{0}, c \mathrm{I}), \boldsymbol{\Delta \sim \mathrm { N }}(\mathbf{0},[0])$ & $c I$ \\
\hline $\begin{array}{l}\text { Damped Least } \\
\text { Squares }\end{array}$ & $\begin{array}{c}\boldsymbol{M} \sim \mathrm{N}(\mathbf{0}, c \mathrm{I}), \Delta \sim \mathrm{N}\left(\mathbf{0}, c \varepsilon^{2} \mathrm{I}\right), \text { or } \\
\boldsymbol{M} \sim \mathrm{N}\left(\mathbf{0}, c \varepsilon^{-2} \mathrm{I}\right), \Delta \sim \mathrm{N}(\mathbf{0}, c \mathrm{I})\end{array}$ & $\begin{array}{l}c\left[I-A^{T}\left(A A^{T}+\mathcal{E}^{2} I\right)^{-1} A\right], \text { or } \\
c \mathcal{E}^{-2}\left[I-A^{T}\left(A A^{T}+\mathcal{E}^{2} I\right)^{-1} A\right]\end{array}$ \\
\hline $\begin{array}{l}\text { Weighted Least } \\
\text { Squares }\end{array}$ & $\boldsymbol{M} \sim \mathrm{N}(\mathbf{0},[\infty]), \Delta \sim \mathrm{N}\left(\mathbf{0}, c W_{e}^{-1}\right)$ & $c\left(A^{T} W_{e} A\right)^{-1}$ \\
\hline $\begin{array}{l}\text { Weighted Minimum } \\
\text { Norm }\end{array}$ & $\boldsymbol{M} \sim \mathrm{N}\left(\boldsymbol{m}_{p}, c W_{m}^{-1}\right), \Delta \sim \mathrm{N}(\mathbf{0},[0])$ & $c W_{m}^{-1}\left[I-A^{T}\left(A W_{m}^{-1} A^{T}\right)^{-1} A W_{m}^{-1}\right]$ \\
\hline $\begin{array}{l}\text { Weighted Damped } \\
\text { Least Squares }\end{array}$ & $\begin{array}{c}\boldsymbol{M} \sim \mathrm{N}\left(\boldsymbol{m}_{p}, c W_{m}^{-1}\right), \Delta \sim \mathrm{N}\left(\mathbf{0}, c \varepsilon^{2} W_{e}^{-1}\right) \\
\text { Or } \\
\boldsymbol{M} \sim \mathrm{N}\left(\boldsymbol{m}_{p}, c \varepsilon^{-2} W_{m}^{-1}\right), \Delta \sim \mathrm{N}\left(\mathbf{0}, c W_{e}^{-1}\right)\end{array}$ & $\begin{array}{c}c W_{m}^{-1}\left[I-A^{T}\left(A W_{m}^{-1} A^{T}+\varepsilon^{2} W_{e}^{-1}\right)^{-1} A W_{m}^{-1}\right] \\
o r \\
c \varepsilon^{-2} W_{m}^{-1}\left[I-A^{T}\left(A W_{m}^{-1} A^{T}+\varepsilon^{2} W_{e}^{-1}\right)^{-1} A W_{m}^{-1}\right]\end{array}$ \\
\hline
\end{tabular}

Note: 1. The Linear Algebraic Solutions can be obtained by minimizing $E+\varepsilon^{2} L$, where

$E=\left(\boldsymbol{d}_{\boldsymbol{o b s}}-A \boldsymbol{m}\right)^{T} W_{e}\left(\boldsymbol{d}_{\boldsymbol{o b s}}-A \boldsymbol{m}\right)$, and $L=\left(\boldsymbol{m}-\boldsymbol{m}_{p}\right)^{T} W_{m}\left(\boldsymbol{m}-\boldsymbol{m}_{p}\right)$. (See Menke, 1989)

$W_{e}$ : error weighting matrix $\quad W_{m}$ : model weighting matrix $\quad \varepsilon$. damping constant

2. $c$ : arbitrary constant $=$ variance of the uncorrelated data error or variance of the uncorrelated prior

[0]: matrix containing all zeros; $\quad[\infty]$ : matrix containing all $\infty$; $\quad$ I: unit matrix 


\subsubsection{Model Resolution Operator}

Note that the model estimator depends not only on the data $\boldsymbol{d}_{o b s}$ but also on the prior model $\boldsymbol{m}_{p}$. So a different optimal solution is obtained if different values of $\boldsymbol{m}_{p}$ are selected. This seems to be a major drawback of the Bayesian framework. However, subjective parameters, which are more difficult to interpret, also exist in linear algebraic methods such as damped least squares and weighted least squares. $\boldsymbol{m}_{p}$ can be thought of as an engineering judgement, a necessary component in geotechnical engineering. The sensitivity of the optimal estimator on the selection of $\boldsymbol{m}_{p}$ can be studied by looking at the model resolution operator. The concept of a model resolution operator arises when a relationship is sought between the estimated model parameter $\hat{\boldsymbol{m}}$ and true model parameter $\boldsymbol{m}_{\text {true }}$ (Menke, 1989). It is possible to express the estimator in terms of the underlying true model parameter $\boldsymbol{m}_{\text {true }}$ (i.e. $\boldsymbol{d}_{\text {obs }}=A \boldsymbol{m}_{\text {true }}+\boldsymbol{\delta}$ ), such that

$$
\begin{aligned}
\hat{\boldsymbol{m}} & =\boldsymbol{m}_{p}+A_{s t}^{-1}\left(\boldsymbol{d}_{\text {obs }}-A \boldsymbol{m}_{p}\right) \\
& =\boldsymbol{m}_{p}+A_{s t}{ }^{-1}\left(A \boldsymbol{m}_{\text {true }}+\boldsymbol{\delta}-A \boldsymbol{m}_{p}\right) \\
& =A_{s t}{ }^{-1} A \boldsymbol{m}_{\text {true }}+\left(I-A_{s t}{ }^{-1} A\right) \boldsymbol{m}_{p}+A_{s t}{ }^{-1} \boldsymbol{\delta}
\end{aligned}
$$

Note that $\boldsymbol{\delta}$ is the realization of the random vector $\Delta$, representing the error occurring in a particular experiment plus modeling errors. The resolution operator $R$ is defined as

$$
\begin{aligned}
R=A_{s t}^{-1} A & =\left(A^{T} C_{\Delta}^{-1} A+C_{M}{ }^{-1}\right)^{-1} A^{T} C_{\Delta}{ }^{-1} A \\
& =C_{M} A^{T}\left(A C_{M} A^{T}+C_{\Delta}\right)^{-1} A
\end{aligned}
$$

Therefore, the posterior mean and covariance of the model parameters in Eq. (4.20) or Eq. (4.22) can be rewritten in terms of a model resolution operator as

$$
\hat{\boldsymbol{m}}=R \boldsymbol{m}_{\text {true }}+(I-R) \boldsymbol{m}_{p}+A_{s t}^{-1} \boldsymbol{\delta}
$$




$$
C_{M_{e s t}}=(I-R) C_{M}
$$

If the resolution operator is equal to the identity matrix, then the model estimator is equal to the true model parameters plus the error, $A_{s t}{ }^{-1} \delta$, due to data and modeling uncertainties, and the uncertainty of the estimation becomes zero (i.e. $C_{M e s t}$ becomes zero matrix if $C_{M}$ is not infinitive). In such a case, the model is said to be perfectly resolved. Any prior information is transformed to the best estimation with zero uncertainty. The farther the resolution operator is from the identity matrix, the worse the resolution. As discussed in Backus and Gilbert (1968), the resolution operator acts as a filter. The true model and the prior information are filtered by the resolution operator to calculate the estimated model. We can not see the real world, but only a filtered version. However, the resolution operator tells us the structure of the problem. As the resolution operator gets farther from the identity matrix, the model becomes more poorly resolved and prior engineering judgement plays a greater role in the estimation of model parameters.

\subsubsection{Data Covariance and Resolution}

Sometimes we may be interested in the data values predicted from the parameter estimation (e.g. in data-fitting problems). It is also possible to use the data covariance and resolution to analyze the mutual dependence of different data and compare the results predicted by different models. The development of data covariance and resolution operator parallels the derivation of model covariance and resolution operator. Let $\boldsymbol{D}_{p r d}=A \boldsymbol{M}_{\text {est }}$ be the random vector having the posterior probability density of the data. Similar to Eq. (4.20), the posterior mean and covariance for the data are

$$
\begin{aligned}
& E\left\{\boldsymbol{D}_{p r d}\right\}=\hat{d}=A \hat{\boldsymbol{m}}=A \boldsymbol{m}_{p}+A A_{s t}^{-1}\left(\boldsymbol{d}_{\text {obs }}-A \boldsymbol{m}_{p}\right) \\
& \operatorname{Cov}\left\{\boldsymbol{D}_{p r d}\right\}=C_{D_{p r d}}=A C_{M_{e s t}} A^{T}=A\left(C_{M}-A_{s t}^{-1} A C_{M}\right) A^{T}
\end{aligned}
$$


The concept of data resolution operator arises when the relationship is sought between the predicted data (i.e. posterior mean $\hat{\boldsymbol{d}}$ ) and true data $\left(\boldsymbol{d}_{o b s}\right)$. Analogous to Eq. (4.27), the data resolution operator is defined as (Menke, 1989)

$$
\begin{aligned}
Q=A A_{s t}^{-1} & =A\left(A^{T} C_{\Delta}^{-1} A+C_{M}{ }^{-1}\right)^{-1} A^{T} C_{\Delta}^{-1} \\
& =A C_{M} A^{T}\left(A C_{M} A^{T}+C_{\Delta}\right)^{-1}
\end{aligned}
$$

Therefore, analogous to Eq. (4.28), the posterior mean and covariance of the model parameters in Eq. (4.29) can be rewritten in terms of the data resolution operator as

$$
\begin{gathered}
\hat{d}=Q \boldsymbol{d}_{o b s}+(I-Q) A \boldsymbol{m}_{p} \\
=Q \boldsymbol{d}_{\text {true }}+(I-Q) A \boldsymbol{m}_{p}+Q \delta \\
C_{D_{p r d}}=(I-Q) A C_{M} A^{T}=(I-Q)\left(C_{D_{o b s}}-C_{\Delta}\right)
\end{gathered}
$$

The interpretation of data covariance and resolution are similar to model covariance and resolution but in the data space. It is noted that data resolution provides a means for assessing the correctness of a given model or discriminating between several possible models, while model resolution can be used for study of the inverse structure and design of experiment. To summarize and clarify the use of random vectors for the linear Gaussian problem, the results of the analysis are summarized in Table 4.3.

Table 4.3 Summary of the random vectors in the linear Gaussian inverse problem

\begin{tabular}{c|cccc}
\hline Name of $R V$ & $R V$ & Realization & Mean Vector & Covariance Matrix \\
\hline Additive Noise & $\boldsymbol{\Delta}$ & $\boldsymbol{\delta}$ & $\mathbf{0}$ & $\mathrm{C}_{\Delta}$ \\
Prior Model & $\boldsymbol{M}$ & $\boldsymbol{m}$ & $\boldsymbol{m}_{\boldsymbol{p}}$ & $\mathrm{C}_{\mathrm{M}}$ \\
\hline Observed Data & $\boldsymbol{D}_{\text {obs }}$ & $\boldsymbol{d}_{o b s}$ & $\mathrm{~A} \boldsymbol{m}_{\boldsymbol{p}}$ & $C_{D_{\text {obs }}}=A C_{M} A^{T}+C_{\Delta}$ \\
$\boldsymbol{D}_{\text {obs }}=g(\boldsymbol{M})+\boldsymbol{\Delta}$ & & & & \\
\hline
\end{tabular}




\begin{tabular}{|c|c|c|c|c|}
\hline Name of $R V$ & $R V$ & Realization & Mean Vector & Covariance Matrix \\
\hline $\begin{array}{l}\text { Estimated Model } \\
\qquad \boldsymbol{M}_{\boldsymbol{e s} t}=\boldsymbol{M} \mid \boldsymbol{D}_{\text {obs }}\end{array}$ & $\boldsymbol{M}_{e s t}$ & $\boldsymbol{m}_{e s t}$ & $\begin{array}{l}\boldsymbol{m}_{p}+A_{s t}^{-1}\left(\boldsymbol{d}_{o b s}-A \boldsymbol{m}_{p}\right) \\
=A_{s t}^{-1} \boldsymbol{d}_{o b s}+(I-R) \boldsymbol{m}_{p}\end{array}$ & $\begin{aligned} C_{M_{e s t}} & =\left(A^{T} C_{\Delta}^{-1} A+C_{M}^{-1}\right)^{-1} \\
& =C_{M}-A_{s t}^{-1} A C_{M} \\
& =(I-R) C_{M}\end{aligned}$ \\
\hline $\begin{array}{c}\text { Predicted Data } \\
D_{p r e d}=A M_{e s t}\end{array}$ & $\boldsymbol{D}_{\text {pred }}$ & $\boldsymbol{D}_{\text {pred }}$ & $\begin{array}{l}A \boldsymbol{m}_{p}+A A_{s t}^{-1}\left(\boldsymbol{d}_{\text {obs }}-A \boldsymbol{m}_{p}\right) \\
=Q \boldsymbol{d}_{\text {obs }}+(I-Q)\left(A \boldsymbol{m}_{p}\right)\end{array}$ & $\begin{aligned} C_{D_{\text {pred }}} & =A\left(C_{M}-A_{s t}^{-1} A C_{M}\right) A^{T} \\
& =(I-Q) A C_{M} A^{T} \\
& =(I-Q)\left(C_{D_{\text {obs }}}-C_{\Delta}\right)\end{aligned}$ \\
\hline $\begin{aligned} A_{s t}^{-1} & =C_{M} A^{T} \\
& =\left(A^{T} C_{\Delta}\right.\end{aligned}$ & +0 & $\begin{array}{l}\left.C_{\Delta}\right)^{-1} \\
A^{-1} C_{\Delta}^{-1}\end{array}$ & $\begin{array}{l}R=A_{s t}^{-1} A \\
Q=A A_{s t}^{-1}\end{array}$ & \\
\hline
\end{tabular}

\subsubsection{Nonlinear Gaussian Problem}

\subsubsection{Optimal Estimator}

For the nonlinear Gaussian problem, there is no analytical solution for the optimal estimator due to the non-linearity. A numerical solution is needed. The MAP estimator is most common because an efficient optimization algorithm can be used instead of multi-dimension numerical integration. Note that when calculating the MAP estimator for Eq. (4.17), it is not necessary to calculate $p\left(\boldsymbol{d}_{o b s}\right)$ since it is not a function of $\boldsymbol{m}$. In fact, maximizing Eq. (4.17) is equivalent to minimizing the term in the exponential.

$$
\hat{\boldsymbol{m}}_{\boldsymbol{M A P}}=\underset{\boldsymbol{m}}{\arg \min }\left\{\left(\boldsymbol{d}_{\boldsymbol{o b s}}-g(\boldsymbol{m})\right)^{T} C_{\Delta}^{-1}\left(\boldsymbol{d}_{\boldsymbol{o b s}}-g(\boldsymbol{m})\right)+\left(\boldsymbol{m}-\boldsymbol{m}_{\boldsymbol{p}}\right)^{T} C_{M}{ }^{-1}\left(\boldsymbol{m}-\boldsymbol{m}_{p}\right)\right\}
$$

\subsubsection{Uncertainty and Resolution Analysis}

For multi-modal density functions resulting from a highly nonlinear model $g($.$) , the$ covariance matrix may not be very meaningful. In such a case, Eq. (4.8) or Eq. (4.15) has to be numerically integrated to analyze the uncertainty. However, for problems where the nonlinearity is not too strong (i.e. problems where the function $\mathrm{g}(\boldsymbol{m})$ is linearizable in the region of significant posterior probability), the uncertainty analysis can be approximated by solving the model (data) 
covariance and resolution at the peak (Tarantola, 1987). Let $\hat{\boldsymbol{m}}_{\text {MAP }}$ be the MAP estimator obtained by solving Eq. (4.32). The model can be approximated by a first order Taylor's series

$$
\begin{aligned}
& g(\boldsymbol{m}) \cong g\left(\hat{\boldsymbol{m}}_{M A P}\right)+\left[\frac{\partial g}{\partial m}\right]_{\hat{\boldsymbol{m}}_{M A P}}\left(\boldsymbol{m}-\boldsymbol{m}_{M A P}\right) \\
& =g\left(\hat{\boldsymbol{m}}_{M A \boldsymbol{P}}\right)+A_{M A P}\left(\boldsymbol{m}-\hat{\boldsymbol{m}}_{M A P}\right)
\end{aligned}
$$

where $A_{M A P}=\left[\frac{\partial g}{\partial \boldsymbol{m}}\right]_{\hat{\boldsymbol{m}}_{M A P}}$ is the sensitivity operator evaluated at $\boldsymbol{m}=\hat{\boldsymbol{m}}_{M A \boldsymbol{P}} \cdot$ It is evaluated analytically if possible or can be evaluated numerically using a finite difference scheme. Using Eq. (4.33), the posterior probability density Eq. (4.17) can be approximated by a Gaussian distribution

$$
p\left(\boldsymbol{m} \mid \boldsymbol{d}_{\text {obs }}\right) \cong \mathrm{const} \cdot \exp \left\{-\frac{1}{2}\left(\boldsymbol{m}-\hat{\boldsymbol{m}}_{M A P}\right) C_{M_{e s t}}{ }^{-1}\left(\boldsymbol{m}-\hat{\boldsymbol{m}}_{M A P}\right)\right\}
$$

in which $C_{\text {Mest }}$ is obtained by substituting $A=A_{M A P}$ in the linear case, Eq. (4.24). The approximate model covariance and resolution operator become

$$
\begin{aligned}
& C_{M_{e s t}} \cong\left(A_{M A P}{ }^{T} C_{\Delta} A_{M A P}+C_{M}{ }^{-1}\right)^{-1} \\
& R \cong I-C_{M_{e s t}} C_{M}{ }^{-1}
\end{aligned}
$$

In fact, all the results of the analysis of errors and resolution developed for the linear case can be used approximately by substituting $A=A_{M A P}$. 


\subsection{Numerical Examples}

Based on the above theoretical study, an inverse analysis may take the following steps assuming Gaussian distributions for the data error and prior model:

1. Select the data and model parameters by the sensitivity analysis. The sensitivity operator in Eq. (4.33) defines the sensitivity of data to the model parameters. It depicts the relative importance of the different model parameters and how the model parameters affect the observed data. The part of data that are not sensitive to the model parameters and the model parameters that do not have significant contribution to the data can be neglected.

2. Estimate the mean and variance of the data error and prior model.

3. Obtain the optimal estimate using Eq. (4.32) and an appropriate optimization algorithm. The optimization algorithms have been widely studied in the area of mathematical programming (Chong and Zak, 1996). Various methods are available for function optimization. The Nelder-Mead simplex search method (Nelder and Mead, 1965) is used in this study because of its ubiquity in the best selling hand book Numerical Recipe (Press et al., 1992) and in Matlab ${ }^{\mathrm{TM}}$ Optimization Toolbox (Math Works, 1997).

4. Estimate the uncertainty and resolution using Eqs. (4.35) and (4.36).

Gaussian distributions often describe the data errors and the subjective prior information very well. If there is strong evidence that non-Gaussian distribution should be used, then Eq. (4.7) should be derived and used in step 3 and the uncertainty analysis in step 4 requires numerical integration. The Log-Normal distribution (i.e. the log of the variable has a Gaussian distribution) may be used for non-negative model parameters and the procedure will remain the same as long as the model parameters are transformed by the log function before computation.

Two examples are shown below using artificial data generated by the material model and wave propagation model. The numerical data is corrupted by some Gaussian noise before being used for inversion. 


\subsubsection{Inversion of an Impedance Profile}

The first example is related to the calibration of a TDR system. Suppose we have three sections of a uniform non-dispersive and lossless transmission line as shown in Fig. 4.7a. The prior model is assumed to be independent Gaussian with a mean of $70 \mathrm{ohms}$ and a standard deviation of 50 ohms. Ten layers of equal length are used to simulate this non-uniform transmission line. The data are generated using the simulation code developed in Chapter 3 and corrupted by independent noises with standard deviation of 0.005 Volts. The result of the estimate and the waveform matching are shown in Fig. $4.7 \mathrm{~b}$ and 4.7c. The optimal estimate and the standard deviation of the estimate are shown in Table 4.4. The resolution matrix is almost equal to the identity matrix. This is a well-resolved problem, where the optimal estimate is very closed to the true solution and the standard deviation of the estimate is very small.

Table 4.4 The inverse solution of the impedance profile

\begin{tabular}{l|llllllllll}
\hline$M$ & $Z_{1}$ & $Z_{2}$ & $Z_{3}$ & $Z_{4}$ & $Z_{5}$ & $Z_{6}$ & $Z_{7}$ & $Z_{8}$ & $Z_{9}$ & $Z_{10}$ \\
\hline m_true & 50 & 50 & 50 & 100 & 100 & 100 & 100 & 50 & 50 & 50 \\
m_prior & 70 & 70 & 70 & 70 & 70 & 70 & 70 & 70 & 70 & 70 \\
G_prior & 50 & 50 & 50 & 50 & 50 & 50 & 50 & 50 & 50 & 50 \\
m_estimate & 50.001 & 50.009 & 49.989 & 99.986 & 100.028 & 99.951 & 100.032 & 49.996 & 50.004 & 49.986 \\
o_estimate & 0.0098 & 0.0098 & 0.0098 & 0.0219 & 0.0220 & 0.0220 & 0.0271 & 0.0123 & 0.0077 & 0.0125 \\
\hline
\end{tabular}

\subsubsection{Inversion of Mixing Model Parameters}

For this example, we suppose the volumetric mixing model with $\alpha=0.5$ (i.e. refractive index mixing formula) describes the dielectric properties of soils and the dielectric permittivities of soil components are known. The assumed values of the dielectric parameters of soil components are listed in Table 4.5. The density of the soil particles is assumed to be $2.65 \mathrm{~g} / \mathrm{cm}^{3}$. Therefore, there are only three parameters left in the volumetric mixing model: volumetric water content $\theta$, dry density $\rho_{d}$, and effective specific surface area $A_{e s}$. We are interested in estimating 
these three parameters from the simulated dielectric spectrum of the soil. The simulated spectrum is corrupted by noise with a standard deviation of 0.1 .

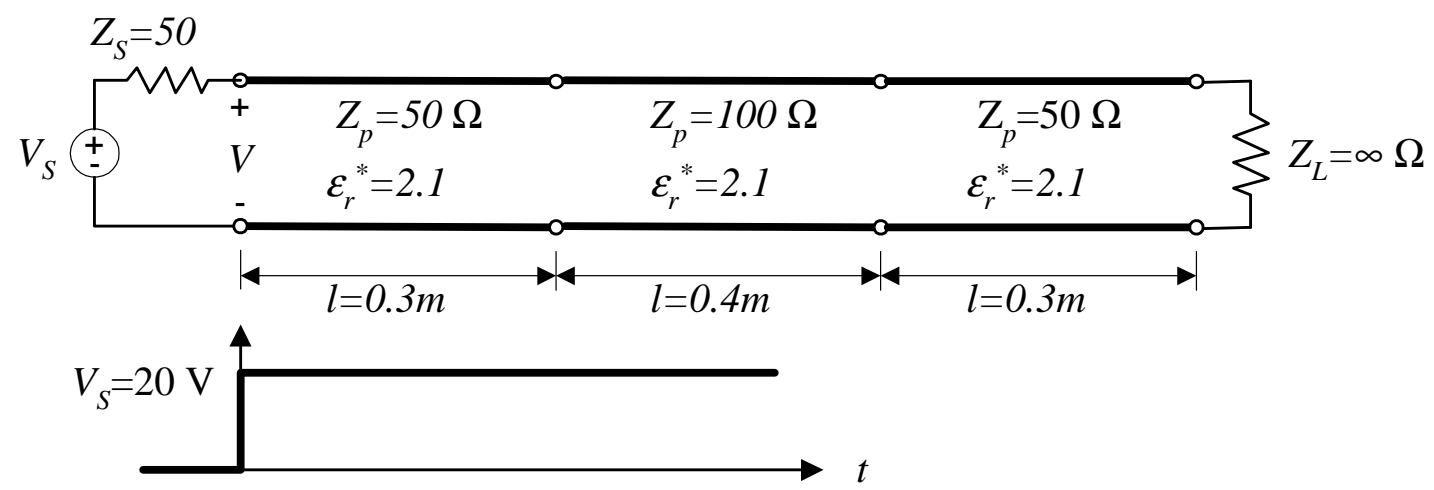

(a) Problem description

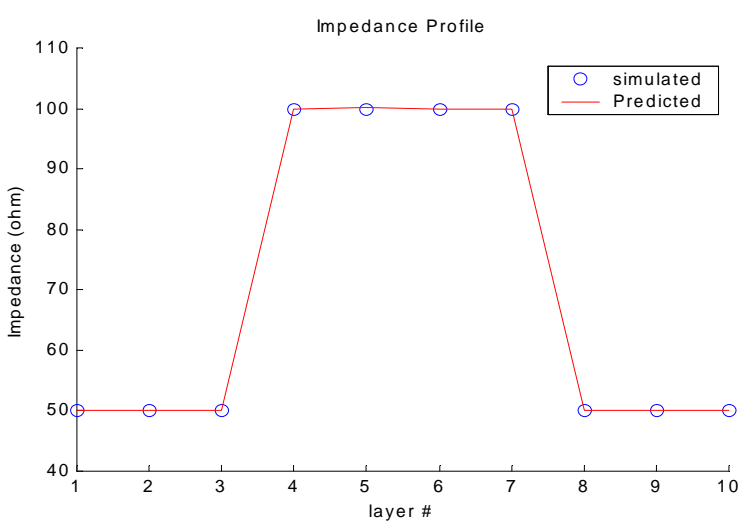

(b) Estimation of impedance profile

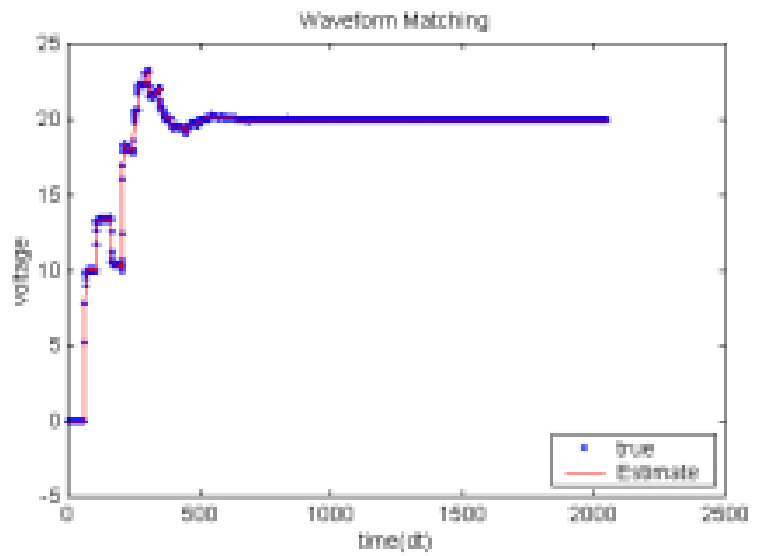

(c) Waveform matching

Fig. 4.7 Example of inversion of impedance profile 
The results of inverse analysis are shown in Table 4.6 and the data matching is shown in Fig. 4.8. The standard error in terms of percentages are $0.6 \%, 1.3 \%, 1.4 \%$ for volumetric water content $\theta$, dry density $\rho_{d}$, and effective specific surface area $A_{e s}$, respectively. The matrix of coefficient of correlation, $C_{r}=\left[\rho_{\mathrm{ij}}\right]$ is calculated as

$$
C_{r}=\left[\begin{array}{ccc}
1.0000 & -0.9986 & 0.9968 \\
-0.9986 & 1.0000 & -0.9948 \\
0.9968 & -0.9948 & 1.0000
\end{array}\right]
$$

The volumetric water content and dry density are highly anti-correlated. This implies that the volumetric water content and density have similar influence on the data. The same data is likely to be observed when the water content increases and the dry density decreases by a certain amount. On the other hand, the water content and effective specific surface area have different influence on the dielectric permittivity because they are highly correlated. The dielectric permittivity increases when the water content increases, but decreases when specific surface area increases. The resolution matrix for this inverse problem is calculated as

$$
R=\left[\begin{array}{ccc}
0.9993 & 0.0003 & 0.0000 \\
0.0125 & 0.9940 & 0.0000 \\
-11482 & 5454 & 0.9982
\end{array}\right]
$$

It can be concluded that this is also a well-resolved inverse problem because the diagonal terms in the resolution matrix are very close to one. Note that the large values of off-diagonal terms on the third row would not contribute much to the estimation of specific surface area because the typical magnitude of the specific surface area is much higher than that of water content or density. Hence, normalizing the model parameters to have the same range of magnitude may enhance the interpretation of the resolution. 


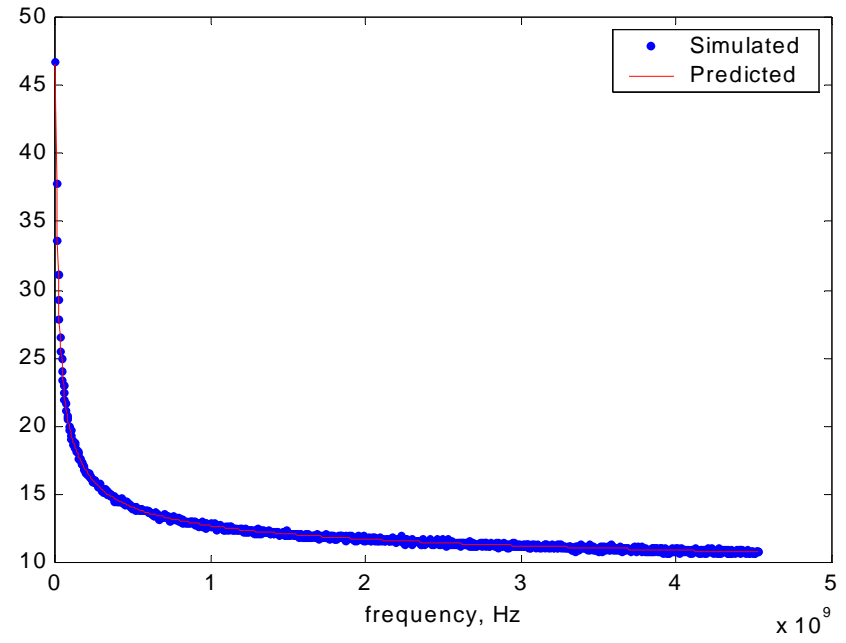

(a) Real part of the dielectric permittivity, $\varepsilon^{\prime}(f)$

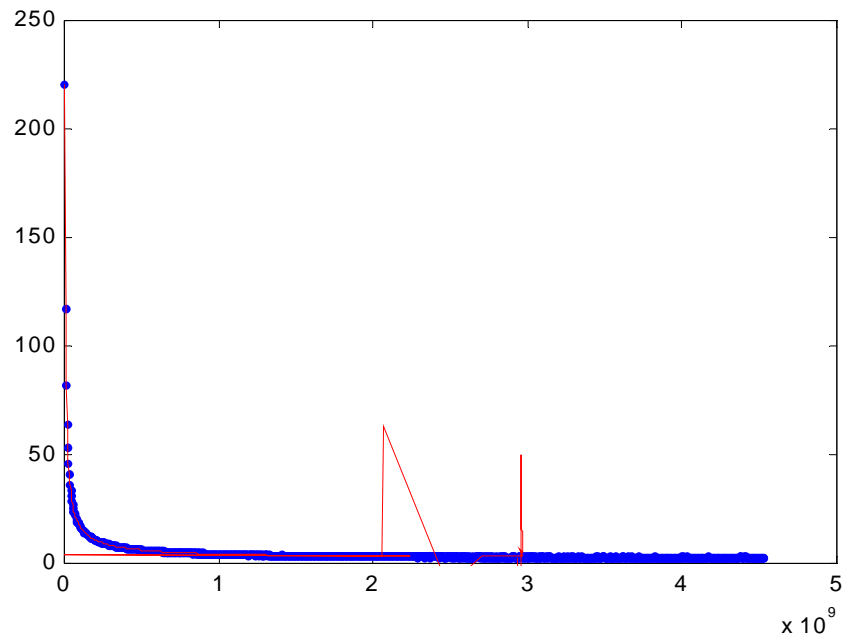

(b) Imaginary part of the dielectric permittivity, $-\varepsilon^{\mathrm{i} i}(f)$

Fig. 4.8 The comparison between simulated spectrum and predicted spectrum 
Table 4.5. Assumed values of dielectric parameters of soil components

\begin{tabular}{l|llll}
\hline $\begin{array}{c}\text { Debye's } \\
\text { Parameters }\end{array}$ & Air & $\begin{array}{c}\text { Soil } \\
\text { Particle }\end{array}$ & $\begin{array}{c}\text { Free } \\
\text { Water }\end{array}$ & $\begin{array}{c}\text { Bound } \\
\text { Water }\end{array}$ \\
\hline$\varepsilon_{s}$ & 1 & 4.7 & 80 & 80 \\
$\varepsilon_{\infty}$ & 1 & 4.7 & 4.22 & 4.22 \\
$f_{r e l}, \mathrm{~Hz}$ & $\ldots$ & $\ldots$ & $17 \times 10^{9}$ & $9 \times 10^{3}$ \\
$\sigma_{d c}, \mathrm{~S} / \mathrm{m}$ & 0 & 0 & 0.05 & 5 \\
\hline
\end{tabular}

Note: (...) denotes no effect

Table 4.6 The inverse solution of the mixing model parameters

\begin{tabular}{|c|c|c|c|}
\hline$m$ & $\theta$ & $\rho_{d}, M g / m^{3}$ & $A_{e s}, \mathrm{~cm}^{2} / \mathrm{g}$ \\
\hline m_true & 0.2500 & 1.8000 & $150.0 \times 10^{4}$ \\
\hline m_prior & 0.2000 & 1.6500 & $100.0 \times 10^{4}$ \\
\hline$\sigma \_$prior & 0.0500 & 0.0300 & $50.0 \times 10^{4}$ \\
\hline m_estimate & 0.2506 & 1.7889 & $150.9 \times 10^{4}$ \\
\hline$\sigma \_$estimate & 0.0014 & 0.0232 & $2.13 \times 10^{4}$ \\
\hline
\end{tabular}

\subsection{Summary}

The application of the TDR technique is an inverse problem. The methods of solving inverse problems are reviewed and a probabilistic framework using the Bayesian statistics is developed and suggested as a general approach for inverse problems in geotechnical engineering. Due to the difficulties of numerical integration at present, the Gaussian distribution is used in the formulation and the interpretation of the inverse solution includes the optimal estimator, uncertainty analysis, and resolution analysis. Nonlinear problems are linearized at the optimal 
estimation of the model parameters for the analysis of uncertainty and resolution. This framework will be applied to TDR system calibration and measurements of dielectric properties in subsequent chapters. 


\section{CHAPTER 5 MEASUREMENT OF DIELECTRIC PROPERTIES}

\subsection{Introduction}

The purpose of the analysis of TDR waveforms is to reveal the information about the dielectric or physical property of the material under test. The dielectric property of the material is what governs the resultant waveform according to the electromagnetic wave propagation theory. The physical property of the material is related to the dielectric property by the dielectric mixing models. The measurement of dielectric properties using TDR will be studied in this chapter, and the incorporation of dielectric mixing models to infer physical properties will be discussed in Chapter 6.

\subsection{TDR Measurement System}

TDR measurements were performed by attaching the probe to a TDR apparatus with 3 feet of Intcom 50- $\Omega$ cable fitted with $50-\Omega$ BNC connectors at each end as shown in Fig. 3.2. The TDR apparatus used in this study was a Tektronix 1502B cable tester equipped with the SP232 serial communication module so that data acquisition could be controlled by a computer.

\subsubsection{TDR Probe System}

A TDR probe system was designed to measure the dielectric properties of soils in a compaction mold and in the field. The TDR probe system consists of a coaxial cable, a coaxial head $(\mathrm{CH})$, and a coaxial cylinder $(\mathrm{CC})$ for laboratory measurements or a multiple rod probe (MRP) for field measurements.

The $\mathrm{CH}$ provides the transition from the coaxial cable to the CC or MRP. It consists of three components, as shown in Fig. 5.1 : 1) a 50- $\Omega$ mating BNC connector; 2) a metal cylindrical head with Delrin ${ }^{\circledR}$ as the insulating material; and 3) multiple rod section consisting of a center rod and three perimeter conducting rods with air as the insulating material. The $\mathrm{CH}$ has four metal studs threaded into the metal head. The spacing between the central stud and outer studs is 66 $\mathrm{mm}(2.588 \mathrm{in})$. The lengths of the central stud and two of the outer studs are the same $21 \mathrm{~mm}$ (0.825 in). The fourth stud is spring loaded to provide adjustable length. 

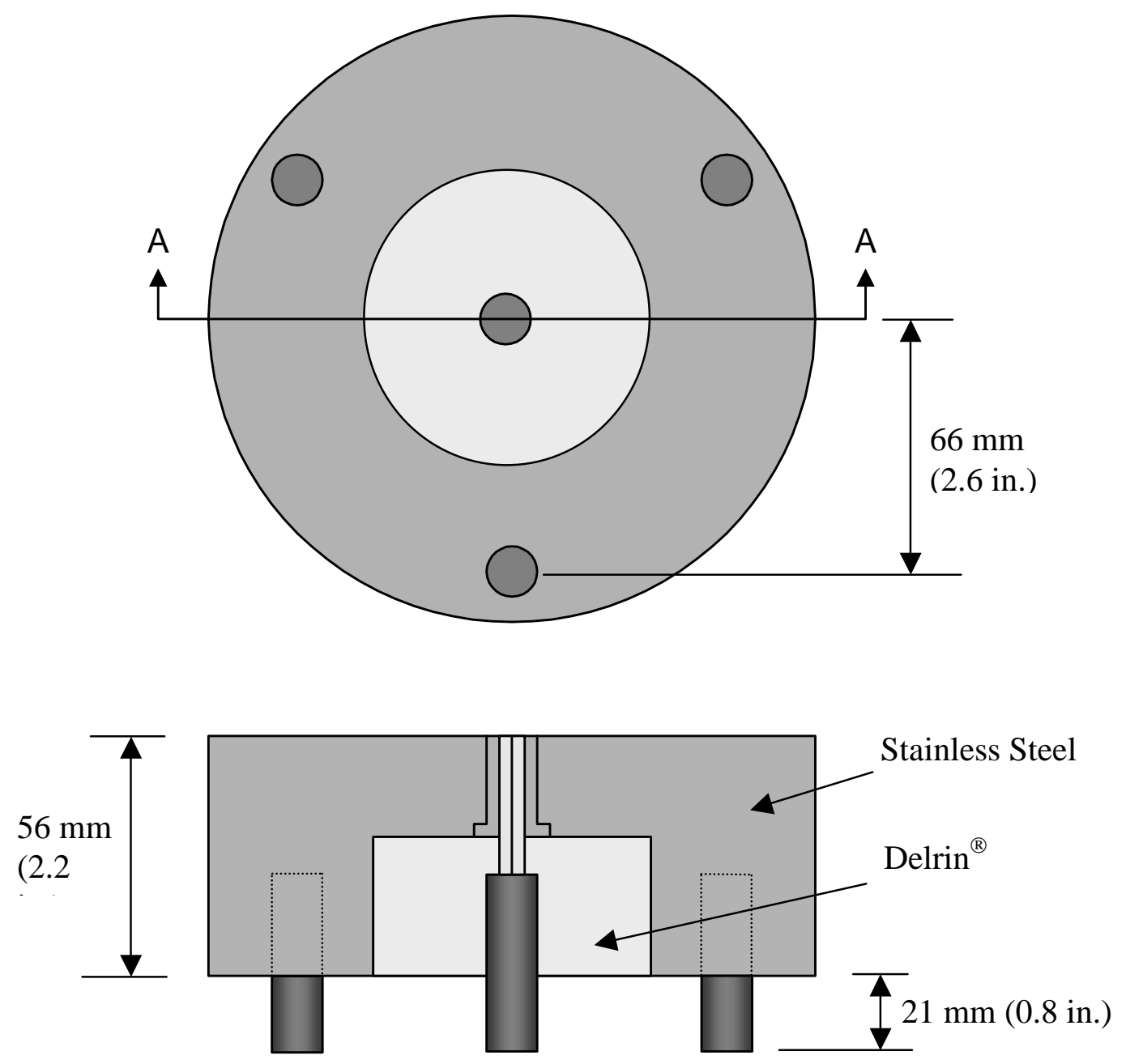

Section A-A

Fig. 5.1 Configuration of coaxial head

The coaxial cylinder transmission line is shown in Fig. 5.2a and consists of a CC mold, a ring and a central rod all made of stainless steel. After the soil is compacted into the coaxial cylinder, a central rod is inserted using a guide plate. The diameter of the central rod and the inside diameter of the CC are $7.94 \mathrm{~mm}$ (5/16 in.) and $102 \mathrm{~mm}$ (4 in.), respectively. The height of the CC mold (or specimen holder) is $203 \mathrm{~mm}$ (8 in.). After the central rod is driven into the soil in the $\mathrm{CC}$ mold, the template is removed and the $\mathrm{CC}$ ring is placed on top of the $\mathrm{CC}$ mold. The $\mathrm{CH}$ is then placed on top of the $\mathrm{CC}$ ring and the threaded stud is adjusted until contact is made. The MRP is shown in Fig. 5.2b and consists of four rods that are common steel spikes, $9.5 \mathrm{~mm}$ 
(3/8 in.) in diameter and $254 \mathrm{~mm}$ (10 in.) long. A guide template is used to guide insertion of the spikes into the ground so that their configuration is the same as the studs on the $\mathrm{CH}$. The guide template is removed after the rods are driven into the soil. The $\mathrm{CH}$ is placed on the heads of the rods so that the central stud sits on top of the central rod and the two equal outer studs sit on top of the two outer rods. The threaded stud is adjusted until contact is made with the remaining rod.

The design of the probe system was based on the following considerations: 1) it must be robust to handle high density compacted soils; 2) the TDR signal must be clear such that the reflections from the surface and the bottom of the soil specimen are recognizable; 3) the connection of the different transmission line sections must be good and consistent. Impedance matching from the $50 \Omega$ cable to the sensor could not be achieved due to the required geometry. So the probe system is a non-uniform transmission line consisting of the cable, the $\mathrm{CH}$, and the CC or the MRP. Details of the design information can be found in Feng et al. (1998).

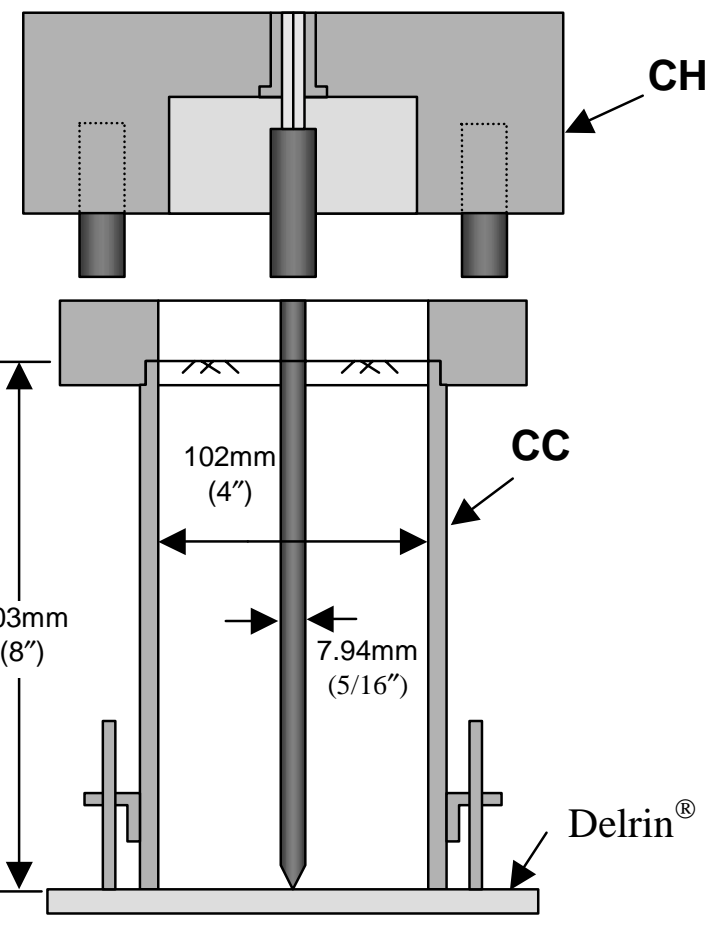

(a)
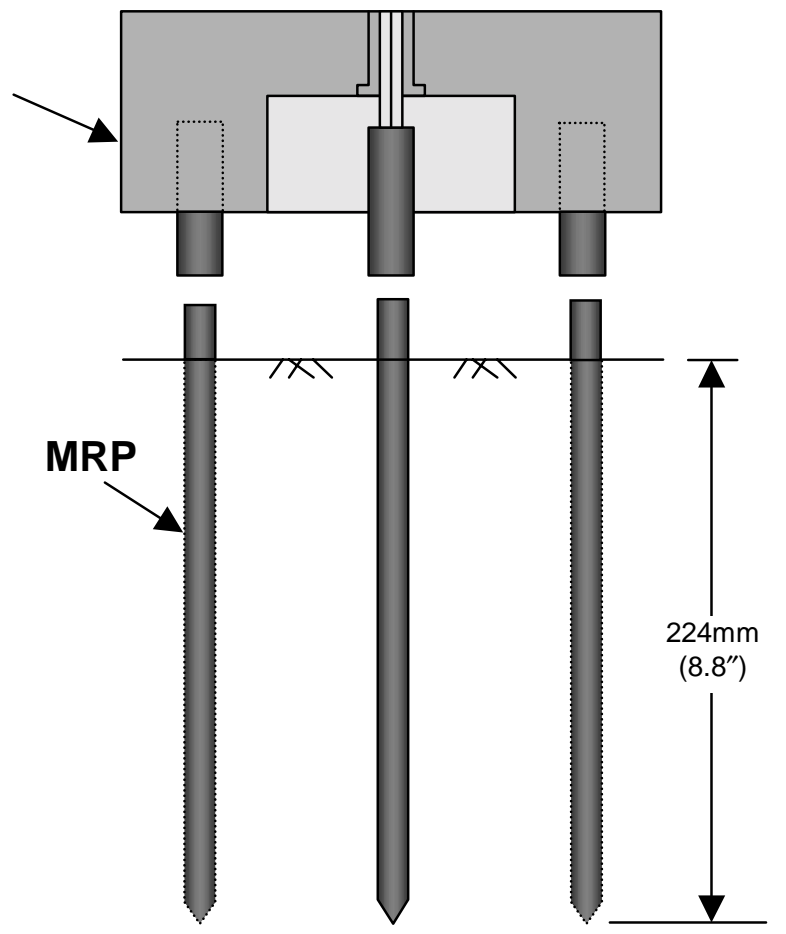

(b)

Fig. 5.2 Configuration of CC and MRP transmission lines 


\subsubsection{Data Acquisition}

A computer connected to the SP232 serial communication module carried out the data acquisition. The computer software, called TDR++, was developed to automate TDR data acquisition (Feng et al., 1998).

When the data is analyzed in the frequency domain, it is important to select an appropriate sample interval and length of the TDR data. The sampling interval is determined by the frequency bandwidth of the TDR measurement system. For the Tektronix 1502B Cable Tester the bandwidth was determined to be from dc up to $1.5 \mathrm{GHz}$ (Heimovaara, 1994). The sample length is selected such that a steady state is reached at the end of the TDR waveform, which means the waveform should achieve a constant value. For a fixed sample interval, this appropriate length depends on the dielectric constant of the material and length of the probe.

The TDR++ program controls the 1502B cable tester and sets the horizontal and vertical scales. For this study, the horizontal scale is set to $0.1 \mathrm{~m}$ per division, and a value of 0.99 is set for the velocity of propagation $\left(V_{p}\right)$. The sampling time interval between two points is $2.7 \mathrm{e}-11 \mathrm{sec}$ under this configuration. The sampling frequency under this scale is $37.1 \mathrm{GHz}$, which is well above twice the TDR frequency bandwidth. The vertical scale was set to 500 mrho (where mrho is one thousandth of one rho and a rho is the reflection coefficient of a TDR cable system). A 13bit data quantization scheme is used where the data range in counts is from 0 to 8192 . The resolution of this scheme is much better than the default $0-128$ setting so that we do not need to change the vertical scale to improve the vertical resolution of the waveform. Three data acquisition modes are available for sampling length (duration) when testing different materials, 1024-point, 2048-point and 4096-point. For most soils, 2048-point waveform length is sufficient. But for liquids, such as water, a 4096-point waveform length is needed to reach a steady state. The frequency resolution depends on the number of sampling points, with $18.0 \mathrm{MHz}$ for 2048 points and 9MHz for 4096 points.

\subsubsection{Characterization of the TDR System}

As discussed in Section 3.6.2.3, the TDR system may be described in the frequency domain by the system function in terms of source voltage and sampled voltage or by the scattering function in terms of incident voltage and reflected voltage of the probe system. The part of output 
waveform pertinent to the probe system is the reflection. Therefore, it may be clearer to describe the probe system by the scattering function rather than the overall system function. The scattering function may be measured experimentally by

$$
S_{11}(f)=\frac{\hat{V}_{r}(f)}{\hat{V}_{\text {in }}(f)}=\frac{\hat{V}_{\text {out }}(f)-\hat{V}_{\text {in }}(f)}{\hat{V}_{\text {in }}(f)}
$$

where $\hat{V}_{\text {out }}(f)$ is the spectrum of the TDR waveform and $\hat{V}_{\text {in }}(f)=\hat{V}_{S}(f) / 2$ is the spectrum of the incident waveform. However, Eq. (5.1) is only appropriate when the signals are noiseless signals. Denote the spectrum of the incident pulse by $X$, the spectrum of the noiseless reflected pulse by $Y$, and the spectrum of the noisy reflected pulse by $Z=Y+N$, where $N$ is the spectrum of the noise. A better estimation of the system function with noise present can be achieved using correlation operations (Bendat and Piersol, 1993). Equation (5.1) becomes

$$
S_{11}(f)=\frac{C_{X Z}(f)}{C_{X X}(f)}
$$

where $C_{X Z}(f)$ is the average cross spectrum between the incident pulse and the measured reflected pulse, and $C_{X X}(f)$ is the average power spectrum of the incident pulse. The cross spectrum is the Fourier transform of the cross-correlation between incident and reflected pulse, and the power spectrum is the Fourier transform of the auto-correlation of the incident pulse. The cross spectrum is averaged from multiple measurements to eliminate uncorrelated noise. This result is described by Bendat and Piersol (1993) as

$$
C_{X Z}(f)=\frac{1}{m} \sum_{m} Z(f) \cdot \operatorname{conj}[X(f)]
$$




$$
C_{X X}(f)=\frac{1}{m} \sum_{m} X(f) \cdot \operatorname{conj}[X(f)]
$$

where $m$ is the number of multiple measurements and $\operatorname{conj}[$ ] is the complex conjugate. The measurements and associated data error of the incident and reflected pulse of the TDR system will be discussed in the next section. The scattering function calculated by the incident pulse and the measured TDR pulse characterizes the whole transmission line of the TDR system. This function can also be derived theoretically in terms of the transmission line parameters as discussed in Chapter 3. Equating the measured scattering function and theoretical scattering function will allow us to measure the dielectric property of the material inside the probe if all other line parameters are known or calibrated in advance. The system calibration and the measurement of dielectric permittivity will be discussed in the subsequent sections.

\subsubsection{Data Error and Bandwidth of the TDR System}

It is straightforward to measure the TDR reflected pulse by connecting the transmission line of the probe system and using the data acquisition device. The incident pulse (i.e. one half of the source voltage) is a step pulse with a rise time of $200 \mathrm{ps}$ according to the specification of the Tektronix 1502B. However, its exact complete waveform is not known. The incident waveform of the TDR system could be obtained experimentally by terminating the cable tester with a $50-\Omega$ impedance block (i.e. there would be no reflection in such a case). Substituting $Z_{\text {in }}(0)=50 \Omega$ into Eq. (3.62), the measured waveform is equal to the incident waveform. Thus,

$$
\hat{V}(0)=\frac{\hat{V}_{S}}{2}=\hat{V}_{\text {in }}
$$

Using the horizontal and vertical scale as specified above, the incident wave from the Tektronix 1502B into the transmission line is obtained by terminating the cable test device with a $50-\Omega$ impedance block and is shown in Fig. 5.3. The waveform is normalized and offset to have a step size of 2000 . The sampling time interval between two points is $2.7 \mathrm{e}-11$ sec, which 


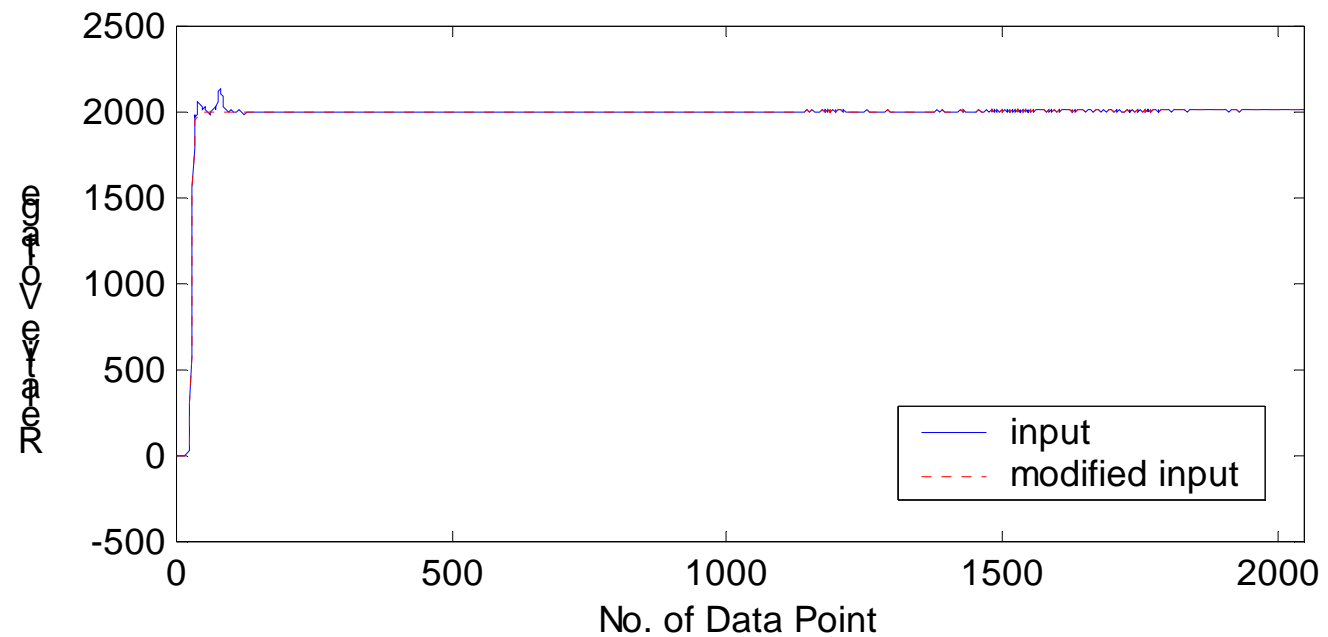

Fig. 5.3 TDR source input

corresponds to the horizontal scale of $0.1 \mathrm{~m}$ per division and $V_{p}=0.99$. The sampling frequency under this scale is $37.1 \mathrm{GHz}$, and the frequency resolution depends on the number of sampling points, with $18.0 \mathrm{MHz}$ for 2048 points and 9MHz for 4096 points. Notice that the bumps following the incident pulse are aberrations from the internal circuitry, and reflections from the front panel. These bumps are not part of the incident step pulse. The measured waveform was modified to eliminate the bumps as shown in Fig. 5.3. The spectrum of the measured incident pulse and corrected incident pulse are shown in Fig. 5.4. The difference between the two spectra

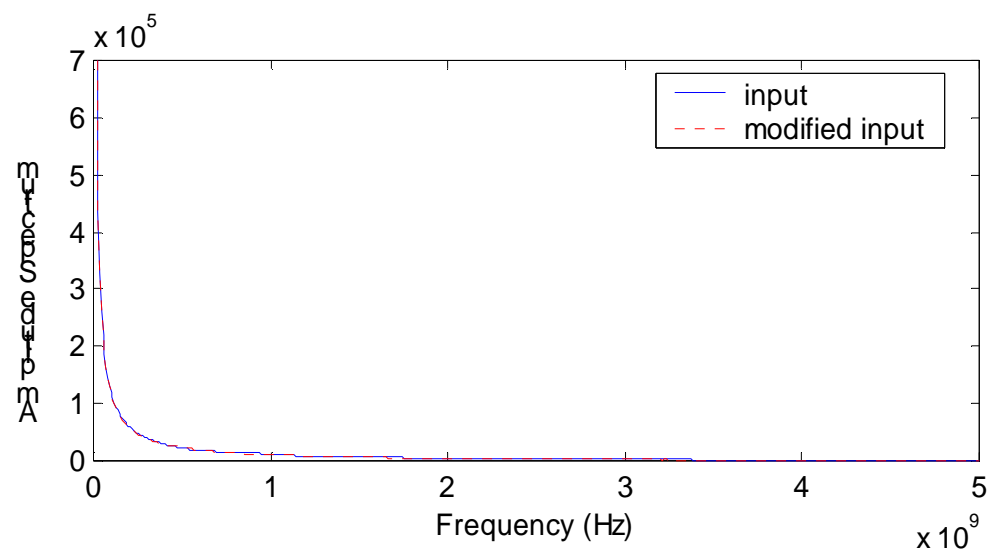

Fig. 5.4 Amplitude spectrum of the incident pulse 
was not noticeable because a large vertical scale was used to cover the large values in the low frequencies. The error in percentage of the spectrum of the incident pulse due to the aberration is shown in Fig. 5.5.

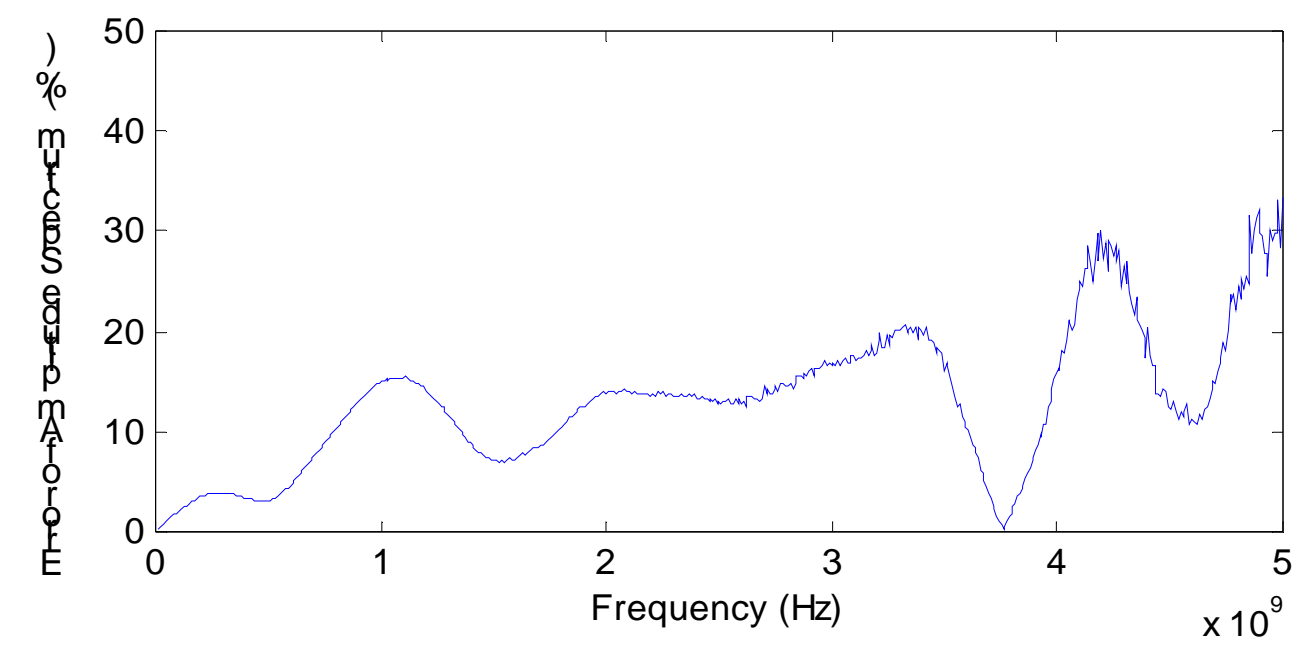

\section{Fig. 5.5 Error of amplitude spectrum $\left(\left(A_{\text {input }}-A_{\text {modified input }}\right) / A_{\text {modified input }}\right)$ due to aberration, where $A$ represent the amplitude spectrum}

It is necessary to estimate the statistics of the data error as discussed in the inverse theory using the probabilistic framework. The reproducibility of the TDR waveform was studied by taking multiple measurements. The probe with air as the filling material was connected to the TDR device, and 10 measurements were taken. The standard deviations of the data in the time domain and frequency domain are shown in Figs. 5.6 and 5.7, respectively. Notice that there are two peaks in Fig. 5.6 due to jitter. Jitter is uncertainty in the timebase (Tektronix, 1993). Its main effect is that the waveform appears to move back and forth a very small amount resulting in larger uncertainties whenever there is a sharp step in the waveform. The data uncertainty will be estimated according to the statistics shown in Fig. 5.6 for inverse analysis in time domain and Fig. 5.7 for inverse analysis in frequency domain

Although the frequency spectrum of the step pulse extends to infinity, the amplitude is quite low at microwave frequencies as shown in Fig. 5.4. Therefore, the capacity of the TDR device to measure a wide range of frequencies is limited. 


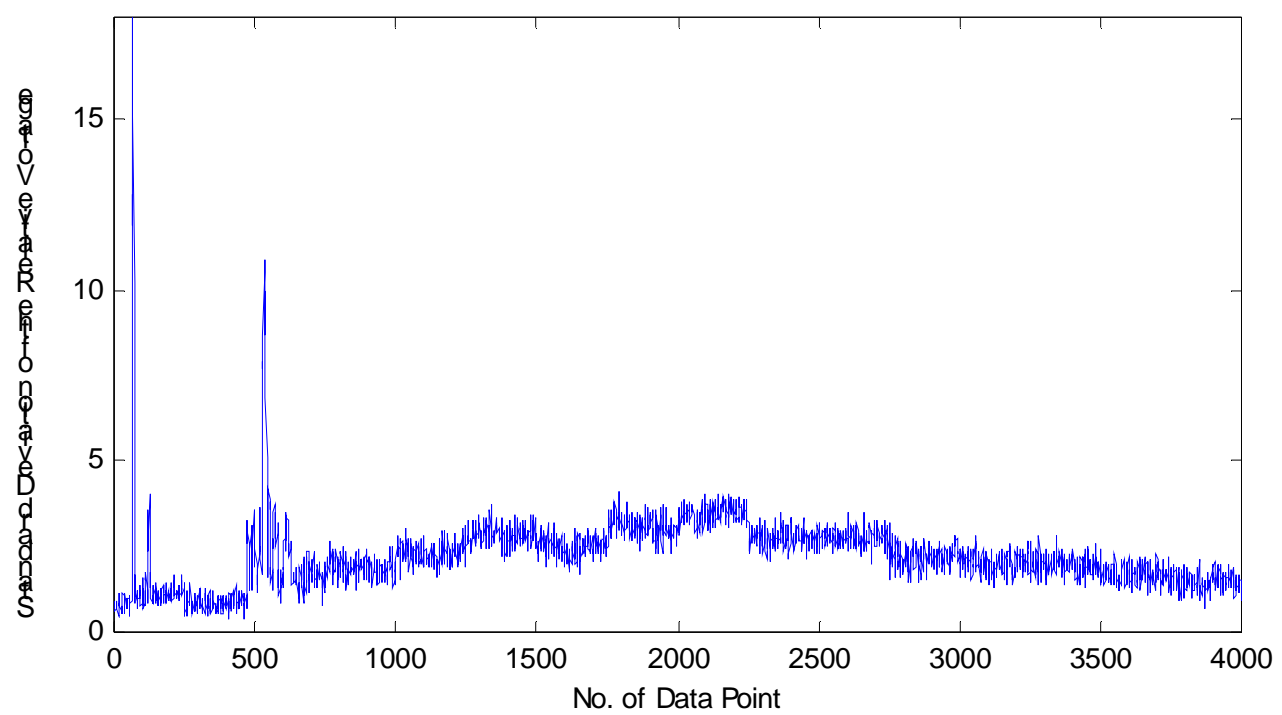

Fig. 5.6 Standard deviation in ten measurements of TDR waveform in air

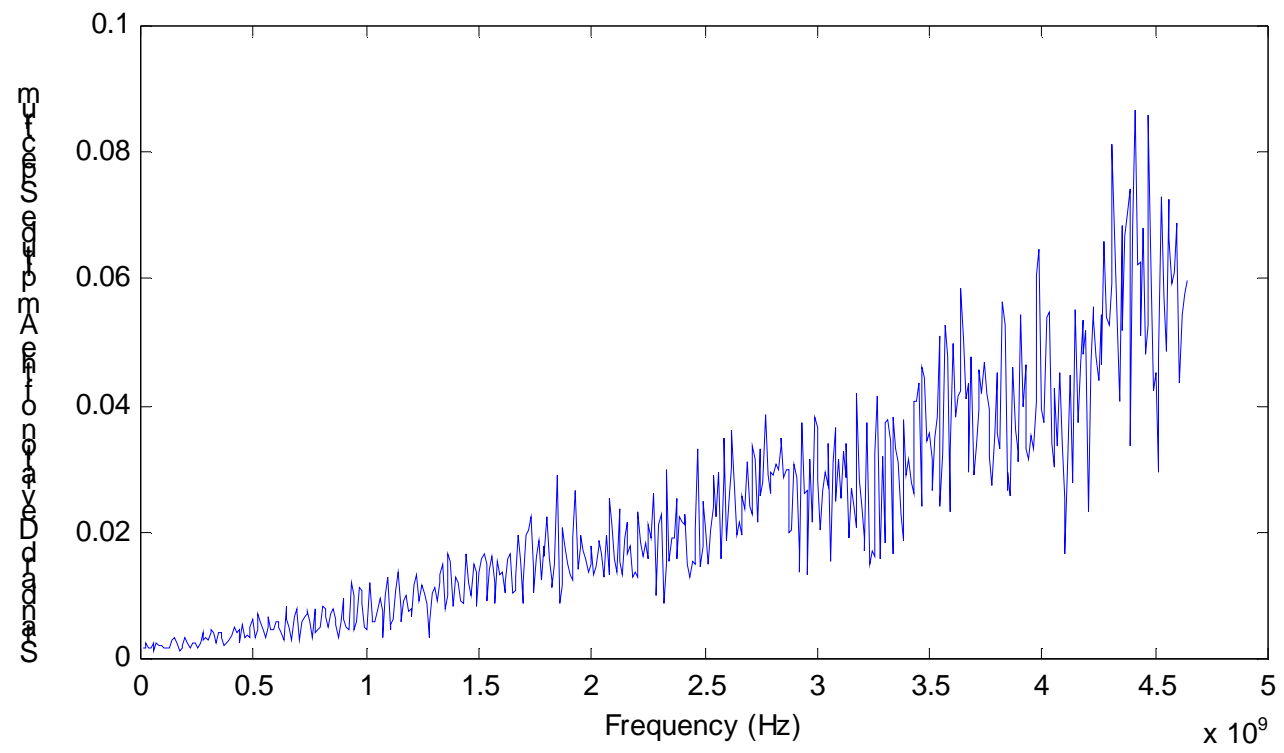

Fig. 5.7 Standard deviation in ten measurements of system function in air

The coherence is often used to test the frequency capacity of a measurement system. The coherence indicates the energy in the measured output that can be related to the input. The 
noiseless output $Y$ is fully caused by the input. Therefore, the coherence $\gamma^{2}(f)$ in terms of the power spectrum becomes (Bendat and Piersol, 1993).

$$
\gamma^{2}(f)=\frac{C_{Y Y}(f)}{C_{Z Z}(f)}
$$

Coherence less than 1.0 indicates noise in the output. The higher value of the coherence the higher the energy in the measured output that was caused by the energy in the input. It can be determined as a function of the average spectra using Eq. (5.2).

$$
\gamma^{2}(f)=\frac{C^{2} X Z(f)}{C_{X X}(f) C_{Z Z}(f)}
$$

A related definition is the Noise-to-Signal Ratio (NSR) which is defined as the ratio between the power spectrum of the noise $N$ to the power spectrum of the noiseless output $Y$,

$$
N S R(f)=\frac{C_{N N}(f)}{C_{Y Y}(f)}
$$

It can be computed from the value of the coherence (Bendat and Piersol, 1993). ,

$$
N S R(f)=\frac{1-\gamma^{2}(f)}{\gamma^{2}(f)}
$$

The range for NSR varies from 0 to infinite. The higher the value, the stronger the noise with respect to the signal. It is important to keep this ratio as low as possible. The higher the values of 
NSR the more difficult it will be to recover information about the system. The coherence and NSR of the ten TDR measurements in air are shown in Fig. 5.8 and Fig. 5.9. It can be concluded from these figures that the frequency capacity of the TDR system is up to about $2 \mathrm{GHz}$.

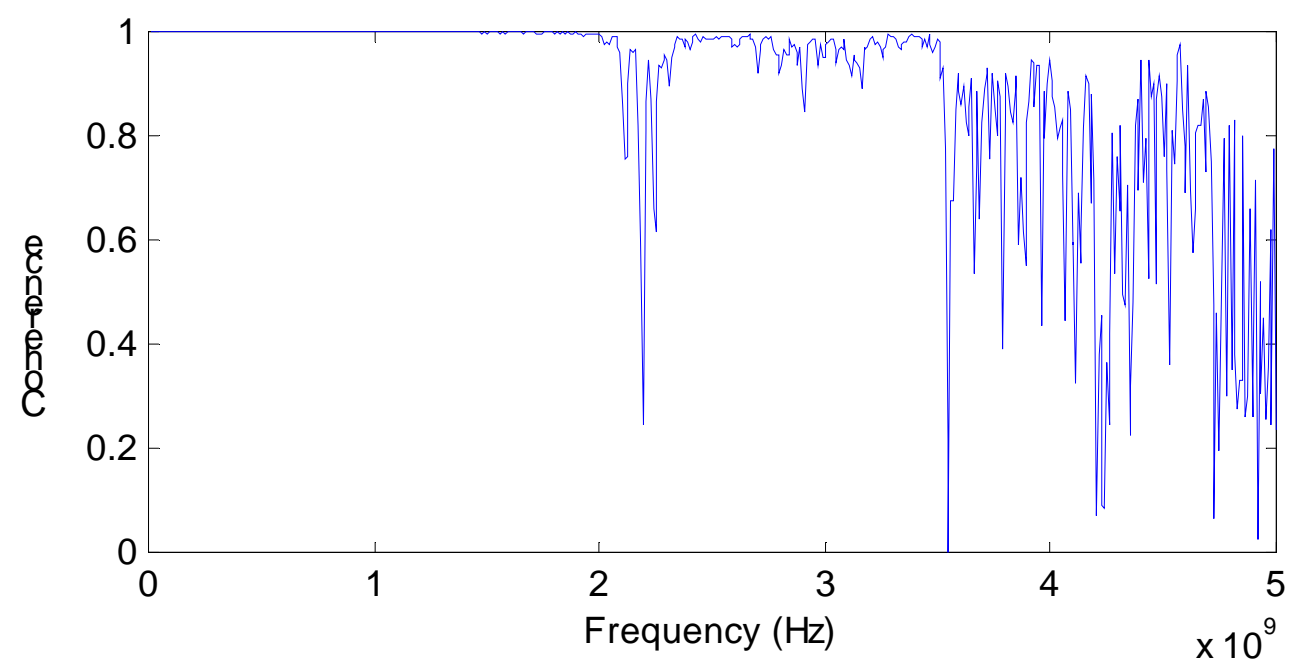

Fig. 5.8 Coherence of ten TDR measurements in air

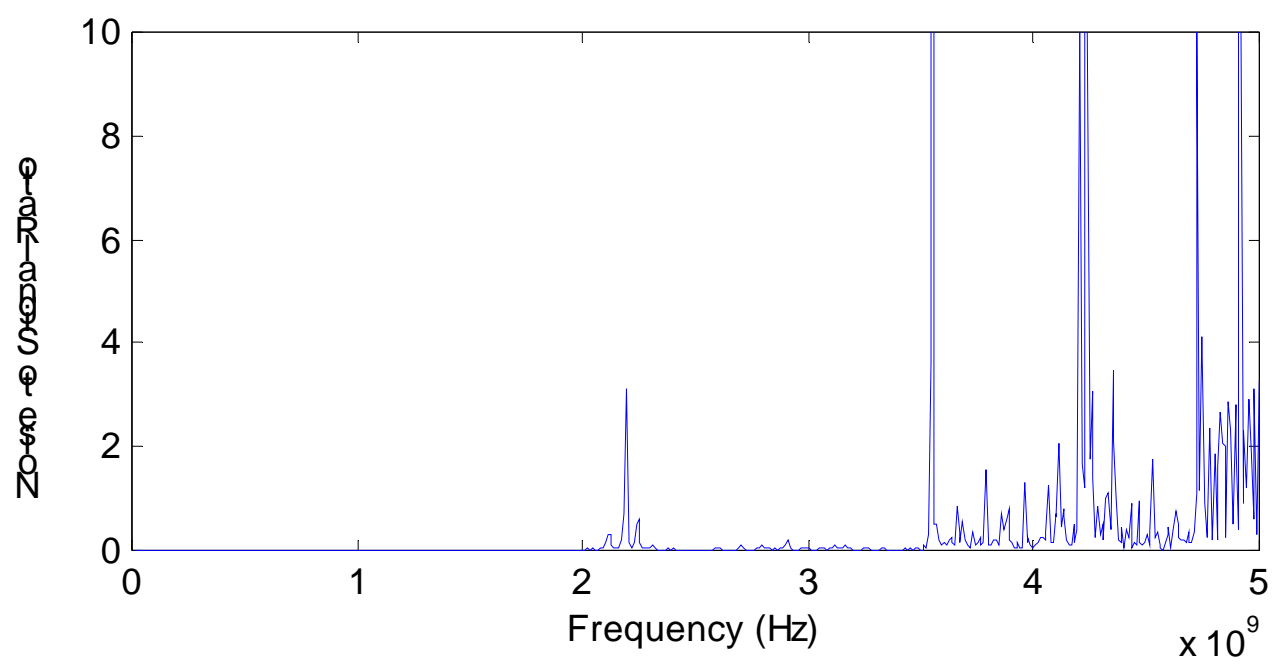

Fig. 5.9 Noise-to-signal ratio of ten TDR measurements in air 


\subsection{Calibration of the TDR System Parameters}

It is necessary to calibrate the TDR probe system when it is used for the first time to measure the dielectric permittivity or physical properties of the material being tested. The system parameters include the boundary conditions and properties of the transmission line. The source impedance $Z_{S}$ is equal to $50 \Omega$ as listed in the specification of the Tektronix 1502B. The TDR probe system is terminated with an open end. It may not be a perfect "open" with a load impedance equal to $\infty$ because of the fringing effect. However, it was argued in the Chapter 3 that the tips of the TDR probes are cone- shaped. In such cases, the fringing effect may be neglected. So we can use a large number to represent the load impedance in place of a perfect open-ended termination. The cone ends of the rods in the probe section may be modeled by several sections of uniform section with different impedances. Alternatively, a uniform transmission line section may be used to model the whole probe section with equivalent length.

The calibration of transmission line parameters can be carried out by the model-based inverse analysis. It was discussed in Chapter 3 that the properties of a non-uniform transmission line are the dielectric permittivity, the reference impedance, and the length of each uniform section. Two characteristics of a wave propagating in a non-uniform transmission line are the reflection/transmission at the interfaces of the mismatches and the propagation delay through each section. The reflection/transmission of waves depends on the impedance of the two sections adjacent to the interface, which are in turn functions of the reference impedance and dielectric permittivity of the insulating medium. The propagation delay is a function of the length of the section and the propagation constant, which is in turn a function of dielectric permittivity of the insulating medium.

Different combinations of reference impedance and dielectric permittivity can result in the same impedance, while different combinations of section length and dielectric constant can result in the same propagation delay. Therefore, there are only two degrees of freedom in the three parameters. Considering the three characteristics of the non-uniform line together in the calibration will result in non-uniqueness. However, for the probe, the material with known material properties will be used for calibration. It is only required then for the coaxial cable and probe head to have equivalent system parameters that will result in the correct waveforms. Hence, 
assuming one of the three parameters for the cable and the probe head, the remaining two parameters can be determined by inversion from the measured waveform in a material with known dielectric permittivity. In order to reduce the complexity and the number of unknowns in the inversion, the TDR probing system is divided into three parts as shown in Fig. 5.10. The transmission line parameters of the front panel, coaxial cable, and TDR probe are calibrated successively.

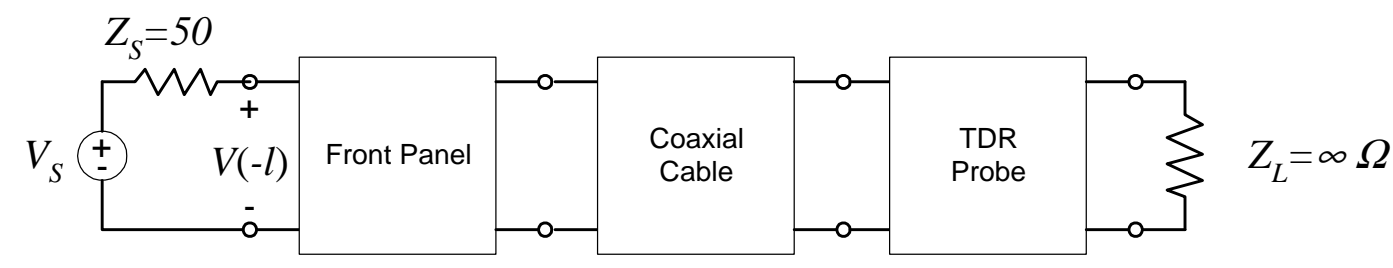

Fig. 5.10 The TDR probe system

\subsubsection{Front Panel Aberration}

The calibration of the front panel aberration is similar to the example of inversion of impedance profile discussed in Chapter 4. But instead of dividing the line into many small sections of equal length, it can be determined from the waveform that twelve uniform sections should be able to model the bumps occurring at the beginning of the step. Assuming the material inside the front panel connector is non-dispersive and has a value of dielectric permittivity equal to one, twelve impedances and twelve lengths were inverted from the waveform measured by connecting the $50-\Omega$ terminating block to the front panel connector. The prior value for each section was set to be $0.02 \mathrm{~m}$ with standard deviation of $0.005 \mathrm{~m}$ for the length and $50 \Omega$ with standard deviation of $10 \Omega$ for the impedance. The impedance profile and the associated error bars of the inversion are shown in Fig. 5.11. The predicted waveform matched the measured TDR input waveform very well as shown in Fig. 5.12. 


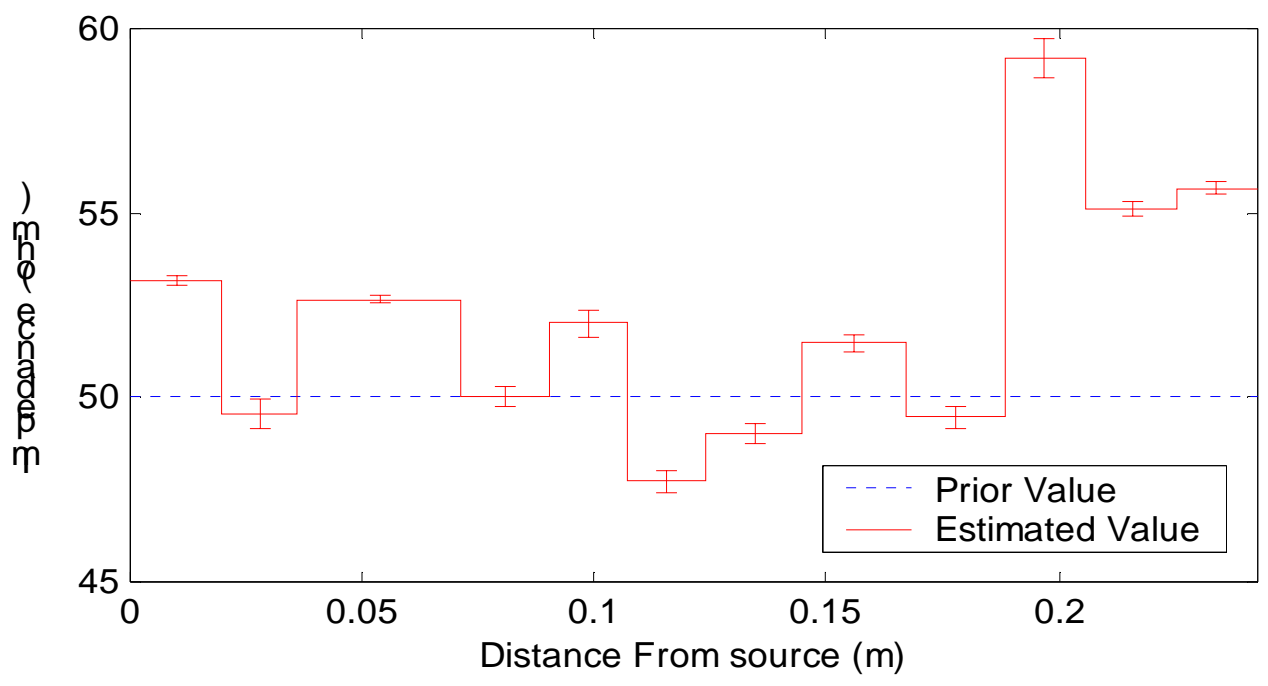

Fig. 5.11 The impedance profile of the front panel aberration

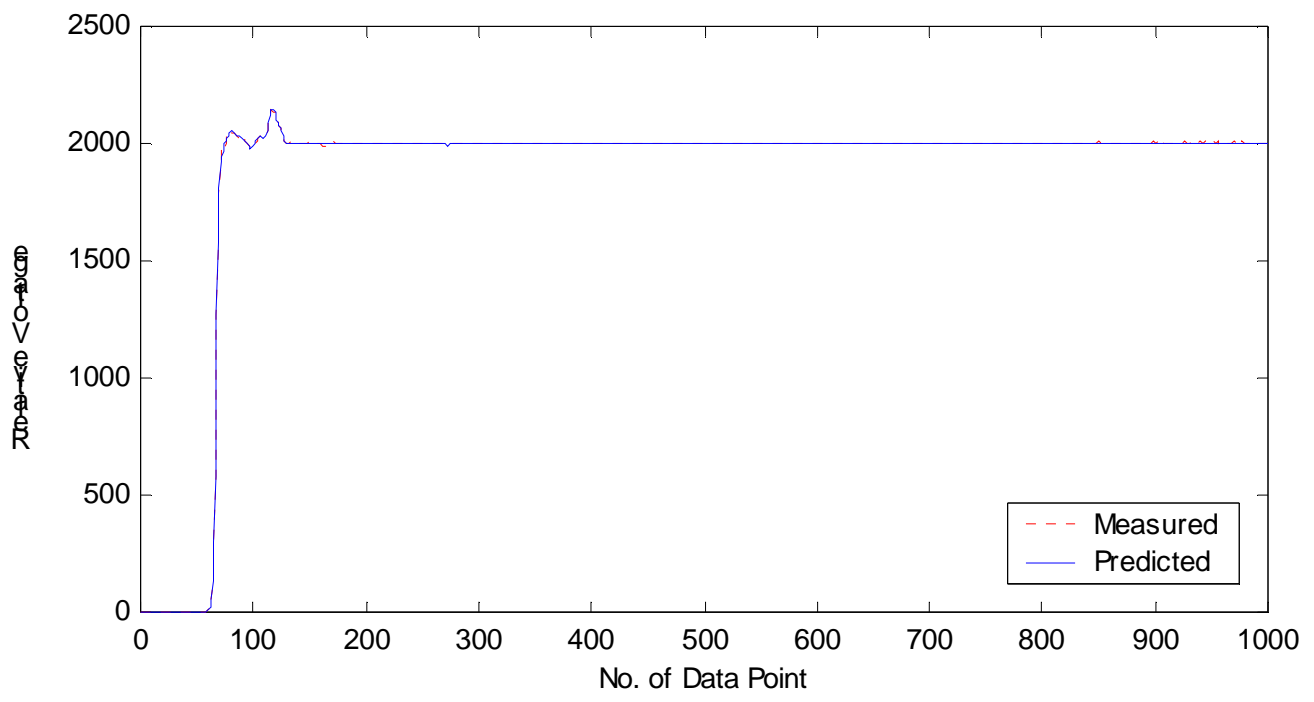

Fig. 5.12 Calibration of the front panel aberration 


\subsubsection{Line Parameters of the Coaxial cable}

The coaxial cable used has nominal impedance of $50 \Omega$. However, The TDR pulses travelling on the coaxial cable are actually dispersive (i.e. the characteristic impedance is a function of frequency) and the rise time increases in duration substantially as the voltage pulse propagate along a cable. Long cables tend to filter out the higher frequencies of the TDR signal, thus reducing the bandwidth in the measurement (Heimovaara, 1993). The dispersion can be taken into account by calibrating the reference impedance and the frequency-dependent dielectric properties of the cable. The frequency-dependent dielectric permittivity may be modeled by the Debye equation. The observed data is the TDR waveform measured by connecting the openended coaxial cable to the TDR apparatus. The transmission line parameters are the reference impedance, the Debye's parameters of the coaxial cable filling material, and the length of the cable. Because of the non-uniqueness of the problem discussed above, the length of the cable was assumed to be known exactly to obtain an equivalent calibration. The prior and the estimated values from the inversion are listed in Table 5.1 and the comparison of the predicted and measured waveforms is shown in Fig. 5.13. The slight difference between predicted waveform and the measured waveform are due to the cable connector at the open end. Note that the

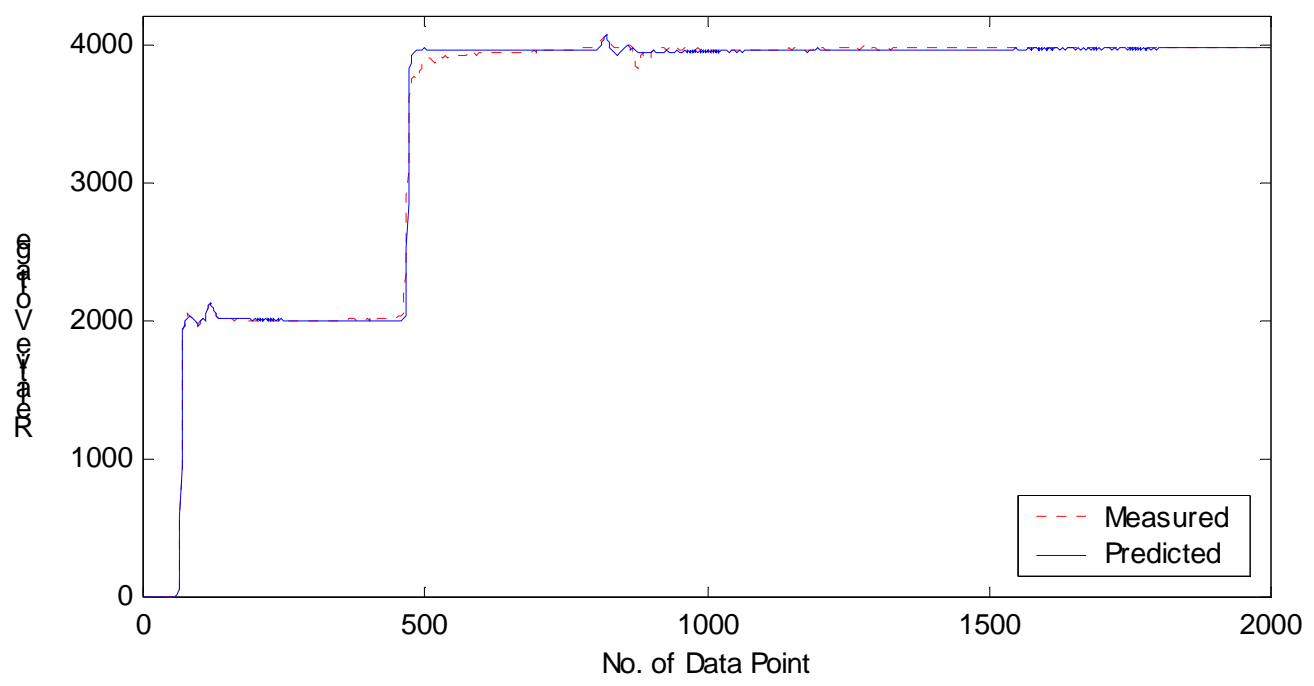

Fig. 5.13 Calibration of the coaxial line 
estimated value of $\varepsilon_{s}$ is several standard deviations away from the prior model. In such a case, the numerical optimization algorithm does not find the MAP point. Instead, it finds a point in the model space that fits the data better than the Eq. (4.32). Strictly speaking, such a solution is not Bayesian (Scales and Snieder, 1997), and the uncertainty is likely to be underestimated.

Table 5.1 The prior and estimated values of the line parameters of the coaxial cable

\begin{tabular}{|c|c|c|c|c|c|}
\hline$m$ & $Z_{p}, \Omega$ & $\varepsilon_{s}$ & $\varepsilon_{\infty}$ & $f_{\text {rel }}, \mathrm{Hz}$ & $l, \mathrm{~m}$ \\
\hline m_prior & 72.5 & 2.2 & 2.1 & $1.0 \times 10^{8}$ & 1 \\
\hline$\sigma \_$prior & 2 & 0.1 & 0.1 & $0.5 \times 10^{8}$ & 0 \\
\hline m_est & 71.2422 & 2.5636 & 2.0412 & $2.3422 \times 10^{6}$ & 1 \\
\hline$\sigma \_$est & 0.0128 & 0.0011 & 0.0001 & $1.2 \times 10^{4}$ & 0 \\
\hline
\end{tabular}

\subsubsection{Line Parameters of the Probe}

The TDR probe consists of three uniform transmission lines as shown in Fig. 5.2: 1) the coaxial head, 2) the air gap, and 3) the probe inserted within the material under test. The air gap in the $\mathrm{CC}$ transmission line actually has two distinct sections, a multiple rod probe section and a coaxial section. However, air has an EM wave velocity of light and the gap is only about $5 \mathrm{~cm}$ (2 in.). The time interval of the wave travelling through the air gap is very small compared to the coaxial line and the probe inserted within the material being tested. Hence, the air gap will be approximated by a single section of uniform transmission line with the TEM field structure.

For each section, one of the three line parameters is determined or assumed and the remaining two parameters are obtained by inversion of the TDR waveform measured in deionized water. Note that some parameters that can be measured directly are left as unknowns to provide the flexibility to equivalently account for the uncertainty of fringing effect due to the discontinuities. The length of the $\mathrm{CH}$ was determined to be $35 \mathrm{~mm}$ (1.38 in) and the reference impedance and dielectric permittivity remained unknown. The dielectric spectrum of Delrin ${ }^{\circledR}$ inside the $\mathrm{CH}$ was modeled by Deybe's equation. The air gap and the probe inserted within the material used for calibration have known dielectric properties. The reference impedance and the length of these two sections were obtained by inversion. The prior and the estimated values of the 
line parameters for the coaxial probe from inversion are listed in Table 5.2 and the comparison of the predicted and measured waveforms is shown in Fig. 5.14.

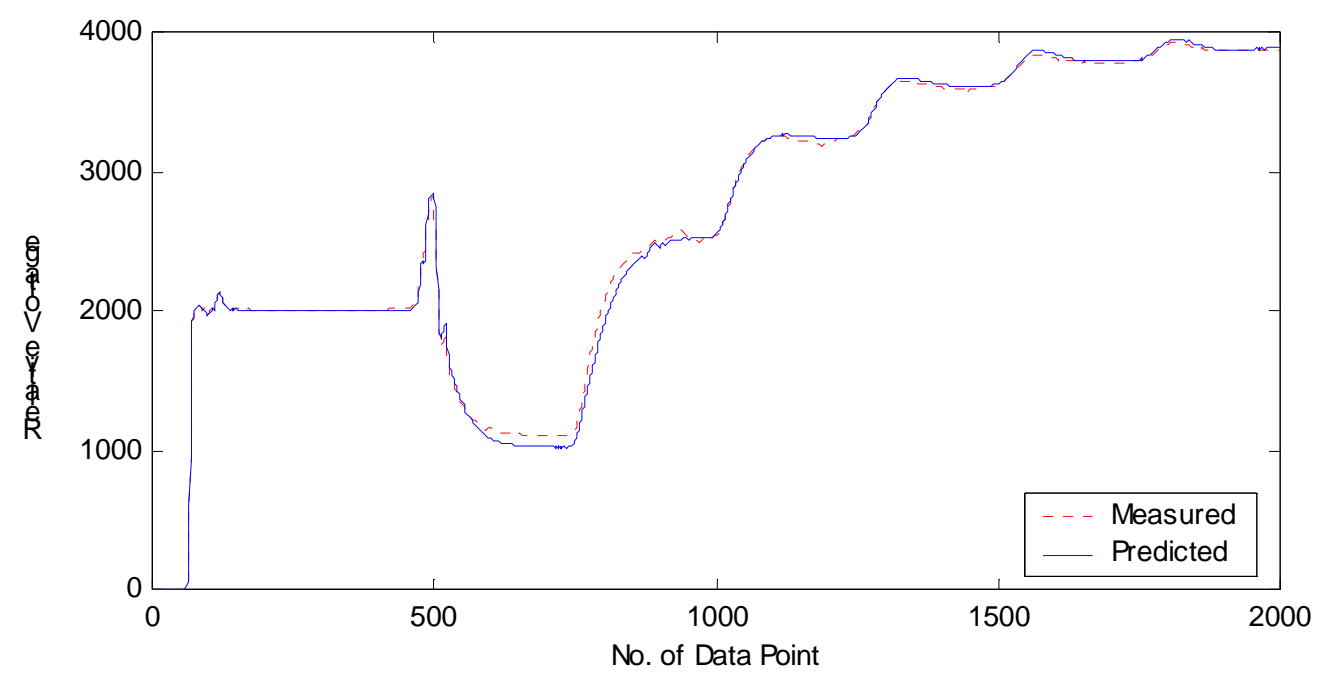

Fig. 5.14 Calibration of TDR probe

Table 5.2 The prior and estimated values of the line parameters for the coaxial probe

\begin{tabular}{|c|c|c|c|c|c|c|c|c|c|c|c|c|c|}
\hline \multirow[b]{2}{*}{$m$} & \multicolumn{5}{|c|}{ Coaxial Head Section } & \multicolumn{3}{|c|}{ Air Gap Section } & \multicolumn{5}{|c|}{ Probe Section } \\
\hline & $Z_{p}, \Omega$ & $\begin{array}{c}l, \\
\mathrm{~mm}\end{array}$ & $\mathcal{E}_{S}$ & $\mathcal{E}_{\infty}$ & $f_{\text {rel }}, \mathrm{Hz}$ & $\begin{array}{c}Z_{p}, \\
\Omega\end{array}$ & $\begin{array}{c}l, \\
\mathrm{~mm}\end{array}$ & $\varepsilon$ & $Z_{p}, \Omega$ & $l, \mathrm{~mm}$ & $\varepsilon_{s}$ & $\varepsilon_{\infty}$ & $\begin{array}{c}f_{\text {rel }}, \\
\mathrm{Hz}\end{array}$ \\
\hline m_prior & 132 & 35.0 & 3.7 & 3.7 & $1 \times 10^{7}$ & 153 & 55.0 & 1 & 152.9 & 116.4 & 79.9 & 4.22 & $17 \times 10^{9}$ \\
\hline$\sigma$ prior & 10 & 0 & 0.5 & 0.5 & $0.5 \times 10^{7}$ & 10 & 2.0 & 0 & 0.1 & 1.0 & 0 & 0 & 0 \\
\hline m_est & 108.2 & 35.0 & 5.9 & 1.8 & $9.9 \times 10^{7}$ & 170.6 & 75.6 & 1 & 153.1 & 115.9 & 79.9 & 4.22 & $17 \times 10^{9}$ \\
\hline$\sigma_{\text {est }}$ & 0.32 & 0 & 0.04 & 0.01 & $9 \times 10^{5}$ & 0.14 & 0.10 & 0 & 0.03 & 0.010 & 0 & 0 & 0 \\
\hline
\end{tabular}

The predicted waveforms matched the measured waveforms reasonably well as shown in Fig. 5.14. The slight difference may be attributed to higher modes and the fringing effect. To further validate the transmission line model and the result of the system calibration, TDR waveforms were measured in some other liquids with known dielectric properties. The dielectric properties of the aqueous samples used are listed in Table 5.3 according to Heimovaara (1994). The predicted waveforms matched the measured waveforms very well in all cases as shown in Fig. 5.15. 


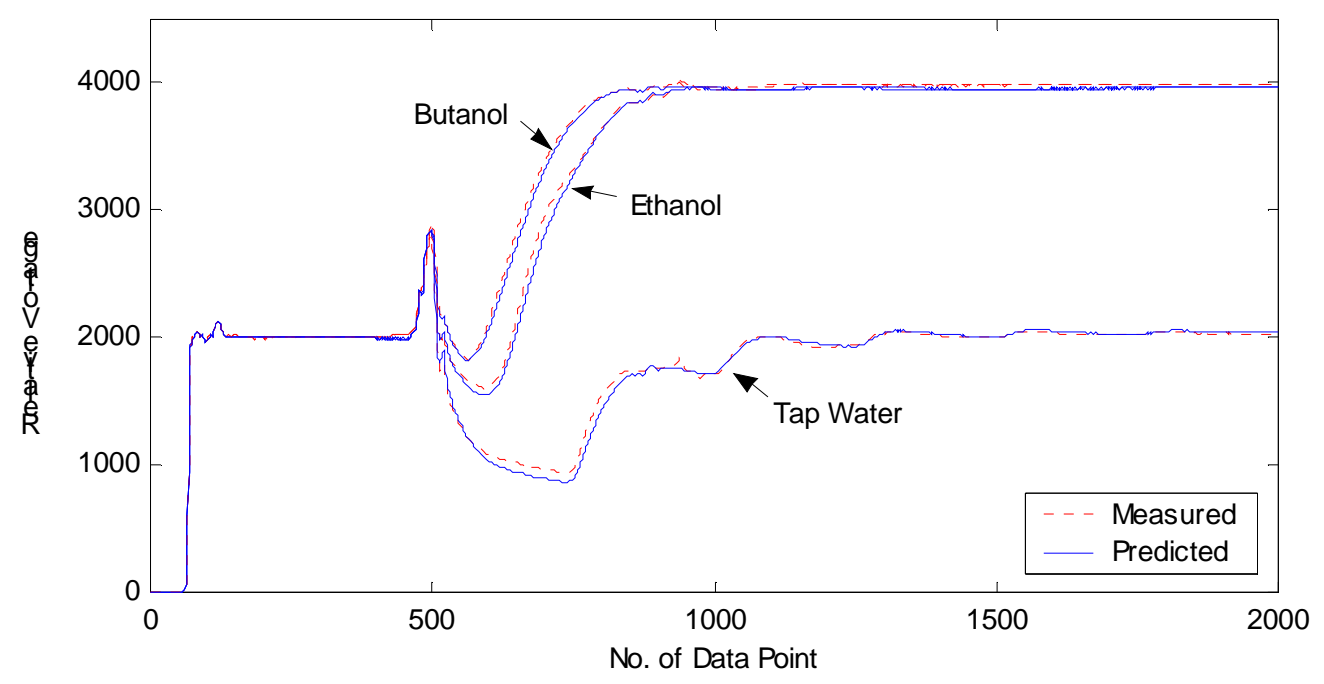

Fig. 5.15 Comparison of the measured and predicted TDR waveforms measured in liquids

Table 5.3 Dielectric properties of aqueous samples

\begin{tabular}{l|lllll}
\hline Aqueous Sample & $T,{ }^{o} \mathrm{C}$ & $\mathcal{E}_{s}$ & $\mathcal{E}_{\infty}$ & $f_{\text {rel }}, \mathrm{GHz}$ & $\sigma_{d c}, \mathrm{Sm}^{-1}$ \\
\hline Butanol & 20.75 & 17.7 & 3.3 & 0.274 & 0 \\
Ethanol & 22.6 & 25.2 & 4.52 & 0.782 & 0 \\
Deionized Water & 20.6 & 79.9 & 4.22 & 17.0 & 0 \\
Tap Water & 19.6 & 80.2 & 4.22 & 17.4 & 0.0666 \\
\hline
\end{tabular}

\subsection{Preprocessing of the TDR Waveforms}

After the system parameters have been calibrated, only the properties of the material in the probe remain unknown and are of interest. Different TDR waveforms obtained by the same probe system are due to different properties of the materials being tested. However, the TDR waveform is a combined result of the probe system and the material being tested. It contains information on both the system parameters and the material properties. It is advantageous for data interpretation to preprocess the measured waveform to remove those multiple reflections not 
related to the material being tested. The waveform after preprocessing should look as if the probe (sample section) is connected to the TDR device directly as shown in Fig. 5.16.

The system function of such a fictitious ideal system is simpler than that of the overall TDR probe system, and the waveform after preprocessing contains only the information of the material under test. To obtain the reduced system function and waveform, the overall input impedance is calculated first by the measured TDR waveform and input waveform using Eq. (3.62) as

$$
Z_{i n}(-L)=\frac{H Z_{S}}{1-H}=\frac{\hat{V}(-L) Z_{S}}{\hat{V}_{S}-\hat{V}(-L)}
$$

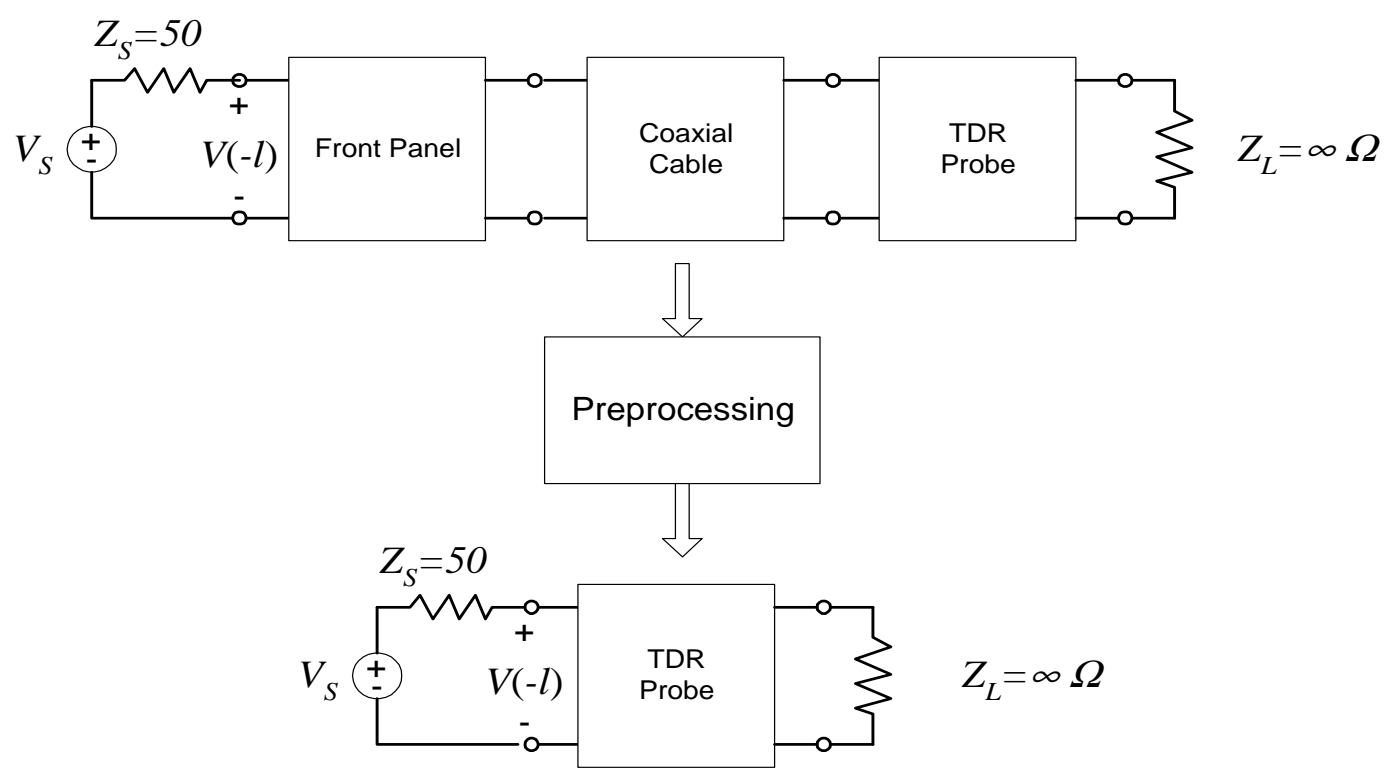

Fig. 5.16 Preprocessing of the TDR waveforms

where $H$ is the system function, $Z_{S}$ is the source impedance, $\hat{V}_{S}$ is the spectrum of the input waveform, and $\hat{V}(-L)$ is the spectrum of the TDR output waveform. A layer-peeling algorithm was formulated by reversing the process of overall input impedance in Eq. (3.71). Using the notation shown in Fig. 3.13, the input impedance looking into the probe section becomes 


$$
\begin{gathered}
Z_{\text {in }}\left(-z_{n-1}\right)=\frac{Z_{c n}\left[Z_{c n} \tanh \left(\gamma_{n} l_{n}\right)-Z_{i n}(-L)\right]}{Z_{i n}(-L) \tanh \left(\gamma_{n} l_{n}\right)-Z_{c n}} \\
\vdots \\
Z_{i n}\left(-z_{1}\right)=\frac{Z_{c 2}\left[Z_{c 2} \tanh \left(\gamma_{2} l_{2}\right)-Z_{i n}\left(-z_{2}\right)\right]}{Z_{i n}\left(-z_{2}\right) \tanh \left(\gamma_{2} l_{2}\right)-Z_{c 2}}
\end{gathered}
$$

The input impedance looking into the sample section, $Z_{i n}\left(-z_{1}\right)$, obtained by the layer-peeling algorithm can then be used to calculate the reduced system function and TDR waveform of the fictitious ideal probe system.

To validate the layer-peeling algorithm, a fabricated example was used. The system parameters of the TDR probe system as calibrated in the preceding section and the dielectric properties of Butanol were used to obtain a computer-simulated TDR waveform. Dielectric property of Butanol was used because it is close to that of soils. The layer-peeling algorithm was applied to the simulated waveform. The scattering function and TDR waveform of the ideal probe system calculated by the layer-peeling algorithm were compared to that of the overall probe system as shown in Figs. 5.17 and 5.18, respectively. The signals moving back and forth in the transmission line are electromagnetic waves that form a standing wave pattern. As a result, an oscillating pattern is observed in the scattering function. The frequency of oscillation depends on the length of the transmission line. The longer the transmission line is the higher the frequency of oscillation is expected. The complexity of the scattering function is reduced significantly by removing the long coaxial cable as shown in Fig. 5.17. In the time domain, peeling off the coaxial cable and probe head essentially scales and offsets the waveforms in the time axis as shown in Fig. 5.18.

The result of layer-peeling preprocessing was verified by comparing to direct simulation of the fictitious ideal probe system as shown in Figs. 5.17 and 5.18. The scattering function and waveform after layer-peeling preprocessing are almost identical to that of direct simulation except that there are very slight differences in scattering function at high frequencies. This may be explained by the fact that the TDR transmission line system acts like an low pass filter that filters out some high frequency components and results in a loss of information. 

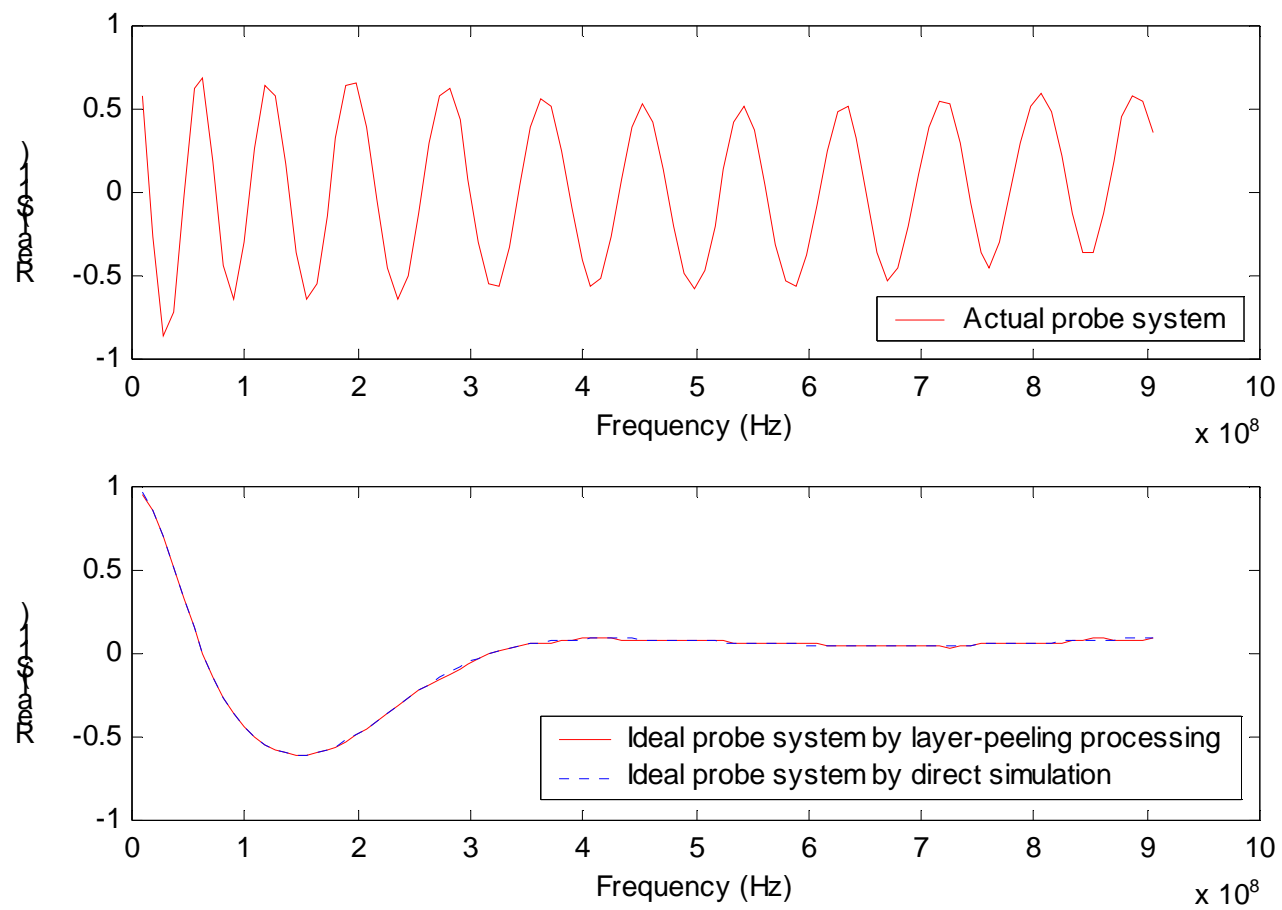

Fig. 5.17 Real part of scattering function after layer-peeling processing
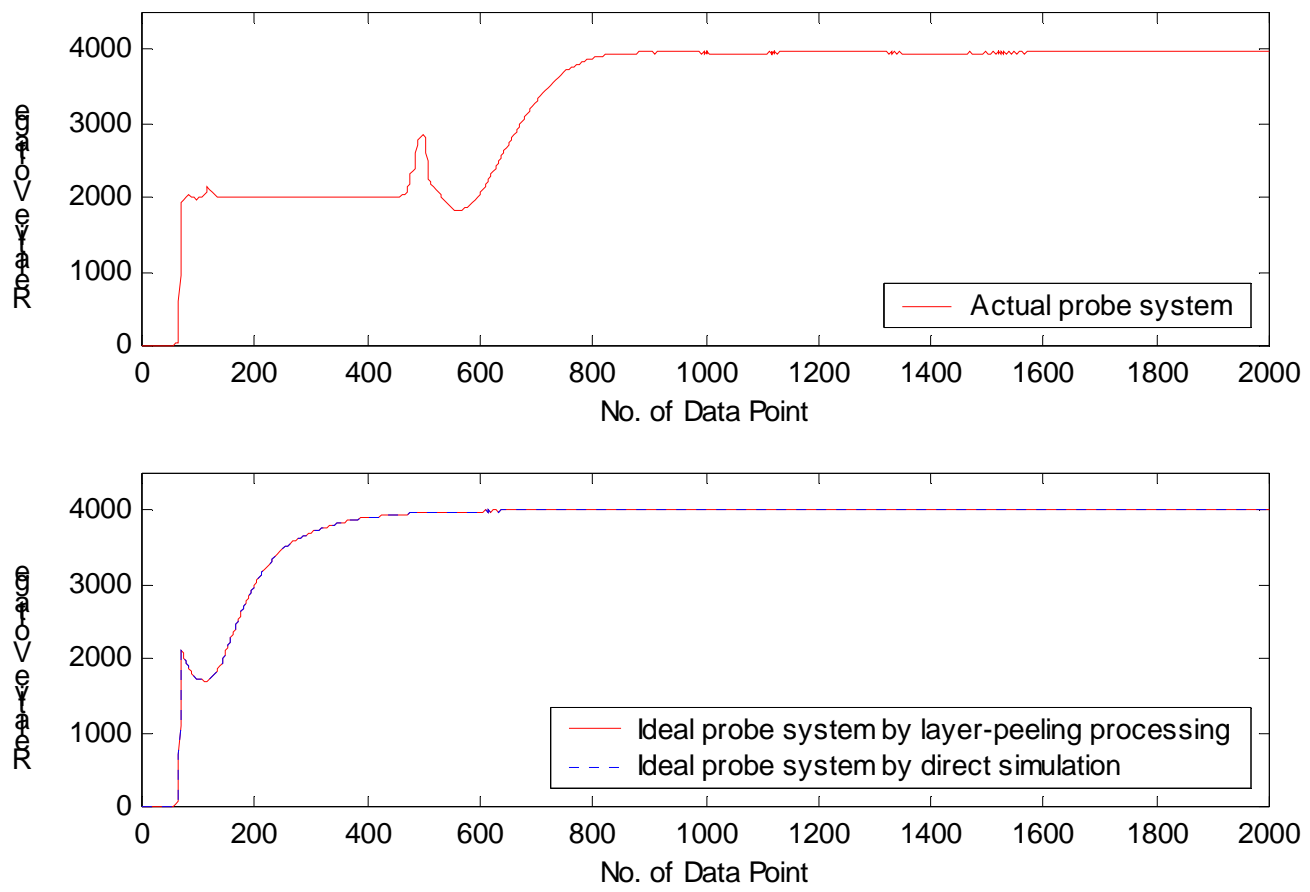

Fig. 5.18 Time-domain waveform after layer-peeling processing 
To support this argument, another example was carried out in which everything remained the same except that the conductivity of the material is increased from 0 to $0.5 \mathrm{~S} / \mathrm{m}$. The comparisons between the direct simulation and layer-peeling processing for the scattering function and time-domain waveform are shown in Fig. 5.19. The distortion in the scattering function increases as the conductivity increases while the time-domain waveform still has a good match. Two scattering functions or system functions with slight difference at high frequencies will not result in a significant difference in the time-domain waveforms because the spectrum of the input has low energy at high frequencies.
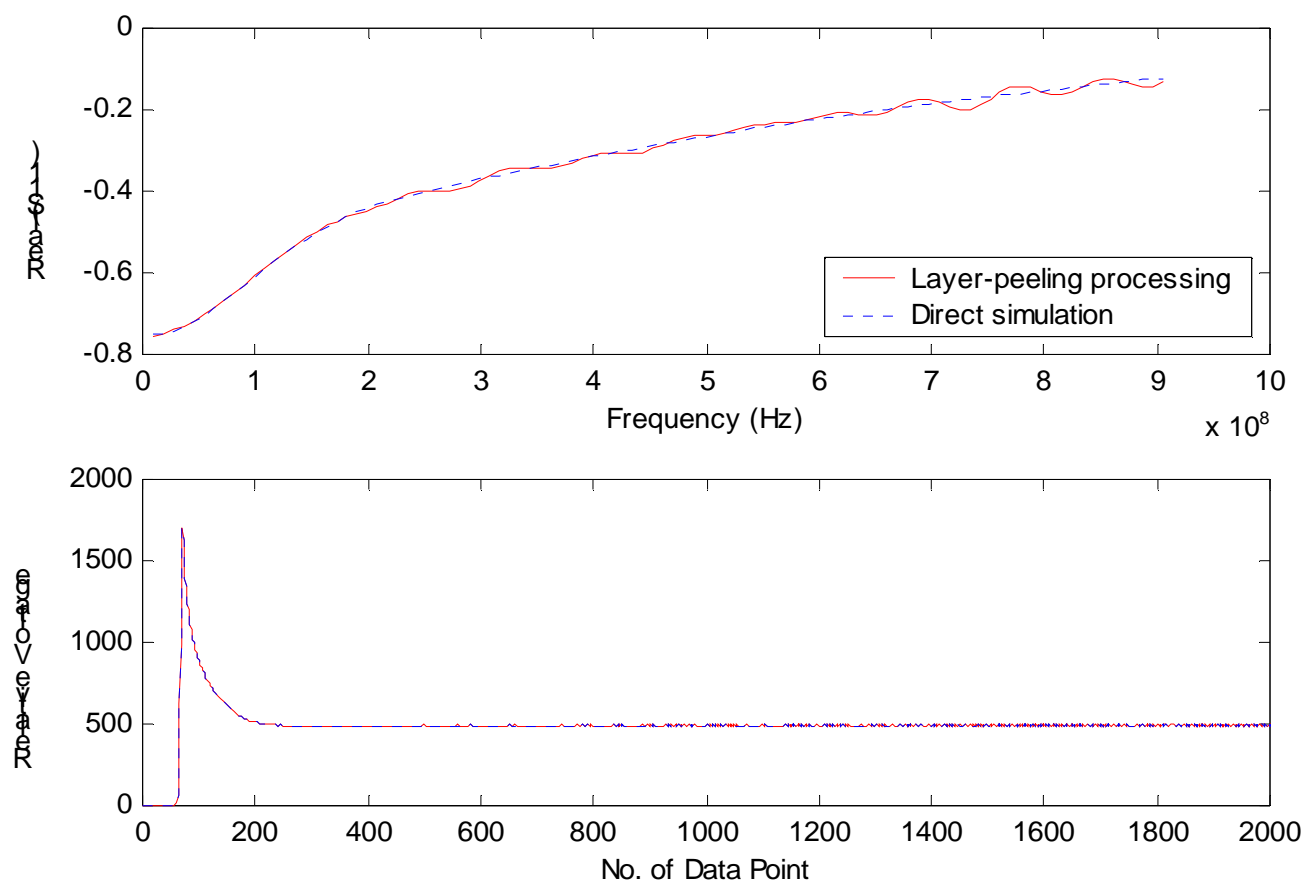

Fig. 5.19 Effect of conductive loss on layer-peeling processing $(\sigma=0.5 \mathrm{~S} / \mathrm{m})$

The TDR waveforms of the aqueous samples shown in Table 5.3 are preprocessed to obtain the reduced system functions in the frequency domain and the reduced waveforms in the time domain. The results are compared to the theoretical values obtained by the applying the TEM transmission line theory to the fictitious ideal system as shown in Figs. 5.20, 5.21, 5.22, and 5.23. It can be observed from these figures that the difference between the measured and 
theoretical scattering functions becomes significant at high frequencies. This may be an evidence of higher mode contribution and that the cutoff frequencies were underestimated.
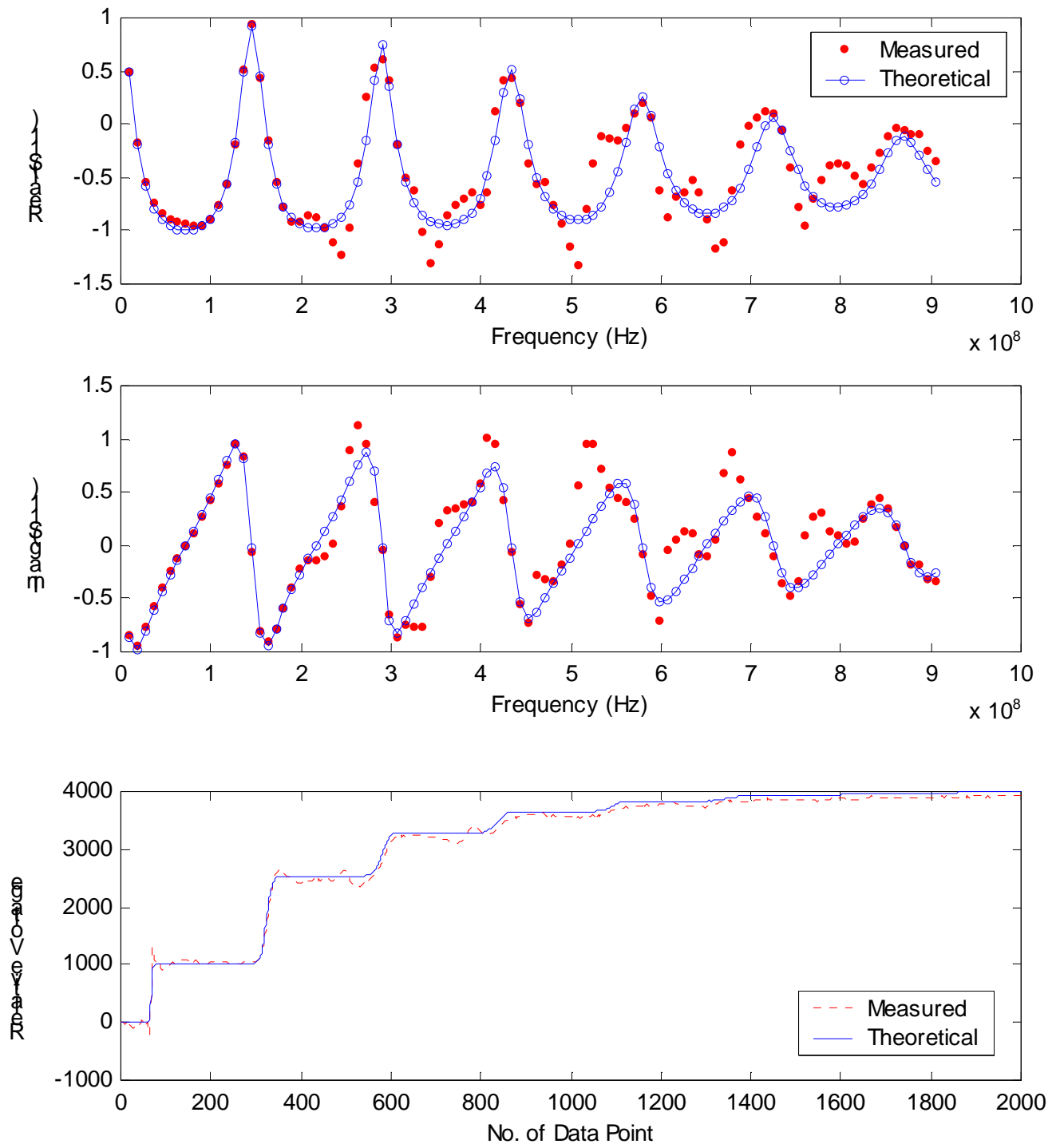

Fig. 5.20 The scattering function and TDR waveform after preprocessing for deionized water 

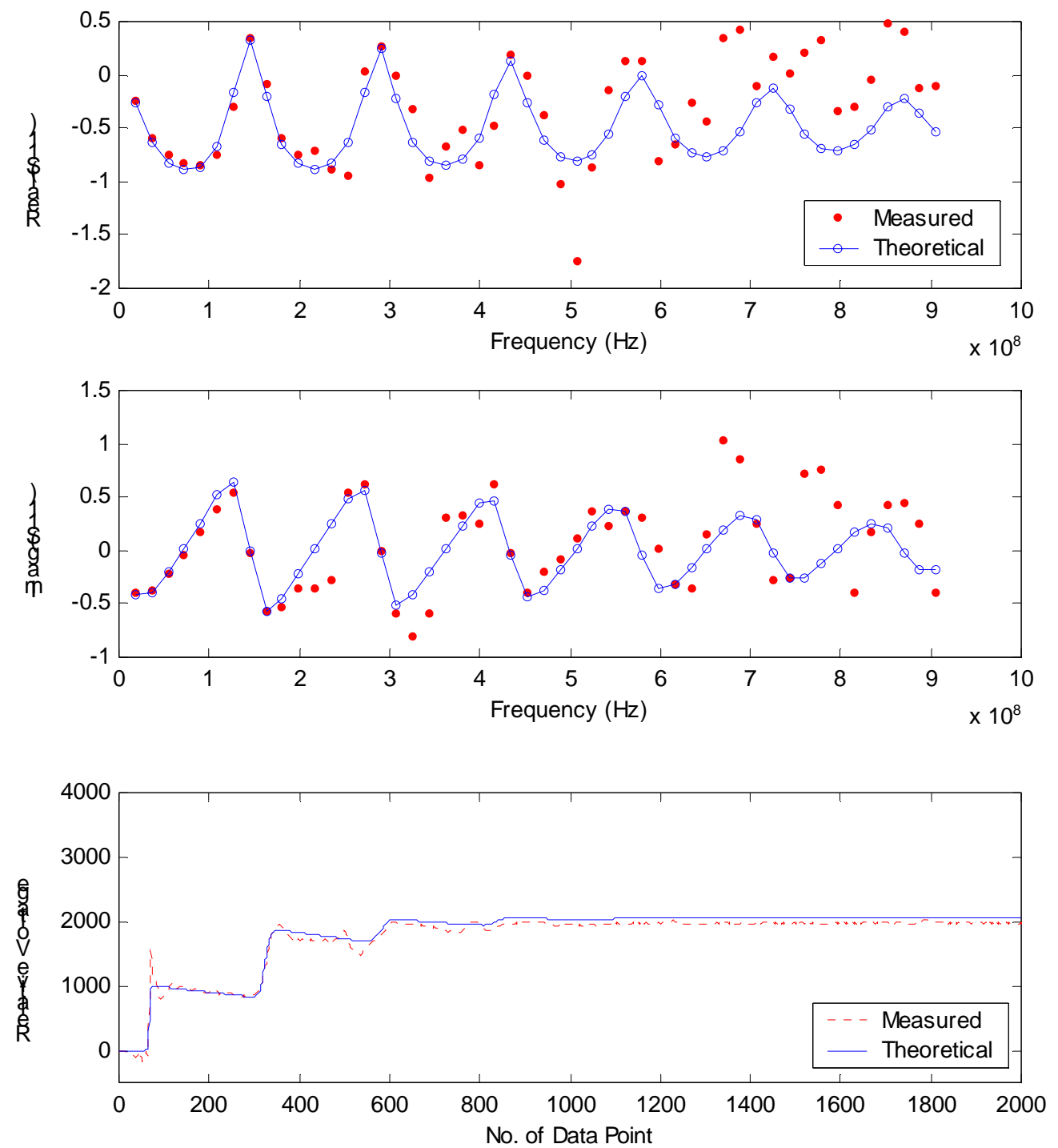

Fig. 5.21 The scattering function and TDR waveform after preprocessing for tap water 

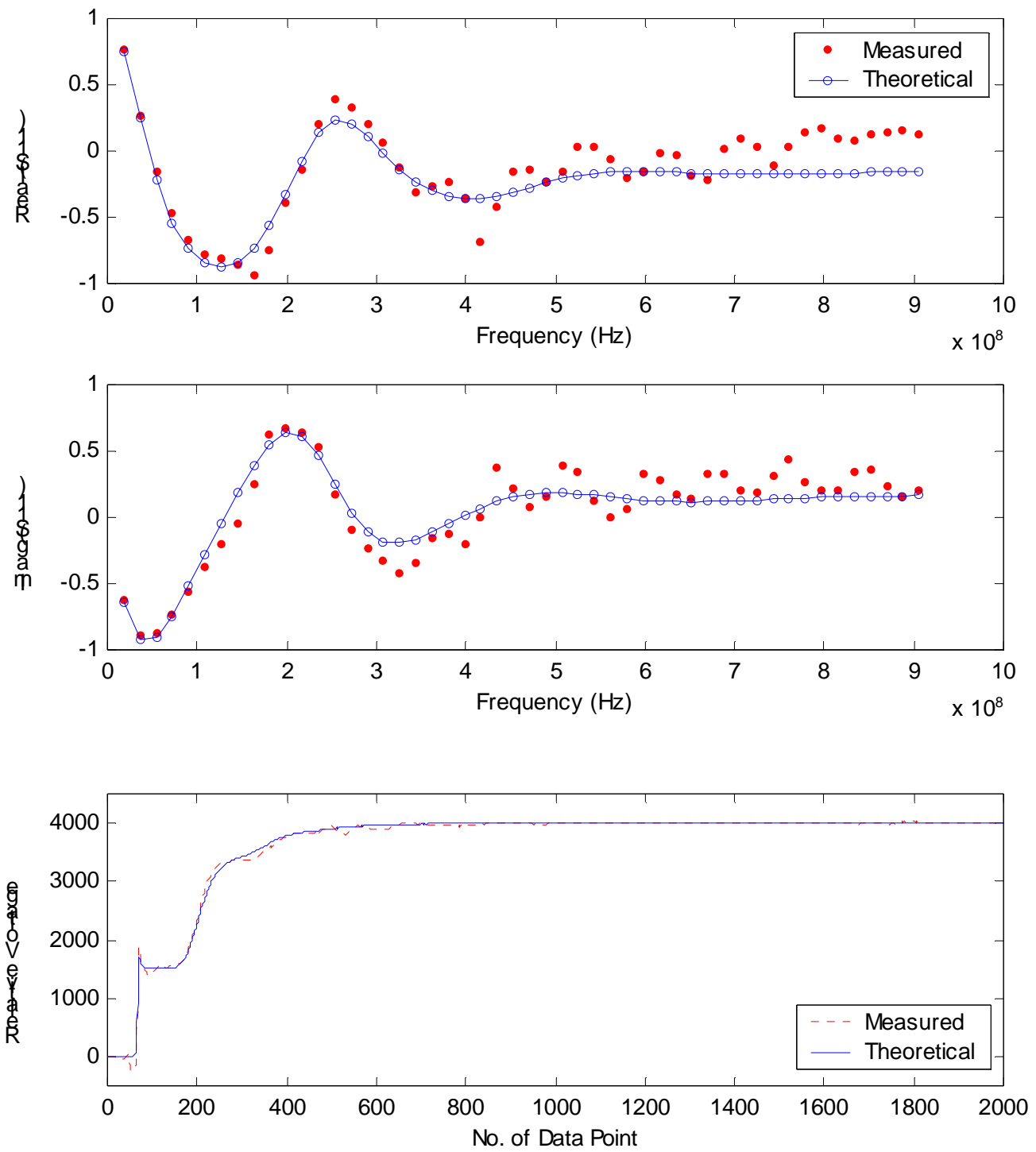

Fig. 5.22 The scattering function and TDR waveform after preprocessing for ethynol alcohol 

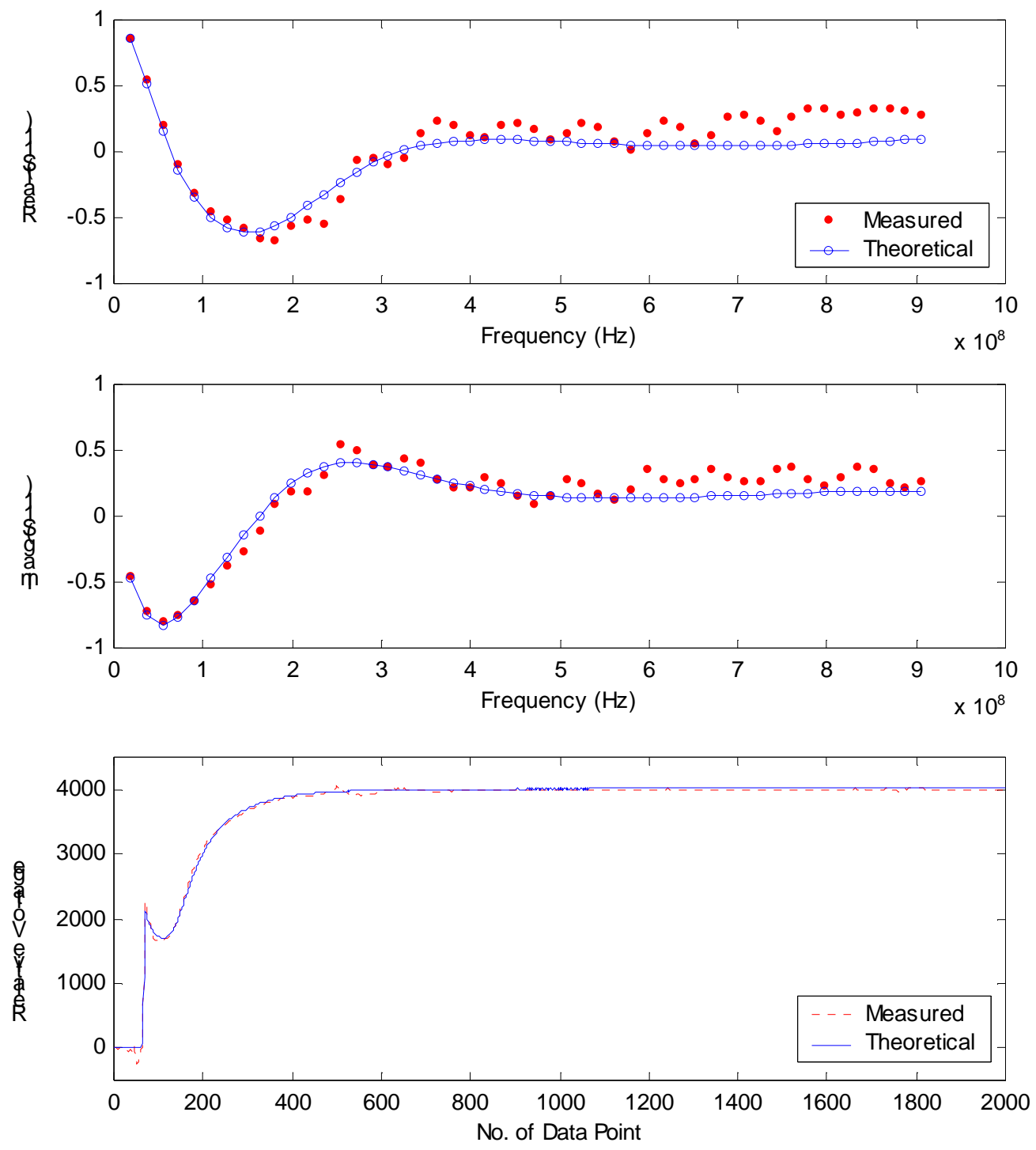

Fig 5.23 The scattering function and TDR waveform after preprocessing for butanol alcohol 

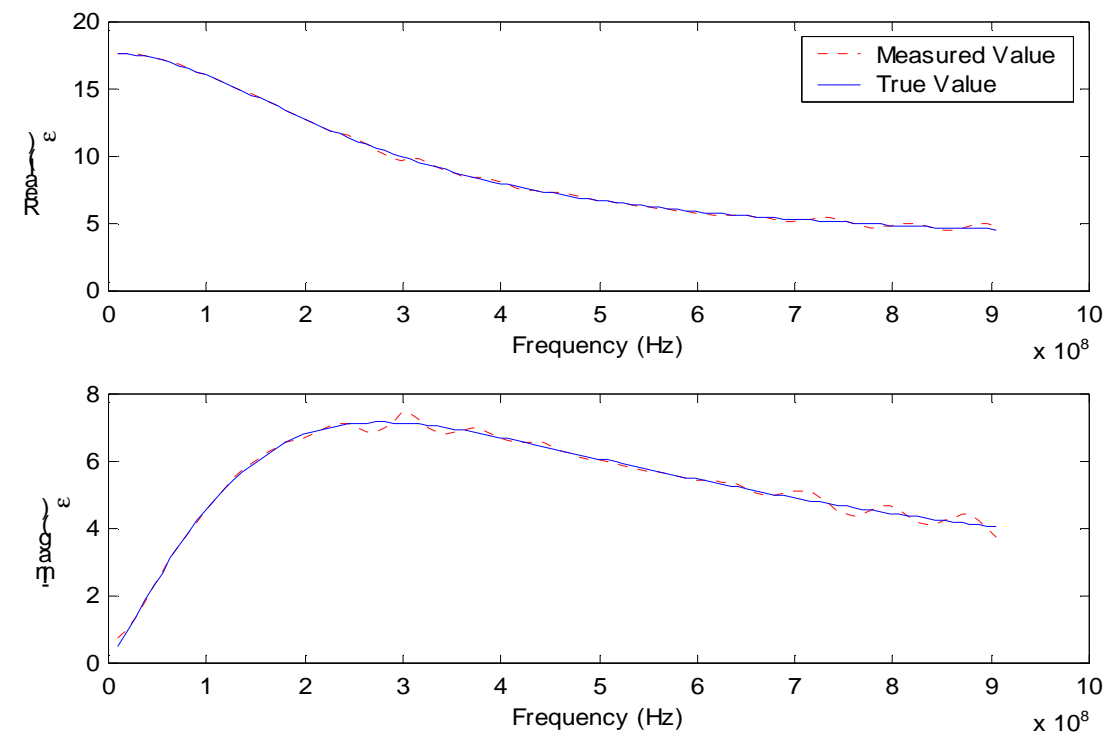

Fig. 5.24 Dielectric spectrum of butanol alcohol with zero conductivity measured by fabricated data

\subsection{Measurement of Dielectric Spectrum}

After the system parameters of the TDR probe are properly calibrated, the only unknown in the TDR transmission line is the dielectric property of the material inside the probe. Although the ultimate interest about the soil inside the probe is the soil physical properties, it is the dielectric properties that govern the response of the TDR probe. If the relationship between soil physical properties and soil dielectric properties (i.e. dielectric mixing model) can be established, a model-based inversion can be carried out to identify soil physical properties directly from the TDR data. The ability to measure the dielectric spectrum of soils will allow us to study or validate the relationship between the soil physical properties and soil dielectric properties.

\subsubsection{Measurement by Solving the Scattering Function}

When working in the frequency domain, it is possible to measure the dielectric spectrum without assuming a dielectric model. As discussed in Section 5.1.3, the dielectric spectrum may be determined by solving equation (5.2) at each frequency (i.e. one equation with one unknown). The left-hand side of Eq. (5.2) represents the theoretical scattering function and the right hand side represents the measured scattering function. Equation (5.2) may be solved by Newton- 
Raphson method. However, the theoretical scattering function for the TDR probe system is highly nonlinear and complex as shown in Fig. 5.17. The solution is very sensitive to the data error and the starting point of Newton-Raphson iteration, especially at high frequencies. The solution diverges after the first few frequency components. Thus, it is necessary to reduce the complexity of the scattering function with the layer-peeling algorithm and solve the equation successively from low frequencies to high frequencies. The starting point of Newton-Raphson iteration at a particular frequency was chosen to be the solution of the previous frequency. This algorithm was tested by fabricated examples. For example, the scattering functions as shown in Fig. 5.17 (butanol alcohol with zero conductivity) and Fig. 5.19 (butanol alcohol with conductivity equal to $0.5 \mathrm{~S} / \mathrm{m}$ ) were used to solve for the dielectric spectra. The results are shown in Fig. 5.24 and 5.25. Very accurate results were obtained for lossless materials. But the measurement is very sensitive to the data error for lossy materials such as occur in a long probe where there is no observable end reflection in the time-domain waveform. Thus, a shorter probe should be used when high-loss material is encountered for better accuracy.
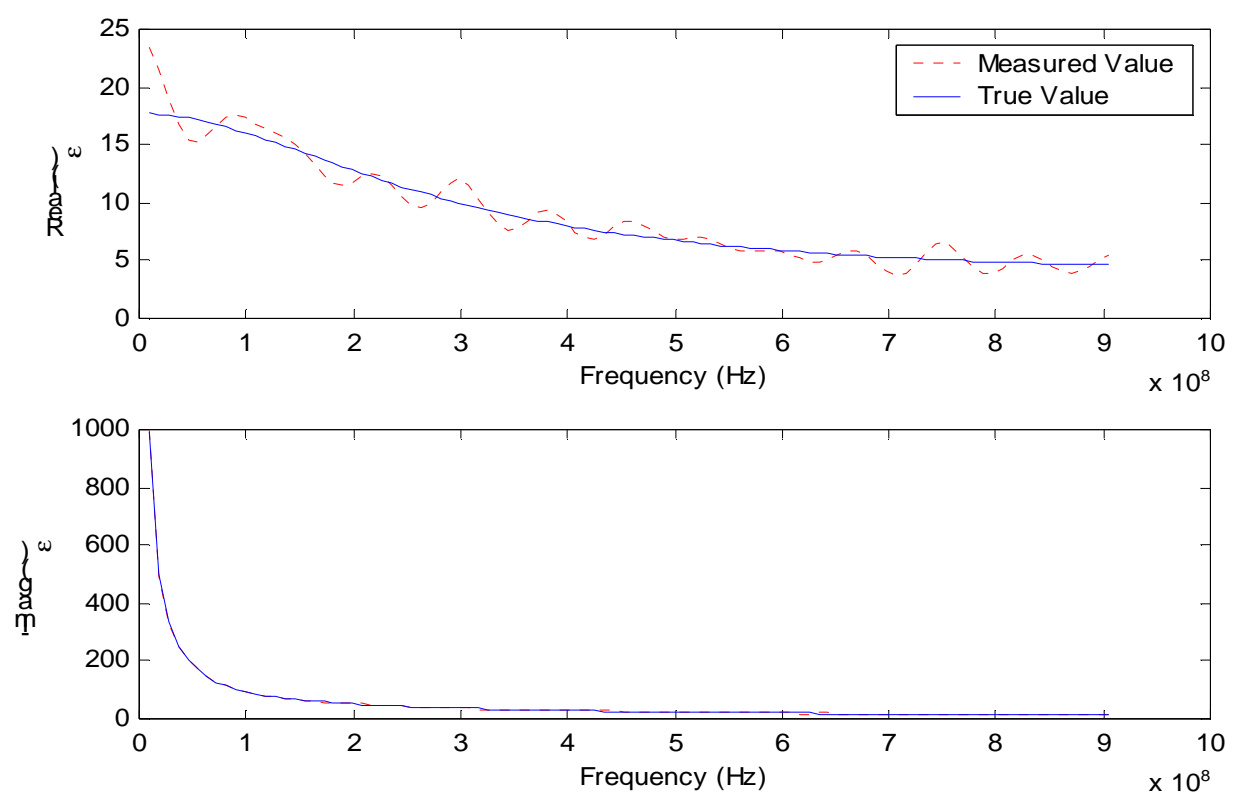

Fig. 5.25 Dielectric spectrum of butanol alcohol with conductivity equal to $0.5 \mathrm{~S} / \mathrm{m}$ measured by fabricated data 
This algorithm works very well on fabricated examples where the data error is small. However, the error in the measured scattering function becomes significant at high frequencies as shown in Figs. 5.20 to 5.23. In order to account for the data error and evaluate the measurement uncertainty, Eq. (5.2) can be solved by the probabilistic inverse framework developed in Chapter 4. In the context of inverse theory, the data at each frequency is the value of the measured scattering function at the particular frequency and the model parameter is the dielectric permittivity at that frequency. Note that both data and model parameters are complex. They can be represented by a $2 \times 1$ vector. The data error was estimated from Figs. 5.20 to 5.23 . The prior dielectric permittivity for the first frequency was chosen to be 40-j1000 with standard deviation of $20-\mathrm{j} 500$. The real part of the prior was chosen to cover the range of the real part of dielectric permittivity from 0 to 80 within two standard deviations, and the imaginary part of the prior was chosen to cover the range of conductivity from 0 to 1 within two standard deviations. The prior dielectric permittivity of the succeeding frequency component was chosen to be the maximum posterior estimate of the preceding frequency component with a standard deviation of 50\%. The dielectric spectra of the aqueous samples were estimated and compared to the expected spectra calculated from Table 5.3 as shown in Figs. 5.26 to 5.29. The standard deviation calculated by linear approximation is also shown on those figures. Because of the data error and the complexity of the scattering functions, the estimation does not give satisfactory results especially at high frequencies. It is expected that the accuracy will improve by reducing the cross-sectional dimension of the probe and discontinuities of the probe to increase the cutoff frequency and to decrease the fringing effect.

Measurement by Waveform Matching It was observed from Figs. 5.20 to 5.23 that although the error in the scattering function at individual frequencies may be significant, the overall matches between the measured value and theoretical value in the scattering function and time-domain waveform are very good. Therefore, it is possible to measure the dielectric spectrum by an inversion based on a dielectric model such as Debye's function. The sensitivities of the TDR waveforms to Debye's parameters and conductivity were evaluated at $\left(\varepsilon_{s}, \varepsilon_{\infty}, f_{r e l}, \sigma\right)=(25$, 15, $30 \mathrm{MHz}, 0.02 \mathrm{~S} / \mathrm{m})$ and $\left(\varepsilon_{s}, \varepsilon_{\infty}, f_{\text {rel }}, \sigma\right)=(25,15,3 \mathrm{GHz}, 0.02 \mathrm{~S} / \mathrm{m})$ and shown in Figs. 5.30 and 5.31, respectively. 

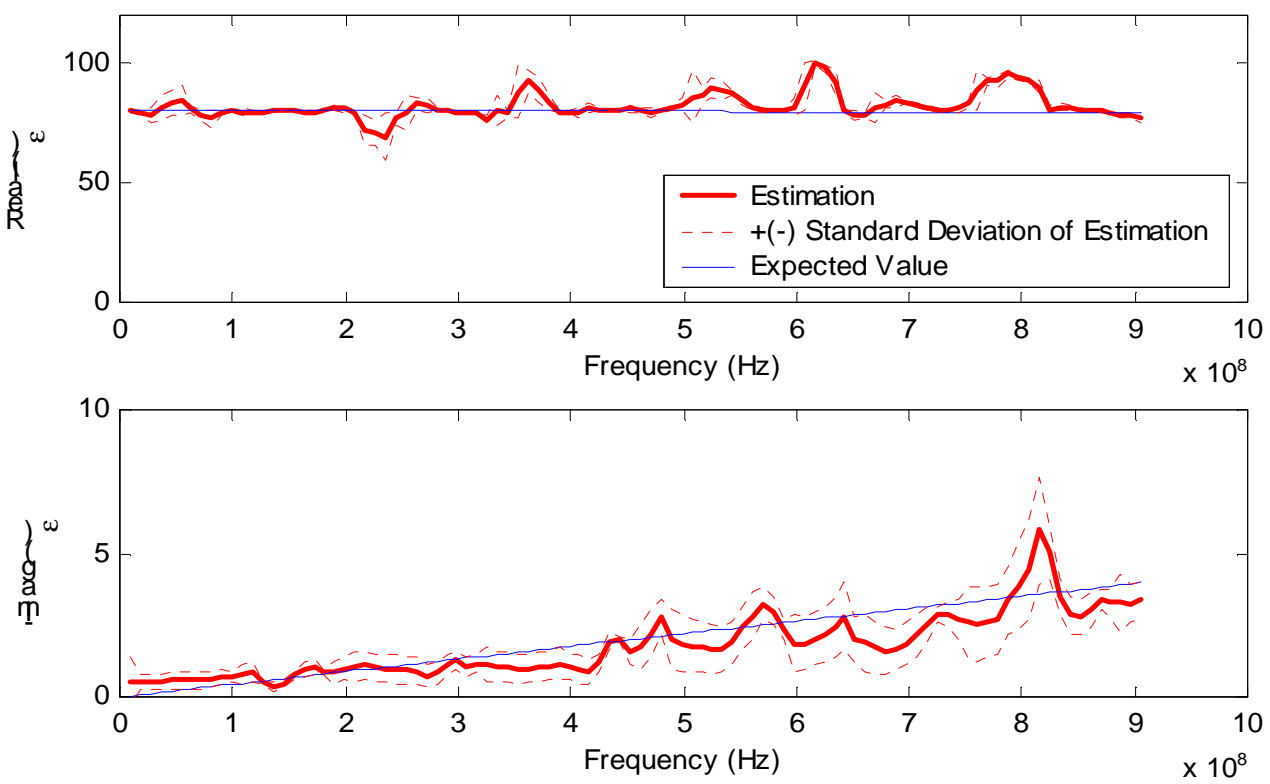

Fig. 5.26 Estimated dielectric spectrum of deionized water
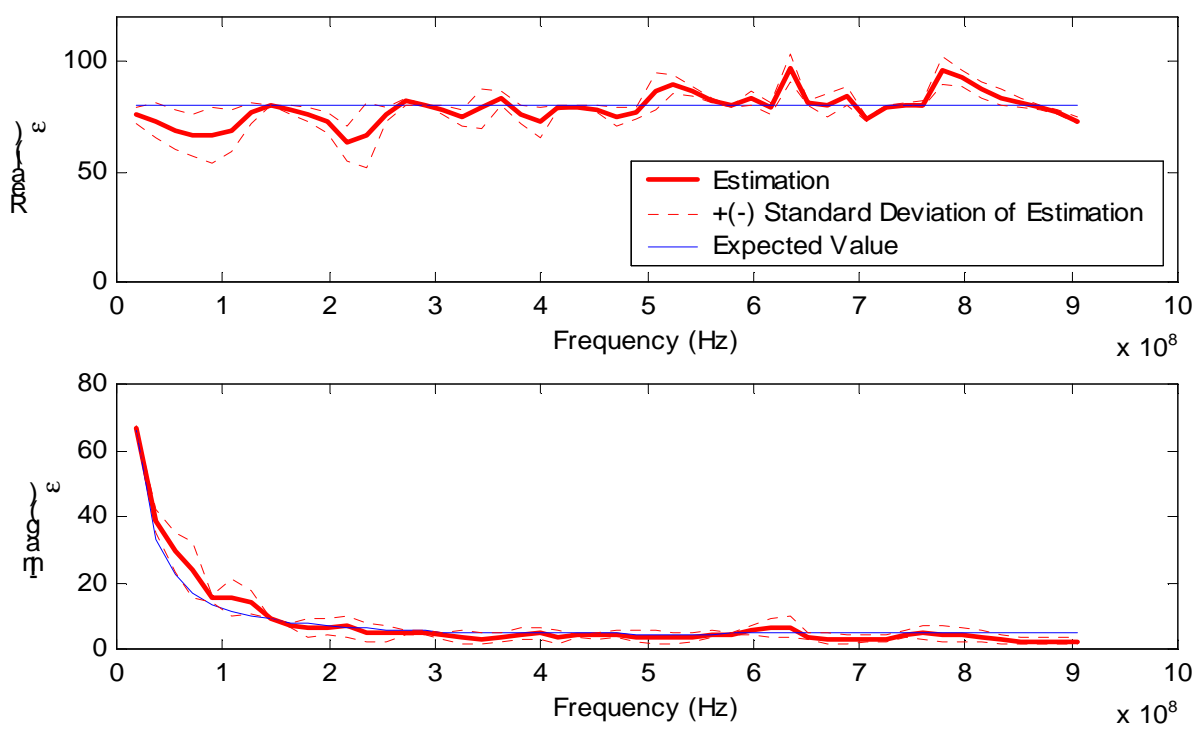

Fig. 5.27 Estimated dielectric spectrum of tap water 

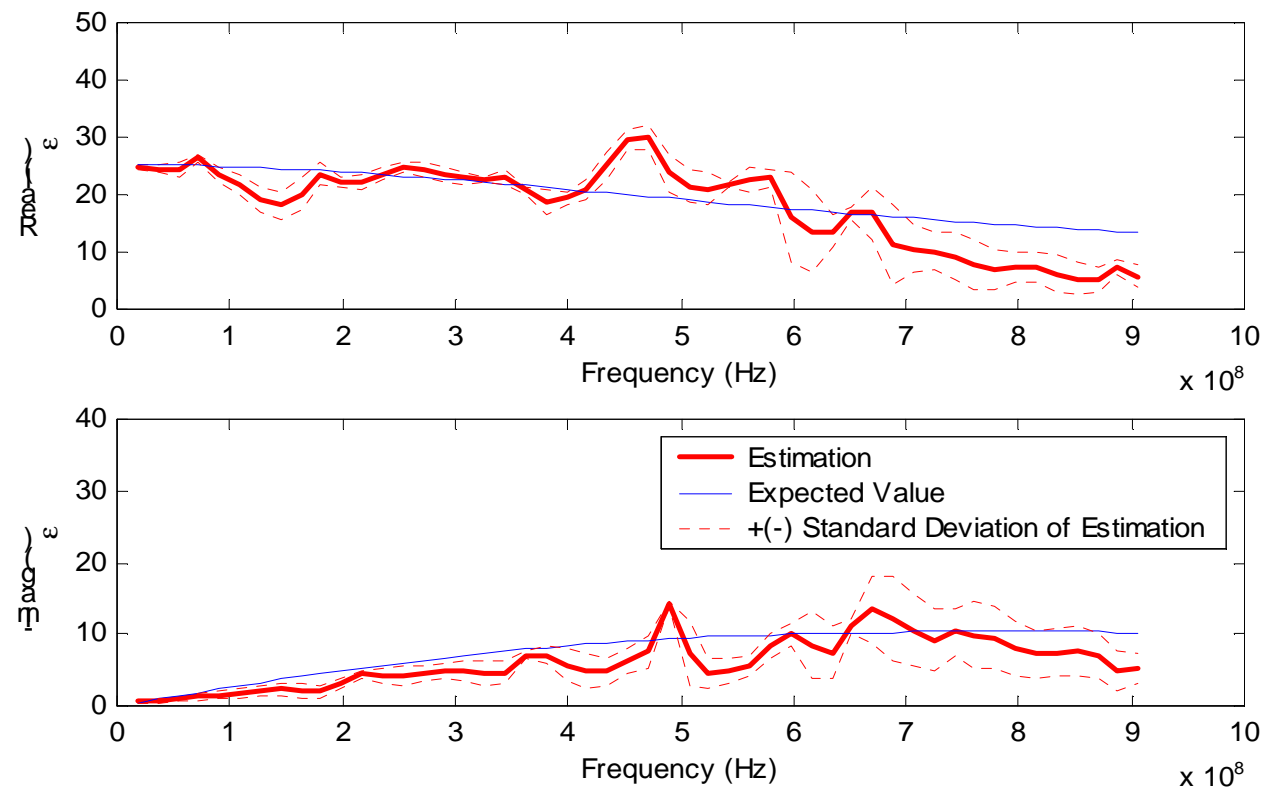

Fig. 5.28 Estimated dielectric spectrum of ethanol alcohol
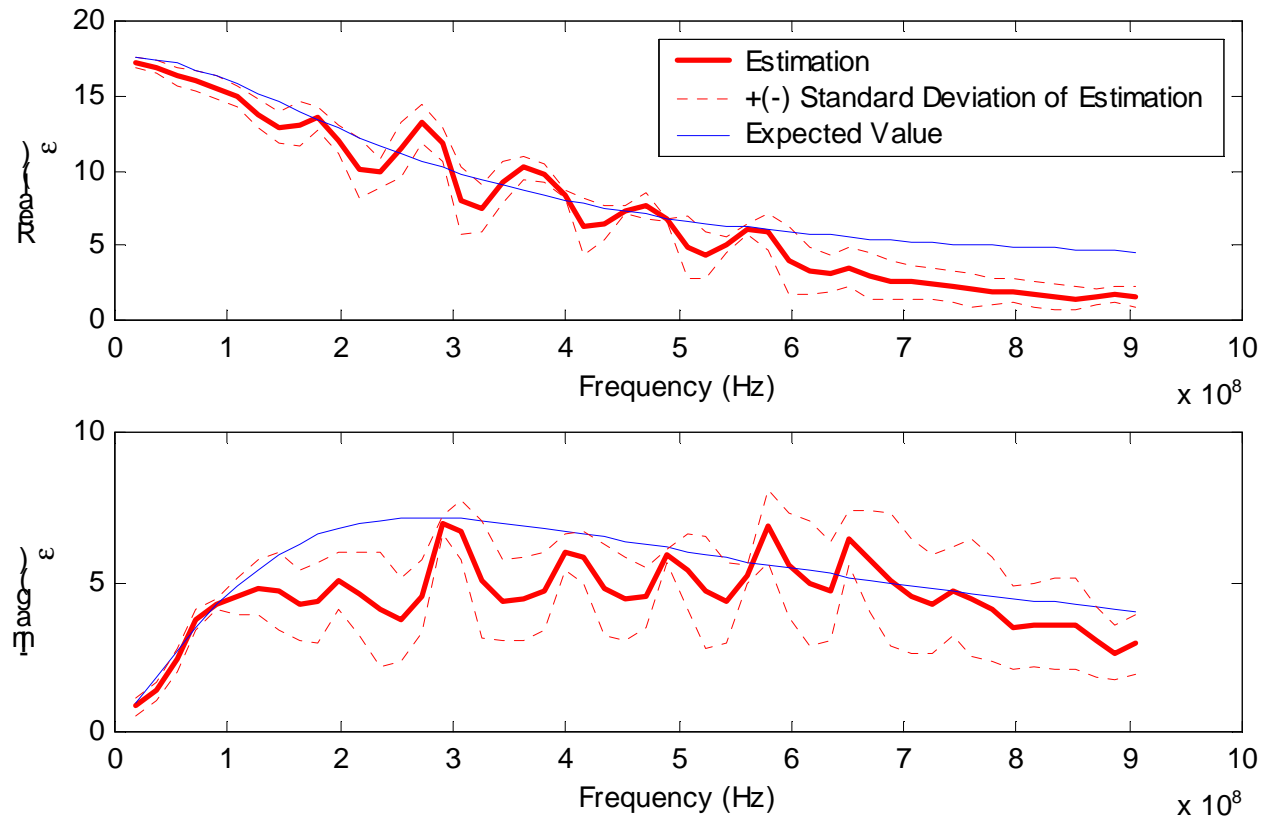

Fig. 5.29 Estimated dielectric spectrum of butanol alcohol 

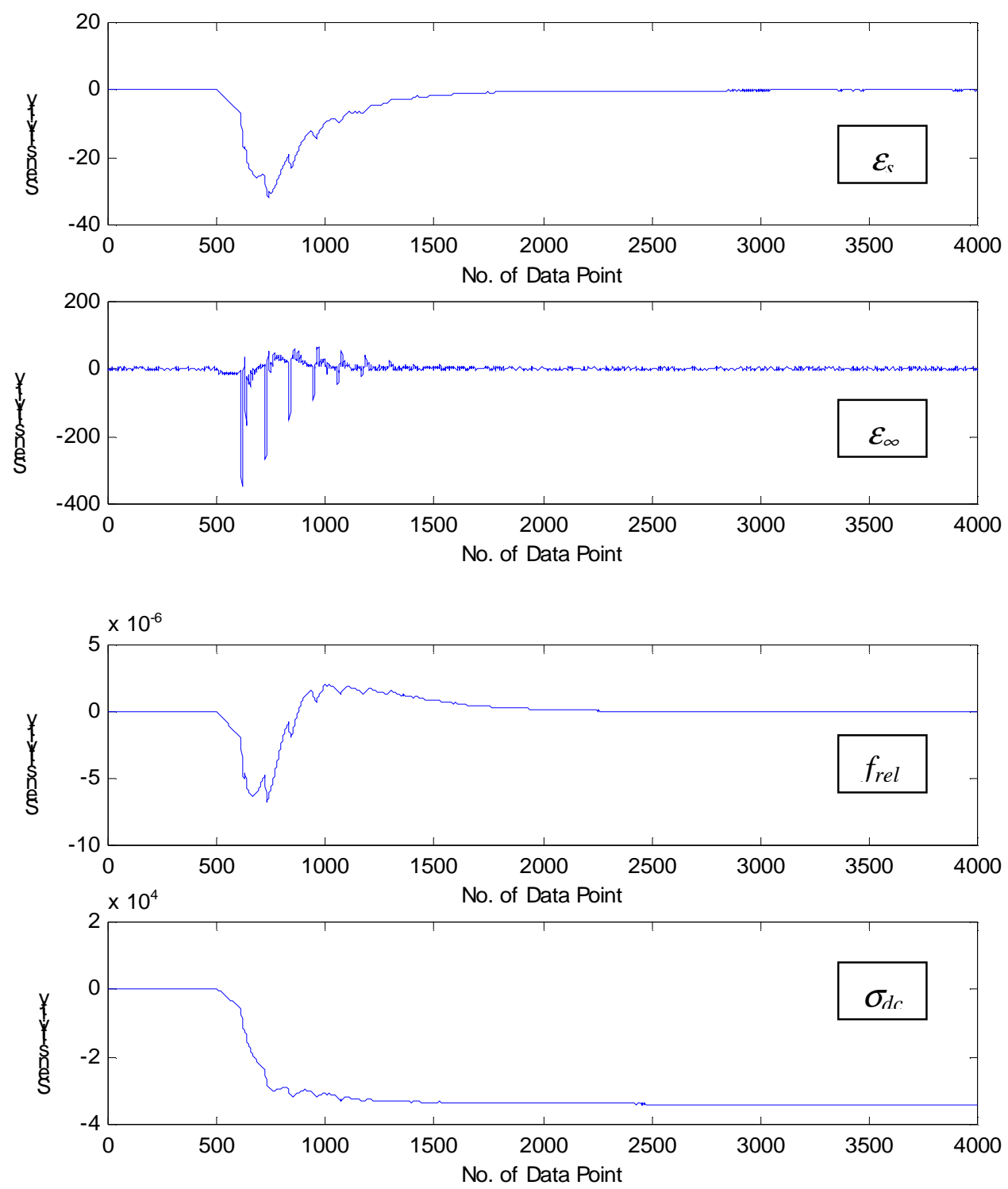

Fig. 5.30 Sensitivity of the TDR waveform on Debye's parameters evaluated at $\left(\varepsilon_{s}, \varepsilon_{\infty}, f_{r e l}, \sigma\right)=(25,15,30 \mathrm{MHz}, 0.02 \mathrm{~S} / \mathrm{m})$

Two different relaxation frequencies were chosen such that one is within the TDR bandwidth and the other beyond the TDR bandwidth. Note that the TDR waveforms at long times are influenced dominantly by the conductivity. The effects of static and infinitive dielectric 
permittivities are at short times. Their relative importance depends on the location of relaxation frequency relative to the TDR bandwidth.
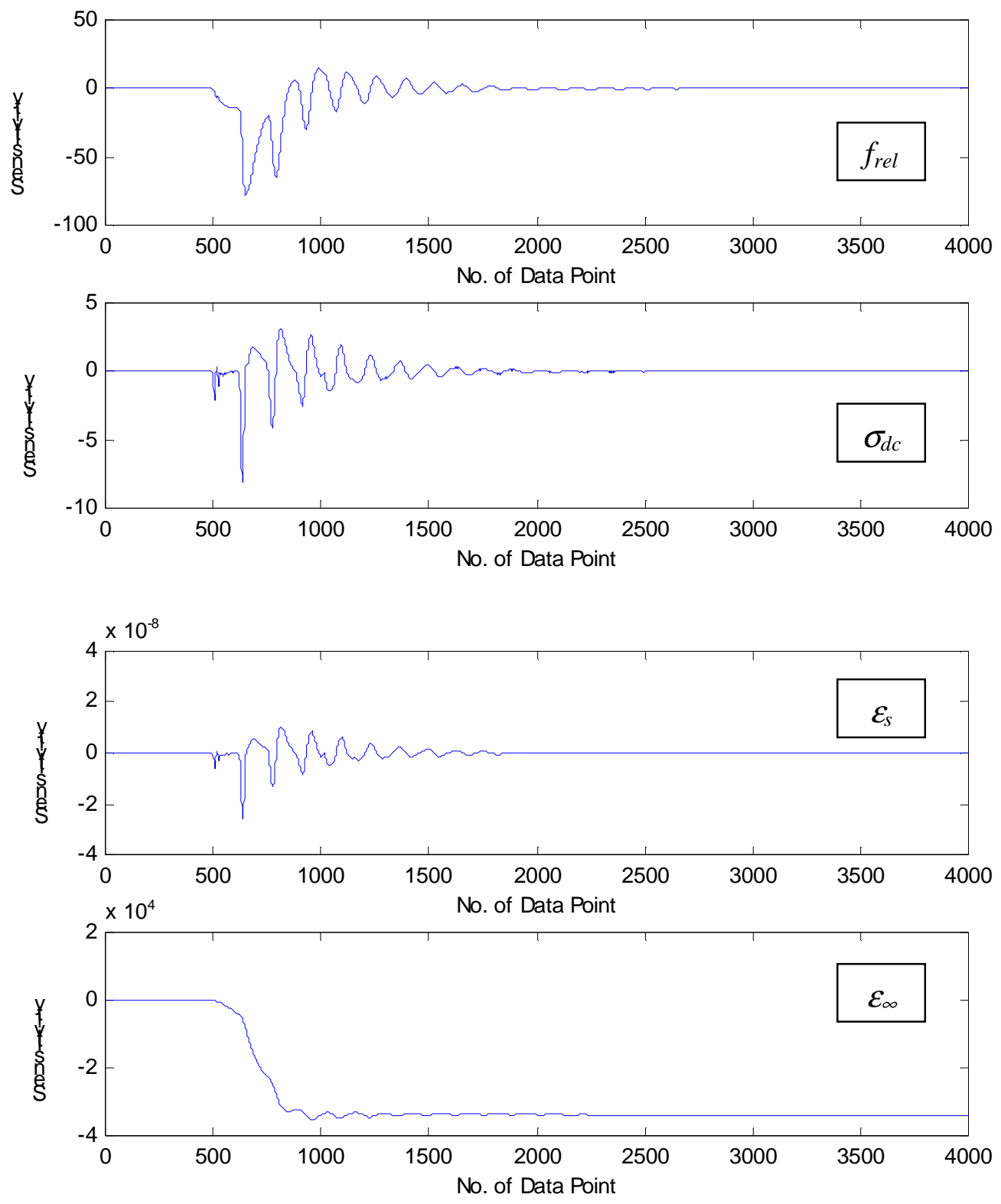

Fig. 5.31 Sensitivity of the TDR waveform on Debye's parameters evaluated at $\left(\varepsilon_{s}, \varepsilon_{\infty}, f_{r e l}, \sigma\right)=(25,15,3 \mathrm{GHz}, 0.02 \mathrm{~S} / \mathrm{m})$

The inversion may be carried out in the frequency domain using the scattering function or in the time domain using the TDR waveform. The inversion in time domain was used because the TDR waveform contains real numbers rather than complex numbers. Both the original TDR 
waveform and the reduced TDR waveform obtained by layer-peeling algorithm may be used. But the inversion based on reduced TDR waveforms is much faster than that based on original TDR waveform because the forward model in the inversion is evaluated using the ideal probe system. The estimation of the dielectric parameters for the aqueous samples is listed in Table 5.4. The associated dielectric spectra are compared to the expected spectra calculated from Table 5.3 are shown in Fig. 5.33. For materials with relaxation frequency well within the TDR bandwidth, the inverse solution gives a very good estimate with a very small standard deviation. When the relaxation frequency of the material such as water gets close to or beyond the TDR bandwidth, the uncertainty of estimation for $\varepsilon_{\infty}$ and $f_{\text {rel }}$ becomes significant. The inverse solution can not resolve the dielectric spectra beyond the TDR bandwidth for a material with relaxation frequency higher than the TDR bandwidth. There are many different values of $\varepsilon_{\infty}$ and $f_{\text {rel }}$ that can give similar dielectric spectra within the TDR bandwidth. However, the inverse solution still provides a very good estimation of dielectric spectra within the TDR bandwidth as shown in Figs. 5.34 and 5.35.
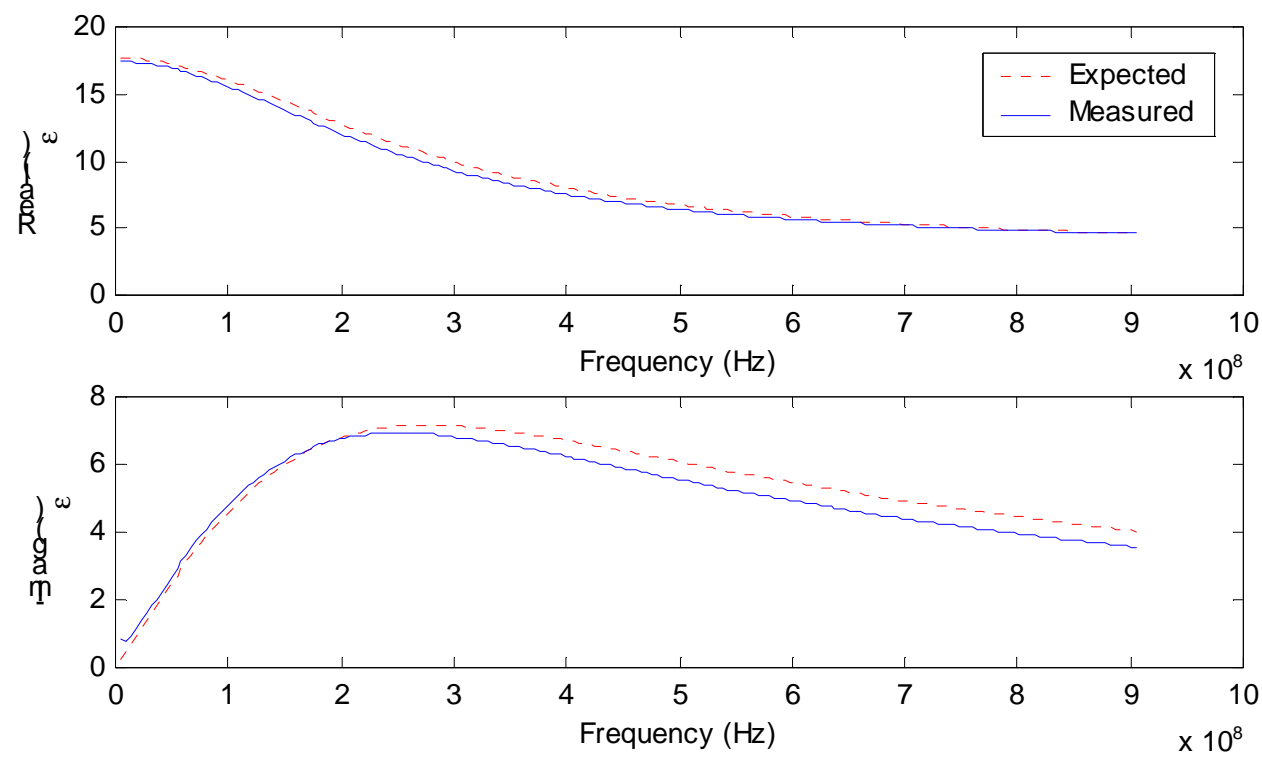

Fig. 5.32 Measured dielectric spectrum of butanol alcohol from the estimation of Debye's parameters 

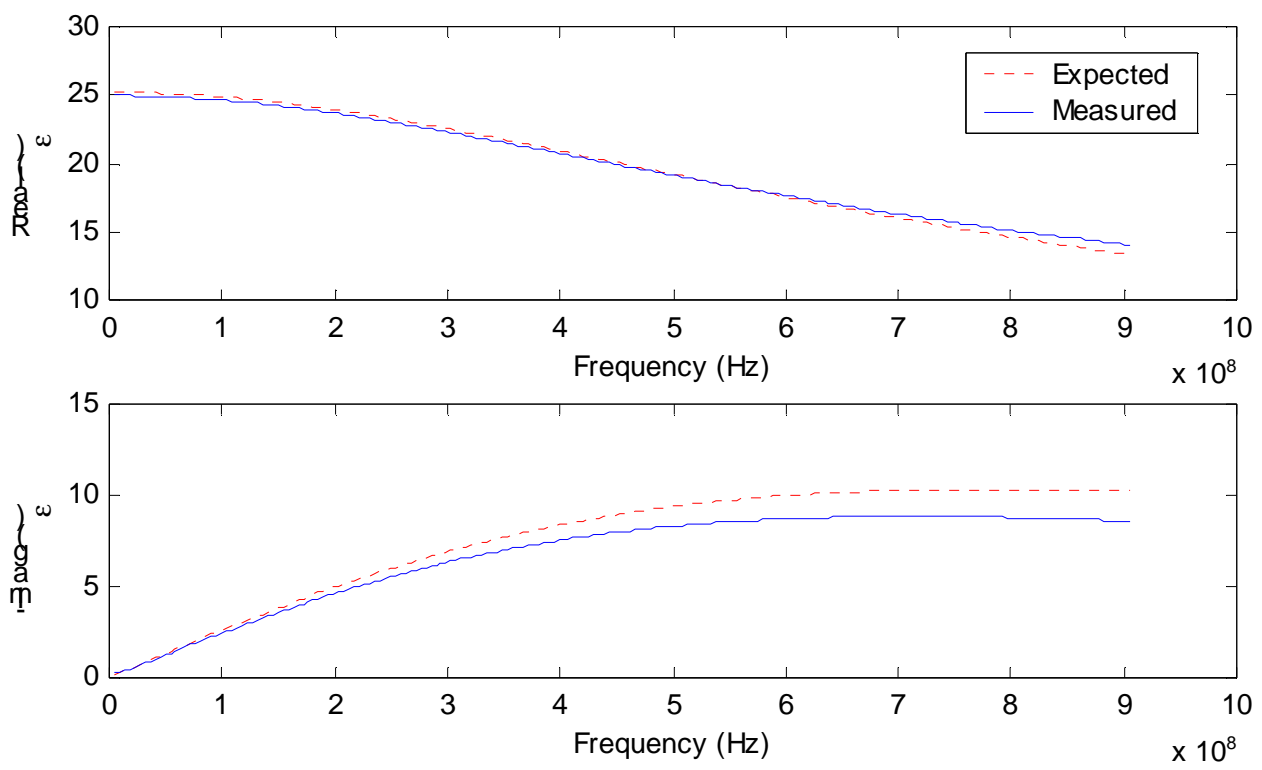

Fig. 5.33 Measured dielectric spectrum of ethanol alcohol from the estimation of Debye's parameters
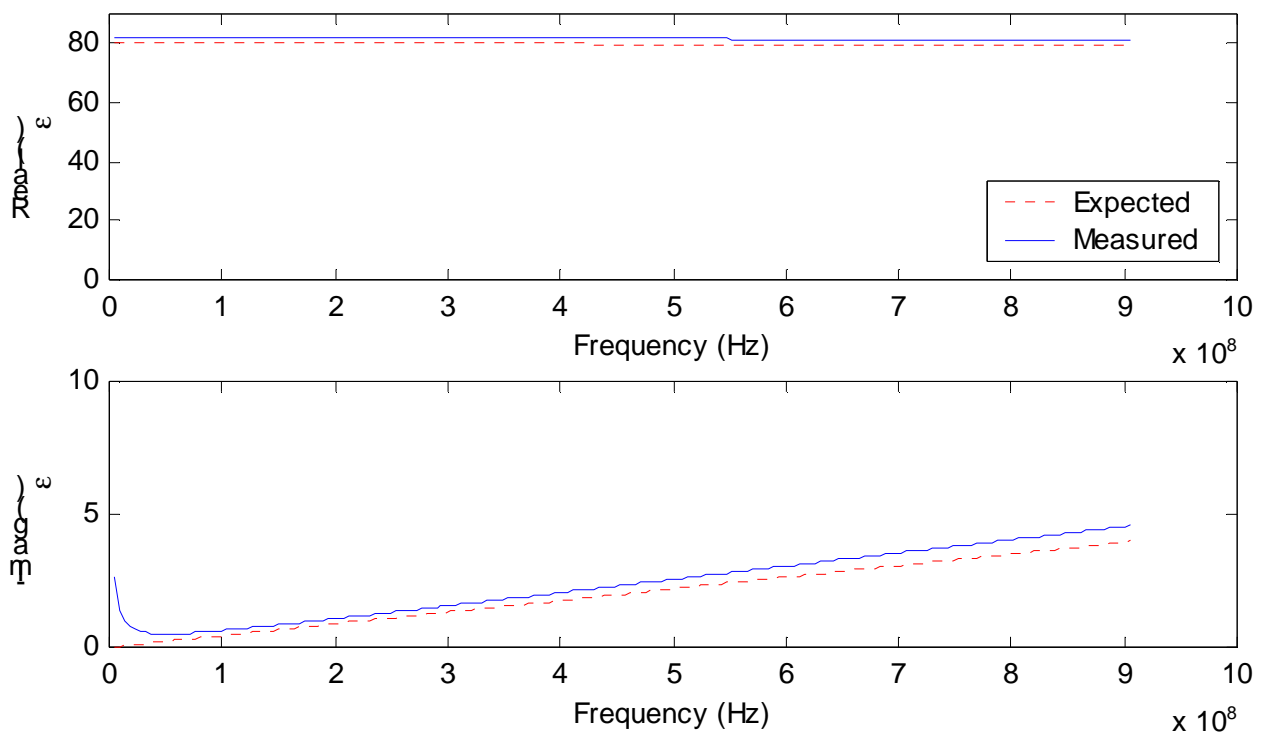

Fig. 5.34 Measured dielectric spectrum of deionized water from the estimation of Debye's parameters 

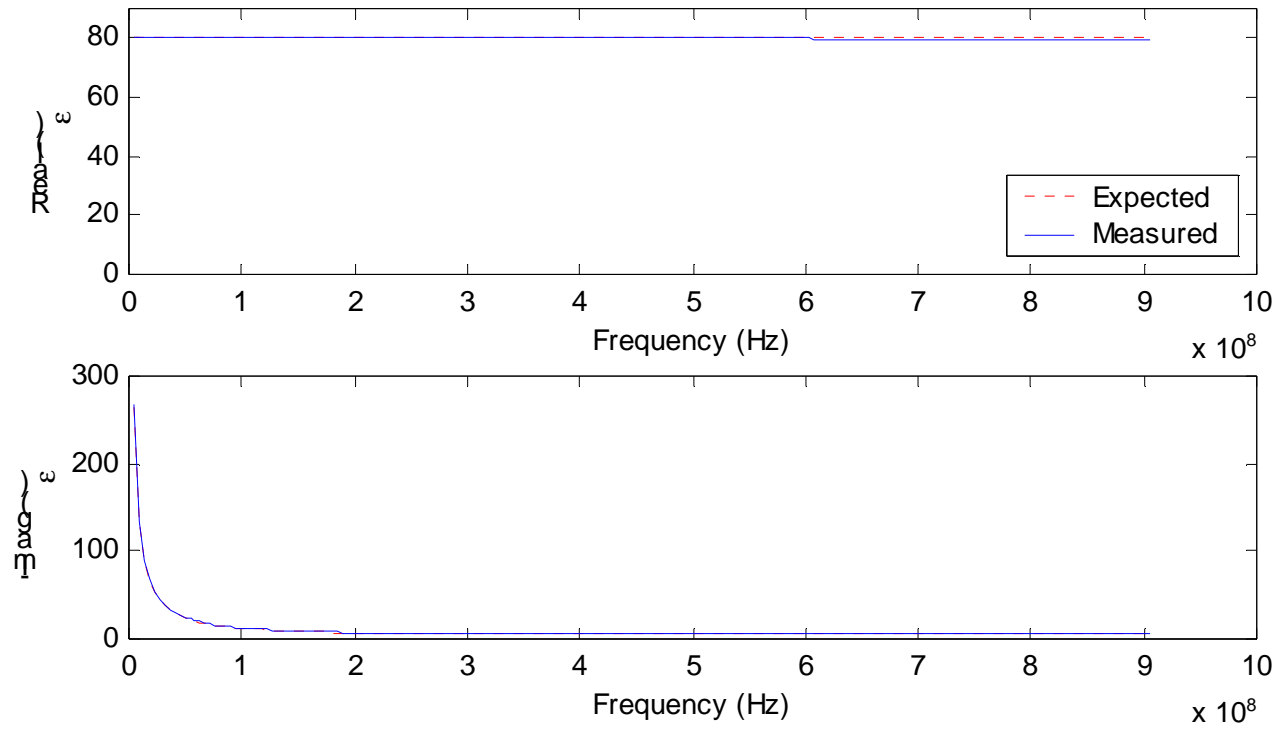

Fig. 5.35 Measured dielectric spectrum of tap water from the estimation of Debye's parameters

Apparently, assuming that the dielectric spectrum can be modeled with a relaxation equation such as the Debye Equation and solving the relaxation parameters by the inverse theory provides much better measurements of dielectric spectra than solving the dielectric spectrum, $\varepsilon^{*}(f)$, directly from Eq. (5.2). Very good estimations were obtained for the aqueous samples because the Debye Equation describes the dielectric spectrum very well. However, it may not be the case for the soils. It was found from the literature that semi-empirical volumetric mixing models could describe the soil dielectric spectrum at least case by case. The parameters in the volumetric mixing model are soil physical parameters and the Debye parameters of each soil phase. The number of parameters in the volumetric mixing model equation is much more than those in a single Debye equation. Although the three parameters in the Debye equation have physical meaning as discussed in Chapter 2, they can also be interpreted as a curve fitting parameters. It would be interesting to know whether the Debye equation can be used as curve fitting formula to approximate the dielectric spectrum of soils. 
Table 5.4 Estimation of the dielectric parameters for the aqueous samples

\begin{tabular}{l|l|llll}
\hline \multicolumn{2}{c|}{ Dielectric parameters } & $\varepsilon_{s}$ & $\varepsilon_{\infty}$ & $f_{\text {rel }}, \mathrm{GHz}$ & $\sigma_{d c}, \mathrm{~S} / \mathrm{m}$ \\
\hline \multirow{2}{*}{ Prior } & Mean & 40 & 4 & 1 & 0.5 \\
& Std. Dev. & 20 & 1 & 0.5 & 0.25 \\
\hline \multirow{3}{*}{ Butanol } & Expected & 17.70 & 3.30 & 0.274 & 0 \\
& Estimated & 17.33 & 3.45 & 0.260 & 0.000015 \\
& Std. Dev. & 0.01 & 0.05 & 0.001 & 0.000004 \\
\hline \multirow{3}{*}{ Ethynol } & Expected & 25.20 & 4.52 & 0.782 & 0 \\
& Estimated & 24.75 & 7.05 & 0.759 & 0.000038 \\
& Std. Dev. & 0.01 & 0.20 & 0.009 & 0.000005 \\
\multirow{2}{*}{ Deionized } & Expected & 79.90 & 4.22 & 17.000 & 0 \\
& Estimated & 80.92 & 3.80 & 16.470 & 0.00067 \\
& Std. Dev. & 0.01 & 2.00 & 0.450 & 0.000003 \\
\hline \multirow{3}{*}{ Tap water } & Expected & 80.20 & 4.22 & 17.400 & 0.0666 \\
& Estimated & 78.64 & 3.54 & 15.500 & 0.0676 \\
& Std. Dev. & 0.03 & 2.00 & 0.470 & 0.00002 \\
\hline
\end{tabular}

Typical values of the volumetric mixing model parameters listed in Table 5.5 were assumed to generate a fabricated TDR waveform by the volumetric mixing formula and the wave propagation model. This waveform, which resembles the typical waveforms measured in soils, was used to perform inversion of Debye parameters. The result of inversion is shown Table 5.6. The comparison between the estimated dielectric spectrum and the actual dielectric spectrum is shown in Fig. 5.36 and the comparison between the simulated waveform and predicted waveform based on the estimated dielectric parameters is shown in Fig. 5.37. It can be seen from Fig. 5.36 that the Debye equation provides a good approximation of the volumetric mixing model overall but not in detail. Typically, the dielectric spectrum of Debye equation crosses over the dielectric spectrum of volumetric mixing equation. As a result, the time-domain waveform based on Debye equation is not as smoothed as the measured waveform of soils or the waveform simulated based on the volumetric mixing model. 
Table 5.5 The assumed values of volumetric mixing parameters

\begin{tabular}{l|c}
\hline \multicolumn{1}{c|}{ Volumetric Mixing Parameters } & Assumed value \\
\hline Soil physical parameters & 20 \\
volumetric water content $\theta, \%$ & 1.65 \\
dry density $\rho_{d}, \mathrm{~g} / \mathrm{cm}^{3}$ & 150 \\
effective specific surface $A_{e s}, \mathrm{~m}^{2} / \mathrm{g}$ & 2.65 \\
density of soil particle $\rho_{s}, \mathrm{~g} / \mathrm{cm}^{3}$ & \\
\hline Dielectric parameters of air & 1 \\
constant $\varepsilon$ & \\
\hline Dielectric parameters of soil particles & 4.7 \\
constant $\varepsilon$ & 80.1 \\
\hline Dielectric parameters of free water & 4.22 \\
static value $\varepsilon_{s}$ & 17.4 \\
high frequency value $\varepsilon_{\infty}$ & 0.0666 \\
Relaxation frequency $f_{r e l}, \mathrm{GHz}$ & \\
Conductivity $\sigma_{d c}$, S/m & 80.1 \\
\hline Dielectric parameters of bound water & 4.22 \\
static value $\varepsilon_{s}$ & 9 \\
high frequency value $\varepsilon_{\infty}$ & 5 \\
Relaxation frequency $f_{r e l}, \mathrm{kHz}$ & \\
Conductivity $\sigma_{d c}$, S/m & \\
\hline
\end{tabular}

Table 5.6 Estimation of Debye parameters and conductivity from the fabricated TDR waveform generated by volumetric mixing model

\begin{tabular}{l|cccc}
\hline Dielectric parameters & \multicolumn{1}{|l}{$\varepsilon_{s}$} & $\varepsilon_{\infty}$ & $f_{\text {rel }}, \mathrm{MHz}$ & $\sigma_{d c}, \mathrm{~S} / \mathrm{m}$ \\
\hline Prior mean & 40 & 15 & 50 & 0.5 \\
Prior Std. Dev. & 20 & 7.5 & 25 & 0.25 \\
Estimated & 32.96 & 10.98 & 32.7 & 0.0425 \\
Std. Dev. of Estimation & 0.05 & 0.01 & 0.13 & 0.000008 \\
\hline
\end{tabular}



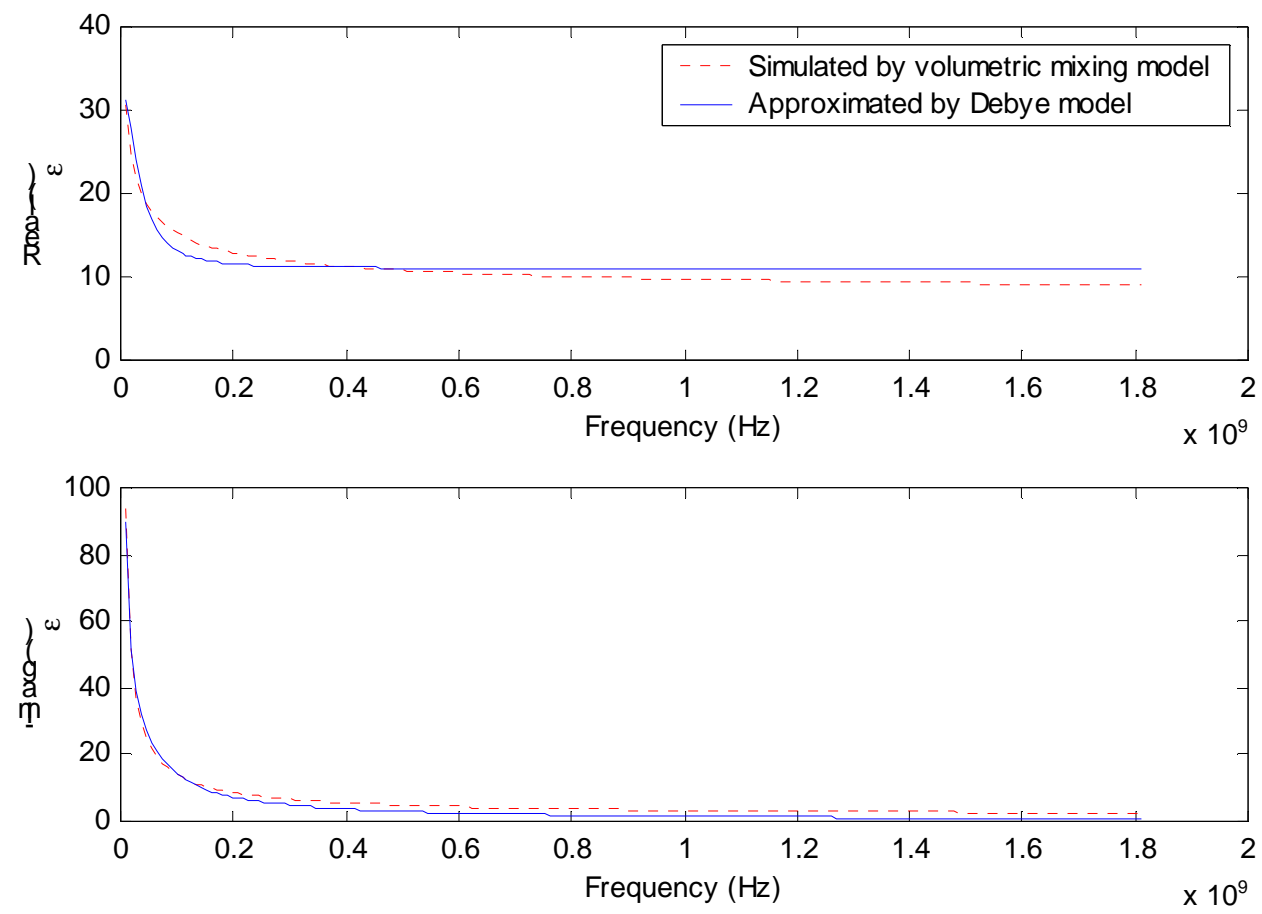

Fig. 5.36 Comparison of the dielectric spectra based on volumetric mixing equation and Debye equation

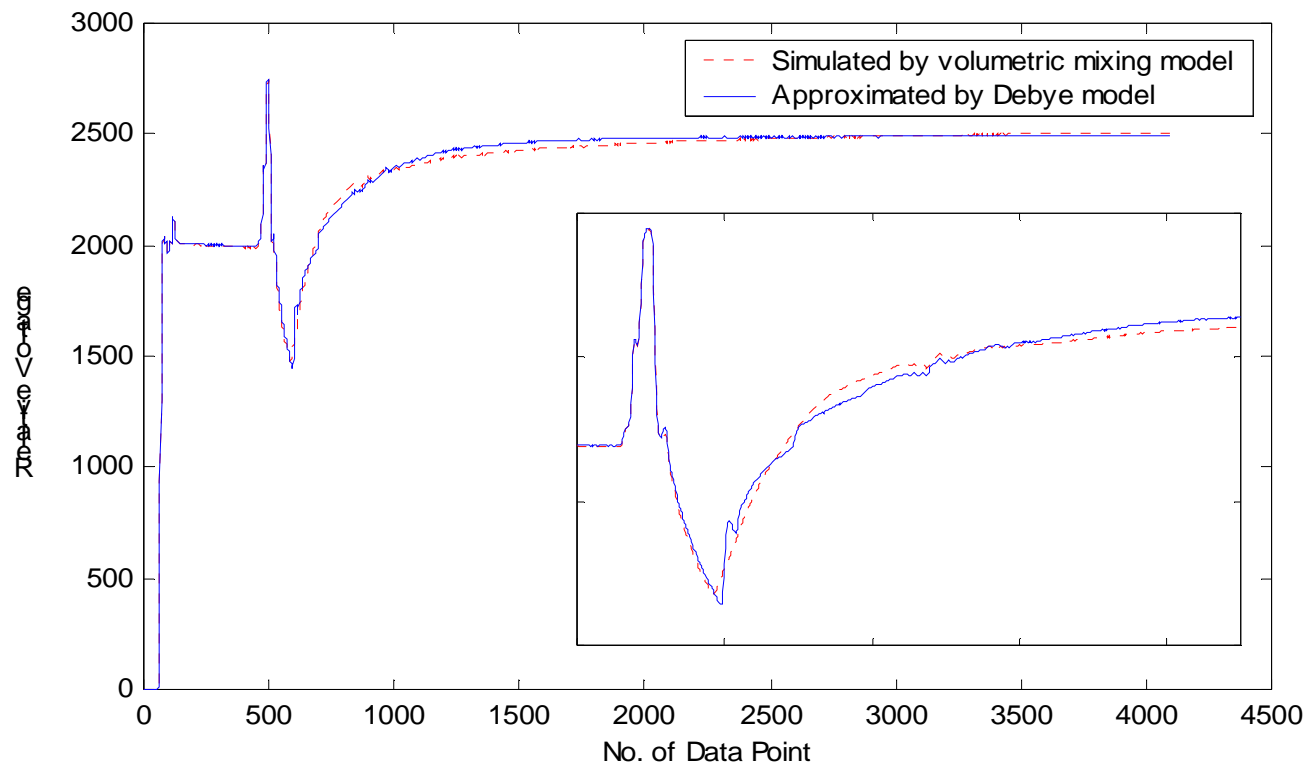

Fig. 5.37 Comparison of the time-domain waveforms based on volumetric mixing equation and Debye equation 


\subsection{Evaluation of Simplified Analysis}

With the ability to simulate TDR waveforms given the transmission line parameters of the probe and dielectric property of the material under test, the simplified analysis discussed in Section 3.2 can be evaluated.

\subsubsection{Analysis}

The simplified dc analysis has been evaluated by a parametric study in which the sample length was varied. Two materials were used for the parametric study. Dielectric parameters of butanol with conductivity equal to $0.01 \mathrm{~S} / \mathrm{m}$ represents the low-loss material while the same dielectric parameters with conductivity equal to $0.1 \mathrm{~S} / \mathrm{m}$ represents the high-loss material. The simplified dc analysis of Eqs. (3.5) and (3.6) was originally developed based on an ideal probe system. An ideal probe system consists of a non-dispersive coaxial cable and a probe without the probe head. Both the TDR probe system described in Section 5.1 and an ideal probe system were used to generate TDR waveforms for the parametric study.

The result of the parametric study for the low-loss material is shown in Fig. 5.38. The dc
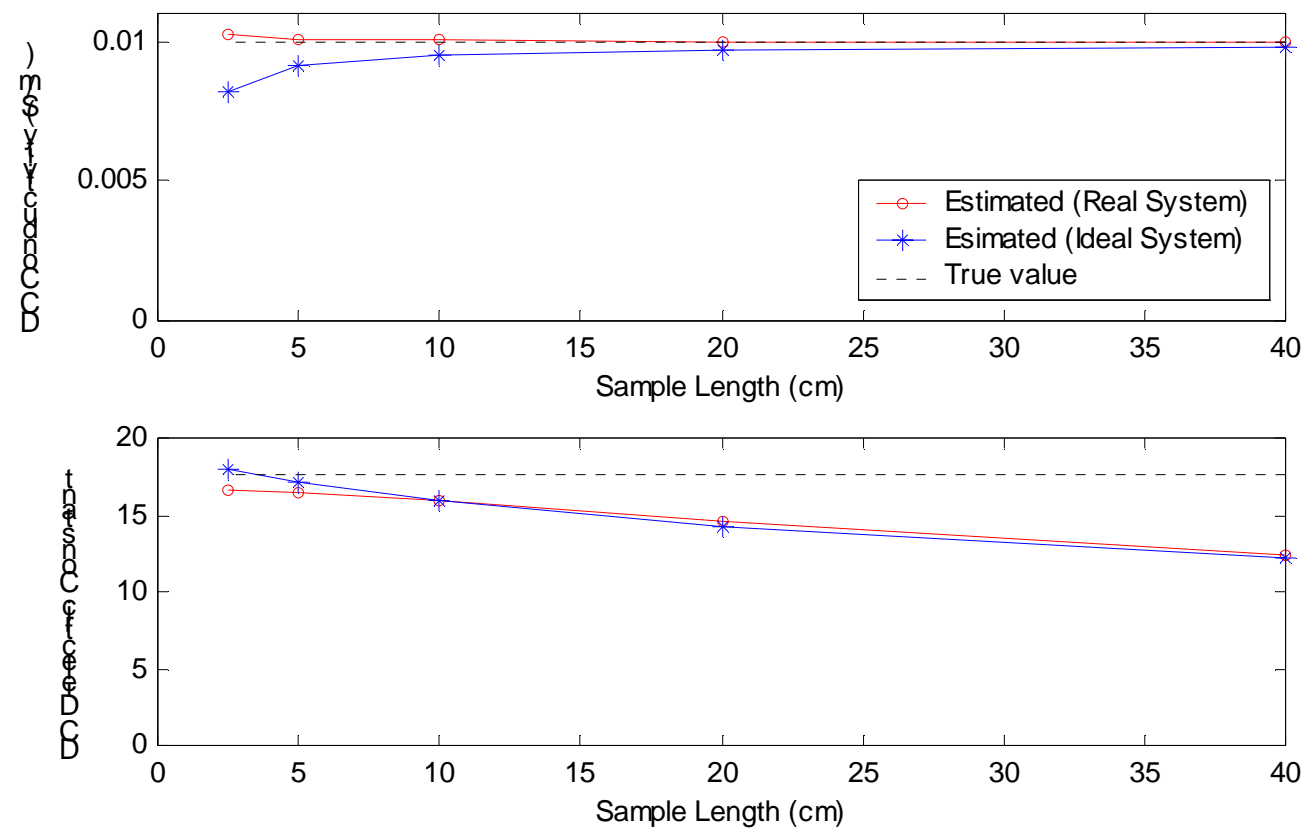

Fig. 5.38 Simplified dc analysis for a low-loss material 
conductivity and dielectric constant obtained from the waveforms in the real TDR probe system and ideal TDR probe system are compared to the true values. It is very interesting to observe that the estimated dc conductivity converges to the true value for long probes while the estimated dc dielectric constant converges to the true value for short probes. Furthermore, the measurement of conductivity is much less sensitive to the sample length than the measurement of dc dielectric constant. The estimation obtained from the real TDR probe system is more accurate in conductivity but slightly less accurate in de dielectric constant than that from the ideal TDR probe system. The result of the parametric study for the high-loss material is shown in Fig. 5.39. The simplified dc analysis still provides very good estimation for dc conductivity but the quality of estimation for dc dielectric constant decreases significantly as the loss of the material increases.
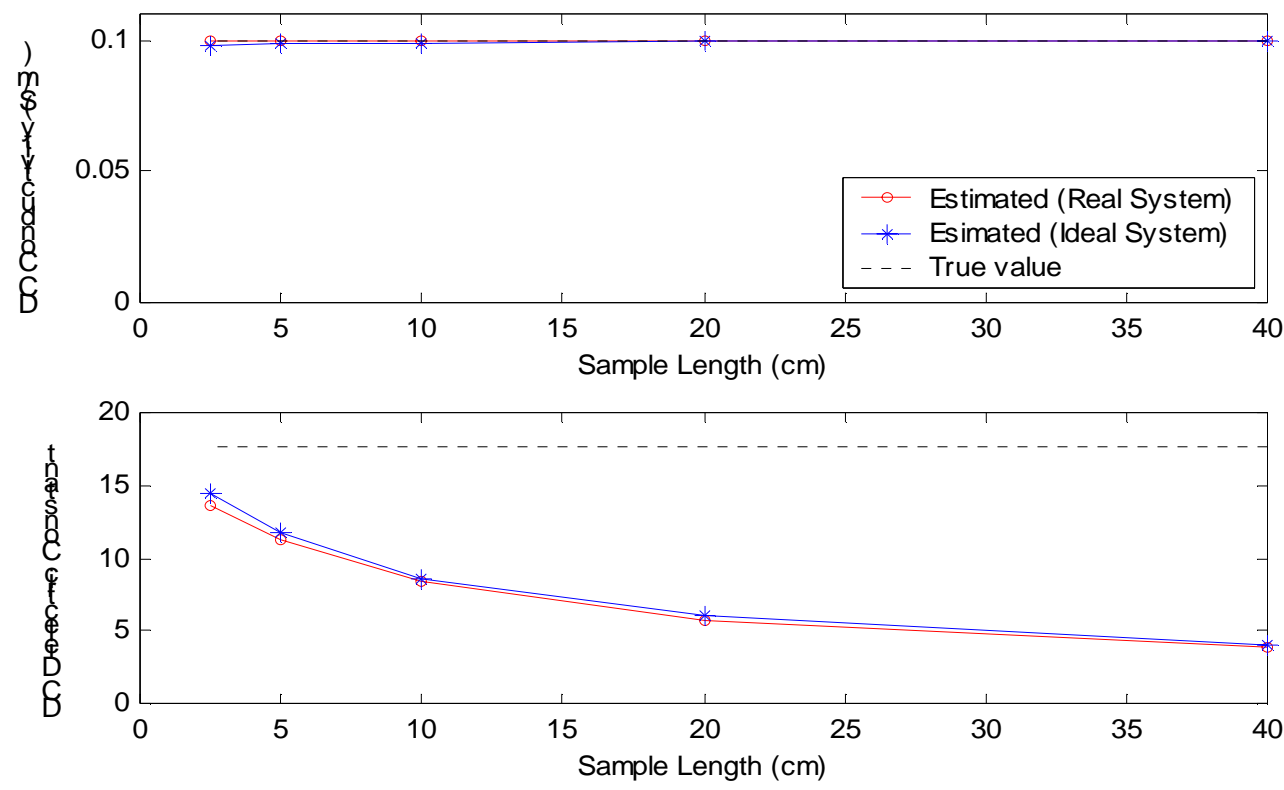

Fig. 5.39 Simplified dc analysis for a high-loss material 
Simplified dc analyses were carried out using real TDR waveforms in aqueous samples. The results are shown in Table 5.7. In all cases, the simplified dc analysis gives very good estimate for the conductivity. But it only gives reasonable estimate for de dielectric constant in lossless samples. Thus, the simplified analysis of dc conductivity by Eq. (3.5) is validated but caution has to be taken when using Eq. (3.6) to estimate the dc dielectric constant.

Table 5.7 The Simplified dc analysis of the aqueous samples

\begin{tabular}{l|c|c|c|c}
\hline \multirow{2}{*}{ Aqueous } & \multicolumn{2}{|c|}{ Expected Value } & \multicolumn{2}{c}{ Simplified DC analysis } \\
\cline { 2 - 5 } \multicolumn{1}{c}{ Sample } & $\mathcal{E}_{s}$ & $\sigma_{d c}$ & $\mathcal{E}_{s}$ & $\sigma_{d c}$ \\
\hline Butanol & 17.7 & 0 & 16.4 & 0.00015 \\
Ethanol & 25.2 & 0 & 23.8 & 0.00004 \\
Deionized Water & 79.9 & 0 & 75.3 & 0.00067 \\
Tap Water & 80.2 & 0.0666 & 38.3 & 0.06768 \\
\hline
\end{tabular}

\subsubsection{Travel Time Analysis}

It has been discussed that the wave propagation in a TDR probe system is a dispersion phenomenon in which each sinusoidal wave component of the step pulse travels at different speed. As a result, we cannot define a velocity of propagation of the wave shape as a whole. The apparent velocity used to calculate the apparent dielectric constant is obtained by the travel-time approximation described in Section 3.2.2. The physical meaning of apparent dielectric constant and its relationship with the dielectric spectrum are studied in the following by the wave propagation and the dielectric-mixing model.

Consider a frequency component of the incident wave $V_{0} e^{j 2 \pi f t}$ travels in a transmission line. At distance $z$ from the incidence it becomes,

$$
V(z)=V_{0} e^{j 2 \pi f t} e^{-\gamma z}=V_{0} e^{-\alpha z} e^{j(2 \pi f t-\beta z)}
$$

in which $\gamma=\alpha+\mathrm{j} \beta$ is the propagation constant defined in Eq. (3.47). The first exponential term in Eq. (5.11) represents the attenuation of the signal and the second exponential term represents 
the phase shift. The loss factor for the wave to travel into the probe and back is $e^{-2 \alpha L}$, where $L$ is the length of the probe. The phase velocity of each frequency component is defined by $v(f)$ $=2 \pi f / \beta$. It can be written as a function of the dielectric permittivity by substituting Eq. (3.47) into the definition.

$$
v(f)=\frac{c}{\operatorname{Re}\left(\sqrt{\varepsilon_{r}{ }^{*}(f)}\right)}=\frac{c}{\sqrt{\frac{\varepsilon_{r}{ }^{\prime}(f)}{2}\left(1+\sqrt{1+\left(\frac{\varepsilon_{r}{ }^{i i}(f)}{\varepsilon_{r}{ }^{\prime}(f)}\right)^{2}}\right)}}
$$

where $\varepsilon_{r}{ }^{\prime}$ and $\varepsilon_{r}{ }^{\prime \prime}$ are the real and imaginary part of the equivalent dielectric permittivity $\varepsilon_{r}{ }^{*}$, respectively.

The denominator in Eq. (5.12) can be defined as the apparent dielectric constant of each frequency component, $\varepsilon_{a}(f)$.

$$
\varepsilon_{a}(f)=\frac{\varepsilon_{r}{ }^{\prime}(f)}{2}\left(1+\sqrt{1+\left(\frac{\varepsilon_{r}{ }^{i i}(f)}{\varepsilon_{r}{ }^{\prime}(f)}\right)^{2}}\right)
$$

Note that the apparent dielectric constant of each frequency component is associated with the corresponding phase velocity and is different from the apparent dielectric constant obtained by the travel-time analysis. Consider a dielectric simulated by the volumetric mixing equation with the parameters listed in Table 5.5. The loss factor $e^{-2 \alpha L}$ and the phase velocity are shown in Fig. 5.40. The wave components with higher frequencies travel faster but also have a higher loss factor. The TDR incident pulse has a broadband spectrum as shown in Fig. 5.4. The loss factor of the transmission line acts like a low-pass filter. The high frequency components within the passed frequency band would travel faster to reach the TDR sampler and be detected as the second reflection point in the travel-time analysis. 

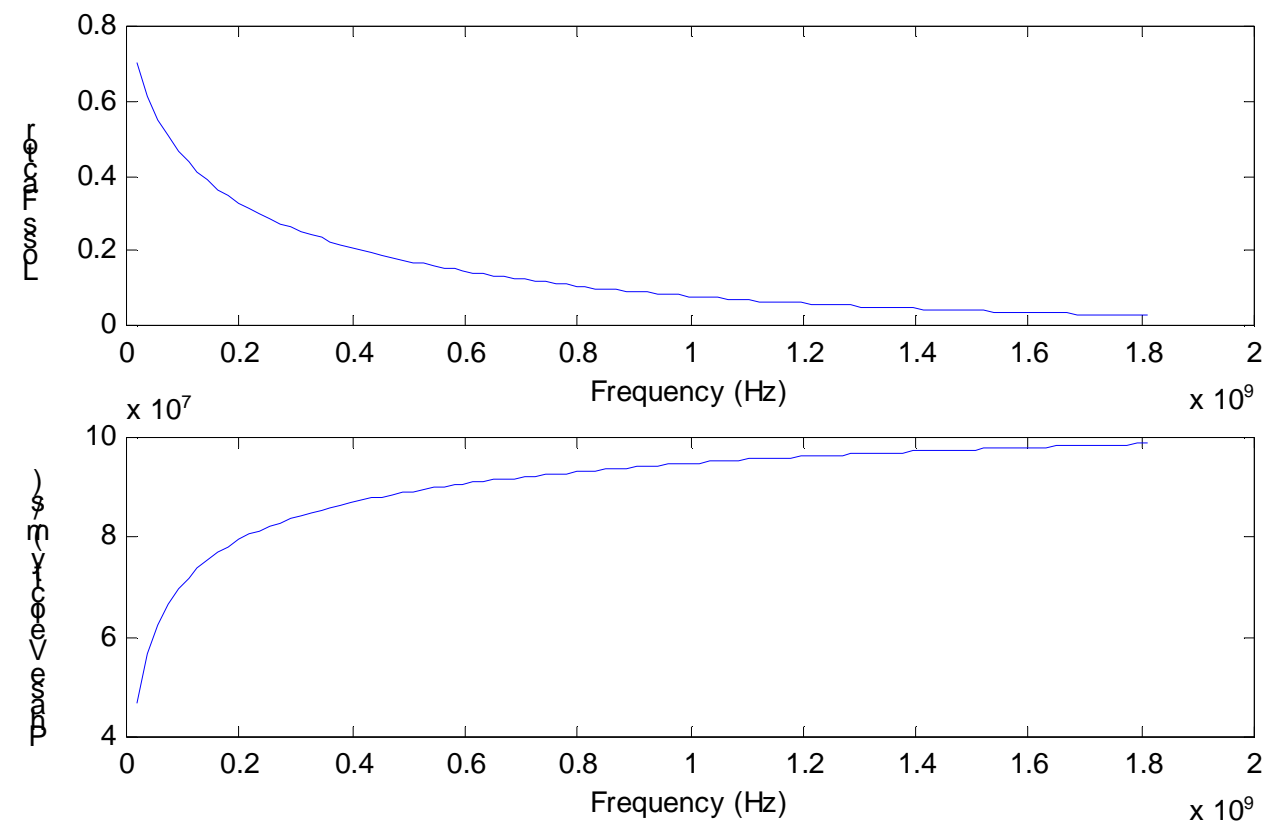

Fig. 5.40 The loss factor and phase velocity of a typical soil dielectric

The apparent dielectric constant of the dielectric was determined from the TDR waveform simulated by the wave propagation model. It is compared with real part of dielectric spectrum, $\operatorname{Re}\left[\varepsilon^{*}(f)\right]$, and the apparent dielectric spectrum, $\varepsilon_{a}(f)$, as shown in Fig. 5.41. Indeed, the traveltime analysis measured the high-frequency part of the apparent dielectric spectrum in the pass band. At high frequencies, the apparent dielectric spectrum is very close to the real part of dielectric spectrum because the imaginary part of dielectric spectrum is much smaller than the real part at high frequencies as shown in Fig. 5.36. Therefore, the apparent dielectric constant is also a measure of the real part of dielectric spectrum at high frequencies within the passing band. The association of the apparent dielectric constant with the dielectric spectrum at high frequencies where soil interface polarization is not significant may have explained its success to estimate volumetric water content independent of soil types.

\subsection{Summary}

A TDR probe system was designed to measure the dielectric properties of soils in a compaction mold and in the field. The error and bandwidth of the TDR system were quantified. The wave propagation model and inverse theory developed in preceding chapters were used to 


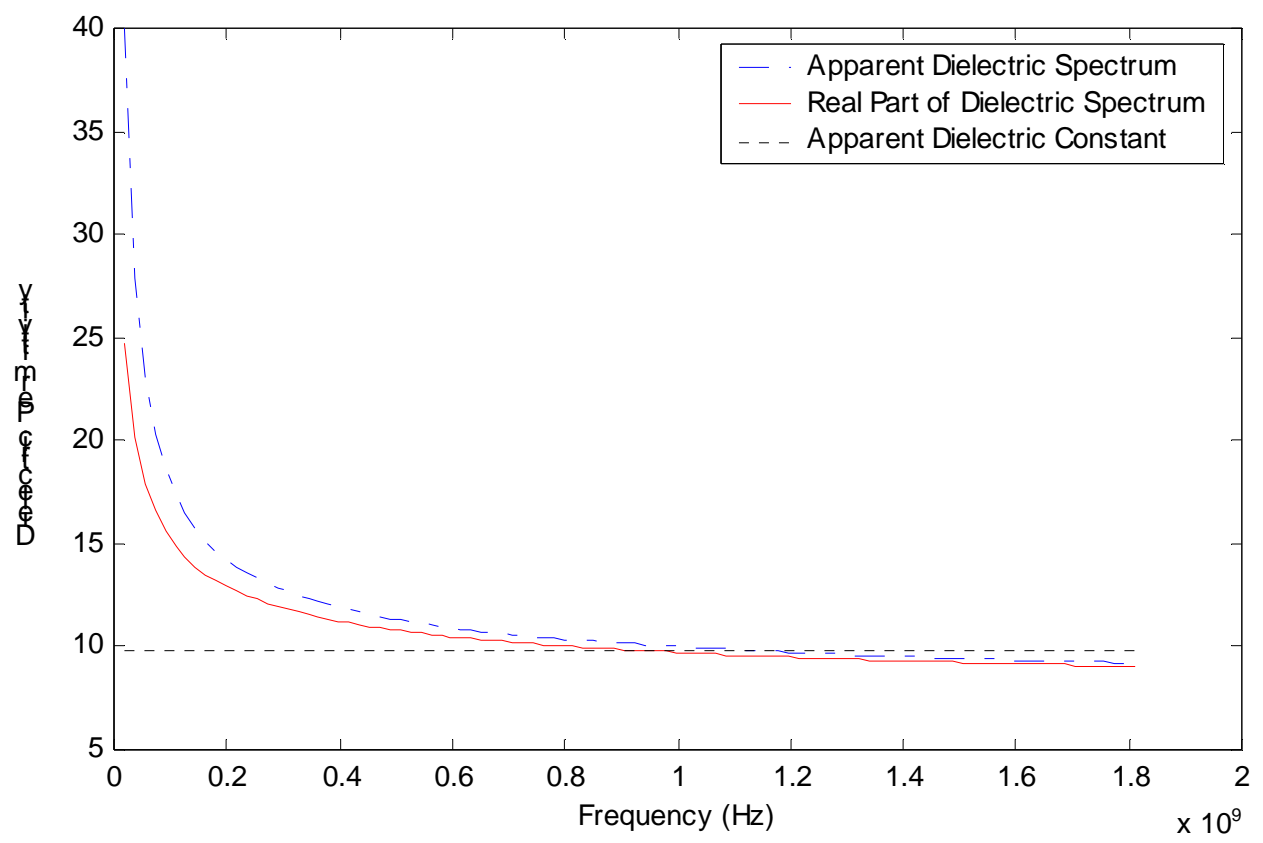

Fig. 5.41 Comparison of apparent dielectric constant with the apparent dielectric spectrum and real part of dielectric

calibrate the TDR probe system. A layer-peeling algorithm was developed to preprocess the TDR waveform to remove the multiple reflections in the waveform due to the probe head and resulted in a waveform of an ideal probe system. Comparison of the measured scattering function with the theoretical scattering function revealed some evidence of higher mode contributions in addition to the TEM mode.

The dielectric spectrum of the material being tested can be estimated either by solving the scattering function for each frequency or by inverting the parameters in an equation describing the dielectric spectrum, such as the Debye equation. It was shown that the accuracy of the measurement by solving the scattering function for each frequency is limited by the data error at higher frequencies and by the non-linearity of the scattering function. Inversion of the dielectric model parameters is a better approach to measure the dielectric spectrum of the material being tested. The Debye equation is an excellent dielectric model for liquids. It also provides an approximate description of the dielectric spectra for soils.

The simplified waveform analyses were evaluated by the wave propagation model. The simplified analysis for the dc conductivity was validated, but it was shown that caution needs to 
be taken when using the simplified analysis for the dc dielectric constant. The apparent dielectric constant obtained by travel-time analysis was found to be associated with the real part of dielectric spectrum at high frequencies. 


\section{CHAPTER 6 EXPERIMENTAL STUDY OF SOIL PROPERTIES}

\subsection{Introduction}

Aqueous materials were used to validate the wave propagation model and develop the methodology to measure dielectric permittivities of materials in Chapter 5. In this chapter, a series of tests are conducted on soils to study the soil properties using the framework developed in the preceding chapters. Both the simplified analysis and full waveform analysis are used to analyze the data.

\subsection{Measurements of TDR Waveforms on Soils}

\subsubsection{Materials and Methods}

Five artificial soils were mixed from three soils, Ottawa sand, natural silt, and Illite to represent a wide range of soil types. The percentage of mixing is listed in Table 6.1. The selection of mixing method was based on the common range of soil types, soil availability, and the objective of studying the dielectric properties of soils with gradually changing soil components. Lab testing on soil properties was performed on each soil, including particle size analysis, specific gravity, and the liquid and plastic limit. The results are shown in Table 6.2.

For each soil, five different water contents were used to prepare samples in five large containers. Soils were oven dried before adding water. The five different water contents were determined by small sample tests to cover the dry side and the wet side of the optimum water content in a compaction test. The water content can be as high as $25 \%$ before the soil becomes non-compactable for clayey soils, while the highest water content that can be retained before the soil gets too soft is around $10 \%$ for sandy soils.

For each water content, water was gradually added to the soil and mixed thoroughly in a large container. The mixed soil was sealed by plastic wraps and allowed to equilibrate for more than 20 hours to achieve a uniform soil specimen. After the equilibration, the soil was mixed again before being compacted into four compaction molds using four different compaction energies. These four compaction methods were selected to obtain four different soil densities for the same gravimetric water content. The first method used a metallic rod to tamp the soil into the 
mold in three layers. The rod was controlled manually to have 25 blows of roughly uniform energy. The second method is called the reduced compaction method, which follows the standard compaction method (ASTM D698) except 15 blows per layer was used instead of 25 blows per layer. The third method used the standard compaction method (ASTM D698) and the last method used the modified compaction method (ASTM 1557). After the soil was compacted into the mold and the surface is trimmed to be even, the weight of the soil and mold were taken. Then a central rod was installed and the 2048-point TDR measurement as describe in Section 5.2 was taken. After the TDR measurement, the soil was oven-dried to obtain the water content.

Table 6.1 Percentage of three components of mixed soils

\begin{tabular}{c|ccc}
\hline $\begin{array}{c}\text { Soil } \\
\text { Name }\end{array}$ & $\begin{array}{c}\text { Percentage of Sand } \\
\text { (Ottawa Sand) }\end{array}$ & $\begin{array}{c}\text { Percentage of Silt } \\
\text { (Natural Silt) }\end{array}$ & $\begin{array}{c}\text { Percentage of Clay } \\
\text { (Illite) }\end{array}$ \\
\hline M1 & $55 \%$ & $35 \%$ & $10 \%$ \\
M2 & $37.5 \%$ & $40 \%$ & $17.5 \%$ \\
M3 & $20 \%$ & $55 \%$ & $25 \%$ \\
M4 & $12.5 \%$ & $47.5 \%$ & $40 \%$ \\
M5 & $5 \%$ & $40 \%$ & $55 \%$ \\
\hline
\end{tabular}

Table 6.2 Engineering characteristics of the mixed soils

\begin{tabular}{c|ccccc}
\hline $\begin{array}{c}\text { Soil } \\
\text { Name }\end{array}$ & $\begin{array}{c}\text { Soil } \\
\text { Classification }\end{array}$ & $\begin{array}{c}\text { Specific } \\
\text { Gravity }\left(G_{s}\right)\end{array}$ & $\begin{array}{c}\mathrm{P}<\# 200 \\
(\%)\end{array}$ & $\begin{array}{c}\text { Liquid } \\
\text { Limit }\end{array}$ & $\begin{array}{c}\text { Plastic } \\
\text { Limit }\end{array}$ \\
\hline M1 & SM-SC & 2.76 & 41.3 & $/$ & $/$ \\
M2 & ML & 2.77 & 52.4 & 16.2 & 5.7 \\
M3 & CL & 2.83 & 72.9 & 28.5 & 16.2 \\
M4 & CL & 2.83 & 78.6 & 33.7 & 14.8 \\
M5 & CL & 2.82 & 84.4 & 41.0 & 21.1 \\
\hline
\end{tabular}

\subsubsection{Results and Discussion}

There were a total of 100 specimens and they were labeled by their soil type, water content, and compaction effort. For example, M3-2-4 denotes the soil specimen with soil type M3 at the second intended water content and compacted by the fourth compaction method. 
In general, the measured TDR waveforms change systematically with change of water content, density, and soil type. However, there are some abnormal waveforms due to poor contact between the coaxial head $(\mathrm{CH})$ and the metallic ring or the central rod. This problem did not occur when measuring the liquids in the previous chapter because a good contact must be established for the central rod to stay in place in the liquids. Specimen M3-1-4 in Fig. 6.1 and M5-5-2 in Fig. 6.2 are typical examples of abnormal waveforms. This operation error does not affect the travel time analysis much but causes disturbances in the overall shape and magnitude of the waveform. Suspicious waveforms were removed from the data in the subsequent discussion and analysis.

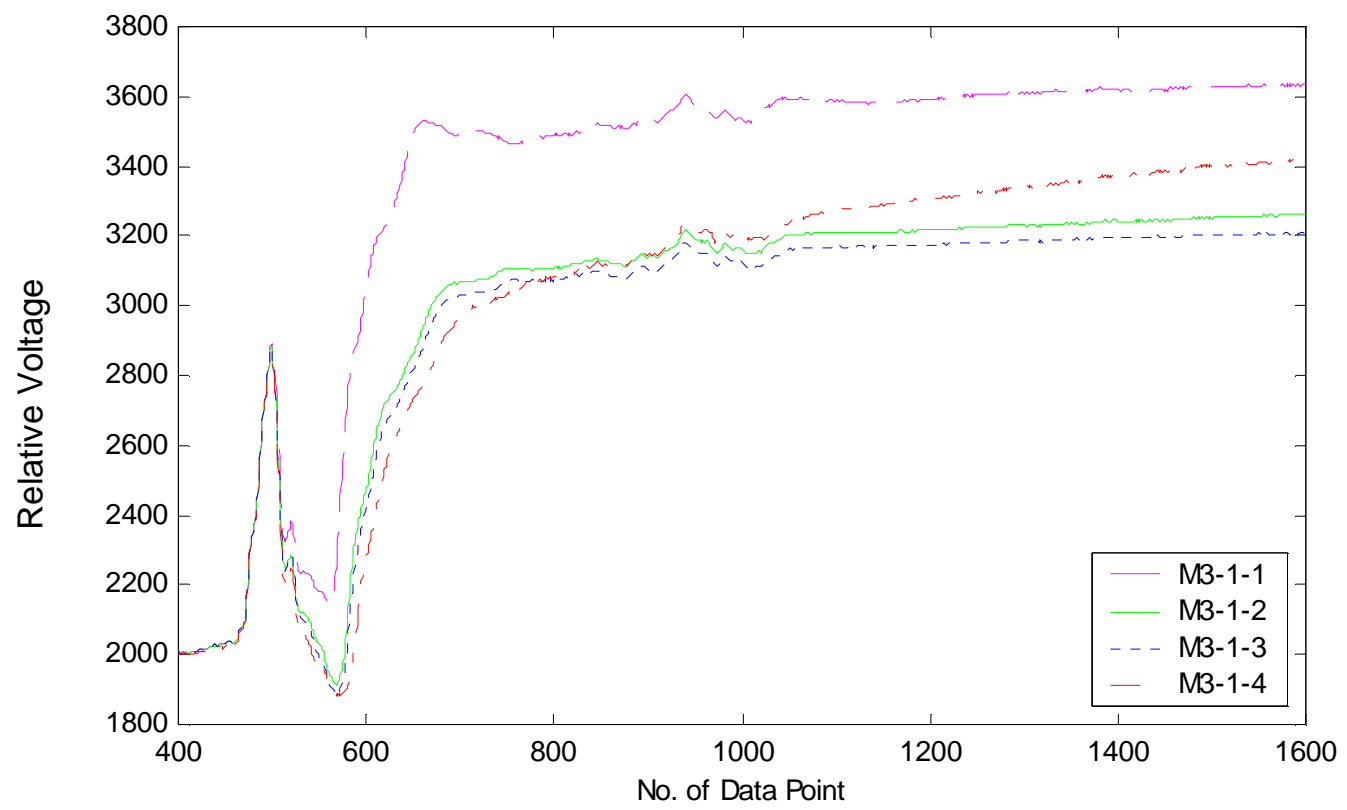

Fig. 6.1 Example of an abnormal waveform in M3-1

Figure 6.3 shows the general trend of TDR waveforms for soils with the same gravimetric water content but different densities (i.e soils prepared with the same water content but compacted by different compaction energy). Both the travel time and the signal attenuation increase as the density increases. As a result, the end reflection points of TDR waveforms move along the dashed line shown in Fig. 6.3. As the water content increases, the group of waveforms and the dashed line shift to the right. Therefore, TDR waveforms contain information on both soil water content and density. 


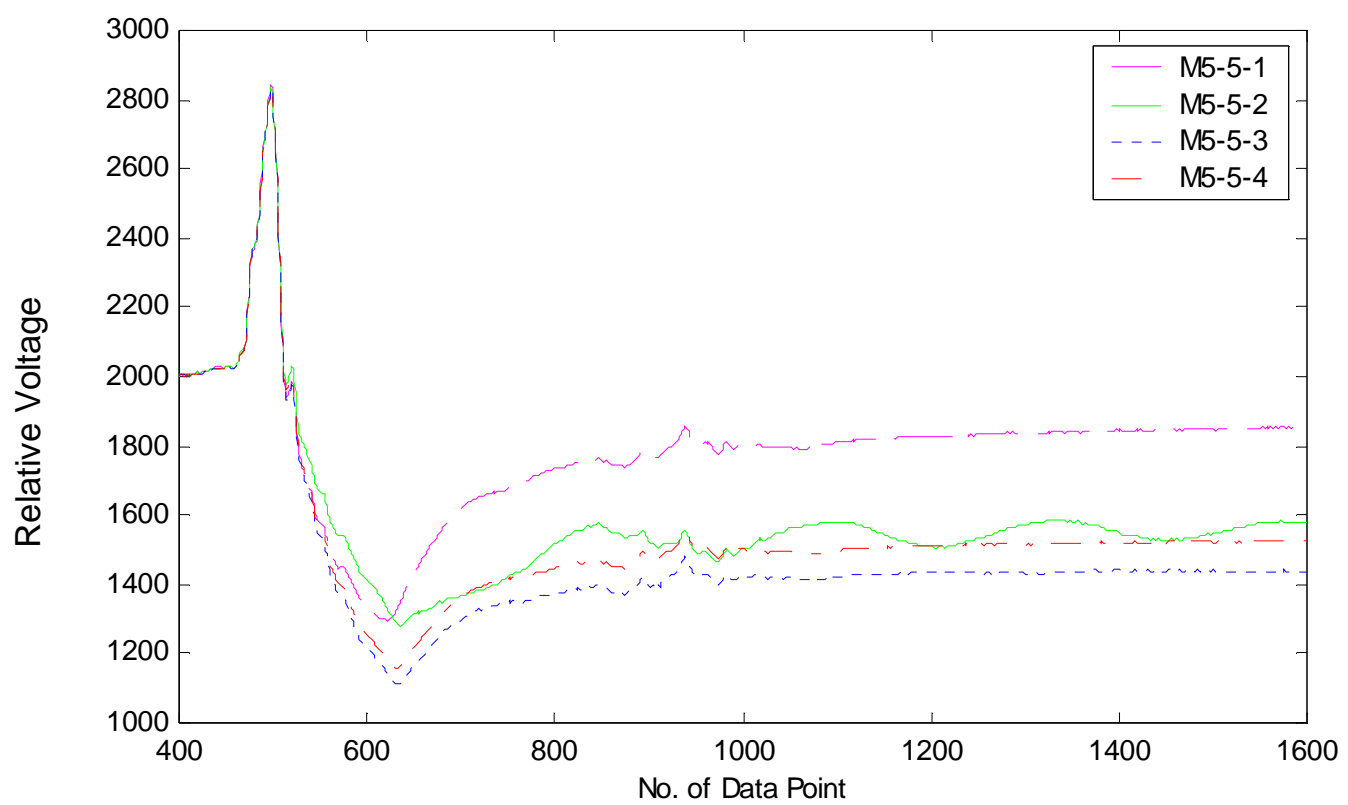

Fig. 6.2 Example of an abnormal waveform in M5-5

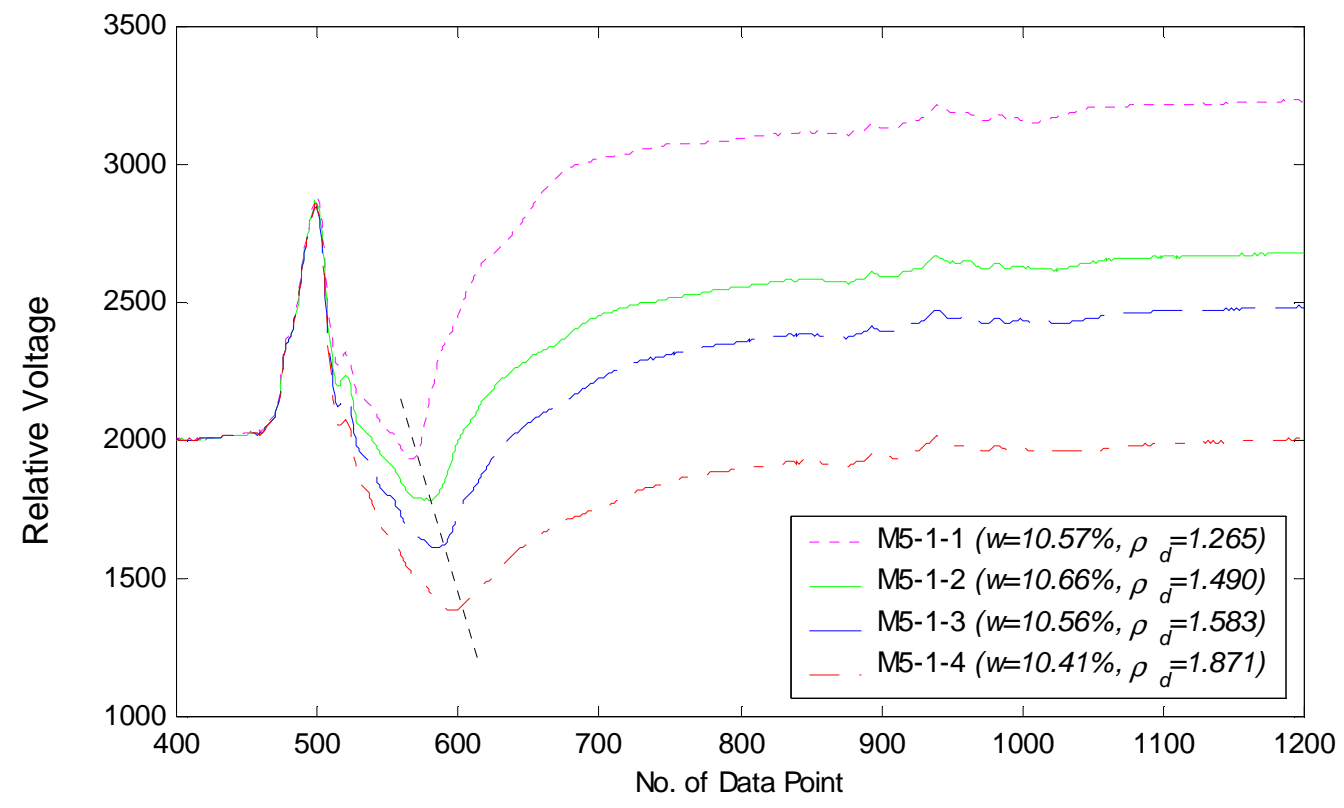

Fig. 6.3 Effect of soil density on TDR waveforms $(w=$ constant $)$ 
As discussed in Chapter 2, the volumetric water content is more appropriate than gravimetric water content when interpreting the change of dielectric permittivity in a soil. The gravimetric water content alone does not represent the volumetric proportion of water in a soil specimen (i.e. $\theta=w \rho_{d} / \rho_{w}$ ). For the same gravimetric water content, the volumetric water content increases as density increases. Therefore, it should be more appropriate to observe the density effect on TDR waveforms when the volumetric water content is kept constant. However, it is not an easy task to prepare soil specimens with the same volumetric water content but different densities. Two specimens in the M2 soil were found with different densities but very close volumetric water content. Their TDR waveforms are shown in Fig. 6.4. It can be observed from this limited amount of data that density affects signal magnitude at long times even though the volumetric water content remains the same.

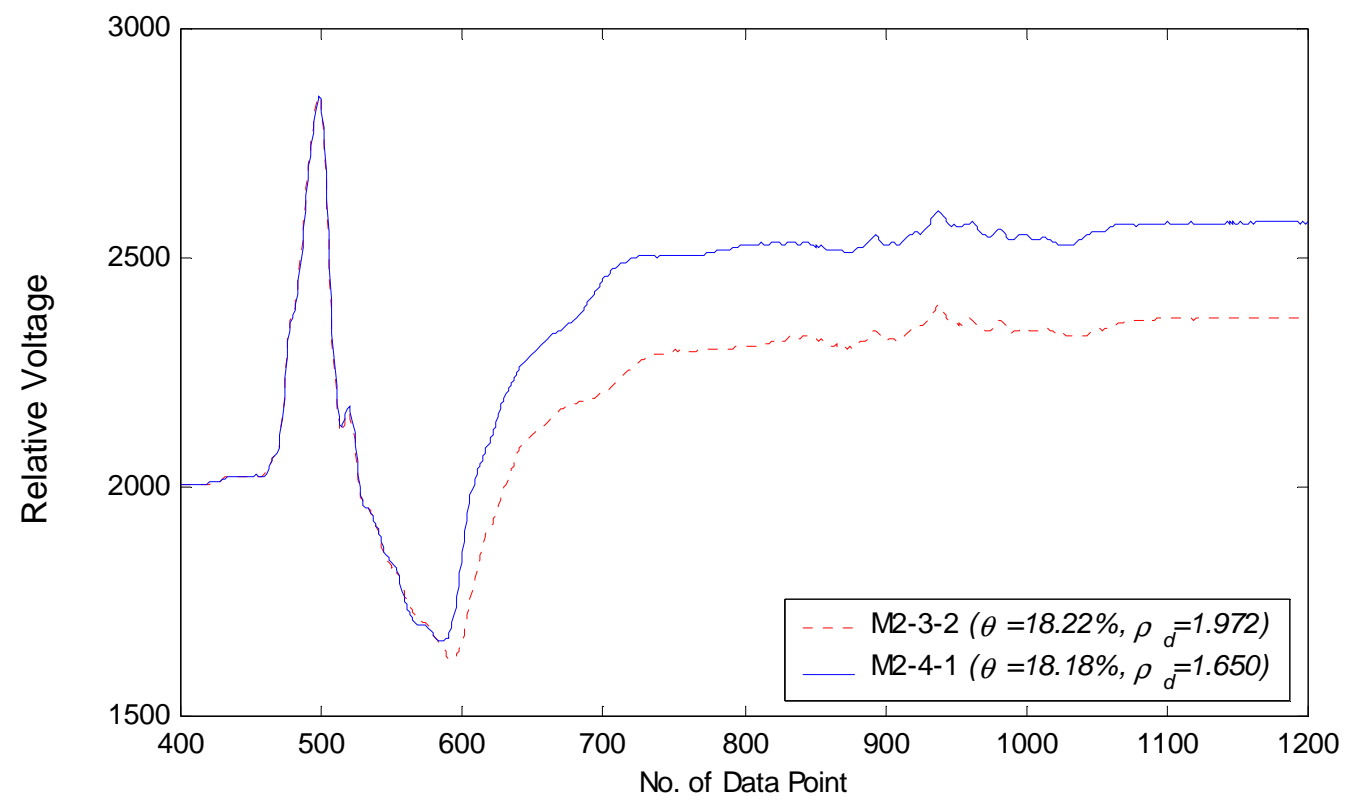

Fig. 6.4 Effect of soil density on TDR waveforms $(\theta=$ constant $)$

The effect of soil type on TDR waveforms may be observed by choosing a specimen from each soil type that had similar volumetric water content and density. This is also not an easy task because of the large increment of intended water content and compaction energy. Nevertheless, specimens in four soil types were found with a similar volumetric water content and density. 
Their TDR waveforms are shown in Fig. 6.5. Qualitatively, the effect of soil type on TDR waveforms is similar to the effect of soil density. More in-depth analyses using the framework developed in the preceding chapters are presented in the subsequent sections.

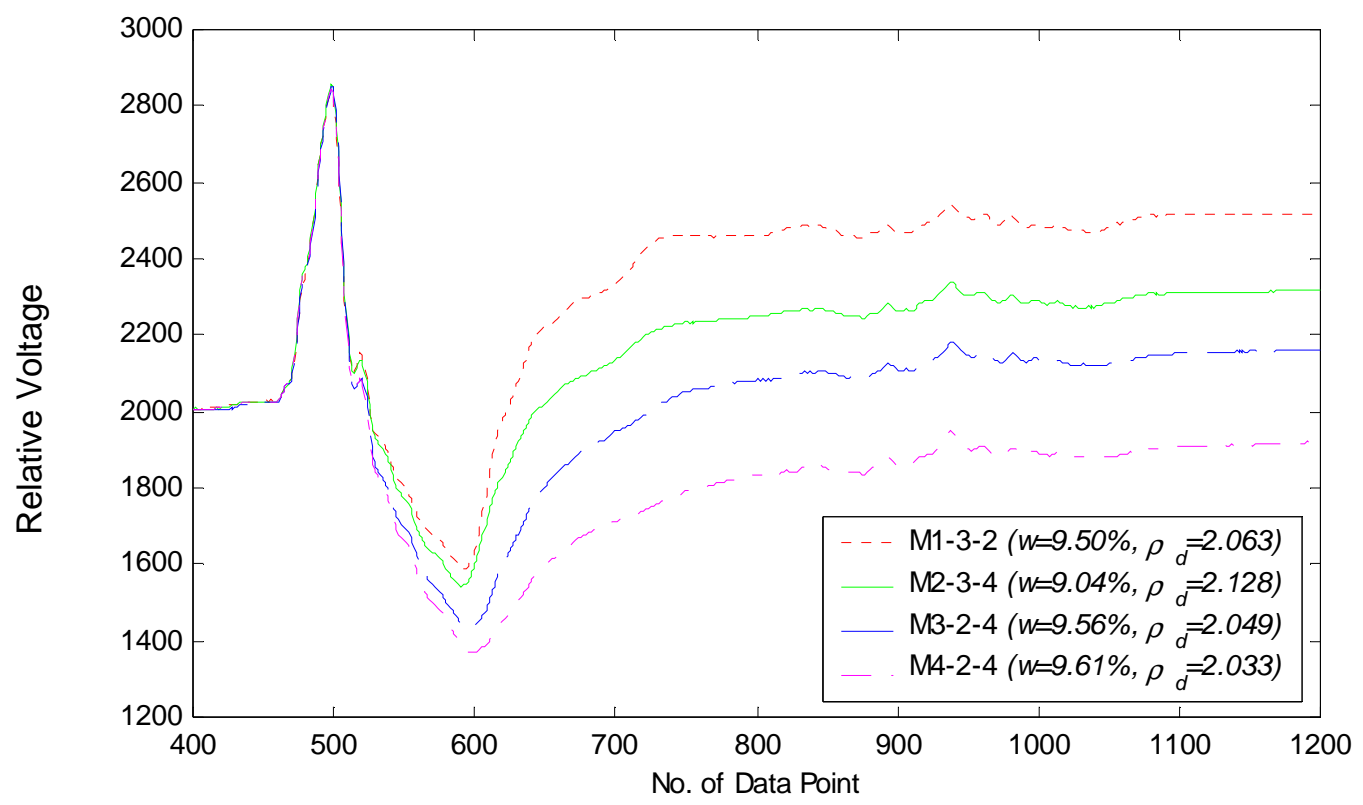

Fig. 6.5 Effect of soil type on TDR waveforms

\subsection{Soil Properties by Simplified Analysis}

\subsubsection{Apparent Dielectric Constant}

It is possible to measure the amount of water in a soil by determining the soil dielectric permittivity since the dielectric permittivity of water is much higher than that of the soil particles. Most previous investigations have measured the soil dielectric permittivity with an impedance bridge in low radio frequency range (see Babb, 1951, and Selig and Mansukhani, 1975). At these frequencies, the dielectric permittivity of soil strongly depends on the soil type and the microstructure due to the interface effects as discussed in Chapter 2. This is one of the major reasons that early work provided only qualitative interpretations. 
More recent studies have focused on using a TDR device to measure the apparent dielectric constant and relate it to soil moisture. TDR has become a popular tool for measuring the volumetric water content in agricultural engineering since Topp et al. (1980) published their empirical equation between apparent dielectric constant and volumetric water content. The results of Topp et al. (1980) showed that the apparent dielectric constant is strongly dependent on the volumetric water content and relatively independent of soil density, texture, and salt content. The empirical equation, known as Topp's equation, was given as

$$
\theta=a+b K_{a}+c K_{a}{ }^{2}+d K_{a}{ }^{3}
$$

where $a=-0.053, b=2.92 \times 10^{-2}, c=-5.5 \times 10^{-4}$, and $d=4.3 \times 10^{-6}$. As discussed in Section 5.6.2, the success of the TDR method compared to the early works may be attributed to the fact that apparent dielectric constant is associated with the real part of dielectric permittivity at high frequencies in which the interface effects are not important. The empirical Topp's equation is sometimes called the "universal calibration equation". The equation was established from the measurements conducted on loose soils that only had 9\% change in dry density ( $\rho_{d}=1.32 \sim 1.44$ $\mathrm{g} / \mathrm{cm}^{3}$ ). However, the densities of soil in geotechnical engineering applications typically have a greater range. Use of this equation for geotechnical engineering applications needs to be verified.

The TDR waveforms presented in the previous section were analyzed by travel time analysis to obtain the apparent dielectric constant. The measured volumetric water contents (i.e. determined from oven-dry gravimetric water content and density) are plotted, along with Topp's equation, against the apparent dielectric constant as shown in Fig. 6.6. It is very obvious that Topp's equation overestimates the volumetric water content for the soils tested. The errors are plotted against the dry densities of the soil tested as shown in Fig. 6.7. Errors are minimal when densities are in the range from 1.3 to $1.5 \mathrm{~g} / \mathrm{cm}^{3}$, the same range in which the Topp's equation was obtained. Therefore, Topp's equation is not really "universal." Calibration is required for geotechnical engineering applications. A new third order polynomial was fitted for the soil tested 
and shown in Fig. 6.6. While this gives an improved relationship for volumetric water content, other approaches, presented subsequently, may be more meaningful for geotechnical use.

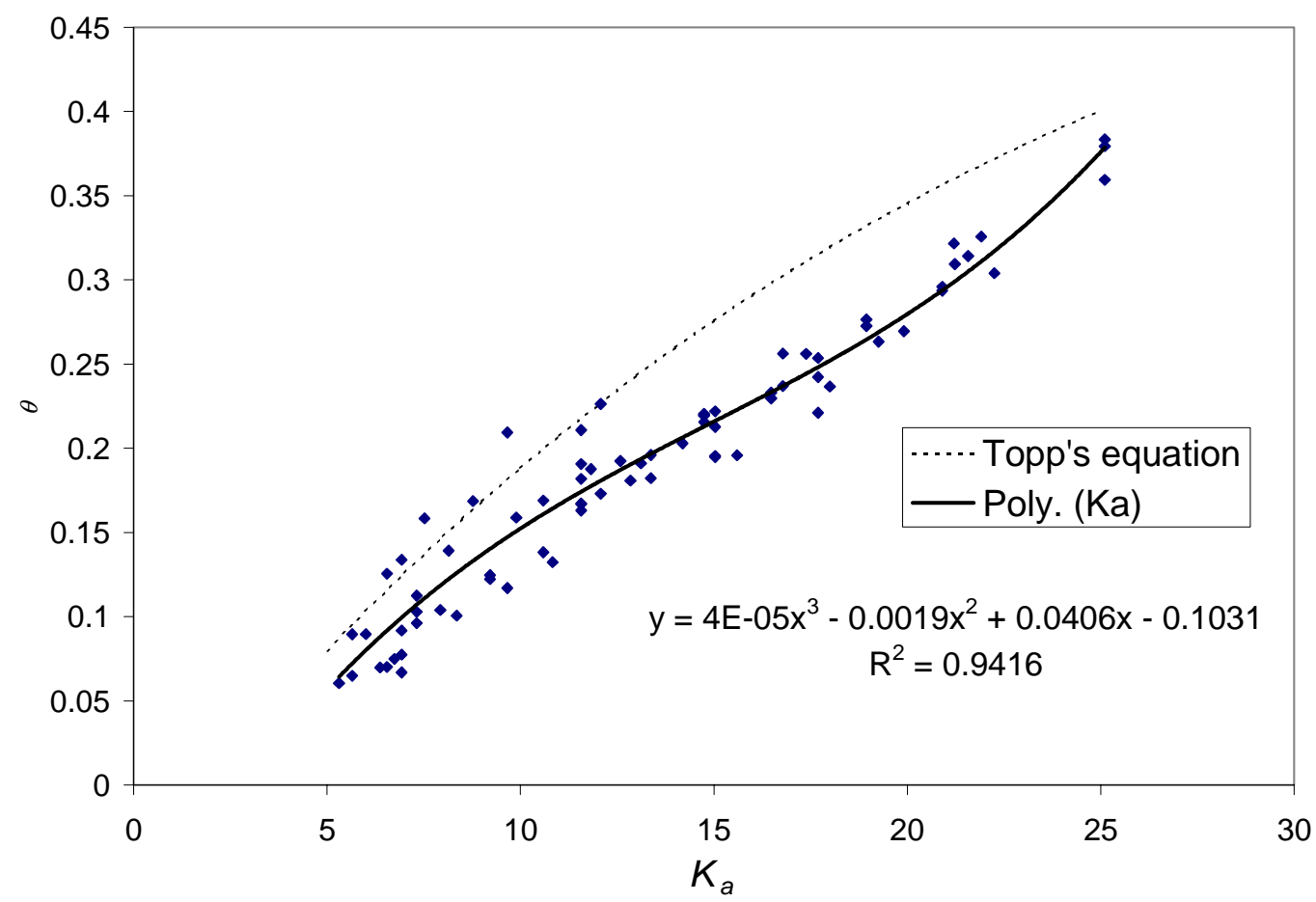

Fig. 6.6 Evaluation of Topp's equation

As an alternative to polynomial fitting, a linear calibration equation was proposed by Ledieu et al. (1986) as

$$
\sqrt{K_{a}}=a+b \theta
$$

Equation (6.2) is a second-order polynomial if expressed in terms of $K_{a}$. Topp's equation (thirdorder polynomial) is essentially the same as Eq. (6.2) in the normal range of water content $(0.05<$ $\theta<0.5)$, with $a=1.56$ and $b=8.47$. The regression analysis for the soil tested using Eq. (6.2) is shown in Fig. 6.7. The coefficient of determination using Eq. (6.2) is slightly less than using Eq. 


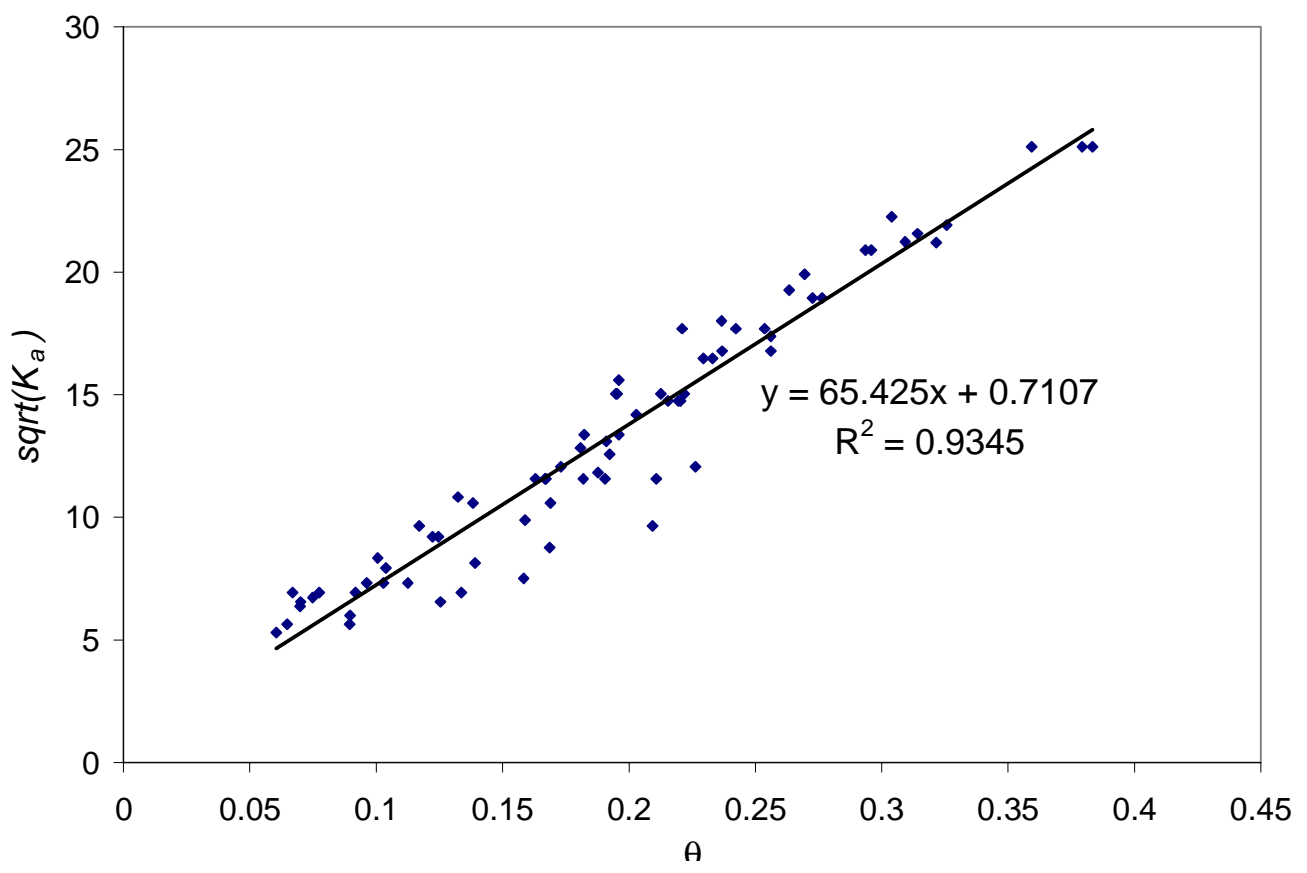

Fig. $6.7 \sqrt{ } K_{a}$ vs. volumetric water content $(\theta)$ relationship

(6.1) because it neglects the third order term. However, Eq. (6.2) is expressed as a linear relationship and much easier to use.

It can be observed from Fig. 6.8 that there seems to be a density effect on the $K_{a}$ vs. $\theta$ relationship.

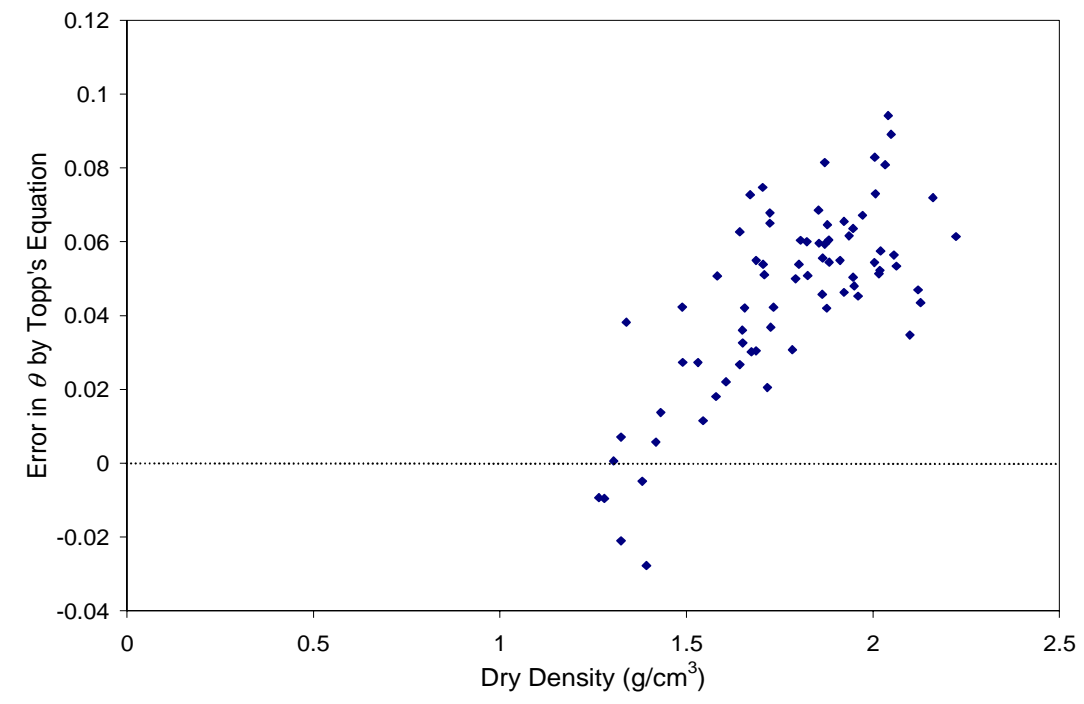

Fig. 6.8 Error in volumetric water content measured by Topp's equation versus dry density 
Ledieu et al. (1986) also reported that the calibration is improved for the soil they tested if bulk dry density is included as

$$
\sqrt{K_{a}}=a \rho_{d}+b \theta+c
$$

where $a, b$, and $c$ were calibration constants obtained by regression analysis. In geotechnical engineering, water content is usually measured in terms of gravimetric water content. It is related to the volumetric water content through the dry density of the soil (i.e. $w=\theta \rho_{w} / \rho_{d}$, where $\rho_{w}$ is the density of water). If expressed in terms of gravimetric water content, Equation (6.3) can be rewritten more conveniently as

$$
\frac{\left(\sqrt{K_{a}}-c\right) \rho_{w}}{\rho_{d}}=a+b w
$$

Regression analysis using Eq. (6.4) was carried out and shown in Fig. 6.9. The constant $c=0.5$ is obtained for the best fit. Siddiqui and Drnevich (1995) tried to normalize the density effect and obtained an equation equivalent to Eq. 6.4 with $c=0$. They expressed the equation in terms of gravimetric water content as

$$
\frac{\sqrt{K_{a}} \rho_{w}}{\rho_{d}}=a+b w
$$

The regression analysis using Eq. (6.5) is shown in Fig. 6.10. The coefficient of determination using Eq. (6.5) is slightly less than that using Eq. (6.4) because the " $c$ term" is neglected, but it is easier to use. It was observed that soil type seems to have an effect on the relationship described 


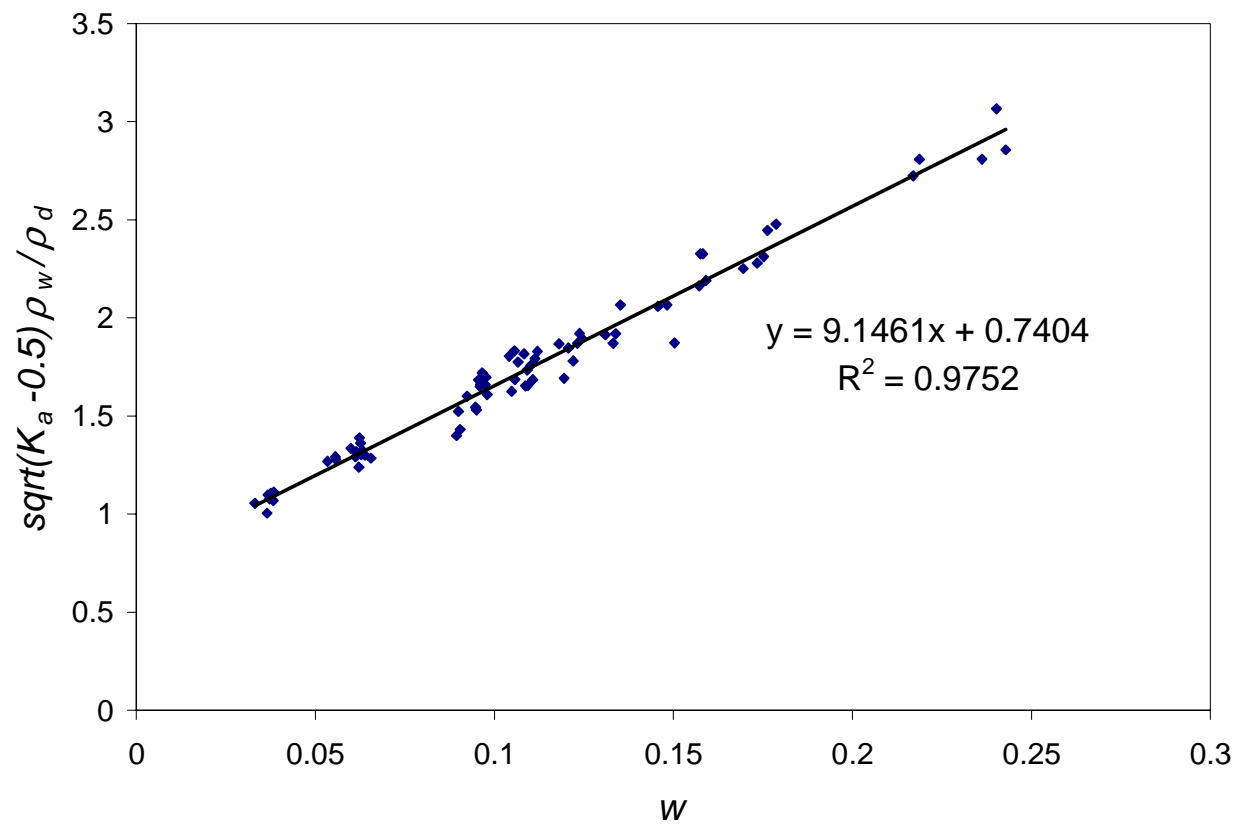

Fig. $6.9\left(\sqrt{ } K_{a}-c\right) \rho_{w} / \rho_{d}$ vs. gravimetric water content $(w)$ relationship

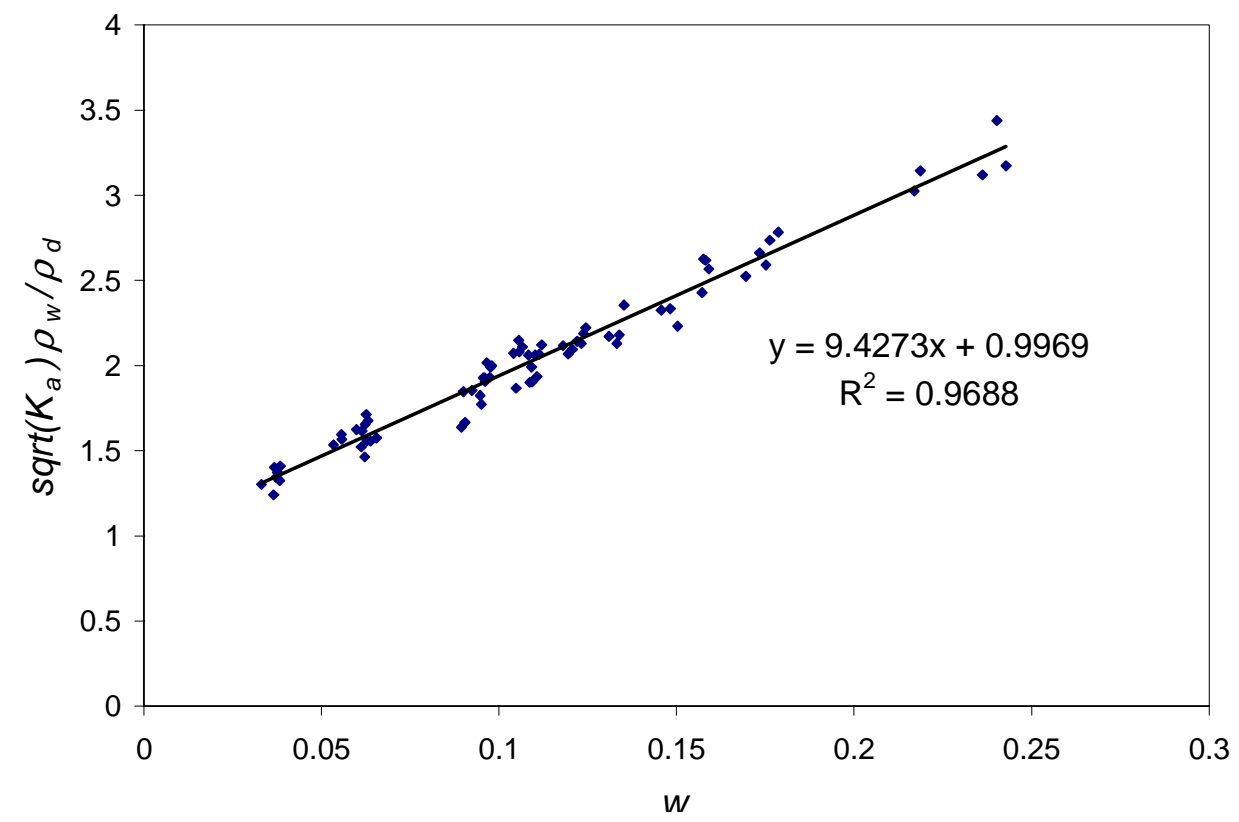

Fig. 6.10 $\left(\sqrt{ } K_{a}\right) \rho_{w} / \rho_{d}$ vs. gravimetric water content (w) relationship

by Eq. (6.4) or Eq. (6.5). For example, Eq. (6.5) was plotted for each soil type. The fitting line moves upward as soil type changes from sand to clay as shown in Fig 6.11. This implies that, for 


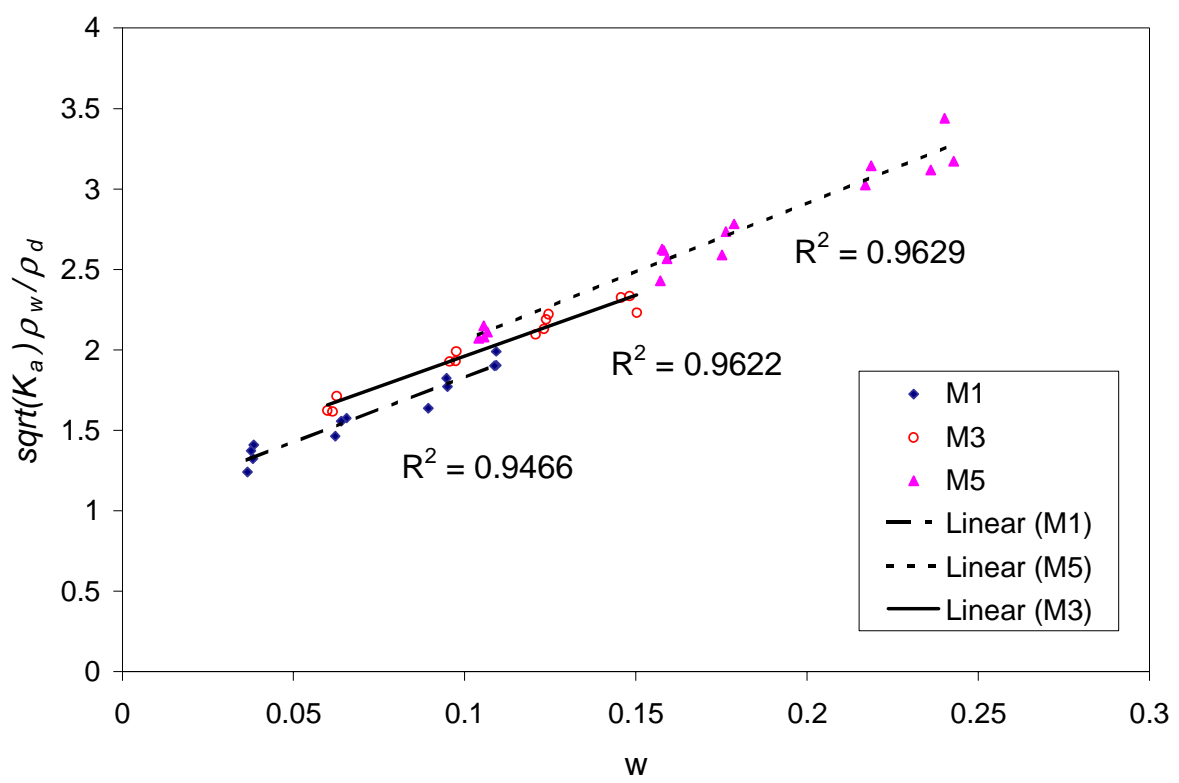

Fig. 6.11 Soil type effect on the relationship between the apparent dielectric constant and water content

the same water content, the apparent dielectric constant is higher in clay than sand, which is contradictory to the common sense that clayey soils have more bound water and hence lower dielectric constant. However, the physical meaning of apparent dielectric constant and its relationship with the dielectric spectrum are not clearly defined. It was found in Section 5.6.2 that the apparent dielectric constant measured the dielectric constant at high frequencies within the pass band. However, the pass band is different for different soils. Clayey soils are lossier than sandy soils and have smaller pass bands. Therefore, the apparent dielectric constants of clayey soils measure higher dielectric constants at lower frequencies than sandy soils. This overcomes the effect of bound water, which is only a very small portion of the water in soils. This can explain why clayey soils have higher dielectric constant than sandy soils at the same water content. The apparent dielectric constant at a particular high frequency (e.g. Eq. (5.13) at 1GHz) may correlate better with water content and density independent of soil type. This will be investigated further in Section 6.4.

\subsubsection{Conductivity}

The volumetric water content of soils may be measured by TDR using Eq. (6.1) or Eq. (6.2). The accuracy of measurement can be improved if the density effect is accounted for by Eq. 
(6.3). However, soil density is not known a priori. It is also needed to calculate the gravimetric water content using from the volumetric water content. Furthermore, it is an important physical parameter for many geotechnical engineering applications.

Siddqui and Drnevich (1995) showed how, by making two separate TDR measurements, it is possible to measure in-place density of soil. A field probe can be used to measure the dielectric constant of soil in place $\left(K_{a, f i e l d}\right)$. Some soil can be quickly taken from the location of the in-place measurement and compacted in a cylindrical mold to measure the dielectric constant of the soil in the mold $\left(K_{a, m o l d}\right)$. Applying the calibration equation to the two measurements, two equations can be obtained

$$
f\left(K_{a, \text { field }}, w_{\text {field }}, \rho_{d, \text { field }}\right)=0
$$

and

$$
f\left(K_{a, \text { mold }}, w_{\text {mold }}, \rho_{d, \text { mold }}\right)=0
$$

where the function $f(K, w, \rho)$ represents a calibration equation such as Eqs. (6.1) to (6.5); $w_{\text {field }}$ and $w_{\text {mold }}$ are the gravimetric water content of the soil in place and in the mold, respectively; $\rho_{d, f i e l d}$ and $\rho_{d, \text { mold }}$ are the dry density of the soil in place and in the mold, respectively. The wet density of the soil in the mold $\left(\rho_{t, \text { mold }}\right)$ can be measured directly using a balance. The dry density of the soil in the mold $\left(\rho_{d, \text { mold }}\right)$ can be calculated as

$$
\rho_{d, \text { mold }}=\frac{\rho_{t, \text { mold }}}{1+w_{\text {mold }}}
$$

By assuming that the gravimetric water content of the soil in the mold is the same as the gravimetric water content of the soil in place (i.e. $\left.w_{\text {field }}=w_{\text {mold }}\right)$, three unknowns $\left(w_{\text {field }}, \rho_{d \text { field }}\right.$, $\rho_{d, \text { mold }}$ ) can then be solved by three equations (Eqs. (6.6) to (6.8)). 
The above procedure requires digging out a sufficient amount of soil from the location of the in-place measurement and uniformly compacting it into a cylinder. It was observed from Figs. 6.3 and 6.4 that the steady-state value of the TDR waveform is also related to the soil water content and density. Since the dc conductivity of a soil can be easily determined from the steadystate value of the TDR waveform using Eq. (3.5), it is possible to express the conductivity in terms of soil density and water content similar to Eq. (6.4) or (6.5). If this additional relationship can be established, then the soil density and water content can be measured nondestructively from a single TDR measurement.

Computer simulation was conducted to gain insight into what the expression may look like for the dc conductivity in terms of soil density and water content. The simulation was based on the volumetric mixing equation and the wave propagation model. Several values of water content, density, and effective specific surface were chosen. The effects of soil density (as characterized by dry density, $\rho_{d}$ ) and soil type (as characterized by the effective specific surface, $\left.A_{e s}\right)$ on the simulated waveforms are shown in Fig. 6.12 and Fig. 6.13, respectively. The behavior is very similar to the experimental results as shown in Fig. 6.3 and Fig. 6.5. Therefore, the expression for the dc conductivity in terms of soil density and water content may be explored using the volumetric mixing equation.

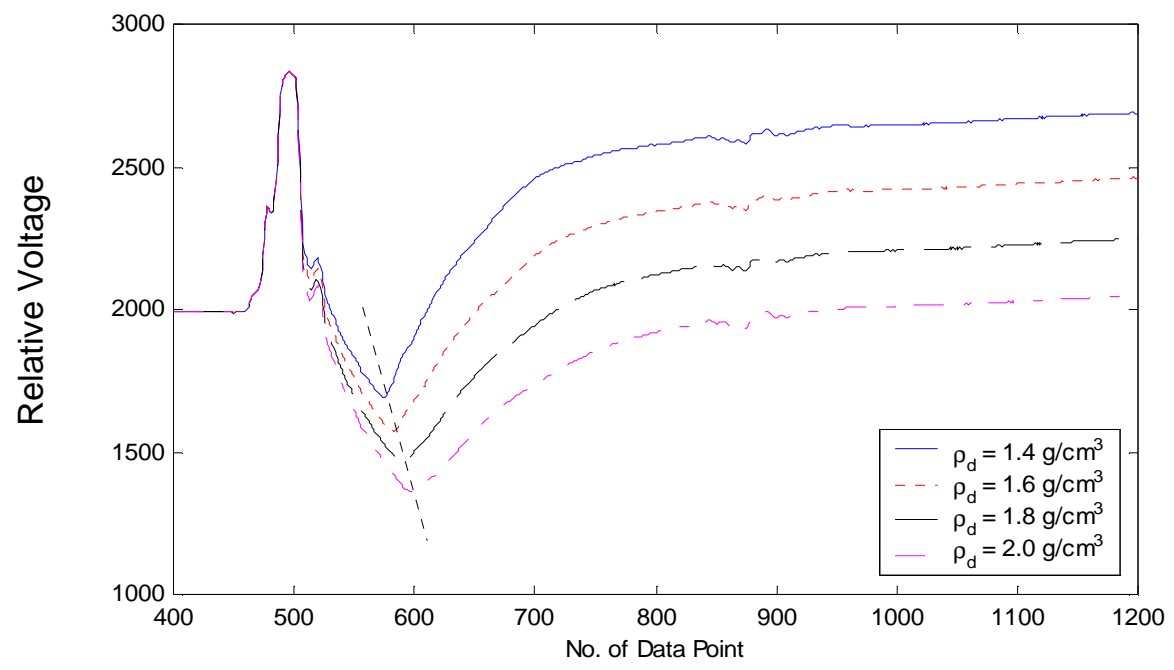

Fig. 6.12 Effect of soil density on simulated TDR waveforms $(w=10 \%)$ 


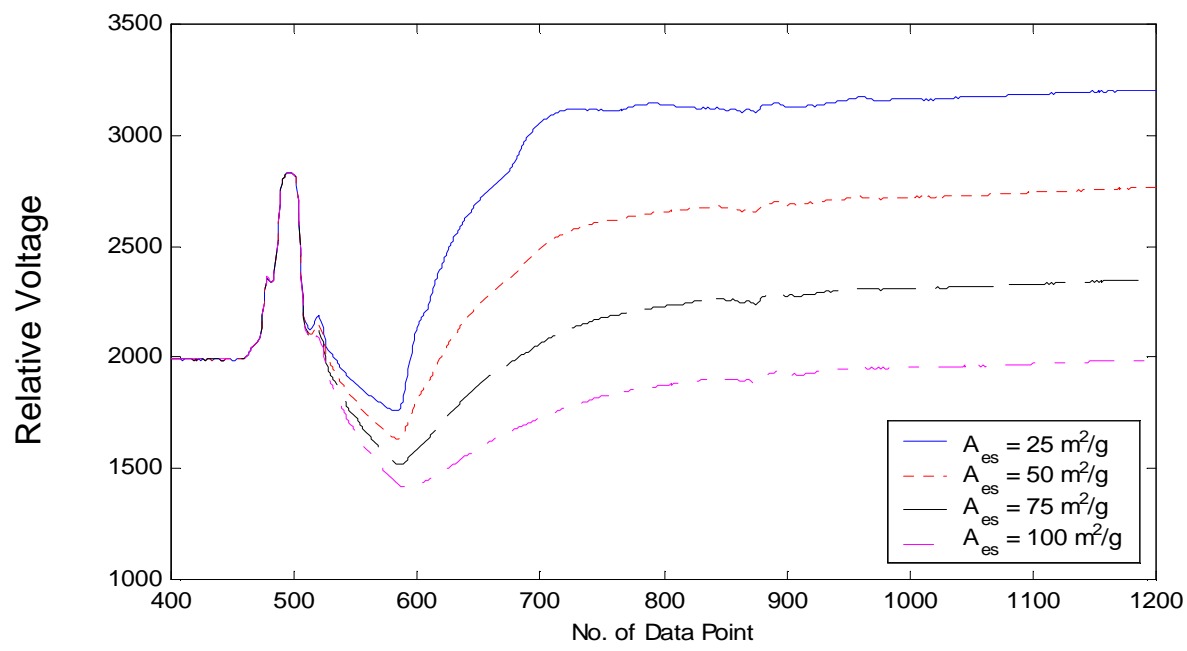

Fig. 6.13 Effect of soil type on simulated TDR waveforms

Conductivities of different soils with different effective specific surfaces, densities, and water contents are calculated from the simulated waveforms using Eq. (3.5). The results are shown in Fig. 6.14. It can be observed that there exists a linear relationship between $\sqrt{ } \sigma_{d c}$ and $\rho_{d}$ for a specific water content and soil type. So the conductivity can be expressed as

$$
\sqrt{\sigma_{d c}}=c_{1}\left(w, A_{e s}\right)+c_{2}\left(w, A_{e s}\right) \rho_{d}
$$

where $c_{1}$ and $c_{2}$ may be dependent on water content and soil type. The expressions for $c_{1}$ and $c_{2}$ as functions of water content are obtained for each soil type from the simulated data in Fig. 6.14 using linear regression. The results are listed in Table 6.3. It can be concluded from Table 6.3 that the Eq. (6.9) can be rewritten as

$\sqrt{\sigma_{d c}}=c+\left[a\left(A_{e s}\right)+b w\right] \rho_{d}$ 

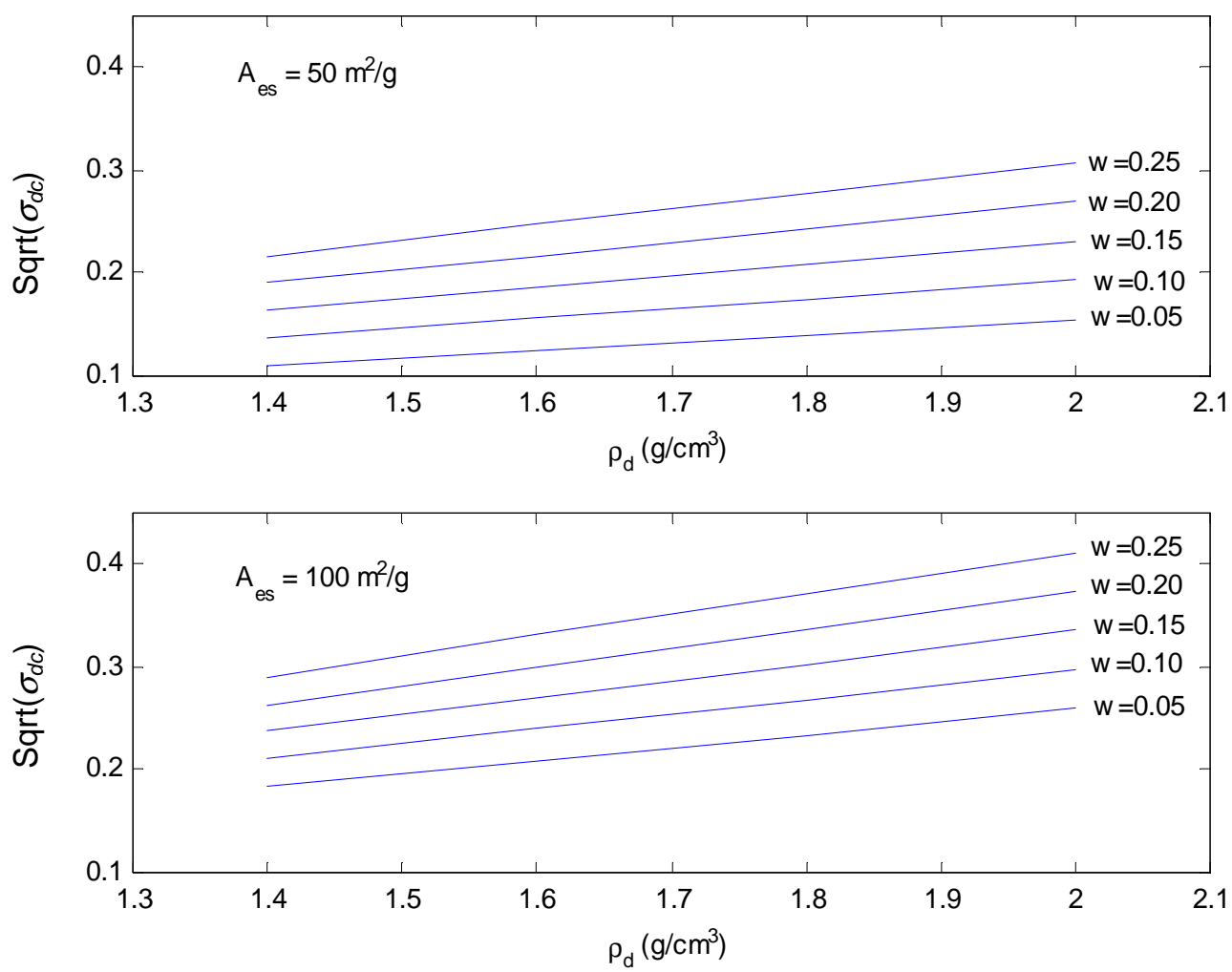

Fig. 6.14 Conductivities calculated from simulated TDR waveforms

Rearranging Eq. (6.10), we obtain a form similar to Eq. (6.4) as

$$
\frac{\left(\sqrt{\sigma_{d c}}-c\right) \rho_{w}}{\rho_{d}}=a\left(A_{e s}\right)+b w
$$

Table 6.3 Results of regression analysis for the constants in Eq. (6.9)

\begin{tabular}{c|cc}
\hline Soil Type & $c_{1}(w)$ & $c_{2}(w)$ \\
\hline$A_{e s}=50 \mathrm{~m}^{2} / \mathrm{g}$ & $c_{1}=0.0067$ & $c_{1}=0.0552+0.3797 \mathrm{w}$ \\
\hline$A_{e s}=100 \mathrm{~m}^{2} / \mathrm{g}$ & $c_{1}=0.0067$ & $c_{1}=0.1074+0.3799 w$ \\
\hline
\end{tabular}


It is shown in Fig. 6.15 that Eqn (6.11) describes the relationship among the conductivity, soil water content, and density very well for the fictitious soils based on the volumetric mixing model.

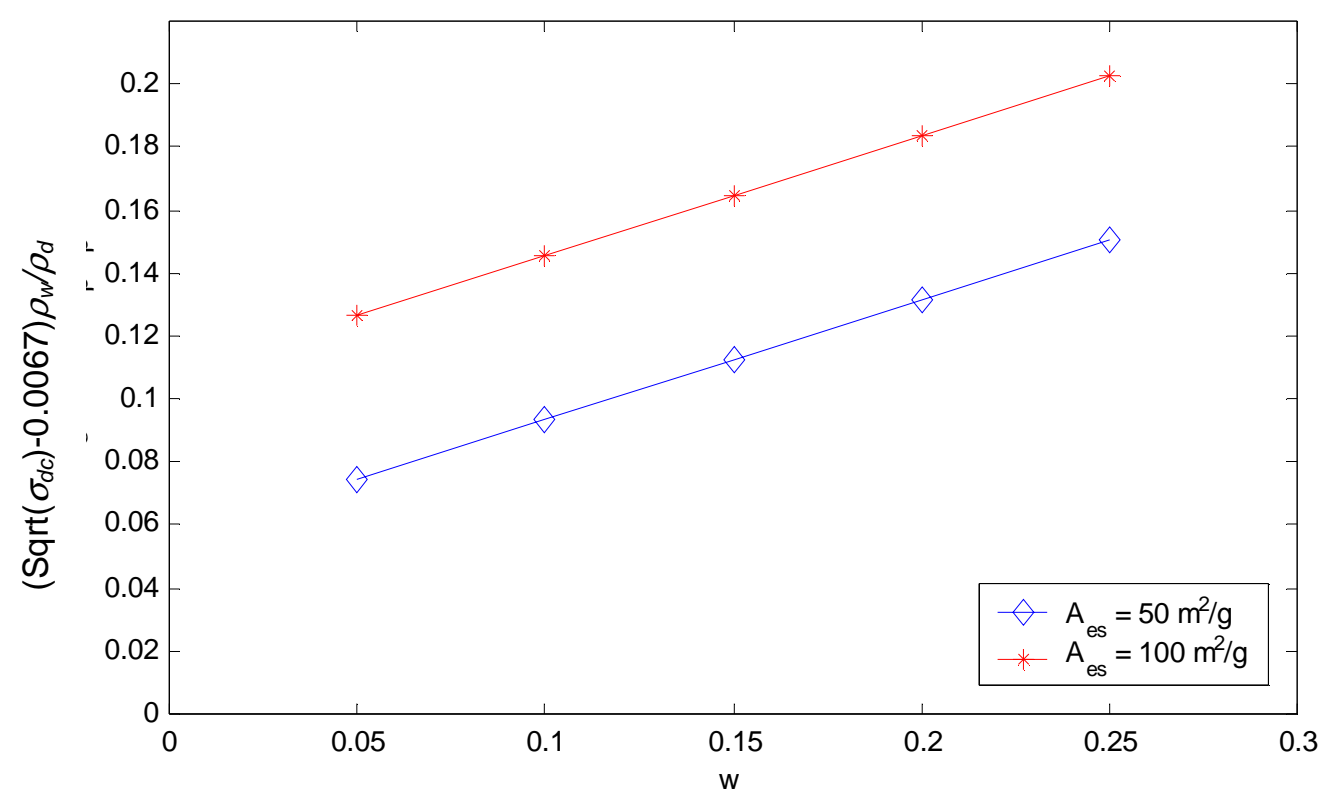

Fig. 6.15 $\left(\sqrt{ } \sigma_{d c}-\mathrm{c}\right) \rho_{w} / \rho_{d}$ vs. $w$ relationship for the fictitious soils based on volumetric mixing model

Conductivities of the mixed soils listed in Table 6.1 were calculated from the measured TDR waveforms. Table 6.4 summarizes the regression analyses using Eq. (6.10). The results are also shown in Fig. 6.16 in the form of Eq. (6.11). Except for M4, the mixed soils seem to have the same behavior as the fictitious soils based on volumetric mixing models where a significantly lower value for the constant $c$ was obtained according to linear regression. This caused the line in Fig. 6.16 for soil M4 to lie above soil M5. If a $c$ value similar to that of other soils is used, then the line for M4 will lie between soil M5 and soil M3, but with more scattering in the data. Unlike the apparent dielectric constant, the data shown in Fig. 6.16 were more subject to measurement error due to the contact between the coaxial head $(\mathrm{CH})$ and the $\mathrm{CC}$ ring. Another source of error could be due to the inadequacy of the volumetric mixing model. For example, the constants in Eq. (6.10) or Eq. (6.11) may be functions of water content and density. Improvement of the probe head and further experimental investigation is required to validate Eqs. (6.10) and (6.11). 


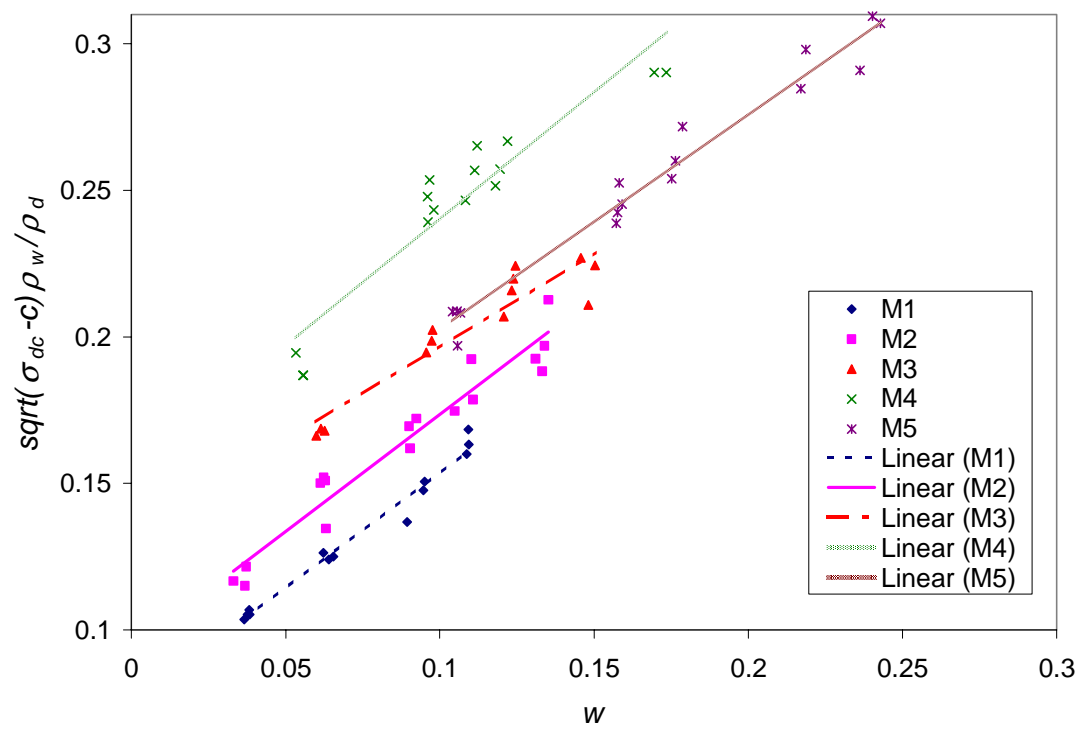

Fig. 6.16 $\left(\sqrt{ } \sigma_{d c}-\mathrm{c}\right) \rho_{w} / \rho_{d}$ vs. $w$ relationship for the mixed soils

Table 6.4 Results of regression analysis for the mixed soils using Eq• (6.10)

\begin{tabular}{c|cccc}
\hline Soil Type & $c$ & $a$ & $B$ & $R^{2}$ \\
\hline M1 & -0.1102 & 0.0746 & 0.7932 & 0.979 \\
M2 & -0.1235 & 0.0955 & 0.7778 & 0.952 \\
M3 & -0.1595 & 0.1340 & 0.6281 & 0.954 \\
M4 & -0.2169 & 0.1514 & 0.8873 & 0.949 \\
M5 & -0.1317 & 0.1310 & 0.7234 & 0.976 \\
\hline
\end{tabular}

\subsection{Soil Properties by the Full Waveform Analysis}

The apparent dielectric constant and dc conductivity were measured by simplified analyses of TDR waveforms and correlated with soil water content and density. The correlation is dependent on soil types, especially for the dc conductivity. A calibration procedure needs to be developed for good measurements. However, the simplified analyses uses only the travel time and steady-state value of the waveform. Much more information is contained in the full TDR waveform. When coupled with the wave propagation model, a good dielectric mixing equation can be used to infer soil physical parameters from the full TDR waveforms. The semi-empirical 
volumetric mixing model is one candidate. The volumetric mixing model and its applications are evaluated in the subsequent section.

\subsubsection{Sensitivity Analysis of the Volumetric Mixing Model}

The volumetric mixing equation involves many parameters as shown in Table 5.5. The soil physical parameters used in Table 5.5 are four independent parameters of the four-phase soil physical model described in Chapter 2. However, only the left-hand side of the phase diagram (i.e. the volumetric fraction of each component) plays a role in the volumetric mixing model. A new term, the volumetric soil content $\left(\theta_{s}\right)$, is defined as the ratio of the volume of soil solid to the total volume of the soil to replace soil dry density and the specific gravity (i.e. density of soil solid). It primarily reflects the soil density since the values of specific gravity for most soil are within a small range (e.g. $2.6 \sim 2.8$ ). Also, the specific surface area $A_{e s}$ is defined as (surface area/volume) instead of (surface area/mass). Thus, there are only three soil physical parameters left. The range and reference value of the volumetric mixing parameters are listed Table 6.5. For those parameters that have a very narrow range, the parameters are assumed to be constants at their typical value. For example, the temperature effect on dielectric parameters of water was neglected because all the experiments were conducted at the same room temperature. The temperature can be easily measured and included into the model for further research. The value of the empirical parameter $\alpha$ is not assumed, so there are a total of six parameters in the volumetric mixing equation: $\theta, \theta_{s}, A_{e s}, \sigma_{f w}, \sigma_{b w}$, and $\alpha$.

To gain insight on how the dielectric mixing parameters affect the TDR waveforms and the dielectric spectrum, parametric studies were carried out by varying the value of each parameter from the reference value listed in Table 6.5 and calculating the corresponding TDR waveform and dielectric spectrum. The results are shown in Figs. 6.17, 6.18, and 6.19 for the TDR waveform, the real part of dielectric spectrum, and the imaginary part of dielectric spectrum, respectively. It can be observed from Fig. 6.17 that the travel time is affected primarily by the volumetric water content and secondarily by the soil density (or volumetric soil content) and the empirical parameter $\alpha$. The volumetric water content causes a time shift in the TDR waveform while the other parameters gradually affect the magnitude with time. In the frequency domain, the real part of dielectric permittivity is affected dominantly by the volumetric water content and secondarily 

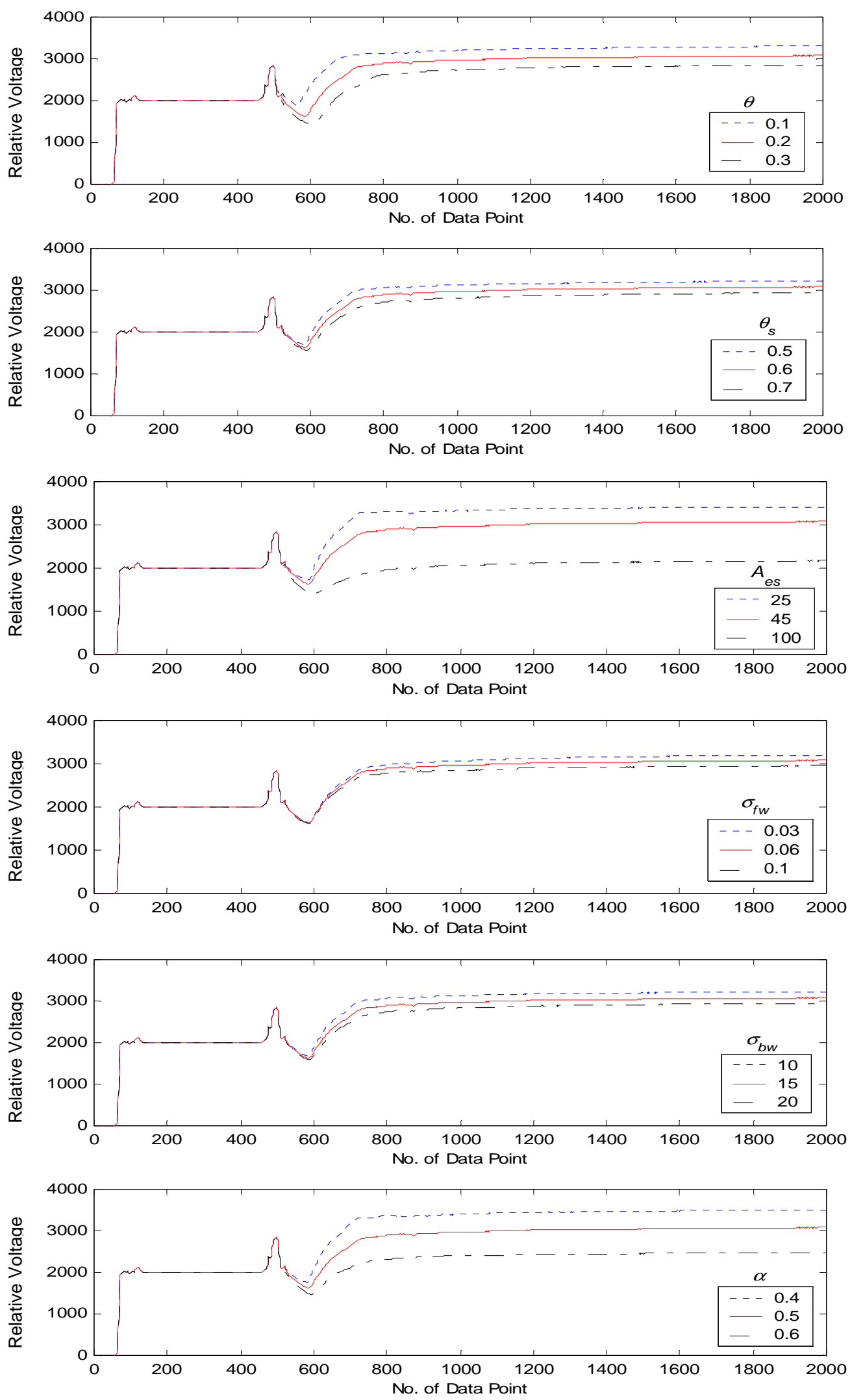

Fig. 6.17 Parametric study of volumetric mixing parameters in TDR 

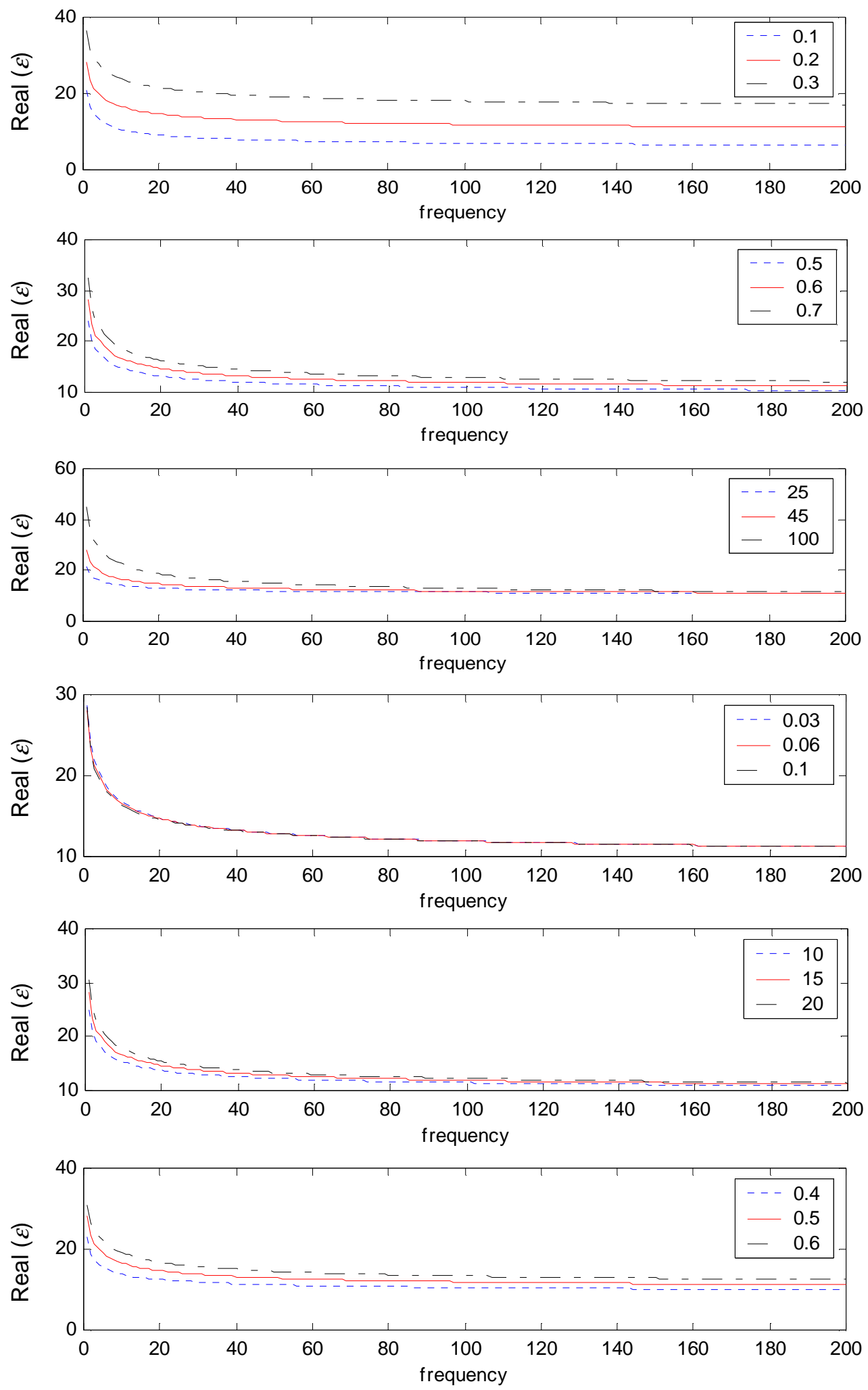

Fig. 6.18 Parametric study of volumetric mixing parameters in $\operatorname{Re}[\varepsilon f f)]$ 

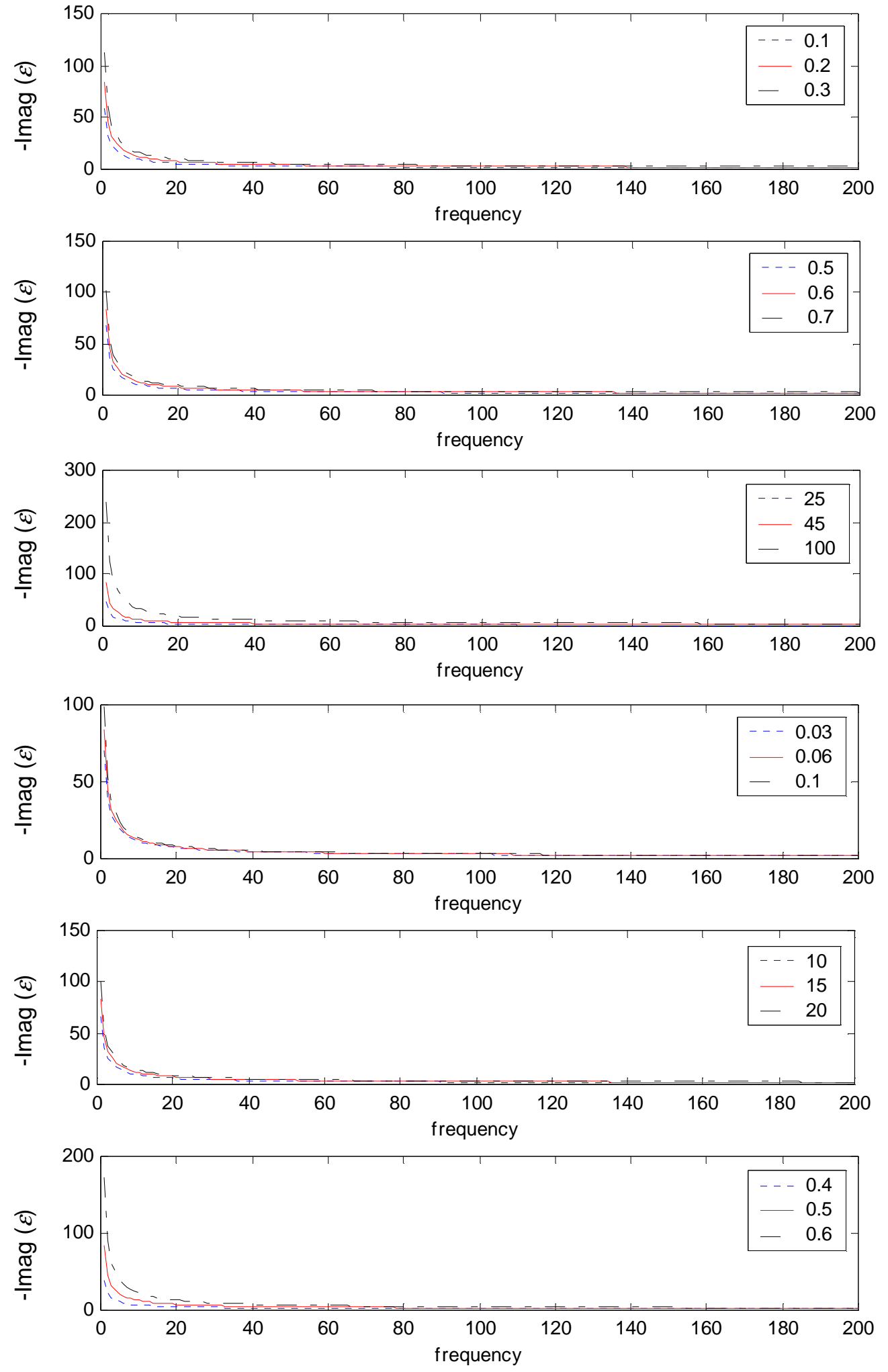

Fig. 6.19 Parametric study of volumetric mixing parameters in $-\operatorname{Im}[\varepsilon(f)]$ 
by the soil density and empirical parameter $\alpha$ as shown in Fig. 6.18. This agrees with the argument that the travel time analysis measures the dielectric permittivity at high frequencies.

Table 6.5 Volumetric mixing parameters

\begin{tabular}{c|cc}
\hline Volumetric Mixing Parameters & Range & Reference value \\
\hline Soil physical parameters & & \\
volumetric water content $\theta, \%$ & $0 \sim 100$ & 20 \\
volumetric soil content $\theta_{s}, \%$ & $0 \sim 100$ & 60 \\
effective specific surface $A_{e s}, 1 / \mu \mathrm{m}$ & $0.1 \sim 1800$ & 45 \\
\hline Dielectric parameters of air & & 1 \\
constant $\varepsilon$ & 1 & 4.7 \\
\hline Dielectric parameters of soil particles & & 80 \\
constant $\varepsilon$ & 4.7 & 4.22 \\
\hline Dielectric parameters of free water & & 17.4 \\
static value $\varepsilon_{s}$ & 80 & 0.06 \\
high frequency value $\varepsilon_{\infty}$ & 4.22 & \\
Relaxation frequency $f_{\text {rel }}, \mathrm{GHz}$ & 17.4 & 80 \\
Conductivity $\sigma_{f w}$, S/m & $0 \sim 2$ & 4.22 \\
Static value $\varepsilon_{s}$ & & 9 \\
high frequency value $\varepsilon_{\infty}$ & 80 & 15 \\
Relaxation frequency $f_{\text {rel }}, \mathrm{kHz}$ & 9.22 & \\
Conductivity $\sigma_{b w}$, S/m & $0.1 \sim 100$ & 0.5 \\
\hline Empirical Parameter & $-1 \sim 1$ & \\
Fitting Parameter $\alpha$ & & \\
\hline
\end{tabular}

To better evaluate the relative importance of each parameter, the normalized sensitivity evaluated at $\boldsymbol{m}=\hat{\boldsymbol{m}}$ is defined as

$$
S_{\hat{\boldsymbol{m}}}=\left[\frac{\partial g / g}{\partial \boldsymbol{m} / \boldsymbol{m}}\right]_{\hat{\boldsymbol{m}}}=\frac{\partial g}{\partial \boldsymbol{m}} \frac{\boldsymbol{m}}{g}
$$

where $\boldsymbol{m}$ denotes the model parameters, and $g(\boldsymbol{m})$ represents the data (e.g. the waveform or dielectric spectrum). The sensitivity analyses for the TDR waveform, the real part of dielectric 
spectrum, and the imaginary part of dielectric spectrum, are shown in Figs. 6.20, 6.21, and 6.22, respectively. It can be observed from Fig. 6.21 that the volumetric water content and the soil density dominate the real part of dielectric permittivity at high frequencies. On the other hand, soil density, specific surface, and the fitting parameter $\alpha$ have more influence on the real part of dielectric permittivity at low frequencies.

\subsubsection{Dielectric Spectrum by Dielectric Mixing Model}

The parameters in the dielectric mixing model can be determined by inversion from the TDR waveform data similar to the inversion for Debye's parameters in Section 5.5.2. Unlike the Debye's parameters, the dielectric mixing parameters are the soil physical parameters. The TDR waveform of the specimen M3_3_2 was randomly picked as an example for inferring volumetric mixing parameters from the TDR waveform. The results of the inversion are summarized in Table 6.6.

There are significant differences between the actual values of the volumetric water content and soil density measured in the lab and the estimated values from inversion. The matrix of coefficients of correlation and the model resolution matrix as defined in Section 4.5 were obtained by linear approximation as discussed in Section 4.5.2 and are shown in Table 6.7 and 6.8, respectively. The volumetric mixing parameters are highly correlated as shown in Table 6.7. This can be expected from Fig. 6.17, in which many parameters have very similar effects on the TDR waveform. The diagonal terms of the resolution matrix in Table 6.8 reveals that the soil density and conductivity of free water are not well resolved and the conductivity of bound water is not resolved at all.

These results show that this is an ill-posed inverse problem. However, regardless of the non-uniqueness of the parameters, the predicted waveform matched the measured TDR waveform almost perfectly as shown in Fig. 6.23. This implies that the inverse solution should provide very good estimation of the dielectric spectrum within the TDR bandwidth. The estimated dielectric spectrum was obtained by substituting the estimated model parameters into the volumetric mixing equation and is shown in Fig. 6.24. Therefore, the 6-parameter volumetric mixing model is better than the Debye equation to infer the dielectric spectra of soils by waveform matching. Because the 

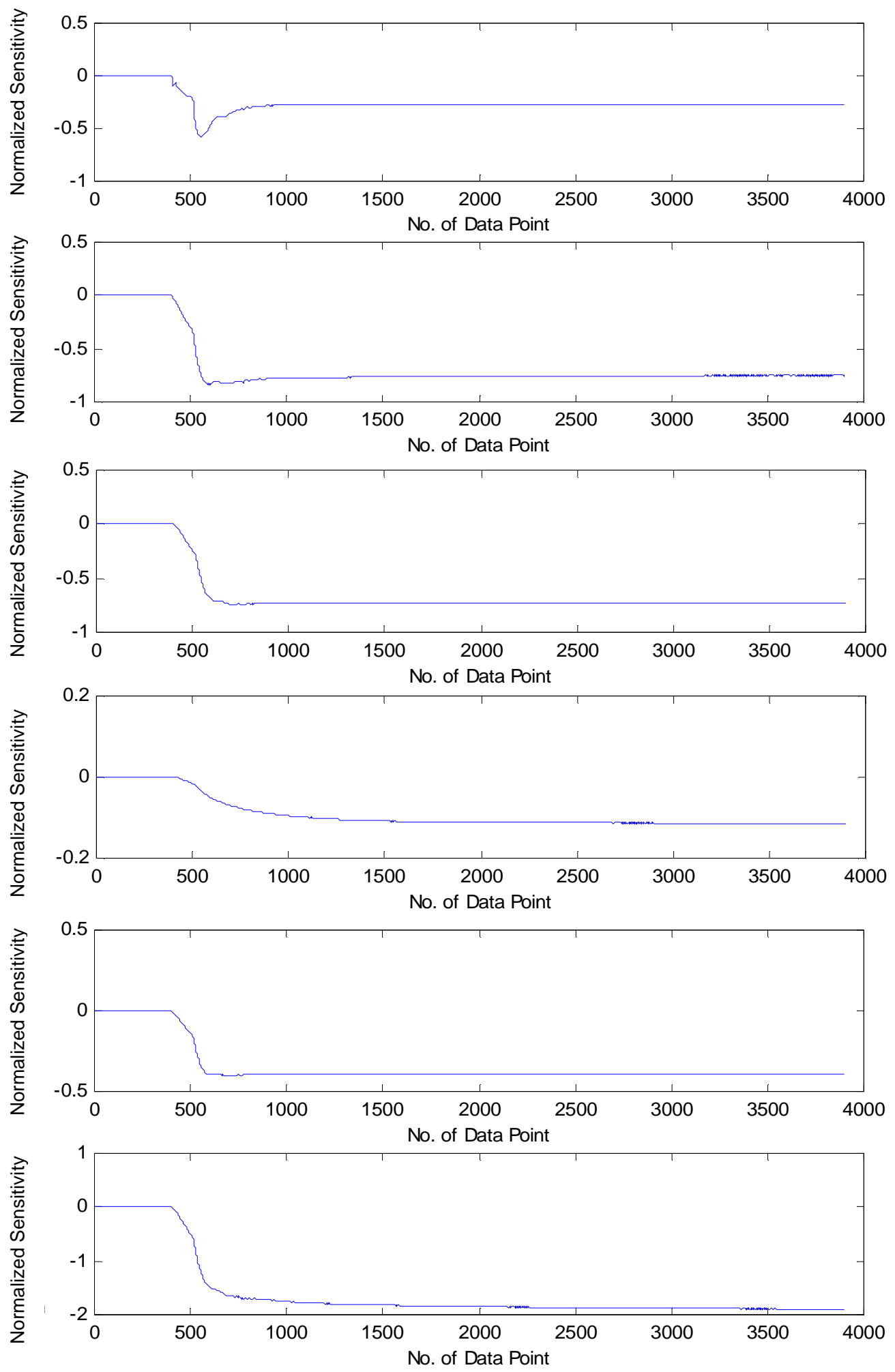

Fig. 6.20 Normalized sensitivity of the TDR waveform to the volumetric mixing parameters 

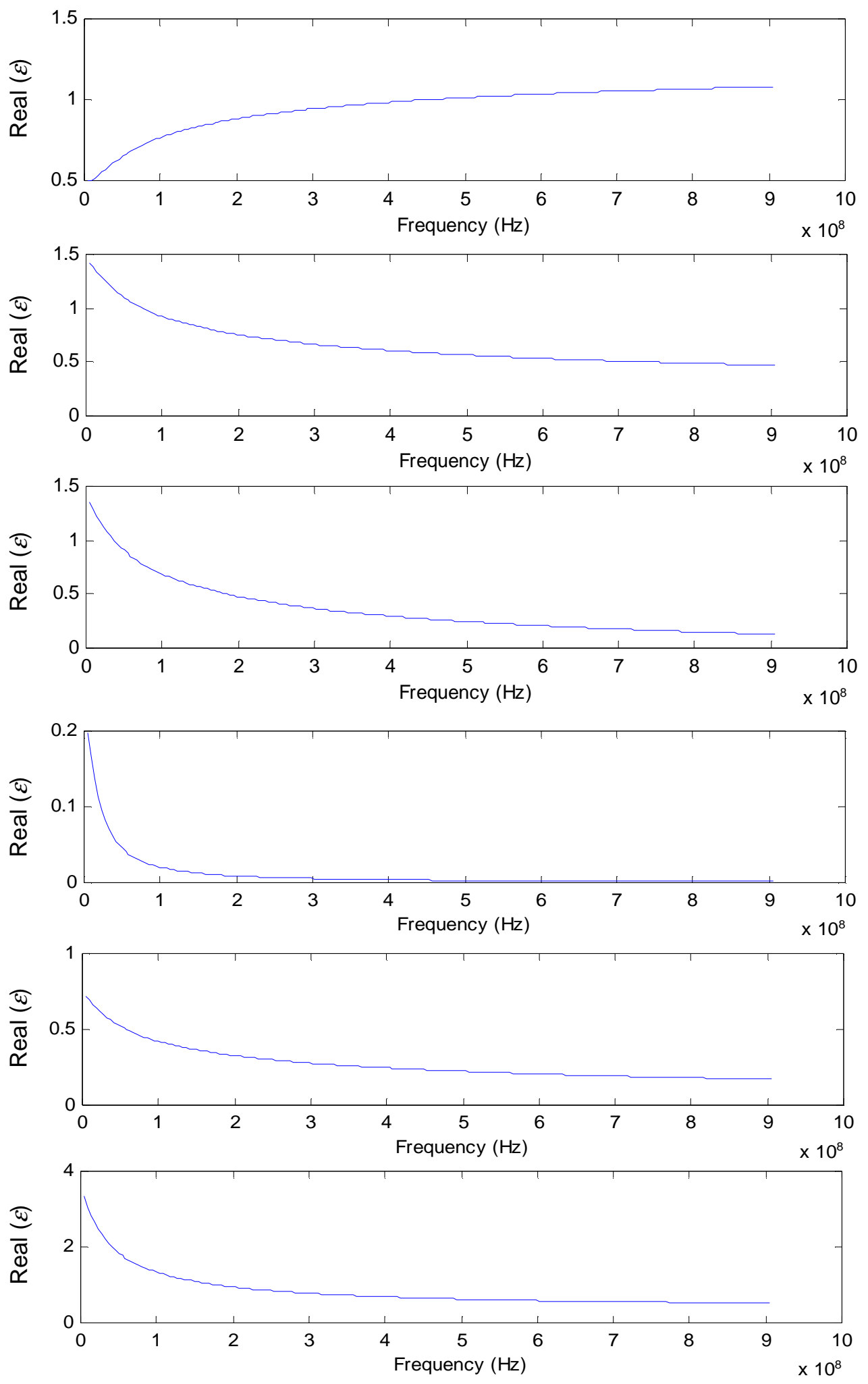

Fig. 6.21 Normalized sensitivity of $\operatorname{Re}[\varepsilon(f)]$ to the volumetric mixing parameters 

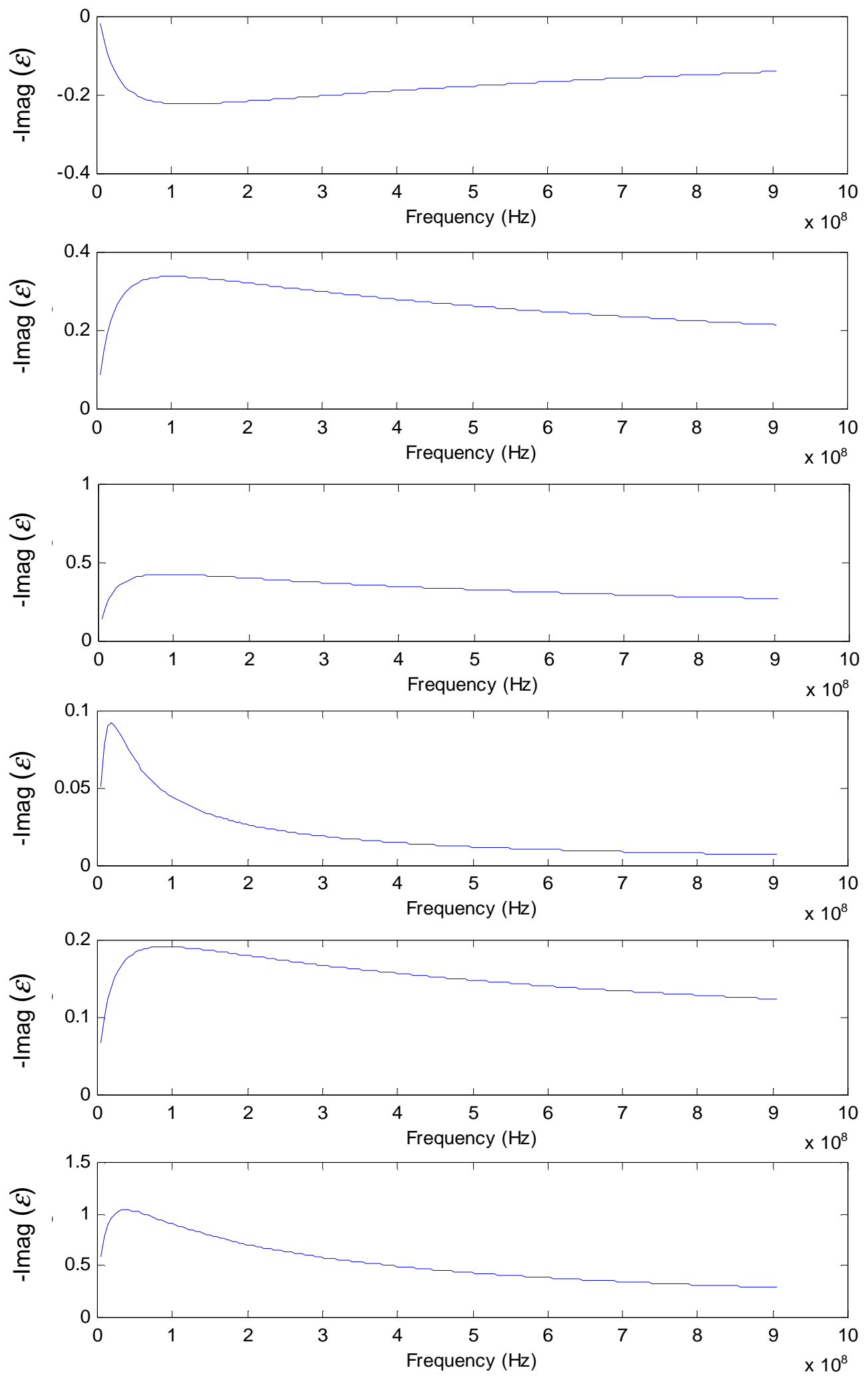

Fig. 6.22 Normalized sensitivity of $-\operatorname{Im}[\varepsilon(f)]$ to the volumetric mixing parameters 


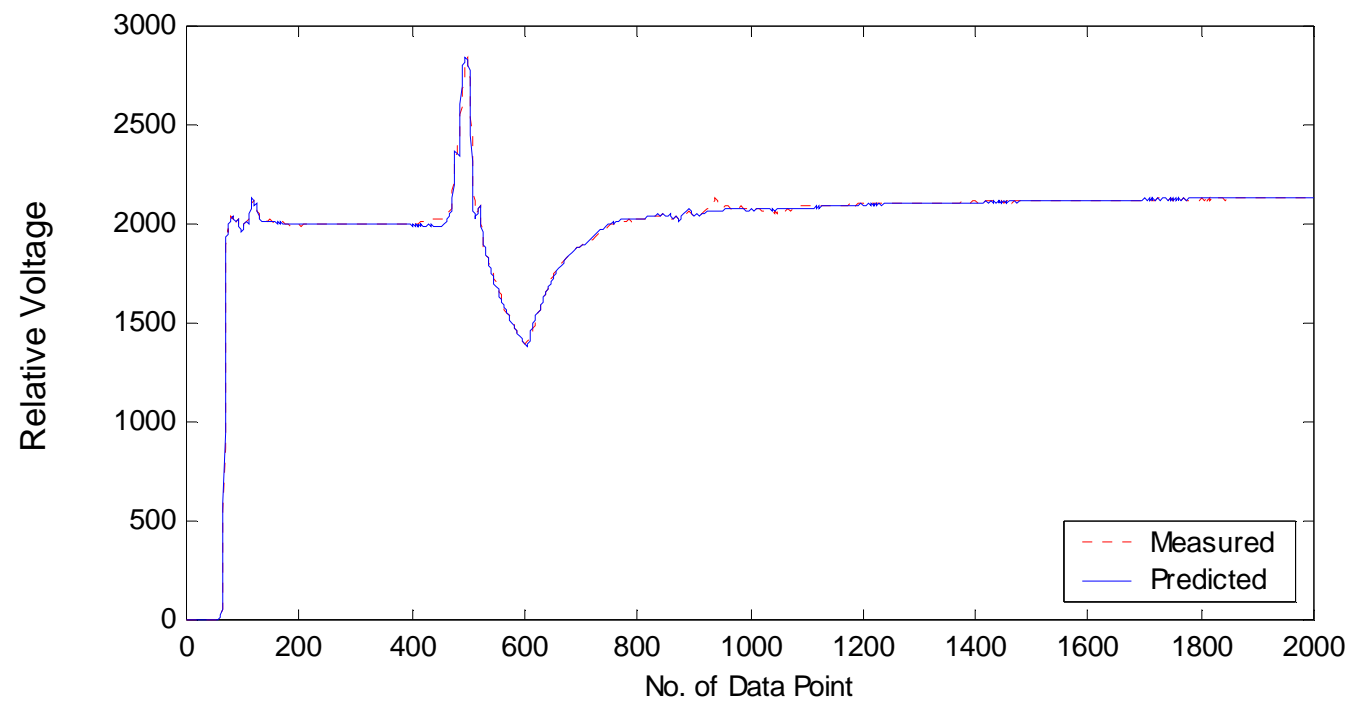

Fig. 6.23 Waveform matching by the volumetric mixing model
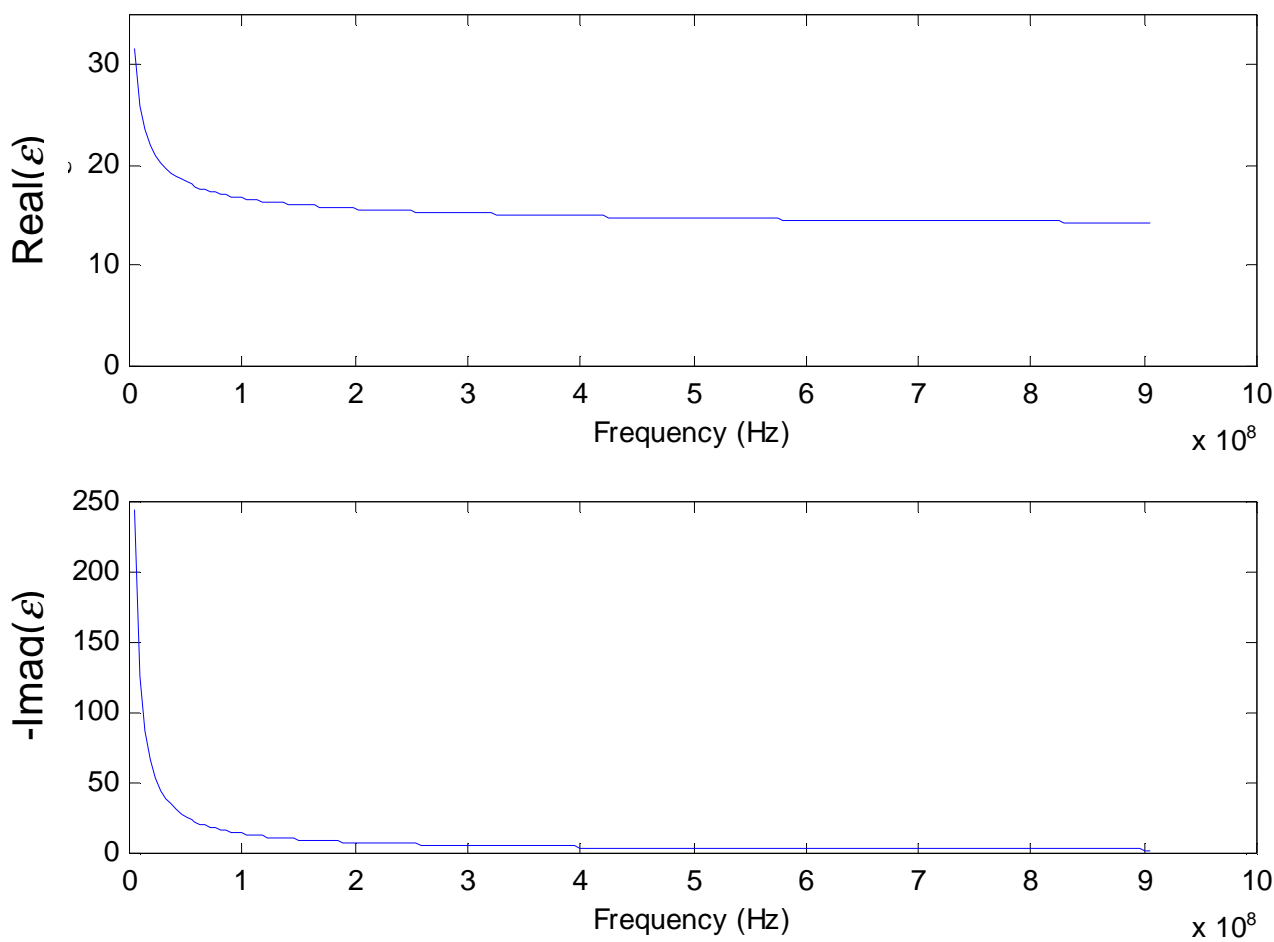

Fig. 6.24 Estimated dielectric spectrum by the volumetric mixing model 
conductivity of bound water is poorly resolved in the inversion and highly correlated with the effective specific surface, it may be eliminated from the model space by using its reference value. The inverse solution of the parameters in the reduced 5-parameter volumetric mixing model gave different estimated values of soil physical parameters but the same dielectric spectrum.

Table 6.6 The prior and estimated values of the volumetric mixing parameters

\begin{tabular}{l|cccccc}
\hline Mixing Parameters & $\theta, \%$ & $\theta_{s}, \%$ & $A_{e s}, 1 / \mu \mathrm{m}$ & $\sigma_{f w}, \mathrm{~S} / \mathrm{m}$ & $\sigma_{b w}, \mathrm{~S} / \mathrm{m}$ & $\alpha$ \\
\hline Prior Mean & 30.0 & 65.0 & 275.0 & 0.060 & 15.0 & 0.5 \\
Prior Std. Dev. & 15.0 & 20.0 & 137.5 & 0.010 & 5.0 & 0.1 \\
Actual Value & 23.0 & 65.5 & N/A & N/A & N/A & N/A \\
Estimated & 19.8 & 52.9 & 77.7 & 0.005 & 15.67 & 0.69 \\
Std. Dev. of Estimation & 1.0 & 11.9 & 26.4 & 0.001 & 4.9 & 0.01 \\
\hline
\end{tabular}

Table 6.7 Matrix of coefficient of correlation for the volumetric mixing model

\begin{tabular}{c|rrrrrr}
\hline$C_{r}$ & \multicolumn{1}{|c}{$\theta$} & \multicolumn{1}{c}{$\theta_{s}$} & \multicolumn{1}{c}{$A_{e s}$} & \multicolumn{1}{c}{$\sigma_{f w}$} & \multicolumn{1}{c}{$\sigma_{b w}$} & \multicolumn{1}{c}{$\alpha$} \\
\hline$\theta$ & 1.000 & -0.947 & 0.875 & -0.425 & -0.345 & 0.238 \\
$\theta_{s}$ & -0.947 & 1.000 & -0.674 & 0.483 & 0.026 & -0.304 \\
$A_{e s}$ & 0.875 & -0.674 & 1.000 & -0.283 & -0.756 & 0.115 \\
$\sigma_{f w}$ & -0.425 & 0.483 & -0.283 & 1.000 & -0.025 & -0.883 \\
$\sigma_{b w}$ & -0.345 & 0.026 & -0.756 & -0.025 & 1.000 & 0.086 \\
$\alpha$ & 0.238 & -0.304 & 0.115 & -0.883 & 0.086 & 1.000 \\
\hline
\end{tabular}

Table 6.8 Resolution matrix for the volumetric mixing model

\begin{tabular}{c|rrrrrr}
\hline$C_{r}$ & \multicolumn{1}{|c}{$\theta$} & \multicolumn{1}{c}{$\theta_{s}$} & \multicolumn{1}{c}{$A_{e s}$} & \multicolumn{1}{c}{$\sigma_{f w}$} & \multicolumn{1}{c}{$\sigma_{b w}$} & \multicolumn{1}{c}{$\alpha$} \\
\hline$\theta$ & 0.996 & 0.027 & 0.000 & 0.037 & 0.001 & 0.000 \\
$\theta_{s}$ & 0.049 & 0.647 & 0.000 & -0.516 & -0.001 & 0.007 \\
$A_{e s}$ & -9.946 & 52.846 & 0.963 & 67.131 & 3.940 & -0.571 \\
$\sigma_{f w}$ & 0.000 & -0.001 & 0.000 & 0.552 & 0.000 & 0.000 \\
$\sigma_{b w}$ & 0.733 & -0.382 & 0.005 & 1.093 & 0.024 & -0.080 \\
$\alpha$ & 0.000 & 0.002 & 0.000 & 0.015 & 0.000 & 0.992 \\
\hline
\end{tabular}


It is possible to reduce the non-uniqueness by reducing the number of unknowns in the inversion. The volumetric water content and soil density can be measured directly in the lab and the remaining three parameters in the 5-parameter volumetric mixing model can be "calibrated" accordingly. If consistent results are obtained for the three remaining parameters, then the example described in Section 4.6.2 can be used to simultaneously measure soil water content, density, and soil type. The results of parameter calibration are summarized in Tables 6.9 to 6.11 for the five mixed soils.

For each soil specimen, the TDR waveform is matched very well by the resulting volumetric mixing parameters. However, the variations in the inferred parameters for each soil type seems to be much more than the experimental error. This implies that the semi-empirical volumetric mixing can fit the TDR waveform and estimate the dielectric spectrum very well for each individual soil specimen, but not good enough in general. The conductivities of free water range from 0.2 to $1.1 \mathrm{~S} / \mathrm{m}$, and the range of fitting parameters is from 0.32 to 0.64 . The averaged values of effective specific surface for each soil types are consistent with their percentages of fine soils. The value of conductivity of free water seems to decrease with increasing water content, which may be explained by decreasing salinity with increasing water content. Further investigation on dielectric properties of soils and an improvement of the TDR probe are needed to explore simultaneous measurement of soil water content, density and soil type.

\subsubsection{Apparent Dielectric Constant Revisited}

The widespread use of TDR has resulted in a number of empirical calibration equations such as Eqs. (6.1) to (6.5). These equations are in different forms and lack a strong theoretical basis. However, it is shown in the following that these empirical equations are unified when considering the apparent dielectric constant in the form of the volumetric mixing equation with $\alpha$ $=0.5$ in Eq. (2.53). Substituting the complex dielectric permittivity with apparent dielectric constant and rearranging, Eq. (2.53) becomes

$$
\sqrt{K_{a}}=\left(\frac{\sqrt{K_{s}}-\sqrt{K_{a i r}}}{\rho_{s}}+\left(\sqrt{K_{b w}}-\sqrt{K_{f w}}\right) \delta A_{e s}\right) \rho_{d}+\left(\sqrt{K_{f w}}-\sqrt{K_{a i r}}\right) \theta+\sqrt{K_{a i r}}
$$

Table 6.9 Results of parameter calibration for mixed soil M1 


\begin{tabular}{|c|c|c|c|c|c|}
\hline \multirow{2}{*}{$\begin{array}{l}\text { Soil } \\
\text { Specime } \\
\mathrm{n}\end{array}$} & \multicolumn{2}{|c|}{$\begin{array}{l}\text { Measured } \\
\text { Parameters }\end{array}$} & \multicolumn{3}{|c|}{ Inferred Parameters } \\
\hline & $\theta$ & $\theta \mathrm{s}$ & $\mathrm{A}_{\mathrm{es}}, 1 / \mu \mathrm{m}$ & $\sigma_{\mathrm{fw}}, \mathrm{S} / \mathrm{m}$ & $\alpha$ \\
\hline M1-1-1 & 0.0648 & 0.6112 & 2.93 & 0.6091 & 0.49 \\
\hline M1-1-2 & 0.0701 & 0.6754 & 9.38 & 0.6599 & 0.52 \\
\hline M1-1-3 & 0.0749 & 0.7101 & 12.30 & 0.5828 & 0.55 \\
\hline M1-1-4 & 0.0774 & 0.7685 & 5.73 & 0.6869 & 0.58 \\
\hline M1-2-1 & 0.1125 & 0.6221 & 1.82 & 0.5555 & 0.51 \\
\hline M1-2-2 & 0.1246 & 0.7065 & 8.66 & 0.6158 & 0.53 \\
\hline M1-2-4 & 0.1383 & 0.8054 & 0.96 & 0.6097 & 0.63 \\
\hline M1-3-1 & 0.1689 & 0.6464 & 1.87 & 0.5408 & 0.53 \\
\hline M1-3-2 & 0.1960 & 0.7475 & 0.41 & 0.5075 & 0.62 \\
\hline M1-3-4 & 0.1877 & 0.7605 & 0.35 & 0.5333 & 0.56 \\
\hline M1-4-1 & 0.2126 & 0.7054 & 0.52 & 0.4973 & 0.64 \\
\hline M1-4-2 & 0.2203 & 0.7304 & 0.39 & 0.4938 & 0.63 \\
\hline M1-4-3 & 0.2195 & 0.7315 & 0.26 & 0.4981 & 0.61 \\
\hline Average & & & 3.51 & 0.5685 & 0.57 \\
\hline
\end{tabular}

Table 6.10 Results of parameter calibration for mixed soil M2

\begin{tabular}{c|cc|rrr}
\hline \multirow{2}{*}{$\begin{array}{c}\text { Soil } \\
\text { Specimen }\end{array}$} & \multicolumn{2}{|c|}{$\begin{array}{c}\text { Measured } \\
\text { Parameters }\end{array}$} & \multicolumn{3}{|c}{ Inferred Parameters } \\
\cline { 2 - 6 } & $\theta$ & \multicolumn{1}{c}{$\theta_{s}$} & $A_{e s}, 1 / \mu m$ & $\sigma_{f w}, S / m$ & $\alpha$ \\
\hline M2-1-1 & 0.0605 & 0.5931 & 68.77 & 0.5352 & 0.41 \\
M2-1-3 & 0.0698 & 0.6773 & 26.94 & 0.8833 & 0.51 \\
M2-1-4 & 0.0669 & 0.7296 & 46.43 & 0.4474 & 0.54 \\
M2-2-1 & 0.0895 & 0.5119 & 45.98 & 0.8340 & 0.37 \\
M2-2-2 & 0.1170 & 0.6780 & 28.82 & 0.6350 & 0.58 \\
M2-2-3 & 0.1223 & 0.7029 & 21.98 & 0.6389 & 0.60 \\
M2-2-4 & 0.1323 & 0.7801 & 20.15 & 0.6904 & 0.63 \\
M2-3-1 & 0.1391 & 0.5578 & 0.79 & 0.7001 & 0.52 \\
M2-3-2 & 0.1822 & 0.7119 & 0.71 & 0.6616 & 0.62 \\
M2-3-4 & 0.1924 & 0.7682 & 0.88 & 0.6855 & 0.60 \\
M2-4-1 & 0.1818 & 0.5957 & 0.88 & 0.5901 & 0.60 \\
M2-4-2 & 0.2218 & 0.7235 & 0.51 & 0.5889 & 0.61 \\
M2-4-4 & 0.2155 & 0.7422 & 0.76 & 0.6199 & 0.62 \\
M2-5-1 & 0.2331 & 0.6224 & 3.25 & 0.5508 & 0.63 \\
M2-5-2 & 0.2560 & 0.6903 & 1.94 & 0.5499 & 0.61 \\
M2-5-3 & 0.2561 & 0.6942 & 0.72 & 0.5188 & 0.60 \\
M2-5-4 & 0.2536 & 0.6989 & 0.50 & 0.5408 & 0.61 \\
\hline Average & & & 15.88 & 0.6277 & 0.57 \\
\hline
\end{tabular}

Table 6.11 Results of parameter calibration for mixed soil M3 


\begin{tabular}{|c|c|c|c|c|c|}
\hline \multirow{2}{*}{$\begin{array}{l}\text { Soil } \\
\text { Specime } \\
\mathrm{n}\end{array}$} & \multicolumn{2}{|c|}{$\begin{array}{l}\text { Measured } \\
\text { Parameters }\end{array}$} & \multicolumn{3}{|c|}{ Inferred Parameters } \\
\hline & $\theta$ & $\theta_{\mathrm{s}}$ & $\mathrm{A}_{\mathrm{es}}, 1 / \mu \mathrm{m}$ & $\sigma_{\mathrm{fw}}, \mathrm{S} / \mathrm{m}$ & $\alpha$ \\
\hline M3-1-1 & 0.0896 & 0.5057 & 95.87 & 0.3461 & 0.41 \\
\hline M3-1-2 & 0.1028 & 0.5915 & 95.20 & 0.3826 & 0.48 \\
\hline M3-1-3 & 0.1039 & 0.6127 & 83.50 & 0.2777 & 0.51 \\
\hline M3-2-2 & 0.1668 & 0.6039 & 56.73 & 0.3858 & 0.57 \\
\hline M3-2-3 & 0.1808 & 0.6558 & 67.50 & 0.3856 & 0.57 \\
\hline M3-2-4 & 0.1959 & 0.7240 & 78.38 & 0.3447 & 0.59 \\
\hline M3-3-1 & 0.1906 & 0.5410 & 20.30 & 0.5182 & 0.55 \\
\hline M3-3-2 & 0.2295 & 0.6551 & 68.67 & 0.3860 & 0.58 \\
\hline M3-3-3 & 0.2369 & 0.6795 & 63.45 & 0.3878 & 0.59 \\
\hline M3-3-4 & 0.2422 & 0.7092 & 30.01 & 0.5365 & 0.58 \\
\hline M3-4-1 & 0.2094 & 0.4922 & 0.38 & 0.5246 & 0.46 \\
\hline M3-4-3 & 0.2764 & 0.6590 & 23.01 & 0.5215 & 0.51 \\
\hline M3-4-4 & 0.2726 & 0.6611 & 18.34 & 0.5888 & 0.56 \\
\hline Average & & & 53.95 & 0.4297 & 0.54 \\
\hline
\end{tabular}

Table 6.12 Results of parameter calibration for mixed soil M4

\begin{tabular}{c|cc|rcc}
\hline \multirow{2}{*}{$\begin{array}{c}\text { Soil } \\
\text { Specimen }\end{array}$} & \multicolumn{2}{|c|}{$\begin{array}{c}\text { Measured } \\
\text { Parameters }\end{array}$} & \multicolumn{3}{|c}{ Inferred Parameters } \\
\cline { 2 - 6 } & \multicolumn{1}{|c}{$\theta$} & \multicolumn{1}{c}{$\theta_{s}$} & $A_{\text {es }}, 1 / \mu \mathrm{m}$ & $\sigma_{\text {fw }}, \mathrm{S} / \mathrm{m}$ & $\alpha$ \\
\hline M4-1-2 & 0.0918 & 0.5834 & 245.60 & 1.0833 & 0.32 \\
M4-1-3 & 0.0961 & 0.6099 & 233.98 & 0.8083 & 0.34 \\
M4-1-4 & 0.1006 & 0.6654 & 152.62 & 0.1904 & 0.48 \\
M4-2-1 & 0.1254 & 0.4523 & 144.85 & 0.9172 & 0.34 \\
M4-2-2 & 0.1630 & 0.5961 & 117.20 & 0.4075 & 0.54 \\
M4-2-3 & 0.1730 & 0.6367 & 115.58 & 0.3948 & 0.55 \\
M4-2-4 & 0.1954 & 0.7184 & 131.26 & 0.4662 & 0.54 \\
M4-3-1 & 0.1583 & 0.4682 & 15.98 & 0.8560 & 0.41 \\
M4-3-2 & 0.1911 & 0.6028 & 113.18 & 0.3654 & 0.56 \\
M4-3-3 & 0.2029 & 0.6442 & 73.85 & 0.6076 & 0.55 \\
M4-3-4 & 0.2210 & 0.7212 & 104.20 & 0.5034 & 0.56 \\
M4-4-1 & 0.1686 & 0.4883 & 13.67 & 0.8612 & 0.45 \\
M4-4-4 & 0.2366 & 0.7085 & 5.62 & 0.8727 & 0.59 \\
M4-5-1 & 0.2263 & 0.4611 & 0.64 & 0.6775 & 0.41 \\
M4-5-4 & 0.3093 & 0.6449 & 20.49 & 0.6445 & 0.57 \\
\hline Average & & & 99.25 & 0.6437 & 0.48 \\
\hline
\end{tabular}

Table 6.13 Results of parameter calibration for mixed soil M1 


\begin{tabular}{|c|c|c|c|c|c|}
\hline \multirow[t]{2}{*}{$\begin{array}{c}\text { Soil } \\
\text { Specimen }\end{array}$} & \multicolumn{2}{|c|}{$\begin{array}{l}\text { Measured } \\
\text { Parameters }\end{array}$} & \multicolumn{3}{|c|}{ Inferred Parameters } \\
\hline & $\theta$ & $\theta_{s}$ & $A_{e s}, 1 / \mu \mathrm{m}$ & $\sigma_{f w}, \mathrm{~S} / \mathrm{m}$ & $\alpha$ \\
\hline M5-1-1 & 0.1337 & 0.4486 & 195.34 & 0.7364 & 0.37 \\
\hline M5-1-2 & 0.1588 & 0.5284 & 37 & 1257 & 0. \\
\hline M5-1-3 & 0.1672 & 0.5613 & 181.17 & 0.5018 & 0. \\
\hline M5-1-4 & 0.1948 & 0.6635 & 180.26 & 0.2972 & 0.52 \\
\hline M5-2-1 & 0.2108 & 0.4699 & 113.68 & 0.5375 & 0.46 \\
\hline M5-2-2 & 0.2633 & 0.5926 & 5.92 & t. & 0.52 \\
\hline M5-2-3 & 0.2696 & 0.6043 & & .37 & 0.5 \\
\hline$-2-4$ & 0.2959 & 0.6674 & 112 & & 0. \\
\hline M5-3-2 & 0.2936 & 0.5826 & 142.50 & 0 & 0. \\
\hline M5-3-3 & 0.3039 & 0.6113 & 30.82 & 0.7 & 0.5 \\
\hline M5-3-4 & 0.3141 & 0.6358 & 116.93 & 0.4696 & 0.53 \\
\hline M5-4-1 & 0.3256 & 0.5280 & 142.96 & 0.4228 & 0.52 \\
\hline M5-4-2 & 0.3594 & 0.5872 & 81.35 & 0.5109 & 0.54 \\
\hline M5-5-1 & 0.3216 & 0.4748 & 168.97 & 0.4043 & 0.48 \\
\hline $5-3$ & 0.3834 & 0.5599 & 4.50 & 0.6283 & 0.57 \\
\hline M5-5-4 & 0.3793 & 0.5695 & 3.39 & 0.6336 & 0.54 \\
\hline Average & & & 104.2 & 0.5737 & 0.51 \\
\hline
\end{tabular}

where $K_{s}, K_{f w}, K_{b w}$, and $K_{a i r}$ are dielectric constants of soil solid, free water, bound water, and air, respectively; $\rho_{s}$ is the density of soil solid; and $\delta$ is the thickness of bound water layer. The dielectric constants of air, water, and soil solid can be assumed invariant in practice. In terms of calibration parameters, the apparent dielectric constant can be expressed as a function of soil type, density, and water content. Equation (6.13) becomes

$$
\sqrt{K_{a}}=a\left(A_{e s}\right) \rho_{d}+b \theta+c
$$

The apparent dielectric constant of soil is affected primarily by the volumetric water content and secondarily by the soil density and soil type. It is interesting to note that the empirical equations are special cases of Eq. (6.14). If the soil-type effect is neglected, Eq. (6.14) reduces to Eq. (6.8). If soil type and density effects are both neglected, Eq. (6.14) becomes Eq. (6.2). 
The dielectric permittivity at high frequencies depends only on the volumetric proportions of each soil phases but not on soil types because the interface effect on dielectric permittivity diminishes at high frequencies. This can also be observed in Fig. 6.18 or Fig. 6.21. Correlation of the apparent dielectric constant with soil water content and density was quite successful because it is associated with the dielectric permittivity at high frequency as discussed in Section 5.6.2. However, significant soil type effects were still observed in Fig. 6.11. It was argued in Section 6.3.1 that this is not because the dielectric permittivity at high frequencies is sensitive to the soil type. Rather, it is because the apparent dielectric constant is associated with dielectric permittivity at different frequencies due to different pass bandwidths for different soil types. Therefore, the apparent dielectric constant at a particular high frequency (e.g. Eq. (5.13) at $1 \mathrm{GHz}$ ) may correlate better with water content and density independent of soil type. The general calibration equation can be rewritten in terms of apparent dielectric constant at $1 \mathrm{GHz}\left(\varepsilon_{a, 1 \mathrm{GHz}}\right)$ as

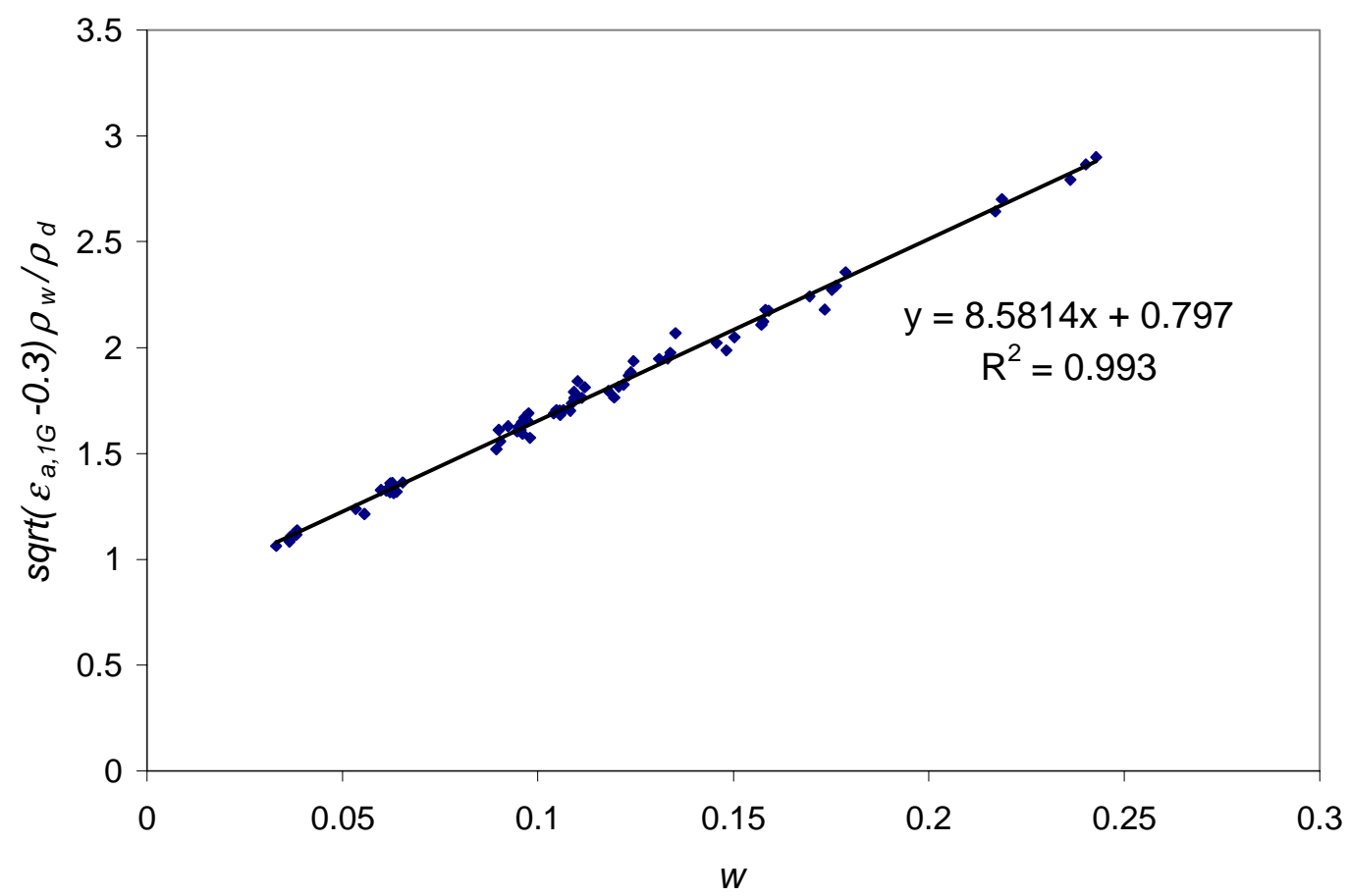

Fig. $6.25\left(\sqrt{ } K_{a^{-}} \mathrm{c}\right) \rho_{w} / \rho_{d}$ vs. $w$ relationship using the apparent dielectric constant at 1 $\operatorname{GHz}\left(\varepsilon_{a, 1 \mathrm{GHz}}\right)$ 


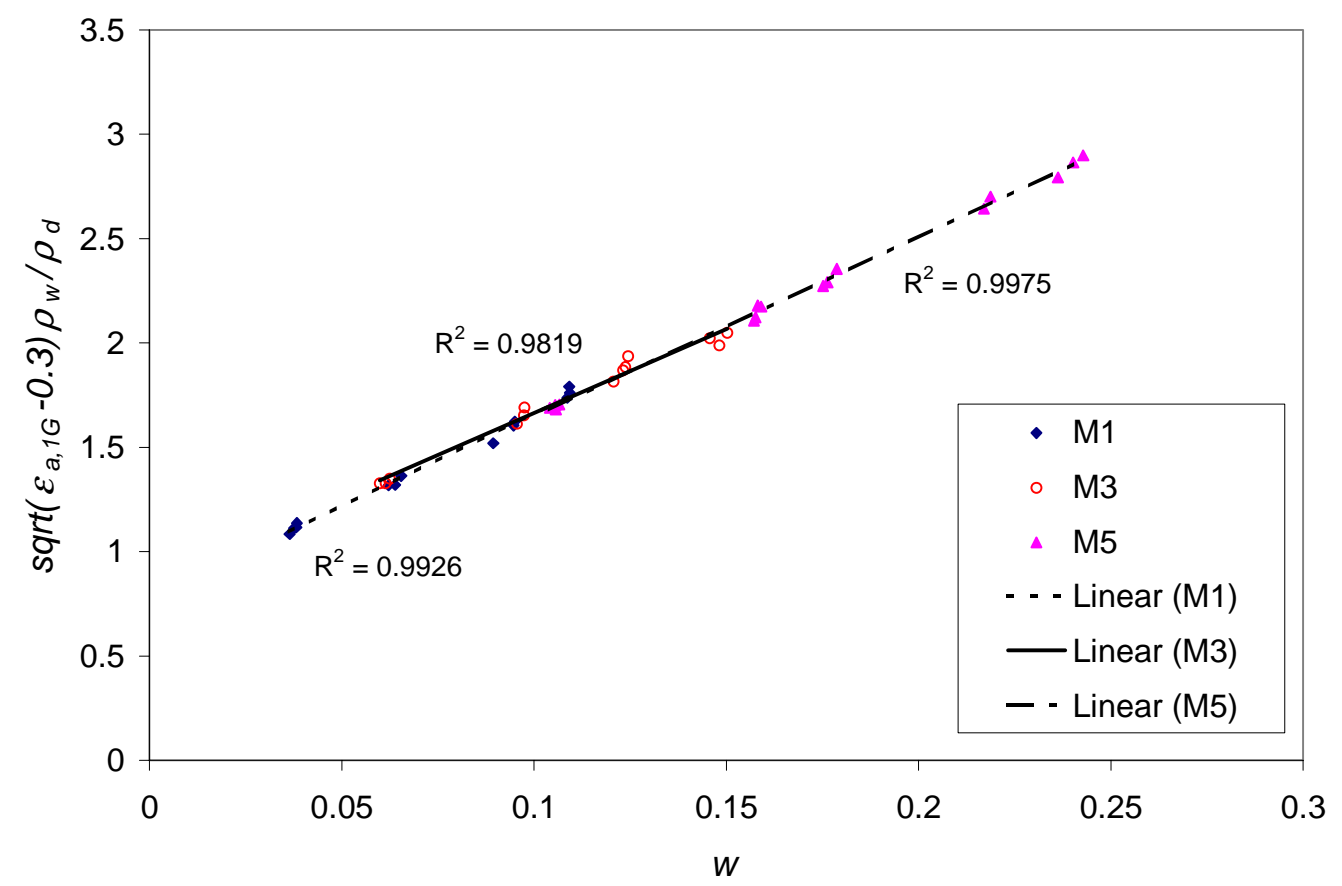

Fig. 6.26 $\left(\sqrt{ } K_{a}-\mathrm{c}\right) \rho_{w} / \rho_{d}$ vs. $w$ relationship using the apparent dielectric constant at 1 $\mathbf{G H z}\left(\varepsilon_{a, 1 \mathrm{GHz}}\right)$ for each soil type

$$
\sqrt{\varepsilon_{a}}=a \rho_{d}+b \theta+c
$$

or

$$
\frac{\left(\sqrt{\varepsilon_{a}}-c\right) \rho_{w}}{\rho_{d}}=a+b w
$$

The ability to measure dielectric spectra of soils by waveform matching based on volumetric mixing model allows us to calculate the apparent dielectric constant at a particular high frequency (e.g. $1 \mathrm{GHz}$ ). The apparent dielectric constants at $1 \mathrm{GHz}$ for the mixed soils were calculated and the regression analysis using Eq. (6.15) or (6.16) was conducted. Equation (6.16) was plotted for all soils in Fig. 6.25. Results for individual soils are shown in Fig. 6.26. Comparing Fig. 6.25 to Fig. 6.10 and Fig. 6.26 to Fig. 6.11, it can be seen that use of apparent dielectric permittivity at $1 \mathrm{GHz}$ essentially removes the soil type effect. The density effect is also better normalized for each individual soil type. In addition, the determination of $\varepsilon_{a, 1 \mathrm{GHz}}$ is more 
objective than the tangent line approximation in $K_{a}$. Equation (6.15) or (6.16) may become a true universal calibration equation.

\subsection{Summary}

A series of tests were conducted to study the soil properties using both the simplified analysis and the full waveform analysis. Empirical calibration equations for apparent dielectric constant in terms of soil physical parameters were evaluated. It was found that none of the empirical equations are universal and calibration is required. Similar to the apparent dielectric constant, a calibration equation for $\mathrm{dc}$ conductivity in terms of soil physical parameters is proposed. It is even more sensitive to soil types and measurement error than the apparent dielectric constant.

The volumetric mixing model was evaluated by the full waveform analysis. The results showed a non-uniqueness in inferring the mixing parameters from the TDR waveform. In its current form, it could not be used to simultaneously measure soil water content, density, and soil type. However, it is a good model that can match the TDR waveform well and measure the dielectric spectrum of individual soils. Because of the ability to measure dielectric spectrum of soils, the apparent dielectric constant at a particular frequency can be calculated. Results showed that the apparent dielectric constant at $1 \mathrm{GHz}$ gave much better correlation with soil water content and density than apparent dielectric constant obtained by a tangent line approximation. The ability to measure dielectric spectra of soils using TDR will assist further study of the dielectric behavior of soils. 


\section{CHAPTER 7 - TEMPERATURE EFFECTS}

\subsection{Background}

Temperature affects the dielectric properties of materials in different ways depending on the material. While this has been known for over 50 years (Frohlich (1949)), details of temperature effects on soils are still not well understood (Wraith and Or (1999)). If the apparent dielectric constant is used as a measure of dielectric properties, the apparent dielectric constant for water decreases with increasing temperature. The apparent dielectric constant for most soil solids is relatively independent of temperature in the range of $4^{\circ} \mathrm{C}$ to $40^{\circ} \mathrm{C}$. It is reasonable to expect that the apparent dielectric constant for mixtures of soil solids and water also would exhibit a decrease in apparent dielectric constant with temperature, but to a lesser extent than for water alone. This holds true for cohesionless soils, but does not hold true for cohesive soils that have significant amounts of clay-sized particles. For these materials, the apparent dielectric constant increases with temperature. Some theories for this behavior were put forth by Wraith and Or (1999) and discussion of the theoretical behavior is beyond the scope of this paper.

This chapter presents the results of an extensive series of tests to measure the apparent dielectric constant on a naturally occurring low plastic clay soil, pure kaolinite clay, pure illite clay, concrete sand, and fine sand. Each of the materials was compacted into a Standard Compaction Test mold (ASTM D698) with standard compaction energy and with different water contents. With use of a guide template, a center conductor was driven into the specimens to form a soil "cable" with length equal to the height of the mold. A ring adapter and a Multiple Rod Probe Head (MRPH) were used to form a connection between the soil cable and a Tektronix ${ }^{\circ} 4102 \mathrm{~B}$ cable tester. Specimens were placed in an environmental chamber allowed to equilibrate to temperatures ranging from $4^{\circ} \mathrm{C}$ to $40^{\circ} \mathrm{C}$. Measurements of apparent dielectric constant were made with time until readings stabilized.

The results of the tests are reported and are analyzed. Recommendations are put forth for correcting measured apparent dielectric constant to values at $20^{\circ} \mathrm{C}$ for cohesionless soils and for cohesive soils. The corrections are typically small and have a minor effect on calculation of water content. 
Results from this work are important for improving the accuracy of the TDR method for measuring the water content and density of soils as described by Feng et al. (1998), Lin et al. (1998), Lin et al. (2000), Siddiqui and Drnevich (1995), Siddiqui et al. (2000), and Drnevich et al. (2001).

\subsection{Test Procedures and Test Results}

\subsubsection{Test Specimens}

Tests were performed on specimens placed in a Standard Compaction Mold (ASTM D698) that had a diameter of $101.6 \mathrm{~mm}$ (4.0 in.) and a height of $116.4 \mathrm{~mm}$ (4.584 in.) giving a volume of $9.19 * 10^{5} \mathrm{~mm}^{3}\left(1 / 30 \mathrm{ft}^{3}\right)$. For all soil specimens, the soil specimens were compacted with standard compaction effort $600 \mathrm{kN}-\mathrm{m} / \mathrm{m}^{3}\left(12,400 \mathrm{ft}-\mathrm{lb} / \mathrm{ft}^{3}\right)$ at a variety of different water contents (gravimetric) ranging from below optimum to above optimum.

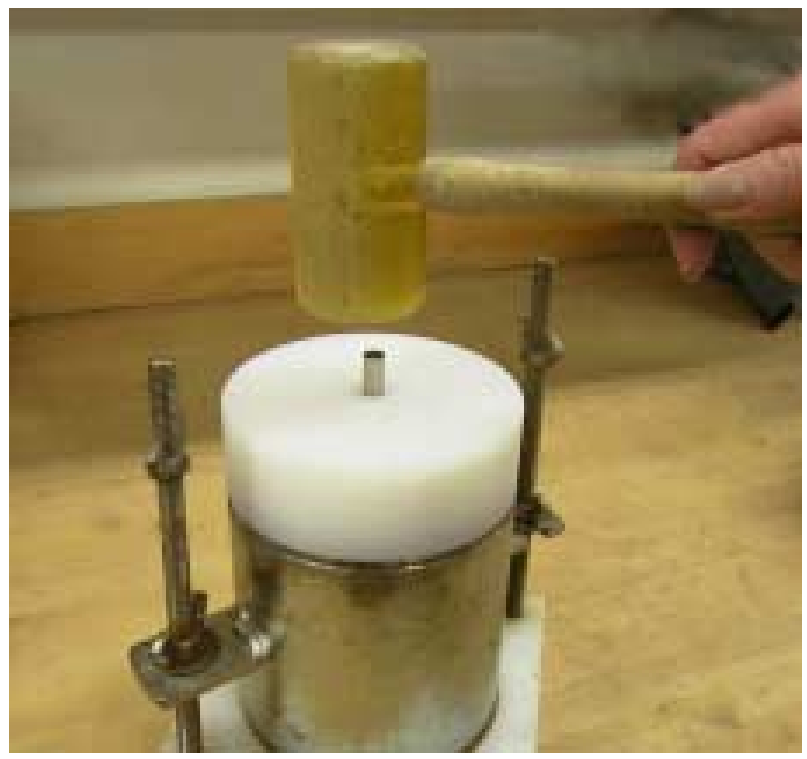

Fig. 7.1. Center Rod Being Driven into Specimen Through Guide Template

Once a specimen was compacted and the mass of the specimen and mold were determined, the metal bottom plate was replaced with a non-metallic plate. Then a guide template was temporarily placed on top of the mold and a stainless steel center rod with diameter of 7.94 $\mathrm{mm}(5 / 16$-in.) was driven into the specimen over its full height (See Fig. 7.1.). When the guide was removed, the rod protruded from the soil surface by approximately $30 \mathrm{~mm}$ (1.2 in.). An adapter ring was then placed on the top of the compaction mold as shown in Fig. 7.2. The ring presented a surface for the outer three legs of the Multiple Rod Probe Head (MRPH) to be supported. The center rod of the MRPH then came in contact with the center rod as shown in Fig. 7.3 .

TDR measurements were made with this arrangement by connecting MRPH with a Tektronix 8102B Cable Tester with a $1 \mathrm{~m}$ (3-ft.) coaxial cable with BNC connectors on each end. 


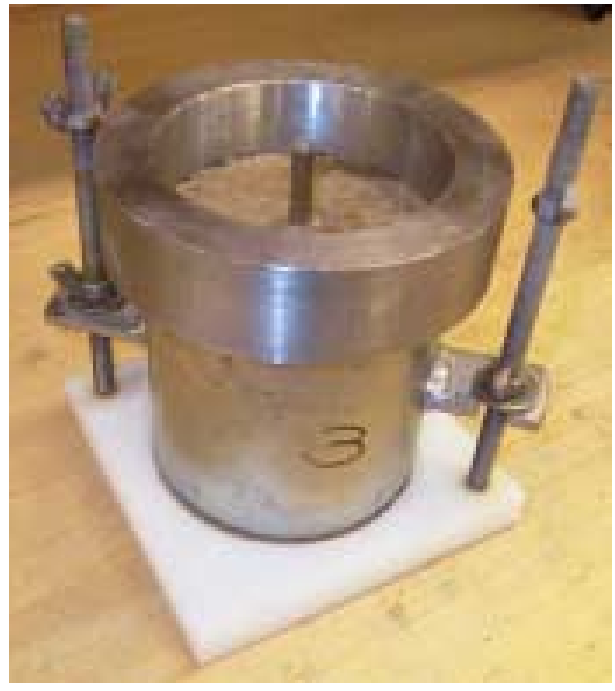

Fig. 7.2. Mold with Center Rod and Adapter Ring Ready for Multiple Rod Probe

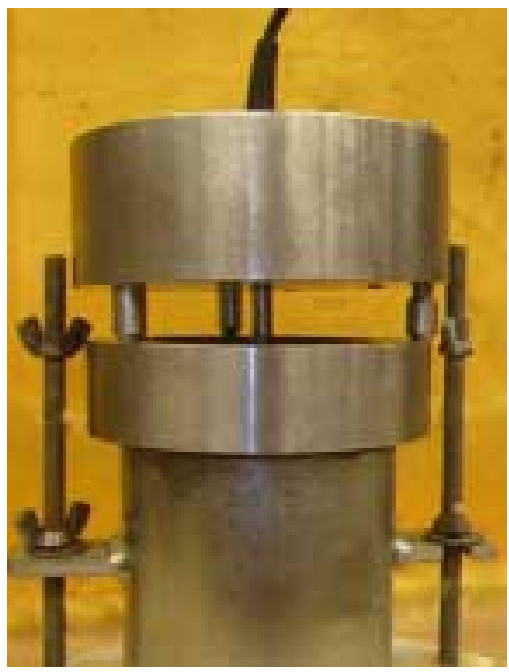

Fig. 7.3. Multiple Rod Probe Head Ready for Making TDR Measurements

Additional information about the use of this equipment for determining the apparent dielectric constant, $K_{a}$, is available in a paper by Drnevich et al. (2001).

One set of tests was done with the mold filled with water to validate that test results were consistent with test reported in the literature.

\subsubsection{Test Environment}

Three separate walk-in environmental test chambers were used for tests at $4^{\circ} \mathrm{C}, 10^{\circ} \mathrm{C}$, and $20^{\circ} \mathrm{C}$ where temperatures were reasonably close to the set temperatures for the test duration. For temperatures of $30^{\circ} \mathrm{C}$ and $40^{\circ} \mathrm{C}$, the specimens were placed in a large drying oven where temperatures were maintained within $\pm 1^{\circ} \mathrm{C}$ of the preset temperature.

Between times when readings were made, the specimens were covered with a plastic film to minimize the amount of moisture lost between readings. Determinations of total mass of the soil, mold, and center rod at the time of each TDR reading allowed for checking any changes in specimen water content. 


\subsubsection{Soils Tested}

The soils tested in this program are described in Table 7.1. The Crosby Till was naturally occurring in the vicinity of the Purdue University campus. The Kaolinite and Illite were pure clay minerals. The concrete sand was washed, naturally occurring, and had subrounded particles. The fine sand was Ottawa sand, nearly pure quartz sand. The Houston Clay was sampled from construction sites in Houston, Texas and is composed mostly of fat clay with some silt. The silt fraction was removed before testing this soil. In Table 7.1, ASTM D2487 was used for Unified Soil Classification, ASTM D4318 was used for Atterberg Limits, and ASTM D422 was used for the Composition. The authors are grateful to Mr. Jie Zhang who performed most of the tests.

Table 7.1 Characteristics of Soils Tested

\begin{tabular}{|c|c|c|c|c|c|c|}
\hline \multirow[b]{2}{*}{ Soil } & \multirow{2}{*}{$\begin{array}{c}\text { Unified } \\
\text { Soil } \\
\text { Classificat } \\
\text { ion }\end{array}$} & \multicolumn{2}{|c|}{ Atterberg Limits } & \multicolumn{3}{|c|}{ Composition } \\
\hline & & $\begin{array}{c}\text { Liquid } \\
\text { Limit }\end{array}$ & $\begin{array}{c}\text { Plastic } \\
\text { Limit }\end{array}$ & $\%$ sand & $\%$ silt & $\%$ clay \\
\hline Crosby Till & CL & 41 & 18 & 16 & 50 & 34 \\
\hline Kaolinite & CL-ML & 30 & 24 & 0 & 0 & 100 \\
\hline Illite & CL-CH & 50 & 22 & 0 & 0 & 100 \\
\hline $\begin{array}{c}\text { Concrete } \\
\text { Sand }\end{array}$ & SW & NA & NA & 100 & 0 & 0 \\
\hline Fine Sand & SP & NA & NA & 100 & 0 & 0 \\
\hline Houston Clay & $\mathrm{CH}$ & 54 & 23 & 0 & $5 *$ & $95 *$ \\
\hline
\end{tabular}

* Estimated

\subsubsection{Testing Sequence}

All specimens were constructed at room temperature, $20^{\circ} \mathrm{C}$ and then placed into the environmental chamber for testing. TDR readings were taken as a function of time to obtain the time required for equilibration. Most of the tests at a given temperature were on specimens tested only at that temperature. An example of this is shown in Fig. 7.4 for tests on Crosby Till at a target water content of 21 percent. Note that it typically took up to 1000 minutes for the readings to stabilize for the size specimens tested in these experiments. 


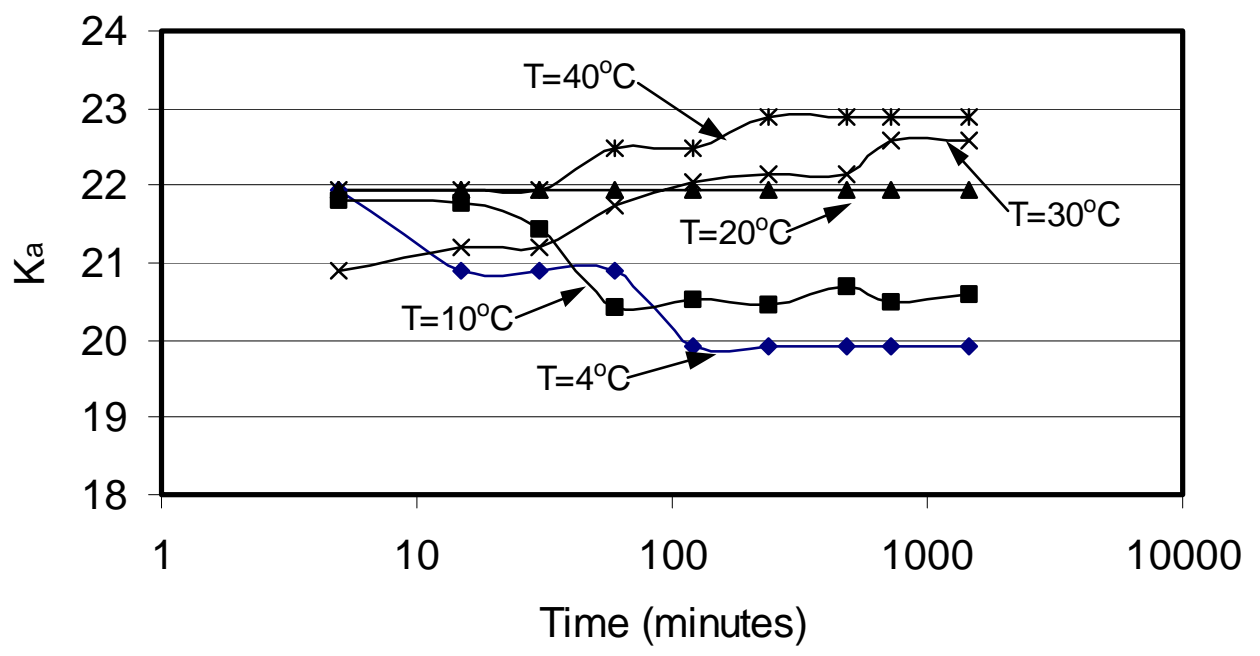

Fig. 7.4 Tests on Crosby Till at a Target Water Content of $21 \%$ with Tests at All Temperatures Done with Separate Soil

A given specimen could be tested at different temperatures without significantly affecting the results and some of the tests were tested at multiple temperatures ranging from $4{ }^{\circ} \mathrm{C}$ to $40^{\circ} \mathrm{C}$. Figure 7.5 gives an example of tests at three temperatures on a specimen of Crosby Till with a water content of 41 percent.

The typical testing process involved testing at $4^{\circ} \mathrm{C}, 10^{\circ} \mathrm{C}, 20^{\circ} \mathrm{C}, 30^{\circ} \mathrm{C}$, and $40^{\circ} \mathrm{C}$. On some of the soils, testing at some of the temperatures was omitted for saving of time since equilibration at each temperature took approximately 24 hours. On several of the clay soils at high water content and at high temperatures, accurate apparent length measurements could not be made because the soil was too lossy, i.e. dissipated the signal so that no reflected signal could be detected.

It has been discovered that insulated probe can prevent energy loss to extend the applicability of TDR technology in field practice (M.A.Mojid (1998)). The Houston Clay was too lossy to get the reflected signal, so an insulated probe was used on this soil.

The mass of soil and compaction mold was measured at each time a TDR measurement was made. At the end of the test after testing at all the temperatures, oven drying was used to determine water content (ASTM D2216) for the end of test condition. The mass measurements 
made along with the TDR measurements were used to calculate the water contents at the time of measurement. Table 7.2 gives the information on water contents, dry densities, and apparent dielectric constant for the soils tested. The water contents in Table 7.2 are gravimetric water contents. Agronomists make extensive use of TDR for measuring the volumetric water content of soil (volume of water as a percentage of the total volume of the soil). The volumetric water content is usually represented by the Greek letter theta, $\theta$. Geotechnical engineers work with the gravimetric water content of soil (mass of the water/mass of dry solids) and it is usually represented by the letter, $w$. Both $\theta$ and $w$ are expressed as percentages. Volumetric and gravimetric water contents are related by

$$
w=\theta \frac{\rho_{w}}{\rho_{d}}
$$

where $\rho_{d}$ is the dry density of the soil and $\rho_{w}$ is the density of water.

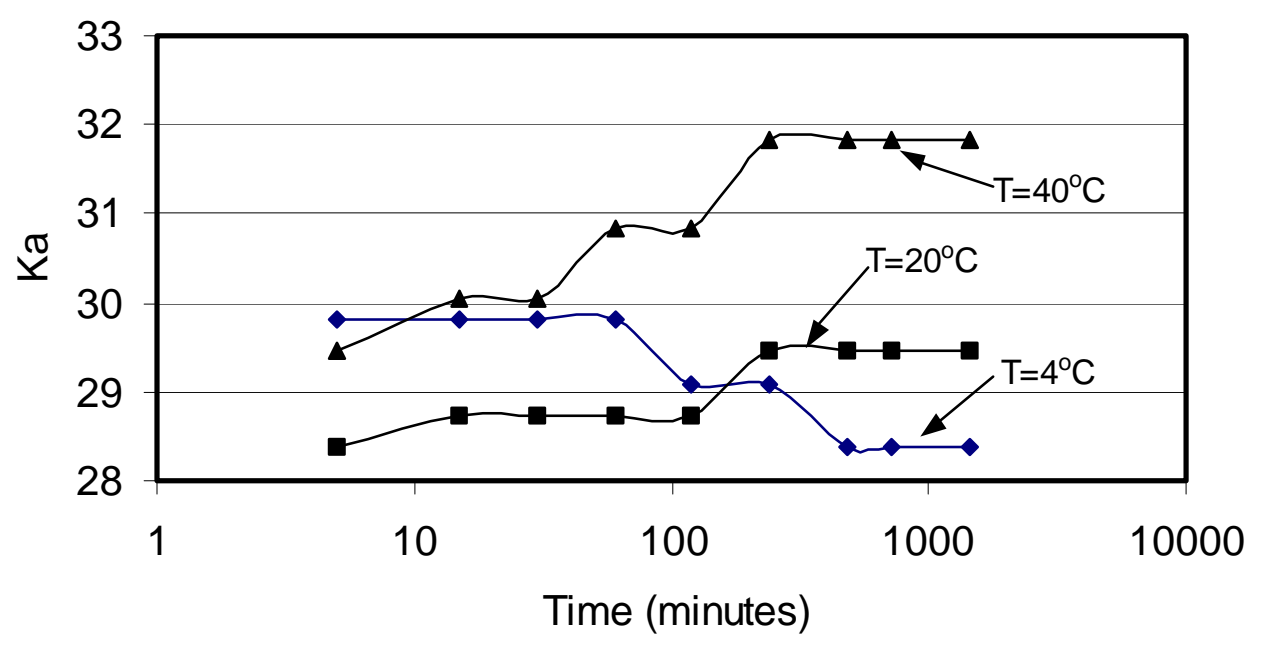

Fig. 7.5 Tests on Crosby Till at a Target Water Content of $41 \%$ with Tests at All Temperatures Done on the Same Soil 


\subsubsection{Tests on Water}

Figure 7.6 presents the results for tests on water. Data from Weast (1986) and Mitchell (1993) also are plotted in this figure. The test results compare fairly well with the greatest discrepancy occurring at a temperature of $40^{\circ} \mathrm{C}$ where the difference is about three percent.

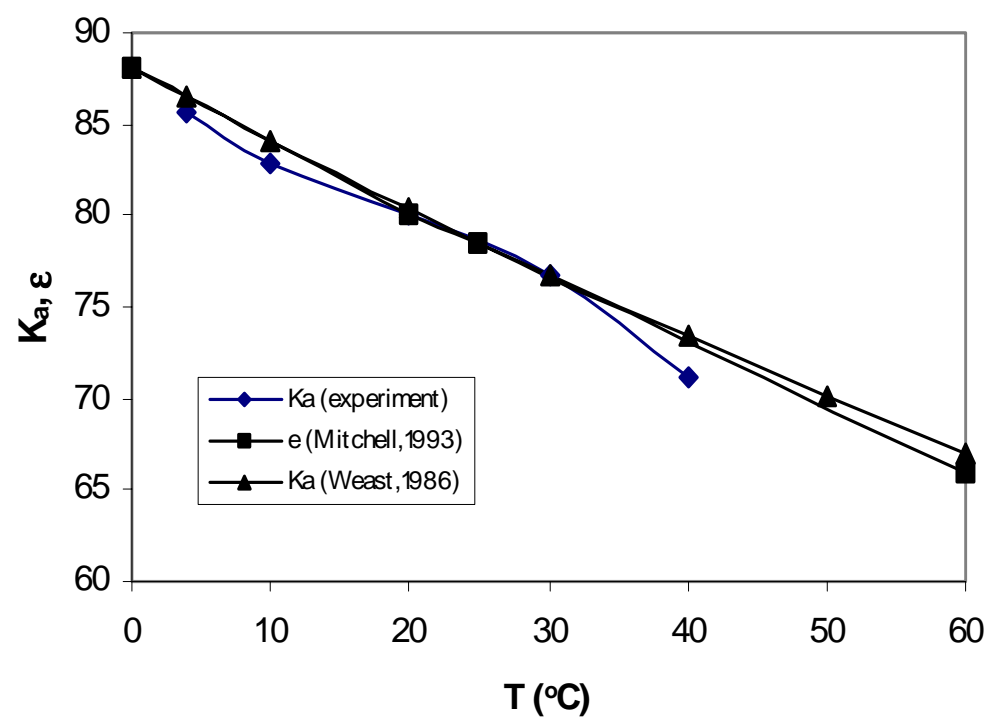

Fig. 7.6 Apparent Dielectric Constant Variation with Temperature for Water

\subsubsection{Calibration of Insulated Probe}

Insulated probe is first calibrated before application. Analysis on energy distribution around TDR probe shows that dielectric constant of composite dielectric medium made up of two medium as shown in Fig. 7.7 can be expressed as:

$$
\frac{\ln (b / a)}{K_{a}}=\frac{\ln \left(r_{1} / a\right)}{K_{1}}+\frac{\ln \left(b / r_{1}\right)}{K_{2}}
$$

Which shows that reciprocal of $K_{a}$ with an insulated probe is a linear relationship to reciprocal of $K_{a}$ by original probe. The equation was used to get a calibration curve. Clean 
Ottawa Sand with different water content and density was used in the calibration process. Readings were taken by both a regular probe and an insulated probe on the same specimen. Corresponding reciprocals of apparent dielectric constant values are plotted on the Fig. 7.8, which gives the calibration equation. The plot shows a fairly good linear relationship, which validated the theoretical prediction. The "true" dielectric constant of material measured with an insulated rod can be obtained from the following hyperbolic equation:

$$
K_{a}=\frac{K_{a, \text { insulated }}}{0.5769+0.014 K_{a, \text { insulated }}}
$$

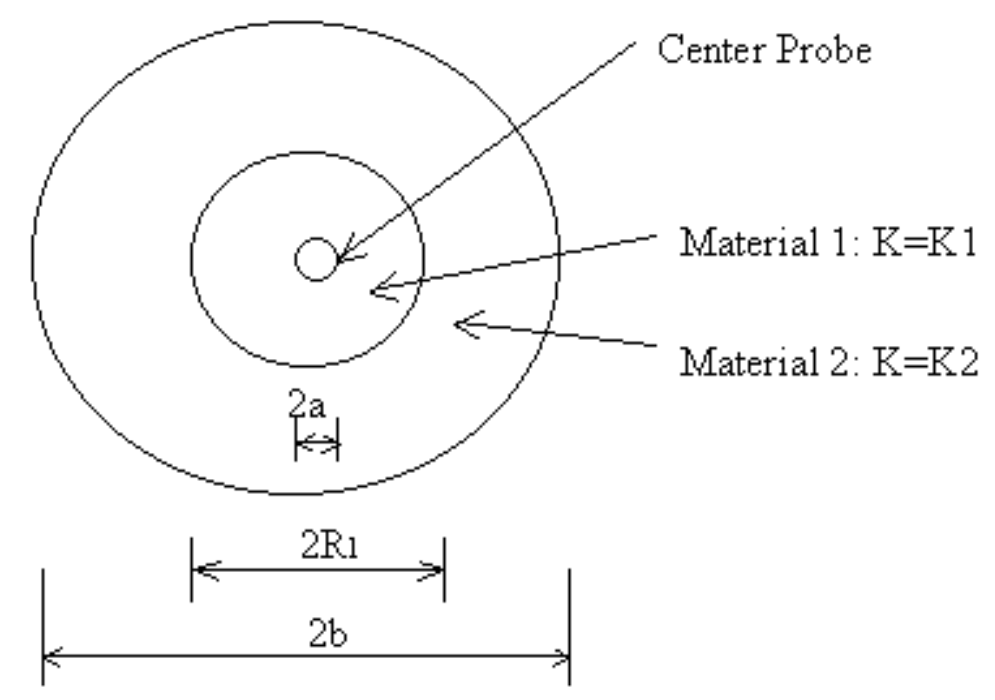

Fig 7.7 Cross Section of TDR Probe with Composite Medium

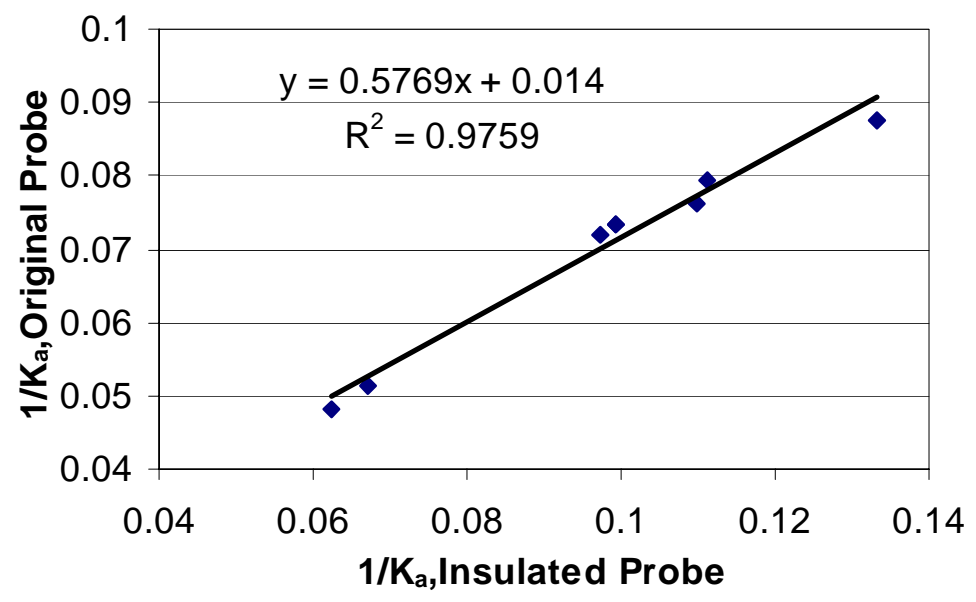

Fig. 7.8 Calibration Curve for Insulated Probe 
Table 7.2 Testing Program and Results of Tests on Soils

\begin{tabular}{|c|c|c|c|c|c|c|c|}
\hline \multirow{3}{*}{ Soil } & \multirow{3}{*}{$\begin{array}{c}\text { Target } \\
\text { Water } \\
\text { Content }(\%) \\
\end{array}$} & Actual Water Content (\%) & \multicolumn{5}{|c|}{ Temperature of Testing $\left({ }^{\circ} \mathrm{C}\right)$} \\
\hline & & Dry Density $\left(\mathrm{Mg} / \mathrm{m}^{3}\right)$ & \multirow{2}{*}{4} & \multirow{2}{*}{10} & \multirow{2}{*}{20} & \multirow{2}{*}{30} & \multirow{2}{*}{40} \\
\hline & & $\mathrm{K}_{\mathrm{a}}$ & & & & & \\
\hline \multirow{21}{*}{$\begin{array}{l}\text { Crosby } \\
\text { Till }\end{array}$} & \multirow{3}{*}{3} & Water Content & 3.1 & & 2.0 & & 3.0 \\
\hline & & Dry Density & 1.543 & & 1.543 & & 1.543 \\
\hline & & $\mathrm{K}_{\mathrm{a}}$ & 4.121 & & 4.335 & & 4.481 \\
\hline & \multirow{3}{*}{12} & Water Content & 11.6 & 12.0 & 11.1 & 11.8 & 11.9 \\
\hline & & Dry Density & 1.713 & 1.713 & 1.713 & 1.722 & 1.706 \\
\hline & & $\mathrm{K}_{\mathrm{a}}$ & 11.940 & 12.348 & 12.740 & 13.660 & 13.976 \\
\hline & \multirow{3}{*}{15} & Water Content & 14.4 & 14.8 & 13.9 & 13.8 & 14.8 \\
\hline & & Dry Density & 1.769 & 1.748 & 1.759 & 1.701 & 1.759 \\
\hline & & $\mathrm{K}_{\mathrm{a}}$ & 15.240 & 16.124 & 16.578 & 17.960 & 18.920 \\
\hline & \multirow{3}{*}{18} & Water Content & 16.8 & 17.2 & 16.6 & 17.1 & 17.9 \\
\hline & & Dry Density & 1.789 & 1.782 & 1.788 & 1.782 & 1.788 \\
\hline & & $\mathrm{K}_{\mathrm{a}}$ & 17.965 & 19.415 & 19.906 & 21.634 & 21.946 \\
\hline & \multirow{3}{*}{21} & Water Content & 20.4 & 20.8 & 20.0 & 20.9 & 20.5 \\
\hline & & Dry Density & 1.713 & 1.694 & 1.715 & 1.660 & 1.715 \\
\hline & & $\mathrm{K}_{\mathrm{a}}$ & 19.906 & 21.430 & 21.946 & 22.367 & 22.875 \\
\hline & \multirow{3}{*}{24} & Water Content & 22.7 & 23.0 & 22.3 & 22.8 & 23.7 \\
\hline & & Dry Density & 1.623 & 1.618 & 1.638 & 1.625 & 1.638 \\
\hline & & $\mathrm{K}_{\mathrm{a}}$ & 20.914 & 21.946 & 23.004 & 25.19 & 26.32 \\
\hline & \multirow{3}{*}{41} & Water Content & 39.0 & & 39.0 & & 38.35 \\
\hline & & Dry Density & 1.280 & & 1.280 & & 1.280 \\
\hline & & $\mathrm{K}_{\mathrm{a}}$ & 28.558 & & 29.458 & & 31.829 \\
\hline \multirow{9}{*}{ Kaolinite } & \multirow{3}{*}{20} & Water Content & 20.6 & & 20.5 & 20.2 & \\
\hline & & Dry Density & 1.505 & & 1.505 & 1.505 & \\
\hline & & $\mathrm{K}_{\mathrm{a}}$ & 20.039 & & 20.982 & 21.301 & \\
\hline & & Water Content & 28.2 & & 28.1 & & \\
\hline & 30 & Dry Density & 1.455 & & 1.455 & & \\
\hline & & $\mathrm{K}_{\mathrm{a}}$ & 22.601 & & 23.602 & & \\
\hline & & Water Content & 43.4 & & 43.2 & 42.6 & \\
\hline & 40 & Dry Density & 1.221 & & 1.221 & 1.221 & \\
\hline & & $\mathrm{K}_{\mathrm{a}}$ & 31.214 & & 31.994 & 33.182 & \\
\hline & & Water Content & 19.2 & & 19.1 & 18.9 & \\
\hline & 20 & Dry Density & 1.626 & & 1.626 & 1.626 & \\
\hline & & $K_{a}$ & 25.998 & & 28.803 & 31.571 & \\
\hline & & Water Content & 45.4 & & & & \\
\hline Illite & 45 & Dry Density & 1.17 & & & & \\
\hline & & $\mathrm{K}_{\mathrm{a}}$ & 36.114 & & & & \\
\hline & & Water Content & 50.1 & & 50.1 & 49.7 & \\
\hline & 50 & Dry Density & 1.15 & & 1.15 & 1.15 & \\
\hline & & $\mathrm{K}_{\mathrm{a}}$ & 47.911 & & 49.037 & 51.096 & \\
\hline & & Water Content & 0.20 & & 0.20 & & 0.20 \\
\hline & 0.2 & Dry Density & 1.785 & & 1.785 & & 1.785 \\
\hline Concrete & & $\mathrm{K}_{\mathrm{a}}$ & 3.643 & & 3.643 & & 3.511 \\
\hline Sand & & Water Content & 14.5 & & 14.5 & & 13.7 \\
\hline & 14.6 & Dry Density & 1.883 & & 1.883 & & 1.883 \\
\hline & & $\mathrm{K}_{\mathrm{a}}$ & 18.515 & & 17.923 & & 16.875 \\
\hline & & Water Content & .08 & & .08 & & .03 \\
\hline & 0.08 & Dry Density & 1.682 & & 1.682 & & 1.682 \\
\hline Fine Sand & & $\mathrm{K}_{\mathrm{a}}$ & 2.66 & & 2.66 & & 2.66 \\
\hline Fine Sand & & Water Content & 19.4 & & 19.4 & & 18.8 \\
\hline & 19.4 & Dry Density & 1.742 & & 1.742 & & 1.742 \\
\hline & & $\mathrm{K}_{\mathrm{a}}$ & 21.622 & & 20.982 & & 19.729 \\
\hline
\end{tabular}




\begin{tabular}{|c|c|c|c|c|c|c|c|}
\hline \multirow{2}{*}{ Soil } & \multirow{2}{*}{$\begin{array}{c}\text { Target } \\
\text { Water } \\
\text { Content } \\
(\%)\end{array}$} & Actual Water & \multicolumn{5}{|c|}{ Temperature of Testing $\left({ }^{\circ} \mathrm{C}\right)$} \\
\hline & & $\begin{array}{l}\text { Dry Density } \\
\left(\mathrm{Mg} / \mathrm{m}^{3}\right)\end{array}$ & 4 & 10 & 20 & 30 & 40 \\
\hline \multirow{18}{*}{$\begin{array}{l}\text { Houston } \\
\text { Clay }\end{array}$} & \multirow{3}{*}{15} & Water Content & 15.4 & 15.4 & 15.4 & 15.2 & 14.7 \\
\hline & & Dry Density & 1.405 & 1.405 & 1.405 & 1.405 & 1.405 \\
\hline & & $\mathrm{K}_{\mathrm{a}}$ & 13.52 & 14.52 & 14.87 & 15.79 & 16.82 \\
\hline & \multirow{3}{*}{20} & Water Content & 20.1 & 20.1 & 20.1 & 19.8 & 19.4 \\
\hline & & Dry Density & 1.488 & 1.488 & 1.488 & 1.488 & 1.488 \\
\hline & & $\mathrm{K}_{\mathrm{a}}$ & 16.82 & 18.11 & 18.83 & 19.67 & 20.97 \\
\hline & \multirow{3}{*}{25} & Water Content & 24.9 & 24.9 & 24.9 & 24.7 & 24.3 \\
\hline & & Dry Density & 1.552 & 1.552 & 1.552 & 1.552 & 1.552 \\
\hline & & $\mathrm{K}_{\mathrm{a}}$ & 21.74 & 23.03 & 23.81 & 24.83 & 27.35 \\
\hline & \multirow{3}{*}{30} & Water Content & 30.3 & 30.3 & 30.3 & 29.9 & 29.3 \\
\hline & & Dry Density & 1.456 & 1.456 & 1.456 & 1.456 & 1.456 \\
\hline & & $\mathrm{K}_{\mathrm{a}}$ & 22.18 & 23.29 & 24.06 & 25.34 & 27.85 \\
\hline & \multirow{3}{*}{35} & Water Content & 35.1 & 35.1 & 35.1 & 34.8 & 34.2 \\
\hline & & Dry Density & 1.335 & 1.335 & 1.335 & 1.335 & 1.335 \\
\hline & & $\mathrm{K}_{\mathrm{a}}$ & 24.83 & 25.59 & 26.80 & 29.80 & 30.76 \\
\hline & \multirow{3}{*}{55} & Water Content & 55.6 & 55.6 & 55.6 & 54.4 & 52.6 \\
\hline & & Dry Density & 1.077 & 1.077 & 1.077 & 1.077 & 1.077 \\
\hline & & $\mathrm{K}_{\mathrm{a}}$ & 27.60 & 28.59 & 30.29 & 31.00 & 34.02 \\
\hline
\end{tabular}

\subsection{Analysis of the Results and Recommendations}

\subsubsection{Normalized Apparent Dielectric Constant}

The apparent dielectric constant data in Table 7.2 were normalized by dividing each of the values of apparent dielectric constant for a soil at a target water content by the corresponding value at $20^{\circ} \mathrm{C}$. The results are plotted in Figs. 7.9a and 7.9b. Also plotted in Fig. 7.9a are the normalized data for water. Note that the curve for water exhibits the most dramatic decrease with increase in temperature. The sand soils also exhibit a decrease in apparent dielectric constant with increase in temperature but the decrease is less dramatic. For sands with near zero water content, there is no appreciable change in apparent dielectric constant with temperature.

The behavior of cohesive soils in Figs. 7.9a and 7.9b shows just the opposite trends from those of water and sands; the dielectric constant increases with increasing temperature. Wraith and Or (1999) and other suggest that this behavior is due to the bound water typically associated with finegrained soils. A detailed discussion of these phenomena is beyond the scope of this paper. 


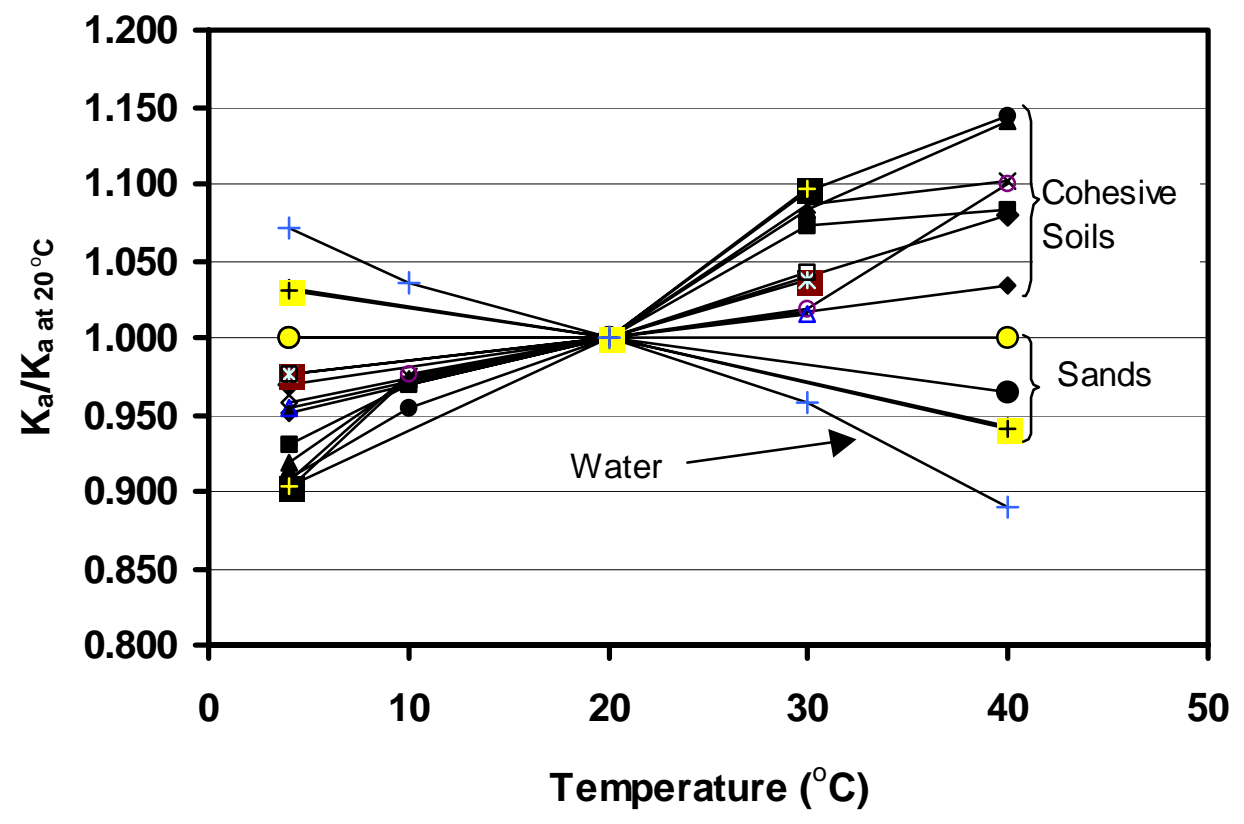

$\longrightarrow$ Crosby Till,3\%

-C Crosby Till, $12 \%$

—-Crosby Till,15\%

$\longrightarrow$ Crosby Till,18\%

——Crosby Till,21\%

$\longrightarrow$ Crosby Till,24\%

—Crosby Till,41\%

$\triangle$ Kaolinte,20\%

$\neg$ Kaolinte,30\%

- - Kaolinte, $40 \%$

-十- llite,20\%

七- llite, $50 \%$

- Conc. Sand, $0.2 \%$

$\triangle$ Conc. Sand, $14 \%$

-O_ Fine Sand, $0.08 \%$

-+ - Fine Sand, 19\%

-1- Water

Fig. 7.9a Normalized Apparent Dielectric Constants for Water and All Soils Tested.

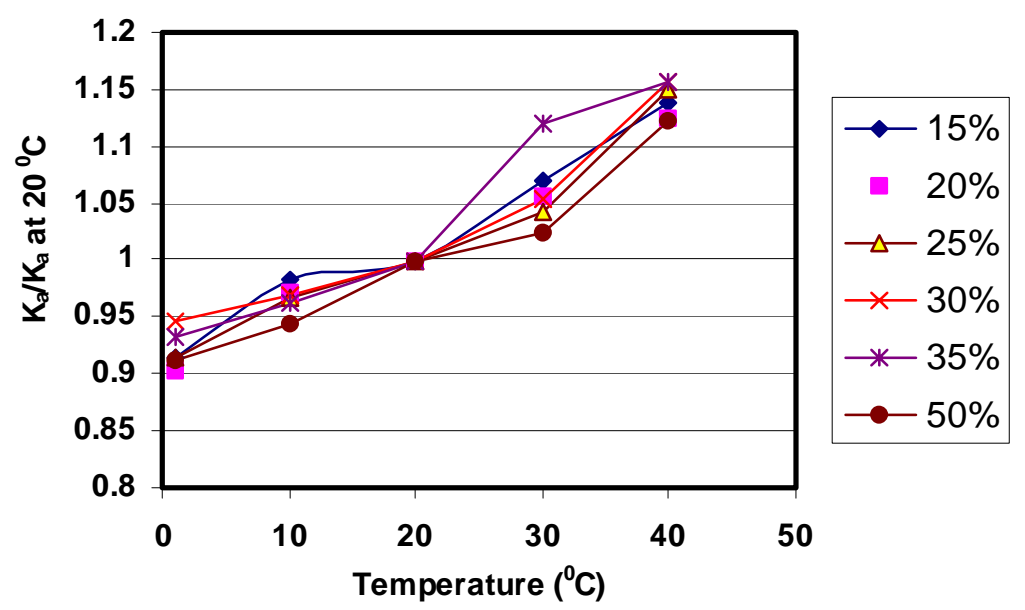

Fig.7.9b Normalized Apparent Dielectric Constants for Houston Clay at Different Water Contents

\subsubsection{Effects of Water Content on Behavior of Cohesive Soils}

For the soils tested, an attempt was made to discern effects of testing at the different water contents on values of apparent dielectric constant. This was done by fitting a straight line by least squares fitting through each of the data sets for the cohesive soils in Fig. 7.9a. Then the slopes of 
each of these lines were plotted versus water content. Figure 7.10 presents the results for Crosby Till. The data in Fig. 7.10 are fitted with a second order polynomial to help accentuate the trend. At low water contents, the slopes were small and increased with increasing water content and then, as water contents got higher, started to decrease. It was argued that a very low water contents,

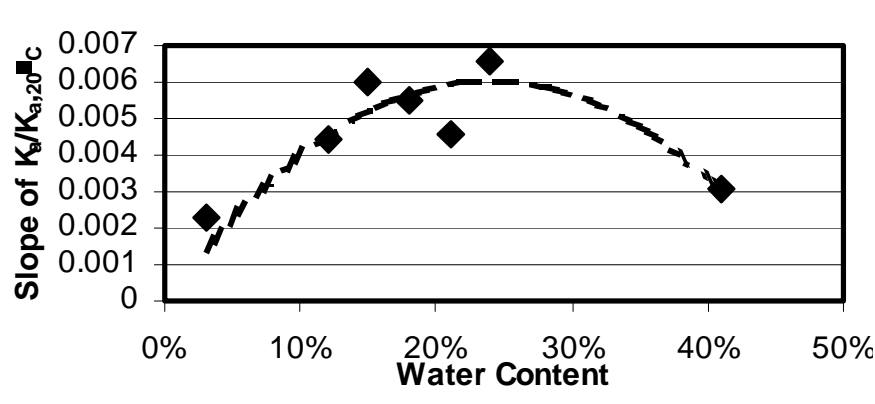

\section{Fig. 7.10 Change of Slope of Normalized Temperature Effects Lines with Wat Content for Crosby Till}

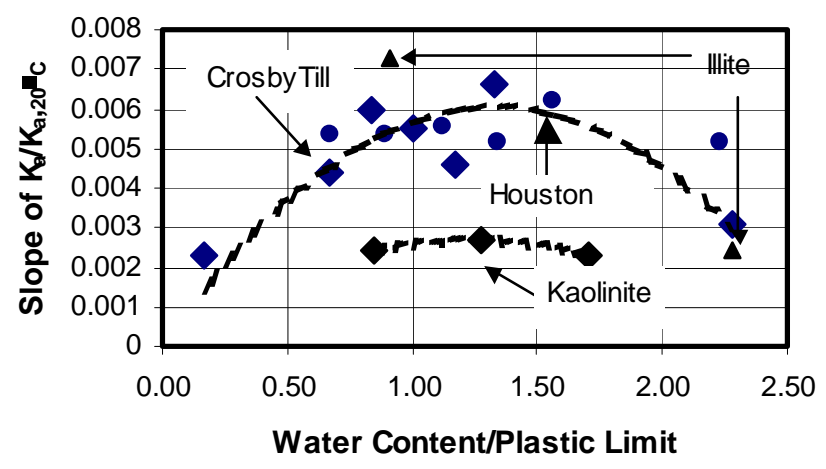
Fig. 7.11 Slope of Normalized Apparent Dielectric Constant with Water Content Normalized by the Plasticity Limit

behavior was dictated by the soil solids and bound water. With increasing water content, free water became an increasingly large component of the total volume and the effects of temperature on free water begin to dominate.

Note that the curve in Fig. 7.10 peaks near the Plastic Limit (ASTM D4318) for this soil, which was 18 percent. It was conjectured that below the Plastic Limit, not much unbound water is available to promote plastic behavior of soil and that this might be related to the amount of water available to affect the dielectric properties with temperature. Hence, the Plastic Limit became a candidate for normalizing the water content for the purposes of studying the behavior of all cohesive soils. In Fig. 7.11 are plotted all of the slope data versus water content normalized by the Plasticity Index for these tests on cohesive soils. While the data available are far too sparse to make any strong conclusions, plotting of future data in the same manner may provide some useful insight to the phenomena. For example, it appears that the peak in the slopes occurs at water contents approximately 1.5 times the Plastic Limit.

Considering that temperature effects on dry soil solids are near zero, the corresponding slope of $\mathrm{K}_{\mathrm{a}} / \mathrm{K}_{\mathrm{a}, 20^{\circ} \mathrm{C}}$ would go through origin. Likewise, as water content gets very large, the slopes 
would become negative and eventually be asymptotic to the slope for water $(-0.354$ after Weast (1986)).

\subsubsection{Temperature Adjustments to Measured Values of Apparent Dielectric Constant}

The values of normalized apparent dielectric constant for the cohesive soils in Fig. 7.9 were averaged at each temperature. These averaged values were then plotted versus temperature to obtain a mean curve that might apply to all of the cohesive soils tested. The same process was applied to all of the sand data in Fig.7.9a as well. The data for both the cohesive soils and the sand soils turned was exceptionally linear.

We are recommending that effects of temperature on apparent dielectric constant can be accommodated by simple linear correction as given in Eq. (7.4).

$$
K_{a, 20^{\circ} \mathrm{C}}=K_{a, T^{\circ} \mathrm{C}} \times T C F
$$

where

$$
\begin{aligned}
T C F & =\text { Temperature Correction Function } \\
& =0.97+0.0015 T_{\text {test }, T^{\circ} \mathrm{C}} \text { for cohesionless soils, } 4^{\circ} \mathrm{C} \leq T_{\text {test, } T^{\circ} \mathrm{C}} \leq 40^{\circ} \mathrm{C} \\
& =1.10-0.005 T_{\text {test }, T^{\circ} \mathrm{C}} \text { for cohesive soils, } 4^{\circ} \mathrm{C} \leq T_{\text {test, } T^{\circ} \mathrm{C}} \leq 40^{\circ} \mathrm{C}
\end{aligned}
$$

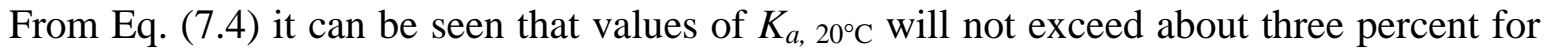
cohesionless soils and ten percent cohesive soils for extremes in temperature covered by this equation.

The theoretical and experimental study by Lin et al. (2000) suggested that the densitycompensating calibration equation proposed by Siddiqui and Drnevich (1995) provides the best relationship between soil water content and apparent dielectric constant. The "Siddiqui-Drnevich" calibration equation accounts for soil density and soil type: 


$$
w=\frac{1}{b}\left[\frac{\rho_{w}}{\rho_{d}} \sqrt{K_{a}}-a\right]
$$

where $\rho_{d}$ is the dry density of soil, $\rho_{w}$ is the density of water, $a$ and $b$ are soil-dependent calibration constants. From tests on a variety of soils the value of $a$ is consistently near unity and the value of $b$ is consistently near eight. Considering Eq. (7.5), we see that water content is related to the square root of $K_{a}$ and hence temperature effects on water content are relatively small. The authors suggest that temperature corrections are not needed for $15^{\circ} \mathrm{C} \leq T_{\text {test }}, T^{\circ} \mathrm{C} \leq 25^{\circ} \mathrm{C}$. This recommendation and the correction recommended in Eq. (7.4) are consistent with the findings of Kuraz (1981) who stated, "Temperature effects may be neglected for fluctuations of $5^{\circ} \mathrm{C}$. For wider changes in temperature, a simple linear correction is required."

\subsection{Summary and Conclusions}

Apparent dielectric constants in soils are somewhat dependent on soil temperature. Data are presented from tests on cohesive and cohesionless soils at various water contents and densities, tested at temperatures ranging from $4^{\circ} \mathrm{C}$ to $40^{\circ} \mathrm{C}$. Tests were performed on the soils compacted by Standard Compaction Effort into a cylindrical mold that is used in compaction testing. Apparent dielectric constants were determined by inserting a steel rod along the axis of the specimen and using a Multiple Rod Head, developed by the authors, along with a Tektronix ${ }^{\circledR} 4102 \mathrm{~B}$ Cable Tester. The entire system was placed in a temperature controlled environmental chamber and measurements were made until readings stabilized, typically less than twenty-four hours. Test results show that apparent dielectric constant for sands was similar to that for water, but less dramatic. For clays, the behavior of apparent dielectric constant with temperature was just the opposite of the behavior for water and sands. The amount of correction for clays depends on the water content and there exists a water content just higher than the Plastic Limit where temperature effects are the largest. Recommendations are made to correct measured values of apparent dielectric constant to values at $20^{\circ} \mathrm{C}$ using simple linear corrections. It is shown that effects of temperature on water content determination are likely to be small and can be neglected for temperatures within $5^{\circ} \mathrm{C}$ of $20^{\circ} \mathrm{C}$. 


\section{CHAPTER 8 - EFFECTS OF TESTING LARGE PARTICLE-SIZED MATERIALS}

\subsection{Background}

The apparent dielectric constant of soils as measured by time domain reflectometry (TDR) is affected by many parameters including: water content, temperature, soil particle size and particle size distribution, and by measurement probe configuration and installation. This chapter is predominantly about the effects of particle size on the insertion of a TDR probe and the resulting measured apparent dielectric constant, especially for soils that contain large particle sizes, a very common occurrence in both manmade fills and naturally occurring soils. This work is part of an ongoing study that is developing and evaluating the Purdue TDR Method (Drnevich et al. 2001) for determination of soil water content and density for use in engineering practice.

Nearly all existing laboratory and field tests for measuring water content and density of soils have limitations on maximum particle size. In many tests such as the Standard Test Method for Laboratory Compaction Characteristics of Soil Using Standard Effort (12,400 ft-lbf/ft ${ }^{3}$ (600 $\left.k N-m / m^{3}\right)$ ), ASTM D698, (1998), for Method B where a $101.6 \mathrm{~mm}$ (4.0-in.) diameter mold is used, the soil to be tested must pass the $9.5-\mathrm{mm}$ (3/8-in.) sieve and no more than 20 percent by weight of the original soil can be retained on the $9.5 \mathrm{~mm}(3 / 8$-in.) sieve. In this case, the soil retained on the 9.5 -mm (3.8-in.) sieve is considered oversize particles and is not used in the test. The Standard Practice for Correction of Unit Weight and Water Content for Soils Containing Oversize Particles, ASTM D4718 (1998) provides procedures for correcting water content and density test results to account for the untested oversized particles. Corrections of results from both the lab and the field tests must be made when oversize particles are encountered. The procedures in ASTM D4718 require measurement of the mass and the water content of both the finer and oversize portions of the sample and they are generally valid when the percentage of oversized particles is typically less than 40 percent. It is expected that there would be similar limitations to the TDR method of measuring water content and density and that procedures of ASTM D4718 (1998) also would apply to results of TDR tests. This chapter focuses on how the TDR results are affected when large particle sizes are present. 
The Purdue TDR Method uses two types of probes, one that is used insitu and one that makes use of a cylindrical mold for the soil. The one for insitu is a multiple rod probe that is formed by driving four steel spikes through a template into the soil surface (See Fig. 8.1), removing the template, and then placing a Multiple Rod Probe Head on these steel spikes. The test in the cylindrical mold uses a metal mold, similar to a compaction mold for the "shield" and

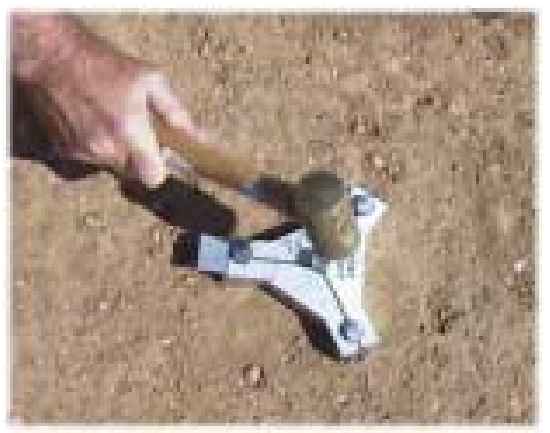

Fig. 8.1 Steel Spikes Forming Multiple Rod Probe

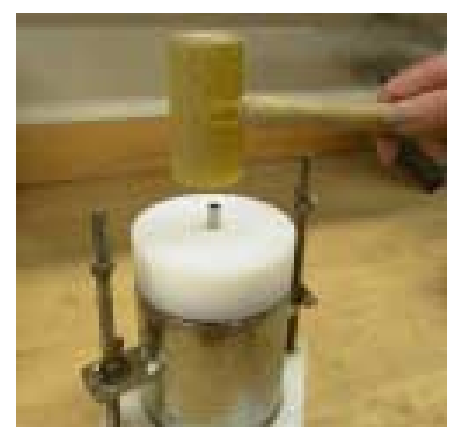

Fig. 8.2 Driving Central Rod Through a Guide Placed on the Mold a central rod that is driven into the soil through a guide placed on top of the mold (See Fig. 8.2). By use of an adapter ring placed on the mold, the same Multiple Rod Probe Head is used to complete the probe arrangement. The testing is done mostly for construction control and the test is frequently performed at multiple locations at a given site.

Steel rods that are separate from the probe head allows for inserting the rods into nearly any soil irrespective of how dense it is or for particle sizes into the gravel range. The rods are reusable, but are sufficiently inexpensive that they could be considered disposable. While this is a very robust system, the question remains on how the rod insertion affects the soil and how representative the measured apparent dielectric constant is of the soil prior to the insertion.

Work done by Siddiqui and Drnevich (1995) and Siddiqui et al. (2000) showed that the Purdue TDR Method was generally applicable for soils with sand sizes and below. They examined various models for densification (or loosening) of the soil by rod insertion and for void creation adjacent to the rods by the rod insertion process. They developed a Spatial Weighting Function (Eq. 8.1) that was a function of $b / a$, the ratio of the radius from the center to the shield (mold or outer rods) to the radius of the inner conductor. The larger the value of this ratio, larger is the influence of the soil immediately adjacent to the inner conductor on the measured apparent dielectric constant. 


$$
F(r)=\frac{\ln (r / a)}{\ln (b / a)}
$$

where

$r=$ the radius to the point of interest from the axis of the inner conductor

$a=$ the radius of the inner conductor

$b=$ the distance from the axis of the inner conductor to the outer conductor

If the Central Area is defined as the area inside the radius $r$, i.e. $\pi r^{2} / 4$, and the Total Area as $\pi b^{2} / 4$ then the Central Area/Total Area is measure of the fraction of the soil adjacent to

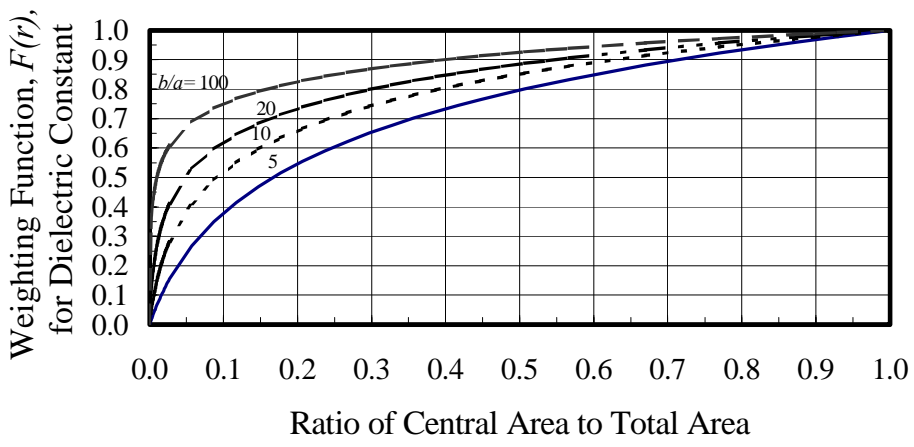

Fig. 8.3. Characteristics of the Spatial Weighting Function for a Coaxial Transmission Line (After Siddiqui et al. (2000)) the inner conductor that contributes to the measured dielectric constant. Figure 8.3 is a plot of the Spatial Weighting Function versus Central Area/Total Area for various values of $b / a$. From Fig. 8.3, when $b / a=10$, the ten percent of soil area adjacent to the inner conductor contributes to half of the apparent dielectric constant.

Based on Fig. 8.3, a small value of $b / a$ ratio is desired to obtain a good representation of the material between the inner and outer conductors. However, small values of $b / a$ are obtained by making the inner conductor quite large relative to the radius to the outer conductor. This has the effect of disturbing a larger volume of soil, especially the soil adjacent to the inner conductor that is critical to the measurement of the apparent dielectric constant. The disturbance is in the form of densification of soils that are relatively loose and loosening of soils (due to dilation) that are relatively dense prior to insertion of the rod. Siddiqui et al. (2000) also developed models for soil densification adjacent to the inner conductor caused by rod insertion and its effect on the apparent dielectric constant. They showed that rod insertion also depends on $b / a$ and causes an increase of the apparent dielectric constant over that before rod insertion. Finally, rod insertion 
generally will cause some air gaps to form adjacent to the inner conductor due to lateral movement or drift of the rod as it is being driven.

These air gaps cause the measured values of apparent dielectric constant to be lower than those when no air gaps exist. Based on their models and on tests of clays, silts, and sands, Siddiqui et al. (2000) suggested that a "b/a ratio of 15 is a good value to use." This is consistent with the findings of Annan (1977), Topp et al. (1980), and Zegelin et al. (1989).

Knight (1992) suggested that for a multiple rod probe, the ratio of the radius of the inner rod to the center-to-center distance between the inner rod and outer rods should be greater than 0.1 [this corresponds to a $b / a$ ratio less than 10] and that the radius should be as large as possible compared to the average pore size of the material. In the Purdue TDR Method, we are using a value of $b / a$ of 13.8 for the multiple-rod probe and a value of $b / a$ of 12.8 for the mold probe. Both of these numbers result from using readily available standard-sized materials and are approximately consistent with the recommendations of Knight (1992) and Siddiqui et al. (2000).

Engineers frequently have to deal with soils where particles range in size from boulders to clays with most sizes in between. Table 8.1 adapted from ASTM D2487-00 Standard Classification of Soils for Engineering Purposes (Unified Soil Classification System) and Lambe and Whitman (1979) gives the names used by geotechnical engineers for different sizes of particles and their size ranges.

For fine-grained soils containing appreciable amounts of clay, the specific surface (surface area per mass typically in units of $\mathrm{m}^{2} / \mathrm{g}$, (Lambe and Whitman, 1979)) of the clay particles is quite large and hence electrical effects have to be considered along with gravitational effects. In clay particles, net negative charges of the particles give rise to adsorbed water and cations on the clay particle surfaces that is described by the Gouy Double Layer theory. A number of researchers (see Or and Wraith (1999), Ponizovsky, et al. (1999)) have documented that apparent dielectric constant of materials within the adsorbed water layer (typically with values 20 to 40 ) is much different from that of free water in the pore space (typically around 80). These effects on the apparent dielectric constant as measured by the Purdue TDR Method is currently under study and is beyond the scope of this chapter. 
Table 8.1 Definitions for Particle Sizes

\begin{tabular}{|c|c|c|}
\hline \multirow[t]{2}{*}{ Name } & \multicolumn{2}{|l|}{ U.S. Standard Sieve Sizes } \\
\hline & Upper Limit Size, mm (in. or sieve No.) & Lower Limit Size, mm (in. or sieve No.) \\
\hline boulders & & $300 \mathrm{~mm}$ (12 in.) \\
\hline cobbles & $300 \mathrm{~mm}(12$ in. $)$ & $75 \mathrm{~mm}$ (3 in.) \\
\hline gravels & $75 \mathrm{~mm}$ (3 in.) & $4.75 \mathrm{~mm}$ (No. 4) \\
\hline sands & $4.75 \mathrm{~mm}$ (No. 4) & $0.075 \mathrm{~mm}$ (No. 200) \\
\hline silts $^{*}$ & $0.075 \mathrm{~mm}$ (No. 200) & $0.002 \mathrm{~mm}\left(8.0 \times 10^{-5}\right.$ in. $)$ \\
\hline clays $^{* *}$ & $0.002 \mathrm{~mm}\left(8.0 \times 10^{-5}\right.$ in. $)$ & \\
\hline
\end{tabular}

For coarse-textured soils, the shape of the particles also can have an effect on measured dielectric properties. Particle shapes typically are described as angular, subangular, subrounded, rounded, and well-rounded (Lambe and Whitman, 1979)). Coarse-textured soils, particularly those containing gravel or rock, occur at many sites. The roundness of large particles would allow for probe rods to slide off these particles and move them aside while for angular particles, there would be an increased tendency of the pointed probe rods to drag the particle along, creating a void along the sides of the rod and disrupting the soil near the rod.

\subsection{Materials and Methods}

\subsubsection{Materials Tested}

Eight different materials were tested. They are described in Table 8.2 and the particle size distributions are shown in Fig. 8.4a and Fig. 8.4b (Code key: CT= Crosby Till, MG = Medium Gravel, $\mathrm{SG}=$ Small Gravel, $\mathrm{CS}=$ Concrete Sand.) The Crosby Till is a local silty-clay soil with a Unified Soil Classification of CL. The rock was a sub-rounded glacial outwash gravel material. Fractions of it by size were mixed with the Crosby Till and the concrete sand to obtain the remaining six of the eight soils for testing. We tested two additional materials, both classified as Indiana 53, with one from naturally occurring gravel-sand mixtures and one a crushed stone. 


\section{Table 8.2 Compositions in Percent by Weight}

\begin{tabular}{|c|c|c|c|c|c|}
\hline Soil Code & $\begin{array}{c}\text { Medium Gravel } \\
\begin{array}{c}9.5 \mathrm{~mm}(3 / 8-\mathrm{in} .) \\
\text { to19 mm (3/4-in.) } \\
(\%)\end{array}\end{array}$ & $\begin{array}{c}\text { Small Gravel } \\
4.75 \mathrm{~mm}(\text { No. 4) to } \\
9.5 \mathrm{~mm}(3 / 8-\mathrm{in} .) \\
(\%)\end{array}$ & $\begin{array}{c}\text { Sand } \\
(\%)\end{array}$ & $\begin{array}{c}\text { Silt } \\
(\%)\end{array}$ & $\begin{array}{c}\text { Clay } \\
(\%)\end{array}$ \\
\hline Crosby Till & 0 & 0 & 12 & 59 & 29 \\
\hline MG-SG-CT50\% & 20 & 30 & 6 & 30 & 14 \\
\hline SG-CT50\% & 0 & 50 & 6 & 30 & 14 \\
\hline MG-SG-CT70\% & 20 & 10 & 9 & 41 & 20 \\
\hline SG-CT70\% & 0 & 30 & 9 & 41 & 20 \\
\hline Concrete Sand & 0 & 0 & 100 & 0 & 0 \\
\hline Stone (Indiana 53) & 20 & 16 & 45 & 8 & 0 \\
\hline Gravel (Indiana 53) & 15 & 19 & 46 & 9 & 0 \\
\hline
\end{tabular}

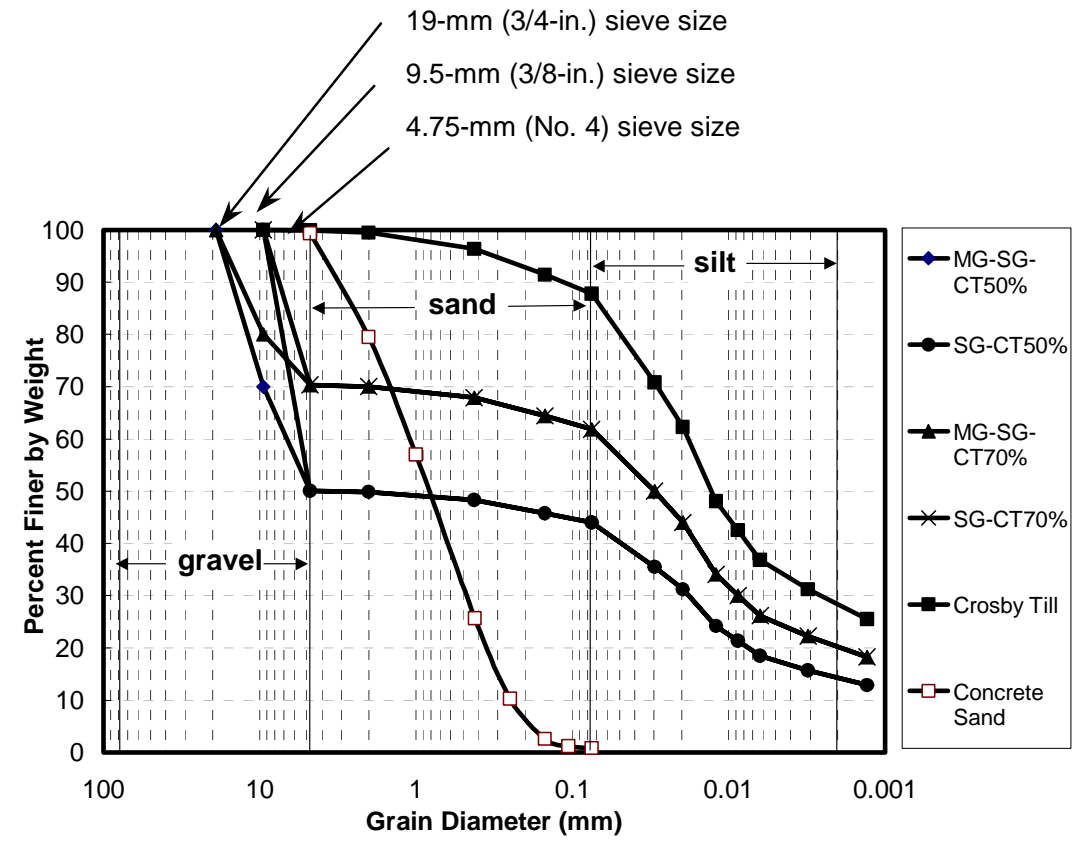

Fig. 8.4a Particle Size Distribution Curves for the Soils

For these tests, a $233.8 \mathrm{~mm}$ (9.168-in.) height mold (twice the height of the mold specified in ASTM D698) was used. The longer length allows for increased resolution of the signal travel time of the electromagnetic wave and allows for testing a volume of soil roughly equal to the volume tested by the insitu probe (Fig. 8.1). 


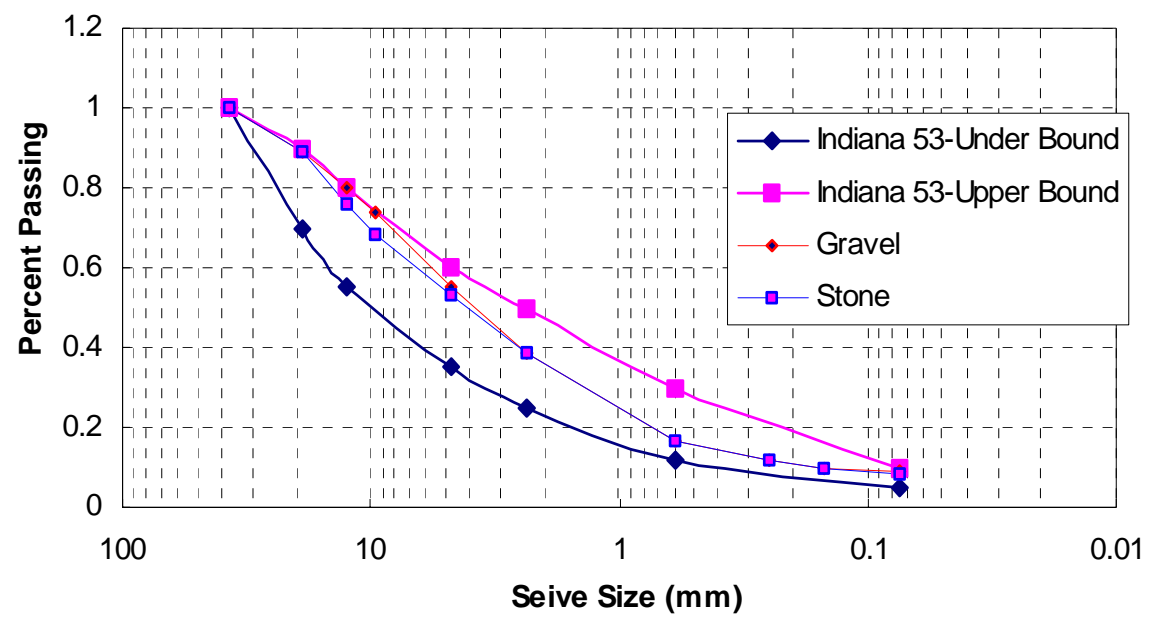

Fig 8.4b Particle Size Distribution of Indiana 53 Soil Test

The mold diameter was $101.2 \mathrm{~mm}$ (4.0-in), which is the size that has been used for nearly all Purdue TDR Method tests to date. The procedures in ASTM D698 limit the use of the $101.2 \mathrm{~mm}$ (4.0-in) diameter mold to particles passing the 9.5-mm (3/8-in.) sieve (MethodB) and require the use of a mold with a $152.4 \mathrm{~mm}(6.0$-in.) diameter for the larger-sized particles. Use of a larger diameter mold for TDR tests was thought to be impractical because two sets of molds would have to be carried to locations in the field to conduct tests. Hence, an attempt was made to see if the smaller diameter, but taller mold would provide reasonably accurate results for the field tests where larger sized particles are frequently encountered.

\subsubsection{Test Methods}

For Crosby Till and the four other specimens containing Crosby Till, five specimens of each of the soils were prepared, one each at a variety of water contents from below optimum to above optimum and allowed to stabilize before compacting into the molds. Compaction was done with a manual rammer according to the procedures in ASTM D698 except that six lifts were used rather than three because the mold height was twice that specified by ASTM D698. This provided the same compaction energy per unit volume as with ASTM D698. Upon completion of the compaction, the mass of the soil-filled mold was determined. This was used along with the mass of the empty mold and the volume of the mold to determine the total density of the soil in the mold. The specimen was prepared for TDR measurement by placing the guide 
on the mold, driving the central rod into the mold, removing the guide, installing the adapter ring on the mold, and placing the MRP head onto the adapter ring.

For the concrete sand and for the mixtures of gravels with the concrete sand, typically only two specimens were prepared, one at low water content and one at a high water content. For the Indiana 53 gravel and stone materials, procedures were identical to those used for Crosby Till.

Measurements were made to obtain the TDR curve and from it determine the locations of the first and second reflections as discussed by Drnevich et al. (2001). These were used along with the length of the central rod that penetrated the soil specimen to determine the apparent dielectric constant for the specimen.

For oven drying with large particle sizes, the entire mold filled with soil was put into the oven to meet the ASTM D2216-98 Standard Test Method for Laboratory Determination of Water (Moisture) Content of Soil and Rock by Mass (ASTM D2216, (1998)) criteria of minimum mass of moist test specimen for water content, $2.5 \mathrm{~kg}(5.5 \mathrm{lb})$.

\subsection{Test Results and Analysis}

\subsubsection{Comparison of Experiment Result by Purdue TDR Method Versus Oven Dry Method}

For each of the materials tested, procedures described by Drnevich et al. (2001) were used to determine the soil dependent parameters $a$ and $b$ for use in the equation proposed by Siddiqui and Drnevich (1995) to calculate water content.

$$
w=\frac{1}{b}\left[\sqrt{K_{a}} \frac{\rho_{w}}{\rho_{d}}-a\right]
$$

where $K_{a}$ is the apparent dielectric constant measured by the TDR method, $\rho_{w}$ is the density of water, and $\rho_{d}$ is the bulk dry density of the compacted soil or soil mixture. To obtain $a$ and $b$ for a given soil or soil mixture, values of $\sqrt{K_{a}} \rho_{w} / \rho_{d}$ are plotted versus oven-dry water contents for the tests at different water contents and fitted with a straight line. The zero-water content intercept of the line is the value of $a$ and the slope of the line is the value of $b$. Values of 
$a$ and $b$ are given for each soil in Table 8.3 along with the $R^{2}$ values for the fit. The values of $a$ and $b$ may be used in Eq. 8.2 to calculate values of water content by the TDR method.

Figure 8.5 shows a comparison of water contents determined by TDR compared to those for oven drying for all of the materials tested in Table 8.3. The comparison is quite good except for

\section{Table 8.3 Values of $a, b$, and $R^{2}$ for Crosby Till and Gravel-Crosby Till Mixtures}

\begin{tabular}{|l|c|c|c|}
\hline Soil Code & $a$ & $b$ & $R^{2}$ \\
\hline Crosby Till & 1.34 & 6.93 & 0.996 \\
\hline MG-SG-CT50\% & 1.07 & 8.28 & 0.972 \\
\hline SG-CT50\% & 1.11 & 7.66 & 0.985 \\
\hline MG-SG-CT70\% & 1.03 & 8.41 & 0.999 \\
\hline SG-CT70\% & 1.14 & 7.29 & 0.958 \\
\hline Concrete Sand & 1.05 & 8.19 & 0.999 \\
\hline Stone (Indiana 53) & 0.97 & 9.058 & 0.997 \\
\hline Gravel(Indiana 53) & 0.77 & 11.259 & 0.978 \\
\hline
\end{tabular}

possibly for water contents between 10 percent and 13 percent in Fig. 8.5. The deviation from straight-line behavior appears to be systematic rather than random. For example, in the procedure to determine values of $a$ and $b$, a plot is made of $\sqrt{K_{a}} \rho_{w} / \rho_{d}$ versus water content as shown in Fig. 8.6. Data points at very low water contents always appear on the line or below it.

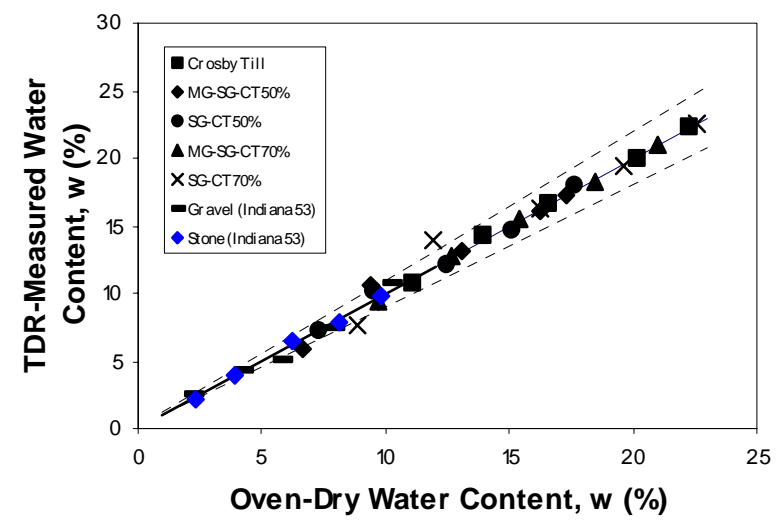

Fig. 8.5. Comparison of TDR-Measured Water Contents with OvenDry-Measured Water Contents

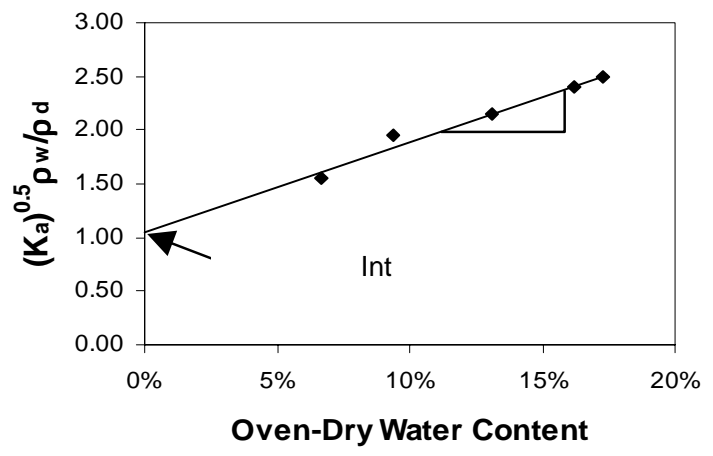

Fig. 8.6. Plot of $\sqrt{K_{a}} \rho_{w} / \rho_{d}$ versus Oven-Dry Water Contents to Obtain $a$ and $b$ 
As water contents increase, the data points then move to above the line and then seem to settle on the line as water contents continue to increase. Data in Fig. 8.6 are fairly consistent with many observations where large particle sizes are tested. There appeared to be a relationship between this behavior and the optimum water content of the soil being tested. To check this out, a normalized plot of data was generated as shown in Fig. 8.7, which includes all of the data for the Crosby Till - gravel mixtures. The abscissa values are normalized by the optimum water content and the ordinate values are the same as in Fig. 8.6, but normalized by $Y_{\text {norm }}$ that is defined as the value of $a+b w_{\text {opt }}$ for each of the soil mixtures tested. The value of $Y_{\text {norm }}$ is the calculated ordinate value at the optimum water content for a given soil. Examination of Fig. 8.7 reveals that the data points consistently fall below the line at 50 to 60 percent of the optimum water content

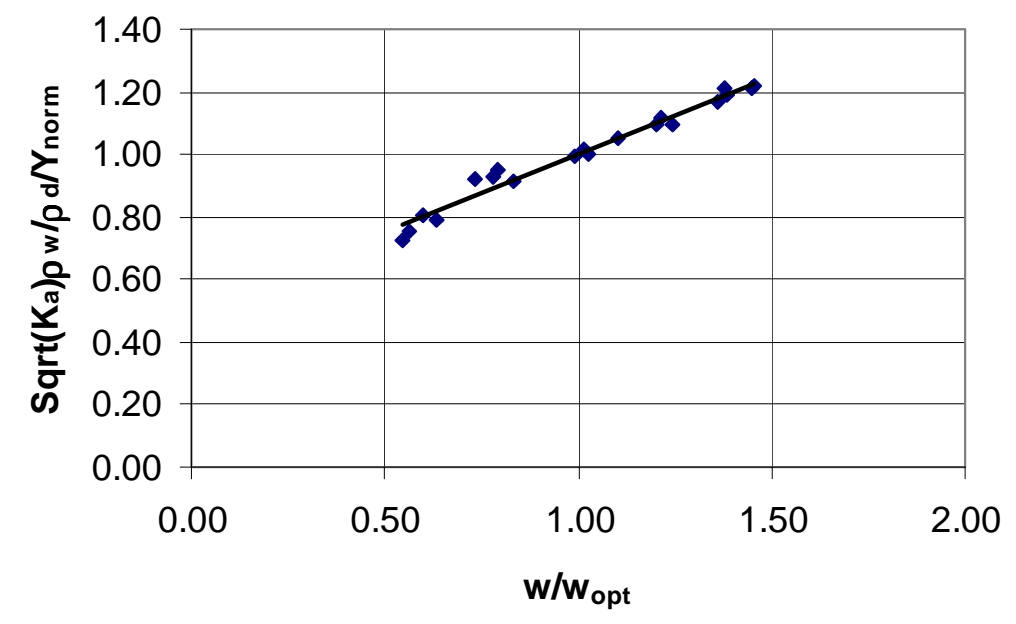

Fig. 8.7. Plot of Normalized Apparent Dielectric Constant versus Water Content Normalized by the Optimum Water Content to Show Behavior of Soil Relative to Standard Compaction Conditions and then consistently fall above the line at 70 to 80 percent of the optimum water content. At and above the optimum water content, the data follow the straight line within experimental error. It is hypothesized that at water contents below the optimum, the soil is quite dense and there is not much water available to lubricate the soil and rock particles as the central rod is

driven into the specimen. The driving of the rod tends to punch into and loosen the soil in the vicinity of the rod. At very low water content, the specimen is not as dense and hence there is a tendency for the rod to densify the soil as it is driven into the specimen. Continued study is warranted on this topic.

For Crosby Till and mixtures of gravels with Crosby Till, the compaction curves are plotted in Fig. 8.8 Values of maximum dry density and optimum water content for each of the 
curves are given in Table 8.4. Note the presence of 70 percent Crosby Till almost totally masks the effects of the gravel on both the maximum dry density and optimum water content.

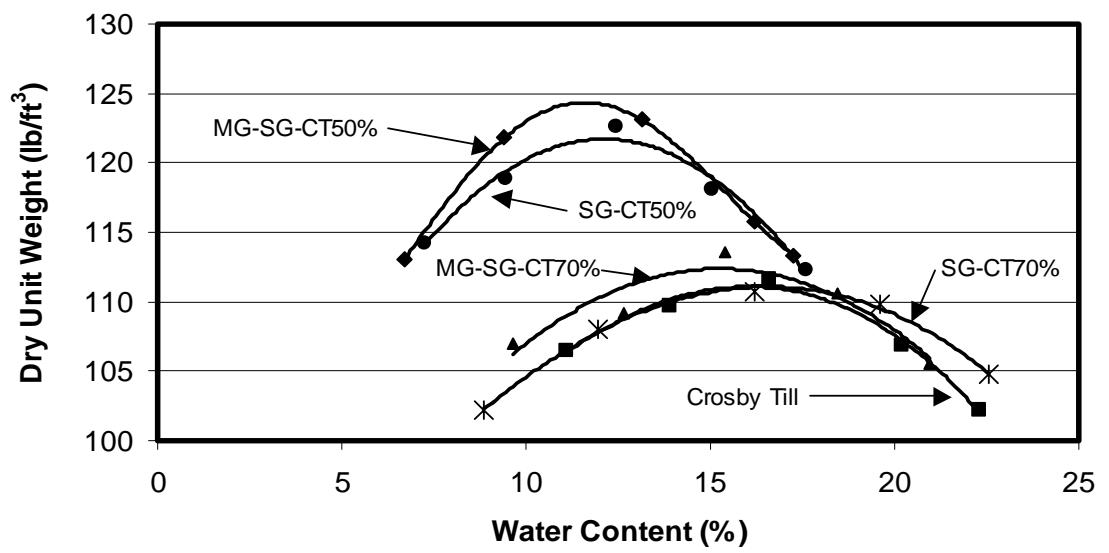

Fig. 8.8. Comparison of Compaction Curves for Crosby Till and Mixtures of Crosby Till with Gravels by Oven Dry Method and Purdue TDR Method

Table 8.4. Optimum Water Contents and Maximum Dry Densities (Unit Weights) for Crosby Till and Mixtures of Gravels with Crosby Till

\begin{tabular}{|c|c|c|c|c|c|c|}
\hline Soil Code & $\begin{array}{c}w_{\text {opt, oven dry }} \\
(\%)\end{array}$ & $\begin{array}{c}w_{\text {opt,TDR }} \\
(\%)\end{array}$ & $\begin{array}{c}\rho_{\text {dmax, oven dry }} \\
\left(\mathrm{Mg} / \mathrm{m}^{3)}\right.\end{array}$ & $\begin{array}{c}\gamma_{\text {dmax, oven } d r y} \\
\left(\mathrm{lbf} / \mathrm{ft}^{3}\right)\end{array}$ & $\begin{array}{c}\rho_{\text {dmax, TDR }} \\
\left(\mathrm{Mg} / \mathrm{m}^{3)}\right.\end{array}$ & $\begin{array}{c}\gamma_{\text {dmax,TDR }} \\
\left(\mathrm{lbf} / \mathrm{ft}^{3}\right)\end{array}$ \\
\hline Crosby Till & 15.8 & 15.5 & 1.781 & 111.1 & 1.772 & 110.6 \\
\hline $\begin{array}{c}\text { MG-SG- } \\
\text { CT50\% }\end{array}$ & 11.9 & 11.3 & 1.983 & 123.7 & 1.952 & 121.8 \\
\hline SG-CT50\% & 12.1 & 12.5 & 1.951 & 121.7 & 1.939 & 121.0 \\
\hline $\begin{array}{c}\text { MG-SG- } \\
\text { CT70\% }\end{array}$ & 15.2 & 15.1 & 1.812 & 113.0 & 1.805 & 112.6 \\
\hline SG-CT70\% & 16.3 & 16.0 & 1.780 & 111.1 & 1.746 & 108.9 \\
\hline $\begin{array}{c}\text { Gravel } \\
\text { (Indiana 53) }\end{array}$ & 6.5 & 6.2 & 2.191 & 136.7 & 2.205 & 137.6 \\
\hline $\begin{array}{c}\text { Stone } \\
\text { (Indiana 53) }\end{array}$ & 5.5 & 5.6 & 2.324 & 145.0 & 2.316 & 144.5 \\
\hline
\end{tabular}

However, decreasing the percentage of Crosby Till to 50 percent markedly increases maximum dry density and decreases the optimum water content. The gravel size, whether a medium gravel 
- small gravel mixture or just all small gravel, appears to have only a minor effect on both the maximum dry density and the optimum water content.

The optimum water contents and maximum dry densities (unit weights) in Table 8.4 were obtained by fitting the compaction curves with parabolas and then solving for the values at the peak. In all tests, the total density of the specimen in the mold was known. Oven-dry water contents were used along with dry densities calculated with oven-dry water contents to obtain the data for the oven-dry items reported in Table 8.4. Similarly, TDR-determined apparent dielectric constants along with soil-specific values of $a$ and $b$ were used to calculate the TDR-determined water contents and these were used along with the total density to calculate the TDR-determined dry densities.

The effects of TDR-determined water contents versus water contents determined by oven drying are shown in Fig. 8.9 for Crosby Till and for a mixture of 50 percent Crosby Till with 30 percent small gravel, and 20 percent medium gravel. The latter is one of the worst case for large

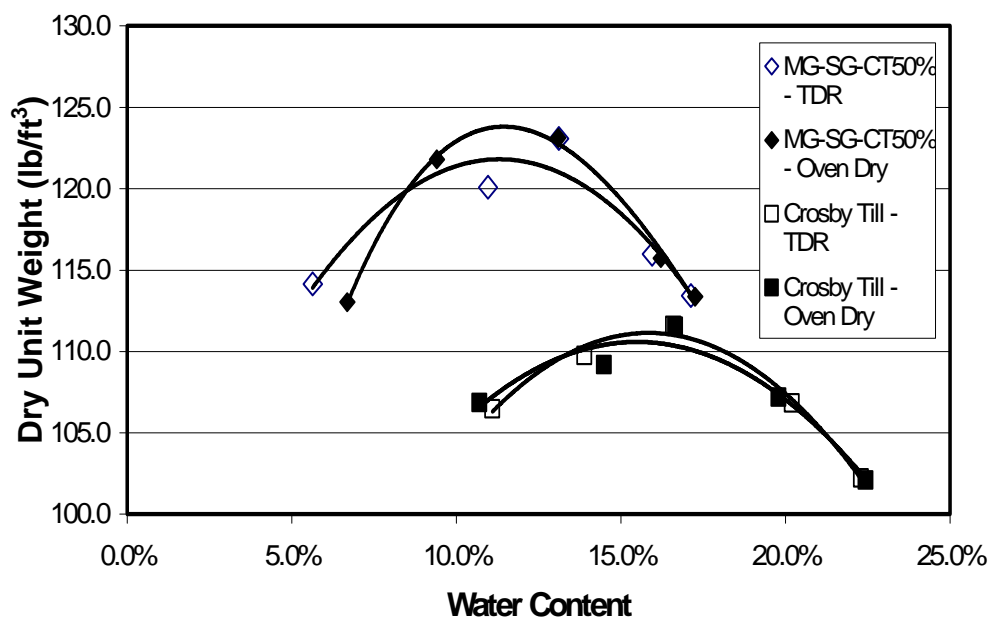

Fig. 8.9 Comparison of Compaction Curves Obtained with Water Contents Determined by OvenDrying to those Obtained with Water Contents Determined by TDR Measurements

particle size tests in this program. Similar information was shown in Fig. 8.10 and Fig. 8.11 for Indiana 53 gravel and stone, respectively.

The data for Crosby Till alone is quite consistent between the two methods. However, the data for the gravel mixture with Crosby Till shows some differences. The TDR water 


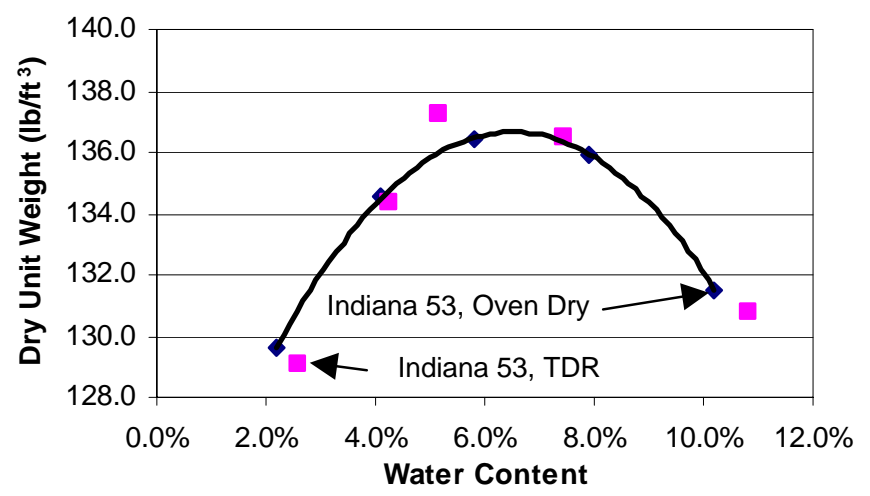

Fig. 8.10. Comparison of Compaction Curves Obtained with Water Contents Determined by Oven Drying to those Determined by Purdue TDR method for Indiana 53 Gravel.

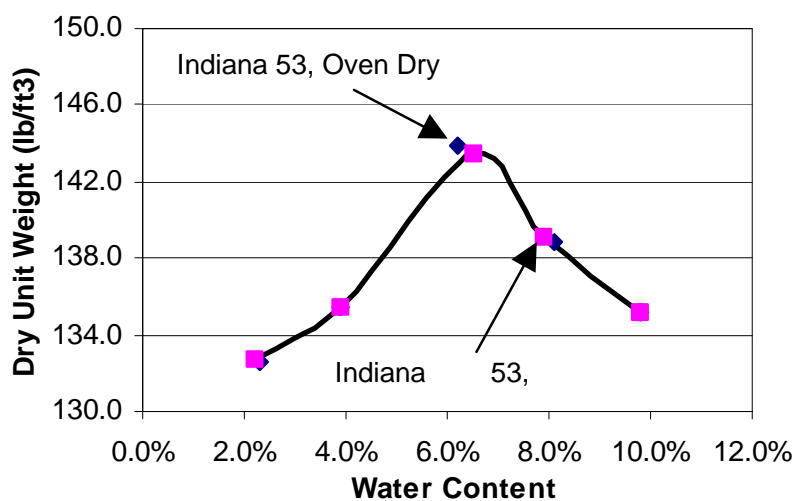

Fig. 8.11 Comparison of Compaction Curves Obtained with Water Contents Determined by Oven Drying to those Determined by Purdue TDR method for Indiana 53 Stone.

contents are too small at the lowest water contents and hence the dry densities are too large. As water contents get closer to the optimum water contents, the TDR water contents are too large and hence the dry densities are too small. Combined, these have the effect of underestimating both the optimum water content and the maximum dry density. For values of water content above the optimum water content, the values are reasonably consistent between the two methods.

For Indiana 53 Gravel and Stone, the compaction curve gives very consistent indication of optimum water content and dry density.

\subsubsection{Evaluation of $a$ from Test of Dry Soils}

For dry soils, $w=0$ and Eq. 8.2 for this situation may be rearranged to provide values of the soil parameter $a$ as shown in Eq. 8.3.

$$
a=\sqrt{K_{a}} \frac{\rho_{w}}{\rho_{d}}
$$

Hence, making a measurement of apparent dielectric constant, $K_{a}$, on a dry soil where the bulk dry density, $\rho_{d}$, is known allows for calculating the value of $a$ directly. To check the accuracy of this, some special tests were performed on concrete sand in the dry state. Two tests were performed on the dry concrete sand in a very loose state. The first test was performed in the usual way by placing the guide on top of the mold containing the sand and driving the central rod into the specimen. A determination of the apparent dielectric constant, $K_{a}$, was made and the 
value of $a$ was calculated by Eq. 8.3. The second test was performed by placing the central rod into an empty mold, holding it aligned with a temporary template and then placing the soil into the annular region between the central rod and the mold wall. Again, a determination of $K_{a}$ was made and the value of $a$ was calculated by Eq. 8.3. The results of these two tests are compared in the first two rows of Table 8.5 and it can be seen that while the densities of the loose materials were slightly different, the values of $a$ are the same.

Table 8.5 Results of Tests on Dry Concrete Sand to Determine Values of $a$

\begin{tabular}{|l|l|c|c|}
\hline \multicolumn{1}{|c|}{ State } & \multicolumn{1}{c|}{ Center Rod } & $\begin{array}{c}\gamma_{d} \\
\left(\mathrm{lbf} / \mathrm{ft}^{3}\right)\end{array}$ & $\boldsymbol{a}$ \\
\hline Loose & Driven & 103.8 & 1.07 \\
\hline Loose & $\begin{array}{l}\text { Installed before } \\
\text { soil placement }\end{array}$ & 102.0 & 1.07 \\
\hline $\begin{array}{l}\text { Very } \\
\text { Dense }\end{array}$ & Driven & 118.1 & 0.99 \\
\hline $\begin{array}{l}\text { Very } \\
\text { Dense }\end{array}$ & $\begin{array}{l}\text { Installed before } \\
\text { soil placement }\end{array}$ & 118.1 & 0.98 \\
\hline
\end{tabular}

Two additional tests were performed in a similar fashion on this concrete sand except that the sand was in a very dense state that was achieved by placing the mold on a vibrating table. The resulting dry densities for these two tests are the same and the values of $a$ are about the same for both tests as shown in the third and fourth rows of Table 8.5. However, the values of $a$ are about eight percent smaller than the values for the soil in the loosest state.

The values of $a$ in Table 8.5 may be compared with the value of 1.05 from Table 3 that was obtained from tests at different water contents with standard compaction energy per unit volume where the density varied depending on the water content.

Some preliminary conclusions may be drawn from these tests. One is that for very loose granular materials, some densification takes place by insertion of the central rod. A second is that inserting the rod in a very dense, dry granular material does not appreciably affect its density. The third is that the value of $a$ is relatively independent of whether the rod is driven into the soil or whether the soil is placed into the mold where the rod is already in place. Finally, it appears 
that Eq. 8.3 does not fully account for density effects on the values of $a$. One possibility of accounting for this would be to look at a slightly modified form of Eq. 8.3 such as:

$$
a^{*}=\sqrt{K_{a}}\left(\frac{\rho_{w}}{\rho_{d}}\right)^{n}
$$

For the data on dry concrete sand, the value of $n$ would have to be approximately 0.4 to accommodate the different densities. Study on this issue continues.

\subsection{Summary, Conclusions, And Recommendations}

The TDR testing of soils will frequently encounter soils containing particle sizes that are large relative to the dimensions of the probe rods. In an effort to assess the effects of these, tests were performed on a naturally occurring soil to which gravel-sized particles were mixed. The maximum particle size in these tests was $19 \mathrm{~mm}$ (3/4-in.). These soils were compacted in a mold with a diameter of 101.6-mm (4.0-in.) and a height of 233.8-mm (9.168-in.) using Standard Compaction Energy. They were tested according to the procedures proposed for the Purdue TDR

Method that includes effects of density and soil type in determining water contents through the use of the Siddiqui-Drnevich Equation that involves two soil-dependent constants, $a$ and $b$. For the soil tested, the dry density and optimum water contents are significantly affected by large particle sizes when the large particle sizes constitute more than about 30 percent of the total sample. The tests indicate that the TDR Method gives reasonably accurate information on the maximum dry density and optimum water content, but that there is a systematic small error in water content values for water contents in the range of 50 - 80 percent of the optimum water content. At water contents above the optimum water content, the accuracy was quite good for all tests. A special series of tests on dry soil specimens of concrete sand were performed to evaluate the effects of rod insertion on the $a$ parameter in the Siddiqui-Drnevich Equation used in the Purdue Method. Values of $a$ were relatively independent of whether the rod was inserted into the soil or whether the soil was placed about the rod. However, there was a small, but consistent difference in the determined value of $a$ depending on the density of the specimen and this 
indicates that the accommodation of density in the Siddiqui-Drnevich equation may need to be refined. Preliminary conclusions from this work indicate that the Purdue TDR Method could be used to obtain reasonably accurate results for soil with large particles where less than 30 percent of the total sample is retained on the $4.75 \mathrm{~mm}$ (No. 4) sieve. Additional work is underway to establish whether this preliminary conclusion is applicable to a wide variety of soils and over a wide variety of densities. 


\section{CHAPTER 9 - EFFECTS OF ADDITIVES}

\subsection{Background}

At the request of the Study Advisory Committee, the research effort was expanded to assess whether the TDR method developed as part of this research would apply to soils that contained stabilizing additives such as lime, fly ash, and cement. Mr. Quanghee Yi was the student who was engaged in doing this work. Guidance on this topic was provided by Dr. Jody Tishmack who has done substantial work on these materials as additives. She currently is Purdue University's Ash Management Coordinator.

\subsection{Some Preliminary Findings}

Preliminary tests on pure lime and fly ash indicated that it was possible to obtain TDR data on these materials in most cases, but that reduced specimen length was necessary because these materials were more lossy than unmodified soils. The more lossy soils dissipate electromagnetic energy more rapidly and no reflected signal is sometimes encountered. By shortening the specimen length, the distance traveled in the specimen is reduced so there is less length over which energy is dissipated. However, with shorter specimen lengths, the travel times are reduced and the accuracy of travel time measurement also reduces.

While preliminary tests were encouraging, we quickly came to realize that each of these materials, when added to soil cause some of the water to become hydrated. The hydration process gives off heat which also affects the dielectric properties. As a consequence of hydration and the exothermic behavior, the measured dielectric constant changes with respect to time as shown in Fig. 9.1 where measured water contents are plotted versus the log of time. The water content reduction noted with time after compacting the soil-fly ash mixture in the mold is believed to be due to hydration. That process gives off heat which builds up and is a possible cause of the hump in the TDR curve. As the heat dissipates, the curve again reduces. Heat build up and reduction was documented by making temperature measurements in the same material contained in a Styrofoam coffee cup with a laboratory thermometer placed directly in the soil and left there for the duration of the test. 


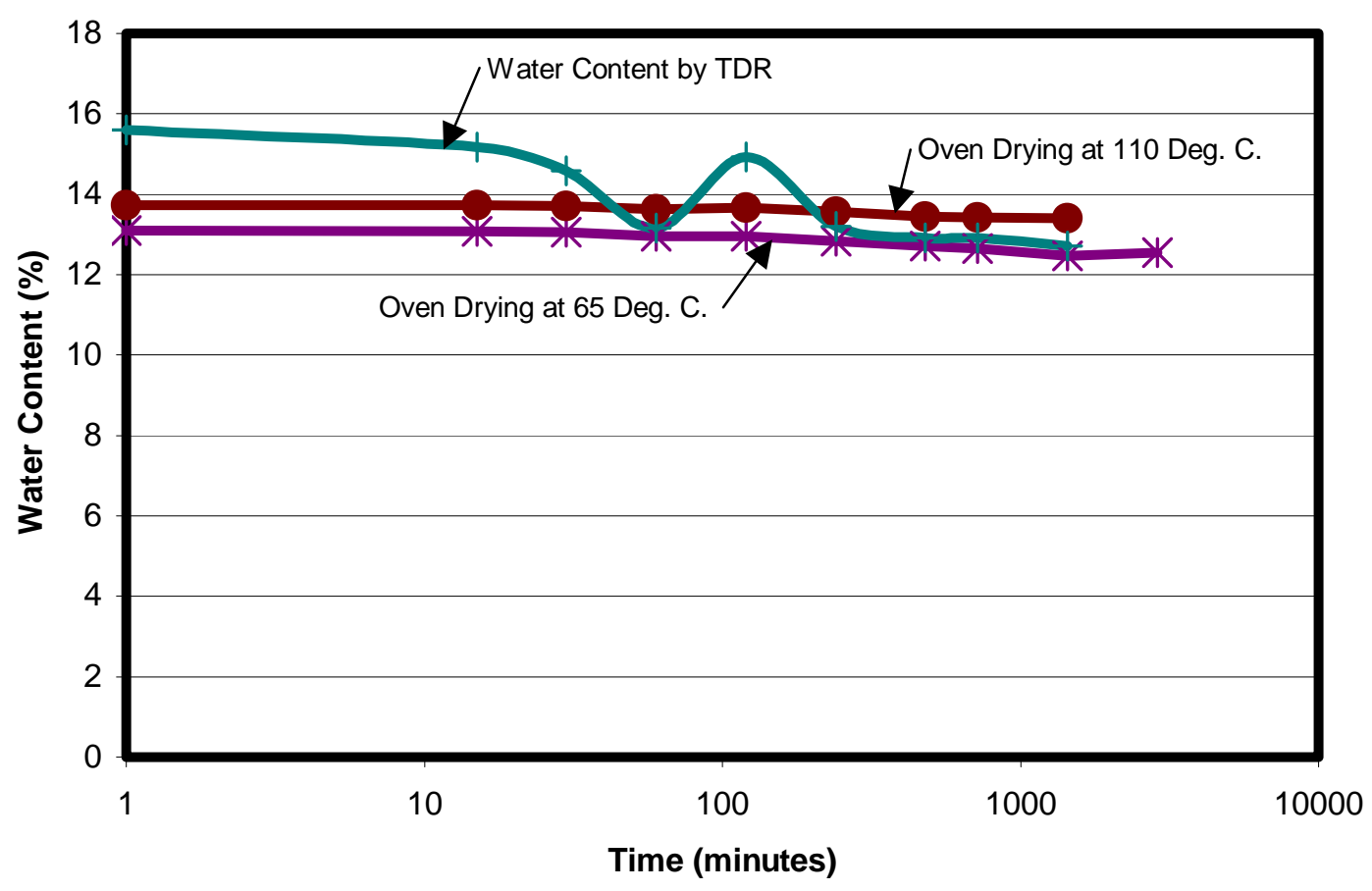

Fig. 9.1 Water Content Comparisons of Fly Ash Stabilized Crosby Till with Oven Drying

Note that for the two oven-dry curves, the specimens were taken from the freshly prepared fly ash-soil mixture at the times noted by the data points, but still required significant time in the oven to dry to a constant weight.

Furthermore, our preliminary tests indicated that values of oven-dried water contents depended on oven temperature. Using the standard oven temperature of $110^{\circ} \mathrm{C}$ gives a higher water content than does using a lower oven temperature such as $65^{\circ} \mathrm{C}$. The difference in ovendried water contents depending on temperature are clearly shown in Fig. 9.1. The difference is expected to vary with type and amount of additive, type of soil, and initial water content.

\subsection{Recommendations}

Water contents by oven-drying of soils modified by cement, lime, and fly ash really cannot provide an accurate description of changes with time of free water availability due to the hydration process because of the drying time required. The TDR method appears to be a good 
candidate for monitoring these changes with time. However, much more work is needed to understand and validate the results from use of the TDR method. This work is continuing in the Beta Testing Project and will be greatly expanded in a future projects that focus on the use of the TDR method for assessing fresh concrete. 


\section{CHAPTER 10 SUMMARY, CONCLUSIONS, AND RECOMMENDATIONS}

\subsection{Summary and Conclusions}

\subsubsection{Fundamental Studies of Electromagnetic Wave Propagation}

The primary objective of this study was to explore the potential use of electromagnetic characteristics of soils using the Time Domain Reflectometry (TDR) technique to identify physical properties of soil. Three fundamental studies in this exploration were the frequencydependent electromagnetic properties of soils, the wave propagation in a TDR system, and the inverse analyses of TDR waveforms. These three fundamental studies provided a sound framework to study the soil properties using time domain reflectometry. The application of these three fundamental studies to the soil dielectric properties indicated the great potential of TDR to develop a non-destructive testing method for soil physical properties.

The fundamental concepts of polarization and dielectric permittivity were highlighted from a macroscopic point of view and their implication with regard to soil dielectric properties examined. The heterogeneity of soils adds to the complexity of their dielectric properties due to interface effects. The three-phase model for soils has been extended to a four-phase model so as to account for interface effects and hence the soil fineness. The physical parameters of the fourphase model are related to the frequency-dependent dielectric permittivity of the soil through a semi-empirical volumetric mixing model.

The TDR basics were reviewed and the insufficiency of current analysis methods was highlighted from the field measurement perspective. The governing wave equations and their limitations were reexamined to obtain a fundamental understanding of transmission lines. A spectral analysis method was developed to simulate wave propagation in a non-uniform and dispersive transmission line. The fast algorithm FFT can implement it very efficiently. This numerical wave propagation model provides a powerful tool for probe design, parametric studies, data interpretation, and inverse analyses.

The application of the TDR technique is an inverse problem. The methods of solving inverse problems were reviewed and a probabilistic framework using the Bayesian statistics was suggested. Gaussian distribution was used in the formulation and the interpretation of the inverse 
solution included the optimal estimator, uncertainty analysis, and resolution analysis. Nonlinear problems were linearized at the optimal estimation of the model parameters for the analysis of uncertainty and resolution.

A TDR probe system was designed to measure the dielectric properties of soils in a compaction mold and in the field. The error and bandwidth of the TDR system were quantified. The developed wave propagation model and inverse theory were used to calibrate the TDR probe system. A layer-peeling algorithm was formulated to preprocess the TDR waveform to remove the multiple reflections in the waveform due to the probe head and result in a waveform of an ideal probe system.

The dielectric spectrum of the material under test can be estimated either by solving the scatter function for each frequency or by inverting the parameters in an equation describing the dielectric spectrum, such as Debye's equation. It was shown that the accuracy of the measurement by solving scattering function for each frequency is limited by the data error at high frequencies and the non-linearity of the scattering function. Inversion of dielectric model parameters is a better approach to measure the dielectric spectrum of the material under test. The Debye equation is an excellent dielectric model for liquids. It also provides an approximate description of dielectric spectra for soils. The wave propagation model validated the simplified analysis of dc conductivity and the apparent dielectric constant obtained by travel-time analysis was found to be associated with the real part of dielectric spectrum at high frequencies. Neural networks were considered, but dismissed as appropriate for use in solving the inverse problem.

A series of tests were conducted to study the soil properties using both the simplified analysis and the full waveform analysis. Empirical calibration equations for apparent dielectric constant in terms of soil physical parameters were evaluated. It was found that none of the empirical equations are universal and calibration is required. Similar to the apparent dielectric constant, a calibration equation for dc conductivity in terms of soil physical parameters is proposed. It is even more sensitive to soil types and measurement error than the apparent dielectric constant.

The volumetric mixing model was evaluated by the full waveform analysis. The results showed a non-uniqueness in inferring the mixing parameters from the TDR waveform. In its 
current form, it could not be used to simultaneously measure soil water content, density, and soil type. However, it is a good model that can match the TDR waveform well and measure the dielectric spectrum of individual soils. Because of the ability to measure dielectric spectrum of soils, the apparent dielectric constant at a particular frequency can be calculated. Results showed that the apparent dielectric constant at $1 \mathrm{GHz}$ gave much better correlation with soil water content and density than apparent dielectric constant obtained by a tangent line approximation in the time domain. The ability to measure dielectric spectra of soils using TDR will assist further study of the dielectric behavior of soils.

\subsubsection{Effects of Temperature, Particle Size and Soil Additives}

This work was done in an expansion to the original project at the suggestion of the Study Advisory Committee. It was of great importance, especially for the follow-on project on Beta Testing of the method.

Temperature of the soil at the time of test has an effect on the measured apparent dielectric constant, and hence, measured water content. The effect is quite small for cohesionless soils and generally can be ignored for test temperatures of $20^{\circ} \mathrm{C} \pm 5^{\circ} \mathrm{C}$. A recommended Temperature Correction Function for use with all cohesionless soils will provide sufficient accuracy for test temperatures between $4^{\circ} \mathrm{C}$ and $40^{\circ} \mathrm{C}$.

This research also confirmed a recently identified "anomaly" in the behavior with temperature for cohesive soils where the apparent dielectric constant increases with temperature rather than decreasing as it does for pure water and cohesionless soils containing water. The source of this behavior is the subject of current research in the TDR community. Work done by Chih-Ping Lin as described in Chapters 2 through 6 should allow for a much for effective study of this phenomena.

Similar to that for cohesionless soils, a Temperature Correction Function also was generated for cohesive soils. This function corrects the values of apparent dielectric constant at the temperature of the soil at the time of test to values at a temperature of $20^{\circ} \mathrm{C}$, assuming that the temperature at the time of test is between $4{ }^{\circ} \mathrm{C}$ and $40^{\circ} \mathrm{C}$. Since the apparent dielectric constant for ice is significantly different from water, the TDR method is not applicable for testing frozen soils. 
For the TDR method to be practical, it must be accurate for wide range of soil types and for soils with larger particle sizes. It was found that the method was applicable for soils having a large particles where less than 30 percent of the total sample is retained on the $4.75 \mathrm{~mm}$ (No. 4) sieve. Additional work is underway to establish whether this preliminary conclusion is applicable to a wide variety of soils and over a wide variety of densities.

Based on earlier work, it was noted that accuracy of the TDR method depends on the central rod having intimate contact with the soil for its entire length. Installation of the central rod may cause some gaps to occur if it impinges on rock fragments and pushes them along as it is driven into the specimen. Effects of this on density tend to counteract the effects of gaps. As a consequence, the method seems to be reasonably accurate for the same range of soil particle sizes for which the laboratory compaction tests and other commonly used field methods for measuring water content and density.

Use of the TDR method for soils with additives appears promising, but is not as simple to address as was originally expected. Introduction of additives like cement, fly ash, and lime generally cause hydration to occur. The hydration process removes some of the free water and it is exothermic. The dielectric properties of hydrated water appear to be different from free water. Because the TDR method is relatively fast and non destructive, it has the ability to monitor changes in the modified soil with time after addition of the additives and compacting the mixture.

\subsection{Recommendations for Further Research}

There is a tremendous potential for the Time Domain Reflectometry to become a powerful non-destructive testing method for soil physical properties. This potential will drive much further research. The three fundamental studies presented in this research will provide a solid framework both for the forward analysis and the inverse analysis in future studies. The followings are some suggestions for further investigation.

1) The TDR Probes developed in this study is subjective to operation error due to the contact between the probe head and the probe. This error does not affect the travel time analysis much but can cause many disturbances in the overall shape and magnitude of the waveform. 
Improvement of the probe design is needed for better measurement of conductivity and full waveform analysis.

2) The apparent dielectric constant at a particular high frequency may result in a universal calibration equation for the current TDR method that can measure the soil water content and density by a single measurement rather than the two measurements in the current procedures. It may also prove to be a powerful way to study temperature effects and effects of soil additives. More research needs to be done to more fully develop these concepts.

3) A more in-depth study of the soil dielectric properties is needed to further explore the use of conductivity and develop a better theoretical mixing model. The method developed in this study for dielectric spectrum measurement using TDR and a systematic experimental program that uses an improved TDR probe will assist in this task.

4) Methodologies developed in this study may be extended to study the properties of layered soils.

5) Procedures for performing the test and analyzing the results could be improved to provide more meaningful information to the engineer.

\subsection{Implementation Efforts}

A follow-on project, SPR 2489, started in September 2000 that will involve twelve agencies/firms/universities around the country in a joint effort to Beta Test the Purdue TDR Method for a variety of soils. A draft ASTM Standard on the method, developed as part of the previous and present projects, is in the balloting process during 2001. A relationship has been established with a major international supplier of TDR equipment to provide custom TDR electronics and software for this method. The Beta Testing project will make use of this equipment. Additional proposals are in the writing stage for further evaluating the TDR method for materials that involve hydration, not only as soil additives, but also in materials such as concrete which was an extension the method suggested by the Study Advisory Committee. 


\section{REFERENCES}

1. Aki, K. and Richards, P. (1980), "Quantitative Seismology," Freeman, San Francisco.

2. Anderson, T. W. (1984), "An Introduction to Multivariate Statistical Analysis," Second Ed., John Wiley \& Sons, New York.

3. Arulanandan, K., and Smith, S. S. (1973), "Electrical Dispersion in Relation to Soil Structure," J. Geotech. Engrg., ASCE, Vol. 99, pp. 1113-1133.

4. $\quad$ ASTM (2001) "Draft Standard Test Method for Water Content and Density of Soil in Place by Time Domain Reflectometry (TDR)," Submitted for D18.08 Committee Ballot, January, $28 \mathrm{p}$.

5. $\quad$ ASTM D422 (1998), "Standard Test Method for Particle-Size Analysis of Soils," Annual Book of ASTM Standards, Vol. 04.08, pp. 10-16.

6. $\quad$ ASTM, D698 (1996), " Standard Test Methods for Laboratory Compaction Characteristics of Soil Using Standard Effort (12,400 ft-lbf/ft3 (600 kN-m/m3))," Annual Book of ASTM Standards, Vol. 04.08, pp. 75-82.

7. ASTM, D1557 (1996), "Standard Test Method for Laboratory Compaction Characteristics of Soil Using Modified Effort (56000 kN-m/m3)," Annual Book of ASTM Standards, Vol. 04.08, pp. 124-131.

8. ASTM D2216 (1998) Standard Test Method for Laboratory Determination of Water (Moisture) Content of Soil and Rock by Mass," Annual Book of ASTM Standards, Vol. 04.08, pp. 188-191.

9. $\quad$ ASTM D2487 (1998), "Standard Classification of Soils for Engineering Purposes (Unified Soil Classification System)," Annual Book of ASTM Standards, Vol. 04.08, pp. 217-238.

10. ASTM, D2922 (1996), "Standard Test Method for Density of Soil and Soil-Aggregate in Place by Nuclear Methods," Annual Book of ASTM Standards, Vol. 04.08, pp. 263-267.

11. ASTM, D3017 (1996), "Standard Test Method for Water Content of Soil and Rock in Place by Nuclear Methods," Annual Book of ASTM Standards, Vol. 04.08, pp. 290-294.

12. ASTM D4318 (1998), "Standard Test Method for Liquid Limit, Plastic Limit, and Plasticity Index of Soils," Annual Book of ASTM Standards, Vol. 04.08, pp. 519-529.

13. Athey, W. T., Stuchly, M. A., and Stuchly S. S. (1982), "Measurement of Radio Frequency Permittivity of Biological Tissues with an Open-Ended Coaxial Line," IEEE Transactions on Microwave Theory and Techniques, Vol. 30, pp. 81-86.

14. Babb, A. T. S. (1951), "A Radio-Frequency Electronic Moisture Meter," Analyst, Vol. 76. 
15. Backus, G. E., and Gilbert, F. (1968), "The resolving power of gross earth data," Geophys. J. R. astr. Soc., Vol. 16, pp. 169-205.

16. Baker, J. M. and Lascano, R. J. (1989), "The Spatial Sensitivity of Time-Domain Reflectometry," Soil Science, Vol. 147, pp. 378-384.

17. Bendat, J. S. and Piersol, A. G. (1993), "Engineering Applications of Correlation and Spectral Analysis," John Wiley \& Sons., New York.

18. Birchak, J. R., Gardner, C. G., Hipp, J. E., and Victor, J. M. (1974), "High Dielectric Constant Microwave Probes for Sensing Soil Moisture," Proc. IEEE, Vol.62, pp. 93-98.

19. Casella, G. and Berger, R. (1990), "Statistical Inference," Wadsworth \& Books, California.

20. Chong, E. K. and Zak, S. H. (1996), "An Introduction to Optimization," John Wiley \& Sons, Inc., New York.

21. Claerbout, J. (1976), "Fundamentals of Geophysical Data Processing," McGraw-Hill, New York.

22. Clarkson, T. S., Glasser, L, Tuxworth, R. W., and Williams, G. (1977), "An Appreciation of Experimental Factors in Time-Domain Spectroscopy," Adv. Mol. Relax. Processes, Vol. 10, pp. 173-202.

23. Dalton, F. N., Herkelrath, W. N., Rawlins, D. S., and Rhoades, J. D. (1984), "Time-domain Reflectometry: Simultaneous Measurement of Soil Water Content and Electrical Conductivity with a Single Probe," Science, Vol. 224, pp. 989-990.

24. Davis, J. L., and Annan, A. P. (1977), "Electromagnetic Detection of Soil Moisture: Progress Report 1," Can. J. Remote Sensing, Vol. 3, No.1, pp. 76-86.

25. De Finetti, B. (1974), "Theory of Probability (Vol. 1)," John Wiley \& Sons, New York.

26. De Finetti, B. (1975), "Theory of Probability (Vol. 2)," John Wiley \& Sons, New York.

27. De Loor, G. P. (1968), "Dielectric Properties of Heterogeneous Mixtures Containing Water," J. Microwave Power, Vol. 3, No. 2, pp. 67-73.

28. Dirkson, C. and Dasberg, S. (1993), "Effects of Bound Water and Bulk Density on Calibration of Soil Water Content Measurements by Time Domain Reflectometry," Soil Sci. Soc. Am. J., Vol. 57, pp. 660-667.

29. Dobson, M.C., Ulaby, F. T., Hallikainen, M. T., and El-Rayes, M. A. (1985), "Microwave Dielectric Behavior of Wet Soil-Part II: Dielectric Mixing Models," IEEE Trans. on Geosci. and Remote Sensing, Vol. 23, No. 1, pp. 35-46. 
30. Drnevich, Vincent P., (2000), "The Purdue Time Domain Reflectometry Method," Geotechnical Engineering Trends in The New Millennium, Central Pennsylvania Section ASCE and the Pennsylvania Department of Transportation, November 1-3, 16 pages.

31. Drnevich, V.P., (2001) "Draft ASTM Standard on Time Domain Reflectometry for In-Place Measurement of Water Content and Density," D18.08.03, ASTM, January, 28 pages.

32. Drnevich, V.P., Lovell, J., and Yi, Q., (2001) "Limitations of the Purdue TDR Method for Soils with Large Particles," Accepted for Second International Conference on Time Domain Reflectometry, Northwestern University, Evanston, September.

33. Drnevich, V.P., Siddiqui, S.I., Lovell, J., and Yi, Q. (2001),"Water Content and Density of Soil Insitu by the Purdue TDR Method," TDR 2001: Innovative Applications of TDR Technology, Infrastructure Technology Institute, Northwestern University, Evanston, IL, September.

34. El-Rayes, M. A. and Ulaby, F. T. (1987), "Microwave Dielectric Spectrum of Vegetation -Part I: Experimental Observations," IEEE Transactions on Geoscience and Remote Sensing, Vol. 25, pp. 541-549.

35. Fellner-Felldegg, J. (1969), "The Measurement of Dielectrics in the Time Domain," J. Phys. Chem., Vol. 73, pp.616-623.

36. Feng, W., Lin, C., Drnevich, V.P., Deschamps, R.J., (1998) "Automation and Standardization of Measuring Moisture Content and Density Using Time Domain Reflectometry," Joint Transportation Research Program Final Report FHWA/IN/JTRP98/4, September, 122 pages.

37. Feng, W. (1999), "Simulation of Time Domain Reflectometry Based on Soil Dielectric Properties," Dissertation in partial fulfillment of the Requirements for the Degree Doctor of Philosophy, Purdue University, May, 163p.

38. Feng, W., Lin, C.P., Deschamps, R.J., and Drnevich, V.P., (1999) "Theoretical Model of a Multi-Section TDR Measurement System," Water Resource Research, Vol. 35, No. 8, Aug., 2321-2331.

39. Frohich, H. (1949). "Theory of Dielectrics," $2^{\text {nd }}$ Ed. The Clarendon Press, Oxford, 180p.

40. Frolik, J. L. and Yagle, A. E. (1997), "Forward and Inverse Scattering for Discrete Layered Lossy and Absorbing Media," IEEE Transaction on Circuits and Systems -- II: Analog and Digital Signal Processing, Vol. 44, No. 9, pp. 710-722.

41. Giese, K. and Tiemann, R. (1975), "Determination of the Complex Permittivity from ThinSample Time Domain Reflectometry: Improved Analysis of the Step Response Wave form," Adv. Mol. Relax. Processes, Vol. 7, pp. 45-59. 
42. Grant, E., Buchanan, T., and Cook, T. (1957), "Dielectric Behavior of Water at Microwave Frequencies," J. Chem. Phys., Vol. 26, pp. 156-161.

43. Hagan, M. T., Demuth, H. B., Beale, M. (1996), "Neural Network Design," PWS Publishing, Boston.

44. Heimovaara, T. J. (1993), "Design of Triple-Wire Time Domain Reflectometry Probes in Practice and Theory," Soil Sci. Soc. Am. J., Vol. 57, pp. 1410-1417.

45. Heimovaara, T. J. (1994), "Frequency Domain Analysis of Time Domain Reflectormetry Waveforms: 1 Measurement of the Complex Dielectric Permittivity of Soils," Water Resources Research, Vol. 30, No. 2, pp. 189-199.

46. Heimovaara, T. J., Bouten, W., and Verstraten, J. M. (1994), "Frequency Domain Analysis of Time Domain Reflectormetry Waveforms: 2. A Four-component Complex Dielectric Mixing Model for Soils," Water Resources Research, Vol. 30, No. 2, pp. 201-209.

47. Heimovaara, T. J., de Winter, E. J. G., van Loon, W. K. P., and Esveld, D. C. (1996), "Frequency-dependency Dielectric Permittivity from 0 to $1 \mathrm{GHz}$ : Time Domain Reflectometry Measurements Compared with Frequency Domain Network analyzer Measurements," Water Resources Research, Vol. 32, No. 12, pp. 3603-3610.

48. Hilhorst, M. A. (1998), "Dielectric Characterization of Soil," Wageningen, Netherlands.

49. Hilhorst, M. A. and Dirkson, C. (1994), "Dielectric Water Content Sensors: Time Domain Versus Frequency Domain," Symposium and Workshop on Time domain Reflectometry in Environmental, Infrastructure, and Mining Applications, Evanston, IL., Spec. Publ. SP 1994, pp. 23-33.

50. Holtz, R. D. and Kovacs, W. D. (1981), "An Introduction to Geotechnical Engineering," Prentice Hall, New Jersey.

51. Hornick, K., Stinchcomebe, M., and White, H. (1989), "Multilayer Feedforward Netwoeks are Universal Approximators." Neural Networks, Vol. 2, pp. 359-366.

52. Jeffrey, L. F., and Yagle, A. E. (1997), "Forward and Inverse Scattering for Discrete Layered Lossy and Absorbing Media," IEEE Transactions on Circuits and Systems. II, Analog and Digital Signal Processing, Vol. 44, pp. 710-722.

53. Klein, L. A., and Swift, C. T. (1977), "An Improved Model for the Dielectric Constant of Sea Water at Microwave Frequencies," IEEE Trans. Antennas Propag., Vol. 25, pp. 104111.

54. Knight, J. H. (1992), "Sensitivity of Time Domain Reflectometry Measurements to Lateral Variations in Soil Water Content," Water Resource Research, Vol. 28, pp. 2345-2352.

55. Krauss, J. D. (1984), "Electromagnetics," McGraw-Hill, New York. 
56. Kuraz, V.(1981). "Testing of a field soil moisture meter." Geotechnical Testing Journal, Vol. 4, No.3, September, pp. 111-116.

57. Ledieu, J., De Ridder, P., De Clerck, P., and Dautrebande, S. (1986), "A Method for Measuring Soil Moisture Content by Time Domain Reflectometry," J. Hydrology, Vol, 88, pp. 319-328.

58. Leviatan, Y. and Adams, A. T. (1982), "The Response of a Two-Wire Transmission Line to Incident Field and Voltage Excitation, Including the Effects of Higher Order Modes," IEEE Trans. on Microwave Theory and Techniques, Vol. 29, pp. 812-817.

59. Feng, W., Lin, C.P., Drnevich, V.P., and Deschamps, R.J., (1998). "Automation and Standardization of Measuring Moisture Content and Density Using Time Domain Reflectometry," Report No.: FHWA/IN/JTRP-98/4, Joint Transportation Research Program, Indiana Department of Transportation - Purdue University, September, 122 p.

60. Lin, C-P. (1999), "Time Domain Reflectometry for Soil Properties," Dissertation in partial fulfillment of the Requirements for the Degree Doctor of Philosophy, Purdue University, August, 226p.

61. Lin, C-P, Siddiqui, S., Feng, W., Drnevich, V. P., and Deschamps, R. J., (2000) "Quality Control of Earth Fills Using Electromagnetic Waves," Constructing and Controlling Compaction of Earth Fills, ASTM STP 1384, D. W. Shanklin, K. R. Rademacher, and J. R. Talbot, Eds., American Society for Testing and Materials, West Conshohocken, PA, pp. 290-310.

62. Lin, C.P., Drnevich, V.P., Feng, W., and Deschamps, R.J., (2000). "Time Domain Reflectometry For Compaction Quality Control," Presented at and publication in Proc. GeoDenver2000, Use of geophysical methods in construction, Soheil Nazarian and John Diehl, Editors, Geotechnical Special Publication No. 108, ASCE GeoInstitute Conference, Denver Colorado, August 5-8, pp. 15-34.

63. Lin, C.P., Drnevich, V.P., Deschamps, R.J., (2001) "Full Waveform Analysis of Nonuniform TDR Measurement Systems: Simulation of TDR Waveforms," Submitted to Water Resources Research, January 2001.

64. Lindell, I. V. (1981), "On the Quasi-TEM Modes in Inhomogeneous Multi-conductor Transmission Lines," IEEE Trans. on Microwave Theory and Techniques, Vol. 23, pp. 753756.

65. Lindley, D. V. (1975), "The Future of Statistics - A Bayesian $21^{\text {st }}$ Century," Supp. Adv. Appl. Prob., Vol. 7, pp. 106-115.

66. Magnusson, P. G., Alexander, G. C., Tripathi, V. K (1992), "Transmission Lines and Wave Propagation," $3^{\text {rd }}$ ed., CRC, Boca Raton.

67. Math Works (1997), "Matlab Optimization Toolbox", Ver. 5, Math Works, Natick, MA. 
68. Menke,W. (1989), "Geophysical Data Analysis: Discrete Inverse Theory", Academic Press, San Diego.

69. Mitchell, J.K. (1993). "Fundamentals of Soil Behavior," $2^{\text {nd }}$ Ed., Wiley, New York, 437p.

70. Mitchell, J. K., and Arulanandan, K. (1968), "Electric Dispersion in Relation to Soil Structure," Journal of Soil Mechanics and Foundations Division, ASCE, Vol. 94, pp. 447471.

71. Mojid, M.A., Wyseure, Guido, C.L., Rose, D.A. (1998), "The Use of Insulated TimeDomain Reflectometry Sensors to Measure Water Content in Highly Saline Soils," Irrigation Science, Vol. 18, pp. 55-61.

72. Nelder, J. A. and Mead, R. (1965), "A simplex Method for Function Minimization," Computer J., Vol. 7, pp. 308-313.

73. Or, D., and Wraith, J.M. (1999), "Temperature Effects on Soil Bulk Dielectric Permittivity Measured by Time Domain Reflectometry: A Physical Model," Water Resources Research, Vol. 35, No. 2, February, pp. 371-383.

74. Papoulis, A. (1991), "Probability, Random Variables, and Stochastic Processes," McGrawHill, New York.

75. Paul, C. R. (1994), "Analysis of Multi-Conductor Transmission Lines," John Wiley Interscience, NY.

76. Poor, H. V. (1988), "An Introduction to Signal Detection and Estimation," Springer-Verlag, New York.

77. Press, W. H., Flannery, P., Teukolsky, S. A., and Vettering, W. T. (1992), "Numerical Recipes in C," $2^{\text {nd }}$ Ed., Cambridge University Press, Cambridge, UK.

78. Prokis, J. G. and Manolakis, D. G. (1992), "Digital Signal Processing: Principles, Algorithms, and Applications," $3^{\text {rd }}$ ed., Prentice Hall, New Jersey.

79. Proponotarios, E. N. and Wing, O. (1967), "Analysis and intrinsic properties of the general non-uniform transmission lines," IEEE Trans. Microwave Theory Tech, Vol. 15, pp. 142150 .

80. Ramo, S., Whinnery, J. R., and Van Duzer, T. (1994), "Fields and Waves in Communication Electronics," $3^{\text {rd }}$ ed., John Wiley, New York.

81. Roth, K., Schulin, H., Fluhler, H.,, and Attinger, W. (1990), "Calibration of Time Domain Reflectometry for Water Content Measurement Using a Composite Dielectric Approach," Water Resource Research, Vol. 26, pp.2267-2273. 
82. Rumelhart, D. E., Hinton, G. E., and Williams, R. J. (1986), "Learning Internal Representations by Error Propagation." In Parallel Distributed Processing, Vol. 1: Foundations, D. E., Rumelhart, and J. L., McClelland, Eds., The MIT Press.

83. Santamarina, J. C. and Fam M. (1997), "Dielectric Permittivity of Soils Mixed with Organic and Inorganic Fluids," Journal of Environmental \& Engineering Geophysics, Vol. 2, No. 1, pp. 37-71.

84. Santamarina, J. D., and Fratta, D. (1997), "Discrete Signals and Inverse Problems In Civil Engineering," ASCE Press, NY.

85. Savage, L. J. (1972), "The Foundations of Statistics," Dover Publications, New York.

86. Scales, J. A. and Snieder, R. (1997), "To Bayes or not to Bayes?" Geophysicas, Vol. 62, pp. 1045-1046.

87. Selig, E. T., and Mansukhani, S. (1975), "Relationship of Soil Water Moisture to the Dielectric Property," J. Geotech, Engrg. Div., Vol. 101, pp. 755-770.

88. Selker, J. S, Graff, L., and Steenhuis, T. (1993), "Non-invasive Time Domain Reflectometry Moisture Measurement Probe," Soil Sci. Soc. Am. J., Vol. 57, pp.934-936.

89. Sen, M, and Stoffa, P.L. (1995), "Global Optimization Methods in Geophysical Inversion," Elsevier, New York.

90. Sen, M, and Stoffa, P.L. (1996), "Bayesian Inference, Gibbs' sampler and uncertainty estimation in geophysical inversion," Geophysical Prospecting, Vol. 44, pp. 313-350.

91. Siddiqui, S.I. (1995), "A New Method of Measuring Density and Moisture Content of Soil Using the Technique of Time Domain Reflectometry," Dissertation in partial fulfillment of the Requirements for the Degree Doctor of Philosophy, Purdue University, December, 253p.

92. Siddiqui, S.I., Drnevich, V.P., (1995) "A New Method of Measuring Density and Moisture Content of Soil Using the Technique of Time Domain Reflectometry," Joint Highway Research Project Final Report FHWA/IN/JHRP-95-9, February 12, 271p.

93. Siddiqui, S.I., Drnevich, V.P., and Deschamps, R., (2000) "Time Domain Reflectometry Development for Use in Geotechnical Engineering," Geotechnical Testing Journal, ASTM, Vol. 23, No. 1, March, pp. 9-20.

94. Sihvola, A. H. and Lindell, I. V. (1992), "Polarizability Modeling of Heterogeneous Media," Progress in Electromagnetics Research (PIER 6), Dielectric Properties of Heterogeneous Materials, Priou, A. (ed.), Elsevier, Amsterdam.

95. Sneddon, I. N. (1951), "Fourier Transforms," McGraw-Hill, NY. 
96. Somlo, P.I., (1967), "The Computation of Coaxial Line Step Capacitances," IEEE Transactions on Microwave Theory and Techniques, Vol. 15, pp. 48-53.

97. Stogryn, A. (1971), "Equations for Calculating the Dielectric Constant of Saline Water," IEEE Trans. Microwave Theory Techn., Vol. 19, pp. 733-736.

98. Tarantola, A. (1987), "Inverse Problem Theory," Elsevier, Amsterdam.

99. Tektronix (1993), "1502B Metallic Time Domain Reflectometry Operator Manul," Tektronix, Inc., Redmond, Oregon.

100. Tinga, W. R. (1992), "Mixture Laws and Microwave-Material Interactions," Progress in Electromagnetics Research (PIER 6), Dielectric Properties of Heterogeneous Materials, Priou, A. (ed.), Elsevier, Amsterdam.

101. Tinga, W. R., Voss, W. A. G., and Blossey, D. F. (1973), "Generalized Approach to Multiphase Dielectric Mixture Theory," J. of Applied Physics, Vol. 44, No. 9, pp. 38973902.

102. Topp, G. C., Davis, J. L., and Annan, A. P. (1980), “Electromagnetic Determination of Soil Water Content and Electrical Conductivity Measurement Using Time Domain Reflectometry," Water Resources Research, Vol. 16, pp. 574-582.

103. Topp, G. C., Yanuka, M., Zebchuk, W. D., and Zegelin, S. (1988), "Determination of Electrical Conductivity Using Time Domain Reflectometry: Soil and Water Experiments in Coaxial Lines," Water Resources Research, Vol. 24, pp. 945-952.

104. Von Hippel, A. (1954), Dielectric Materials and Applications, John Wiley \& Sons, New York

105. Weast, R.C. (1986), CRC Handbook of Chemistry and Physics, 67th ed., CRC Press.

106. Wraith, J.M., and Or, D. (1999), "Temperature Effects on Soil Bulk Dielectric Permittivity Measured by Time Domain Reflectometry: Experimental Evidence and Hypothesis Development," Water Resources Research, Vol. 35, No. 2, February, pp. 361-369.

107. Yagle, A. E., and Levy, B. C. (1983), "Application of the Schur Algorithm to the Inverse Problems for a Layered Acoustic Medium," J. Acous. Soc. Am., Vol. 76, pp. 301-308.

108. Yagle, A. E., and Levy, B. C. (1985), "A Layered-stripping Solution of the Inverse Problem for a One-dimensional Elastic Medium," Geophysics, Vol. 50, pp. 425-433.

109. Yi, Q., Drnevich, V.P., and Lovell, J., (2001), "Effects of Particle Size Distribution on the Purdue TDR Method with Rock and Silty Clay Soil," TDR 2001: Innovative Applications of TDR Technology, Infrastructure Technology Institute, Northwestern University, Evanston, IL, September. 
110. Zegelin, S. J., White, I., and Jenkins, D. R. (1989), "Improved Field Probes for Soil Water Content and Electrical Conductivity Measurement Using Time Domain Reflectometry," Water Resources Research, Vol. 25, pp. 2367- 2376. 


\section{APPENDIX A. List of Presentations Resulting from this Research}

1. "A New Technology for Soil Water Content and Density Measurement for Construction Control", University of Washington, Seattle, April, 1996.

2. "Quality Control of Earth Fills Using Electromagnetic Waves," University of South Florida, September 3, 1999.

3. "Quality Control of Earth Fills Using Electromagnetic Waves," Cincinnati-Dayton Geotechnical Group, ASCE, Cincinnati Ohio, March 7, 2000.

4. "Time Domain Reflectometry for Construction Quality Control," Use of Geophysical Methods in Construction - GeoDenver2000, conference sponsored by the GeoInstitute of ASCE, Denver, CO, August 6, 2000. (with Chih-Ping Lin)

5. Demonstration of Purdue Time Domain Reflectometry Method at Field Demonstration Site associated with GeoDenver2000, conference sponsored by the GeoInstitute of ASCE, Denver, CO, August 8, 2000. (with Chih-Ping Lin)

6. "The Purdue Time Domain Reflectometry Method," Kentucky Geotechnical Engineering Group, ASCE, Frankfort, KY, September 7, 2000.

7. The Purdue Time Domain Reflectometry Method," Central Pennsylvania ASCE/PennDoT Conference, November 3, 2000.

8. "Soil Properties from Electromagnetic Wave Propagation," Graduate Seminar in Civil and Environmental Engineering, Carnegie Mellon University, Pittsburgh, PA, December $3,2000$.

9. "The Purdue Time Domain Reflectometry Method," GAI Consultants, Inc., Monroeville, PA, December 14, 2000.

10. "The Purdue Time Domain Reflectometry Method," Gannett Fleming Consultants, Inc., Pittsburgh, PA, December 19, 2000.

11. "Water Content and Density of Soil in Place by the Purdue Time Domain Reflectometry Method," (made by Barry Newman, GAI Consultants, Inc.), Committee A2K02, Transportation Research Board Meeting, January 9, 2001."Soil Properties from Electromagnetic Wave Propagation," Graduate Seminar in Civil and Environmental Engineering, Geotechnical Seminar, School of Civil Engineering, Purdue University, January 17, 2001."Water Content and Density of Soil in Place by Time Domain Reflectometry (TDR)," Task Group D18.08.03, ASTM, Reno Nevada, January 24, 2001."Water Content And Density Of Soil Insitu by the Purdue TDR Method," TDR 2001: Innovative Applications of TDR Technology, Infrastructure Technology Institute, 
Northwestern University, Evanston, IL, September 2001. (Co-authored with: Siddiqui, S.I., Lovell, J., and Yi, Q.)

15. "Temperature Effects on Dielectric Constant Determined by Time Domain Reflectometry," TDR 2001: Innovative Applications of TDR Technology, Infrastructure Technology Institute, Northwestern University, Evanston, IL, September 2001. (Presented by Xiong Yu, co-authored with Lovell, J., Tishmack, J., and Yu, X.)

16. "Limitations of the Purdue TDR Method for Soils with Large Particles," TDR 2001: Innovative Applications of TDR Technology, Infrastructure Technology Institute, Northwestern University, Evanston, IL, September 2001. (Co-authored with: Yi, Q. and Lovell, J.) 


\section{APPENDIX B. Draft ASTM Standard}

The following 28 pages contain the Draft Standard Test Method for Water Content and Density of Soil in Place by Time Domain Reflectometry (TDR) currently in the balloting process within ASTM. At the D18.08 Subcommittee level, the ballot provided no negative votes and the Subcommittee recommended that the Draft be submitted for both D18 Main Committee Ballot and Society Ballot in the Fall 2001. This same Draft has been submitted to AASHTO for consideration as standard in that organization as well. 
THIS DOCUMENT IS NOT AN ASTM STANDARD; IT IS UNDER CONSIDERATION WITHIN AN ASTM TECHNICAL COMMITTEE BUT HAS NOT RECEIVED ALL APPROVALS REQUIRED TO BECOME AN ASTM STANDARD. IT SHALL NOT BE REPRODUCED OR CIRCULATED OR QUOTED, IN WHOLE OR IN PART, OUTSIDE OF ASTM COMMITTEE ACTIVITIES EXCEPT WITH THE APPROVAL OF THE CHAIRPERSON OF THE COMMITTEE HAVING J URISDICTION AND THE PRESIDENT OF THE SOCIETY. COPYRIGHT ASTM, 100 Barr Harbor Drive, West Conshohocken PA 19428 ALL RIGHTS RESERVED

\title{
Standard Test Method for Water Content and Density of Soil in Place by Time Domain Reflectometry (TDR)
}

\begin{abstract}
This standard is issued under the fixed designation X XXXX; the number immediately following the designation indicates the year of original adoption or, in the case of revision, the year of last revision. A number in parentheses indicates the year of last reapproval. A superscript epsilon $(\varepsilon)$ indicates an editorial change since the last revision or reapproval.
\end{abstract}

\section{Scope}

1.1 This test method may be used to determine the water content of soils and the in-place density of soils using a TDR apparatus.

1.2 This test method applies to soils that have $30 \%$ or less by weight of their particles retained on the 19.0-mm (3/4-in) sieve.

1.3 This test method is suitable for use as a means of acceptance for compacted fill or embankments.

1.4 This method may not be suitable for organic and highly plastic soils.

1.5 Units - The values stated in either SI units or inch-pound units are to be regarded separately as standard. The values stated in each system may not be exact equivalents; therefore, each system shall be used independently of the other. Combining values from the two systems may result in non-conformance with the standard.

\footnotetext{
${ }^{1}$ This test method is under the jurisdiction of ASTM Committee D18 on Soil and Rock and is the direct responsibility of Subcommittee D18.08 Special and Construction Control Tests.

Current edition approved XXX. XX, XXXX. Published XX XXXX.
} 
1.6 All observed and calculated values shall conform to the guidelines for significant digits and rounding established in Practice D 6026."

1.6.1 The method used to specify how data are collected, calculated, or recorded in this standard is not directly related to the accuracy to which the data can be applied in design or other uses, or both. How one applies the results obtained using this standard is beyond its scope.

1.7 This standard does not purport to address all of the safety concerns, if any, associated with its use. It is the responsibility of the user of this standard to establish appropriate safety and health practices and to determine the applicability of regulatory limitations prior to use.

\section{Referenced Documents}

\subsection{ASTM Standards:}

D653 Terminology Relating to Soil, Rock, and Contained Fluids

D698 Test Methods for Moisture-Density Relations of Soils and Soil-Aggregate Mixtures, Using $5.5 \mathrm{lb}(2.49 \mathrm{~kg})$ Rammer and 12-in. (305-mm) Drop

D1556 Test Method for Moisture-Density of Soil In-Place by the Sand-Cone Method

D1557 Test Methods for Moisture-Density Relations of Soils and Soil-Aggregate Mixtures, Using $10 \mathrm{lb}$ (4.54kg) Rammer and 18-in. (457-mm) Drop

D2167 Test Method for Density of Soil In-Place by the Rubber-Balloon Method

D2216 Method for Laboratory Determination of Water (Moisture) Content of Soil, Rock, and SoilAggregate Mixtures

D2922 Test Methods for Density of Soil and Soil Aggregate and Rock in Place by Nuclear Methods (Shallow Depth)

D2937 Test Method for Density of Soil In-Place by the Drive-Cylinder Method 
D3017 Test Method for Water Content of Soil and Rock In-Place by Nuclear Methods (Shallow Depth)

D3740 Practice for Minimum Requirements for Agencies Engaged in the Testing and/or Inspection of Soil and Rock as Used in Engineering Design and Construction

D4643 Method for Determination of Water (Moisture) Content of Soil by the Microwave Oven Method

D4718 Practice for Correction of Unit Weight and Water Content for Soils Containing Oversize Particles

D4753 Specification for Evaluating, Selecting, and Specifying Balances and Scales for Use in Soil and Rock Testing

D4914 Test Method for Density and Unit Weight of Soil and Rock in Place by the Sand Replacement Method

D4944 Test Method for Field Determination of Water (Moisture) Content of Soil by the Calcium Carbide Gas Pressure Tester Method

D4959 Test Method for Determination of Water (Moisture) Content of Soil by Direct Heating Method D5030 Test Methods for Density and Unit Weight of Soil and Rock in Place by the Water Replacement Method

D5080 Standard Test Method for Rapid Determination of Percent Compaction D6026 Practice for Using Significant Digits in Geotechnical Data

E 1 Specification for ASTM Thermometers

E 11 Specification for Wire-Cloth Sieves for Testing Purposes ${ }^{2}$

E 380 Practice for Use of the International System of Units (SI) (the Modernized Metric System) ${ }^{2}$

\footnotetext{
${ }^{2}$ Annual Book of ASTM Standards, Vol. 14.02
} 


\section{Terminology}

3.1 Definitions: Refer to Terminology D653 for standard definitions of terms.

\subsection{Description of Terms Specific to This Standard:}

3.2.2 Apparent length, $l_{a}$ - On a plot of electromagnetic wave signal versus scaled distance measured by a TDR apparatus as shown in Fig. 1, it is the horizontal distance between the point on the waveform due to the reflection from the surface of the soil where the probe is inserted into the soil to the point on the waveform due to the reflection from the end of the probe.

3.2.2 Apparent dielectric constant, $K_{\text {insitu }}, K_{\text {mold }}$ - The squared ratio of the velocity of light in air to the apparent velocity of electromagnetic wave propagation in the soil measured by a TDR apparatus in place and in the cylindrical mold, respectively.

3.2.3 Coaxial Head, $\mathrm{CH}^{3}$ - A device that forms a transition from the coaxial cable connected to the TDR apparatus to the Multiple Rod Probe or to a Cylindrical Mold Probe.

3.2.4 Cylindrical Mold Probe, $C M P^{3}$ - A probe formed by a cylindrical metal mold as the outer conductor having a non-metallic end plate, filled with compacted soil, and with an inner conductor consisting of a rod driven into the soil along the axis of the mold.

3.2.5 Multiple rod probe, $M R P^{3}$ - A probe formed by driving four rods of equal length into the soil in a pattern where three of the rods define the outer conductor of a "coaxial cable" and one of the rods is the inner conductor.

3.2.6 Probe length, $L$ - The length of the TDR probe that is below the surface of the soil.

3.2.7 Scaled distance, $l$ - The product of the velocity of light in air and electromagnetic wave travel time in the soil divided by two.

\footnotetext{
${ }^{3}$ The apparatus is covered by patents. Interested parties are invited to submit information regarding the identification of alternative(s) to this patented item to the ASTM Headquarters. Your comments will receive careful consideration at a meeting of the responsible technical committee, which you may attend.
} 


\section{Summary of Test Method}

4.1 The dielectric constant of the soil in-place is determined using a multiple rod probe (MRP), a coaxial head $(\mathrm{CH})$, and TDR apparatus. The soil at the location of the in-situ measurement is then excavated and compacted in a mold. By measurement of the mass of the mold and soil and with the mass and volume of the mold known, the wet density of the soil in the mold is determined. A rod driven into the soil along the axis of the mold creates a cylindrical mold probe (CMP). Using the same coaxial head $(\mathrm{CH})$, an adapter ring, and the TDR apparatus the dielectric constant of the soil in the mold is measured. The water content of the soil in the mold is determined using a correlation between the dielectric constant, moisture content and soil density. The correlation requires two constants that are somewhat soil specific. It is assumed that the water content of the soil in place is the same as the water content in the mold. The density of the soil in place is determined from the density of the soil in the mold and the dielectric constants measured in the mold and in place.

\section{Significance and Use}

5.1 This test method can be used to determine the density and water content of naturally occurring soils and of soils placed during the construction of earth embankments, road fills, and structural backfills.

5.2 Time domain reflectometry (TDR) measures the apparent dielectric constant of soil. The dielectric constant is affected significantly by the water content and density of soil, and to a lesser extent by the chemical composition of soil and pore water and by temperature.

5.3 Soil and porewater characteristics are accounted for in this method with two calibration constants that are determined for a given soil by performing compaction tests as described in Annex A.2 where water content also is measured by use of ASTM D2216. 
5.4 The water content is the average value over the length of the cylindrical mold and the density is the average value over the length of the multiple-rod probe embedded in the soil.

Note 1 -The quality of the result produced by this standard is dependent on the competence of the personnel performing it, and the suitability of the equipment and facilities used. Agencies that meet the criteria of Practice D 3740 are generally considered capable of competent and objective testing/sampling/inspection/etc. Users of this standard are cautioned that compliance with Practice D 3740 does not in itself assure reliable results. Reliable results depend on many factors; Practice D 3740 provides a means of evaluating some of those factors.

\section{Interferences}

6.1 Quality and accuracy of the test results significantly depend on soil having contact with the inner conductor of the probes. To assist this, when installing the rods of the MRP, the rod that forms the inner conductor must be the last rod installed. If in the installation process, the rod hits upon a large particle that causes it to drift from vertical alignment, all rods should be removed and the test conducted in a new location at least $0.2-\mathrm{m}$ (8-in) from the previous test location.

6.2 The quality of the signal read by the TDR apparatus depends on having clean contacts between the $\mathrm{CH}$ and the MRP and the CMP. The contacting surfaces should be wiped with a clean cloth prior to placing the $\mathrm{CH}$ on the MRP and the CMP. Once placed, observe the signal on the TDR apparatus. If the characteristic signal is not present, the $\mathrm{CH}$ may have to be slightly rotated about its axis to make better contact.

6.3 This test method only applies to non-frozen soil. The apparent dielectric constant is slightly temperature dependent for soils and depends on soil type. For soil temperatures between $15^{\circ} \mathrm{C}$ and $25^{\circ} \mathrm{C}$ $\left(59^{\circ} \mathrm{F}\right.$ and 77$)$, no temperature corrections are needed for most soils. A simple temperature adjustment for water content determination is part of the test method. 


\section{Apparatus}

7.1. TDR apparatus: A Metallic Time Domain Reflectometer with a scaled length resolution of at least 2.4-mm (0.10-in) (this corresponds approximately to a time between data points less than or equal to sixteen picoseconds $\left(16 \times 10^{-12} \mathrm{~s}\right)$. A portable computer with a serial communication port to the TDR is suggested for controlling the apparatus, acquiring and saving the data, and for making the calculations as the test proceeds.

\subsection{Multiple Rod Probe (MRP) $)^{3}$ with Coaxial Head $(\mathrm{CH})^{3}$ :}

7.2.1. The MRP consists of four steel spikes, typically $250-\mathrm{mm}$ (10-in.) in length and uniform diameters of 9.5 -mm (3/8-in.). (Other length spikes, but with the same diameter, may be used but in no case should they have lengths less than 150-mm (6-in.). For lengths longer than 250-mm (10-in.), drift in the alignment of the spikes and loss of reflected signal from the end of the MRP may occur.)

7.2.2. A MRP guide template (See Fig. 2) is used to guide the spikes as they are driven into the soil. The template must allow for its removal after the spikes are driven and before a TDR measurement is made. (The radius from the central spike to the outer spikes must be within the range of 5 to 7.5 times the diameter of the central spike.)

7.2.3. The Coaxial Head $(\mathrm{CH})^{3}$ (See Fig. 3) forms a transition from the coaxial cable coming from the TDR apparatus to the MRP.

7.3. Cylindrical Mold Probe (CMP) ${ }^{3}$ : The CMP consists of a cylindrical mold, a guide template, a central rod, and a ring collar. Details for these items are shown in Fig. 4.

7.3.1. The central rod is a stainless steel rod with a diameter of 8.0-mm (5/16-in.) and a length of 234-mm (9.2-in.) in length. 
7.4. Balances or scales: Meeting Specification GP10 of ASTM D4753 to determine the mass of the soil and the cylindrical mold. A battery-operated balance or scale having a minimum capacity of $10 \mathrm{~kg}$ is suitable when an apparatus with the dimension given in Fig. 3 is used.

7.5. Driving tools: A brass-headed hammer for driving spikes for the MRP and the central rod into the cylindrical mold. A resin-headed hammer also may be used for driving the central rod into the cylindrical mold. (Use of these hammers prevents peening of the driving end of the steel rods from repeated use.)

7.6. Tamping rod: An aluminum rod with flat ends, a diameter of $37-\mathrm{mm}$ (1.5-in.), and a length of 380-mm (15-in.).

7.7. Thermometer: 0 to $50^{\circ} \mathrm{C}$ range, $0.5^{\circ} \mathrm{C}$ graduations, confirming to requirements of Specification $\mathrm{E}$ 1.

7.8. Vernier or Dial Caliper-having a measuring range of at least 0 to $250-\mathrm{mm}$ (0 to 10-in.) and readable to at least $0.02-\mathrm{mm}(0.001-\mathrm{in}$.$) .$

7.9. Miscellaneous tools: A battery-powered hand drill with a spare battery and charger and with a 25$\mathrm{mm}$ (1-in.) diameter auger bit (alternatively, a small pick will work.), straight edge for smoothing the surface of the soil for the in-place test and for smoothing the surface of the soil in the cylindrical mold, pliers for removing the spikes and central rod, small scoop or spoon for removal of the loosened soil and for placement in the cylindrical mold, and a brush for removing excess soil from around the base of the cylindrical mold prior to determining its mass.

\section{Preparation of Apparatus}

8.1 Charge or replace, as appropriate batteries in the TDR apparatus, the hand drill, and the balance.

\section{Calibration and Standardization}


9.1 Determine the average length of the spikes that will penetrate into the soil surface in the in-place test, $L_{\text {insitu }}, \mathrm{m}$ (in.), by inserting each spike into the MRP guide template and measuring the length that each spike protrudes from the template when fully inserted. All measured lengths should be equal to the average length within $0.5-\mathrm{mm}(0.020$-in).

9.2 Determine the volume of the cylindrical mold, $V_{\text {mold }}, \mathrm{m}^{3}$ (in. ${ }^{3}$ ), in accordance with ANNEX A1. VOLUME OF CYLINDRICAL MOLD.

9.3 Determine the mass of the empty and clean cylindrical mold including the base, but without the ring collar, $M_{2}$, $\mathrm{kg}(\mathrm{lbf})$, by placing on a calibrated balance.

9.4 Determine the length of the central rod for insertion into the compaction mold, $L_{\text {central rod, }} \mathrm{m}$ (in.).

9.5 Determine the values of $a$ and $b$ for the soils to be tested in the field by procedures in ANNEX

\section{A.2. DETERMINATION OF PARAMETERS $a$ AND $b$}

\section{Procedure}

10.1 Measure the apparent length in place:

10.1.1 Prepare the surface at the test location so that it is plane and level.

10.1.2 Seat the MRP guide template on the plane surface.

10.1.3 Drive the outer spikes through the guide holes so that the bottom surfaces of the spike heads touch the template. Drive the central spike last. (See Fig. 5)

10.1.4 Remove the template as shown in Fig. 6. Check that all spikes are driven properly without any air gap around the spikes where they penetrate the soil.

10.1.5 Connect the coaxial cable to the $\mathrm{CH}$ and the TDR device. Turn on the device.

10.1.6 Wipe the top surfaces of the spike heads and ends of the studs on the $\mathrm{CH}$ and place the $\mathrm{CH}$ on the spikes, centering the $\mathrm{CH}$ on the heads of all the spikes as shown in Fig 7. 
10.1.7 Determine and record the apparent length, $l_{\text {insitu }}, \mathrm{m}$ (in.) with the TDR equipment $\square$

10.1.8 Remove the spikes using the pliers.

10.2 Measure the apparent length in the cylindrical mold:

10.2.1 Assemble and secure the cylindrical mold to the base plate and attach the ring collar.

10.2.2 With the use of the power drill or other suitable digging implement, dig out soil from the between the holes left by the outer rods of the MRP and to a depth corresponding to the rod penetration and place the soil into the cylindrical mold in 6 uniform lifts applying 10 blows per lift using the aluminum-tamping rod. Soil should be taken uniformly over the entire depth of inplace measurement and placed directly and quickly into the cylindrical mold to minimize moisture loss. Remove the ring collar and strike the surface level with the straight edge after compaction. Remove any spilled soil from around the exterior of the base plate with the brush.

10.2.3 Make sure the balance is leveled, measure and record the mass of the soil-filled cylindrical mold including the base plate, $M_{l}, \mathrm{~kg}$ (lbf).

10.2.4 Mount the cylindrical mold guide template on to the cylindrical mold.

10.2.5 Using the brass-headed or resin-headed hammer, drive the central rod through the guide hole into the soil until the top of rod is flush with the template.

10.2.6 Remove the guide template from the cylindrical mold.

10.2.7 Determine and record the length of the central rod above the soil surface, $L_{\text {rod exposed }}, \mathrm{m}$ (in.).

10.2.8 Place the ring collar on the cylindrical mold.

\footnotetext{
${ }^{4}$ Automated procedures for doing this are usually contained in a program on the portable computer. Algorithms for various procedures are discussed by Baker and Allmaras (1990), Feng et al. (1998), Heimovaara and Bouten (1990), and Wraith, J.M., and Or, D., (1999).
} 
10.2.9 Wipe the top surface of the ring collar, the central rod and the ends of studs of the $\mathrm{CH}$ and then place the $\mathrm{CH}$ on the ring collar, centering the central stud on the central rod as shown in Fig. 8 .

10.2.10 Determine and record the apparent length, $l_{\text {mold }}, \mathrm{m}$, with the TDR device ${ }^{4}$.

10.2.11 Remove the central rod from the mold.

10.2.12 If the soil is a cohesive soil and if the temperature of the soil is estimated to be outside the range of $15^{\circ} \mathrm{C}$ to $25^{\circ} \mathrm{C}\left(59^{\circ} \mathrm{F}\right.$ to $\left.77^{\circ} \mathrm{F}\right)$, insert a metal thermometer into the hole created by the central rod, wait until the temperature stabilizes, and record the temperature, ${ }^{\circ} \mathrm{C}$.

10.2.13 Remove the soil from the cylindrical mold.

\section{Calculation or Interpretation of Results}

11.1 Calculate the apparent dielectric constant of the soil in place as follows:

$$
K_{\text {insitu }}=\left(\frac{l_{\text {insitu }}}{L_{\text {insitu }}}\right)^{2}
$$

where

$K_{\text {insitu }}=$ apparent dielectric constant of the soil in place,

$l_{\text {insitu }}=$ measured apparent length insitu, $\mathrm{m}$ (in.),

$L_{\text {insitu }}=$ length of the spikes inserted into the soil, m (in.).

11.2 Calculate the dielectric constant of the soil in the mold as follows:

$$
K_{\text {mold }}=\left(\frac{l_{\text {mold }}}{L_{\text {mold }}}\right)^{2}
$$

where

$K_{\text {mold }}=$ apparent dielectric constant of soil in the mold, $l_{\text {mold }}=$ measured apparent length in the mold, m (in.), 


$$
\begin{aligned}
L_{\text {mold }} & =\text { length of the rod inserted into the soil in the mold, } \mathrm{m} \text { (in.) } \\
& =L_{\text {central rod }}-L_{\text {rod exposed }}
\end{aligned}
$$

11.3 Calculate the wet density of the soil in mold as follows:

$$
\rho_{t, \text { mold }}=\frac{M_{1}-M_{2}}{V_{\text {mold }}}
$$

where

$\rho_{t, \text { mold }}=$ wet density of the soil in the mold, $\mathrm{kg} / \mathrm{m}^{3}\left(\mathrm{lbf} / \mathrm{ft}^{3}\right)$,

$M_{1}=$ mass of the soil-filled mold, and base plate, $\mathrm{kg}$ (lbf),

$M_{2}=$ mass of the empty mold and base plate, $\mathrm{kg}$ (lbf),

$V_{\text {mold }}=$ volume of the mold, $\mathrm{m}^{3}\left(\mathrm{ft}^{3}\right)$.

11.4 Calculate the Apparent Dielectric Constant of the soil in the mold at $20^{\circ} \mathrm{C}$ from:

$$
K_{\text {mold }, 20^{\circ} \mathrm{C}}=K_{\text {mold }, T^{\circ} \mathrm{C}} \times \mathrm{TCF}
$$

where

$T C F=$ Temperature Correction Factor

$=0.97+0.0015 T_{\text {mold }, T^{\circ} \mathrm{C}}$ for cohesionless soils, $4^{\circ} \mathrm{C} \leq T_{\text {mold }, T^{\circ} \mathrm{C}} \leq 40^{\circ} \mathrm{C}$

$=1.10-0.005 T_{\text {mold }, ~} T^{\circ} \mathrm{C}$ for cohesive soils, $4^{\circ} \mathrm{C} \leq T_{\text {mold, }} T^{\circ} \mathrm{C} \leq 40^{\circ} \mathrm{C}$.

11.5 Calculate the water content of the soil in the mold and in place as follows

$w_{\text {insitu }}=w_{\text {mold }}=\frac{\sqrt{K_{\text {mold }, 20^{\circ} \mathrm{C}}}-a \rho_{t, \text { mold }} / \rho_{w}}{b \rho_{t, \text { mold }} / \rho_{w}-\sqrt{K_{\text {mold }, 20^{\circ} \mathrm{C}}}} \times 100$

where

$w_{\text {mold }}=$ water content of the soil in the mold, $\%$,

$w_{\text {insitu }}=$ water content of the soil in place, $\%$,

$\rho_{w}=$ density of water $=1000 \mathrm{~kg} / \mathrm{m}^{3}\left(62.4 \mathrm{lbf} / \mathrm{ft}^{3}\right)$,

$a=$ calibration constant, (See Annex A.2),

$b=$ calibration constant, (See Annex A.2). 
11.5 Calculate the in place dry density of the soil as follows:

$\rho_{d, \text { insitu }}=\frac{\sqrt{K_{\text {insitu }}}}{\sqrt{K_{\text {mold }}}} \times \frac{\rho_{t, \text { mold }}}{1+w_{\text {mold }} / 100}$

where

$\rho_{d, \text { insitu }}=$ dry density of the soil in place, $\mathrm{kg} / \mathrm{m}^{3}\left(\mathrm{lbf} / \mathrm{ft}^{3}\right)$.

\section{Report}

12.1 The report shall include the following:

12.1.1 Test site identification.

12.1.2 Date and time of test.

12.1.3 Name of the operator(s).

12.1.4 Make, model and serial number of the TDR apparatus.

12.1.5 Average length of the spikes that penetrated into the soil surface in the in-place test, $L_{\text {insitu }}$, m (in.),

12.1.6 Volume of the cylindrical mold, $V_{\text {mold }}, \mathrm{m}^{3}\left(\mathrm{ft}^{3}\right)$.

12.1.7 Length of the central rod, $L_{c e n t r a l ~ r o d}, \mathrm{~m}$ (in.), the length of the central rod exposed, $L_{\text {rod }}$ exposed, $\mathrm{m}$ (in.), and inserted length of the central rod in the mold, $L_{\text {mold }}, \mathrm{m}$ (in.).

12.1.8 Temperature of the soil in the mold, $T_{m o l d, T^{\circ} \mathrm{C}}$.

12.1.9 Visual description of material tested.

12.1.10 Values of apparent dielectric constant for the in-place test, $K_{\text {insitu }}$, and the test in the mold, $K_{\text {mold }}$

12.1.11 Water content in percent, $w$, from $\mathrm{Eq}(5)$. 


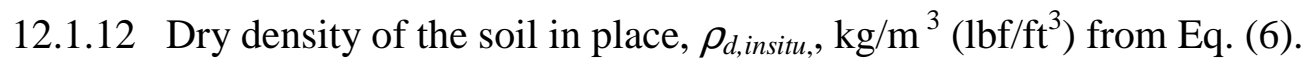

12.1.13 Calibration constants $a$ and $b$.

12.1.14 Other comments as appropriate.

\section{Precision and Bias}

13.1 Precision - Test data on precision is not presented due to the nature of this test method. It is either not feasible or too costly at this time to have ten or more agencies participate in an in situ testing program at a given site. The Subcommittee (D18.08) is seeking any data from the users of this test method that might be used to make a limited statement on precision.

13.2 Bias-There is no accepted reference values for this test method, therefore, bias cannot be determined.

\section{Keywords}

14.1 compaction test; construction control; density; dielectric constant; electrical permittivity; electromagnetic waves; field control; field tests; inspection; moisture content; quality control; soil compaction; soil density, time domain reflectometry, water content 


\title{
ANNEX
}

\section{(Mandatory Information)}

\section{A1. VOLUME OF CYLINDRICAL MOLD}

\begin{abstract}
A1.1 Scope
A1.1.1 This annex describes the procedure for determining the volume of a compaction mold.

A1.1.2 The volume is determined by a water-filled method and checked by a linearmeasurement method.
\end{abstract}

\section{A1.2 Apparatus}

A1.2.1 In addition to the apparatus listed in Section 7 the following items are required:

A1.2.1.1 Vernier or Dial Caliper-having a measuring range of at least 0 to $250-\mathrm{mm}$ (0 to 10 -in.) and readable to at least $0.02-\mathrm{mm}(0.001-\mathrm{in}$.$) .$

A1.2.1.2 Inside Micrometer - having a measuring range of at least 100 to 150 -mm (4 to 5-in.) and readable to at least $0.02-\mathrm{mm}$ (0.001-in.).

A1.2.1.3 Plastic or Glass Plates_-Two plastic or glass plates approximately 200 by 200-mm by 6 -mm thick ( 8 -in. by 8 -in. by $1 / 4$-in. thick).

A1.2.1.4 Thermometer -0 to $50^{\circ} \mathrm{C}$ range, $0.5^{\circ} \mathrm{C}$ graduations, conforming to the requirements of Specification E 1 .

A1.2.1.5 Stopcock grease or similar sealant.

A1.2.1.6 Miscellaneous equipment-Bulb syringe, towels, etc. 


\section{A1.3 Precautions}

A1.3.1 Perform this method in an area isolated from drafts or extreme temperature fluctuations.

\section{A1.4 Procedure}

\section{A1.4.1 Water-Filling Method:}

A1.4.1.1 Lightly grease the bottom of the compaction mold and place it on one of the plastic or glass plates. Lightly grease the top of the mold. Be careful not to get grease on the inside of the mold. If it is necessary to use the base plate, place the greased mold onto the base plate and secure with the locking studs.

A14.1.2 Determine the mass of the greased mold and either plastic or glass plates to the nearest 1-g (0.01-lbm) and record. When the base plate is being used in lieu of the bottom plastic or glass plate determine the mass of the mold, base plate and a single plastic or glass plate to be used on top of the mold to the nearest $1-\mathrm{g}(0.01-\mathrm{lbm})$ and record.

A1.4.1.3 Place the mold and the bottom plastic or glass plate on a firm, level surface and fill the mold with water to slightly above its rim.

A1.4.1.4 Slide the second plate over the top surface of the mold so that the mold remains completely filled with water and air bubbles are not entrapped. Add or remove water as necessary with a bulb syringe.

A1.4.1.5 Completely dry any excess water from the outside of the mold and plates.

A1.4.1.6 Determine the mass of the mold, plates and water and record to the nearest 1-g (0.01-lbm). 
A144.1.7 Determine the temperature of the water in the mold to the nearest $1^{\circ} \mathrm{C}$ and record. Determine and record the absolute density of water from Table A1.1.

A 1.4.1.8 Calculate the mass of water in the mold by subtracting the mass determined in A1.4.1.2 from the mass determined in A1.4.1.6.

A1.4.1.9 Calculate the volume of water by dividing the mass of water by the density of water and record to the nearest $1 \mathrm{~cm}^{3}\left(0.0001 \mathrm{ft}^{3}\right)$.

A1.4.1.10 When the base plate is used for the calibration of the mold volume repeat A1.4.1.3A1.4.1.9.

\section{A1.4.2 Linear Measurement Method:}

A1.4.2.1 Using either the vernier caliper or the inside micrometer, measure the diameter of the mold 6 times at the top of the mold and 6 times at the bottom of the mold, spacing each of the six top and bottom measurements equally around the circumference of the mold. Record the values to the nearest $0.02-\mathrm{mm}(0.001-\mathrm{in}$.$) .$

A1.4.2.2 Using the vernier caliper, measure the inside height of the mold by making three measurements equally spaced around the circumference of the mold. Record values to the nearest 0.02-mm (0.001-in.).

A1.4.2.3 Calculate the average top diameter, average bottom diameter and average height.

A1.4.2.4 Calculate the volume of the mold and record to the nearest $1 \mathrm{~cm}^{3}\left(0.0001 \mathrm{ft}^{3}\right)$ as

follows:

$$
\mathrm{V}=\frac{\pi\left(d_{t}+d_{b}\right)^{2} h}{16} \times\left(\frac{1 f t^{3}}{1728 \mathrm{in}^{3}}\right) \text { (inch-pound system) }
$$




$$
\mathrm{V}=\frac{\pi\left(d_{t}+d_{b}\right)^{2} h}{16} \times\left(\frac{1 \mathrm{~m}^{3}}{10^{9} \mathrm{~mm}^{3}}\right)(\mathrm{SI})
$$

where:

$$
\begin{array}{lll}
V & = & \text { Volume of mold, } \mathrm{m}^{3}\left(\mathrm{ft}^{3}\right), \\
h & = & \text { Average height, } \mathrm{mm} \text { (in.), } \\
d_{t} & = & \text { Average top diameter, mm. (in), } \\
d_{b} & = & \text { Average bottom diameter, mm. (in), }
\end{array}
$$

\section{A1.5 Comparison of Results}

A1.5.1 The volume obtained by either method should be within the volume tolerance requirements of 6.1.1 and 6.1.2.

A1.5.2 The difference between the two methods should not exceed $0.5 \%$ of the nominal volume of the mold.

A1.5.3 Repeat the determination of volume if these criteria are not met.

A1.5.4 Failure to obtain satisfactory agreement between the two methods, even after several trials, is an indication that the mold is badly deformed and should be replaced.

A1.5.5 Use the volume of the mold determined using the water-filling method as the assigned volume value for calculating the wet density (see 11.3).

\section{A2 DETERMINATION OF PARAMETERS $a$ AND $b$}

\section{A2.1 Scope}

A2.1.1 This annex describes the procedure for determining the soil specific parameters $a$ and $b$ for use in Eq. (5) of Section 11. 
A2.1.2 The determination requires that five tests at different water contents be performed using the cylindrical mold probe.

\section{A2.2 Apparatus}

A2.2.1 See items 7.1, 7.3, 7.4, 7.5, 7.6, 7.7, and 7.9.

\section{A2.3 Precautions}

A2.3.1 Perform this method in an area where the ambient temperatures and the temperature of the soil are within the range of $15^{\circ} \mathrm{C}$ to $25^{\circ} \mathrm{C}\left(59^{\circ} \mathrm{F}\right.$ to $\left.77^{\circ} \mathrm{F}\right)$.

\section{A2.4 Procedure}

A2.4.1 Air-dry the soil sample.

A2.4.2 Thoroughly break up the soil clumps in such a manner as to avoid breaking individual particles. Pass the material through a No. 4 sieve.

A2.4.3 Prepare five specimens having water contents such that they bracket the estimated field water content and vary by about $2 \%$. Preparation procedure should be as specified in Section 10.2 or 10.3 of ASTM D 698

A2.4.4 Determine the volume of the cylindrical mold in accordance with ANNEX A.1, VOLUME OF CYLINDRICAL MOLD.

A2.4.5 Determine and record the mass of the cylindrical mold and base plate, and the length of the central rod.

A2.4.6 Assemble and secure the cylindrical mold and ring collar to the base plate.

A2.4.7 Place the soil into cylindrical mold in 6 uniform lifts applying 10 blows per lift using the aluminum-tamping rod. Remove the ring collar and strike the surface level with the 
straight edge after compaction. Remove any soil from around the exterior of the base plate with the brush.

A2.4.8 Do procedure 10.2.3 to 10.2.13

A2.4.9 Remove the soil from the cylindrical mold. Obtain a portion of the sample for water content determination by slicing the compacted specimen axially through the center and removing at least $0.500 \mathrm{~kg}(1 \mathrm{lbf})$ of soil from the cut faces. Obtain the water content in accordance with Test Method D2216.

A2.4.10 Repeat A2.5.5 to A2.5.8 for each soil specimen.

\section{A2.5 Calculation}

A2.5.1 For each of the soil specimens, calculate the dielectric constant of soil in the cylindrical mold $\left(K_{\text {mold }}\right)$ using Eq. (2).

A2.5.2 Calculate water content, $w_{\text {oven }}$ ry, in accordance with Test Method D2216.

A2.5.3 Calculate the wet density of the soil in the cylindrical mold using Eq. (3).

A2.5.4 Calculate the dry density of the soil in the cylindrical mold as follows:

$$
\rho_{d}=\frac{\rho_{t}}{1+w_{\text {ovendry }} / 100}
$$

where:

$\rho_{d}=$ dry density of the soil in the cylindrical mold, $\mathrm{kg} / \mathrm{m}^{3}\left(\mathrm{lbf} / \mathrm{ft}^{3}\right)$,

$\rho_{t}=$ wet density of the soil in the cylindrical mold, $\mathrm{kg} / \mathrm{m}^{3}\left(\mathrm{lbf} / \mathrm{ft}^{3}\right)$,

$w_{\text {oven } d r y}=$ oven dry water content from step A2.5.2, \%. 
A2.5.5 Calculate the calibration coefficients as follows:

Plot $\sqrt{K_{\text {mold }}} \frac{\rho_{w}}{\rho_{d}}$ vs. $w_{\text {oven } d r y}$

where:

$K_{\text {mold }}=$ dielectric constant of soil in the cylindrical mold.

$\rho_{d}=$ dry density of the soil in the cylindrical mold, $\mathrm{kg} / \mathrm{m}^{3}\left(\mathrm{lbf} / \mathrm{ft}^{3}\right)$, from Eq. (A2.1)

$\rho_{w}=$ density of water $=1000 \mathrm{~kg} / \mathrm{m}^{3}\left(62.4 \mathrm{lbf} / \mathrm{ft}^{3}\right)$,

$w_{\text {oven } d r y}=$ oven dry water content, $\%$, from step A2.4.2.

A2.5.6 Find the zero intercept and slope of the best-fit straight line where:

$a=$ zero intercept of the best-fit straight line

$b=$ slope of the best-fit straight line

Note: Values of $\boldsymbol{a}$ typically are between 0.7 and 1.3 and values of $\boldsymbol{b}$ are typically between 7.5 and 11 for commonly encountered natural soils. 


\section{APPENDIX}

\section{A3 REFERENCES}

1. Baker, J.M., and Allmaras, R.W., (1990) "System for Automating and Multiplexing Soil Moisture Measurement by Time-Domain Reflectometry," Journal of the Soil Science Society of America, Vol. 54, pp. 1-6.

2. Feng, W., Lin, C.P., Drnevich, V.P., and Deschamps, R.J., (1998). "Automation and Standardization of Measuring Moisture Content and Density Using Time Domain Reflectometry," Report No.: FHWA/IN/JTRP-98/4, Joint Transportation Research Program, Indiana Department of Transportation - Purdue University, September, $122 \mathrm{p}$.

3. Feng, W., Lin, C.P., Deschamps, R.J., and Drnevich, V.P., (1999). "Theoretical Model of a Multi-Section TDR Measurement System," Water Resource Research, Vol. 35, No. 8, Aug., pp. 2321-2331.

4. Heimovaara, T.J., and Bouten, W., (1990), "A Computer-Controlled 36-Channel Time Domain Reflectometry System for Monitoring Soil Water Contents," Water Resources Research, Vol. 26, pp. 2311-2316.

5. Lin, C.P., Drnevich, V.P., Feng, W., and Deschamps, R.J., (2000). "Time Domain Reflectometry For Compaction Quality Control,' Presented at and publication in Proc. GeoDenver2000, Use of geophysical methods in construction, Soheil Nazarian and John Diehl, Editors, Geotechnical Special Publication No. 108, ASCE GeoInstitute Conference, Denver Colorado, August 5-8, pp. 15-34.

6. Lin, C-P., Siddiqui, S. I., Feng, W., Drnevich, V. P., and Deschamps, R. J., (2000). "Quality Control of Earth Fills Using Electromagnetic Waves," Constructing and Controlling Compaction of Earth Fills, ASTM STP 1384, D. W. Shanklin, K. R. Rademacher, and J. R. Talbot, Eds., American Society for Testing and Materials, West Conshohocken, PA, pp. 290310.

7. Siddiqui, S.I., and Drnevich, V.P., (1995). “A New Method of Measuring Density and Moisture Content of Soil Using the Technique of Time Domain Reflectometry," Report No.: FHWA/IN/JTRP-95/9, Joint Transportation Research Program, Indiana Department of Transportation - Purdue University, February, 271 p.

8. Siddiqui, S.I., Drnevich, V.P., and Deschamps, R.J., (2000). "Time Domain Reflectometry Development for Use in Geotechnical Engineering," Geotechnical Testing Journal, GTJODJ, Vol. 23, No. 1, March, pp. 9-20. 


\section{泡》 D XXXX}

9. Wraith, J.M., and Or, D., (1999) "Temperature Effects on Soil Bulk Dielectric Permittivity Measured by Time Domain Reflectometry: Experimental Evidence and Hypothesis Development," Water Resources Research, Vol. 35, No. 2, February, pp. 361369. 


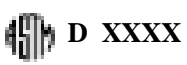

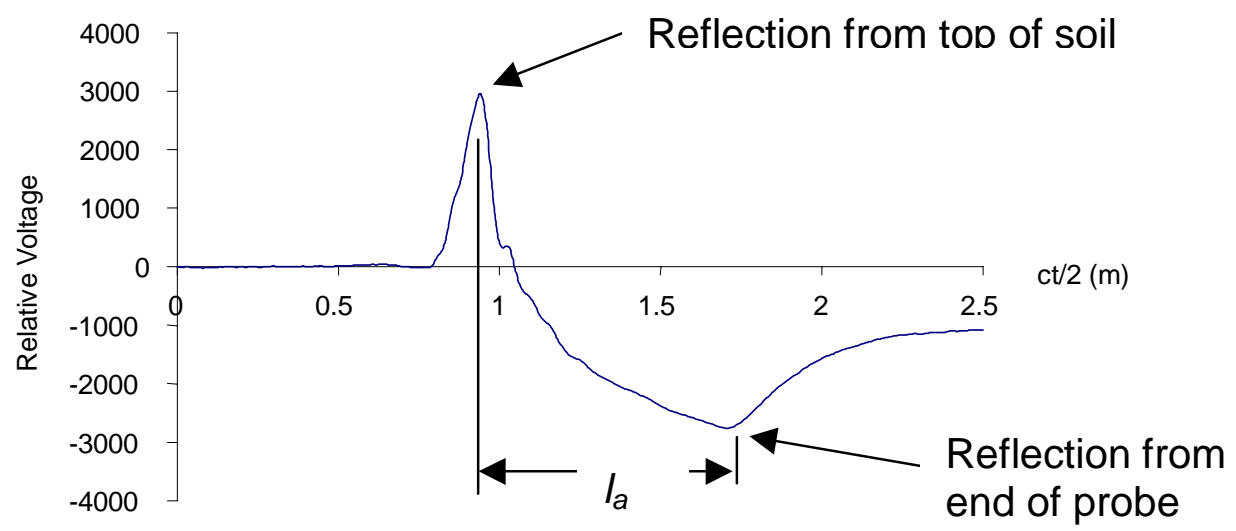

Fig. 1. Typical TDR Curve Showing Reflection Points and Apparent Length, $I_{a}$. 


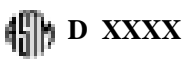

Fig. 2. MRP Guide Template
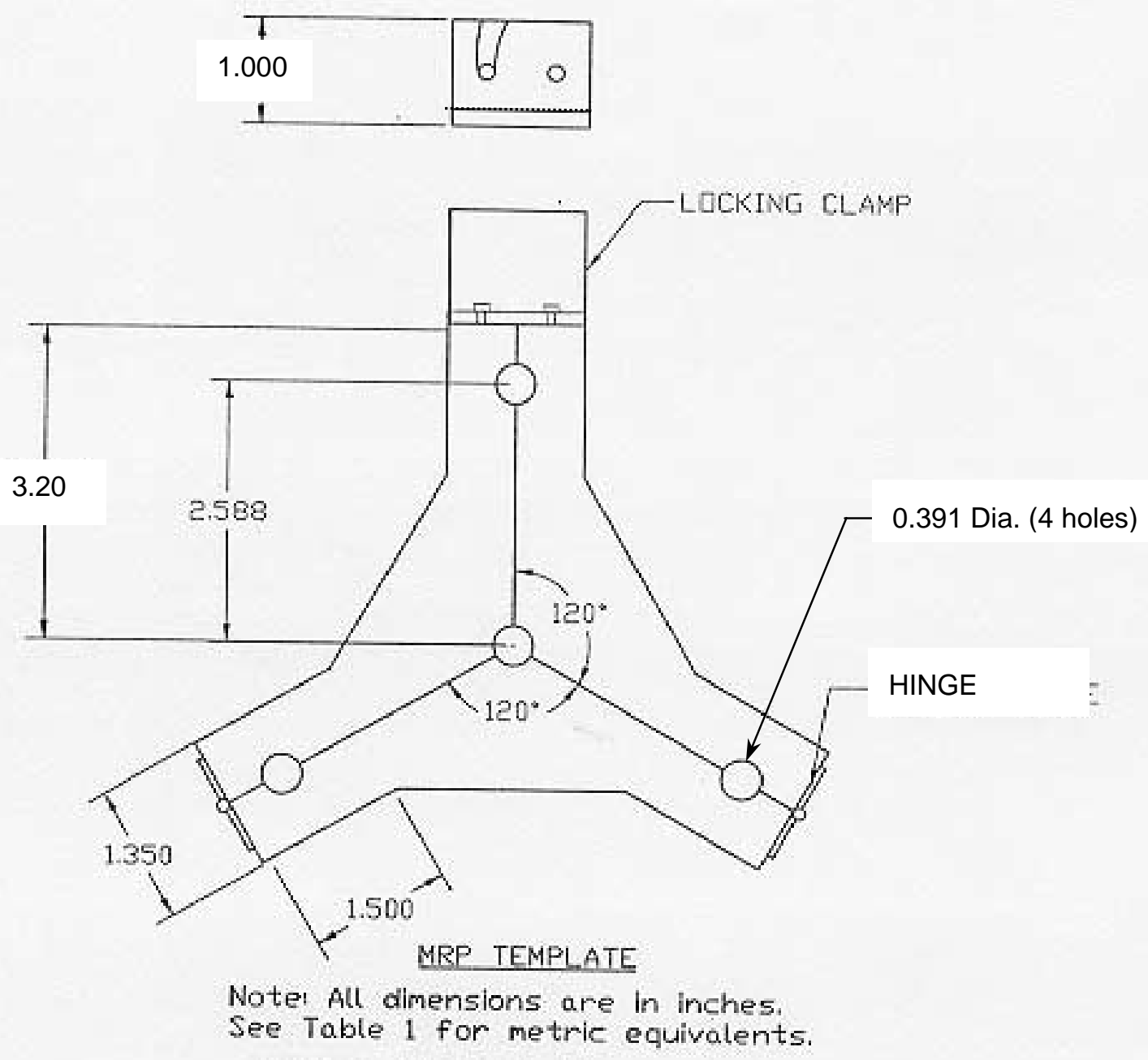
D XXXX

Fig. 3. Coaxial Head $(\mathrm{CH})$

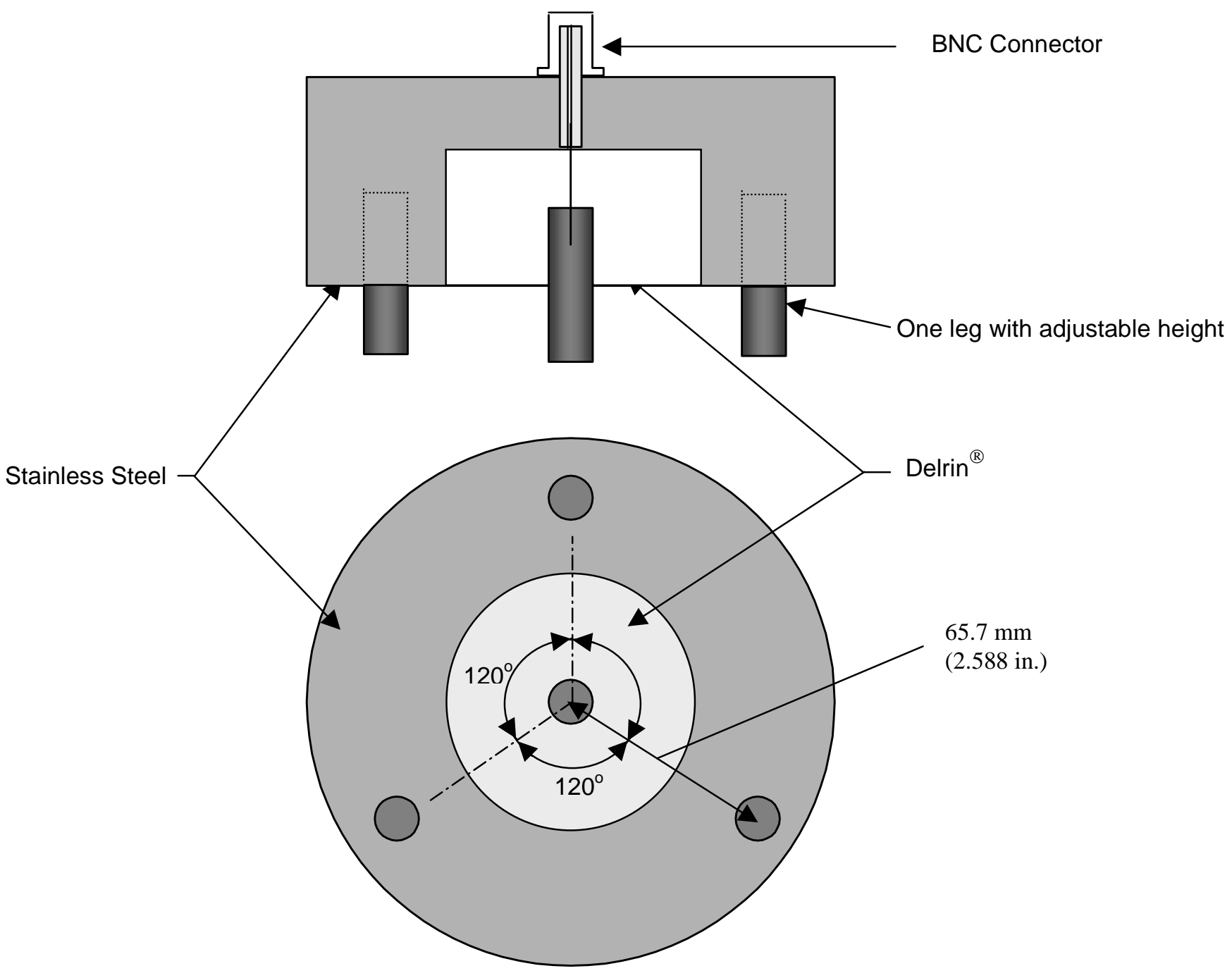


Fig. 4. Cylindrical Mold, Ring Collar, and Guide Template

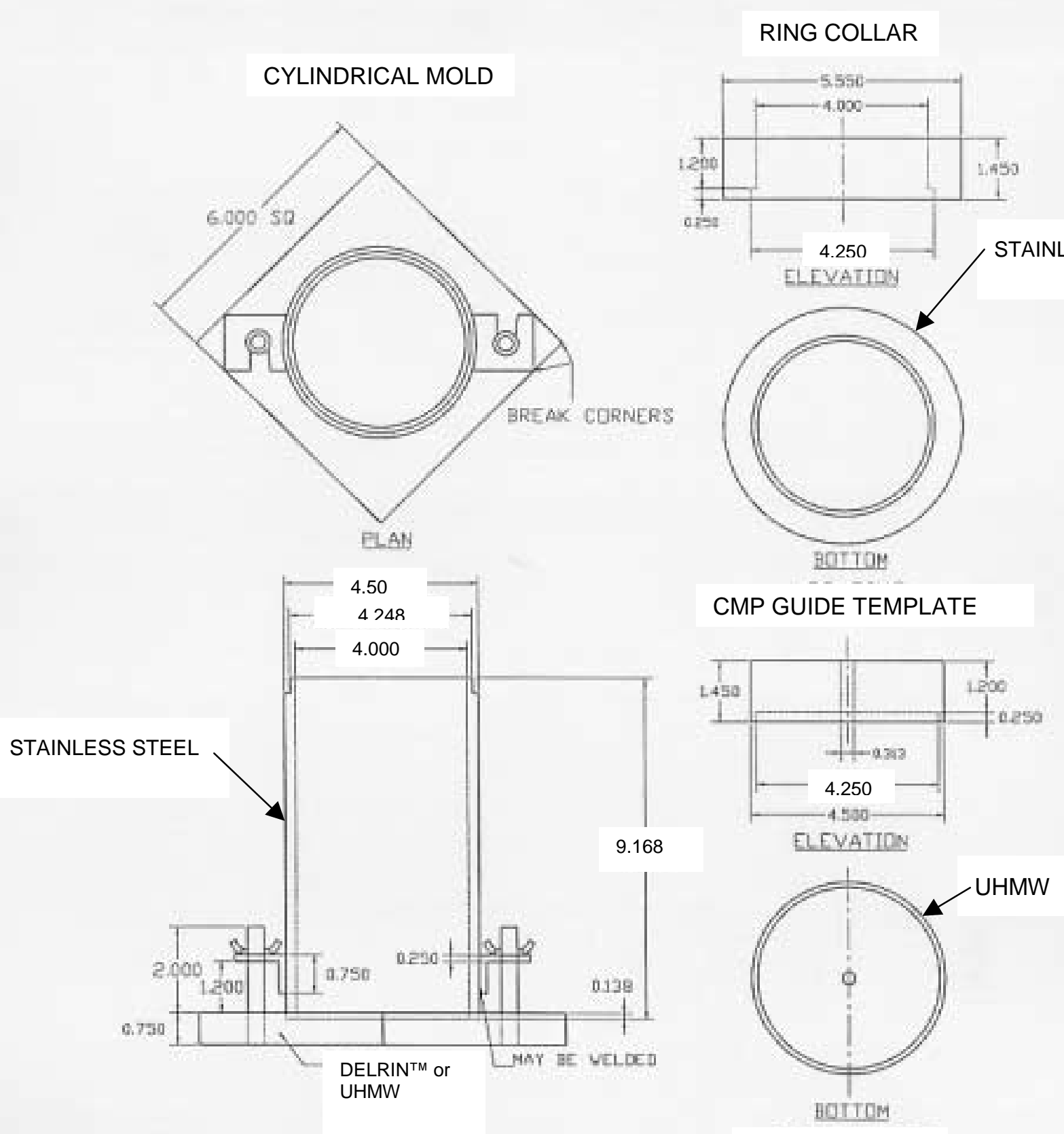

Note: All dimensions are in inches. See Table 2 for metric equivalents. 
Table 1. Metric Equivalents for Dimensions in Fig. 2.

\begin{tabular}{|r|l|c|l|}
\hline (in.) & Tol. (in.) & (mm) & Tol. $(\mathrm{mm})$ \\
\hline 0.391 & \pm 0.002 & 10.00 & \pm 0.05 \\
\hline 1.000 & \pm 0.005 & 25.00 & \pm 0.15 \\
\hline 1.350 & \pm 0.015 & 34.30 & \pm 0.40 \\
\hline 1.500 & \pm 0.015 & 38.00 & \pm 0.40 \\
\hline 2.588 & \pm 0.005 & 65.70 & \pm 0.13 \\
\hline 3.200 & \pm 0.020 & 80.00 & \pm 0.50 \\
\hline
\end{tabular}

Table 2. Metric Equivalents for Dimensions in Fig. 4.

\begin{tabular}{|c|c|c|c|}
\hline (in.) & Tol. (in.) & (mm) & Tol. $(\mathrm{mm})$ \\
\hline $0.138 \pm 0.005$ & $3.50 \pm 0.13$ \\
\hline $0.250 \pm 0.005$ & $6.30 \pm 0.13$ \\
\hline $0.313+0.002,-0.000$ & $7.88+0.05,-0.00$ \\
\hline $0.750 \pm 0.010$ & $18.90 \pm 0.25$ \\
\hline $1.000 \pm 0.010$ & $25.00 \pm 0.25$ \\
\hline $1.200 \pm 0.002$ & $30.24 \pm 0.05$ \\
\hline $1.450 \pm 0.005$ & $36.54 \pm 0.13$ \\
\hline $2.000 \pm 0.020$ & $50.00 \pm 0.50$ \\
\hline $4.000 \pm 0.016$ & $100.00 \pm 0.40$ \\
\hline $4.248+0.000,-0.003$ & $107.90+0.00,-0.08$ \\
\hline $4.250+0.003,-0.000$ & $107.95+0.08,-0.00$ \\
\hline $4.500 \pm 0.020$ & $115.00 \pm 0.50$ \\
\hline $5.500 \pm 0.020$ & $140.00 \pm 0.50$ \\
\hline $6.000 \pm 0.020$ & $150.00 \pm 0.50$ \\
\hline $9.168 \pm 0.020$ & $231.00 \pm 0.50$ \\
\hline
\end{tabular}




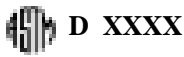

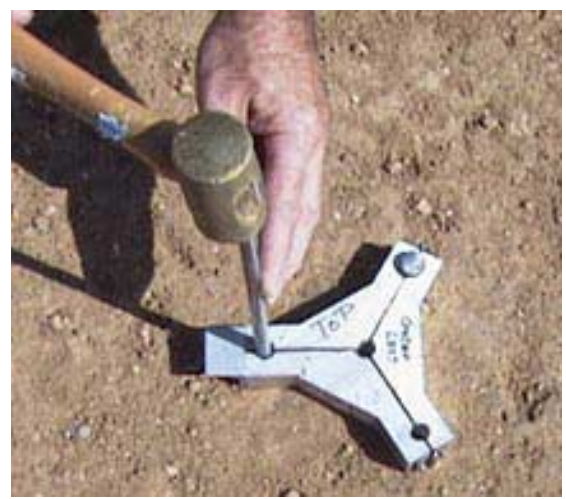

Fig. 5. Driving Spikes Through Template

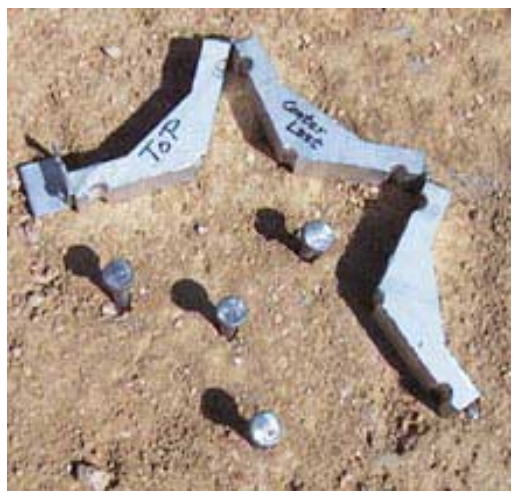

Fig. 6. Removal of Template After Driving Spikes

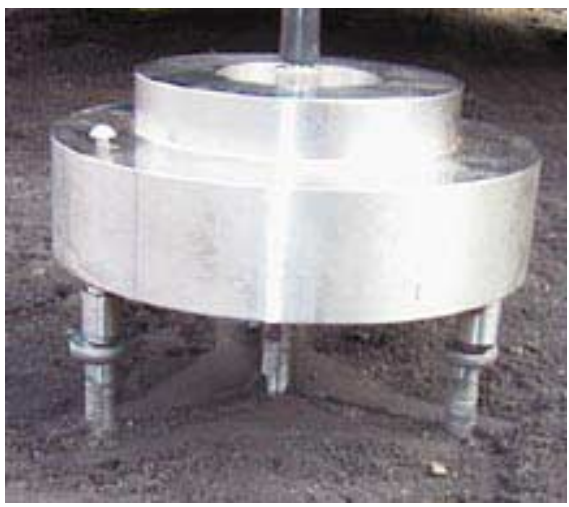

Fig. 7. Placement of Coaxial Head on Spikes 
物的 D XXXX

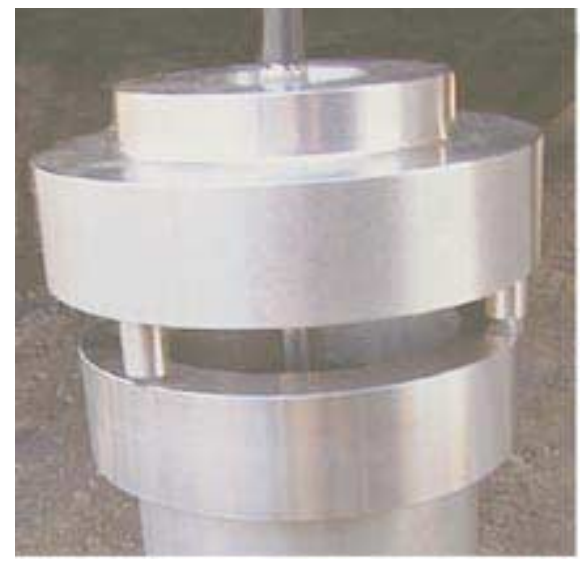

Fig. 8. Coaxial Head on Ring Collar 